

\section{Tank Characterization Report for Double-Shell Tank 241-AN-104}

T. A. Hu

Lockheed Martin Hanford Corp., Richland, WA 99352

U.S. Department of Energy Contract DE-ACO6-87RL10930

EDT/ECN: EDT-617664

Org Code: 74620

B\&R Code: EW 3120074
UC: 2070

Charge Code: N4G4C

Total Pages: $3 / 2$

Key Words: Waste Characterization, Double-Shell Tank, DST, Tank 241-AN104. Tank AN-104, AN-104. AN Farm, Tank Characterization Report, TCR, Waste Inventory, TPA Mi Testone M-44

Abstract: This document summarizes the information on the historical uses, present status, and the sampling and analysis results of waste stored in Tank 241-AN-104. This report supports the requirements of the Tri-Party Agreement Mi Testone M-44-10.

TRADEMARK DISCLAIMER. Reference herein to any specific commercial product, process, or service by trade name, trademark, manufacturer, or otherwise, does not necessarily constitute or imply its endorsement, recommendation, or favoring by the United States Government or any agency thereof or $i$ ts contractors or subcontractors.

Printed in the United States of America. To obtain copies of this document, contact: WHC/BCS Document Control Services, P.O. Box 1970, Mailstop H6-08, Richland WA 99352, Phone (509) 372-2420; Fax (509) 376-4989.
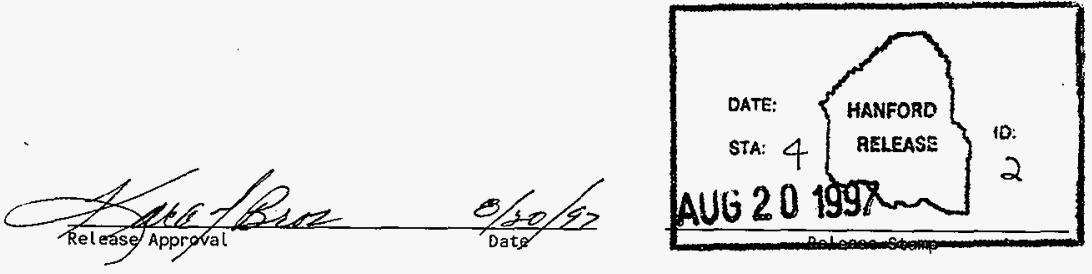


\section{Tank Characterization Report for Double-Shell Tank 241-AN-104}

T. A. Hu

Lockheed Martin Hanford Corporation

L. W. Shelton, Jr.

Numatec Hanford Corporation

T. L. Welsh

B\&W Protec, Inc.

Date Published

August 1997

Prepared for the U.S. Department of Energy

Assistant Secretary for Environmental Management

Project Hanford Management Contractor for the

U.S. Department of Energy under Contract DE-AC06-95RL13200

Approved for public release; distribution is unlimited 


\section{CONTENTS}

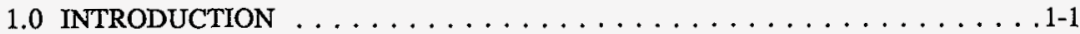

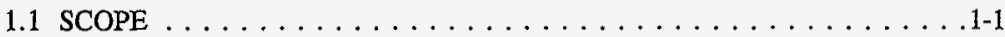

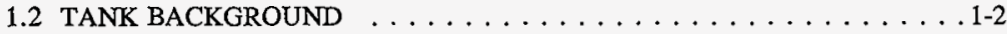

2.0 RESPONSE TO TECHNICAL ISSUES $\ldots \ldots \ldots \ldots \ldots \ldots \ldots \ldots \ldots \ldots . \ldots \ldots$

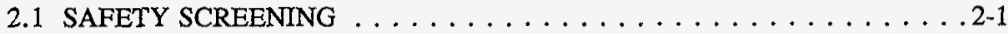

2.1.1 Exothermic Conditions (Energetics) . . . . . . . . . 2-2

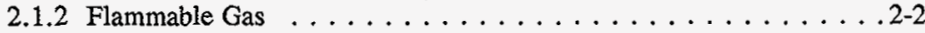

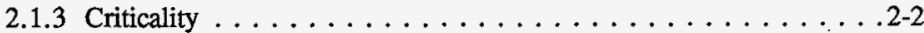

2.2 FLAMMABLE GAS TANK SAFETY EVALUATION $\ldots \ldots \ldots \ldots .2-3$

2.3 ORGANIC SOLVENTS SCREENING $\ldots \ldots \ldots \ldots \ldots \ldots \ldots \ldots .4$

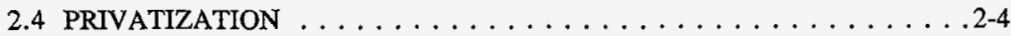

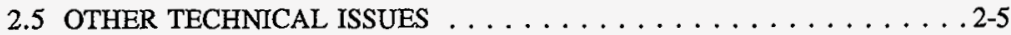

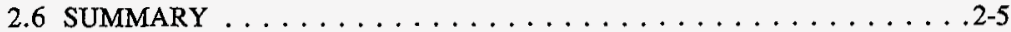

3.0 BEST-BASIS STANDARD INVENTORY ESTIMATE $\ldots \ldots \ldots \ldots \ldots \ldots$. $\ldots \ldots$

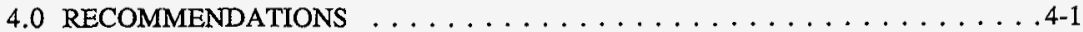

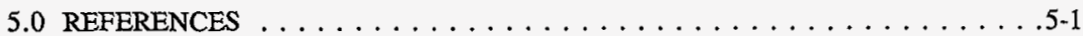

\section{APPENDIXES}

APPENDIX A: HISTORICAL TANK INFORMATION $\ldots \ldots \ldots \ldots \ldots \ldots$ A-1

A1.0 CURRENT TANK STATUS $\ldots \ldots \ldots \ldots \ldots \ldots \ldots \ldots \ldots \ldots \ldots \ldots$

A2.0 TANK DESIGN AND BACKGROUND $\ldots \ldots \ldots \ldots \ldots \ldots \ldots \ldots$ A-4

A3.0 PROCESS KNOWLEDGE $\ldots \ldots \ldots \ldots \ldots \ldots \ldots \ldots \ldots \ldots$ A . . . . . . . . . .

A3.1 WASTE TRANSFER HISTORY . . . . . . . . . . . . A-9

A3.2 HISTORICAL ESTIMATION OF TANK CONTENTS $\ldots \ldots \ldots \ldots$. . . A-11

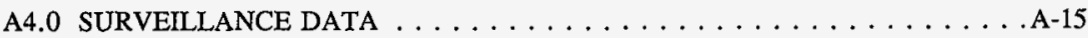

A4.1 SURFACE LEVEL READINGS $\ldots \ldots \ldots \ldots \ldots \ldots \ldots \ldots$ A $\ldots \ldots$

A4.2 INTERNAL TANK TEMPERATURES $\ldots \ldots \ldots \ldots \ldots \ldots$ A-15

A4.3 TANK 241 -AN-104 PHOTOGRAPHS $\ldots \ldots \ldots \ldots \ldots \ldots \ldots$ A-18

A5.0 APPENDIX A REFERENCES . . . . . . . . . . . . . . . . A-19 


\section{CONTENTS (Continued)}

APPENDIX B: SAMPLING OF TANK $241-A N-104 \ldots \ldots \ldots \ldots \ldots$ B-1

B1.0 TANK SAMPLING OVERVIEW $\ldots \ldots \ldots \ldots \ldots \ldots \ldots \ldots \ldots$ B-3

B1.1 DESCRIPTION OF 1996 SAMPLING EVENT . . . . . . . . . . B-3

B1.2 SAMPLE HANDLING OF THE 1996 SAMPLING EVENT $\ldots \ldots \ldots$ B-5

B1.3 SAMPLE ANALYSIS OF 1996 SAMPLING EVENT $\ldots \ldots \ldots \ldots \ldots$. . . . . . .

B1.4 DESCRIPTION OF HISTORICAL SAMPLING EVENT $\ldots \ldots \ldots \ldots$. . 13

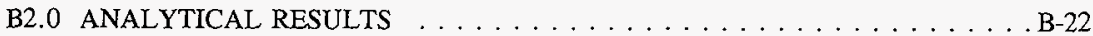

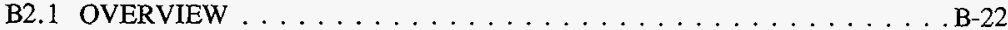

B2.2 SOLID AND LIQUID SAMPLES RESULTS FROM 1996 CORE

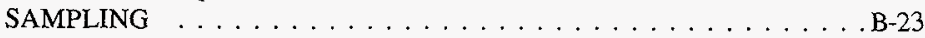

B2.2.1 Inorganic Analyses . . . . . . . . . . . . . . B-23

B2.2.2 Total Inorganic Carbon/Total Organic Carbon . . . . . . . . . B-24

B2.2.3 Hydroxide . . . . . . . . . . . . . . . . . . B-24

B2.2.4 Thermodynamic Analyses . . . . . . . . . . . . . B-24

B2.2.5 Density and Specific Gravity $\ldots \ldots \ldots \ldots \ldots \ldots \ldots$ B-25

B2.2.6 Radionuclide Analysis . . . . . . . . . . . . . . B-25

B2.3 RETAINED GAS SAMPLE RESULTS FORM 1996 CORE

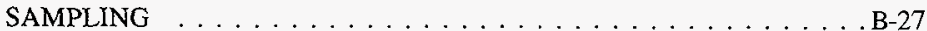

B2.3.1 Retained Gas Concentration . . . . . . . . . . . . . B-27

B2.3.2 Retained Gas Inventory . . . . . . . . . . . . . . . . . . . B-29

B2.3.3 Waste Density . . . . . . . . . . . . . . . . . . . . B-30

B2.4 1996 HEADSPACE VAPOR MEASUREMENT . . . . . . . . . . B-31

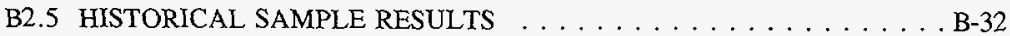

B2.6 ANALYTICAL DATA TABLES FROM 1996 SAMPLING . . . . . . . . B-34

B3.0 ASSESSMENT OF CHARACTERIZATION RESULTS . . . . . . . . . . B-179

B3.1 FIELD OBSERVATIONS . . . . . . . . . . . . . . . . B-179

B3.1.1 Evaluation of Hydrostatic Head Fluid Contamination . . . . . . B-179

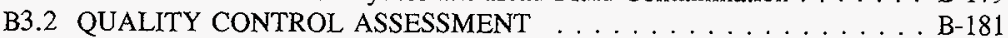

B3.3 DATA CONSISTENCY CHECKS . . . . . . . . . . . . . . B-182

B3.3.1 Comparison of Results from Different Analytical Methods . . B-182

B3.3.2 Mass and Charge Balance ................ B-183

B3.4 MEAN CONCENTRATIONS AND CONFIDENCE INTERVALS ... B-185

B3.4.1 Mean Concentrations . . . . . . . . . . . . . B-186

B3.4.2 Analysis of Variance Model . . . . . . . . . . . B-208

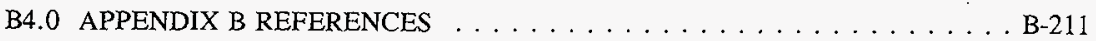




\section{CONTENTS (Continued)}

APPENDIX C: STATISTICAL ANALYSIS FOR ISSUE RESOLUTION $\ldots \ldots \ldots$ C-1 C1.0 STATISTICS FOR SAFETY SCREENING DATA QUALITY OBJECTIVES . . . C-3 C1.1 DIFFERENTIAL SCANNING CALORIMETRY IN DRY . . . . . C C-3 C1.2 TOTAL ALPHA $\ldots \ldots \ldots \ldots \ldots \ldots \ldots \ldots \ldots \ldots \ldots$

C2.0 APPENDIX C REFERENCES . . . . . . . . . . . . . C-8 APPENDIX D: EVALUATION TO ESTABLISH BEST-BASIS INVENTORY FOR TANK $241-A N-104 \ldots \ldots \ldots \ldots \ldots$ D-1

D1.O CHEMICAL INFORMATION SOURCES $\ldots \ldots \ldots \ldots \ldots$ D-3

D2.0 COMPARISON OF COMPONENT INVENTORY VALUES $\ldots \ldots \ldots \ldots$ D-3

D3.0 COMPONENT INVENTORY EVALUATION $\ldots \ldots \ldots \ldots \ldots \ldots$ D-7

D4.0 DEFINE THE BEST-BASIS AND ESTABLISH COMPONENT

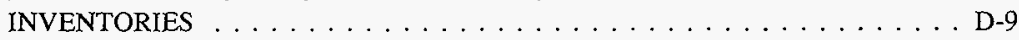

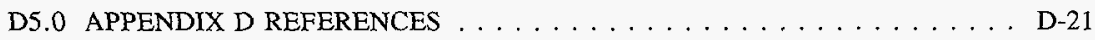

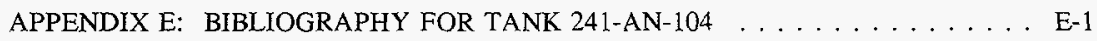




\section{LIST OF FIGURES}

A2-1 Riser Configuration for Tank 241-AN-104 . . . . . . . . . . . A-7

A2-2 Tank 241-AN-104 Cross Section and Schematic . . . . . . . . . . . A-8

A3-1 Tank Layer Model $\ldots \ldots \ldots \ldots \ldots \ldots \ldots \ldots \ldots$. . . . . . . . . . . . . . .

A4-1 Tank 241-AN-104 Level History $\ldots \ldots \ldots \ldots \ldots \ldots \ldots \ldots$. . . . . . . . . .

A4-2 Tank 241-AN-104 Weekly High Temperature Plot . . . . . . . . . . . A-17

A4-3 Tank 241-AN-104 Temperature Profile - Riser 4A . . . . . . . . . . . A-18

\section{LIST OF TABLES}

1-2 Description of Tank 241-AN-104 . . . . . . . . . . . . . . 1-4

2-1 Heat Load for Tank 241-AN-104 Based on Radionuclide Content $\ldots \ldots \ldots$. . . .5

2-2 Summary of Safety Screening and Historical Flammable Gas . . . . . . . . 2-6

3-1 Best-Basis Total Inventory Estimates for Nonradioactive Components in Tank 241-AN-104 as of May $31,1997 \ldots \ldots \ldots \ldots \ldots$. . . . . . . . . . .

3-2 Best-Basis Inventory Estimates for Radioactive Components in Tank 241-AN-104 as of May 31, $1997 \ldots \ldots \ldots \ldots \ldots$. . . . . . . . . . .

4-1 Acceptance of Tank 241-AN-104 Sampling and Analysis . . . . . . . . . . 4-2

4-2 Acceptance of Evaluation of Characterization Data and Information for Tank $241-\mathrm{AN}-104 \ldots \ldots \ldots \ldots \ldots \ldots$. . . . . . . . . . .

A1-1 Tank Contents Status Summary as of May $31,1997 \ldots \ldots \ldots \ldots \ldots$ A-4

A2-1 Tank 241-AN-104 Risers $\ldots \ldots \ldots \ldots \ldots \ldots \ldots \ldots \ldots \ldots$ A-6

A3-1 Tank 241-AN-104 Major Waste Transfers . . . . . . . . . . . . . . . . A-10

B1-1 Integrated Data Quality Objective Requirements for Tank 241-AN-104 . . . . B-5

B1-2 Sample Receipt and Extrusion Information for Tank 241-AN-104 . . . . . . B-6 


\section{LIST OF TABLES (Continued)}

B1-3 Tank 241-AN-104 Summary of Sample Analyses . . . . . . . . . . B-14

B1-4 Analytical Procedures $\ldots \ldots \ldots \ldots \ldots \ldots \ldots$ B-21

B2-1 Analytical Presentation Tables . . . . . . . . . . . . B

B2-2 Concentrations of Gas Constituents from the Retained Gas Sampler in Tank 241-AN-104 . . . . . . . . . . . . . . . . . . . . . . B-28

B2-3 Gas Inventory in Tank 241-AN-104 at Standard Temperature and Pressure . . . B-29

B2-4 Comparison of Bulk Density for the Retained Gas Sampler and Non-Retained Gas Sampler Segments for Tank 241-AN-104 . . . . . . . . B-31

B2-5 Tank 241-AN-104 Vapor Sampling Results . . . . . . . . . . . . B-31

B2-6 Tank 241-AN-104 Sampling Results for July 1984 and January $1985 \ldots \ldots$. . B-32

B2-7 Tank 241-AN-104 Analytical Results: Aluminum (ICP) . . . . . . . . . . B-35

B2-8 Tank 241-AN-104 Analytical Results: Antimony (ICP) $\ldots \ldots \ldots \ldots$. . . . . . .

B2-9 Tank 241-AN-104 Analytical Results: Arsenic (ICP) . . . . . . . . . . . B-41

B2-10 Tank 241-AN-104 Analytical Results: Barium (ICP) . . . . . . . . . . . . B-44

B2-11 Tank 241-AN-104 Analytical Results: Beryllium (ICP) $\ldots \ldots \ldots \ldots \ldots$. . B-47

B2-12 Tank 241-AN-104 Analytical Results: Bismuth (ICP) . . . . . . . . . . . B-50

B2-13 Tank 241-AN-104 Analytical Results: Boron (ICP) . . . . . . . . . . B-53

B2-14 Tank 241-AN-104 Analytical Results: Cadmium (ICP) $\ldots \ldots \ldots \ldots$. . . . . . .

B2-15 Tank 241-AN-104 Analytical Results: Calcium (ICP) . . . . . . . . . . B-59

B2-16 Tank 241-AN-104 Analytical Results: Cerium (ICP) $\ldots \ldots \ldots \ldots$. . . . . B-62

B2-17 Tank 241-AN-104 Analytical Results: Chromium (ICP) . . . . . . . . . . B-65

B2-18 Tank 241-AN-104 Analytical Results: Cobalt (ICP) $\quad \ldots \ldots \ldots \ldots$. . . . B-68 


\section{LIST OF TABLES (Continued)}

B2-19 Tank 241-AN-104 Analytical Results: Copper (ICP) . . . . . . . . . . . . . B-71

B2-20 Tank 241-AN-104 Analytical Results: Iron (ICP) $\ldots \ldots \ldots \ldots \ldots$. . . . . B-74

B2-21 Tank 241-AN-104 Analytical Results: Lanthanum (ICP) $\ldots \ldots \ldots \ldots$. . . B-77

B2-22 Tank 241-AN-104 Analytical Results: Lead (ICP) . . . . . . . . . . . . . B-80

B2-23 Tank 241-AN-104 Analytical Results: Lithium (ICP) $\ldots \ldots \ldots \ldots \ldots$. . . . . . .

B2-24 Tank 241-AN-104 Analytical Results: Magnesium (ICP) . . . . . . . . . . B-86

B2-25 Tank 241-AN-104 Analytical Results: Manganese (ICP) $\ldots . . \ldots \ldots \ldots$. . B-89

B2-26 Tank 241-AN-104 Analytical Results: Molybdenum (ICP) . . . . . . . . . B-92

B2-27 Tank 241-AN-104 Analytical Results: Neodymium (ICP) $\ldots \ldots \ldots \ldots \ldots$. . . B-95

B2-28 Tank 241-AN-104 Analytical Results: Nickel (ICP) . . . . . . . . . . . . B-98

B2-29 Tank 241-AN-104 Analytical Results: Phosphorus (ICP) . . . . . . . . . . B-101

B2-30 Tank 241-AN-104 Analytical Results: Potassium (ICP) . . . . . . . . . . . B-104

B2-31 Tank 241-AN-104 Analytical Results: Samarium (ICP) . . . . . . . . . . B-106

B2-32 Tank 241-AN-104 Analytical Results: Selenium (ICP) . . . . . . . . . . B-109

B2-33 Tank 241-AN-104 Analytical Results: Silicon (ICP) . . . . . . . . . . . B B-112

B2-34 Tank 241-AN-104 Analytical Results: Silver (ICP) . . . . . . . . . . . . B-115

B2-35 Tank 241-AN-104 Analytical Results: Sodium (ICP) . . . . . . . . . . . B B-118

B2-36 Tank 241-AN-104 Analytical Results: Strontium (ICP) . . . . . . . . . B-121

B2-37 Tank 241-AN-104 Analytical Results: Sulfur (ICP) . . . . . . . . . . . B B-124

B2-38 Tank 241-AN-104 Analytical Results: Thallium (ICP) $\ldots \ldots \ldots \ldots$. . . B-127

B2-39 Tank 241-AN-104 Analytical Results: Titanium (ICP) . . . . . . . . . B-130 


\section{LIST OF TABLES (Continued)}

B2-40 Tank 241-AN-104 Analytical Results: Total Uranium (ICP) . . . . . . . . . B-133

B2-41 Tank 241-AN-104 Analytical Results: Vanadium (ICP) . . . . . . . . . . . B-136

B2-42 Tank 241-AN-104 Analytical Results: Zinc (ICP) . . . . . . . . . . . B-139

B2-43 Tank 241-AN-104 Analytical Results: Zirconium (ICP) . . . . . . . . . . B-142

B2-44 Tank 241-AN-104 Analytical Results: Bromide (IC) . . . . . . . . . . . B-145

B2-45 Tank 241-AN-104 Analytical Results: Chloride (IC) . . . . . . . . . . B B-147

B2-46 Tank 241-AN-104 Analytical Results: Fluoride (IC) . . . . . . . . . . . B-149

B2-47 Tank 241-AN-104 Analytical Results: Formate (IC) $\ldots \ldots \ldots \ldots$. . . . B-151

B2-48 Tank 241-AN-104 Analytical Results: Nitrate (IC) . . . . . . . . . . B-153

B2-49 Tank 241-AN-104 Analytical Results: Nitrite (IC) . . . . . . . . . . . B-155

B2-50 Tank 241-AN-104 Analytical Results: Phosphate (IC) . . . . . . . . . . . B-157

B2-51 Tank 241-AN-104 Analytical Results: Sulfate (IC) . . . . . . . . . . . . . B-159

B2-52 Tank 241-AN-104 Analytical Results: Acetate (IC) . . . . . . . . . . . . B-161

B2-53 Tank 241-AN-104 Analytical Results: Oxalate (IC) . . . . . . . . . . B-163

B2-54 Tank 241-AN-104 Analytical Results: Hexavalent Chromium $(\mathrm{Cr}+6) \ldots$. . B-165

B2-55 Tank 241-AN-104 Analytical Results:

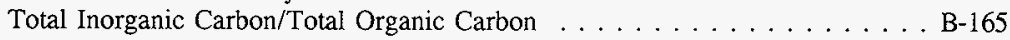

B2-56 Tank 241-AN-104 Analytical Results: Hydroxide (OH Direct) . . . . . . . B-165

B2-57 Tank 241-AN-104 Analytical Results: Percent Water

(Thermogravimetric Analysis) $\ldots \ldots \ldots \ldots \ldots \ldots \ldots$. . . . . . . . . . . . .

B2-58 Tank 241-AN-104 Analytical Results: Exotherm - transition 1

(Differential Scanning Calorimetry) . . . . . . . . . . . . . B-168

B2-59 Tank 241-AN-104 Analytical Results: Bulk Density and Specific Gravity . . B B-170 


\section{LIST OF TABLES (Continued)}

B2-60 Tank 241-AN-104 Analytical Results: Total Alpha (Alpha Radiation) . . . . B-172

B2-61 Tank 241-AN-104 Analytical Results: Total Beta (Alpha) . . . . . . . . . . B-174

B2-62 Tank 241-AN-104 Analytical Results: Strontium-89/90 . . . . . . . . . B-174

B2-63 Tank 241-AN-104 Analytical Results: Americium-241

(Gamma Energy Analysis) $\ldots \ldots \ldots \ldots \ldots \ldots \ldots$. . . . . . . . . . . .

B2-64 Tank 241-AN-104 Analytical Results: Cesium-137

(Gamma Energy Analysis) . . . . . . . . . . . . . . B-175

B2-65 Tank 241-AN-104 Analytical Results: Cobalt-60 (Gamma Energy Analysis) - B-175

B2-66 Tank 241-AN-104 Analytical Results: Europium-154

(Gamma Energy Analysis) $\ldots \ldots \ldots \ldots \ldots \ldots$. . . . . . . . . . . . . . . . .

B2-67 Tank 241-AN-104 Analytical Results: Europium-155

(Gamma Energy Analysis) . . . . . . . . . . . . . . . . . B-176

B2-68 Tank 241-AN-104 Analytical Results: Total Uranium . . . . . . . . . . B-176

B2-69 Tank 241-AN-104 Analytical Results: Plutonium-239/40 . . . . . . . . . B-176

B2-70 Tank 241-AN-104 Analytical Results: Iodine-129 . . . . . . . . . . . . . B-177

B2-71 Tank 241-AN-104 Analytical Results: Tritium (Liquid Scintillation) . . . . . B-177

B2-72 Tank 241-AN-104 Analytical Results: Neptunium-237 . . . . . . . . . . B-177

B2-73 Tank 241-AN-104 Analytical Results: Technetium-99 . . . . . . . . . . . B-177

B2-74 Tank 241-AN-104 Analytical Results: Americium-241

(Alpha Energy Analysis) $\ldots \ldots \ldots \ldots \ldots \ldots \ldots$. . . . . . . . . . . .

B2-75 Tank 241-AN-104 Analytical Results: Cm-243/244

(Alpha Energy Analysis) . . . . . . . . . . . . . . . . . . . B-178

B3-1 Weight Percent Water Correction Based on Bromide Results

for Tank 241-AN-104 . . . . . . . . . . . . . . . . . . . . . . . B-180

B3-2 Comparison of Phosphate/Phosphorous and Sulfate/Sulfur . . . . . . . B 183 


\section{LIST OF TABLES (Continued)}

B3-3 Mass and Charge Balance for Tank 241-AN-104 . . . . . . . . . . . B-184

B3-4 Summary Statistics - Supernate (nested ANOVA) $\ldots \ldots \ldots \ldots$. . . . B-187

B3-5 Summary Statistics - Supernate (one-way ANOVA) $\ldots \ldots \ldots \ldots \ldots$. . . . . . . .

B3-6 Analytes (Supernate) with $>50$ Percent "Less Than" Values . . . . . . . B B-189

B3-7 Summary Statistics - Supernate Composite (core 164, segments 1 to 13) . . . B-190

B3-8 Supernate Composite (core 164 , segments 1 to 13 )

Analytes with $>50$ Percent "Less Than" Values . . . . . . . . . . B-191

B3-9 Summary Statistics (per g basis) - Slurry (nested ANOVA with riser term) . . . B-194

B3-10 Summary Statistics (per g basis) - Slurry

(nested ANOVA without riser) $\ldots \ldots \ldots \ldots \ldots \ldots$. . . . . . . . . . . .

B3-11 Analytes (Slurry - per g basis) with $>50$ Percent "Less Than" Values . . . , B-198

B3-12 Summary Statistics - Slurry Composite (core 164, segments 13 to 20) . . . B-200

B3-13 Slurry Composite (core 164, segments 13 to 20 )

Analytes with $>50$ percent "Less Than" Values . . . . . . . . . . B-202

B3-14 Summary Statistics (per g basis) - Crust

(nested ANOVA with riser term) $\ldots \ldots \ldots \ldots \ldots \ldots \ldots$. . . . . . . . . . .

B3-15 Summary Statistics (per g basis) - Crust

(nested ANOVA without riser term) $\ldots \ldots \ldots \ldots \ldots$. . . . . . . . . . . . . .

B3-16 Analytes (Crust - per g basis) with $>50$ Percent "Less Than" Values . . . . . B-206

C1-1 Tank 241-AN-104 Differential Scanning Calorimetry Results (J/g dry) . . . . . C-4

C1-2 Tank 241-AN-104 Total Alpha Results . . . . . . . . . . . . . . C C-6

D2-1 Sampling And Hanford Defined Waste Model Inventory Estimates for

Nonradioactive Components in Double-Shell Tank 241-AN-104 . . . . . . . . D-4

D2-2 Sampling and Hanford Defined Waste Model Inventory Estimates for Radioactive Components in Double-Shell Tank 241-AN-104 . . . . . . . . D-5 


\section{LIST OF TABLES (Continued)}

D3-1 Comparison of September 1996 Core Sample Concentrations . . . . . . . . . D-7

D4-1 Best-Basis Inventory Estimates for Nonradioactive Components in Tank 241-AN-104 Supernatant as of May 31, $1997 \ldots \ldots \ldots \ldots$. . . . . . . . .

D4-2 Best-Basis Inventory Estimates for Radioactive Components in Tank 241-AN-104 Supernatant as of May 31, $1997 \ldots \ldots \ldots \ldots \ldots$ D-11

D4-3 Best-Basis Inventory Estimates for Nonradioactive Components in Tank 241-AN-104 Salt Slurry as of May 31, $1997 \ldots \ldots \ldots \ldots$. . . . . . . .

D4-4 Best-Basis Inventory Estimates for Radioactive Components in Tank 241-AN-104 Salt Slurry as of May 31, $1997 \ldots \ldots \ldots$. . . . . D-13

D4-5 Best-Basis Inventory Estimates for Nonradioactive Components in Tank 241-AN-104 Crust as of May 31, $1997 \ldots \ldots \ldots \ldots \ldots \ldots$ D-14 . . . . . . .

D4-6 Best-Basis Inventory Estimates for Radioactive Components in

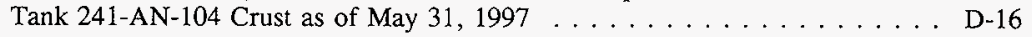

D4-7 Best-Basis Total Inventory Estimates for Nonradioactive Components in Tank 241-AN-104 as of May 31, $1997 \ldots \ldots \ldots \ldots$. . . . . . . . . . . .

D4-8 Best-Basis Inventory Estimates for Radioactive Components in Tank 241-AN-104 as of May 31, $1997 \ldots \ldots \ldots \ldots \ldots$. . . . . . . . . . . . 


\section{LIST OF TERMS}

\begin{tabular}{|c|c|}
\hline ANOVA & analysis of variance \\
\hline $\mathrm{Btu} / \mathrm{hr}$ & British thermal units per hour \\
\hline $\mathrm{CI}$ & confidence interval \\
\hline $\mathrm{cm}$ & centimeter \\
\hline DQO & data quality objective \\
\hline DSC & differential scanning calorimetry \\
\hline $\mathrm{ft}$ & feet \\
\hline $\mathrm{ft}^{2}$ & square feet \\
\hline $\mathrm{ft}^{3}$ & cubic feet \\
\hline GEA & gamma energy analysis \\
\hline $\mathrm{g}$ & gram \\
\hline $\mathrm{g} \mathrm{C} / \mathrm{L}$ & grams of carbon per liter \\
\hline $\mathrm{g} / \mathrm{cm}^{3}$ & grams per cubic centimeter \\
\hline $\mathrm{g} / \mathrm{L}$ & grams per liter \\
\hline $\mathrm{g} / \mathrm{mL}$ & grams per milliliter \\
\hline HDW & Hanford defined waste \\
\hline IC & ion chromatography \\
\hline ICP & inductively coupled plasma spectroscopy \\
\hline in. & inch \\
\hline $\mathrm{J} / \mathrm{g}$ & joules per gram \\
\hline $\mathrm{kg}$ & kilogram \\
\hline $\mathrm{kgal}$ & kilogallon \\
\hline $\mathrm{kL}$ & kiloliter \\
\hline $\mathrm{kW}$ & kilowatt \\
\hline LFL & lower flammability limit \\
\hline LH & lower half \\
\hline LL & lower limit \\
\hline $\mathrm{m}$ & meter \\
\hline $\mathrm{m}^{2}$ & square meters \\
\hline$m^{3}$ & cubic meters \\
\hline$M$ & moles per liter \\
\hline $\mathrm{mL}$ & milliliter \\
\hline $\mathrm{mg}$ & milligram \\
\hline $\mathrm{mm}$ & millimeter \\
\hline $\mathrm{mR} / \mathrm{hr}$ & millirems per hour \\
\hline NA & not available \\
\hline $\mathrm{n} / \mathrm{a}$ & not applicable \\
\hline NR & not reported \\
\hline
\end{tabular}




\section{LIST OF TERMS (Continued)}

$\begin{array}{ll}\text { PHMC } & \text { Project Hanford Management Contractor } \\ \text { ppm } & \text { parts per million } \\ \text { PUREX } & \text { Plutonium Uranium Extraction (Plant) } \\ \text { QC } & \text { quality control } \\ \text { REML } & \text { restricted maximum likelihood estimation methods } \\ \text { RGS } & \text { retained gas sampler } \\ \text { RPD } & \text { relative percent difference } \\ \text { SMM } & \text { supernatant mixing model } \\ \text { SMMA2 } & \text { A solid layer of A2 waste calculated by the SMM model where A2 is } \\ \text { SpG } & \text { the concentrate of the } 1981 \text { to } 1988 \text { Evaporator Campaign } \\ \text { TCR } & \text { specific gravity } \\ \text { TGA } & \text { tank characterization report } \\ \text { TIC } & \text { thermogravimetric analysis } \\ \text { TLM } & \text { total inorganic carbon } \\ \text { TOC } & \text { tank layer model } \\ \text { TWRS } & \text { total organic carbon } \\ \text { UH } & \text { Tank Waste Remediation System } \\ \text { UL } & \text { upper half } \\ \text { VFI } & \text { upper limit } \\ \text { W/Ci } & \text { void fraction instrument } \\ \text { WSTRS } & \text { watts per curies } \\ \text { wt } \% & \text { Waste Status and Transaction Record Summary } \\ { }^{\circ} \mathrm{C} & \text { weight percent } \\ { }^{\circ} \mathrm{F} & \text { degrees Celsius } \\ \mu \mathrm{Ci} / \mathrm{g} & \text { degrees Fahrenheit } \\ \mu \mathrm{Ci} / \mathrm{L} & \text { microcuries per gram } \\ \mu \mathrm{Ci} / \mathrm{mL} & \text { microcuries per liter } \\ \mu \mathrm{eq} / \mathrm{g} & \text { microcuries per milliliter } \\ \mu \mathrm{g} & \text { microequivalents per gram } \\ \mu \mathrm{g} / \mathrm{mL} & \text { microgram } \\ \mu \mathrm{gC} / \mathrm{g} & \text { micrograms per milliliter } \\ \mu \mathrm{g} / \mathrm{g} & \text { micrograms of carbon per gram } \\ \mu \mathrm{mole} / \mathrm{L} & \text { micrograms per gram } \\ & \text { micromole per liter } \\ & \end{array}$




\subsection{INTRODUCTION}

A major function of the Tank Waste Remediation System (TWRS) is to characterize wastes in support of waste management and disposal activities at the Hanford Site. Analytical data from sampling and analysis, along with other available information about a tank, are compiled and maintained in a tank characterization report (TCR). This report and its appendixes serve as the TCR for double-shell tank 241-AN-104.

The objectives of this report are: 1) to use characterization data in response to technical issues associated with tank 241-AN-104 waste and 2) to provide a standard characterization of this waste in terms of a best-basis inventory estimate. Section 2.0 summarizes the response to technical issues, Section 3.0 provides the best-basis inventory estimate, Section 4.0 makes recommendations regarding safety status and additional sampling needs. The appendixes contain supporting data and information. This report also supports the requirements of the Hanford Federal Facility Agreement and Consent Order (Ecology et al. 1996), Milestone M-44-10.

\subsection{SCOPE}

The characterization information in this report originated from sample analyses and known historical sources. Although only the results of recent sample events will be used to fulfill the requirements of the data quality objectives (DQOs), other information can be used to support (or question) conclusions derived from these results. Appendix A provides historical information about tank 241-AN-104 including surveillance information, records pertaining to waste transfers and tank operations, and expected tank contents derived from a process knowledge model. Appendix B summarizes the recent sampling events (see Table 1-1), vapor sniff test results, and sample data obtained before 1989 , and the sampling results. The results of the 1996 sampling events, reported in the 222-S Laboratory data package (Steen 1997), satisfied the data requirements specified in the tank sampling and analysis plan for this tank (Winkleman 1996). Also seven segments from the 1996 core sampling are selected as retained gas samples. From the study of retained gas samples, tank gas composition and quantities of the retained gas are estimated by Shekarriz et al. (1997). Appendixes B and C report the statistical analysis and numerical manipulation of data used in issue resolution. Appendix D contains the evaluation to establish the best basis for the inventory estimate. Appendix $E$ is a bibliography resulting from an in-depth literature search of all known information sources applicable to tank 241-AN-104 and its respective waste types. The reports listed in Appendix $\mathrm{E}$ can be found in the Tank Characterization Safety and Resource Center. 
Table 1-1. Summary of Recent Sampling.

\begin{tabular}{|c|c|c|c|c|}
\hline 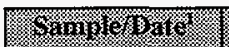 & Chrixso & Wosining & Gicineringuor & 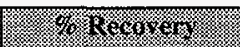 \\
\hline $\begin{array}{l}\text { Core } 164: \text { total } \\
\text { of } 21 \text { segments } \\
\text { sampled. } \\
\text { Segment } 6 \text { and } \\
21 \text { failed. } \\
8 / 8 / 96 \text { to } 8 / 16 / 96\end{array}$ & $\begin{array}{l}\text { Solid liquid } \\
\text { and retained } \\
\text { gas }\end{array}$ & Riser 12A & $\begin{array}{l}\text { Solid in half } \\
\text { segment, liquid as } \\
\text { whole. Retained } \\
\text { gas sampler in } \\
\text { segment } 18 .\end{array}$ & $\begin{array}{l}\text { Most segments are } \\
100 \% \text { recovered } \\
\text { except segment } 2 \text {, } \\
3,4,10 \text {, and } 18 \\
\text { ranged } 79 \% \text { and } \\
99 \% .\end{array}$ \\
\hline \begin{tabular}{|l|} 
Core $163:$ total \\
of 21 segments \\
sampled. \\
$9 / 9 / 96$ to $9 / 11 / 96$
\end{tabular} & $\begin{array}{l}\text { Solid liquid } \\
\text { and retained } \\
\text { gas }\end{array}$ & Riser 10A & $\begin{array}{l}\text { Solid in half } \\
\text { segment. liquid as } \\
\text { whole and retained } \\
\text { gas segment } 3,13 \text {, } \\
15,17,19 \text {, and } 21 \text {. }\end{array}$ & $\begin{array}{l}\text { There are } 11 \\
\text { segments with } \\
100 \% \text { recovery. } \\
\text { The rest of the } \\
\text { segments ranged } \\
\text { from } 83 \% \text { and } 94 \% \\
\text { except segment } 1 \text { is } \\
12 \% \text {. }\end{array}$ \\
\hline $\begin{array}{l}\text { Vapor sample } \\
9 / 9 / 96 \text { to } 9 / 9 / 96\end{array}$ & Gas & $\begin{array}{l}\text { Tank } \\
\text { headspace, } \\
\text { risers } 12 \mathrm{~A} \text { and } \\
10 \mathrm{~A}, 6.1 \mathrm{~m} \\
(20 \mathrm{ft}) \text { below } \\
\text { top of risers. }\end{array}$ & $n / a$ & $n / a$ \\
\hline
\end{tabular}

Notes:

$\mathrm{n} / \mathrm{a}=$ not applicable

${ }^{1}$ Dates are in the month/day/year format.

\subsection{TANK BACKGROUND}

Tank 241-AN-104 is located in the 200 East Area AN Tank Farm on the Hanford Site. The tank went into service in 1982 and received water. The tank received double-shell slurry feed waste from tank 241-AW-102 in the fourth quarter of 1982 and the first quarter of 1984. In the fourth quarter of 1983, the tank received PUREX waste. In the first quarter of 1984, waste was sent to tanks 241-AZ-102 and 241-AN-105. In the third quarter of 1984, $82 \mathrm{kgal}$ of flush water was added to the tank, and waste was sent to tank 241-AN-103. From the fourth quarter to the second quarter of 1985 , the tank received double-shell slurry feed waste from tank 241-AW-102 via the evaporator (Agnew et al. 1997b). The last transfer for this tank was a small amount of flush water added in 1996. 
Table 1-2 summarizes a description of tank 241-AN-104. The tank has an operating capacity of 4,390 kL (1,160 kgal) and contains an estimated 3,994 kL (1,055 kgal) of double-shell slurry feed waste (Hanlon 1997). This tank is still active and when waste transfers take place, the data shown here will be changed. The tank is on the Flammable Gas Watch List (Public Law 101-510). 
Table 1-2. Description of Tank 241-AN-104.

\begin{tabular}{|c|c|}
\hline ( IXKR & WIOYY \\
\hline Type & Double-shell \\
\hline Constructed & 1980 to 1981 \\
\hline In-service & 1982 \\
\hline Diameter & $22.9 \mathrm{~m}(75.0 \mathrm{ft})$ \\
\hline Operating depth & $10.7 \mathrm{~m}(35 \mathrm{ft})$ \\
\hline Capacity & $4,390 \mathrm{~kL}(1,160 \mathrm{kgal})$ \\
\hline Bottom shape & Flat \\
\hline Ventilation & Active \\
\hline $\operatorname{lin}_{4}$ & 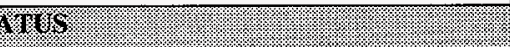 \\
\hline Waste classification & Double-shell slurry feed ${ }^{t}$ \\
\hline Total waste volume & $3,994 \mathrm{~kL}(1,055 \mathrm{kgal})$ \\
\hline Supernatant volume ${ }^{2}$ & $2,273 \mathrm{~kL}(600.5 \mathrm{kgal})$ \\
\hline Saltcake volume $^{2}$ & $21 \mathrm{~kL}(5.5 \mathrm{kgal})$ \\
\hline Sludge volume & $1700 \mathrm{~kL}(449 \mathrm{kgal})$ \\
\hline Drainable interstitial liquid volume & $182 \mathrm{~kL}(48 \mathrm{kgal})$ \\
\hline Waste surface level (May 31, 1997) & $974 \mathrm{~cm}(383.6 \mathrm{in})$. \\
\hline Temperature (March 1994 to May 1997) & $21.7^{\circ} \mathrm{C}\left(71.06^{\circ} \mathrm{F}\right)$ to $51.2^{\circ} \mathrm{C}\left(126.16^{\circ} \mathrm{F}\right)$ \\
\hline Integrity & Sound \\
\hline Watch List & Flammable gas \\
\hline 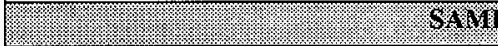 & 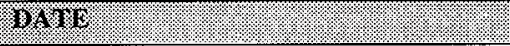 \\
\hline $\begin{array}{l}\text { Core samples and tank headspace } \\
\text { flammability }\end{array}$ & August/September 1996 \\
\hline 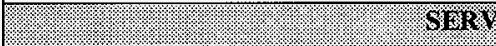 & 14110 \\
\hline Active $^{3}$ & present \\
\hline
\end{tabular}

Notes:

'Double-shell slurry feed is waste that exceeds the sodium aluminate saturation boundary in the evaporator without exceeding the receiver tank composition limit. This waste contains less than $10 \mathrm{~g} / \mathrm{L}$ organic and is considered noncomplexed waste.

${ }^{2}$ Based on extrusion results of 1996 core samples (see Appendix D).

${ }^{3}$ The data shown in the table will be changed when waste transfer occurs. 


\subsection{RESPONSE TO TECHNICAL ISSUES}

Four technical issues have been identified for tank 241-AN-104 (Brown et al. 1997). They are:

- Safety screening: Does the waste pose or contribute to any recognized potential safety problems?

- Flammable gas: Data from the core samples are needed to provide an understanding of the tank contents so that 1) insight may be obtained on the mechanisms for gas generation, retention, and release; 2) models of waste behavior can be developed to support safety analysis and development of mitigation methods; 3 ) simulants for waste studies can be developed; and 4) modeling of the gas releases and subsequent potential for ignition in the headspace can be performed to support hazard analyses.

- Organic solvents screening: Does an organic solvent pool exist that may cause a fire or ignition or organic solvents in entrained waste solids?

- Privatization: Do the September 1996 core samples taken from tank 241-AN-104 and the subsequent laboratory analysis meet the needs of the privatization low-activity waste DQO (Jones and Wiemers 1996)?

The tank 241-AN-104 characterization plan (Winkelman 1996b) and the analysis plan (Winkleman 1996a) integrate all applicable DQOs and describe the sampling and analysis used to address the above issues. Data from the recent analysis of two core samples, tank headspace flammability measurements, and historical information were the means used to respond to the issues. This response is described in the sections below. For sample and analysis data for tank 241-AN-104, see Appendix B.

\subsection{SAFETY SCREENING}

The data needed to screen tank 241-AN-104 waste for potential safety problems is documented in Tank Safety Screening Data Quality Objective (Dukelow et al. 1995). The potential safety problems include exothermic conditions in the waste, flammable gases in the waste and/or tank headspace, and criticality conditions in the waste. Each condition is addressed separately. 


\subsubsection{Exothermic Conditions (Energetics)}

The first requirement outlined in Dukelow et al. (1995) is to ensure there are not sufficient exothermic constituents in tank 241-AN-104 to cause a safety hazard. Because of this requirement, energetics in tank $241-\mathrm{AN}-104$ waste were evaluated. The safety screening DQO required that solid samples be tested for energetics every $24 \mathrm{~cm}(9.5$ in.) to determine whether the energetics exceed the safety threshold limit.

Historically, evidence exists showing exothermic behavior in the waste. Waste transfer records indicate the major waste type expected in the tank is double-shell slurry feed (Agnew et al. 1997b). The waste types are expected to have organic constituents (Sasaki 1984). A sample analysis taken in 1984 also showed evidence of exotherms (Mauss 1984).

The 1996 sampling results obtained using differential scanning calorimetry (DSC) indicated that exotherms were well below notification limits of $480 \mathrm{~J} / \mathrm{g}$ on a dry weight basis with the maximum value of $175 \mathrm{~J} / \mathrm{g}$ (Steen 1996). Furthermore, the upper limits (UL) of the one-sided 95 percent confidence interval (CI) for the DSC results were below notification limit except for one sample from segment 16, upper half of core 163. The DSC results in the dry weight basis of this sample and a duplicate are 204 and $62.8 \mathrm{~J} / \mathrm{g}$, and the resulting UL of the one-sided 95 percent $\mathrm{CI}$ is $579 \mathrm{~J} / \mathrm{g}$. Because the DSC results of the sample and a duplicate are well below the criteria of $480 \mathrm{~J} / \mathrm{g}$ on a dry weight basis, no rerun was performed on this sample. These results suggest that no energetic concern exists in this tank for the waste. However, the waste does generate and retain flammable gases which are released episodically at various intervals (see Section 2.2).

\subsubsection{Flammable Gas}

Vapor phase measurements, which were taken in the tank headspace during the core sampling in September 1996, indicated 0.013 percent of the lower flammability limit (LFL). This value is well below the concern level of 25 percent LFL. Appendix B provides data from these vapor phase measurements. Data obtained from on-line gas monitors are summarized in Section 2.2.

\subsubsection{Criticality}

The safety threshold limit is $1 \mathrm{~g}{ }^{239} \mathrm{Pu}$ per liter of waste. Assuming all alpha is from ${ }^{239} \mathrm{Pu}$, with a maximum density of $1.76 \mathrm{~g} / \mathrm{mL}$ for salt slurry, the safety threshold limit is equivalent to $34.9 \mu \mathrm{Ci} / \mathrm{g}$ of salt slurry and $61.5 \mu \mathrm{Ci} / \mathrm{mL}$ of liquid of alpha activity. The core samples were analyzed according to Dukelow et al. (1995). 
Waste samples were tested for total alpha activity for each core sample. Concentrations in all samples were well below this limit with the maximum value of $0.0558 \mu \mathrm{Ci} / \mathrm{g}$.

Additionally, as required by the DQO, the UL of the one-sided 95 percent CI for these results was less than $37 \mu \mathrm{Ci} / \mathrm{g}$ with the maximum value of $0.0849 \mu \mathrm{Ci} / \mathrm{g}$. Appendix $\mathrm{C}$ describes the method used to calculate confidence limits. The data show that no criticality concern exists for the waste in this tank.

\subsection{FLAMMABLE GAS TANK SAFETY EVALUATION}

The purpose of the flammable gas tank safety evaluation is to obtain data to support tank behavior models needed for making safety analyses, to support evaluations of mechanisms for gas production and release, and to develop mitigation methods (McDuffie and Johnson 1995). These data needs were met by the 1996 core sampling event which included the analytical results of core segments samples in the solid/liquid phase (Steen 1997), the retained gas composition and quantities (Shekarriz et al. 1997) from core segments of the retained gas sampler (RGS), and by tank headspace monitoring data (Wilkins et al. 1997). Using analytical data and available models, a calculation was carried out on the hydrogen release rate at steady state on the tank headspace ( $\mathrm{Hu} \mathrm{1997)} \mathrm{to} \mathrm{evaluate} \mathrm{the} \mathrm{flammable} \mathrm{gas}$ hazard at various ventilation rates for all double-shell tanks.

In the RGS study (Shekarriz et al. 1997), the total volume of gas in the vapor phase is $212 \mathrm{~m}^{3}$ with $163 \mathrm{~m}^{3}$ dissolved in the liquid phase. The three major gases in the vapor phase are hydrogen $\left(98.6 \mathrm{~m}^{3}\right)$, nitrogen $\left(69.9 \mathrm{~m}^{3}\right)$, and nitrous oxide $\left(40.5 \mathrm{~m}^{3}\right)$. About $143 \mathrm{~m}^{3}$ of ammonia is dissolved in the liquid phase, and $0.048 \mathrm{~m}^{3}$ in the vapor phase. The remaining gases include methane and other hydrocarbons. For detailed gas compositions in tank 241-AN-104, see Appendix B.

The extraction results of RGS segments (Shekarriz et al. 1997) show the insoluble gases were retained primarily in the lower, nonconvective layer. Based on the estimated solubilities and RGS measurements of gas concentrations, about 5.7 percent by volume (in-situ) of the nonconvective layer was filled with free gas, and 0.5 percent by volume (in-situ) of the convective (upper) layer was filled with free gas. The void fractions calculation, based on RGS data, were in close agreement with the void fraction instrument (VFI) results for all segments except segment 21 which had no VFI data. The maximum void fraction measured with RGS was approximately 13.3 percent from segment 21 of core 163 . The x-ray imaging method provides an estimate of waste density for the RGS segments. The density is $1.41 \mathrm{~g} / \mathrm{mL}$ for the supernatant layer, and 1.72 to $2.09 \mathrm{~g} / \mathrm{mL}$ in the nonconvective layer.

Waste level drops in tank 241-AN-104 were observed in past years (Reynolds 1994) indicating the gas release behavior for this tank. Recently, a standard hydrogen monitoring system was installed in the tank, and the hydrogen concentrations of several gas release events have been recorded subsequently. The maximum hydrogen concentration (Wilkins et al. 1997) of the gas release events were $3,000 \mathrm{ppm}$ on November 6, 1994, 2,088 ppm on November 16, 1995, 480 ppm on August 3, 1995, 3,200 ppm on October 2, 1995 , 
1,000 ppm on October 5, 1995, 5,000 ppm on October 8, 1995 and 6,109 ppm on May 3, 1996. No concentrations reached 25 percent of LFL which is equivalent to a hydrogen concentration of $9,300 \mathrm{ppm}$. Although gas release events were observed, no net waste level increases have been noticed in the past three years.

Based on the study of real waste samples and waste simulants (Pederson and Bryan 1996), three major mechanisms are identified that generate the hydrogen gas: radiolysis of water and organic compounds, chemical reaction (thermolysis), and corrosion of carbon steel. According to the calculation of $\mathrm{Hu}$ (1997), the hydrogen generation rate for tank 241-AN-104 is $242 \mathrm{~L}$ per day: 71 percent from radiolysis, 17 percent from thermolysis, and 12 percent from corrosion. In the calculation, the analytical results (Steen 1997) of total organic carbon (TOC), $\mathrm{NO}_{3}, \mathrm{NO}_{2}, \mathrm{Al}$, heat load (radionuclide inventory), bulk density, and moisture content are used to determine the hydrogen generation rate. This model-derived hydrogen generation rate agrees reasonably with the field-data derived hydrogen generation rate of $178 \mathrm{~L}$ per day.

If active ventilation is lost, $\mathrm{Hu}$ (1997) shows that it takes 59 days to reach 25 percent of the LFL in the headspace, and the hydrogen concentration can reach as high as

3.8 percent. The evaluation also shows the required minimum ventilation rate is 0.63 cubic feet per minute to keep the headspace below the 25 percent LFL.

\subsection{ORGANIC SOLVENTS SCREENING}

The data required to support the organic solvent screening issue are documented in the Defense Nuclear Facility Safety Board 93-5 implementation plan (DOE-RL 1996). A new DQO is currently being developed to address the organic solvent issue. In the interim, tanks are to be sampled for total nonmethane hydrocarbon to determine whether an organic extractant pool greater than $1 \mathrm{~m}^{2}\left(10.8 \mathrm{ft}^{2}\right.$ ) exists (Cash 1996). The purpose of this assessment is to ensure that an organic solvent pool fire or ignition of organic solvents cannot occur. Vapor analyses for total nonmethane organic hydrocarbon have not been conducted. Vapor samples are scheduled for fiscal year 1999. However, the size of the organic extractant pool will be estimated and reported by the Organic Safety Project, based on the sniff test TOC results, the tank headspace temperature, and tank ventilation rate.

\subsection{PRIVATIZATION}

Tank 241-AN-104 is in the scope of the privatization low-activity waste DQO (Jones and Wiemers 1996). However, sampling was performed before this DQO was issued. The sampling and analytical results are being assessed by the privatization program to determine whether the 1996 core sampling results meet the needs of the privatization low-activity waste DQO. Results will be reported in a future revision to this report or additional sampling and analyses may be required. 


\subsection{OTHER TECHNICAL ISSUES}

A factor in assessing tank safety is the heat generation and temperature of the waste. Heat is generated in the tanks from radioactive decay. An estimate of the tank heat load can be calculated based on the results from the 1996 sample event. The heat load value calculated was $11.5 \mathrm{~kW}(39,150 \mathrm{Btu} / \mathrm{hr}$ ) (see Table 2-1). The heat load estimate, based on the tank process history, was $6.5 \mathrm{~kW}(22,200 \mathrm{Btu} / \mathrm{hr}$ ) (Agnew et al. 1997a). The heat load estimate, based on the tank headspace temperature, was $11.7 \mathrm{~kW}(40,000 \mathrm{Btu} / \mathrm{hr})$ (Kummerer 1995). These estimates are well below the maximum heat load of $20.5 \mathrm{~kW}(70,000 \mathrm{Btu} / \mathrm{hr})$ allowed for double-shell tanks (Heubach 1996).

Table 2-1. Heat Load for Tank 241-AN-104 Based on Radionuclide Content.

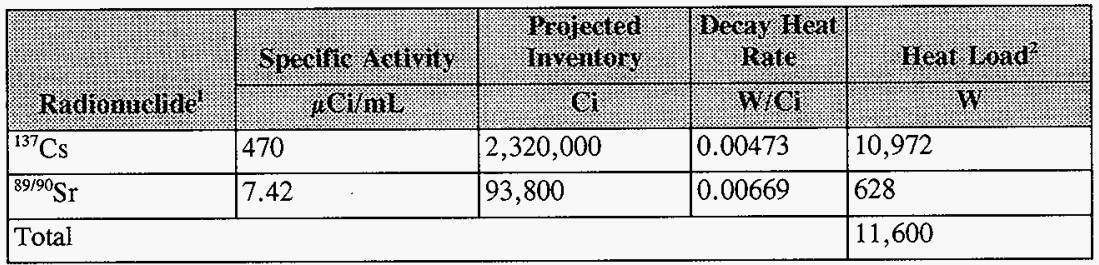

Notes:

'Includes daughter radionuclides.

${ }^{2}$ Only analytes contributing to the heat generation rate above $100 \mathrm{~W}$ are included.

\subsection{SUMMARY}

The results of all analyses performed to address potential safety issues showed that no primary analyte exceeded safety decision threshold limits. The analyses for flammable gas was completed to support tank behavior models needed for making safety analyses, to support evaluations of mechanisms for gas production and release, and to obtain data to develop mitigation methods. Table $2-2$ shows the results of the analyses. 
Table 2-2. Summary of Safety Screening and Historical Flammable Gas.

\begin{tabular}{|c|c|c|}
\hline 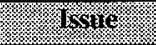 & 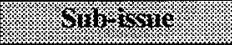 & ( \\
\hline \multirow[t]{3}{*}{$\begin{array}{l}\text { Safety } \\
\text { screening }\end{array}$} & Energetics & $\begin{array}{l}\text { All exothermic reaction were well below } 480 \mathrm{~J} / \mathrm{g} \text { in dry } \\
\text { weight basis. Highest } 95 \% \mathrm{CI} \mathrm{UL}=579 \mathrm{~J} / \mathrm{g} \text { because } \\
\text { of the high RPD of the sample and duplicate results of } \\
204 \text { and } 62.8 \mathrm{~J} / \mathrm{g} \text {. }\end{array}$ \\
\hline & Flammable gas & $\begin{array}{l}\text { Vapor measurement was reported at } 0.013 \% \text { of LFL } \\
\text { (combustible gas meter). }\end{array}$ \\
\hline & Criticality & $\begin{array}{l}\text { All analyses were well below } 39.5 \mu \mathrm{Ci} / \mathrm{g} \text { total alpha } \\
\text { (within } 95 \% \text { confidence limit on each sample). }\end{array}$ \\
\hline \multirow[t]{4}{*}{$\begin{array}{l}\text { Flammable } \\
\text { gas }\end{array}$} & $\begin{array}{l}\text { Mechanism of gas } \\
\text { generation, } \\
\text { retention, and } \\
\text { released }\end{array}$ & $\begin{array}{l}\text { Preliminary assessments of flammable gas generation, } \\
\text { retention, and release mechanisms; and waste behavior } \\
\text { modeling results are reported in Meyer et al. (1997) }\end{array}$ \\
\hline & $\begin{array}{l}\text { Waste behavior } \\
\text { model }\end{array}$ & $\begin{array}{l}\text { Additional evaluations to assess potential impacts and } \\
\text { waste behavior in tank } 241-\mathrm{AN}-104 \text { are in progress. }\end{array}$ \\
\hline & $\begin{array}{l}\text { Simulant } \\
\text { development }\end{array}$ & $\mathrm{n} / \mathrm{a}$ \\
\hline & $\begin{array}{l}\text { Model to support } \\
\text { hazard analysis }\end{array}$ & Being developed. \\
\hline $\begin{array}{l}\text { Organic } \\
\text { solvent }\end{array}$ & $\begin{array}{l}\text { Total nonmethane } \\
\text { hydrocarbon }\end{array}$ & $\begin{array}{l}\text { Evaluation in progress is based on TOC sniff test } \\
\text { results. }\end{array}$ \\
\hline Privatization & Low-activity waste & $\begin{array}{l}\text { Results of } 1996 \text { core sample are being evaluated to } \\
\text { determine whether they meet the needs of privatization. }\end{array}$ \\
\hline
\end{tabular}




\subsection{BEST-BASIS STANDARD INVENTORY ESTIMATE}

Information about the chemical and/or physical properties of tank wastes is used to perform safety analyses, engineering evaluations, and risk assessments associated with waste management activities, as well as to address regulatory issues. Waste management activities include overseeing tank farm operations and identifying, monitoring, and resolving safety issues associated with these operations and with the tank wastes. Disposal activities involve designing equipment, processes, and facilities for retrieving wastes and processing the wastes into a form that is suitable for long-term storage.

Chemical inventory information generally is derived using two approaches: 1) component inventories are estimated using the results of sample analyses, and 2) component inventories are predicted using a model based on process knowledge and historical information. The most recent model was developed by the Los Alamos National Laboratory (Agnew et al. 1997a). Information derived from these two approaches is often inconsistent. An effort is underway to provide waste inventory estimates that will serve as standard characterization information for the waste management activities (Hodgson and LeClair 1996). As part of this effort, an evaluation of available chemical information for tank 241-AN-104 was performed that included:

- Steen (1997) and Appendix B of this report, which provides characterization results from the September 1996 core sampling event.

- A feed projection document that supported grout treatment facility studies provides estimates of the waste in tank 241-AN-104 based on 242-A Evaporator slurry product data (Hendrickson 1994).

- The Hanford defined waste (HDW) model document (Agnew et al. 1997a) provides tank content estimates in terms of component concentrations and inventories.

Inventories based on the September 1996 sampling event should serve as the basis for the best-estimate inventory to tank 241-AN-104 for the following reasons:

1. The September 1996 sampling event provides the most recent data for the waste.

2. Estimates based on 242-A Evaporator product sample data agrees with the September 1996 analytical data.

3. The HDW model estimates, although in reasonable agreement with the sampling data, do not agree as closely with the evaporator product data. 
Tables 3-1 and 3-2 show the best-basis inventory estimates for tank 241-AN-104. Radionuclide values are decayed to January 1, 1994. The HDW model inventories for radionuclides were used when no sample data were available

Table 3-1. Best-Basis Total Inventory Estimates for Nonradioactive Components in Tank 241-AN-104 as of May 31, 1997. ${ }^{1}$ (2 sheets)

\begin{tabular}{|c|c|c|c|}
\hline (1) & 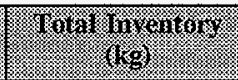 & 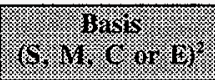 & Corinent \\
\hline $\mathrm{Al}$ & 143,000 & $S$ & \\
\hline $\mathrm{Bi}$ & $<667$ & $S$ & \\
\hline $\mathrm{Ca}$ & $<855$ & $S$ & \\
\hline$\overline{\mathrm{Cl}}$ & 29,900 & $S$ & \\
\hline TIC as $\mathrm{CO}_{3}$ & 210,000 & $S$ & \\
\hline $\mathrm{Cr}$ & 5,190 & $S$ & \\
\hline $\mathbf{F}$ & 2,160 & $S$ & \\
\hline$\overline{F e}$ & $<395$ & $S$ & \\
\hline $\bar{K}$ & 24,900 & $S$ & \\
\hline $\mathrm{La}$ & $<334$ & $S$ & \\
\hline $\mathrm{Mn}$ & $<74.6$ & $S$ & \\
\hline $\mathrm{Na}$ & $1.19 \mathrm{E}+06$ & $S$ & \\
\hline $\mathrm{Ni}$ & $<226$ & 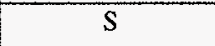 & \\
\hline $\mathrm{NO}_{2}$ & 448,000 & $S$ & \\
\hline $\mathrm{NO}_{3}$ & 680,000 & $S$ & \\
\hline$\widehat{\mathrm{OH}}$ & 650,000 & $\mathrm{C}$ & sample-based $=255,000$ \\
\hline $\mathrm{Pb}$ & $<382$ & $\bar{S}$ & \\
\hline $\mathrm{PO}_{4}$ & 17,100 & $S$ & \\
\hline $\mathrm{Si}$ & 1,590 & $\mathrm{~S}$ & \\
\hline $\mathrm{SO}_{4}$ & 37,100 & $S$ & \\
\hline $\mathrm{Sr}$ & $<66.7$ & $\bar{S}$ & \\
\hline TOC & 18,700 & $S$ & \\
\hline $\mathbf{U}_{\text {TOTAL }}$ & $<3,340$ & $S$ & \\
\hline
\end{tabular}


pəseq-1

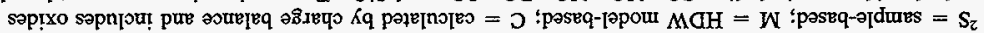

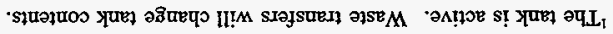

\begin{tabular}{|c|c|c|c|}
\hline & $S$ & St*I & Tu/8 'K1!̣suəCI \\
\hline & $S$ & $t 80$ & Іәңем \% \\
\hline & $S$ & $z+9$ & $I Z$ \\
\hline (1) & (3) & . & 83. \\
\hline
\end{tabular}

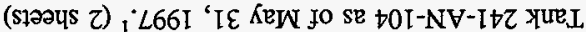

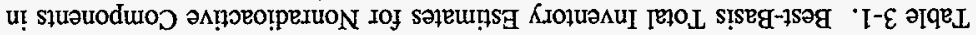


HNF-SD-WM-ER-690 Rev. 0

Table 3-2. Best-Basis Inventory Estimates for Radioactive Components in Tank 241-AN-104 as of May 31, 1997 (Decayed to January 1, 1994). ${ }^{1}$ (2 sheets)

\begin{tabular}{|c|c|c|c|}
\hline 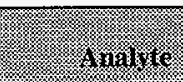 & 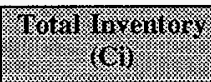 & 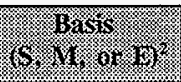 & Somient \\
\hline${ }^{3} \mathrm{H}$ & 43.3 & $\mathrm{~S}$ & Slurry/crust only \\
\hline${ }^{14} \mathrm{C}$ & 104 & $\mathrm{M}$ & \\
\hline${ }^{59} \mathrm{Ni}$ & 5.61 & $\mathrm{M}$ & \\
\hline${ }^{60} \mathrm{Co}$ & $<149$ & $S$ & \\
\hline${ }^{63} \mathrm{Ni}$ & 552 & $\mathbf{M}$ & \\
\hline${ }^{79} \mathrm{Se}$ & 11.3 & $\bar{M}$ & \\
\hline${ }^{90} \mathrm{Sr}$ & 93,800 & $S$ & \\
\hline${ }^{90} \mathrm{Y}$ & 93,800 & $\mathrm{~S}$ & Referenced to ${ }^{90} \mathrm{Sr}$ \\
\hline${ }^{93} \mathrm{Zr}$ & 55.3 & $\mathrm{M}$ & \\
\hline${ }^{93 \mathrm{~m}} \mathrm{Nb}$ & 40.2 & $\overline{\mathrm{M}}$ & \\
\hline${ }^{99} \mathrm{Tc}$ & 345 & $S$ & \\
\hline${ }^{106} \mathrm{Ru}$ & 0.0323 & $\mathbf{M}$ & \\
\hline${ }^{113 m} \mathrm{Cd}$ & 290 & $\bar{M}$ & \\
\hline${ }^{125} \mathrm{Sb}$ & 745 & $\mathrm{M}$ & \\
\hline${ }^{126} \mathrm{Sn}$ & 17.2 & $\mathrm{M}$ & \\
\hline${ }^{129} \mathrm{Y}$ & $<7.53$ & $S$ & \\
\hline${ }^{134} \mathrm{Cs}$ & 123 & $\mathbf{M}$ & \\
\hline${ }^{137} \mathrm{Cs}$ & $2.32 \mathrm{E}+06$ & $\mathrm{~S}$ & \\
\hline${ }^{137 \mathrm{~m}} \mathrm{Ba}$ & $2.20 \mathrm{E}+06$ & $\mathrm{~S}$ & Referenced to ${ }^{137} \mathrm{Cs}$ \\
\hline${ }^{151} \mathrm{Sm}$ & 40,000 & $\bar{M}$ & \\
\hline${ }^{152} \mathrm{Eu}$ & 14.5 & $\mathrm{M}$ & \\
\hline${ }^{154} \mathrm{Eu}$ & $<676$ & $\mathrm{~S}$ & \\
\hline${ }^{155} \mathrm{Eu}$ & $<4,730$ & $\mathrm{~S}$ & \\
\hline${ }^{226} \mathrm{Ra}$ & $4.63 \mathrm{E}-04$ & $\bar{M}$ & \\
\hline${ }^{227} \mathrm{Ac}$ & 0.00286 & $\mathrm{M}$ & \\
\hline${ }^{228} \mathrm{Ra}$ & 1.03 & $M$ & \\
\hline
\end{tabular}


Table 3-2. Best-Basis Inventory Estimates for Radioactive Components in Tank 241-AN-104 as of May 31, 1997 (Decayed to January 1, 1994). ${ }^{1}$ (2 sheets).

\begin{tabular}{|c|c|c|c|}
\hline 1. & 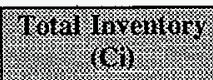 & 5. & 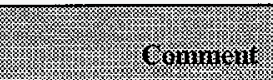 \\
\hline${ }^{229} \mathrm{Th}$ & 0.0239 & $\mathrm{M}$ & \\
\hline${ }^{231} \mathrm{~Pa}$ & 0.0128 & $\bar{M}$ & \\
\hline${ }^{232} \mathrm{Th}$ & 0.109 & $\mathrm{M}$ & \\
\hline${ }^{232} \mathrm{U}$ & 3.17 & $\bar{M}$ & \\
\hline${ }^{233} \mathrm{U}$ & 12.1 & $\mathrm{M}$ & \\
\hline${ }^{234} \mathrm{U}$ & 3.59 & $\mathrm{M}$ & \\
\hline${ }^{235} \mathrm{U}$ & 0.140 & $M$ & \\
\hline${ }^{236} \mathrm{U}$ & 0.193 & $\mathrm{M}$ & \\
\hline${ }^{237} \mathrm{~Np}$ & $<30.7$ & $S$ & \\
\hline${ }^{238} \mathrm{Pu}$ & 7.97 & $\mathrm{M}$ & \\
\hline${ }^{238} \mathrm{U}$ & 3.86 & $\mathrm{M}$ & \\
\hline${ }^{239 / 240} \mathrm{Pu}$ & 9.70 & $S$ & \\
\hline${ }^{241} \mathrm{Am}$ & $<50.0$ & $S$ & Slurry $=9.04 \mathrm{Ci}$ \\
\hline${ }^{241} \mathrm{Pu}$ & 664 & $\mathbf{M}$ & \\
\hline${ }^{242} \mathrm{Cm}$ & 0.524 & $\mathbf{M}$ & \\
\hline${ }^{242} \mathrm{Pu}$ & 0.00306 & $\mathrm{M}$ & \\
\hline${ }^{243} \mathrm{Am}$ & 0.00124 & $\mathbf{M}$ & \\
\hline $243 \mathrm{Cm}$ & $<0.48$ & $S$ & \\
\hline${ }^{244} \mathrm{Cm}$ & $<12.0$ & $S$ & \\
\hline
\end{tabular}

Note:

'The tank is active. Waste transfers will change tank contents.

${ }^{2} \mathrm{~S}=$ composite sample-based, no segment data, $\mathrm{M}=\mathrm{HDW}$ model-based, $\mathrm{E}=$ engineering assessment-based 
HNF-SD-WM-ER-690 Rev, 0

This page intentionally left blank. 


\subsection{RECOMMENDATIONS}

All analytical results for the safety screening DQO (Dukelow et al. 1995) were well within the safety notification limit. The exotherms were well below the threshold of $480 \mathrm{~J} / \mathrm{g}$ on a dry weight basis with a maximum value of $175 \mathrm{~J} / \mathrm{g}$. The vapor phase measurement in tank headspace indicated 0.013 percent of LFL, well below the flammability concern level of 25 percent. The maximum value of total alpha is $0.0808 \mu \mathrm{Ci} / \mathrm{g}$ for solids, well below the criticality criteria of $34.9 \mu \mathrm{Ci} / \mathrm{g}$. The sampling and analysis activities for tank $241-\mathrm{AN}-104$ have met all the requirements for the flammable gas tank safety DQO (McDuffie and Johnson 1995). Other documents that have addressed the flammable gas safety issues include the following: the 1996 core sampling analytical results in solid and liquid phases (Steen 1997), the 1996 core sampling RGS results for the gas phase (Shekarriz et al. 1997), tank headspace monitoring data (Wilkins et al. 1997), studies of the gas generation mechanism by Meyer et al. (1997) and Pederson and Bryan (1996), and a calculation of hydrogen generation rate in steady state (Hu 1997). Additional evaluation of RGS results and the flammable gas issue to determine the safety status of the tank are in progress. To address the organic solvents screening issue (DOE-RL 1996), the Organic Safety Project will estimate the size of the organic safety extractant pool. Vapor analyses for total nonmethane organic hydrocarbon have not been conducted.

Table 4-1 summarizes the status of the Project Hanford Management Contractor (PHMC) TWRS Program review and acceptance of the sampling and analysis results reported in this TCR. Column 1 of Table 4-1 lists all DQO issues required to be addressed by sampling and analysis. Column 2 indicates whether the requirements of the DQO were met by the sampling and analysis activities performed and is answered with a "yes" or a "no." Column 3 indicates concurrence and acceptance by the program in PHMC TWRS that is responsible for the DQO that the sampling and analysis activities performed adequately meet the needs of the DQO. A "yes" or "no" in column 3 indicates acceptance or disapproval of the sampling and analysis information presented in the TCR. If the results/information have not been reviewed, "N/R" is shown; if the results/information have not been determined, "N/D" is shown. 
Table 4-1. Acceptance of Tank 241-AN-104 Sampling and Analysis.

\begin{tabular}{|c|c|c|}
\hline Fin & (1) & 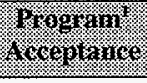 \\
\hline Safety screening DQO & Yes & Yes \\
\hline Flammable Gas Tank Safety Program & Yes & Yes \\
\hline Organic solvents screening & No & No \\
\hline Privatization low-activity waste $\mathrm{DQO}^{2}$ & $\mathrm{~N} / \mathrm{D}$ & $\mathrm{N} / \mathrm{D}$ \\
\hline
\end{tabular}

Notes:

'PHMC TWRS

'Jones and Wiemers (1996)

Table 4-2 summarizes the status of the PHMC TWRS Program review and acceptance of the evaluations and other characterization information contained in this report. The evaluations outlined in this report required to determine whether the tank is safe, conditionally safe, or unsafe. Column 1 lists the evaluations performed in this report. Columns 2 and 3 are in the same format as Table 4-1. The manner in which concurrence and acceptance are summarized is also the same as that in Table 4-1.

Table 4-2. Acceptance of Evaluation of Characterization Data and Information for Tank 241-AN-104.

\begin{tabular}{|c|c|c|}
\hline Issue- & 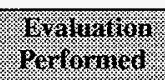 & $\begin{array}{l}\text { Program! } \\
\text { Acceptrinee }\end{array}$ \\
\hline Safety categorization & No & $\mathrm{N} / \mathrm{D}$ \\
\hline Flammable Gas Tank Safety Program & In progress & $\mathrm{N} / \mathrm{D}$ \\
\hline Organics solvents screening & No & No \\
\hline Applicability of privatization data & In progress & $\mathrm{N} / \mathrm{D}$ \\
\hline
\end{tabular}

Note:

'PHMC TWRS

The waste in tank 241-AN-104 should be monitored continuously because it generates and retains gases that are released episodically at various intervals. The safety of operations that will retrieve and dispose of the waste have not been assessed. Vapor samples to further assess the organic solvent screening issue are scheduled for Fiscal Year 1999. No additional liquid and solid characterization efforts are needed at this time. 


\subsection{REFERENCES}

Agnew, S. F., J. Boyer, R. A. Corbin, T. B. Duran, J. R. Fitzpatrick, K. A. Jurgensen, T. P. Ortiz, and B. L. Young, 1997a, Hanford Tank Chemical and Radionuclide Inventories: HDW Model Rev. 4, LA-UR-96-3680, Los Alamos National Laboratory, Los Alamos, New Mexico.

Agnew, S. F., R. A. Corbin, T. B. Duran, K. A. Jurgensen, T. P. Ortiz, and B. L. Young, 1997b, Waste Status and Transaction Record Summary WSTRS Rev. 4, LA-UR-97-311, Rev. 0, Los Alamos National Laboratory, New Mexico.

Brown, T. M., J. W. Hunt, and L. J. Fergestrom, 1997, Tank Characterization Technical Basis, HNF-SD-WM-TA-164, Rev. 3, Lockheed Martin Corp. for Fluor Daniel Hanford Inc., Richland, Washington.

Cash, R. J., 1996, Scope Increase of "Data Quality Objective to Support Resolution of the Organic Complexant Safery Issue" Rev. 2, (internal memorandum 79300-96-029 to S. J. Eberlein, July 12), Westinghouse Hanford Company, Richland, Washington.

DOE-RL, 1996, Recommendation 93-5, DOE/RL-94-0001, Rev. 1, U.S. Department of Energy, Richland Operations, Richland, Washington.

Dukelow, G. T., J. W. Hunt, H. Babad, and J. E. Meacham, 1995, Tank Safety Screening Data Quality Objective, WHC-SD-WM-SP-004, Rev. 2, Westinghouse Hanford Company, Richland, Washington.

Ecology, EPA, and DOE, 1996, Hanford Federal Facility Agreement and Consent Order, as amended, Washington State Department of Ecology, U.S. Environmental Protection Agency, and U.S. Department of Energy, Olympia, Washington.

Hanlon, B. M., 1997, Waste Tank Summary Report for Month Ending May 31, 1997, WHC-EP-0182-109, Lockheed Martin Corp. for Fluor Daniel Hanford Inc., Richland, Washington.

Heubach, E. C., 1996, Double-Shell Tank Interim Operational Safety Requirements, WHC-SD-WM-OSR-016, Rev. OE, Westinghouse Hanford Company, Richland Washington.

Hendrickson, D. W., 1994, Grout Treatment Facility Waste Feed Projections, WHC-SD-WM-TI-528, Rev. 1, Westinghouse Hanford Company, Richland, Washington. 
Hodgson, K. M., and M. D. LeClair, 1996, Work Plan for Defining a Standard Inventory Estimate for Wastes Stored in Hanford Site Underground Tanks, WHC-SD-WM-WP-311, Rev. 1, Lockheed Martin Hanford Corp. for Fluor Daniel Hanford, Inc., Richland, Washington.

Hu, T. A., 1997, Calculations of Hydrogen Release Rate at Steady State for Double-Shell Tanks, HNF-SD-WM-CN-117, Rev. 0, Lockheed Martin Hanford Corp. for Fluor Daniel Hanford, Inc., Richland, Washington.

Jones, T. E., and K. D. Wiemers, 1996, Data Requirments for TWRS Privatization Characterization of Potential Low-Activity Waste Feed, WHC-SD-WM-SP-023, Rev. 0, Westinghouse Hanford Company, Richland, Washington.

Kummerer, M., 1995, Heat Removal Characteristics of Waste Storage Tanks, WHC-SD-WM-SARR-010, Rev. 1, Westinghouse Hanford Company, Richland, Washington.

Mauss, B. M., 1984, Chemical Compositions of 102-AY, 101-AW, 105-AN, and 104-AW, (letter 65453-84-348 to E. G. Gratny, November 9), Rockwell Hanford Operation, Richland, Washington.

McDuffie, N. G., and G. D Johnson, 1995, Flammable Gas Tank Safety Program: Data Requirements for Core Sample Analysis Developed Through the Data Quality Objectives (DQO) Process, WHC-SD-WM DQO-004, Rev. 2, Westinghouse Hanford Company, Richland, Washington.

Meyer, P. A., L. R. Pederson, M. E. Brewster, C. W. Steward, S. A. Bryan, G. Terrones, and G. Chen, 1997, Gas Retention and Release Behavior in Hanford Double-Shell Waste Tanks, PNNL-11536, Rev. 1, Pacific Northwest National Laboratory, Richland, Washington.

Pederson, L. R., and S. A. Bryan, 1996, Status and Integration of Studies of Gas Generation in Hanford Wastes, PNNL-11297, Rev. 0, Pacific Northwest National Laboratory, Richland, Washington.

Public Law 101-510, 1990, "Safety Measures for Waste Tanks at Hanford Nuclear Reservation," Section 3137 of National Defense Authorization Act for Fiscal Year 1991.

Sasaki, L. M., 1984, Results of Organic Analysis of Double-Shell Slurry, (letter 65611-84-053 to D. J. Fisher, April 5), Rockwell Hanford Operation, Richland, Washington. 
Shekarriz, A., D. R. Rector, N. S. Cannon, L. A. Mahoney, B. E. Hey, M. A. Chieda, C. G. Linshooten, J. M. Bates, F. J. Reitz, R. E. Bauer, and E. R. Siciliano, 1997, Composition and Quantities of Retained Gas Measured in Hanford Waste Tanks 241-AW-101, $A-101, A N-105, A N-104$, and $A N-103$, PNNL-11450, Rev. 1, Pacific Northwest National Laboratory, Richland, Washington.

Steen, F. H., 1996, Tank 241-AN-104, Cores 163 and 164, Analytical Results for The 45-Day Report, HNF-SD-WM-DP-226, Rev. 0, Westinghouse Hanford Company, Richland, Washington.

Steen, F. H., 1997, Tank 241-AN-104, Cores 163 and 164 Analytical Results for the Final Report, HNF-SD-WM-DP-266, Rev, 1A, Rust Federal Service of Hanford, Inc. for Fluor Daniel Hanford, Inc., Richland, Washington.

Wilkins, N. E., R. E. Bauer, and D. M. Ogden, 1997, Results of Vapor Space Monitoring of Flammable Gas Watch List Tanks, HNF-SD-WM-TI-797, Rev. 1, Lockheed Martin Hanford Corp. for Fluor Daniel Hanford, Inc., Richland, Washington.

Winkelman, W. D., 1996a, Tank 241-AN-104 Push Mode Core Sampling and Analysis Plan, WHC-SD-WM-TSAP-086, Rev. 0, Westinghouse Hanford Company, Richland, Washington.

Winkelman, W. D., 1996b, Tank 241-AN-104 Tank Characterization Plan, WHC-SD-WM-SD-TP-384, Rev. 3, Westinghouse Hanford Company, Richland, Washington. 
HNF-SD-WM-ER-690 Rev. 0

This page intentionally left blank. 
HNF-SD-WM-ER-690 Rev. 0

APPENDIX A

HISTORICAL TANK INFORMATION

A-1 
HNF-SD-WM-ER-690 Rev. 0

This page intentionally left blank.

A-2 


\section{APPENDIX A}

\section{HISTORICAL TANK INFORMATION}

Appendix A describes tank 241-AN-104 based on historical information. For this report, historical information includes information about the fill history, waste types, surveillance, or modeling data about the tank. This information is necessary for providing a balanced assessment of sampling and analytical results.

This appendix contains the following information:

- Section A1.0: Current status of the tank, including the current waste levels as well as the stabilization and isolation status of the tank.

- Section A2.0: Information about the tank design.

- $\quad$ Section A3.0: Process knowledge of the tank, that is, the waste transfer history and the estimated contents of the tank based on modeling data.

- Section A4.0: Surveillance data for tank 241-AN-104 including surface-level readings, temperatures, and a description of the waste surface based on photographs.

- Section A5.0: References for Appendix A.

Historical sampling results (results from samples obtained before 1989) are provided in Appendix B.

\section{A1.0 CURRENT TANK STATUS}

As of May 31, 1997, tank 241-AN-104 contained an estimated 3,994 kL (1,055 kgal) of waste classified as double-shell slurry feed (Hanlon 1997). Liquid waste volumes are estimated using a combination of a level measurement gauge and a manual tape. The solid waste volumes are estimated using a sludge level measurement device. The solid waste volume was last updated on March 31, 1997. Table A1-1 shows the estimated volumes of the waste phases in the tank. 
Tank 241-AN-104 is categorized as sound and is actively ventilated. The tank is classified as a concentrated waste holding tank. The tank is on the Flammable Gas Watch List (Hanlon 1997). All monitoring systems were in compliance with documented standards as of May 31, 1997 (Hanlon 1997).

Table A1-1. Tank Contents Status Summary as of May 31, 1997.1,2

\begin{tabular}{|c|c|}
\hline l) & 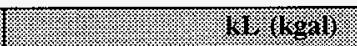 \\
\hline Total waste & $3,994(1,055)$ \\
\hline Supernatant liquid & $2,273(600.5)$ \\
\hline Double-shell slurry & $0(0)$ \\
\hline Sludge & $1,700(449)$ \\
\hline Saltcake & $21(5.5)$ \\
\hline Drainable interstitial liquid & $182(48)$ \\
\hline Drainable liquid remaining & $2,476(654)$ \\
\hline Pumpable liquid remaining & $2,392(632)$ \\
\hline
\end{tabular}

Notes:

Hanlon (1997)

${ }^{2}$ The tank is still active and waste transfers that occur will change the tank inventory.

\section{A2.0 TANK DESIGN AND BACKGROUND}

The 241-AN Tank Farm was constructed in 1980 to 1981 in the 200 East Area. The tank farm contains seven double-shell tanks. These tanks have a capacity of $4,391 \mathrm{~kL}$ $(1,160 \mathrm{kgal})$ and a diameter of $23 \mathrm{~m}$ (75 ft). These tanks were designed to hold boiling waste with a maximum design temperature of $177^{\circ} \mathrm{C}\left(350^{\circ} \mathrm{F}\right)$ (Brevick et al. 1997).

Tank 241-AN-104 was constructed with a primary carbon steel liner (heat-treated and stressrelieved), a secondary carbon steel liner (not heat-treated), and a reinforced concrete shell. The bottom of the primary liner is $13 \mathrm{~mm}(0.5 \mathrm{in}$.$) thick, the lower portion of the sides is$ $19 \mathrm{~mm}(0.75 \mathrm{in}$.) thick, the upper portion of the sides is $13 \mathrm{~mm}(0.5 \mathrm{in}$.) thick, and the dome liner is $9.5 \mathrm{~mm}(0.375 \mathrm{in}$.) thick. The secondary liner is $9.5 \mathrm{~mm}(0.375 \mathrm{in}$.) thick. The concrete walls are $450 \mathrm{~mm}(1.5 \mathrm{ft})$ thick and the dome is $380 \mathrm{~mm}(1.25 \mathrm{ft})$ thick (Brevick et al. 1997). The tank has a flat bottom. The bottoms of the primary and secondary liners are separated by an insulating concrete layer. There is a grid of drain slots in the concrete foundation beneath the secondary steel liner. The grid's function is to collect waste that may leak from the tank and divert it to the leak detection well. 
Tank 241-AN-104 has 22 risers; ranging in diameter from $100 \mathrm{~mm}$ (4 in.) to $1.1 \mathrm{~m} \mathrm{(42} \mathrm{in.),}$ which access the tank and 37 risers which access the annulus. Table A2-1 shows numbers, diameters, and descriptions of the risers (annular risers not included). Figure A2-1 shows the riser configuration. Risers $125(10 \mathrm{~A}), 131(15 \mathrm{~A})$, and $155(21 \mathrm{~A})$ (each $100 \mathrm{~mm}$ [4 in.] in diameter) and riser 127 (21A) (300 mm [12 in.] in diameter) are available for use (Lipnicki 1997). Figure A2-2 is a tank cross section showing the approximate waste level and a schematic of tank equipment. 
Table A2-1. Tank 241-AN-104 Risers. 1, 2,3

\begin{tabular}{|c|c|c|c|}
\hline 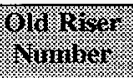 & 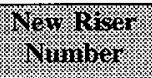 & 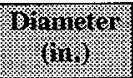 & 1. \\
\hline $1 \mathrm{~A}$ & 102 & 4 & Sludge measurement port \\
\hline $1 \mathrm{~B}$ & 103 & 4 & Sludge measurement port (12 in. cover) \\
\hline $1 \mathrm{C}$ & 101 & 4 & Sludge measurement port \\
\hline $2 \mathrm{~A}$ & 104 & 4 & Liquid level, level indicating transmitter \\
\hline $3 \mathrm{~A}$ & 015 & 12 & Supernatant pump, central pump pit \\
\hline $4 \mathrm{~A}$ & 106 & 4 & Thermocouple tree \\
\hline $5 \mathrm{~A}$ & 108 & 42 & Manhole \\
\hline $5 \mathrm{~B}$ & 107 & 42 & Manhole \\
\hline $7 \mathrm{~A}$ & 112 & 12 & Spare (12 in. cover) \\
\hline $7 \mathrm{~B}$ & 111 & 12 & Tank ventilation \\
\hline $10 A^{4}$ & 125 & 4 & Spare \\
\hline $11 \mathrm{~A}$ & 126 & 42 & Slurry distributor, central pump pit \\
\hline $12 \mathrm{~A}^{4}$ & 127 & 12 & Observation port, spare \\
\hline $13 \mathrm{~A}$ & 128 & 4 & Tank pressure \\
\hline $14 \mathrm{~A}$ & 129 & 4 & Supernate return, central pump pit \\
\hline $15 \mathrm{~A}^{4}$ & 131 & 4 & Spare (12 in. cover) \\
\hline $15 B$ & 130 & 4 & High liquid-level sensor \\
\hline $16 \mathrm{~A}$ & 134 & 4 & Sludge measurement port \\
\hline 16B & 132 & 4 & Sludge measurement port \\
\hline $16 \mathrm{C}$ & 133 & 4 & Sludge measurement port \\
\hline $21 \mathrm{~A}^{4}$ & 155 & 4 & Spare \\
\hline $22 \mathrm{~A}$ & 156 & 4 & Sludge measurement port \\
\hline
\end{tabular}

Notes:

${ }^{1}$ Salazar (1994)

${ }^{2} \mathrm{~T}$ ran (1993)

${ }^{3}$ WHC (1992)

${ }^{4}$ Indicates risers tentatively available for sampling (Lipnicki 1997) 
Figure A2-1. Riser Configuration for Tank 241-AN-104.

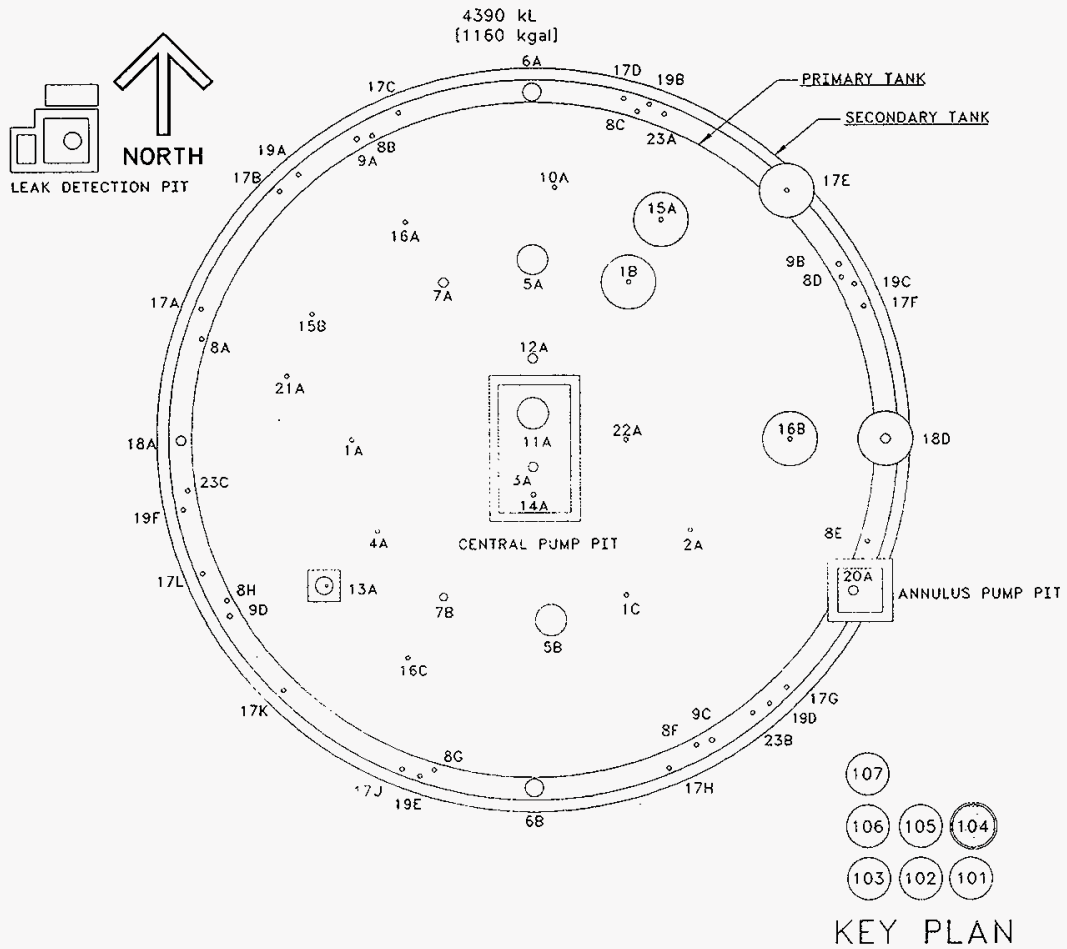


Figure A2-2. Tank 241-AN-104 Cross Section and Schematic.

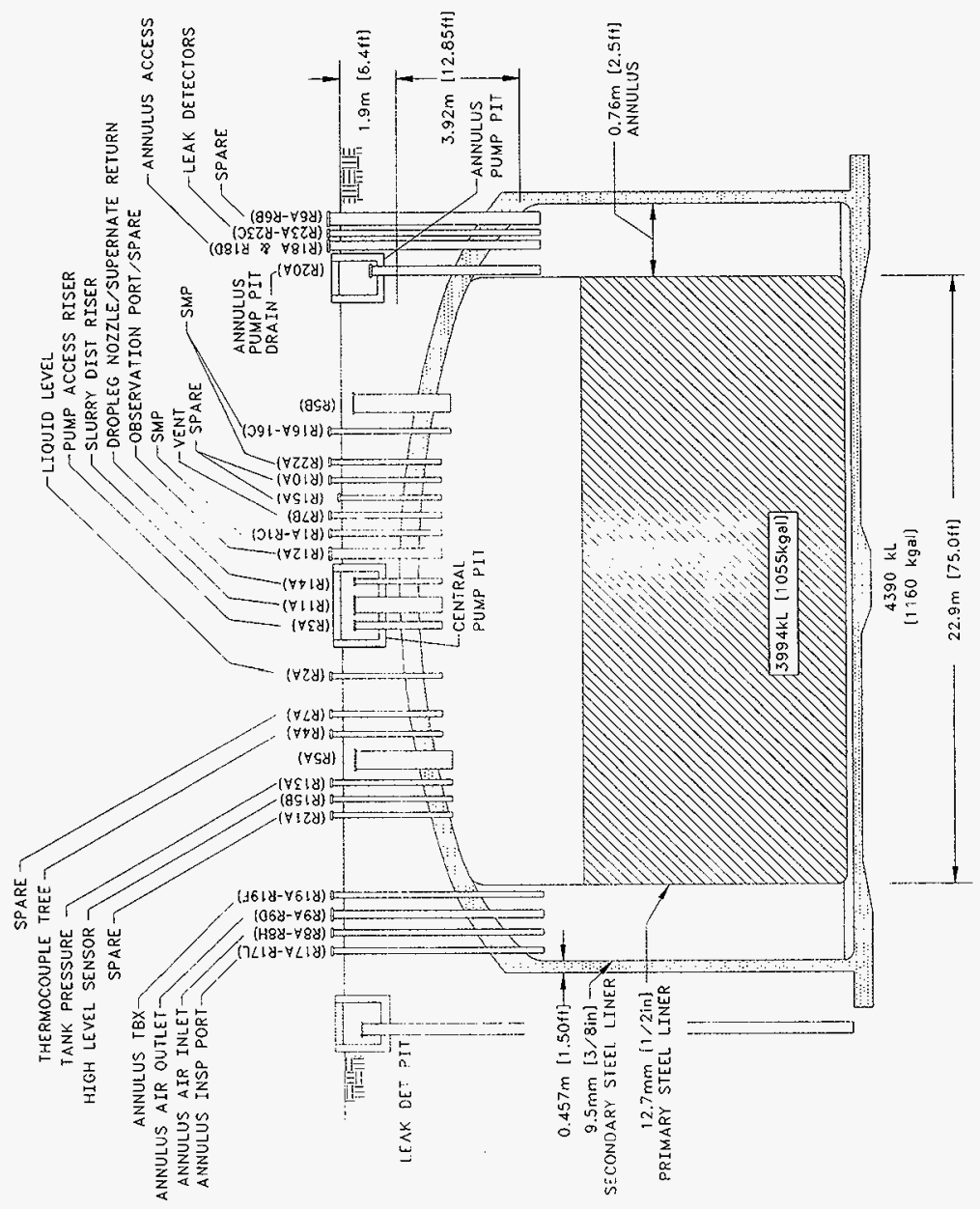




\section{A3.0 PROCESS KNOWLEDGE}

The following sections 1) provide information about the waste transfer history of tank 241-AN-104, 2) describe the process wastes that were transferred, and 3) provide an estimate of current tank contents based on waste transfer history.

\section{A3.1 WASTE TRANSFER HISTORY}

Table A3-1 summarizes the waste transfer history of tank 241-AN-104 (Agnew et al. 1997b). Tank 241-AN-104 received flush water in the second quarter of 1982, then received dilute noncomplexed waste from tank 241-AW-102 in the fourth quarter of 1982. The tank received waste from miscellaneous PUREX streams in the fourth quarter of 1983. Waste from the tank was sent to tanks 241-AZ-102 and 241-AN-105 in the first quarter of 1984 . Also during that time, tank 241-AN-104 received additional dilute noncomplexed waste from tank 241-AW-102.

In the third quarter of 1984 , the tank received $82 \mathrm{kgal}$ of flush water from miscellaneous sources, and waste was sent to tank 241-AN-103. Tank 241-AW-102 sent dilute noncomplexed waste to tank 241-AN-104 during the fourth quarter of 1984 and the second quarter of 1985. Flush water from miscellaneous sources was added to the tank. From the second quarter of 1982 to the present, waste level changes have occurred because of gas generation and venting in the waste. 
Table A3-1. Tank 241-AN-104 Major Waste Transfers. ${ }^{1,2,3}$

\begin{tabular}{|c|c|c|c|c|c|}
\hline \multirow{2}{*}{ Thuster: } & \multirow{2}{*}{ 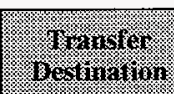 } & \multirow{2}{*}{ 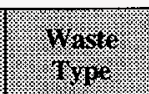 } & \multirow[b]{2}{*}{ Arine Ierruta } & \multicolumn{2}{|c|}{ 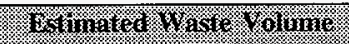 } \\
\hline & & & & Kroliors. & (Filogallows \\
\hline 241-AW-102 & & DN & 1982 & 4,206 & 1,111 \\
\hline \multirow[t]{3}{*}{ PUREX } & & PXMSC & 1983 & 19 & 5 \\
\hline & 241-AZ-102 & $\mathrm{DN}$ & 1984 & $-2,158$ & -570 \\
\hline & 241-AN-105 & $\mathrm{DN}$ & 1984 & $-1,889$ & -499 \\
\hline \multirow[t]{2}{*}{ 241-AW-102 } & & DN & 1984 & 1,098 & 290 \\
\hline & 241-AN-103 & DN & 1984 & -731 & -193 \\
\hline $241-A W-102$ & & DN & 1984 & 2,650 & 700 \\
\hline $241-A W-102$ & & $\mathrm{DN}$ & 1985 & 458 & 121 \\
\hline \begin{tabular}{|l|} 
Miscellaneous \\
sources
\end{tabular} & & Flush water & 1996 & 8 & 2 \\
\hline
\end{tabular}

Notes:

DN $=\quad$ Dilute noncomplexed waste (that is, contains no complexants) defined as waste with TOC $<1 w t \%(10 \mathrm{~g} / \mathrm{L})$

PXMSC $=$ Dilute noncomplexed waste from miscellaneous PUREX streams

'Agnew et al. (1997b)

${ }^{2}$ Because only major transfers are listed, the sum of these transfers will not equal the current tank waste volume.

${ }^{3}$ Waste volumes and types are best estimates based on historical data. 


\section{A3.2 HISTORICAL ESTIMATION OF TANK CONTENTS}

The historical transfer used for this estimate are from the following sources:

- Waste Status and Transaction Record Summary for the Northeast Quadrant (WSTRS) (Agnew et al. 1997b). WSTRS is a tank-by-tank quarterly summary spreadsheet of waste transactions.

- Hanford Tank Chemical and Radionuclide Inventories: HDW Model Rev. 4 (Agnew et al. 1997a) This document contains the Hanford defined waste (HDW) list, the supernatant mixing model (SMM), and the tank layer model (TLM).

- The HDW list which is comprised of approximately 50 waste types defined by concentrations for major analytes/components for sludge and supernatant layers.

- Tank layer model (TLM). The TLM defines the sludge and saltcake layers in each tank using waste composition and waste transfer information.

Supernatant mixing model (SMM). This is a subroutine within the HDW model that calculates the volume and composition of certain supernatant blends and concentrates.

Using these records, the TLM defines the sludge and saltcake layers in each tank. The SMM uses information from both the WSTRS and the TLM to describe the supernatants and concentrates in each tank. Together, the WSTRS, TLM, and SMM determine each tank's inventory estimate. These model predictions are considered estimates that require further evaluation using analytical data.

Based on the TLM and SMM, tank 241-AN-104 contains 4,012 kL (1,060 kgal) of waste comprised of a bottom solids layer of $999 \mathrm{~kL}$ ( $264 \mathrm{kgal})$ of concentrated supernatant solids waste (SMMA2) and 3,013 kL (796 kgal) of supernatant liquid above the surface of the solid waste. As of May 31, 1997, the tank contains a bottom sludge layer, a middle supernatant layer, and a top crust layers with a volume of $1,700 \mathrm{~kL}$ (449 kgal), 2,273 (600.5 kgal), and $21 \mathrm{~kL}(5.5 \mathrm{kgal})$, respectively. This historical estimate is for information only. Figure A3-1 is a graphical representation of the estimated waste types and volumes for each tank layer. Table A3-3 provides an estimate of the expected waste constituents and concentrations. 
HNF-SD-WM-ER-690 Rev. 0

Figure A3-1. Tank Layer Model. ${ }^{1}$

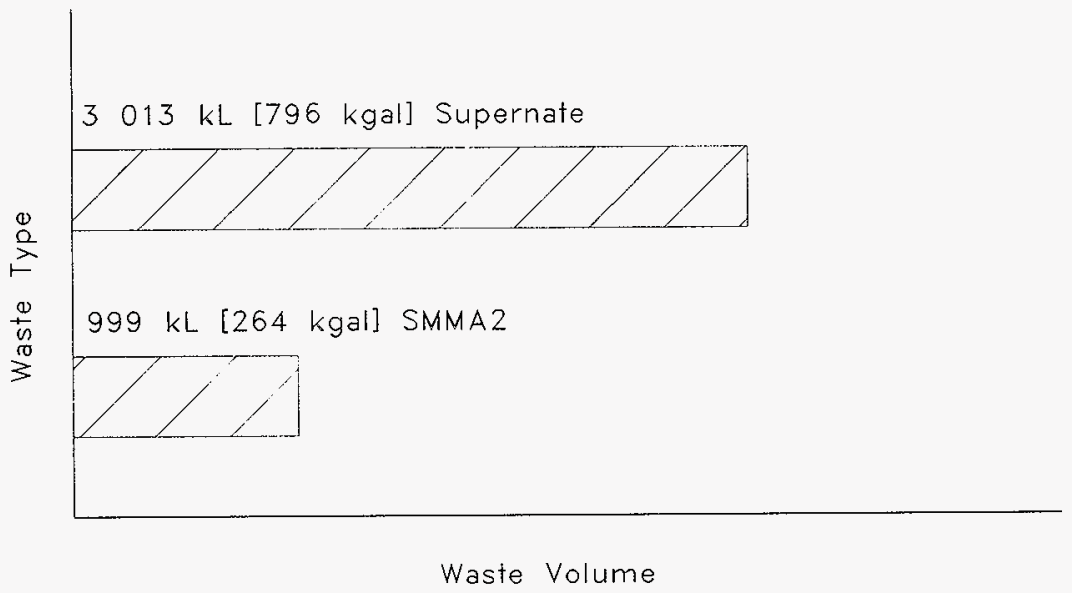

'This historical estimate does not reflect the current volume of sludge and supernatant. It should be used for information only. 
Table A3-2. Historical Tank Inventory Estimate. ${ }^{1,2}$ (2 sheets)

\begin{tabular}{|c|c|c|c|c|c|}
\hline 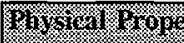 & 1395: & 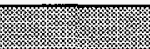 & 8 & J 95 . & 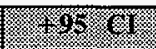 \\
\hline Total waste & $5.89 \mathrm{E}+06(\mathrm{~kg})$ & $\begin{array}{l}(1.06 \mathrm{E}+03 \\
\mathrm{kgal})\end{array}$ & $\cdots$ & --- & -- \\
\hline Heat load & $6.06(\mathrm{~kW})$ & $\begin{array}{l}(2.07 \mathrm{E}+04 \\
\text { Btu/hr) }\end{array}$ & -- & 5.54 & 6.58 \\
\hline Bulk density ${ }^{3}$ & $1.47 \mathrm{~g} / \mathrm{cm}^{3}$ & - & - & 1.43 & 1.51 \\
\hline Water wt\% ${ }^{3}$ & 42.8 & -- & -- & 40.1 & 45.3 \\
\hline $\begin{array}{l}\text { TOC wt\% C } \\
\text { (wet) }^{3}\end{array}$ & 1.05 & --- & $-\cdots$ & 0.666 & 1.44 \\
\hline $\begin{array}{l}\text { Ciremingl } \\
\text { Cinustiments }\end{array}$ & (1) & (1) & $\sqrt{18}$ & $900.9(1 / 4)$ & $4^{193}=$ \\
\hline $\mathrm{Na}^{+}$ & 11.1 & $1.73 \mathrm{E}+05$ & $1.02 \mathrm{E}+06$ & 10.2 & 12.0 \\
\hline $\mathrm{Al}^{3+}$ & 1.26 & $2.31 E+04$ & $1.36 \mathrm{E}+05$ & 1.08 & 1.33 \\
\hline $\mathrm{Fe}^{3+}$ (total $\left.\mathrm{Fe}\right)$ & $9.06 \mathrm{E}-03$ & 343 & $2.02 E+03$ & $8.19 \mathrm{E}-03$ & $9.92 \mathrm{E}-03$ \\
\hline $\mathrm{Cr}^{3+}$ & $8.91 \mathrm{E}-02$ & $3.14 \mathrm{E}+03$ & $1.85 \mathrm{E}+04$ & $7.92 \mathrm{E}-02$ & $9.83 \mathrm{E}-02$ \\
\hline $\mathrm{Bi}^{3+}$ & $9.49 \mathrm{E}-04$ & 134 & 793 & $8.90 \mathrm{E}-04$ & $1.01 \mathrm{E}-03$ \\
\hline $\mathrm{La}^{3+}$ & $1.27 \mathrm{E}-05$ & 1.19 & 7.04 & $9.38 \mathrm{E}-06$ & $1.60 \mathrm{E}-05$ \\
\hline $\mathrm{Hg}^{2+}$ & $9.15 \mathrm{E}-06$ & 1.25 & 7.34 & $7.76 \mathrm{E}-06$ & $9.43 \mathrm{E}-06$ \\
\hline $\begin{array}{l}\mathrm{Zr} \text { (as } \\
\left.\mathrm{ZrO}(\mathrm{OH})_{2}\right)\end{array}$ & $7.49 \mathrm{E}-04$ & 46.3 & 273 & $4.22 \mathrm{E}-04$ & $8.29 \mathrm{E}-04$ \\
\hline $\mathrm{Pb}^{2+}$ & $9.48 \mathrm{E}-04$ & 133 & 785 & $7.49 \mathrm{E}-04$ & $1.15 \mathrm{E}-03$ \\
\hline $\mathrm{Ni}^{2+}$ & $5.71 \mathrm{E}-03$ & 227 & $1.34 \mathrm{E}+03$ & $5.56 \mathrm{E}-03$ & $5.83 \mathrm{E}-03$ \\
\hline $\mathrm{Sr}^{2+}$ & 0 & 0 & 0 & 0 & 0 \\
\hline $\mathrm{Mn}^{4+}$ & $6.70 \mathrm{E}-03$ & 250 & $1.47 \mathrm{E}+03$ & $4.54 \mathrm{E}-03$ & $8.55 \mathrm{E}-03$ \\
\hline $\mathrm{Ca}^{2+}$ & $3.19 \mathrm{E}-02$ & 868 & $5.11 \mathrm{E}+03$ & $2.97 \mathrm{E}-02$ & $3.41 \mathrm{E}-02$ \\
\hline $\mathrm{K}^{+}$ & 0.100 & $2.65 \mathrm{E}+03$ & $1.56 \mathrm{E}+04$ & $5.45 \mathrm{E}-02$ & 0.148 \\
\hline$\widehat{\mathrm{OH}^{-}}$ & 7.38 & $8.51 \mathrm{E}+04$ & $5.02 \mathrm{E}+05$ & 6.50 & 7.99 \\
\hline $\mathrm{NO}_{3}$ & 3.71 & $1.56 \mathrm{E}+05$ & $9.19 \mathrm{E}+05$ & 3.49 & 3.93 \\
\hline $\mathrm{NO}_{2}^{-}$ & 1.72 & $5.35 \mathrm{E}+04$ & $3.16 \mathrm{E}+05$ & 1.39 & 1.97 \\
\hline $\mathrm{CO}_{3}{ }^{2-}$ & 0.504 & $2.05 E+04$ & $1.21 \mathrm{E}+05$ & 0.434 & 0.564 \\
\hline$\overline{\mathrm{PO}_{4}{ }^{3-}}$ & 0.115 & $7.42 \mathrm{E}+03$ & $4.37 \mathrm{E}+04$ & 9.01E-02 & 0.140 \\
\hline $\mathrm{SO}_{4}{ }^{2-}$ & 0.210 & $1.37 \mathrm{E}+04$ & $8.08 \mathrm{E}+04$ & 0.173 & 0.238 \\
\hline $\mathrm{Si}\left(\right.$ as $\left.\mathrm{SiO}_{3}{ }^{2-}\right)$ & $6.10 \mathrm{E}-02$ & $1.16 \mathrm{E}+03$ & $6.85 \mathrm{E}+03$ & $5.30 \mathrm{E}-02$ & $6.88 \mathrm{E}-02$ \\
\hline
\end{tabular}


Table A3-2. Historical Tank Inventory Estimate. ${ }^{1,2}$ (2 sheets)

\begin{tabular}{|c|c|c|c|c|c|}
\hline \multicolumn{2}{|c|}{ 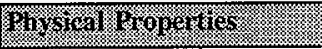 } & & & \multicolumn{2}{|c|}{95.612} \\
\hline $\mathrm{F}^{-}$ & $9.99 \mathrm{E}-02$ & $1.29 \mathrm{E}+03$ & $7.59 \mathrm{E}+03$ & $5.28 \mathrm{E}-02$ & 0.116 \\
\hline $\mathrm{Cl}^{-}$ & 0.190 & $4.56 \mathrm{E}+03$ & $2.69 \mathrm{E}+04$ & 0.168 & 0.206 \\
\hline $\mathrm{C}_{6} \mathrm{H}_{5} \mathrm{O}_{7}{ }^{3-}$ & $2.61 \mathrm{E}-02$ & $3.34 \mathrm{E}+03$ & $1.97 \mathrm{E}+04$ & $2.21 E-02$ & $3.00 \mathrm{E}-02$ \\
\hline EDTA $^{4}$ & $2.12 \mathrm{E}-02$ & $4.14 \mathrm{E}+03$ & $2.44 \mathrm{E}+04$ & $6.95 \mathrm{E}-03$ & $3.56 \mathrm{E}-02$ \\
\hline HEDTA $^{3-}$ & $3.78 \mathrm{E}-02$ & $7.02 \mathrm{E}+03$ & $4.14 \mathrm{E}+04$ & $9.34 \mathrm{E}-03$ & $6.67 \mathrm{E}-02$ \\
\hline glycolate & 0.142 & $7.22 \mathrm{E}+03$ & $4.25 \mathrm{E}+04$ & $8.90 \mathrm{E}-02$ & 0.195 \\
\hline acetate $^{-}$ & $1.46 \mathrm{E}-02$ & 584 & $3.44 \mathrm{E}+03$ & $1.15 \mathrm{E}-02$ & $1.94 \mathrm{E}-02$ \\
\hline oxalate $^{2-}$ & $1.66 \mathrm{E}-05$ & 0.991 & 5.84 & $1.48 \mathrm{E}-05$ & $1.84 \mathrm{E}-05$ \\
\hline$\overline{\mathrm{DBP}}$ & $1.97 \mathrm{E}-02$ & $2.81 \mathrm{E}+03$ & $1.65 \mathrm{E}+04$ & $1.68 \mathrm{E}-02$ & $2.41 \mathrm{E}-02$ \\
\hline butanol & $1.97 \mathrm{E}-02$ & 989 & $5.83 \mathrm{E}+03$ & $1.68 \mathrm{E}-02$ & $2.41 \mathrm{E}-02$ \\
\hline $\mathrm{NH}_{3}$ & 0.206 & $2.37 \mathrm{E}+03$ & $1.40 \mathrm{E}+04$ & $4.68 \mathrm{E}-02$ & 0.371 \\
\hline $\mathrm{Fe}(\mathrm{CN})_{6}^{4}$ & 0 & 0 & 0 & 0 & 0 \\
\hline
\end{tabular}

Notes:

${ }^{1}$ Unknowns in tank solids inventory are assigned by the TLM.

${ }^{2}$ Historical data is not validated and should be used with caution.

${ }^{3}$ Water wt\% derived from the difference of density and total dissolved species. 


\section{A4.0 SURVEMLANCE DATA}

Tank 241-AN-104 surveillance includes surface level measurements (liquid and solid) and temperature monitoring inside the tank (waste and headspace). The tank annulus is equipped with leak-detection instrumentation consisting of continuous air monitors and conductivity probes (Johnson 1995). Surveillance data provide the basis for determining tank integrity.

For double-shell tanks, the leak detection instruments are the primary means of detecting a leak from the primary tank; liquid-level measurements may be used to confirm a possible leak detected by the annulus instruments (Johnson 1995). Solid surface level measurements can indicate physical changes and consistency of the solid layers.

\section{A4.1 SURFACE LEVEL READINGS}

A Food Instrument Corporation gauge was used to monitor the waste surface level in tank 241-AN-104 through riser 2A in the automatic mode until July 31, 1995, and in the manual mode until May 25, 1995. A manual ENRAF ${ }^{1}$ system began recording on August 4, 1995. On May 31, 1997, the waste surface level was $9.76 \mathrm{~m}$ (383.81 in.) as measured by the ENRAF $^{\mathrm{TM}}$ system. Figure A4-1 is a level history graph of the volume measurements.

\section{A4.2 INTERNAL TANK TEMPERATURES}

Tank 241-AN-104 has a single thermocouple tree, located in riser 4A, with 18 thermocouples to monitor the waste temperature. Temperature data recorded from March 14, 1994, through October 24, 1996, were obtained from the Surveillance Analysis Computer System (LMHC 1997) for all thermocouples. The average temperature of the Surveillance Analysis Computer System data is $42.7^{\circ} \mathrm{C}\left(108.9^{\circ} \mathrm{F}\right)$, the minimum is $31.8^{\circ} \mathrm{C}\left(89.2^{\circ} \mathrm{F}\right)$, and the maximum is $51.2^{\circ} \mathrm{C}\left(124.2^{\circ} \mathrm{F}\right)$. The average temperature of the these data over the last year (October 1995 through October 1996) was $41.3^{\circ} \mathrm{C}\left(106.4^{\circ} \mathrm{F}\right)$, the minimum was $31.8^{\circ} \mathrm{C}\left(89.2^{\circ} \mathrm{F}\right)$, and the maximum was $50.1^{\circ} \mathrm{C}\left(122.2^{\circ} \mathrm{F}\right)$. The maximum temperature on October 24,1996 , was $46.7^{\circ} \mathrm{C}\left(116.1^{\circ} \mathrm{F}\right)$ on thermocouple 4 , and the minimum was $35.2^{\circ} \mathrm{C}\left(95.4^{\circ} \mathrm{F}\right)$ on thermocouple 18 . Thermocouple 4 is located in the waste, and thermocouple 18 is in the headspace. Figure A4-2 is a graph of the weekly high temperatures. Plots of the individual thermocouple readings can be found in the Supporting Document for the Historical Tank Content Estimate for AN Tank Farm (Brevick et al. 1997).

The tank has a multifunctional instrument tree with 22 thermocouples located in riser 15A. Temperature data from June 10,1996 to the present is recorded manually weekly and ranges from $31.7^{\circ} \mathrm{C}\left(89.1^{\circ} \mathrm{F}\right)$ to $47.2^{\circ} \mathrm{C}\left(116.9^{\circ} \mathrm{F}\right)$ with average around $42.3^{\circ} \mathrm{C}\left(108.2^{\circ} \mathrm{F}\right)$.

\footnotetext{
${ }^{1}$ ENRAF is a trademark of the ENRAF Corporation, Houston, Texas.
} 


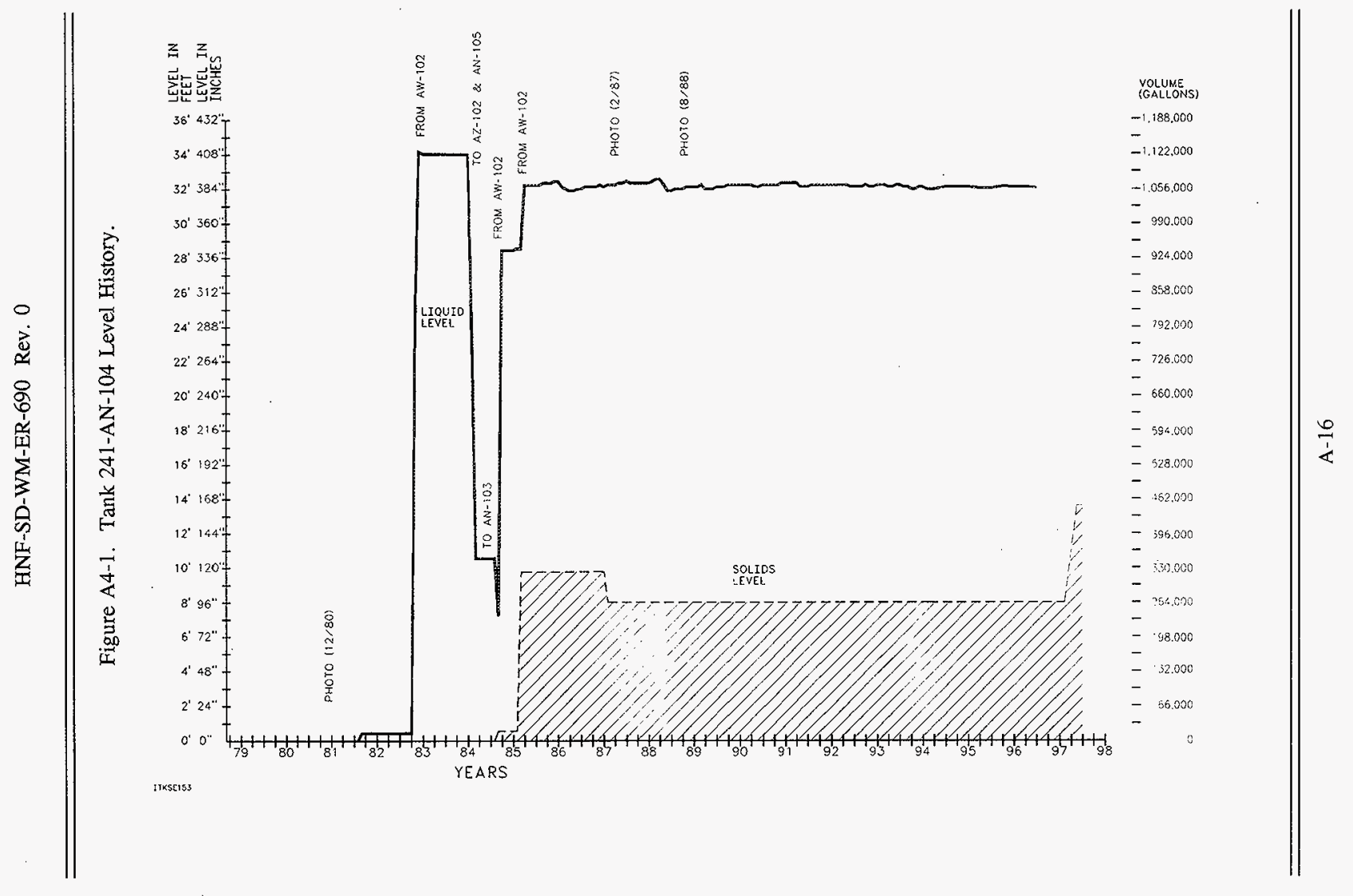


HNF-SD-WM-ER-690 Rev. 0

Figure A4-2. Tank 241-AN-104 Weekly High Temperature Plot.

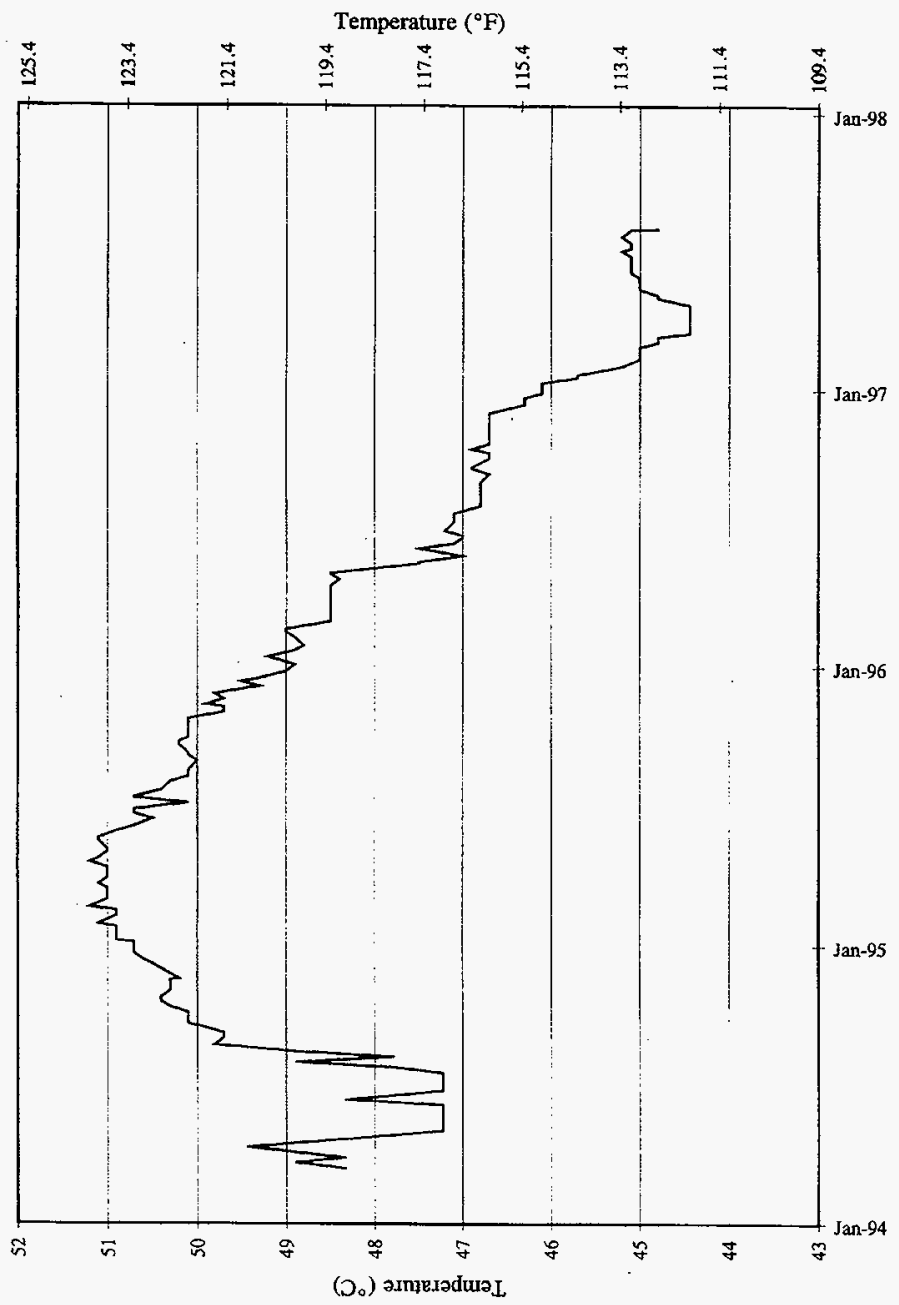


Figure A4-3 shows a typical temperature profile of tank AN-104 from riser 4A (October 22, 1996. The temperature profile indicates the nonconductive sludge layer (the curve line below the elevation of $190 \mathrm{in}$.) and the convective supernatant layer (the straight line between 190 and $380 \mathrm{in.).} \mathrm{This} \mathrm{is} \mathrm{roughly} \mathrm{consistent} \mathrm{with} \mathrm{observations} \mathrm{from} \mathrm{the} \mathrm{sample} \mathrm{extrusion} \mathrm{results}$ (see Table B1-2) where segments 13 to 21 are sludge and segments 2 to 12 are supernatant. The top segment is crust. Notice the temperature is lower on the top and bottom of the sludge layer because of the thermal dissipation by the supernatant and the tank bottom heat sink.

Figure A4-3. Tank 241-AN-104 Temperature Profile - Riser 4A.

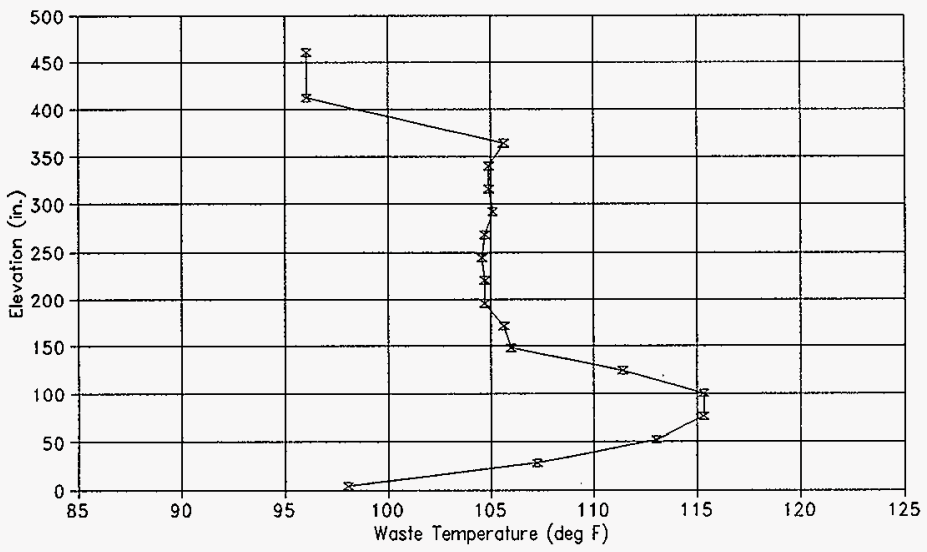

$z-10 / 22 / 96$

\section{A4.3 TANK 241-AN-104 PHOTOGRAPHS}

A video camera is located in riser 5B. A video (Harding 1996) was recorded in February 27,1996 , that shows the waste surface cover with light brown, cream-colored crust with small pools of dark liquid scattering around. A lot of these liquid pools are formed because of the pipe line breaking through. 


\section{A5.0 APPENDIX A REFERENCES}

Agnew, S. F., J. Boyer, R. A. Corbin, T. B. Duran, J. R. Fitzpatrick, K. A. Jurgensen, T. P. Ortiz, and B. L. Young, 1997a, Hanford Tank Chemical and Radionuclide Inventories: HDW Model Rev. 4, LA-UR-96-3860, Rev. 0, Los Alamos National Laboratory, Los Alamos, New Mexico.

Agnew, S. F., P. Baca, R. A. Corbin, T. B. Duran, and K. A. Jurgensen, 1997b, Waste Status and Transaction Record Summary, (WSTRS), Rev. 4, LA-UR-97-311, Rev. 0, Los Alamos National Laboratory, Los Alamos, New Mexico.

Brevick, C. H., J. L. Stroup, and J. W. Funk, 1997, Supporting Document for the Historical Tank Content Estimate for AN Tank Farm, HNF-SD-WM-ER-314, Rev. 1, Fluor Daniel Northwest, Inc. for Fluor Daniel Hanford, Inc., Richland, Washington.

Hanlon, B. M., 1997, Waste Tank Summary Report for Month Ending May 31, 1997, HNF-EP-0182-110, Lockheed Martin Hanford Corp. for Fluor Daniel Hanford, Inc., Richland, Washington.

Harding, R. H., 1996, "AN-103, 104, and 105 Tank Surface Scan," Videograph 200-1470, Westinghouse Hanford Company, Richland, Washington.

Johnson, M. G., 1995, Technical Bases for Leak Detection Surveillance of Waste Storage Tanks, WHC-SD-WM-TI-573, Rev, 1, Westinghouse Hanford Company, Richland, Washington.

Lipnicki, J., 1997, Waste Tank Risers Available for Sampling, HNF-SD-WM-TI-710, Rev. 4, Lockheed Martin Hanford Corp. for Fluor Daniel Hanford, Inc., Richland, Washington.

LMHC, 1997, SACS: Surveillance Analysis Computer System. In: SQL Server/Visual Basic (Mainframe). Available: HLAN, Lockheed Martin Hanford Company, Richland, Washington.

Tran, T. T., 1993, Thermocouple Status Single Shell \& Double Shell Waste Tanks, WHC-SD-WM-TI-553, Rev. 0, Westinghouse Hanford Company, Richland, Washington.

Salazar, B. E., 1994, Double-Shell Underground Waste Storage Tanks Riser Survey, WHC-SD-RE-TI-093, Rev. 4, Westinghouse Hanford Company, Richland, Washington.

WHC, 1992, Piping Plan Tank AN-104, Drawing H-2-71994, Rev. 8, Westinghouse Hanford Company, Richland, Washington. 
HNF-SD-WM-ER-690 Rev. 0

This page intentionally left blank. 
HNF-SD-WM-ER-690 Rev. 0

\section{APPENDIX B}

SAMPLING OF TANK 241-AN-104 
HNF-SD-WM-ER-690 Rev. 0

This page intentionally left blank. 


\section{APPENDIX B}

\section{SAMPLING OF TANK 241-AN-104}

Appendix B provides sampling and analysis information for each known sampling event for tank 241-AN-104, and it assesses the core sample results.

- Section B1.0: Tank Sampling Overview

- Section B2.0: Analytical Results

- Section B3.0: Assessment of Characterization Results

- $\quad$ Section B4.0: References for Appendix B

Future sampling of tank 241-AN-104 will be appended to the above list.

\section{B1.0 TANK SAMPLING OVERVIEW}

This section describes the August/September 1996 sampling and analysis events for tank 241-AN-104. Core samples were obtained to satisfy the requirements of the Tank Safety Screening Data Quality Objective (Dukelow et al. 1995) and the Flammable Gas Safety Program: Data Requirements for Core Sample Analysis Developed through the Data Quality Objectives (DQO) Process (McDuffie and Johnson 1995). The sampling and analyses were performed according to the Tank 241-AN-104 Push Mode Core Sampling and Analysis Plan (Winkleman 1996a). The plan required that 4.0 liters of supernatant from the tank be obtained as part of the push mode core sampling event. The sample will be used to support the privatization initiative. Two supernatant samples were obtained from this tank in 1984 (Jansky 1984b) to support Evaporator Campaign Run 84-5. One supernatant sample was obtained in August 1984 to support Evaporator Campaign Run 85-3.

\section{B1.1 DESCRIPTION OF 1996 SAMPLING EVENT}

Twenty push mode core segments were removed from tank 241-AN-104 riser 12A (core 164) between August 8, 1996, and August 14, 1996. The segments were received by the 222-S Laboratory between August 16, 1996, and August September 17, 1996. Segment 18 was sampled using the RGS and extruded by the Process Chemistry and Statistical Analysis Group. Segment 21 was not recovered from the tank because of a broken sampler. 
The second core, consisting of 21 push mode core segments, was removed from tank 241-AN-104 riser 10A (core 163) between September 9, 1996, and September 12, 1996. Segments were received by the 222-S Laboratory between September 12, 1996, and September 27, 1996. Selected segments $(3,13,15,17,19$, and 21) were sampled using the RGS and extruded by the Process Chemistry and Statistical Analysis Group.

A field blank was provided to the $222-S$ Laboratory with core 164 . It underwent the same analysis as the drainable liquid as instructed by Winkleman (1996a).

A lithium bromide solution, used as hydrostatic head fluid (HHF) during sampling, was provided to the 222-S Laboratory with core 164 . It underwent inductively coupled plasma spectroscopy (ICP) and ion chromatography (IC) analyses as instructed by Winkleman 1996a).

Safety screening analyses included the following: total alpha to determine criticality, DSC to determine the fuel energy value, and thermogravimetric analysis (TGA) to determine the total moisture content. In addition, combustible gas meter readings in the tank headspace were performed to measure flammability. The current revision of the safety screening DQO also requires bulk density measurements.

Tank 241-AN-104 also was evaluated for the Flammable Gas Safety Project. The specified analyses included the following: DSC, TGA, bulk density, viscosity, void fraction, total inorganic carbon (TIC) TOC, formate, oxalate, ICP, IC, and radiochemistry.

Table B1-1 summarizes the sampling and analytical requirements of the safety screening and flammable gas DQOs. 
Table B1-1. Integrated Data Quality Objective Requirements for Tank 241-AN-104. ${ }^{1}$

\begin{tabular}{|c|c|c|c|}
\hline (1) & Wollowillo & 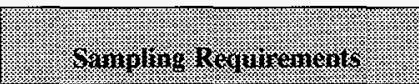 & W. \\
\hline \multirow[t]{2}{*}{ Core sampling } & Safety screening & $\begin{array}{l}\text { Core samples from a minimum of } \\
\text { two risers separated radially to the } \\
\text { maximum extent possible. }\end{array}$ & $\begin{array}{l}\text { - Energetics } \\
\text { - Moisture content } \\
\text { - Total alpha }\end{array}$ \\
\hline & Flammable gas & At least one complete core & $\begin{array}{l}\text { - Energetics } \\
\text { - Moisture content } \\
\text { - Bulk density } \\
\text { - Viscosity } \\
\text { - Void fraction } \\
\text { - TIC/TOC } \\
\text { - Formate } \\
\text { - Oxalate } \\
\text { - OH } \\
\text { - ICP } \\
\text { - IC. } \\
\text { - Radiochemistry }\end{array}$ \\
\hline $\begin{array}{l}\text { Combustible } \\
\text { gas meter } \\
\text { reading }\end{array}$ & Safety screening & $\begin{array}{l}\text { Measurement in a minimum of one } \\
\text { location within tank headspace. }\end{array}$ & $\begin{array}{l}\text { - Flammable gas } \\
\text { concentration }\end{array}$ \\
\hline Vapor & $\begin{array}{l}\text { Organic solvent } \\
\text { screening }^{2}\end{array}$ & Steel canisters & - Organic vapor \\
\hline
\end{tabular}

Notes:

'Winkieman (1996a)

${ }^{2}$ Sampling for organic solvent screening has not been performed.

\section{B1.2 SAMPLE HANDLING OF THE 1996 SAMPLING EVENT}

The core samples were shipped to the 222-S Laboratory for subsampling and analysis. Samples were assigned LABCORE numbers and were subjected to visual inspection for color, clarity, and solids content. The radiation dose rate on contact was also measured. The salt slurry samples were divided into upper half (UH) and lower half (LH) segments. The material was homogenized and subsampled for laboratory analyses and archiving. A subsample of each half-segment was recombined and subsampled for core composite analyses. Table B1-2 provides the subsampling scheme and sample description for cores 163 and 164 . 
Table B1-2. Sample Receipt and Extrusion Information for Tank 241-AN-104. (7 sheets)

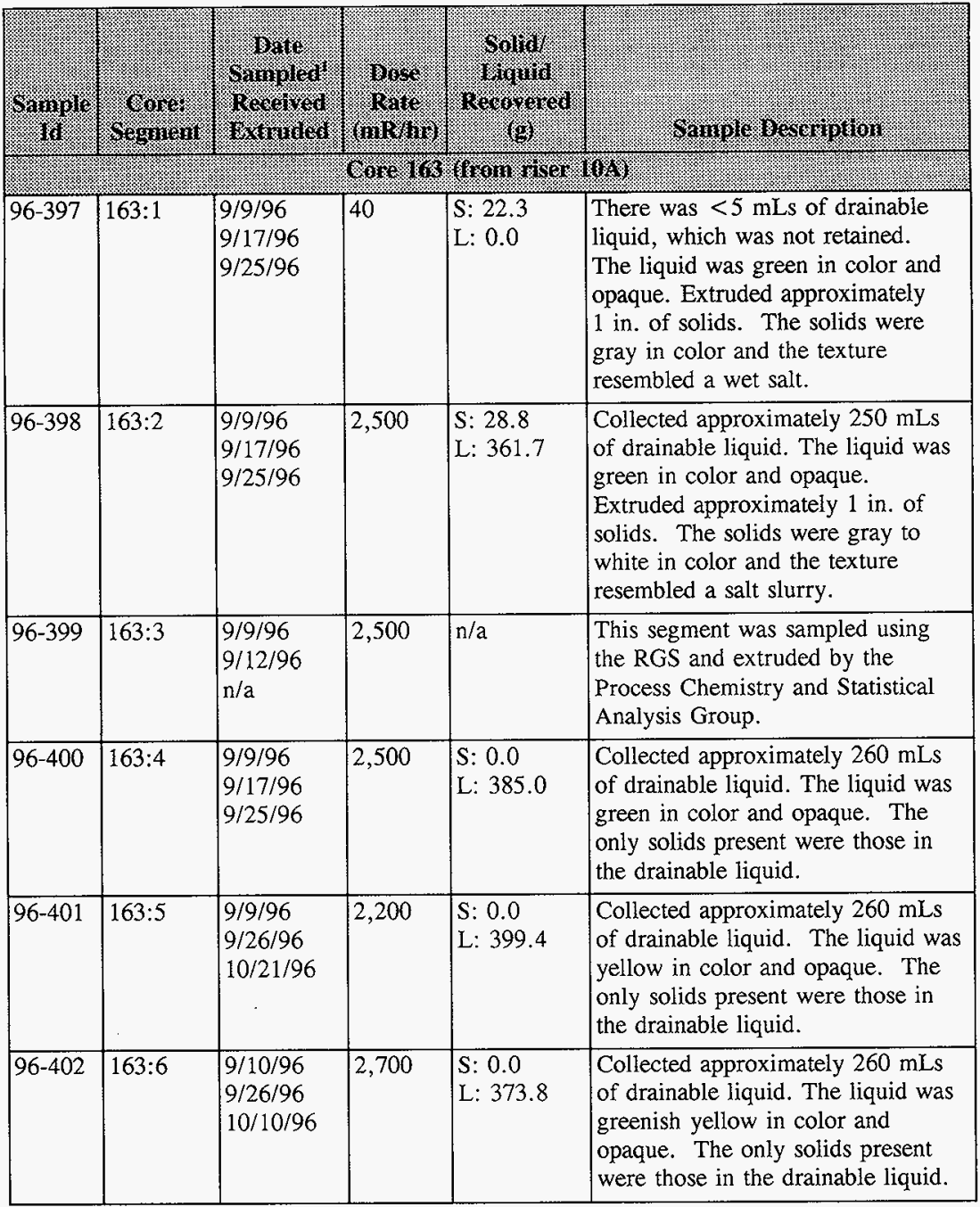


Table B1-2. Sample Receipt and Extrusion Information for Tank 241-AN-104. (7 sheets)

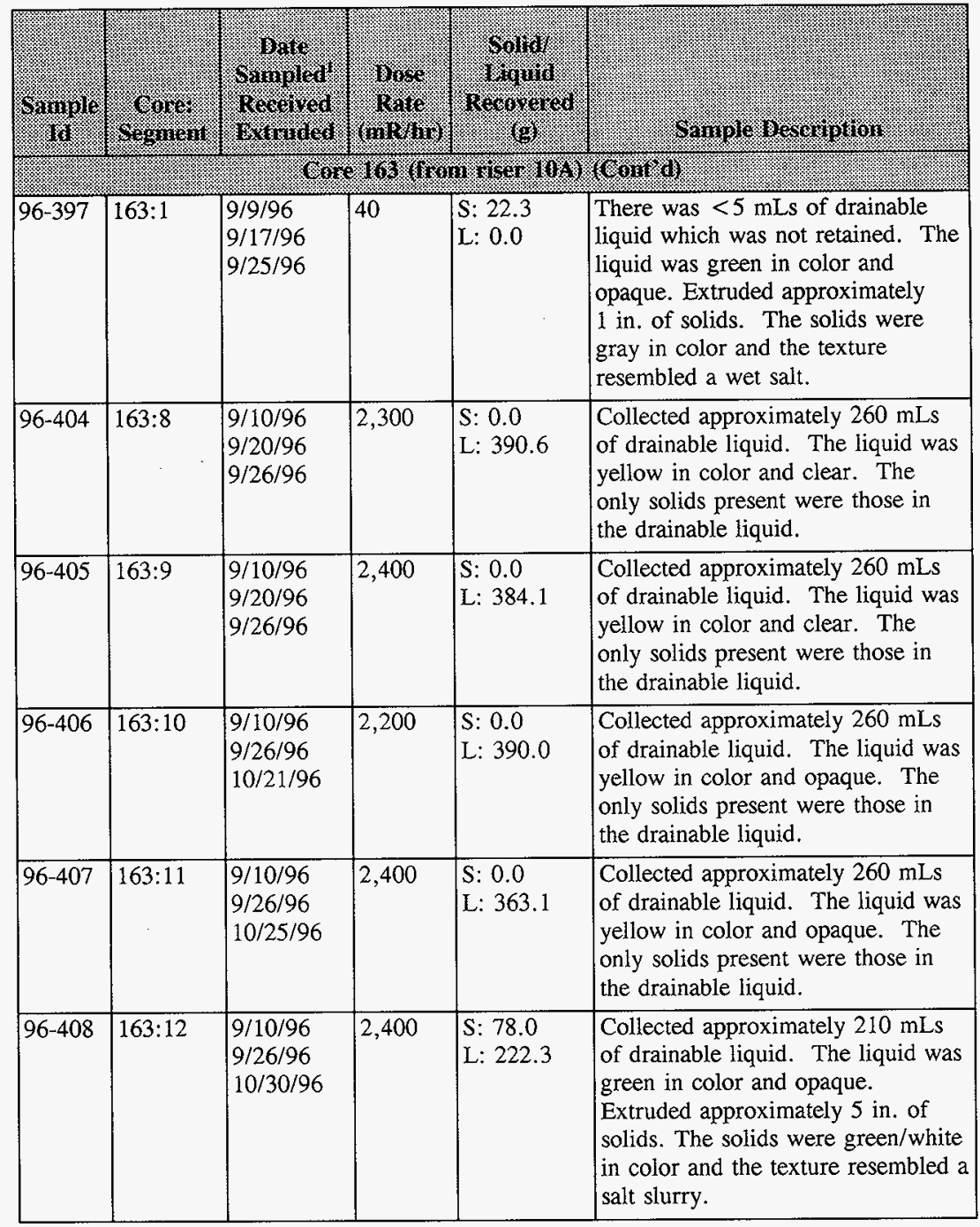




\begin{tabular}{|c|c|c|c|c|c|}
\hline 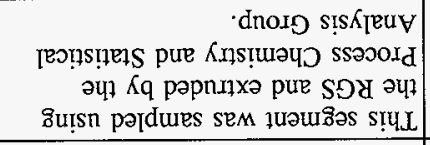 & $\mathrm{e} / \mathrm{u}$ & $008^{6} \mathrm{I}$ & $\begin{array}{r}\mathrm{e} / \mathrm{u} \\
96 / \mathrm{L} / 6 \\
96 / \mathrm{L} / 6 \\
\end{array}$ & $6[: \varepsilon 9 I$ & $\varsigma[\nabla-96$ \\
\hline 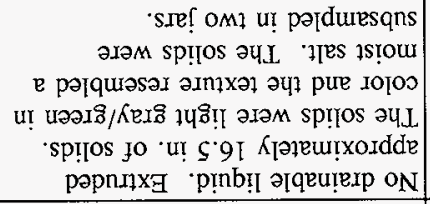 & $\begin{aligned} & 0.0: T \\
& L S 0 t: S \\
&\end{aligned}$ & $006^{\circ} \mathrm{I}$ & $\begin{array}{r}96 / 0 \varepsilon / 0 I \\
96 / 9 Z / 6 \\
96 / \text { I } / 6 \\
\end{array}$ & $8 \mathrm{I}: \mathcal{E} 9 \mathrm{I}$ & $t[t-96$ \\
\hline 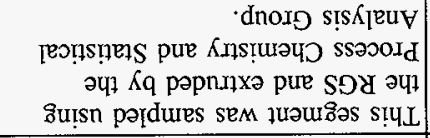 & $\mathrm{e} / \mathrm{u}$ & $000^{\prime} z$ & $\begin{array}{r}\mathrm{e} / \mathrm{u} \\
96 / \mathrm{Ll} / 6 \\
96 / 6 / 6 \\
\end{array}$ & $\angle I: \varepsilon 9 \mathrm{I}$ & EIt-96 \\
\hline 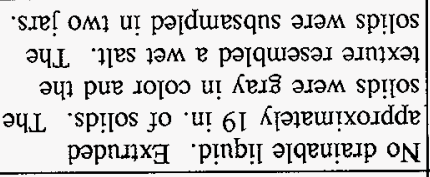 & $\begin{array}{r}0 \cdot 0: T \\
+86 \varepsilon: S \\
\end{array}$ & $00 \tau^{\prime} \tau$ & $\begin{array}{l}96 / 9 z / 6 \\
96 / 0 Z / 6 \\
96 / I I / 6\end{array}$ & $9 I: \varepsilon 9 I$ & $z$ z \\
\hline 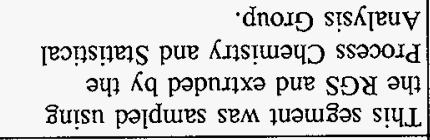 & $\mathrm{e} / \mathrm{u}$ & $00 s^{\prime} \tau$ & $\begin{array}{r}\mathrm{e} / \mathrm{U} \\
96 / Z \mathrm{I} / 6 \\
96 / \mathrm{II} / 6 \\
\end{array}$ & SI:E9I & I It -96 \\
\hline 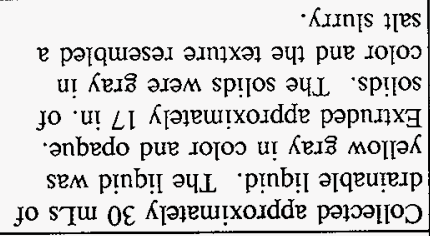 & $\begin{array}{r}L \mathcal{E} t: T \\
9 \subseteq \mathcal{S}: S \\
\end{array}$ & $00 s^{\prime} z$ & $\begin{array}{r}96 / \mathrm{SZ} / 0 \mathrm{I} \\
96 / 9 \mathrm{Z} / 6 \\
96 / \mathrm{II} / 6 \\
\end{array}$ & $t I: \varepsilon 9 I$ & $01 t-96$ \\
\hline 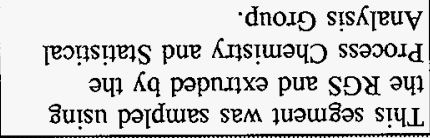 & $\mathrm{E} / \mathrm{U}$ & $006^{\circ} \mathrm{I}$ & $\begin{array}{r}\text { e/u } \\
96 / Z I / 6 \\
96 / \mathrm{IL} / 6 \\
\end{array}$ & $\mathcal{E}[: \mathcal{E} 9 \mathrm{I}$ & $60 t-96$ \\
\hline (10. 110 & (M) & max & (3) & & \\
\hline 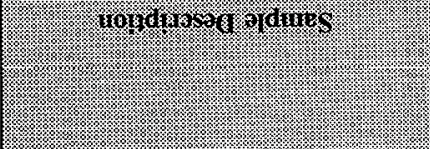 & 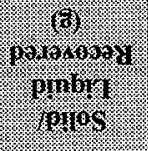 & 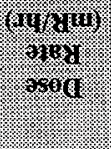 & 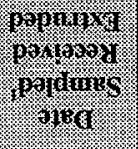 & (1) & monos \\
\hline
\end{tabular}

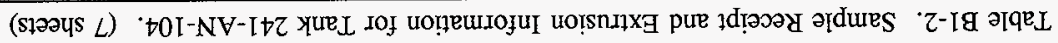




\begin{tabular}{|c|c|c|c|c|c|}
\hline 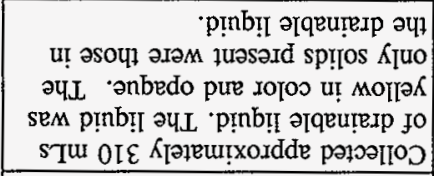 & $\begin{array}{r}960 t: 7 \\
00: 5 \\
\end{array}$ & $00 z^{6} z$ & $\begin{array}{r}96 / 8 Z / 8 \\
96 / 9 Z / 8 \\
96 / 8 / 8 \\
\end{array}$ & $7: t 9 I$ & IZヤ-96 \\
\hline 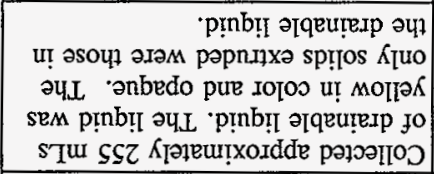 & $\begin{array}{r}68 \mathcal{} 58: T \\
0^{\circ} 0: S \\
\end{array}$ & $00 \mathcal{E}^{\prime} z$ & $\begin{array}{r}96 / 8 乙 / 8 \\
96 / \varepsilon 乙 / 8 \\
96 / 8 / 8 \\
\end{array}$ & $\varepsilon:+9 I$ & $0 z t-96$ \\
\hline 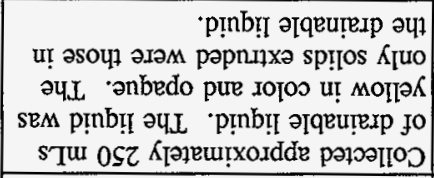 & $\begin{array}{r}L 69 \varepsilon: T \\
0^{\circ} 0: S \\
\end{array}$ & $00 \varepsilon^{\prime} z$ & $\begin{array}{r}96 / 8 Z / 8 \\
96 / \mathcal{L} / 8 \\
96 / 8 / 8 \\
\end{array}$ & $\tau: \downarrow 9 I$ & $6 \mathrm{It}-96$ \\
\hline 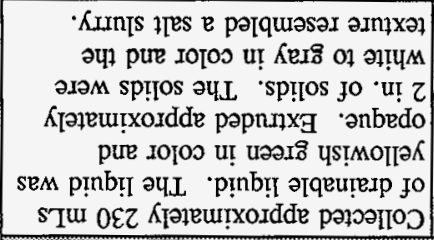 & $\begin{array}{r}9^{\circ} 282: 1 \\
\nabla^{\circ} \mathrm{SE}: 5\end{array}$ & $00 s^{\prime} z$ & $\begin{array}{r}96 / 8 Z / 8 \\
96 / \mathcal{E Z} / 8 \\
96 / 8 / 8\end{array}$ & $I: t 9 I$ & $8 I t-96$ \\
\hline 3. & 185.610 .62 & 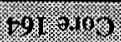 & & & \\
\hline 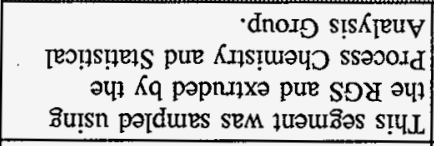 & $\mathrm{e} / \mathrm{u}$ & $\mathrm{e} / \mathrm{u}$ & $\begin{array}{r}\mathrm{e} / \mathrm{U} \\
96 / L \mathrm{~L} / 6 \\
96 / 6 / 6 \\
\end{array}$ & $\mathrm{IZ}: \mathcal{E 9 I}$ & LIt -96 \\
\hline 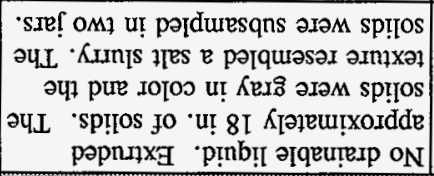 & $\begin{array}{r}00: \mathrm{T} \\
\mathrm{I} 88 \mathcal{E}: \mathrm{S}\end{array}$ & $\mathrm{e} / \mathrm{u}$ & $\begin{array}{l}96 / Z / 0 \mathrm{~L} \\
96 / L Z / 6 \\
96 / \mathrm{L} / 6\end{array}$ & $0 Z: \varepsilon 9 \mathrm{I}$ & $9 I t-96$ \\
\hline 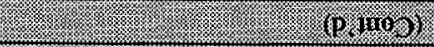 & (1) & 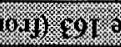 & 19. & & \\
\hline 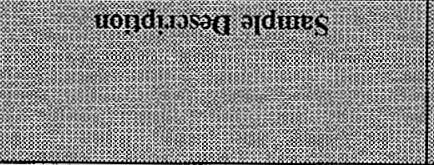 & 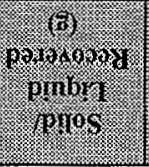 & 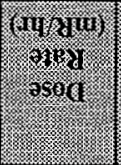 & 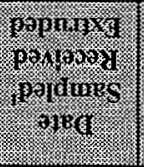 & 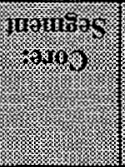 & (16) \\
\hline
\end{tabular}

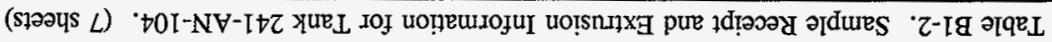




\begin{tabular}{|c|c|c|c|c|c|}
\hline 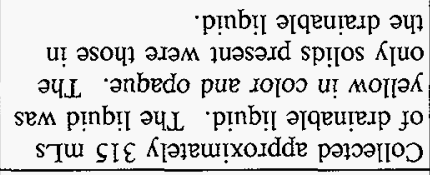 & $\begin{array}{r}\text { I ZZ॰ :T } \\
000: S \\
\end{array}$ & $008^{\prime} z$ & $\begin{array}{r}96 / 6 \mathcal{C Z} / 8 \\
96 / \mathcal{E Z} / 8 \\
96 / 6 / 8\end{array}$ & II:t9I & $827-96$ \\
\hline 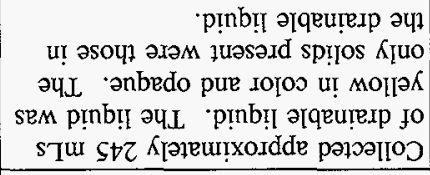 & $\begin{array}{r}\tau \cdot \angle 9 \varepsilon: T \\
0.0: S \\
\end{array}$ & $00 L^{\prime} z$ & $\begin{array}{r}96 / \mathrm{I} \text { I/6 } \\
96 / 9 / 6 \\
96 / 6 / 8 \\
\end{array}$ & $0 I:+9 I$ & $L Z t-96$ \\
\hline 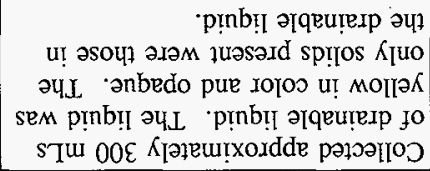 & $\begin{array}{r}I \mathcal{E} I t: \mathrm{T} \\
0: 0: \mathrm{S}\end{array}$ & $00 L^{\prime} z$ & $\begin{array}{l}96 / 6 / 6 \\
96 / \varepsilon / 6 \\
96 / 6 / 8\end{array}$ & $6: \nabla 9 I$ & $9 z \nabla-96$ \\
\hline 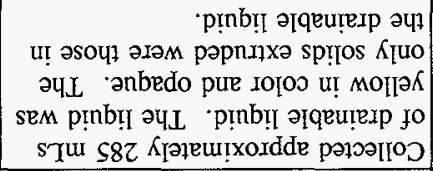 & $\begin{array}{r}0.81 t: 1 \\
0.0: S\end{array}$ & $00 L^{\prime} z$ & $\begin{array}{l}96 / 6 / 6 \\
96 / \mathcal{E} / 6 \\
96 / 6 / 8 \\
\end{array}$ & $8:+9 I$ & szt-96 \\
\hline 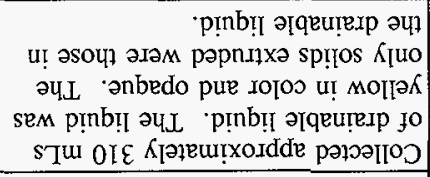 & $\begin{array}{r}9.0 z t: 1 \\
0.0: S \\
9.0 z t \\
\end{array}$ & $00 S^{\prime} z$ & $\begin{array}{r}96 / 6 Z / 8 \\
96 / 97 / 8 \\
96 / 8 / 8 \\
\end{array}$ & $L: \$ 9 I$ & $\nabla z \downarrow-96$ \\
\hline 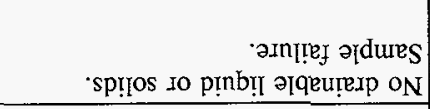 & $\begin{array}{ll}0.0 & : 1 \\
0.0 & : S \\
\end{array}$ & $\varepsilon \varepsilon$ & $\begin{array}{r}96 / 6 Z / 8 \\
96 / 9 z / 8 \\
96 / 8 / 8 \\
\end{array}$ & $9: t 91$ & $\varepsilon z \downarrow-96$ \\
\hline 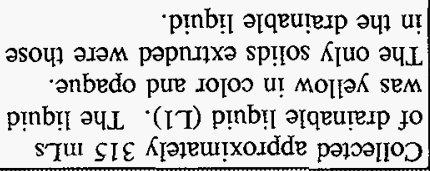 & $\begin{array}{r}\varsigma^{\circ} 9 \mathcal{E} t: T \\
0^{\circ} 0: S\end{array}$ & $00 z^{\prime} z$ & $\begin{array}{l}96 / 6 / 6 \\
96 / \mathcal{E} / 6 \\
96 / 8 / 8\end{array}$ & $s: t 9 I$ & $22 \downarrow-96$ \\
\hline 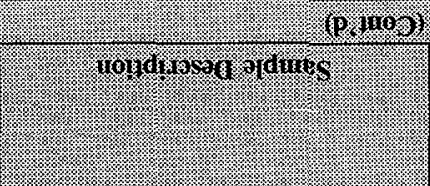 & 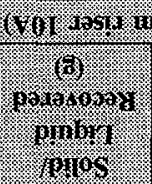 & 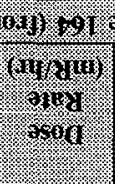 & 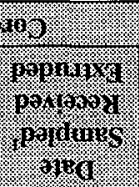 & 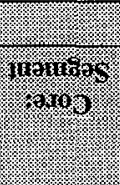 & 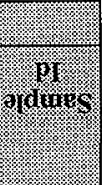 \\
\hline
\end{tabular}

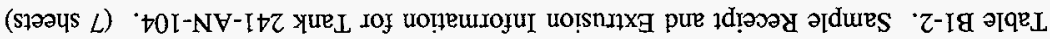




\begin{tabular}{|c|c|c|c|c|c|}
\hline 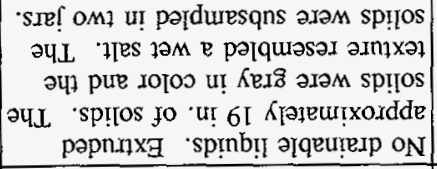 & $\begin{array}{r}00: 7 \\
816 \varepsilon: S \\
\end{array}$ & $00 z^{6} z$ & $\begin{array}{r}96 / Z \mathrm{LI} / 6 \\
96 / 9 / 6 \\
96 / \mathcal{L I} / 8 \\
\end{array}$ & $91:+9 I$ & $\varepsilon \varepsilon \nabla-96$ \\
\hline 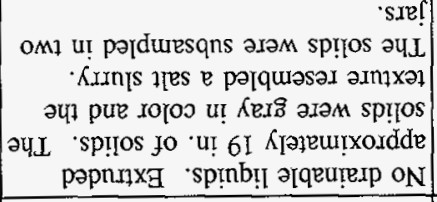 & $\begin{array}{r}00: 7 \\
569 \mathcal{S}: \mathrm{S} \\
\end{array}$ & $005^{\prime} 2$ & $\begin{array}{r}96 / \mathcal{I}[/ 6 \\
96 / 9 / 6 \\
96 / \mathcal{E}[/ 8 \\
\end{array}$ & SI:t9I & $z \varepsilon+-96$ \\
\hline 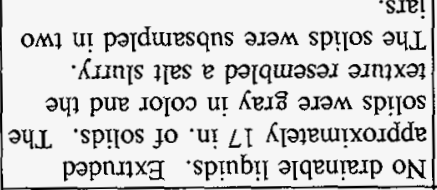 & $\begin{array}{r}0.0: 1 \\
9^{\circ} 19 \varepsilon: S \\
\end{array}$ & $00 s^{\circ} z$ & $\begin{array}{r}96 /[\mathrm{I} / 6 \\
96 / 9 / 6 \\
96 / \mathcal{E}[/ 8 \\
\end{array}$ & $D[\because 69 I$ & IEt-96 \\
\hline 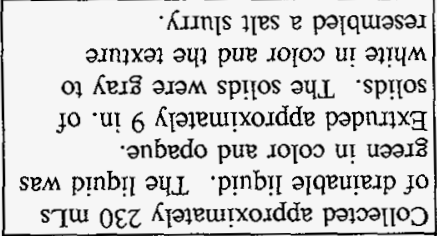 & $\begin{array}{r}t I I \mathcal{L}: \mathrm{T} \\
t \cdot 26: \mathrm{s}\end{array}$ & $00 \operatorname{s}^{\prime} z$ & $\begin{array}{l}96 / 6 Z / 8 \\
96 / \varepsilon 乙 / 8 \\
96 / \mathcal{E L} / 8\end{array}$ & $\varepsilon I: \neq 9[$ & $0 \varepsilon \nabla-96$ \\
\hline 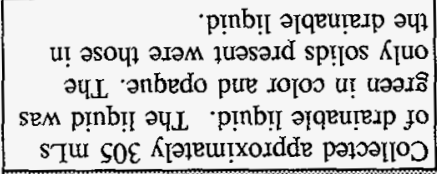 & $\begin{array}{r}8^{\circ} \varepsilon I t: T \\
0.0: S\end{array}$ & $000^{\prime} z$ & $\begin{array}{l}96 / 6 Z / 8 \\
96 / \varepsilon Z / 8 \\
96 / \varepsilon \backslash / 8\end{array}$ & $21:+9 I$ & $62 t-96$ \\
\hline . & (x) & (3) & Yis & & \\
\hline (10) & 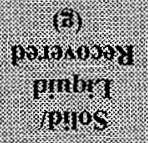 & 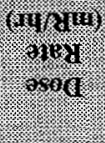 & 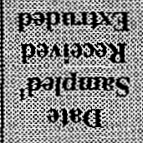 & 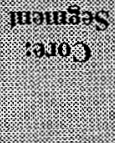 & 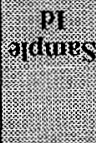 \\
\hline
\end{tabular}

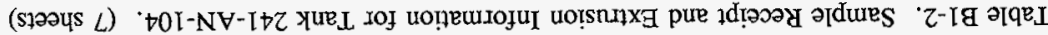


Table B1-2. Sample Receipt and Extrusion Information for Tank 241-AN-104. (7 sheets)

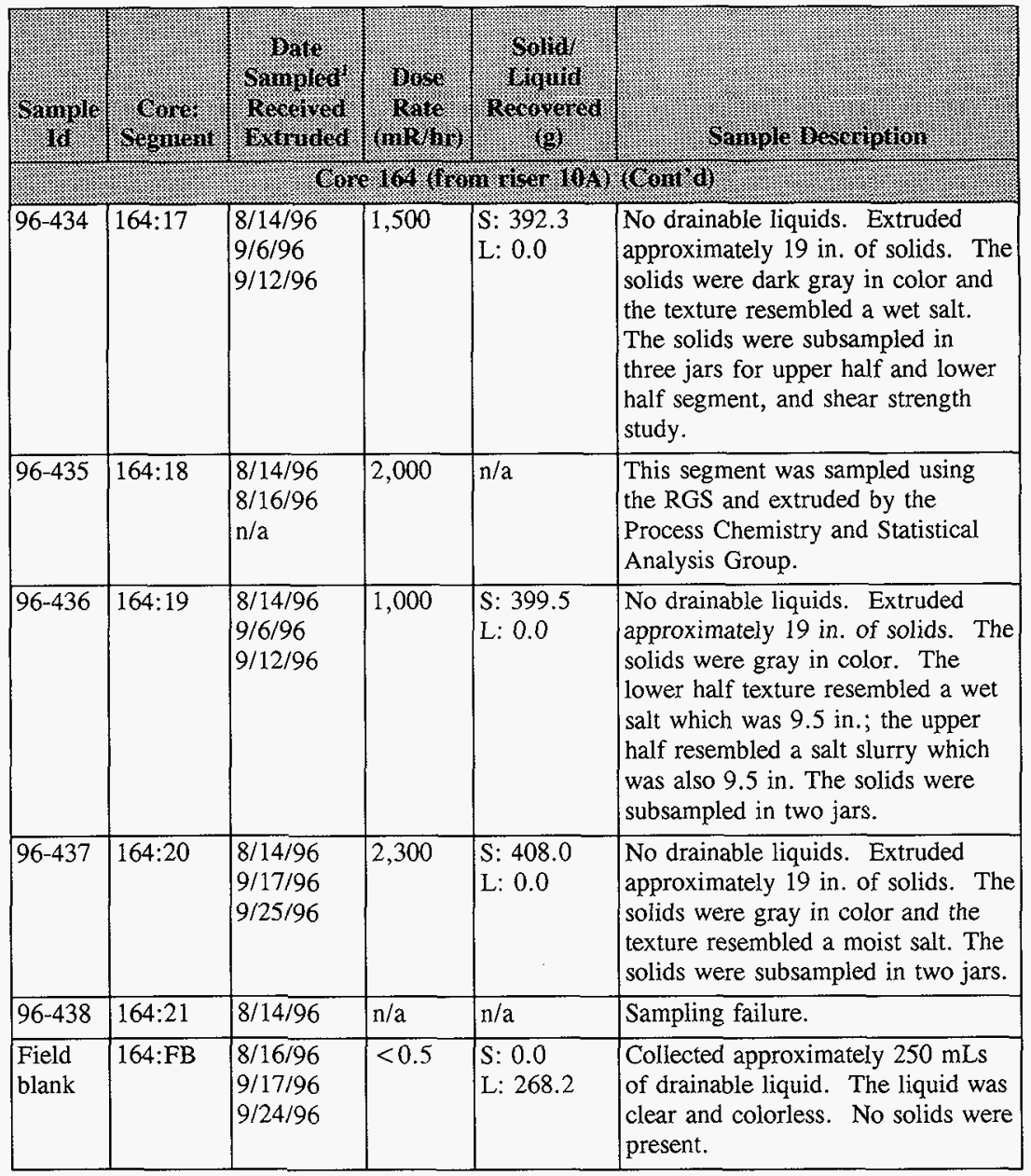

Notes:
$\mathfrak{L}=$ liquid
$S=$ solid

'Dates are provided in the month/day/year format. 


\section{B1.3 SAMPLE ANALYSIS OF 1996 SAMPLING EVENT}

The analyses performed on the core samples were limited to those required by the safety screening and the flammable gas DQOs (McDuffie and Johnson 1995). The safety screening DQO (Dukelow et al. 1995) required analyses for thermal properties by DSC, moisture content by TGA, and content of fissile material by total alpha activity analysis. The flammable gas DQO required a full set of analytes be analyzed by IC, ICP, TOC, TIC, and various radionuclides.

Differential scanning calorimetry and TGA were performed on $8.665-\mathrm{mg}$ to $45.550-\mathrm{mg}$ samples. Quality control (QC) tests included performing the analyses in duplicate and using standards. Moisture content was also measured by a gravimetric method.

Total alpha activity measurements were performed on samples that had been fused in a matrix of potassium hydroxide and dissolved in acid. The resulting solution was dried on a counting planchet and counted in an alpha proportional counter. Quality control tests included standards, spikes, blanks, and duplicate analyses.

Ion chromatography was performed on samples that had been prepared by water digestion. Quality control tests included standards, spikes, blanks, and duplicate analyses. The sampling and analysis plan (Winkelman 1996a) required the full suite of IC analytes be measured.

Inductively coupled plasma spectrometry was performed on samples that had been prepared by a fusion procedure, followed by dissolution in acid. Quality control tests included standards, blanks, spikes, and duplicate analyses. The sampling and analysis plan required the full suite of ICP elements be analyzed.

All analyses were performed in accordance with approved laboratory procedures. Table B1-3 lists sample numbers and applicable analyses. Table B1-4 lists sample preparation procedure numbers and analysis procedure numbers.

\section{B1.4 DESCRIPTION OF HISTORICAL SAMPLING EVENT}

Analyses of two sampling events for tank 241-AN-104 were obtained from historical records. The dates of these events were January 18, 1985, and July 2, 1984. An aliquot from a tank 241-AN-104 sample was analyzed then mixed with waste from tank 241-AW-102 to determine waste compatibility for use in the 242-A Evaporator (Mauss and Jansky 1985). A sample of tank 241-AN-104 waste was analyzed after being stored at elevated temperatures for a period (Jansky 1984a). The sample contained approximately 10 percent white granular solids when received. An aliquot was stored at $40{ }^{\circ} \mathrm{C}$ for two weeks to note changes in volume percent settled solids, but none were observed. The aliquot was centrifuged and the supernate and solids prepared for analysis. Table B2-6 shows the analytical results. No 
information was available regarding sample handling for this tank. The reason for the sampling was to support the 242-A Evaporator Campaign.

Table B1-3. Tank 241-AN-104 Summary of Sample Analyses. (7 sheets)

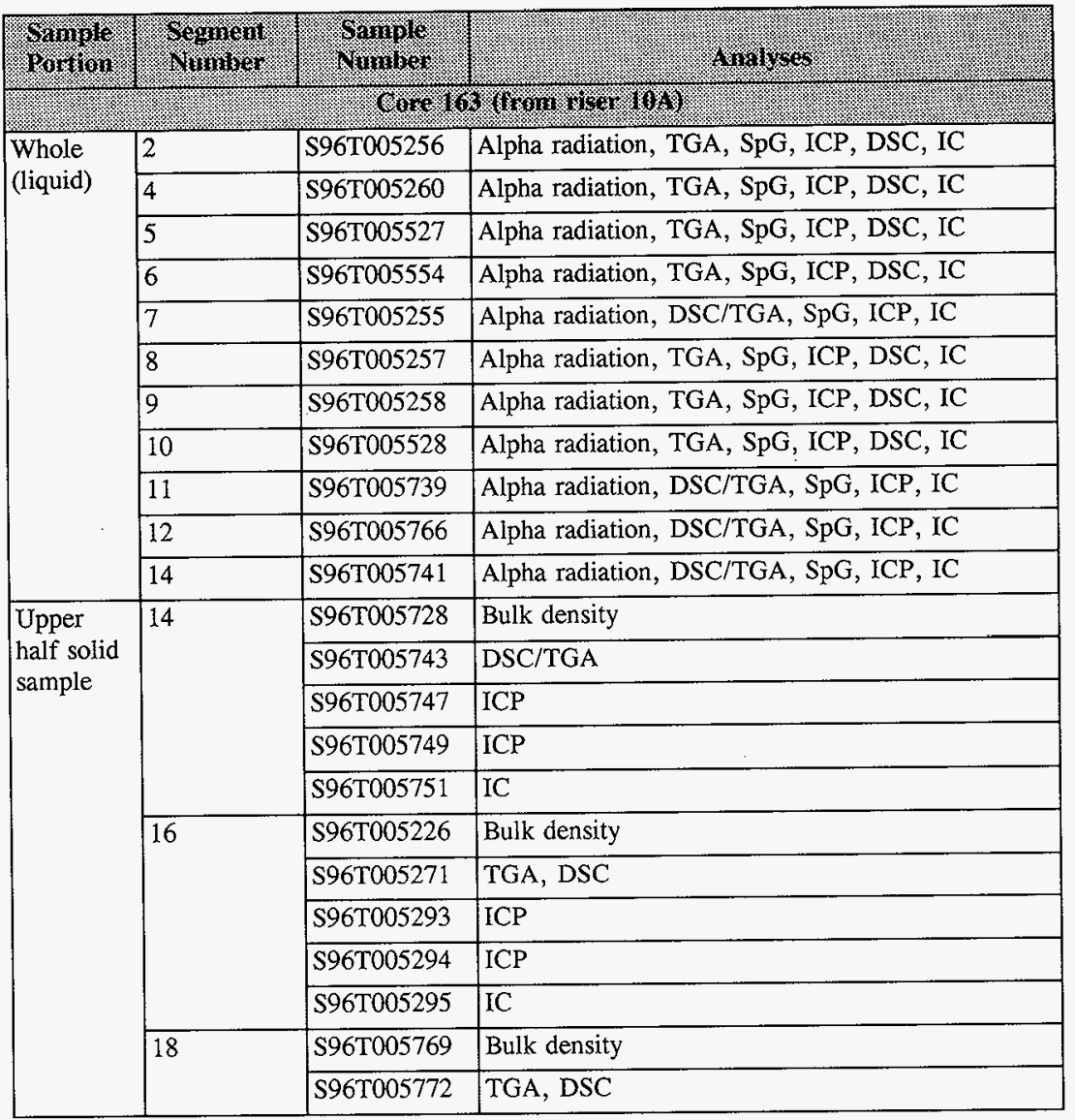


Table B1-3. Tank 241-AN-104 Summary of Sample Analyses. (7 sheets)

\begin{tabular}{|c|c|c|c|}
\hline Srmple & Suegnent & 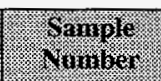 & 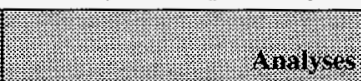 \\
\hline & 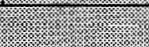 & r. & 3. fromin fiser IOA) \\
\hline Upper & 18 & S96T005777 & ICP \\
\hline half solid & & S96T005778 & ICP \\
\hline & & S96T005779 & IC \\
\hline & 20 & S96T005548 & DSC/TGA \\
\hline & & S96T005552 & Bulk density \\
\hline & & S96T005556 & $\overline{\mathrm{ICP}}$ \\
\hline & & \begin{tabular}{|l|} 
S9T005557 \\
\end{tabular} & ICP \\
\hline & & S96T005558 & IC \\
\hline Lower & 1 & $\$ 96 T 005269$ & $\overline{D S C} / \mathrm{TGA}$ \\
\hline half solid & & S96T005284 & Alpha, ICP \\
\hline & & S96T005287 & ICP \\
\hline & & S96T005290 & IC \\
\hline & 2 & S967005270 & DSC/TGA \\
\hline & & $\longdiv { \text { S96T005285 } }$ & Alpha, ICP \\
\hline & & $\$ 967005288$ & ICP \\
\hline & & $\widehat{\text { S96T005291 }}$ & IC \\
\hline & 12 & S96T005768 & Bulk density \\
\hline & & S96T005771 & TGA, DSC \\
\hline & & $\overline{\text { S96T005780 }}$ & Alpha, ICP \\
\hline & & S96T005782 & ICP \\
\hline & & S96T005784 & IC \\
\hline & 14 & S96T005729 & Bulk density \\
\hline & & S96T005744 & $\mathrm{DSC} / \mathrm{TGA}$ \\
\hline & & S96T005748 & Alpha, ICP \\
\hline
\end{tabular}


Table B1-3. Tank 241-AN-104 Summary of Sample Analyses. (7 sheets)

\begin{tabular}{|c|c|c|c|}
\hline $\begin{array}{l}\text { Srminge } \\
\text { Iromion }\end{array}$ & 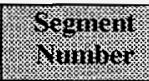 & (Tomple: & 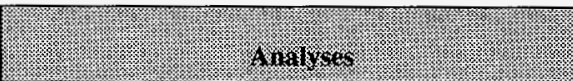 \\
\hline \multicolumn{4}{|c|}{ 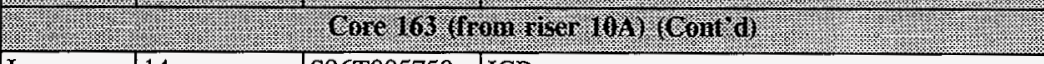 } \\
\hline \multirow{17}{*}{$\begin{array}{l}\text { Lower } \\
\text { half solid } \\
\text { sample }\end{array}$} & \multirow[t]{2}{*}{14} & S96T005750 & ICP \\
\hline & & S96T005752 & IC \\
\hline & \multirow[t]{5}{*}{16} & \$96T005227 & Bulk density \\
\hline & & S96T005272 & TGA, DSC \\
\hline & & S96T005286 & Alpha, ICP \\
\hline & & S96T005289 & ICP \\
\hline & & $\overline{\text { S96'T005292 }}$ & IC \\
\hline & \multirow[t]{5}{*}{18} & S96T005770 & Bulk density \\
\hline & & S96T005773 & TGA, DSC \\
\hline & & S96T005781 & Alpha, ICP \\
\hline & & S96T005783 & ICP \\
\hline & & \$96T005785 & IC \\
\hline & \multirow[t]{5}{*}{20} & S96T005549 & DSC/TGA \\
\hline & & S96T005553 & Bulk density \\
\hline & & S96r005559 & Alpha, ICP \\
\hline & & S96T005560 & ICP \\
\hline & & S96T005561 & IC \\
\hline & & Corrol & 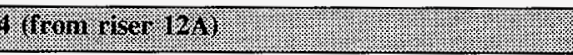 \\
\hline \multirow[t]{5}{*}{ Whole } & \multirow{5}{*}{$\begin{array}{l}\text { Solid core } \\
\text { composite }\end{array}$} & S96T005970 & Bulk density \\
\hline & & S96T005972 & TIC/TOC, TGA, DSC \\
\hline & & S96T005975 & $\begin{array}{l}{ }^{239 / 240} \mathrm{Pu} \text { Alpha, GEA, Te, }{ }^{89 / 90} \mathrm{Sr} \mathrm{U},{ }^{99} \mathrm{Tc},{ }^{241} \mathrm{Am} \text {, } \\
{ }^{84344} \mathrm{Cm} \text {, Total beta }\end{array}$ \\
\hline & & S96T005976 & ICP \\
\hline & & S96T005977 & ${ }^{129} \mathrm{I}, \mathrm{Cr}+6, \mathrm{OH}, \mathrm{IC},{ }^{3} \mathrm{H}$ \\
\hline
\end{tabular}


Table B1-3. Tank 241-AN-104 Summary of Sample Analyses. (7 sheets)

\begin{tabular}{|c|c|c|c|}
\hline Shom & Hinger & 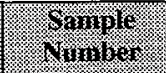 & 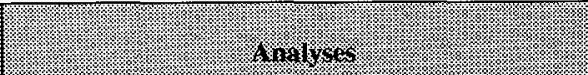 \\
\hline (1: & $\left(\frac{1}{4}\right.$ & (6, w, w, & 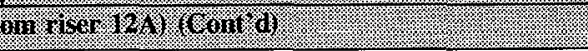 \\
\hline \multirow[t]{20}{*}{ Whole } & \multirow{6}{*}{$\begin{array}{l}\text { Liquid core } \\
\text { composite }\end{array}$} & S96T005978 & Alpha radiation, TIC/TOC, DSC/TGA \\
\hline & & S96T005979 & Alpha radiation, ICP, GEA, IC \\
\hline & & $\$ 96 \mathrm{~T} 005980$ & ${ }^{239 / 240} \mathrm{Pu},{ }^{237} \mathrm{~Np},{ }^{241} \mathrm{Am}, \mathrm{Cr}+6, \mathrm{Sr}$ \\
\hline & & S96T005981 & $\begin{array}{l}\text { Uranium gross (Kinetic Phosphoresence), OH } \\
\text { direct, SpG }\end{array}$ \\
\hline & & \$97T000022 & ${ }^{129} \mathrm{I}, \mathrm{Te}$ \\
\hline & & S97T000389 & Scintillation \\
\hline & 1 & S96T004774 & Alpha radiation, $\mathrm{TGA}, \mathrm{SpG}, \mathrm{ICP}, \mathrm{DSC}, \mathrm{IC}$ \\
\hline & 2 & \$96T004778 & Alpha radiation, TGA, SpG, ICP, DSC, IC \\
\hline & 3 & $\$ 967004779$ & Alpha radiation, TGA, SpG, ICP, DSC, IC \\
\hline & 4 & \$96T004780 & Alpha radiation, TGA, SpG, ICP, DSC, IC \\
\hline & 5 & S96T004976 & Alpha radiation, TGA, SpG, ICP, DSC, IC \\
\hline & 7 & S96T004781 & Alpha radiation, TGA, $\mathrm{SpG}, \mathrm{ICP}, \mathrm{DSC}, \mathrm{IC}$ \\
\hline & 8 & \$96T004977 & Alpha radiation, TGA, SpG, ICP, DSC, IC \\
\hline & 9 & S96T004978 & Alpha radiation, DSC/TGA, TGA, SpG, ICP, IC \\
\hline & 10 & \$96T004979 & Alpha radiation, DSC/TGA, SpG, ICP, IC \\
\hline & 11 & S96T004782 & Alpha radiation, TGA, SpG, ICP, DSC, IC \\
\hline & 12 & S96T004783 & Alpha radiation, DSC/TGA, SpG, ICP, IC \\
\hline & 13 & S96T004784 & Alpha radiation, DSC/TGA, SpG, ICP, IC \\
\hline & HHF Blank & S96T004916 & $\mathrm{ICP}, \mathrm{IC}$ \\
\hline & Field Blank & $\$ 96 \mathrm{~T} 005253$ & Alpha radiation, DSC/TGA, SpG, ICP, IC \\
\hline \multirow{4}{*}{$\begin{array}{l}\text { Upper } \\
\text { half solid } \\
\text { sample }\end{array}$} & \multirow[t]{4}{*}{14} & S96T004965 & Bulk density \\
\hline & & S96T004984 & DSC/TGA \\
\hline & & S96T005035 & ICP \\
\hline & & S96T005042 & $\mathrm{ICP}$ \\
\hline
\end{tabular}


Table B1-3. Tank 241-AN-104 Summary of Sample Analyses. (7 sheets)

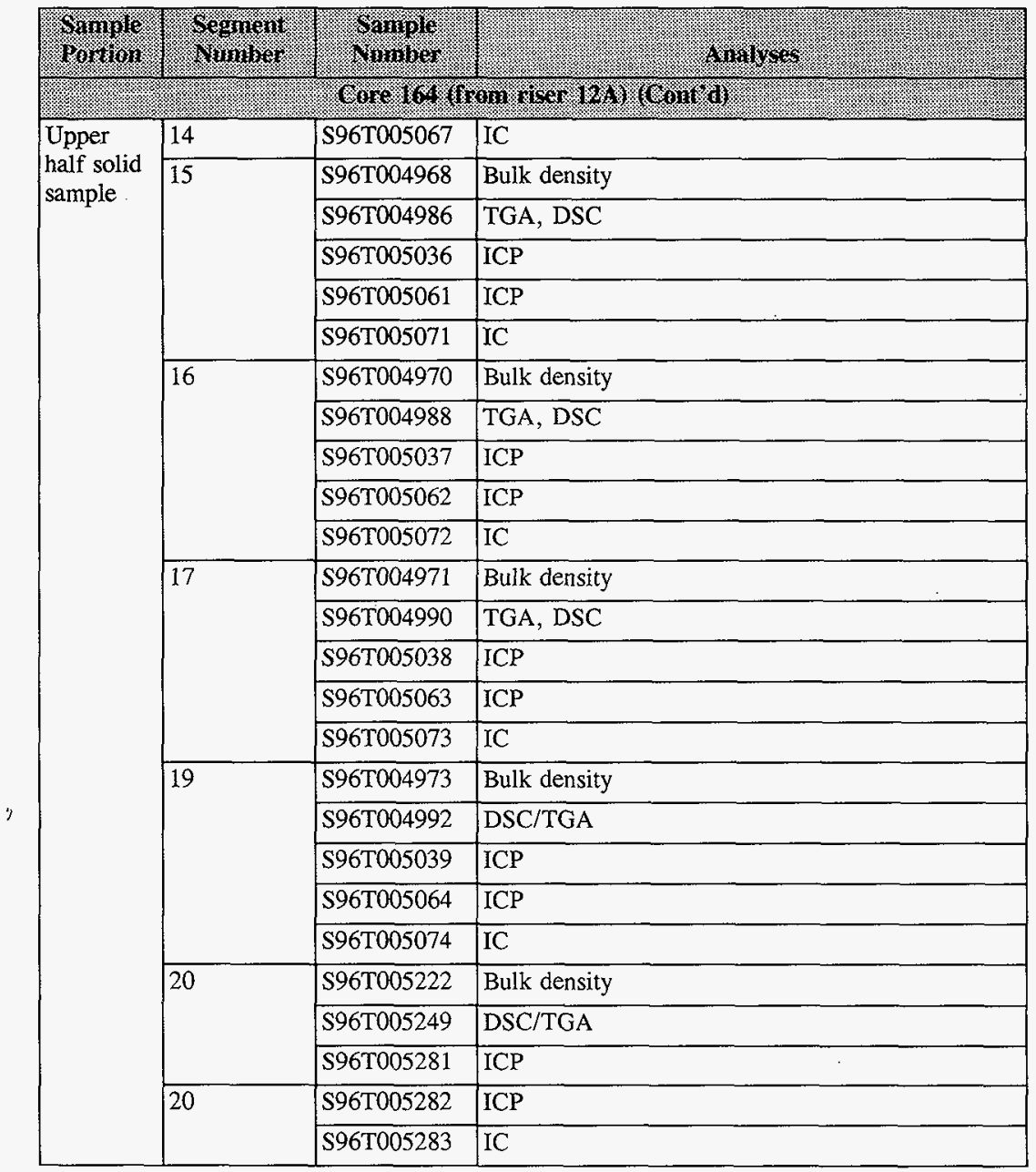


Table B1-3. Tank 241-AN-104 Summary of Sample Analyses. (7 sheets)

\begin{tabular}{|c|c|c|c|c|}
\hline $\begin{array}{l}\text { Simple } \\
\text { rortion }\end{array}$ & $\begin{array}{l}\text { Segment } \\
\text { Jumber. }\end{array}$ & $\begin{array}{l}\text { Sarmple } \\
\text { Sinn }\end{array}$ & 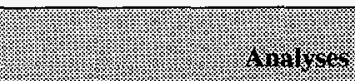 & \\
\hline (3. & $1:$ & (6) & 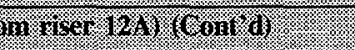 & \\
\hline Lower & 1 & S96T004772 & $\mathrm{DSC} / \mathrm{TGA}$ & \\
\hline half solid & & S96r004795 & Alpha, ICP & \\
\hline & & S96T004797 & ICP & \\
\hline & & S96T004799 & IC & \\
\hline & & S97T000648 & IC & \\
\hline & 13 & S96T004771 & Bulk density & \\
\hline & & S96T004773 & $\mathrm{DSC} / \mathrm{TGA}$ & \\
\hline & & S96T004796 & Alpha, ICP & \\
\hline & & S96T004798 & ICP & \\
\hline & & S96T004800 & IC & \\
\hline & & S97T000649 & $\overline{\mathrm{IC}}$ & \\
\hline & 14 & S96T004966 & Bulk density & \\
\hline & & S96T004985 & $\overline{\mathrm{DSC} / \mathrm{TGA}}$ & \\
\hline & & S96T005030 & Alpha, ICP & \\
\hline & & S96T005040 & ICP & \\
\hline & & S96T005065 & $\mathrm{IC}$ & \\
\hline & 15 & S96T004967 & Bulk density & \\
\hline & & S96T004987 & TGA, DSC & \\
\hline & & S96T005032 & Alpha, ICP & \\
\hline & & S96T005043 & ICP & \\
\hline & & S96T005068 & IC & \\
\hline Lower & 16 & S96T005033 & Alpha, ICP & \\
\hline half solid & & S96T004969 & Bulk density & \\
\hline & & S96T004989 & TGA, DSC & \\
\hline & & S96T005044 & ICP & \\
\hline & & S96T005069 & IC & \\
\hline
\end{tabular}


Table B1-3. Tank 241-AN-104 Summary of Sample Analyses. (7 sheets)

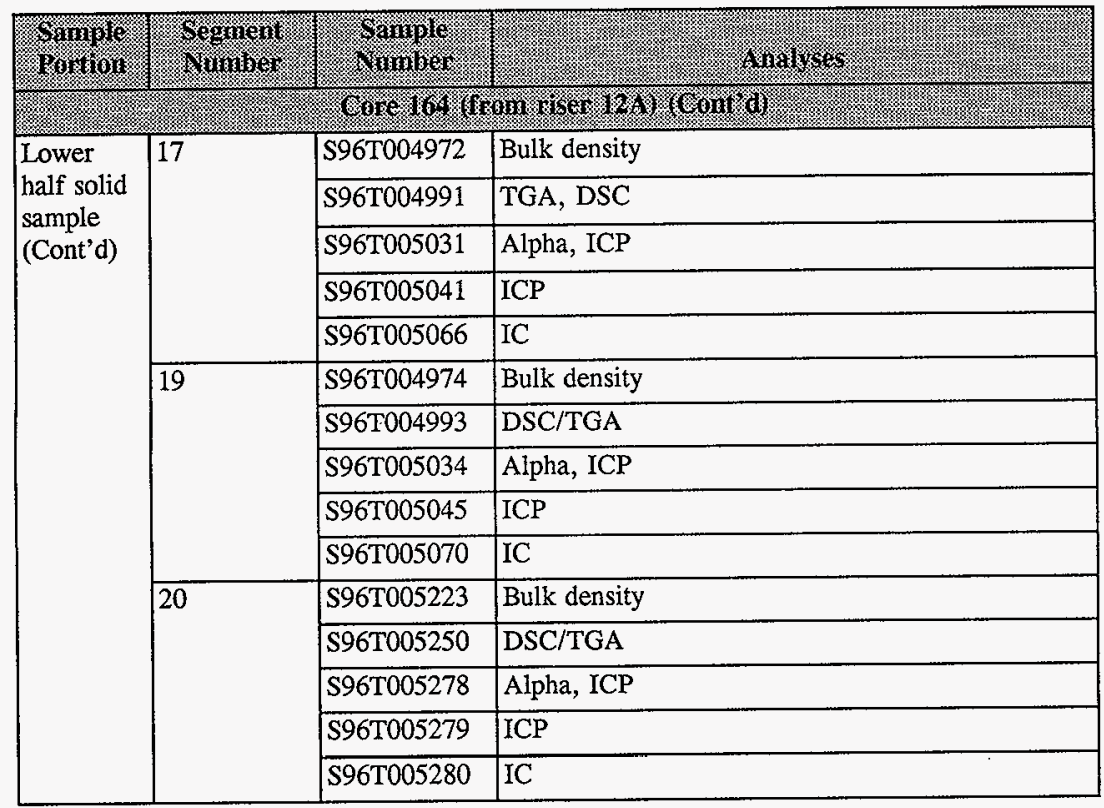

Notes:

$$
\begin{aligned}
\text { SpG } & =\text { specific gravity } \\
\text { GEA } & =\text { gamma energy analysis }
\end{aligned}
$$


Table B1-4. Analytical Procedures.

\begin{tabular}{|c|c|c|c|}
\hline : vinusis & 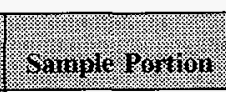 & 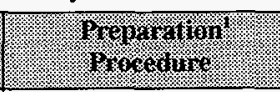 & 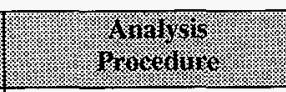 \\
\hline DSC & Solid/liquid & $\mathrm{n} / \mathrm{a}$ & $\begin{array}{l}\text { LA-514-114 Rev. C-1 } \\
\text { LA-514-113 Rev. C-1 }\end{array}$ \\
\hline TGA & Solid/liquid & $\mathrm{n} / \mathrm{a}$ & $\begin{array}{l}\text { LA-514-114 Rev. C-1 } \\
\text { LA-560-112 Rev. B-1 }\end{array}$ \\
\hline Bulk density & Solid & $n / a$ & LO-160-103 Rev. B-0 \\
\hline SpG & Liquid & $\mathrm{n} / \mathrm{a}$ & LA-510-112 Rev. C-3 \\
\hline Uranium & Solid/liquid & LA-549-141 Rev. F-0 & LA-925-009 Rev. A-1 \\
\hline IC & Solid/liquid & LA-504-101 Rev. E-0 & LA-533-105 Rev. D-1 \\
\hline ICP & Solid/liquid & $\begin{array}{l}\text { LA-505-159 Rev. D-0 } \\
\text { LA-549-141 Rev. F-0 }\end{array}$ & \begin{tabular}{|l|} 
LA-505-151 Rev. D-3 \\
LA-505-161 Rev. B-1
\end{tabular} \\
\hline $\mathrm{OH}-$ & Solid/liquid & LA-504-101 Rev. E-0 & LA-211-102 Rev. C-0 \\
\hline TIC/TOC & Solid/liquid & $n / a$ & LA-342-100 Rev. D-0 \\
\hline$\overline{\mathrm{Cr}(\mathrm{VI})}$ & Solid/liquid & LA-504-101 Rev. E-0 & LA-265-101 Rev. B-0 \\
\hline TOC & Liquid & $n / a$ & LA-344-105 Rev. D-1 \\
\hline${ }^{3} \mathrm{H}$ & Solid/liquid & LA-504-101 Rev. E-0 & LA-218-114 Rev. B-0 \\
\hline${ }^{129} \mathrm{I}$ & Solid/liquid & LA-504-101 Rev. E-0 & LA-378-103 Rev. C-0 \\
\hline${ }^{99} \mathrm{Tc}$ & Solid/liquid & LA-549-141 Rev. F-0 & LA-438-101 Rev. D-2 \\
\hline${ }^{237} \mathrm{~Np}$ & Solid/liquid & LA-549-141 Rev. F-0 & LA-933-141 Rev. H-1 \\
\hline $\begin{array}{l}\begin{array}{l}\text { Total alpha/ } \\
\text { total beta }\end{array} \\
\end{array}$ & Solid/liquid & LA-549-101 Rev. F-0 & LA-508-101 Rev. D-2 \\
\hline$\overline{\text { GEA }}$ & Solid/liquid & LA-549-101 Rev. F-0 & LA-548-121 Rev. E-0 \\
\hline${ }^{241} \mathrm{Am} / /^{244} \mathrm{Cm}$ & Solid/liquid & LA-549-101 Rev. F-0 & LA-953-103 Rev. B-0 \\
\hline${ }^{90} \mathrm{Sr}$ & Solid/liquid & LA-549-141 Rev. F-0 & LA-220-101 Rev. D-1 \\
\hline${ }^{239} \mathrm{Pu}$ & Solid/liquid & LA-549-101 Rev. F-0 & LA-943-128 Rev. B-0 \\
\hline
\end{tabular}

Note:

'Procedures are listed for solid sample preparation. Liquid samples are direct samples. 


\section{B2.0 ANALYTICAL RESULTS}

\section{B2.1 OVERVIEW}

This section summarizes the sampling and analytical results associated with the August/September 1996 core samples and two historical samples from tank 241-AN-104. Table B2-1 shows the location and analytical results associated with this tank. Steen (1997) documents the detailed analytical results of solid and liquid phase samples from the 1996 core sampling, and Shekarriz et al. (1997) documents detailed retained gas sample results from 1996 core sampling. Sections B2.2 through B2.5 discuss the analyses and analytical results, and Section B2.6 tabulates all analytical measurements for 1996 core samples in the solid and liquid phase.

Table B2-1. Analytical Presentation Tables.

\begin{tabular}{|l|l|}
\hline Concentration of retained gas sample & $\mathrm{B} 2-2$ \\
\hline Gas inventories of RGS & $\mathrm{B} 2-3$ \\
\hline Waste density of RGS & $\mathrm{B} 2-4$ \\
\hline Vapor sampling results (sniff data) & $\mathrm{B} 2-5$ \\
\hline Historical sampling results (1984 and 1985) & $\mathrm{B} 2-6$ \\
\hline Cations analysis (ICP) & $\mathrm{B} 2-7$ through $\mathrm{B} 2-43$ \\
\hline Anions analysis (IC) & $\mathrm{B} 2-44$ through $\mathrm{B} 2-53$ \\
\hline Hexavalent chromium (Cr VI) & $\mathrm{B} 2-54$ \\
\hline TIC/TOC and OH & $\mathrm{B} 2-55$ through $\mathrm{B} 2-56$ \\
\hline Thermodynamic analyses (DSC and TGA) & $\mathrm{B} 2-57$ and $\mathrm{B} 2-58$ \\
\hline Density and specific gravity (bulk density/specific & $\mathrm{B} 2-59$ \\
\hline Total alpha activities and total beta & $\mathrm{B} 2-60$ through $\mathrm{B} 2-61$ \\
\hline${ }^{89190} \mathrm{Sr}$ & $\mathrm{B} 2-62$ \\
\hline Radionuclides by GEA & $\mathrm{B} 2-63$ through $\mathrm{B} 2-67$ \\
\hline Total uranium and ${ }^{239 / 240} \mathrm{Pu}$ & $\mathrm{B} 2-68$ and $\mathrm{B} 2-69$ \\
\hline Radionuclides of ${ }^{131} \mathrm{I},{ }^{3} \mathrm{H},{ }^{237} \mathrm{~Np},{ }^{99} \mathrm{Tc}$ & $\mathrm{B} 2-70$ through $\mathrm{B} 2-73$ \\
\hline Radionuclides by alpha energy analysis & $\mathrm{B} 2-74$ through $\mathrm{B} 2-75$ \\
\hline
\end{tabular}




\section{B2.2 SOLID AND LIQUDD SAMPLES RESULTS FROM 1996 CORE SAMPLING}

The analysis of core samples in solid and liquid phase from the 1996 push mode sampling were performed in 222-S Laboratory. The analysis results are discussed in sections below.

\section{B2.2.1 Inorganic Analyses}

The ICP and IC analyses were performed for anions and cations, respectively. A table for each analysis is provided. In each table, the "Mean" column is the average of result and duplicate values. All values were average including those below the detection level (denoted by the less-than symbol, "<"). If both sample and duplicate values were nondetected, the mean is expressed as a nondetected value. If one value was detected, but the other was not, the mean is expressed as a detected value. If both values were detected, the mean is expressed as a detected value.

B2.2.1.1 Inductively Coupled Plasma. The ICP analyses were performed according to procedures LA-505-161, Rev. B-1 or LA-505-151, Rev. D-3 depending on the ICP instrument used. A full suite of analytes was reported. Phosphorus was analyzed as a cross check for the phosphate results reported from IC analyses. The liquid subsamples were prepared for analysis by an acid adjustment of the direct subsample. Solid subsamples were prepared for analysis by performing an acid digest and a fusion. The solid core composite sample was prepared by fusion, water, and acid digestion before analysis.

Although a full suite of analytes were reported on the core samples, the analytes of interest were $\mathrm{Li}, \mathrm{Na}, \mathrm{Al}, \mathrm{Cr}, \mathrm{Ca}, \mathrm{Fe}, \mathrm{K}, \mathrm{U}, \mathrm{Zn}, \mathrm{Zr}, \mathrm{Ba}, \mathrm{Si}, \mathrm{B}, \mathrm{Bi}, \mathrm{Mn}, \mathrm{Ni}$. These were evaluated against the $\mathrm{QC}$ requirements. Lithium was required to evaluate contamination by $\mathrm{HHF}$ and/or wash water used during sampling. The remaining analytes of interest are requested by the flammable gas DQO. Additional ICP data were collected on an opportunistic basis (Kristofzski 1996) and are reported without the QC evaluation. The potassium and nickel results for ICP fusion analyses should be disregarded because the samples were prepared in a nickel crucible by fusion using potassium hydroxide.

B2.2.1.2 Ion Chromatography. The IC analyses were performed on direct subsamples of liquid samples. The solid subsamples were prepared for analysis by performing a water digest. Samples for IC were performed in duplicate according to procedure LA-533-105, Rev. D-1. All analytes reported by the IC instrument were requested.

High RPDs ( $>20$ percent) were reported for several analytes and can be attributed to sample inhomogeneity. Spike recoveries outside of the required range ( 75 percent to 125 percent) were reported for acetate, $\mathrm{Cl}^{-}, \mathrm{F}^{-}, \mathrm{NO}_{2}^{-}$, and $\mathrm{NO}_{3}^{-}$ions. The chemist noted that the spike failures were caused by matrix interferences from organic acids. Bromide was detected in 22 of 51 subsamples submitted for analysis and indicated HHF intrusion into the samples. The standard recoveries for IC analysis were within the required limits. 
B2.2.1.3 Chromium VI (CrVI). The Cr(VI) analyses were performed on direct liquid core composite subsamples. The solid core composite subsamples were prepared for analysis by performing a water digest. The standard recoveries, spike recoveries, and RPDs for this analysis were within required limits. The $\mathrm{Cr}$ (VI) results are $200 \mu \mathrm{g} / \mathrm{g}$ and $212.5 \mu \mathrm{g} / \mathrm{mL}$ for solids and liquids, respectively.

\section{B2.2.2 Total Inorganic Carbon/Total Organic Carbon}

The TIC/TOC by persulfate/coulometry analyses were performed on the core composite samples. No result exceeded the TOC notification limit of $30,000 \mu \mathrm{gC} / \mathrm{g}$. Low concentrations of TIC and TOC were noted in some preparation blanks; however, the levels of contamination were inconsequential when compared to the result of the sample and do not impact sample data quality. The results shows one high RPD (TOC on a liquid sample) and one low spike (TIC on a solid sample). The TIC values were $14,650 \mu \mathrm{g} / \mathrm{g}$ for solids and $2,165 \mu \mathrm{g} / \mathrm{mL}$ for liquids. The TOC results were $4,625 \mu \mathrm{g} / \mathrm{g}$ for solids and $3,405 \mu \mathrm{g} / \mathrm{mL}$ for liquids.

\section{B2.2.3 Hydroxide}

The hydroxide analyses were performed on direct liquid core composite subsamples and the field blank. The solid core composite subsamples were prepared for analysis by performing a water digest. The standard recoveries and RPDs for this analysis were within the required limits. The hydroxide results are $37,150 \mu \mathrm{g} / \mathrm{g}$ for solids and $70,900 \mu \mathrm{g} / \mathrm{mL}$ for liquids.

\section{B2.2.4 Thermodynamic Analyses}

As required by the safety screening (Dukelow et al. 1995) and flammable gas DQOs (McDuffie and Johnson 1995), TGA and DSC were performed on the solids and liquids.

B2.2.4.1 Thermogravimetric Analysis. Thermogravimetric analysis measures the mass of a sample while its temperature is increased at a constant rate. Nitrogen is passed over the sample during heating to remove any released gases. Any decrease in the weight of a sample during TGA represents a loss of gaseous matter from the sample through evaporation or through a reaction that forms gas phase products. The moisture content is estimated by assuming that all TGA sample weight loss up to a certain temperature (typically 150 to $200^{\circ} \mathrm{C}$ ) is caused by water evaporation. The temperature limit for moisture loss is chosen by the operator at an inflection point on the TGA plot. Other volatile matter fractions can often be differentiated by inflection points as well. Tank 241-AN-104 samples were analyzed by TGA using procedure LA-514-114, Rev. D-0 on a Perkin-Elmer ${ }^{1}$ TGA 7 instrument, or

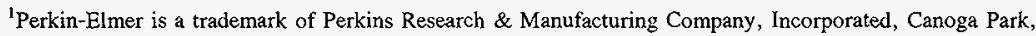
California.
} 
procedure LA-560-112, Rev. B-1 on a Mettler ${ }^{2}$ TG 50 instrument. All samples exhibited a large weight loss between the ambient temperature and $200^{\circ} \mathrm{C}\left(392^{\circ} \mathrm{F}\right)$. The weight percent water values were between 47.35 and 52.44 for liquid samples, and 36.54 and 51.34 for solid samples. The standard recoveries and RPDs for this analysis were within the required limits.

B2.2.4.2 Differential Scanning Calorimetry. In a DSC analysis, heat absorbed or emitted by a substance is measured while the temperature of the sample is heated at a constant rate. Nitrogen is passed over the sample material to remove any gases being released. The onset temperature for an endothermic or exothermic event is determined graphically. The DSC analyses for tank 241-AN-104 were performed using procedure LA-514-113, Rev. C-1 on a Mettler ${ }^{\mathrm{TM}}$ DSC 20 instrument or procedure LA-514-114, Rev. D-0 on a Perkin-Elmer ${ }^{\mathrm{TM}}$ DSC 7 instrument. The highest individual sample or duplicate exothermic results for liquids and solids were 39.3 and $106.6 \mathrm{~J} / \mathrm{g}$, respectively. The exothermic results of solid core composite samples were 101 and $105 \mathrm{~J} / \mathrm{g}$.

Most of transitions 1 and 2 represent endothermic events, both of which are caused by water evaporation. High RPDs ( $>20$ percent) in the exotherms were reported for both liquid and solid subsamples. The observed exotherm results were far below the threshold of $480 \mathrm{~J} / \mathrm{g}$, and no reruns were requested. The standard recoveries for this analysis were within the required limits.

\section{B2.2.5 Density and Specific Gravity}

Bulk density measurements were performed on all solid subsegments except segments 1 and 2 of core 163 and segment 1 of core 164 . In these cases, the degree of recovery for solid samples were insufficient to perform the analysis. The subsegment-level results for salt slurry densities ranged from 1.52 to $1.76 \mathrm{~g} / \mathrm{mL}$ with mean of $1.60 \mathrm{~g} / \mathrm{mL}$. The specific gravity measurements for liquid samples ranged from 1.34 to 1.49 with mean of 1.40 .

\section{B2.2.6 Radionuclide Analysis}

B2.2.6.1 Total Alpha. Total alpha activity analyses were performed on direct liquid core composite subsamples and the field blank. The solid core composite subsamples were prepared for analysis by performing a fusion digest in duplicate. The highest results were $0.0259 \mu \mathrm{Ci} / \mathrm{mL}$ and $0.0808 \mu \mathrm{Ci} / \mathrm{g}$ for liquids and solids, respectively. High RPD ( $>20$ percent) were reported for the solid core composite subsamples and can be attributed to sample inhomogeneity. No reruns were requested because of low alpha activity in the samples. The standard recoveries and spike recoveries for this analysis were within the required limits (Steen 1997).

\footnotetext{
${ }^{2}$ Mettler is a trademark of Mettler Instrument Corporation, Anaheim, California.
} 
B2.2.6.2 Total Beta. Total beta activity analyses were performed on direct liquid core composite subsamples. The solid core composite subsamples were prepared for analysis by performing a fusion digest in duplicate. The standard recoveries, spike recoveries, and RPDs for this analysis were within the required limits. The sample results are $400 \mu \mathrm{Ci} / \mathrm{g}$ for solids and $509 \mu \mathrm{Ci} / \mathrm{mL}$ for liquids.

B2.2.6.3 Strontium 90. Strontium 90 analyses were performed on direct liquid core composite subsamples. The solid core composite subsamples were prepared for analysis by performing a fusion digest. The standard recoveries and RPDs for this analysis were within the required limits. Strontium 90 analytical results are $34.25 \mu \mathrm{Ci} / \mathrm{g}$ for solids and $0.338 \mu \mathrm{Ci} / \mathrm{g}$ for liquids.

B2.2.6.4 Gamma Energy Analysis. Gamma energy analysis, procedure number LA-548-121, was used to measure the activity of ${ }^{137} \mathrm{Cs},{ }^{241} \mathrm{Am},{ }^{154 / 155} \mathrm{Eu}$ and ${ }^{60} \mathrm{Co}$. The GEA analyses were performed on direct liquid core composite subsamples and the field blank. The solid core composite subsamples were prepared for analysis by performing a fusion digest in duplicate. All results were below detection limit except ${ }^{137} \mathrm{Cs}$, which was $349.2 \mu \mathrm{Ci} / \mathrm{g}$ for solids and $570 \mu \mathrm{Ci} / \mathrm{mL}$ for liquids.

Actual detection limits for GEA analyses are not currently available. The latest GEA software does not currently report a minimum detectable activity. If an analysis is reported as "less than," the value reported is the detection limit.

B2.2.6.5 Total Uranium. Uranium analyses were performed on direct liquid core composite subsamples. The solid core composite subsamples were prepared for analysis by performing a fusion digest. The standard recoveries, spike recoveries, and RPDs for this analysis were within the required limits. Total uranium analytical results were $180.5 \mu \mathrm{g} / \mathrm{g}$ and $3.68 \mu \mathrm{g} / \mathrm{mL}$ for solids and liquids, respectively.

B2.2.6.6 Plutonium. The ${ }^{239 / 240} \mathrm{Pu}$ analyses were performed on direct liquid core composite subsamples. The solid core composite subsamples were prepared for analysis by performing a fusion digest. The standard recoveries and RPDs for this analysis were within the required limits. Plutonium analytical results were $0.00365 \mu \mathrm{Ci} / \mathrm{g}$ for solids and below the detection limit of $1.75 \mathrm{E}-04 \mu \mathrm{Ci} / \mathrm{mL}$ for liquids.

B2.2.6.7 Iodine 129. The ${ }^{129} \mathrm{I}$ analyses were performed on direct liquid core composite subsamples and the field biank. The solid core composite subsamples were prepared for analysis by performing a water digest. Iodine analytical results were $2.075 \mathrm{E}-04 \mu \mathrm{Ci} / \mathrm{g}$ for solids and below the detection limit of $2.7 \mathrm{E}-03 \mu \mathrm{Ci} / \mathrm{mL}$ for liquids.

B2.2.6.8 Neptunium ${ }^{237} \mathrm{~Np}$. The ${ }^{237} \mathrm{~Np}$ analyses were performed on direct liquid core composite subsamples and the field blank. The solid core composite subsamples were prepared for analysis by performing a water digest. The standard recoveries and RPDs for this analysis were within required limits. Neptunium analytical results were below the detection limit of $0.0116 \mu \mathrm{Ci} / \mathrm{g}$ for solids and $2.97 \mathrm{E}-05 \mu \mathrm{Ci} / \mathrm{mL}$ for liquid. 
B2.2.6.9 Tritium ${ }^{3} \mathrm{H}$. The ${ }^{3} \mathrm{H}$ analyses were performed on direct liquid core composite subsamples and the field blank. The solid core composite subsamples were prepared for analysis by performing a water digest. Tritium analytical results were $0.01 \mu \mathrm{Ci} / \mathrm{g}$ for solids and $1.5 \mathrm{E}-03 \mu \mathrm{Ci} / \mathrm{mL}$ for liquids.

B2.2.6.10 Technetium ${ }^{99} \mathrm{Tc}$. The ${ }^{99} \mathrm{Tc}$ analyses were performed on direct liquid core composite subsamples and the field blank. The solid core composite subsamples were prepared for analysis by performing a fusion digest in duplicate. Technetium analytical results are $0.13 \mu \mathrm{Ci} / \mathrm{g}$ for solids and $2.195 \mathrm{E}-04 \mu \mathrm{Ci} / \mathrm{mL}$ for liquids.

B2.2.6.11 Alpha Energy Analyses. The analyses were performed for ${ }^{241} \mathrm{Am}$ and ${ }^{243 / 244} \mathrm{Cm}$. The ${ }^{241} \mathrm{Am}$ analyses were performed on direct liquid core composite subsamples and the field blank. The solid core composite subsamples were prepared for analysis by performing a fusion digest in duplicate. High RPDs were reported for the solid core composite samples. This can be attributed to the heterogenous nature of the samples. No rerun was requested because of the low americium activity in the samples. The standard recoveries for this analysis were within the required limits. Americium analytical results were $0.0192 \mu \mathrm{Ci} / \mathrm{g}$ for solids and below the detection limit of $3.470 \mathrm{E}-04 \mu \mathrm{Ci} / \mathrm{mL}$ for liquid.

The ${ }^{243 / 244} \mathrm{Cm}$ analyses were performed on direct liquid core composite subsamples and the field blank. The solid core composite subsamples were prepared for analysis by performing a fusion digest in duplicate. The RPDs for this analysis were within the required limits. Curium analytical results were below the detection limits of $0.00388 \mu \mathrm{Ci} / \mathrm{g}$ for solids and 3.47E-04 $\mu \mathrm{Ci} / \mathrm{mL}$ for liquid.

\section{B2.3 RETAINED GAS SAMPLE RESULTS FORM 1996 CORE SAMPLING}

Seven segments from 1996 push mode core samples were selected as retained gas samples for the gas composition and quantities study of tank retained gas.

\section{B2.3.1 Retained Gas Concentration}

The extraction results show insoluble gases were primarily retained in the lower, nonconvective layer. Based on the estimated solubilities and RGS measurements of gas concentrations, approximately 5.7 percent by volume (in-situ) of the nonconvective layer was filled with free gas, and 0.5 percent by volume (in-situ) of the convective (upper) layer was free gas. The calculated hydrogen inventory in both phases of the nonconvective and convective layers of tank 241-AN-104 is $102 \mathrm{~m}^{3}\left(3,600 \mathrm{ft}^{3}\right)$ based on integrated RGS measurements.

Table B2-2 shows the estimated concentrations of the insoluble/low-solubility and soluble gases obtained from the RGS in tank 241-AN-104. No correction for air entrainment has been made in the data. Such a correction would consist of removing all the $\mathrm{O}_{2}$ and Ar and 


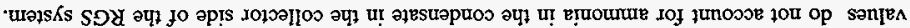

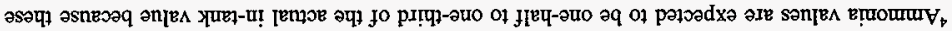

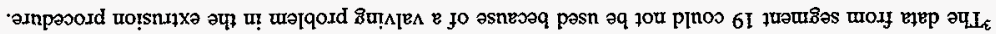

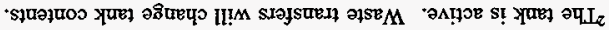

(L66I) 'TE ze z!ureyaqS

: $\$$

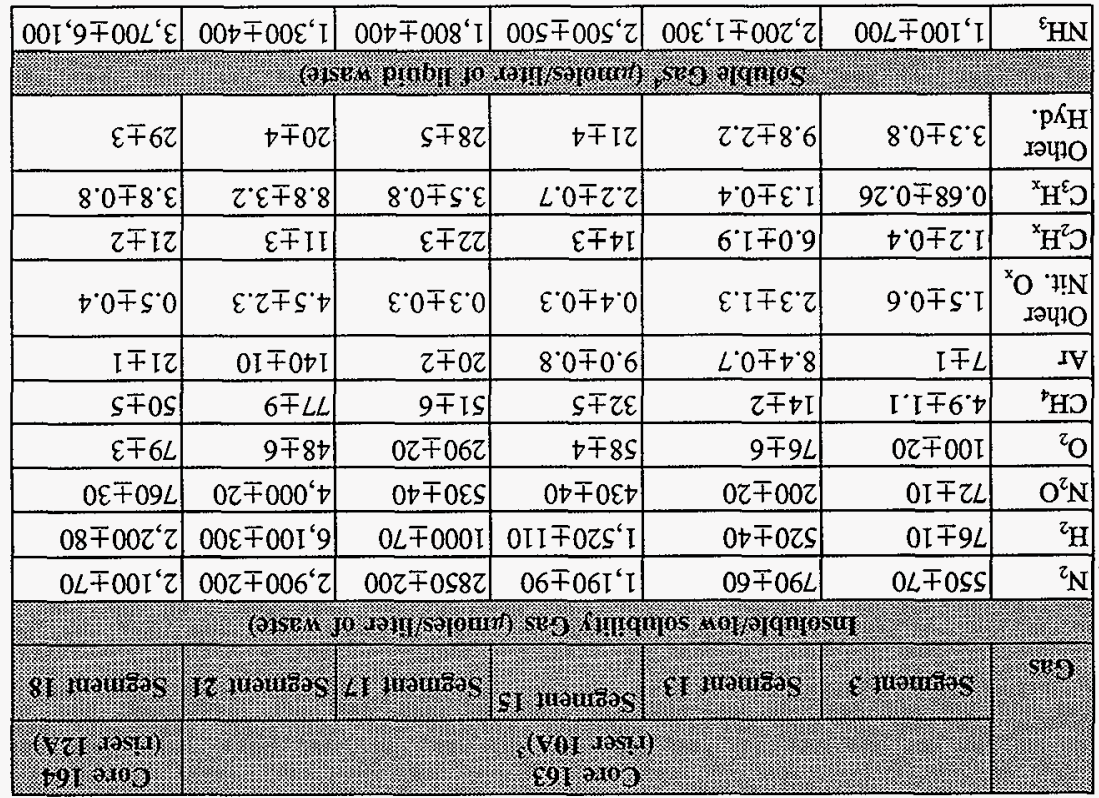

$\tau$ ' ‘

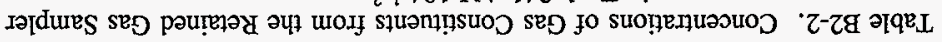

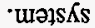

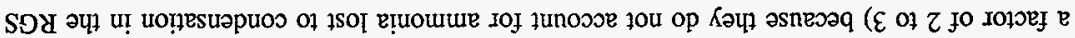

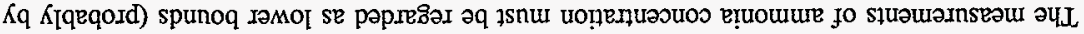

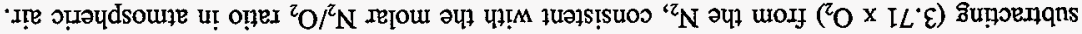




\begin{tabular}{|c|c|c|}
\hline 0 & $\left(\% 9^{\circ} 0\right) \varepsilon 200^{\circ} \mp 6 \mathrm{SO}^{\circ} 0$ & ${ }^{x} \mathrm{H}^{2} \mathrm{D}$ \\
\hline$\left(\% I^{\circ} 0\right) 100^{\circ} \mp 90^{\circ} 0$ & $\left(\% L^{\circ} I\right)+0.0 \mp 8 I^{\circ} 0$ & 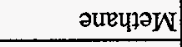 \\
\hline$(\% \mathrm{I} \varsigma) \varsigma 0 \mp 0 \cdot \varepsilon$ & $\left(\% S^{\circ}+\right)^{\circ} 80^{\circ} \mp \angle t^{\circ} 0$ & әp!xo snonț \\
\hline$\left(\% 8^{\circ} \mathrm{I}\right) \tau \cdot 0 \mp \mathrm{I}^{\circ} \mathrm{I}$ & $\left(\% I^{*} \subseteq z\right) \varepsilon t^{*} 0 \mp 9^{\circ} Z$ & 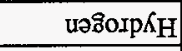 \\
\hline$(\% \tau \cdot z) 9^{\circ} 0 \mp \varepsilon^{\circ} \mathrm{I}$ & $(\% 9 \cdot$ (9) I $\varepsilon \mp 6 \%$ & นə8ิంम!ก \\
\hline$(\% 8 \cdot 06) \varsigma \varepsilon \mp \emptyset \varsigma$ & $\left(\% 20^{\circ} 0\right) 100^{\circ} 0 \mp 200^{\circ} 0$ & ह!uoururv \\
\hline \multicolumn{3}{|c|}{ 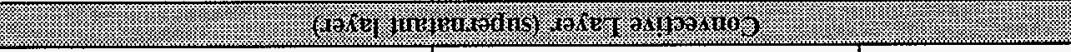 } \\
\hline $8 L \mp \varepsilon 0 \mathrm{~L}$ & $9 z \mp z 0 z$ & [E]OL \\
\hline 0 & $\left(\% \varsigma^{\circ} 0\right) \tau z^{\circ} 0 \mp \downarrow 6^{\circ} 0$ & $\rightarrow$ 工әчto \\
\hline 0 & $\left(\% \mathrm{I}^{\circ} 0\right) \mathrm{s} 0^{\circ} 0 \mp 9 \mathrm{I}^{\circ} \mathrm{O}$ & ${ }^{x} \mathrm{H}^{\varepsilon} \mathrm{O}$ \\
\hline 0 & $(\% \varepsilon \cdot 0) \varepsilon I^{\circ} 0 \mp 9^{\circ} 0$ & ${ }^{x} \mathrm{H}^{\tau} \mathrm{O}$ \\
\hline$\left(\%+0^{\circ} 0\right) \angle 00^{\circ} 0 \mp \varepsilon+0^{\circ} 0$ & $(\% 60) \varepsilon 0 \mp 8 \cdot I$ & әUечрәN \\
\hline$(\% \mathrm{I} O \mathrm{OI})+\mathrm{I}$ FOI & $\left(\% 8^{\circ} 6 I\right)[\cdot \varsigma \mp 0 t$ & әp!xo snon!n \\
\hline$(\% \tau \cdot \tau) \varepsilon \cdot 0 \mp \tau \cdot \tau$ & $(\% \varepsilon L \downarrow) Z I \mp 96$ & uว $80 \operatorname{sp}_{\mathrm{H}}$ \\
\hline$\left(\% 8^{\circ} 0\right) \tau I^{\circ} 0 \mp 98^{\circ} 0$ & $(\% 乙 \cdot I \varepsilon) \varsigma^{\prime} 8 \mp \varepsilon 9$ & นวชิอม!ก $\mathrm{N}$ \\
\hline$\left(\% 8^{\circ} 98\right) 9 L \mp 68$ & $\left(\% 20^{\circ} 0\right) 9+0^{\circ} 0 \mp 9+0^{\circ} 0$ & Ẹฺouนty \\
\hline 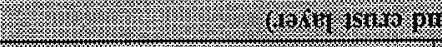 & 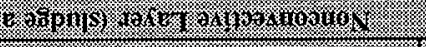 & \\
\hline 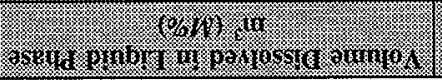 & 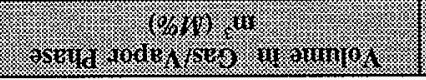 & $\sqrt{103}$ \\
\hline
\end{tabular}

(şəəus $\tau$ )

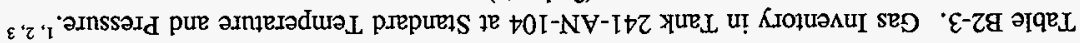

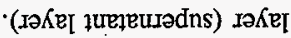

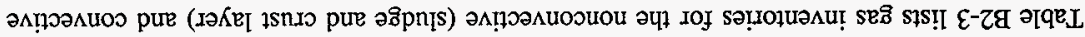

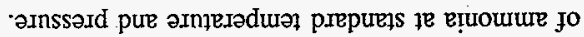

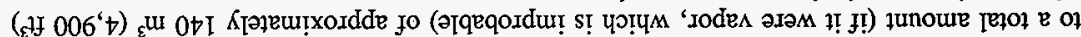

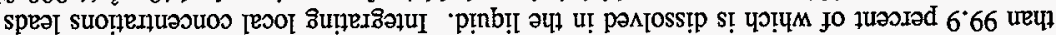

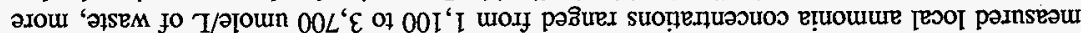
әЧ.L 'suoqreวo

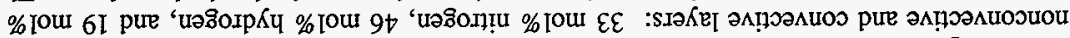

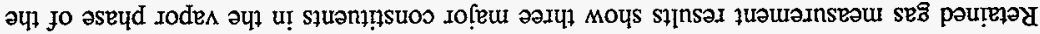

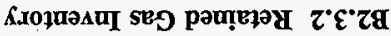


Table B2-3. Gas Inventory in Tank 241-AN-104 at Standard Temperature and Pressure. ${ }^{1,2,3}$ ( 2 sheets)

\begin{tabular}{|c|c|c|}
\hline 1. (6.S & 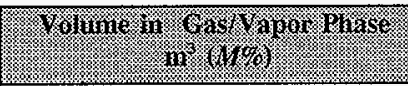 & 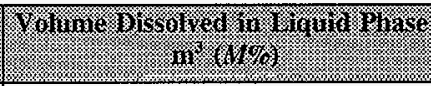 \\
\hline $\mathrm{C}_{3} \mathrm{H}_{\mathrm{x}}{ }^{4}$ & $0.033 \pm 0.013(0.3 \%)$ & 10 \\
\hline Other $^{4}$ & $0.24 \pm 0.07(2.3 \%)$ & 0 \\
\hline Total & $10 \pm 3.8$ & $60 \pm 36$ \\
\hline
\end{tabular}

Notes:

'Shekarriz et al. (1997)

${ }^{2}$ The error bands in the table represent the uncertainty that carries through from instrument error and uncertainty in layer interface location. Temporal and lateral variability are not included, and the resulting inventories may not be conservative.

${ }^{3}$ The tank is active. Waste transfers will change tank contents.

${ }^{4}$ These gases were assumed to be entirely insoluble.

The gas inventories listed in Table B2-3 were calculated from RGS data with corrections for entrained air; these agree with the inventories calculated from the VFI data by Stewart et al. (1996).

\section{B2.3.3 Waste Density}

Table B2-4 lists waste density, bulk density, and specific gravity from a non-RGS segment. The waste density for RGS was determined by the X-ray imaging method (Shekarriz et al. 1997). The X-ray imaging result of RGS for solid samples are about 5 percent to 20 percent higher than the solid density of the non-RGS segment at the same approximate depth of waste. 
Table B2-4. Comparison of Bulk Density for the Retained Gas Sampler and Non-Retained Gas Sampler Segments for Tank 241-AN-104. ${ }^{1}$

\begin{tabular}{|c|c|c|c|c|c|}
\hline 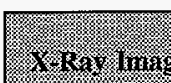 & gerviluc & IU⿴囗十) & 15 and & Ch Gravin Res & STroin: \\
\hline 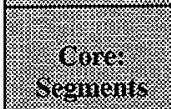 & 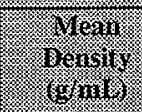 & 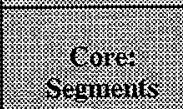 & I: & 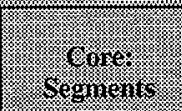 & Henvit \\
\hline $163: 3$ & 1.41 & 163:4 (Liquid) & 1.38 & 164:4 (Liquid) & 1.41 \\
\hline $163: 13$ & 1.79 & $163: 12$ (Solid) & 1.76 & 164:13 (Solid) & 1.53 \\
\hline $163: 15$ & 1.72 & 163:14 (Solid) & 1.63 & $164: 15$ (Solid) & 1.56 \\
\hline $163: 17$ & 2.09 & $163: 16$ (Solid) & 1.57 & $164: 17$ (Solid) & 1.54 \\
\hline 163:19 & 1.74 & $163: 18$ (Solid) & 1.65 & $164: 19$ (Solid) & 1.57 \\
\hline $163: 21$ & 1.85 & $163: 20$ (Solid) & 1.64 & $164: 20$ (Solid) & 1.60 \\
\hline
\end{tabular}

Notes:

${ }^{1}$ The tank is active. Waste transfers will change tank contents.

${ }^{2}$ Shekarriz et al. (1997)

${ }^{3}$ See Table B2-59. Solid density is the mean of the upper and lower segment.

\section{B2.4 1996 HEADSPACE VAPOR MEASUREMENT}

During August and September 1996, tank headspace gas samples were obtained from tank 241-AN-104. These measurements supported the safety screening DQO (Dukelow et al. 1995). The vapor phase screening was taken to assess flammability issues. The vapor phase measurements were obtained $20 \mathrm{ft}$ below risers $10 \mathrm{~A}$ and $12 \mathrm{~A}$ in the headspace of the tank, and results were obtained in the field (that is, no gas sample was sent to the laboratory for analysis).

Table B2-5. Tank 241-AN-104 Vapor Sampling Results. ${ }^{1}$

\begin{tabular}{|l|l|}
\hline \multicolumn{1}{|c|}{ Measureaurit } \\
\hline Total Organic Carbon & 9.0 parts per million by volume (ppmv) \\
\hline Lower explosive limit & 0.013 percent of the lower explosive limit \\
\hline Oxygen & 21.0 percent \\
\hline Ammonia & 100 ppmv \\
\hline
\end{tabular}

Note:

${ }^{1}$ The tank is active. Waste transfers will change tank contents. 


\section{B2.5 HISTORICAL SAMPLE RESULTS}

Table B2-6 shows the results of the July 1984 (Jansky 1984a) and January 1985 (Mauss and Jansky 1985) sampling events. Tank 241-AN-104 was used as a receiver tank for the 242-A Evaporator. The samples were analyzed for specific constituents, and the results showed high concentrations of aluminum, hydroxide, nitrate, and nitrite. Sodium was found in high concentration in the second sampling event. These data have not been validated and should be used with caution.

Table B2-6. Tank 241-AN-104 Sampling Results for July 1984 and January $1985 .{ }^{1}$ (2 sheets)

\begin{tabular}{|c|c|c|c|}
\hline & \multicolumn{2}{|c|}{ Why $198 \% ?$} & Mrininy 1985 \\
\hline Conipurients & Superinate & Solugro & Singerunger \\
\hline 1: & \multicolumn{2}{|c|}{ Minsied squa } & $\sqrt{2}$ \\
\hline Specific gravity & 1.464 & & $1.38^{4}$ \\
\hline Bulk density ${ }^{5}$ & & 1.76 & \\
\hline \multicolumn{4}{|c|}{ 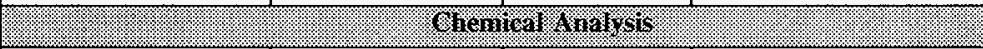 } \\
\hline $\mathrm{Na}$ & NA & & $11.22 M$ \\
\hline $\mathrm{A} 1$ & $1.81 M$ & & $1.56 M$ \\
\hline $\mathrm{Cr}^{+3}$ & $0.013 M$ & & NA \\
\hline $\mathrm{Fe}^{+2,3}$ & $0.0002 M$ & & NA \\
\hline $\mathrm{K}^{+}$ & NA & & $0.18 M$ \\
\hline $\mathrm{Cl}^{-}$ & NA & & $0.17 M$ \\
\hline $\mathrm{NO}_{3}^{-}$ & $2.72 M$ & $29 \%$ & $3.00 M$ \\
\hline $\mathrm{NO}_{2}^{-}$ & $1.87 M$ & $35 \%$ & $1.96 M$ \\
\hline $\mathrm{CO}_{3}^{2-}$ & $0.28 M$ & $33 \%$ & $0.32 M$ \\
\hline $\mathrm{SO}_{4}^{2 \cdot}$ & $0.016 M$ & & $0.025 M$ \\
\hline $\mathrm{PO}_{4}^{33}$ & $0.007 M$ & & $0.020 M$ \\
\hline $\mathrm{OH}^{-}$ & $4.00 M$ & & $3.98 M$ \\
\hline TOC & $3.830 \mathrm{~g} \mathrm{C} / \mathrm{L}$ & & $2.88 M^{6}$ \\
\hline
\end{tabular}


Table B2-6. Tank 241-AN-104 Sampling Results

for July 1984 and January 1985.1 (2 sheets)

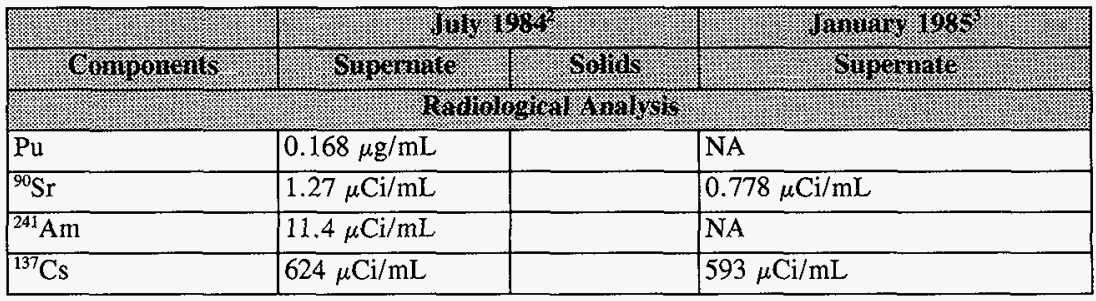

Notes:

$\mathrm{NA}=$ not available

'Pre-1989 analytical data have not been validated and should be used with caution.

${ }^{2} J a n s k y$ (1984a)

${ }^{3}$ Mauss and Jansky (1985)

${ }^{4}$ Estimated values

${ }^{s}$ Bulk density of centrifugal solids

${ }^{6}$ The unit is probably $\mathrm{g} C / \mathrm{L}$. 


\section{B2.6 ANALYTICAL DATA TABLES FROM 1996 SAMPLING}

For most analyses (except for some physical and rheological measurements), the data tables consist of six columns. The first column lists the sample number. For each primary/duplicate pair, the sample number is for the primary result. The second column lists the core from which the samples were derived. The third column lists the sample portion from which the aliquots were taken. The final three columns display the primary and duplicate analytical values and a mean for each sample/duplicate pair.

The four QC parameters assessed in conjunction with tank 241-AN-104 samples were standard recoveries, spike recoveries, duplicate analyses (RPDs), and blanks. The QC criteria specified in Winkleman (1996a) for the safety screening DQO were 90 to 110 percent recovery for standards and spikes and $\leq 10$ percent for RPDs. The only QC parameter, for which limits are not specified in Winkelman, is blank contamination. The limits for blanks are in guidelines followed by the laboratory, and all data results in this report have met those guidelines. Sample and duplicate pairs, in which any QC parameter was outside the limits, are footnoted in the sample mean column of the data summary tables with an $a, b, c, d, e$, or $f$ as follows:

- $\quad$ "a" indicates that the standard recovery was below the QC range.

- $\quad \mathrm{b}$ " indicates that the standard recovery was above the $\mathrm{QC}$ range.

- $\quad \mathrm{c} "$ indicates that the spike recovery was below the $\mathrm{QC}$ range.

- $\quad \mathrm{d}$ " indicates that the spike recovery was above the $\mathrm{QC}$ range.

- $\quad$ "e" indicates that the RPD was greater than the QC limit range.

- $\quad \mathrm{f}$ " indicates that there was blank contamination. 
Table B2-7. Tank 241-AN-104 Analytical Results: Aluminum (ICP). (3 sheets)

\begin{tabular}{|c|c|c|c|c|c|}
\hline owion & 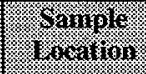 & \%ornolo & Weall & Holligh & yexin \\
\hline \multicolumn{3}{|c|}{ 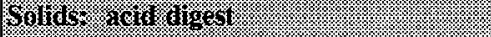 } & 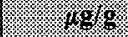 & 498 & 6 㴗 \\
\hline S96T005287 & $163: 1$ & Lower half & 20,900 & 20,600 & $20,750^{Q C: c}$ \\
\hline S96T005288 & $163: 2$ & Lower half & 17,100 & 17,500 & $17,300^{\text {QC:d }}$ \\
\hline S96T005782 & $163: 12$ & Lower half & 21,200 & 20,100 & 20,650 \\
\hline S96T005749 & \multirow[t]{2}{*}{$163: 14$} & Upper half & 20,000 & 20,500 & 20,250 \\
\hline S96T005750 & & Lower half & 21,300 & 21,500 & 21,400 \\
\hline S96T005294 & \multirow[t]{2}{*}{$163: 16$} & Upper half & 18,600 & 19,600 & 19,100 \\
\hline S96T005289 & & Lower half & 19,500 & 20,700 & $20,100^{\text {eC:d }}$ \\
\hline S96T005778 & \multirow[t]{2}{*}{$163: 18$} & Upper half & 17,800 & 18,000 & 17,900 \\
\hline S96T005783 & & Lower half & 17,900 & 17,300 & 17,600 \\
\hline S96T005557 & \multirow[t]{2}{*}{$163: 20$} & Upper half & 17,500 & 17,900 & 17,700 \\
\hline S96T005560 & & Lower half & 18,800 & 15,300 & $17,050^{\mathrm{QC}: \mathrm{c}, \mathrm{c}}$ \\
\hline S96T004797 & $164: 1$ & Lower half & 20,300 & 20,500 & $20,400^{\mathrm{QC} ; \mathrm{d}}$ \\
\hline S96T004798 & $164: 13$ & Lower half & 21,800 & 23,100 & $22,450^{\mathrm{QC}: \mathrm{d}}$ \\
\hline S96T005042 & \multirow[t]{2}{*}{$164: 14$} & Upper half & 21,700 & 21,700 & 21,700 \\
\hline S96T005040 & & Lower half & 22,300 & 22,700 & 22,500 \\
\hline \$96T005061 & \multirow[t]{2}{*}{$164: 15$} & Upper half & 21,000 & 21,300 & 21,150 \\
\hline S96T005043 & & Lower half & 21,200 & 20,900 & 21,050 \\
\hline \$96T005062 & \multirow[t]{2}{*}{$164: 16$} & Upper half & 23,800 & 24,000 & 23,900 \\
\hline S96T005044 & & Lower half & 22,400 & 22,300 & 22,350 \\
\hline S96T005063 & \multirow[t]{2}{*}{$164: 17$} & Upper half & 22,700 & 23,900 & 23,300 \\
\hline S96T005041 & & Lower half & 21,800 & 21,900 & $21,850^{\mathrm{eC:d}}$ \\
\hline \$96T005064 & \multirow[t]{2}{*}{$164: 19$} & Upper half & 20,900 & 21,700 & 21,300 \\
\hline S96T005045 & & Lower half & 22,800 & 22,800 & 22,800 \\
\hline \$96T005282 & \multirow[t]{2}{*}{$164: 20$} & Upper half & 20,200 & 21,000 & 20,600 \\
\hline \$96T005279 & & Lower half & 19,000 & 21,400 & $20,200^{\mathrm{QC}: \mathrm{c}}$ \\
\hline S96T005976 & Core 164 & Composite & 20,600 & 20,900 & $20,750^{\mathrm{ec}: \mathrm{a}}$ \\
\hline
\end{tabular}


Table B2-7. Tank 241-AN-104 Analytical Results: Aluminum (ICP). (3 sheets)

\begin{tabular}{|c|c|c|c|c|c|}
\hline gandirar & 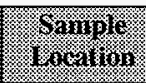 & (1) & I. & Bingingur & 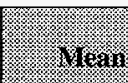 \\
\hline 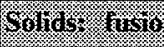 & 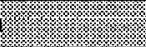 & S & (3) & (6) & $f_{*} \cdot$. \\
\hline \$96T005284 & $163: 1$ & Lower half & 21,700 & 20,300 & 21,000 \\
\hline S96T005285 & $163: 2$ & Lower half & 16,100 & 13,800 & 14,950 \\
\hline S96T005780 & $163: 12$ & Lower half & 21,500 & 21,700 & 21,600 \\
\hline S96T005747 & \multirow[t]{2}{*}{$163: 14$} & Upper half & 20,200 & 20,000 & 20,100 \\
\hline S96T005748 & & Lower half & 21,000 & 21,100 & 21,050 \\
\hline \$96T005293 & \multirow[t]{2}{*}{$163: 16$} & Upper half & 19,500 & 20,400 & 19,950 \\
\hline S96T005286 & & Lower half & 21,000 & 18,800 & 19,900 \\
\hline S96T005777 & \multirow[t]{2}{*}{$163: 18$} & Upper half & 25,900 & 18,900 & $22,400^{\mathrm{QC} c \mathrm{c}}$ \\
\hline S96T005781 & & Lower half & 21,400 & 22,600 & 22,000 \\
\hline S96T005556 & \multirow[t]{2}{*}{$163: 20$} & Upper half & 21,700 & 17,500 & $19,600^{0 \mathrm{C}: \mathrm{c}}$ \\
\hline S96T005559 & & Lower half & 16,700 & 21,400 & $19,050^{\mathrm{QC:e}}$ \\
\hline \$96T004795 & $164: 1$ & Lower half & 22,800 & 22,000 & 22,400 \\
\hline S96T004796 & $164: 13$ & Lower half & 23,000 & 21,800 & 22,400 \\
\hline S96T005035 & \multirow[t]{2}{*}{$164: 14$} & Upper half & 22,400 & 20,200 & 21,300 \\
\hline S96T005030 & & Lower half & 22,900 & 21,800 & 22,350 \\
\hline \$96T005036 & \multirow[t]{2}{*}{$164: 15$} & Upper half & 22,200 & 22,600 & 22,400 \\
\hline S96T005032 & & Lower half & 21,700 & 21,400 & 21,550 \\
\hline S96T005037 & \multirow[t]{2}{*}{$164: 16$} & Upper half & 23,400 & 22,900 & 23,150 \\
\hline S96T005033 & & Lower half & 22,700 & 22,700 & 22,700 \\
\hline S96T005038 & \multirow[t]{2}{*}{$164: 17$} & Upper half & 22,600 & 22,300 & 22,450 \\
\hline S96T005031 & & Lower half & 20,400 & 21,700 & 21,050 \\
\hline S96T005039 & \multirow[t]{2}{*}{$164: 19$} & Upper half & 20,800 & 19,900 & 20,350 \\
\hline S96T005034 & & Lower half & 21,600 & 21,000 & 21,300 \\
\hline S96T005281 & \multirow[t]{2}{*}{$164: 20$} & Upper half & 17,400 & 17,300 & 17,350 \\
\hline S96T005278 & & Lower half & 19,800 & 19,200 & 19,500 \\
\hline
\end{tabular}


Table B2-7. Tank 241-AN-104 Analytical Results: Aluminum (ICP). (3 sheets)

\begin{tabular}{|c|c|c|c|c|c|}
\hline 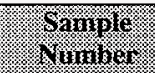 & 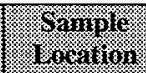 & 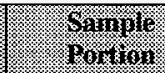 & How & 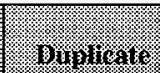 & (.). Yuar. \\
\hline yrgurin: & 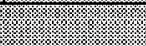 & 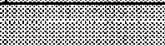 & (V) & (2. & f\% \\
\hline S96T005256 & $163: 2$ & Liquid & 37,500 & 40,600 & 39,050 \\
\hline \$96T005260 & $163: 4$ & Liquid & 40,200 & 37,500 & $38,850^{\mathrm{QC:c}}$ \\
\hline S96T005527 & $163: 5$ & Liquid & 37,300 & 36,800 & $37,050^{\mathrm{eC:c}}$ \\
\hline S96T005554 & $163: 6$ & Liquid & 38,700 & 38,400 & 38,550 \\
\hline S96T005255 & $163: 7$ & Liquid & 41,000 & 43,200 & $42,100^{\mathrm{QC:d}}$ \\
\hline S96T005257 & $163: 8$ & Liquid & 36,800 & 37,700 & 37,250 \\
\hline S96T005258 & $163: 9$ & Liquid & 39,200 & 37,500 & 38,350 \\
\hline S96T005528 & $163: 10$ & Liquid & 35,400 & 35,900 & 35,650 \\
\hline S96T005739 & $163: 11$ & Liquid & 37,400 & 37,200 & 37,300 \\
\hline S96T005766 & $163: 12$ & Liquid & 40,400 & 40,000 & 40,200 \\
\hline \$96T005741 & $163: 14$ & Liquid & 37,300 & 37,100 & 37,200 \\
\hline \$96T004774 & $164: 1$ & Liquid & 40,600 & 41,500 & $41,050^{Q C: d}$ \\
\hline S96T004778 & $164: 2$ & Liquid & 35,400 & 41,600 & 38,500 \\
\hline S96T004779 & $164: 3$ & Liquid & 40,600 & 39,600 & 40,100 \\
\hline$\$ 96 T 004780$ & $164: 4$ & Liquid & 37,300 & 38,300 & $37,800^{\mathrm{QC:d}}$ \\
\hline$\$ 96 \mathrm{~T} 004976$ & $164: 5$ & Liquid & 40,100 & 37,800 & $38,950^{Q C: c}$ \\
\hline S96T004781 & $164: 7$ & Liquid & 36,900 & 38,000 & $37,450^{\mathrm{ec:d}}$ \\
\hline S96T004977 & $164: 8$ & Liquid & 43,500 & 37,200 & $40,350^{\mathrm{QC}: \mathrm{c}}$ \\
\hline \$96T004978 & $164: 9$ & Liquid & 41,100 & 34,700 & 37,900 \\
\hline \$96T004979 & $164: 10$ & Liquid & 38,700 & 37,200 & 37,950 \\
\hline S96T004782 & $164: 11$ & Liquid & 36,500 & 38,500 & $37,500^{0 \mathrm{c}: \mathrm{d}}$ \\
\hline S96T004783 & $164: 12$ & Liquid & 39,000 & 43,400 & 41,200 \\
\hline \$96T004784 & $164: 13$ & Liqquid & 36,200 & 38,000 & $37,100^{Q C: d}$ \\
\hline S96T005979 & Core 164 & Composite & 42,500 & 40,900 & $41,700^{\mathrm{QC}: \mathrm{c}}$ \\
\hline
\end{tabular}


Table B2-8. Tank 241-AN-104 Analytical Results: Antimony (ICP). (3 sheets)

\begin{tabular}{|c|c|c|c|c|c|}
\hline $\begin{array}{l}\text { Sampla } \\
\text { Hamplo }\end{array}$ & 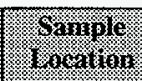 & 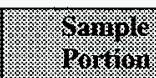 & Resulu & 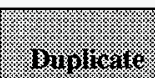 & (3) \\
\hline \multicolumn{3}{|c|}{ 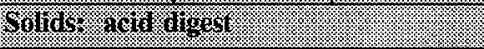 } & l. & 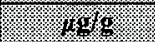 & 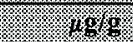 \\
\hline S96T005287 & $163: 1$ & Lower half & $<22.7$ & $<23.6$ & $<23.15$ \\
\hline S96T005288 & $163: 2$ & Lower half & $<26.6$ & $<22.5$ & $<24.55$ \\
\hline S96T005782 & $163: 12$ & Lower half & $<27.4$ & $<27.7$ & $<27.55$ \\
\hline S96T005749 & \multirow[t]{2}{*}{$163: 14$} & Upper half & $<26.9$ & $<26.2$ & $<26.55$ \\
\hline S96T005750 & & Lower half & $<27.5$ & $<27.1$ & $<27.3$ \\
\hline S96T005294 & \multirow[t]{2}{*}{$163: 16$} & Upper half & $<24.8$ & $<22.2$ & $<23.5$ \\
\hline S96T005289 & & Lower half & $<27.9$ & $<25.6$ & $<26.75$ \\
\hline S96T005778 & \multirow[t]{2}{*}{$163: 18$} & Upper half & $<28.9$ & $<27.3$ & $<28.1$ \\
\hline S96T005783 & & Lower half & $<28.5$ & $<28.3$ & $<28.4$ \\
\hline S96T005557 & \multirow[t]{2}{*}{$163: 20$} & Upper half & $<22.8$ & $<24.1$ & $<23.45$ \\
\hline S96T005560 & & Lower half & $<26.5$ & $<22.4$ & $<24.45$ \\
\hline \$96T004797 & $164: 1$ & Lower half & $<28.1$ & $<28.3$ & $<28.2$ \\
\hline S96T004798 & $164: 13$ & Lower half & $<29$ & $<28.6$ & $<28.8$ \\
\hline S96T005042 & \multirow[t]{2}{*}{$164: 14$} & Upper half & $<28.5$ & $<28.8$ & $<28.65$ \\
\hline S96T005040 & & Lower half & $<28.6$ & $<28.2$ & $<28.4$ \\
\hline S96T005061 & \multirow[t]{2}{*}{$164: 15$} & Upper half & $<29$ & $<28.8$ & $<28.9$ \\
\hline S96T005043 & & Lower half & $<27.8$ & $<28.6$ & $<28.2$ \\
\hline \$96T005062 & \multirow[t]{2}{*}{$164: 16$} & Upper half & $<90.4$ & $<89.6$ & $<90$ \\
\hline S96T005044 & & Lower half & $<88.5$ & $<90$ & $<89.25$ \\
\hline S96T005063 & \multirow[t]{2}{*}{$164: 17$} & Upper half & $<83.6$ & $<85.7$ & $<84.65$ \\
\hline S96T005041 & & Lower half & $<91.5$ & $<90.7$ & $<91.1$ \\
\hline S96T005064 & \multirow[t]{2}{*}{$164: 19$} & Upper half & $<90.8$ & $<92.1$ & $<91.45$ \\
\hline S96T005045 & & Lower half & $<87$ & $<86.4$ & $<86.7$ \\
\hline S96T005282 & \multirow[t]{2}{*}{$164: 20$} & Upper half & $<23.6$ & $<21.6$ & $<22.6$ \\
\hline S96T005279 & & Lower half & $<27.2$ & $<22.7$ & $<24.95$ \\
\hline S96T005976 & Core 164 & Composite & $<23.9$ & $<23.9$ & $<23.9$ \\
\hline
\end{tabular}


Table B2-8. Tank 241-AN-104 Analytical Results: Antimony (ICP). (3 sheets)

\begin{tabular}{|c|c|c|c|c|c|}
\hline 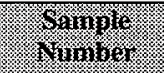 & Woris & 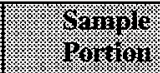 & Pexin) & Broluterto & 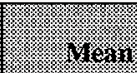 \\
\hline \multicolumn{3}{|c|}{ Soluds } & moso & 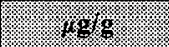 & 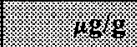 \\
\hline S96T005284 & $163: 1$ & Lower half & $<1,120$ & $<1,120$ & $<1,120$ \\
\hline \$96T005285 & $163: 2$ & Lower half & $<1,070$ & $<1,150$ & $<1,110$ \\
\hline \$96T005780 & $163: 12$ & Lower half & $<1,130$ & $<1,140$ & $<1,135$ \\
\hline S96T005747 & \multirow[t]{2}{*}{$163: 14$} & Upper half & $<1,340$ & $<1,350$ & $<1,345$ \\
\hline S96T005748 & & Lower half & $<1,120$ & $<1,160$ & $<1,140$ \\
\hline S96T005293 & \multirow[t]{2}{*}{$163: 16$} & Upper half & $<1,060$ & $<1,140$ & $<1,100$ \\
\hline \$96T005286 & & Lower half & $<1,190$ & $<1,100$ & $<1,145$ \\
\hline \$96T005777 & \multirow[t]{2}{*}{$163: 18$} & Upper half & $<1,230$ & $<1,190$ & $<1,210$ \\
\hline S96T005781 & & Lower half & $<1,280$ & $<1,280$ & $<1,280$ \\
\hline \$96T005556 & \multirow[t]{2}{*}{$163: 20$} & Upper half & $<1,110$ & $<1,160$ & $<1,135$ \\
\hline \$96T005559 & & Lower half & $<1,170$ & $<1,170$ & $<1,170$ \\
\hline S96T004795 & $164: 1$ & Lower half & $<1,230$ & $<1,210$ & $<1,220$ \\
\hline \$96T004796 & $164: 13$ & Lower half & $<1,240$ & $<1,240$ & $<1,240$ \\
\hline S96T005035 & \multirow[t]{2}{*}{$164: 14$} & Upper half & $<1,220$ & $<1,240$ & $<1,230$ \\
\hline \$96T005030 & & Lower half & $<1,230$ & $<1,220$ & $<1,225$ \\
\hline \$96T005036 & \multirow[t]{2}{*}{$164: 15$} & Upper half & $<1,210$ & $<1,180$ & $<1,195$ \\
\hline \$96T005032 & & Lower half & $<1,180$ & $<1,200$ & $<1,190$ \\
\hline S96T005037 & \multirow[t]{2}{*}{$164: 16$} & Upper half & $<1,150$ & $<1,100$ & $<1,125$ \\
\hline S96T005033 & & Lower half & $<1,150$ & $<1,120$ & $<1,135$ \\
\hline S96T005038 & \multirow[t]{2}{*}{$164: 17$} & Upper half & $<1,160$ & $<1,140$ & $<1,150$ \\
\hline S96T005031 & & Lower half & $<1,310$ & $<1,300$ & $<1,305$ \\
\hline S96T005039 & \multirow[t]{2}{*}{$164: 19$} & Upper half & $<1,170$ & $<1,200$ & $<1,185$ \\
\hline S96T005034 & & Lower half & $<1,280$ & $<1,300$ & $<1,290$ \\
\hline S96T005281 & \multirow[t]{2}{*}{$164: 20$} & Upper half & $<1,030$ & $<1,110$ & $<1,070$ \\
\hline \$96T005278 & & Lower half & 1,220 & 1,590 & $1,405^{\mathrm{QC:c}}$ \\
\hline
\end{tabular}


Table B2-8. Tank 241-AN-104 Analytical Results: Antimony (ICP). (3 sheets)

\begin{tabular}{|c|c|c|c|c|c|}
\hline (3) & 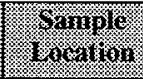 & J & ne ainin & 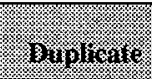 & m $m$ \\
\hline Wring & & & (6\% & \% & \% \\
\hline S96T005256 & $163: 2$ & Liquid & $<36.1$ & $<36.1$ & $<36.1$ \\
\hline S96T005260 & $163: 4$ & Liquid & $<36.1$ & $<36.1$ & $<36.1$ \\
\hline S96T005527 & $163: 5$ & Liquid & $<36.1$ & $<36.1$ & $<36.1$ \\
\hline$\$ 96 \mathrm{~T} 005554$ & $163: 6$ & Liquid & $<36.1$ & $<36.1$ & $<36.1$ \\
\hline S96T005255 & $163: 7$ & Liquid & $<36.1$ & $<36.1$ & $<36.1$ \\
\hline S96T005257 & $163: 8$ & Liquid & $<36.1$ & $<36.1$ & $<36.1$ \\
\hline S96T005258 & $163: 9$ & Liquid & $<36.1$ & $<36.1$ & $<36.1$ \\
\hline \$96T005528 & $163: 10$ & Liquid & $<36.1$ & $<36.1$ & $<36.1$ \\
\hline S96T005739 & 163:11 & Liquid & $<36.1$ & $<36.1$ & $<36.1$ \\
\hline S96T005766 & $163: 12$ & Liquid & $<36.1$ & $<36.1$ & $<36.1$ \\
\hline S96T005741 & $163: 14$ & Liquid & $<36.1$ & $<36.1$ & $<36.1$ \\
\hline S96T004774 & $164: 1$ & Liquid & $<72.1$ & $<72.1$ & $<72.1$ \\
\hline S96T004778 & $164: 2$ & Liquid & $<72.1$ & $<72.1$ & $<72.1$ \\
\hline S96T004779 & $164: 3$ & Liquid & $<72.1$ & $<72.1$ & $<72.1$ \\
\hline S96T004780 & $164: 4$ & Liquid & $<36.1$ & $<36.1$ & $<36.1$ \\
\hline S96T004976 & $164: 5$ & Liquid & $<36.1$ & $<36.1$ & $<36.1$ \\
\hline \$96T004781 & $164: 7$ & Liquid & $<36.1$ & $<36.1$ & $<36.1$ \\
\hline S96T004977 & $164: 8$ & Liquid & $<36.1$ & $<36.1$ & $<36.1$ \\
\hline S96T004978 & $164: 9$ & Liquid & $<36.1$ & $<36.1$ & $<36.1$ \\
\hline S96T004979 & $164: 10$ & Liquid & $<36.1$ & $<36.1$ & $<36.1$ \\
\hline S96T004782 & $164: 11$ & Liquid & $<36.1$ & $<36.1$ & $<36.1$ \\
\hline S96T004783 & $164: 12$ & Liquid & $<36.1$ & $<36.1$ & $<36.1$ \\
\hline S96T004784 & $164: 13$ & Liquid & $<36.1$ & $<36.1$ & $<36.1$ \\
\hline S96T005979 & Core 164 & Composite & $<72.1$ & $<72.1$ & $<72.1$ \\
\hline
\end{tabular}


HNF-SD-WM-ER-690 Rev. 0

Table B2-9. Tank 241-AN-104 Analytical Results: Arsenic (ICP). (3 sheets)

\begin{tabular}{|c|c|c|c|c|c|}
\hline (2) & Grivple & Wornos & (hesil & 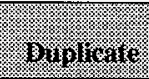 & $4=2$ \\
\hline \multicolumn{2}{|c|}{ Goinds acingingst } & W & \% & (1) & (1) \\
\hline S96T005287 & 163:1 & Lower half & $<37.9$ & $<39.3$ & $<38.6$ \\
\hline S96T005288 & $163: 2$ & Lower half & $<44.3$ & $<37.5$ & $<40.9$ \\
\hline \$96T005782 & $163: 12$ & Lower half & $<45.7$ & $<46.1$ & $<45.9$ \\
\hline \$96T005749 & \multirow[t]{2}{*}{ 163:14 } & Upper half & $<44.8$ & $<43.6$ & $<44.2$ \\
\hline S96T005750 & & Lower half & $<45.9$ & $<45.2$ & $<45.55$ \\
\hline S96T005294 & \multirow[t]{2}{*}{$163: 16$} & Upper half & $<41.3$ & $<37$ & $<39.15$ \\
\hline S96T005289 & & Lower half & $<46.4$ & $<42.6$ & $<44.5$ \\
\hline S96T005778 & \multirow[t]{2}{*}{$163: 18$} & Upper half & $<48.1$ & $<45.4$ & $<46.75$ \\
\hline S96T005783 & & Lower half & $<47.4$ & $<47.2$ & $<47.3$ \\
\hline S96T005557 & \multirow[t]{2}{*}{$163: 20$} & Upper half & $<38$ & $<40.1$ & $<39.05$ \\
\hline S96T005560 & & Lower half & $<44.2$ & $<37.3$ & $<40.75$ \\
\hline S96T004797 & $164: 1$ & Lower half & $<46.9$ & $<47.1$ & $<47$ \\
\hline S96T004798 & $164: 13$ & Lower half & $<48.4$ & $<47.7$ & $<48.05$ \\
\hline S96T005042 & \multirow[t]{2}{*}{$164: 14$} & Upper half & $<47.4$ & $<48$ & $<47.7$ \\
\hline \$96T005040 & & Lower half & $<47.7$ & $<47$ & $<47.35$ \\
\hline S96T005061 & \multirow[t]{2}{*}{$164: 15$} & Upper half & $<48.3$ & $<48$ & $<48.15$ \\
\hline S96T005043 & & Lower half & $<46.4$ & $<47.7$ & $<47.05$ \\
\hline S96T005062 & \multirow[t]{2}{*}{$164: 16$} & Upper half & $<151$ & $<149$ & $<150$ \\
\hline S96T005044 & & Lower half & $<147$ & $<150$ & $<148.5$ \\
\hline S96T005063 & \multirow[t]{2}{*}{$164: 17$} & Upper half & $<139$ & $<143$ & $<141$ \\
\hline S96T005041 & & Lower half & $<152$ & $<151$ & $<151.5$ \\
\hline S96T005064 & \multirow[t]{2}{*}{$164: 19$} & Upper half & $<151$ & $<153$ & $<152$ \\
\hline S96T005045 & & Lower half & $<145$ & $<144$ & $<144.5$ \\
\hline S96T005282 & \multirow[t]{2}{*}{$164: 20$} & Upper half & $<39.3$ & $<36.1$ & $<37.7$ \\
\hline \$96T005279 & & Lower half & $<45.4$ & $<37.8$ & $<41.6$ \\
\hline S96T005976 & Core 164 & Composite & $<39.8$ & $<39.8$ & $<39.8^{\mathrm{QC}: \mathrm{a}}$ \\
\hline
\end{tabular}


Table B2-9. Tank 241-AN-104 Analytical Results: Arsenic (ICP). (3 sheets)

\begin{tabular}{|c|c|c|c|c|c|}
\hline 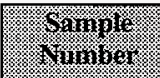 & 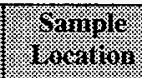 & 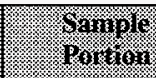 & Rysing & 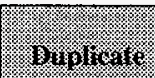 & 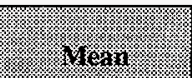 \\
\hline \multicolumn{3}{|c|}{ \%) } & 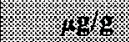 & \%o & 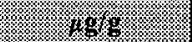 \\
\hline S96T005284 & $163: 1$ & Lower half & $<1,870$ & $<1,870$ & $<1,870$ \\
\hline S96T005285 & $163: 2$ & Lower half & $<1,790$ & $<1,910$ & $<1,850$ \\
\hline S96T005780 & $163: 12$ & Lower half & $<1,880$ & $<1,900$ & $<1,890$ \\
\hline S96T005747 & \multirow[t]{2}{*}{$163: 14$} & Upper half & $<2,240$ & $<2,250$ & $<2,245$ \\
\hline S96T005748 & & Lower half & $<1,870$ & $<1,930$ & $<1,900$ \\
\hline S96T005293 & \multirow[t]{2}{*}{$163: 16$} & Upper half & $<1,770$ & $<1,900$ & $<1,835$ \\
\hline S96T005286 & & Lower half & $<1,990$ & $<1,830$ & $<1,910$ \\
\hline S96T005777 & \multirow[t]{2}{*}{$163: 18$} & Upper half & $<2,050$ & $<1,980$ & $<2,015$ \\
\hline \$96T005781 & & Lower half & $<2,130$ & $<2,140$ & $<2,135$ \\
\hline S96T005556 & \multirow[t]{2}{*}{$163: 20$} & Upper half & $<1,850$ & $<1,940$ & $<1,895$ \\
\hline S96T005559 & & Lower half & $<1,950$ & $<1,950$ & $<1,950$ \\
\hline S96T004795 & $164: 1$ & Lower half & $<2,040$ & $<2,010$ & $<2,025$ \\
\hline \$96T004796 & $164: 13$ & Lower half & $<2,060$ & $<2,070$ & $<2,065$ \\
\hline \$96T005035 & \multirow[t]{2}{*}{$164: 14$} & Upper half & $<2,040$ & $<2,060$ & $<2,050$ \\
\hline \$96T005030 & & Lower half & $<2,050$ & $<2,040$ & $<2,045$ \\
\hline S96T005036 & \multirow[t]{2}{*}{$164: 15$} & Upper half & $<2,010$ & $<1,970$ & $<1,990$ \\
\hline S96T005032 & & Lower half & $<1,970$ & $<2,000$ & $<1,985$ \\
\hline S96T005037 & \multirow[t]{2}{*}{$164: 16$} & Upper half & $<1,920$ & $<1,830$ & $<1,875$ \\
\hline S96T005033 & & Lower half & $<1,910$ & $<1,870$ & $<1,890$ \\
\hline S96T005038 & \multirow[t]{2}{*}{$164: 17$} & Upper half & $<1,930$ & $<1,900$ & $<1,915$ \\
\hline \$96T005031 & & Lower half & $<2,180$ & $<2,170$ & $<2,175$ \\
\hline S96T005039 & \multirow[t]{2}{*}{$164: 19$} & Upper half & $<1,940$ & $<2,000$ & $<1,970$ \\
\hline S96T005034 & & Lower half & $<2,140$ & $<2,170$ & $<2,155$ \\
\hline S96T005281 & \multirow[t]{2}{*}{$164: 20$} & Upper half & $<1,720$ & $<1,850$ & $<1,785$ \\
\hline S96T005278 & & Lower half & $<1,890$ & $<1,930$ & $<1,910$ \\
\hline
\end{tabular}


Table B2-9. Tank 241-AN-104 Analytical Results: Arsenic (ICP). (3 sheets)

\begin{tabular}{|c|c|c|c|c|c|}
\hline 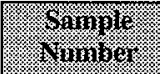 & oringer & 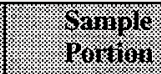 & 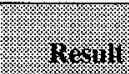 & 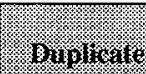 & mera \\
\hline 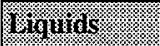 & & & F. & ( & /. \\
\hline S96T005256 & $163: 2$ & Liquid & $<60.1$ & $<60.1$ & $<60.1$ \\
\hline S96T005260 & $163: 4$ & Liquid & $<60.1$ & $<60.1$ & $<60.1$ \\
\hline S96T005527 & $163: 5$ & Liquid & $<60.1$ & $<60.1$ & $<60.1$ \\
\hline S96T005554 & $163: 6$ & Liquid & $<60.1$ & $<60.1$ & $<60.1$ \\
\hline S96T005255 & $163: 7$ & Liquid & $<60.1$ & $<60.1$ & $<60.1$ \\
\hline S96T005257 & $163: 8$ & Liquid & $<60.1$ & $<60.1$ & $<60.1$ \\
\hline S96T005258 & $163: 9$ & Liquid & $<60.1$ & $<60.1$ & $<60.1$ \\
\hline S96T005528 & $163: 10$ & Liquid & $<60.1$ & $<60.1$ & $<60.1$ \\
\hline$\$ 967005739$ & $163: 11$ & Liquid & $<60.1$ & $<60.1$ & $<60.1$ \\
\hline \$96T005766 & $163: 12$ & Liquid & $<60.1$ & $<60.1$ & $<60.1$ \\
\hline S96T005741 & $163: 14$ & Liquid & $<60.1$ & $<60.1$ & $<60.1$ \\
\hline S96T004774 & $164: 1$ & Liquid & $<120$ & $<120$ & $<120$ \\
\hline S96T004778 & $164: 2$ & Liquid & $<120$ & $<120$ & $<120$ \\
\hline S96T004779 & $164: 3$ & Liquid & $<120$ & $<120$ & $<120$ \\
\hline S96T004780 & $164: 4$ & Liquid & $<60.1$ & $<60.1$ & $<60.1$ \\
\hline S96T004976 & $164: 5$ & Liquid & $<60.1$ & $<60.1$ & $<60.1$ \\
\hline S96T004781 & $164: 7$ & Liquid & $<60.1$ & $<60.1$ & $<60.1$ \\
\hline S96T004977 & $164: 8$ & Liquid & $<60.1$ & $<60.1$ & $<60.1$ \\
\hline S96T004978 & $164: 9$ & Liquid & $<60.1$ & $<60.1$ & $<60.1$ \\
\hline S96T004979 & 164:10 & Liquid & $<60.1$ & $<60.1$ & $<60.1$ \\
\hline S96T004782 & $164: 11$ & Liquid & $<60.1$ & $<60.1$ & $<60.1$ \\
\hline S96T004783 & $164: 12$ & Liquid & $<60.1$ & $<60.1$ & $<60.1$ \\
\hline S96T004784 & $164: 13$ & Liquid & $<60.1$ & $<60.1$ & $<60.1$ \\
\hline S96T005979 & Core 164 & Composite & $<120$ & $<120$ & $<120$ \\
\hline
\end{tabular}


Table B2-10. Tank 241-AN-104 Analytical Results: Barium (ICP). (3 sheets)

\begin{tabular}{|c|c|c|c|c|c|}
\hline forion & Sompinger & 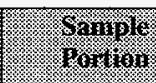 & (2) & 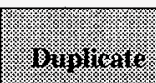 & (3) \\
\hline Sowdis rou & Hogs & & \% & (89) & (4) \\
\hline S96T005287 & $163: 1$ & Lower half & $<18.9$ & $<19.6$ & $<19.25$ \\
\hline S96T005288 & $163: 2$ & Lower half & $<22.2$ & $<18.7$ & $<20.45$ \\
\hline \$96T005782 & $163: 12$ & Lower half & $<22.8$ & $<23$ & $<22.9$ \\
\hline S96T005749 & \multirow[t]{2}{*}{$163: 14$} & Upper half & $<22.4$ & $<21.8$ & $<22.1$ \\
\hline S96T005750 & & Lower half & $<23$ & $<22.6$ & $<22.8$ \\
\hline S96T005294 & \multirow[t]{2}{*}{$163: 16$} & Upper half & $<20.6$ & $<18.5$ & $<19.55$ \\
\hline S96T005289 & & Lower half & $<23.2$ & $<21.3$ & $<22.25$ \\
\hline S96T005778 & \multirow[t]{2}{*}{$163: 18$} & Upper half & $<24.1$ & $<22.7$ & $<23.4$ \\
\hline \$96T005783 & & Lower half & $<23.7$ & $<23.6$ & $<23.65$ \\
\hline S96T005557 & \multirow[t]{2}{*}{$163: 20$} & Upper half & $<19$ & $<20.1$ & $<19.55$ \\
\hline S96T005560 & & Lower half & $<22.1$ & $<18.7$ & $<20.4$ \\
\hline S96T004797 & $164: 1$ & Lower half & $<23.4$ & $<23.5$ & $<23.45$ \\
\hline S96T004798 & $164: 13$ & Lower half & $<24.2$ & $<23.8$ & $<24$ \\
\hline S96T005042 & \multirow[t]{2}{*}{$164: 14$} & Upper half & $<23.7$ & $<24$ & $<23.85$ \\
\hline S96T005040 & & Lower half & $<23.9$ & $<23.5$ & $<23.7$ \\
\hline S96T005061 & \multirow[t]{2}{*}{$164: 15$} & Upper half & $<24.2$ & $<24$ & $<24.1$ \\
\hline S96T005043 & & Lower half & $<23.2$ & $<23.9$ & $<23.55$ \\
\hline S96T005062 & \multirow[t]{2}{*}{$164: 16$} & Upper half & $<75.3$ & $<74.7$ & $<75$ \\
\hline S96T005044 & & Lower half & $<73.7$ & $<75$ & $<74.35$ \\
\hline S96T005063 & \multirow[t]{2}{*}{$164: 17$} & Upper half & $<69.7$ & $<71.4$ & $<70.55$ \\
\hline \$96T005041 & & Lower half & $<76.2$ & $<75.6$ & $<75.9$ \\
\hline S96T005064 & \multirow[t]{2}{*}{$164: 19$} & Upper half & $<75.6$ & $<76.7$ & $<76.15$ \\
\hline S96T005045 & & Lower half & $<72.5$ & $<72$ & $<72.25$ \\
\hline S96T005282 & \multirow[t]{2}{*}{$164: 20$} & Upper half & $<19.7$ & $<18$ & $<18.85$ \\
\hline S96T005279 & & Lower half & $<22.7$ & $<18.9$ & $<20.8$ \\
\hline S96T005976 & Core 164 & Composite & $<19.9$ & $<19.9$ & $<19.9^{\mathrm{QC}: \mathrm{a}}$ \\
\hline
\end{tabular}


Table B2-10. Tank 241-AN-104 Analytical Results: Barium (ICP). (3 sheets)

\begin{tabular}{|c|c|c|c|c|c|}
\hline Hampl & (1) & Sognole & 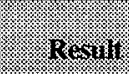 & 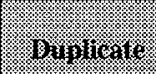 & \%or \\
\hline \multicolumn{2}{|c|}{ Soldas o finsiom } & & 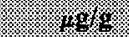 & 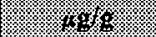 & \% \\
\hline S96T005284 & 163:1 & Lower half & $<937$ & $<936$ & $<936.5$ \\
\hline S96T005285 & $163: 2$ & Lower half & $<894$ & $<956$ & $<925$ \\
\hline S96T005780 & $163: 12$ & Lower half & $<940$ & $<950$ & $<945$ \\
\hline S96T005747 & \multirow[t]{2}{*}{$163: 14$} & Upper half & $<1,120$ & $<1,130$ & $<1,125$ \\
\hline S96T005748 & & Lower half & $<934$ & $<963$ & $<948.5$ \\
\hline S96T005293 & \multirow[t]{2}{*}{$163: 16$} & Upper half & $<887$ & $<949$ & $<918$ \\
\hline S96T005286 & & Lower half & $<993$ & $<917$ & $<955$ \\
\hline S96T005777 & \multirow[t]{2}{*}{ 163:18 } & Upper half & $<1,030$ & $<992$ & $<1,011$ \\
\hline S96T005781 & & Lower half & $<1,060$ & $<1,070$ & $<1,065$ \\
\hline S96T005556 & \multirow[t]{2}{*}{$163: 20$} & Upper half & $<924$ & $<968$ & $<946$ \\
\hline S96T005559 & & Lower half & $<977$ & $<973$ & $<975$ \\
\hline \$96T004795 & $164: 1$ & Lower half & $<1,020$ & $<1,000$ & $<1,010$ \\
\hline \$96T004796 & $164: 13$ & Lower half & $<1,030$ & $<1,040$ & $<1,035$ \\
\hline S96T005035 & \multirow[t]{2}{*}{$164: 14$} & Upper half & $<1,020$ & $<1,030$ & $<1,025$ \\
\hline S96T005030 & & Lower half & $<1,020$ & $<1,020$ & $<1,020$ \\
\hline S96T005036 & \multirow[t]{2}{*}{$164: 15$} & Upper half & $<1,000$ & $<983$ & $<991.5$ \\
\hline S96T005032 & & Lower half & $<987$ & $<1,000$ & $<993.5$ \\
\hline \$96T005037 & \multirow[t]{2}{*}{$164: 16$} & Upper half & $<961$ & $<915$ & $<938$ \\
\hline S96T005033 & & Lower half & $<957$ & $<933$ & $<945$ \\
\hline S96T005038 & \multirow[t]{2}{*}{$164: 17$} & Upper half & $<966$ & $<949$ & $<957.5$ \\
\hline S96T005031 & & Lower half & $<1,090$ & $<1,080$ & $<1,085$ \\
\hline S96T005039 & \multirow[t]{2}{*}{$164: 19$} & Upper half & $<972$ & $<1,000$ & $<986$ \\
\hline S96T005034 & & Lower half & $<1,070$ & $<1,090$ & $<1,080$ \\
\hline S96T005281 & \multirow[t]{2}{*}{$164: 20$} & Upper half & $<860$ & $<925$ & $<892.5$ \\
\hline S96T005278 & & Lower half & $<943$ & $<966$ & $<954.5$ \\
\hline
\end{tabular}


Table B2-10. Tank 241-AN-104 Analytical Results: Barium (ICP). (3 sheets)

\begin{tabular}{|c|c|c|c|c|c|}
\hline (1) & Whanger & 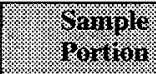 & $\mathrm{H}^{2} \mathrm{sin}$ & Biylimatc & How \\
\hline I) & & & \% & 4rim & 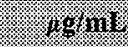 \\
\hline S96T005256 & $163: 2$ & Liquid & $<30.1$ & $<30.1$ & $<30.1$ \\
\hline S96T005260 & $163: 4$ & Liquid & $<30.1$ & $<30.1$ & $<30.1$ \\
\hline \$96T005527 & $163: 5$ & Liquid & $<30.1$ & $<30.1$ & $<30.1$ \\
\hline \$96T005554 & $163: 6$ & Liquid & $<30.1$ & $<30.1$ & $<30.1$ \\
\hline S96T005255 & $163: 7$ & Liquid & $<30.1$ & $<30.1$ & $<30.1$ \\
\hline S96T005257 & $163: 8$ & Liquid & $<30.1$ & $<30.1$ & $<30.1$ \\
\hline \$96T005258 & $163: 9$ & Liquid & $<30.1$ & $<30.1$ & $<30.1$ \\
\hline S96T005528 & $163: 10$ & Liquid & $<30.1$ & $<30.1$ & $<30.1$ \\
\hline S96T005739 & $163: 11$ & Liquid & $<30.1$ & $<30.1$ & $<30.1$ \\
\hline S96T005766 & $163: 12$ & Liquid & $<30.1$ & $<30.1$ & $<30.1$ \\
\hline S96T005741 & $163: 14$ & Liquid & $<30.1$ & $<30.1$ & $<30.1$ \\
\hline S96T004774 & $164: 1$ & Liquid & $<60.1$ & $<60.1$ & $<60.1$ \\
\hline S96T004778 & $164: 2$ & Liquid & $<60.1$ & $<60.1$ & $<60.1$ \\
\hline S96T004779 & $164: 3$ & Liquid & $<60.1$ & $<60.1$ & $<60.1$ \\
\hline \$96T004780 & $164: 4$ & Liquid & $<30.1$ & $<30.1$ & $<30.1$ \\
\hline \$96T004976 & $164: 5$ & Liquid & $<30.1$ & $<30.1$ & $<30.1$ \\
\hline S96T004781 & $164: 7$ & Liquid & $<30.1$ & $<30.1$ & $<30.1$ \\
\hline \$96T004977 & $164: 8$ & Liquid & $<30.1$ & $<30.1$ & $<30.1$ \\
\hline S96T004978 & $164: 9$ & Liquid & $<30.1$ & $<30.1$ & $<30.1$ \\
\hline S96T004979 & $164: 10$ & Liquid & $<30.1$ & $<30.1$ & $<30.1$ \\
\hline S96T004782 & $164: 11$ & Liquid & $<30.1$ & $<30.1$ & $<30.1$ \\
\hline S96T004783 & $164: 12$ & Liquid & $<30.1$ & $<30.1$ & $<30.1$ \\
\hline S96T004784 & $164: 13$ & Liquid & $<30.1$ & $<30.1$ & $<30.1$ \\
\hline \$96T005979 & Core 164 & Composite & $<60.1$ & $<60.1$ & $<60.1$ \\
\hline
\end{tabular}


Table B2-11. Tank 241-AN-104 Analytical Results: Beryllium (ICP). (3 sheets)

\begin{tabular}{|c|c|c|c|c|c|}
\hline 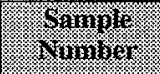 & $\begin{array}{l}\mathrm{l}_{\mathrm{f}} \\
\mathrm{f}\end{array}$ & \%on & Resimit & 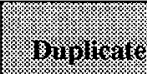 & yesu \\
\hline Woldis\% ach & 1968 & 12 & 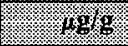 & 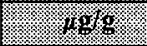 & 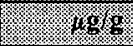 \\
\hline S96T005287 & $163: 1$ & Lower half & $<1.89$ & $<1.96$ & $<1.925$ \\
\hline S96T005288 & $163: 2$ & Lower half & $<2.22$ & $<1.87$ & $<2.045$ \\
\hline$\$ 96 \mathrm{~T} 005782$ & $163: 12$ & Lower half & $<2.28$ & $<2.3$ & $<2.29$ \\
\hline S96T005749 & \multirow[t]{2}{*}{$163: 14$} & Upper half & $<2.24$ & $<2.18$ & $<2.21$ \\
\hline S96T005750 & & Lower half & $<2.3$ & $<2.26$ & $<2.28$ \\
\hline \$96T005294 & \multirow[t]{2}{*}{$163: 16$} & Upper half & $<2.06$ & $<1.85$ & $<1.955$ \\
\hline S96T005289 & & Lower half & $<2.32$ & $<2.13$ & $<2.225$ \\
\hline S96T005778 & \multirow[t]{2}{*}{$163: 18$} & Upper half & $<2.41$ & $<2.27$ & $<2.34$ \\
\hline S96T005783 & & Lower half & $<2.37$ & $<2.36$ & $<2.365$ \\
\hline S96T005557 & \multirow[t]{2}{*}{$163: 20$} & Upper half & $<1.9$ & $<2.01$ & $<1.955$ \\
\hline S96T005560 & & Lower half & $<2.21$ & $<1.87$ & $<2.04$ \\
\hline S96T004797 & $164: 1$ & Lower half & $<2.34$ & $<2.35$ & $<2.345$ \\
\hline S96T004798 & $164: 13$ & Lower half & $<2.42$ & $<2.38$ & $<2.4$ \\
\hline S96T005042 & \multirow[t]{2}{*}{$164: 14$} & Upper half & $<2.37$ & $<2.4$ & $<2.385$ \\
\hline S96T005040 & & Lower half & $<2.39$ & $<2.35$ & $<2.37$ \\
\hline S96T005061 & \multirow[t]{2}{*}{$164: 15$} & Upper half & $<2.42$ & $<2.4$ & $<2.41$ \\
\hline S96T005043 & & Lower half & $<2.32$ & $<2.39$ & $<2.355$ \\
\hline S96T005062 & \multirow[t]{2}{*}{$164: 16$} & Upper half & $<7.53$ & $<7.47$ & $<7.5$ \\
\hline S96T005044 & & Lower half & $<7.37$ & $<7.5$ & $<7.435$ \\
\hline S96T005063 & \multirow[t]{2}{*}{$164: 17$} & Upper half & $<6.97$ & $<7.14$ & $<7.055$ \\
\hline$\$ 96 \mathrm{~T} 005041$ & & Lower half & $<7.62$ & $<7.56$ & $<7.59$ \\
\hline S96T005064 & \multirow[t]{2}{*}{$164: 19$} & Upper half & $<7.56$ & $<7.67$ & $<7.615$ \\
\hline S96T005045 & & Lower half & $<7.25$ & $<7.2$ & $<7.225$ \\
\hline S96T005282 & \multirow[t]{2}{*}{$164: 20$} & Upper half & $<1.97$ & $<1.8$ & $<1.885$ \\
\hline S96T005279 & & Lower half & $<2.27$ & $<1.89$ & $<2.08$ \\
\hline S96T005976 & Core 164 & Composite & $<1.99$ & $<1.99$ & $<1.99^{\mathrm{QC}: \mathrm{a}}$ \\
\hline
\end{tabular}


Table B2-11. Tank 241-AN-104 Analytical Results: Beryllium (ICP). (3 sheets)

\begin{tabular}{|c|c|c|c|c|c|}
\hline (1) & Whor & ( & (n. & 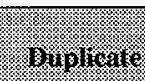 & (1) \\
\hline 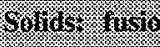 & & li & 18. & (1.6. & 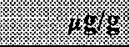 \\
\hline S96T005284 & $163: 1$ & Lower half & $<93.7$ & $<93.6$ & $<93.65$ \\
\hline S96T005285 & $163: 2$ & Lower half & $<89.4$ & $<95.6$ & $<92.5$ \\
\hline S96T005780 & $163: 12$ & Lower half & $<94$ & $<95$ & $<94.5$ \\
\hline S96T005747 & \multirow[t]{2}{*}{$163: 14$} & Upper half & $<112$ & $<113$ & $<112.5$ \\
\hline \$96T005748 & & Lower half & $<93.4$ & $<96.3$ & $<94.85$ \\
\hline S96T005293 & \multirow[t]{2}{*}{$163: 16$} & Upper half & $<88.7$ & $<94.9$ & $<91.8$ \\
\hline S96T005286 & & Lower half & $<99.3$ & $<91.7$ & $<95.5$ \\
\hline S96T005777 & \multirow[t]{2}{*}{$163: 18$} & Upper half & $<103$ & $<99.2$ & $<101.1$ \\
\hline S96T005781 & & Lower half & $<106$ & $<107$ & $<106.5$ \\
\hline S96T005556 & \multirow[t]{2}{*}{$163: 20$} & Upper half & $<92.4$ & $<96.8$ & $<94.6$ \\
\hline S96T005559 & & Lower half & $<97.7$ & $<97.3$ & $<97.5$ \\
\hline S96T004795 & $164: 1$ & Lower half & $<102$ & $<100$ & $<101$ \\
\hline S96T004796 & $164: 13$ & Lower half & $<103$ & $<104$ & $<103.5$ \\
\hline S96T005035 & \multirow[t]{2}{*}{$164: 14$} & Upper half & $<102$ & $<103$ & $<102.5$ \\
\hline S96T005030 & & Lower half & $<102$ & $<102$ & $<102$ \\
\hline S96T005036 & \multirow[t]{2}{*}{$164: 15$} & Upper half & $<100$ & $<98.3$ & $<99.15$ \\
\hline S96T005032 & & Lower half & $<98.7$ & $<100$ & $<99.35$ \\
\hline S96T005037 & \multirow[t]{2}{*}{$164: 16$} & Upper half & $<96.1$ & $<91.5$ & $<93.8$ \\
\hline S96T005033 & & Lower half & $<95.7$ & $<93.3$ & $<94.5$ \\
\hline S96T005038 & \multirow[t]{2}{*}{$164: 17$} & Upper half & $<96.6$ & $<94.9$ & $<95.75$ \\
\hline S96T005031 & & Lower half & $<109$ & $<108$ & $<108.5$ \\
\hline S96T005039 & \multirow[t]{2}{*}{$164: 19$} & Upper half & $<97.2$ & $<100$ & $<98.6$ \\
\hline \$96T005034 & & Lower half & $<107$ & $<109$ & $<108$ \\
\hline S96T005281 & \multirow[t]{2}{*}{$164: 20$} & Upper half & $<86$ & $<92.5$ & $<89.25$ \\
\hline \$96T005278 & & Lower half & $<94.3$ & $<96.6$ & $<95.45$ \\
\hline
\end{tabular}


Table B2-11. Tank 241-AN-104 Analytical Results: Beryllium (ICP). (3 sheets)

\begin{tabular}{|c|c|c|c|c|c|}
\hline VArinible & Garrole & 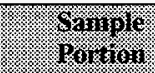 & (1) & $\frac{1}{1 .}$. & 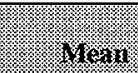 \\
\hline Gogings & & (3) & 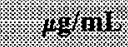 & \%gring & \%gring \\
\hline \$96T005256 & $163: 2$ & Liquid & $<3$ & $<3$ & $<3$ \\
\hline S96T005260 & $163: 4$ & Liquid & $<3$ & $<3$ & $<3$ \\
\hline S96T005527 & $163: 5$ & Liquid & $<3$ & $<3$ & $<3$ \\
\hline S96T005554 & $163: 6$ & Liquid & $<3$ & $<3$ & $<3$ \\
\hline S96T005255 & $163: 7$ & Liquid & $<3$ & $<3$ & $<3$ \\
\hline \$96T005257 & $163: 8$ & Liquid & $<3$ & $<3$ & $<3$ \\
\hline S96T005258 & $163: 9$ & Liquid & $<3$ & $<3$ & $<3$ \\
\hline S96T005528 & $163: 10$ & Liquid & $<3$ & $<3$ & $<3$ \\
\hline S96T005739 & $163: 11$ & Liquid & $<3$ & $<3$ & $<3$ \\
\hline S96T005766 & $163: 12$ & Liquid & $<3$ & $<3$ & $<3$ \\
\hline S96T005741 & $163: 14$ & Liquid & $<3$ & $<3$ & $<3$ \\
\hline S96T004774 & 164:1 & Liquid & $<6$ & $<6$ & $<6$ \\
\hline S96T004778 & $164: 2$ & Liquid & $<6$ & $<6$ & $<6$ \\
\hline S96T004779 & $164: 3$ & Liquid & $<6$ & $<6$ & $<6$ \\
\hline S96T004780 & $164: 4$ & Liquid & $<3$ & $<3$ & $<3$ \\
\hline S96T004976 & $164: 5$ & Liquid & $<3$ & $<3$ & $<3$ \\
\hline S96T004781 & $164: 7$ & Liquid & $<3$ & $<3$ & $<3$ \\
\hline S96T004977 & $164: 8$ & Liquid & $<3$ & $<3$ & $<3$ \\
\hline S96T004978 & $164: 9$ & Liquid & $<3$ & $<3$ & $<3$ \\
\hline S96T004979 & $164: 10$ & Liquid & $<3$ & $<3$ & $<3$ \\
\hline S96T004782 & $164: 11$ & Liquid & $<3$ & $<3$ & $<3$ \\
\hline S96T004783 & $164: 12$ & Liquid & $<3$ & $<3$ & $<3$ \\
\hline S96T004784 & $164: 13$ & Liquid & $<3$ & $<3$ & $<3$ \\
\hline S96T005979 & Core 164 & Composite & $<6$ & $<6$ & $<6$ \\
\hline
\end{tabular}


Table B2-12. Tank 241-AN-104 Analytical Results: Bismuth (ICP). (3 sheets)

\begin{tabular}{|c|c|c|c|c|c|}
\hline (1) & (8) & ornore & rew1il & Bumlicale & $19 \%$ \\
\hline Whot 1 ato & Hex. & (1) & 12.7. & $17 \%$ & \% \\
\hline S96T005287 & 163:1 & Lower half & $<37.9$ & $<39.3$ & $<38.6$ \\
\hline S96T005288 & $163: 2$ & Lower half & $<44.3$ & $<37.5$ & $<40.9$ \\
\hline S96T005782 & $163: 12$ & Lower half & $<45.7$ & $<46.1$ & $<45.9$ \\
\hline S96T005749 & \multirow[t]{2}{*}{$163: 14$} & Upper half & $<44.8$ & $<43.6$ & $<44.2$ \\
\hline S96T005750 & & Lower half & $<45.9$ & $<45.2$ & $<45.55$ \\
\hline S96T005294 & \multirow[t]{2}{*}{$163: 16$} & Upper half & $<41.3$ & $<37$ & $<39.15$ \\
\hline S96T005289 & & Lower half & $<46.4$ & $<42.6$ & $<44.5$ \\
\hline S96T005778 & \multirow[t]{2}{*}{$163: 18$} & Upper half & $<48.1$ & $<45.4$ & $<46.75$ \\
\hline S96T005783 & & Lower half & $<47.4$ & $<47.2$ & $<47.3$ \\
\hline S96T005557 & \multirow[t]{2}{*}{$163: 20$} & Upper half & $<38$ & $<40.1$ & $<39.05$ \\
\hline S96T005560 & & Lower half & $<44.2$ & $<37.3$ & $<40.75$ \\
\hline S96T004797 & 164:1 & Lower half & $<46.9$ & $<47.1$ & $<47$ \\
\hline S96T004798 & $164: 13$ & Lower half & $<48.4$ & $<47.7$ & $<48.05$ \\
\hline S96T005042 & \multirow[t]{2}{*}{$164: 14$} & Upper half & $<47.4$ & $<48$ & $<47.7$ \\
\hline \$96T005040 & & Lower half & $<47.7$ & $<47$ & $<47.35$ \\
\hline S96T005061 & \multirow[t]{2}{*}{$164: 15$} & Upper half & $<48.3$ & $<48$ & $<48.15$ \\
\hline S96T005043 & & Lower half & $<46.4$ & $<47.7$ & $<47.05$ \\
\hline$\$ 96 \mathrm{~T} 005062$ & \multirow[t]{2}{*}{$164: 16$} & Upper half & $<151$ & $<149$ & $<150$ \\
\hline S96T005044 & & Lower half & $<147$ & $<150$ & $<148.5$ \\
\hline S96T005063 & \multirow[t]{2}{*}{$164: 17$} & Upper half & $<139$ & $<143$ & $<141$ \\
\hline S96T005041 & & Lower half & $<152$ & $<151$ & $<151.5$ \\
\hline \$96T005064 & \multirow[t]{2}{*}{$164: 19$} & Upper half & $<151$ & $<153$ & $<152$ \\
\hline S96T005045 & & Lower half & $<145$ & $<144$ & $<144.5$ \\
\hline S96T005282 & \multirow[t]{2}{*}{$164: 20$} & Upper half & $<39.3$ & $<36.1$ & $<37.7$ \\
\hline S96T005279 & & Lower half & $<45.4$ & $<37.8$ & $<41.6$ \\
\hline S96T005976 & Core 164 & Composite & $<39.8$ & $<39.8$ & $<39.8^{\mathrm{QC}: \mathrm{a}}$ \\
\hline
\end{tabular}


Table B2-12. Tank 241-AN-104 Analytical Results: Bismuth (ICP). (3 sheets)

\begin{tabular}{|c|c|c|c|c|c|}
\hline 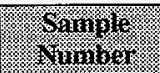 & Symple & 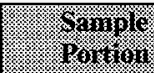 & Pravill & Bullitale & ingan \\
\hline \multicolumn{2}{|c|}{ 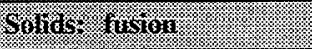 } & \% & yso & (1) & $4.96 \%$ \\
\hline \$96T005284 & $163: 1$ & Lower half & $<1,870$ & $<1,870$ & $<1,870$ \\
\hline S96T005285 & $163: 2$ & Lower half & $<1,790$ & $<1,910$ & $<1,850$ \\
\hline S96T005780 & $163: 12$ & Lower half & $<1,880$ & $<1,900$ & $<1,890$ \\
\hline S96T005747 & \multirow[t]{2}{*}{$163: 14$} & Upper half & $<2,240$ & $<2,250$ & $<2,245$ \\
\hline \$96T005748 & & Lower half & $<1,870$ & $<1,930$ & $<1,900$ \\
\hline S96T005293 & \multirow[t]{2}{*}{$163: 16$} & Upper half & $<1,770$ & $<1,900$ & $<1,835$ \\
\hline S96T005286 & & Lower half & $<1,990$ & $<1,830$ & $<1,910$ \\
\hline S96T005777 & \multirow[t]{2}{*}{$163: 18$} & Upper half & $<2,050$ & $<1,980$ & $<2,015$ \\
\hline S96T005781 & & Lower half & $<2,130$ & $<2,140$ & $<2,135$ \\
\hline S96T005556 & \multirow[t]{2}{*}{$163: 20$} & Upper half & $<1,850$ & $<1,940$ & $<1,895$ \\
\hline S96T005559 & & Lower half & $<1,950$ & $<1,950$ & $<1,950$ \\
\hline \$96T004795 & $164: 1$ & Lower half & $<2,040$ & $<2,010$ & $<2,025$ \\
\hline \$96T004796 & $164: 13$ & Lower half & $<2,060$ & $<2,070$ & $<2,065$ \\
\hline$\$ 96 \mathrm{~T} 005035$ & \multirow[t]{2}{*}{$164: 14$} & Upper half & $<2,040$ & $<2,060$ & $<2,050$ \\
\hline S96T005030 & & Lower half & $<2,050$ & $<2,040$ & $<2,045$ \\
\hline S96T005036 & \multirow[t]{2}{*}{$164: 15$} & Upper half & $<2,010$ & $<1,970$ & $<1,990$ \\
\hline$\$ 96 T 005032$ & & Lower half & $<1,970$ & $<2,000$ & $<1,985$ \\
\hline \$96T005037 & \multirow[t]{2}{*}{$164: 16$} & Upper half & $<1,920$ & $<1,830$ & $<1,875$ \\
\hline$\$ 96 T 005033$ & & Lower half & $<1,910$ & $<1,870$ & $<1,890$ \\
\hline S96T005038 & \multirow[t]{2}{*}{$164: 17$} & Upper half & $<1,930$ & $<1,900$ & $<1,915$ \\
\hline S96T005031 & & Lower half & $<2,180$ & $<2,170$ & $<2,175$ \\
\hline S96T005039 & \multirow[t]{2}{*}{$164: 19$} & Upper half & $<1,940$ & $<2,000$ & $<1,970$ \\
\hline S96T005034 & & Lower half & $<2,140$ & $<2,170$ & $<2,155$ \\
\hline S96T005281 & \multirow[t]{2}{*}{$164: 20$} & Upper half & $<1,720$ & $<1,850$ & $<1,785$ \\
\hline S96T005278 & & Lower half & $<1,890$ & $<1,930$ & $<1,910$ \\
\hline
\end{tabular}




\begin{tabular}{|c|c|c|c|c|c|}
\hline $0 Z I>$ & $0 Z I>$ & OZI $>$ & tItsoduro & $\$ 91$ ग100 & $6 \angle 6 \mathrm{~S} 00 \mathrm{~L} 96 \mathrm{~S}$ \\
\hline$I \cdot 09>$ & I.09> & {$[\cdot 09>$} & p!nb!ฺ & $\varepsilon I: \neq 9 I$ & $+8 L+00 \mathrm{~L} 96 \mathrm{~S}$ \\
\hline [.09> & $1.09>$ & $I_{0}^{\circ} 09>$ & pịb!̣ & $2 I: t 9 I$ & $\varepsilon 8 \angle t 00 \mathrm{~L} 96 \mathrm{~S}$ \\
\hline$[.09>$ & {$[\cdot 09>$} & $I^{\circ} 09>$ & pinb! & {$[I:+9 I$} & $z 8 L+00 \mathrm{~L} 96 \mathrm{~S}$ \\
\hline I.09> & {$[.09>$} & $I \cdot 09>$ & p!nb!̣ & OI:t9I & $6 \angle 6+00 \mathrm{~L} 96 \mathrm{~S}$ \\
\hline$[\cdot 09>$ & {$[.09>$} & {$[\cdot 09>$} & p!nb!̣ & $6: 79 I$ & $8 \angle 6+00 . \mathrm{L} 96 \mathrm{~S}$ \\
\hline$I^{\circ} 09>$ & $109>$ & I.09> & prnbriา & $8:+9 \mathrm{I}$ & LL6+00L96S \\
\hline $1.09>$ & I.09> & I.09> & pInb!a & $L:+9 I$ & I8Lt00L96S \\
\hline $1.09>$ & I.09> & {$[.09>$} & prnbryx & $s: t 9 I$ & $9 \angle 6+00 \mathrm{~L} 96 \mathrm{~S}$ \\
\hline$[\cdot 09>$ & {$[\cdot 09>$} & {$[\cdot 09>$} & p!̣b!x & $p: t 9 \mathrm{I}$ & $08 \angle+00 \perp 96 \mathrm{~S}$ \\
\hline $0 Z I>$ & OZI $>$ & $0 Z I>$ & p!nbrit & $\varepsilon:+9 I$ & 6LLt00L96S \\
\hline $0 Z I>$ & $0 Z I>$ & OZI $>$ & pı̣nb!x & $z:+9 I$ & $8 L L+00 L 96 S$ \\
\hline $0 Z I>$ & OZI> & $0 Z I>$ & p!̣b!̣ & $I:+9 I$ & $\nabla L L+00 L 96 \mathrm{~S}$ \\
\hline I.09> & $109>$ & I.09> & p!̣nb!̣ & $\nabla I: \varepsilon 9[$ & $I+\angle S 00 L 96 \mathrm{~S}$ \\
\hline$[\cdot 09>$ & I.09> & I.09> & p!̣nb!̣ & 乙I:६9І & $99 \angle S 00 \mathrm{~L} 96 \mathrm{~S}$ \\
\hline I.09> & $\mathrm{I} \cdot 09>$ & I.09> & p!̣b!̣ & I I:६9I & $6 \mathcal{L} \angle S 00 \mathrm{~L} 96 \mathrm{~S}$ \\
\hline$[\cdot 09>$ & $1 \cdot 09>$ & $109>$ & p!̣nb!x & $0 I: \varepsilon 9 \mathrm{I}$ & $8 z \varsigma 500 \mathrm{~L} 96 \mathrm{~S}$ \\
\hline $1 \cdot 09>$ & I.09> & $109>$ & pinbry & $6: \varepsilon 9 \mathrm{I}$ & $8 \mathrm{SZS00L96S}$ \\
\hline$I \cdot 09>$ & $I \cdot 09>$ & I.09> & prnbry & $8: \varepsilon 9 \mathrm{I}$ & LSZS00J96S \\
\hline$[\cdot 09>$ & I'09> & I.09> & p!̣b!t & $L: E 9 I$ & SSZS00L96S \\
\hline$[\cdot 09>$ & {$[\cdot 09>$} & I.09> & p!̣b!̣T & $9: \varepsilon 9$ I & $\nabla \varsigma \varsigma \varsigma 00 \mathrm{~L} 96 \mathrm{~S}$ \\
\hline I.09> & I.09> & I.09> & prnbbr] & $\varsigma: \varepsilon 9 \mathrm{I}$ & LZSS00X96S \\
\hline$[09>$ & I'09> & {$[09>$} & p!nb! & $\nabla: \varepsilon 9 \mathrm{I}$ & $092500 \mathrm{~L} 96 \mathrm{~S}$ \\
\hline$I 09>$ & {$[\cdot 09>$} & I.09> & p!̣b!ฺ & $\tau: \varepsilon 9 \mathrm{I}$ & $9 \Im 2500 \mathrm{~L} 96 \mathrm{~S}$ \\
\hline 110.8\% & $1010 \%$ & 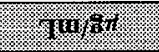 & & & 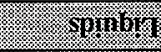 \\
\hline $17+54$ & 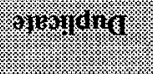 & Intord & moliod & rograros & a \\
\hline
\end{tabular}

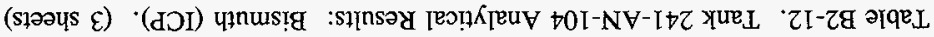


Table B2-13. Tank 241-AN-104 Analytical Results: Boron (ICP). (3 sheets)

\begin{tabular}{|c|c|c|c|c|c|}
\hline Mininer & \%orming & (3) & hentis & 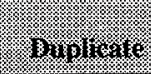 & 19 ext \\
\hline \multicolumn{2}{|c|}{ Solids } & 原 & $\%$ & fing & $\mathrm{H}_{\mathrm{k}}$ \\
\hline S96T005287 & $163: 1$ & Lower half & 155 & 171 & 163 \\
\hline S96T005288 & $163: 2$ & Lower half & 200 & 182 & 191 \\
\hline S96T005782 & $163: 12$ & Lower half & 109 & 95.8 & 102.4 \\
\hline S96T005749 & \multirow[t]{2}{*}{$163: 14$} & Upper half & 86.2 & 89.4 & 87.8 \\
\hline S96T005750 & & Lower half & 104 & 97.3 & 100.65 \\
\hline S96T005294 & \multirow[t]{2}{*}{$163: 16$} & Upper half & 164 & 145 & 154.5 \\
\hline S96T005289 & & Lower half & 217 & 166 & $191.5^{\mathrm{QC}: \mathrm{c}}$ \\
\hline S96T005778 & \multirow[t]{2}{*}{ 163:18 } & Upper half & 124 & 104 & 114 \\
\hline S96T005783 & & Lower half & 95.8 & 85 & 90.4 \\
\hline \$96T005557 & \multirow[t]{2}{*}{$163: 20$} & Upper half & 167 & 167 & 167 \\
\hline S96T005560 & & Lower half & 168 & 156 & 162 \\
\hline S96T004797 & $164: 1$ & Lower half & 146 & 120 & 133 \\
\hline S96T004798 & $164: 13$ & Lower half & 153 & 126 & 139.5 \\
\hline S96T005042 & \multirow[t]{2}{*}{$164: 14$} & Upper half & 119 & 133 & 126 \\
\hline S96T005040 & & Lower half & 119 & 134 & 126.5 \\
\hline S96T005061 & \multirow[t]{2}{*}{$164: 15$} & Upper half & 158 & 128 & $143^{\text {QC:c }}$ \\
\hline S96T005043 & & Lower half & 147 & 127 & 137 \\
\hline S96T005062 & \multirow[t]{2}{*}{$164: 16$} & Upper half & 141 & 114 & $127.5^{\mathrm{QC}: \mathrm{c}}$ \\
\hline S96T005044 & & Lower half & 136 & 115 & 125.5 \\
\hline S96T005063 & \multirow[t]{2}{*}{$164: 17$} & Upper half & 142 & 121 & 131.5 \\
\hline \$96T005041 & & Lower half & 126 & 122 & 124 \\
\hline S96T005064 & \multirow[t]{2}{*}{$164: 19$} & Upper half & 147 & 133 & 140 \\
\hline S96T005045 & & Lower half & 120 & 131 & 125.5 \\
\hline S96T005282 & \multirow[t]{2}{*}{$164: 20$} & Upper half & 170 & 160 & 165 \\
\hline \begin{tabular}{|l|} 
S96T005279 \\
\end{tabular} & & Lower half & 176 & 185 & 180.5 \\
\hline S96T005976 & Core 164 & Composite & 133 & 158 & 145.5 \\
\hline
\end{tabular}


Table B2-13. Tank 241-AN-104 Analytical Results: Boron (ICP). (3 sheets)

\begin{tabular}{|c|c|c|c|c|c|}
\hline 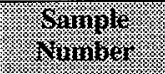 & tom & Promplo & (Resin & 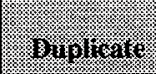 & 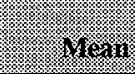 \\
\hline \multicolumn{2}{|l|}{ 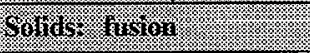 } & & 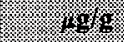 & Was & \% \\
\hline S96T005284 & $163: 1$ & Lower half & $<937$ & $<936$ & $<936.5$ \\
\hline S96T005285 & $163: 2$ & Lower half & $<894$ & $<956$ & $<925$ \\
\hline S96T005780 & $163: 12$ & Lower half & $<940$ & $<950$ & $<945$ \\
\hline S96T005747 & \multirow[t]{2}{*}{$163: 14$} & Upper half & $<1,120$ & $<1,130$ & $<1,125$ \\
\hline S96T005748 & & Lower half & $<934$ & $<963$ & $<948.5$ \\
\hline S96T005293 & \multirow[t]{2}{*}{$163: 16$} & Upper half & $<887$ & $<949$ & $<918$ \\
\hline S96T005286 & & Lower half & $<993$ & $<917$ & $<955$ \\
\hline S96T005777 & \multirow[t]{2}{*}{$163: 18$} & Upper half & $<1,030$ & $<992$ & $<1,011$ \\
\hline$\$ 96 \mathrm{~T} 005781$ & & Lower half & $<1,060$ & $<1,070$ & $<1,065$ \\
\hline S96T005556 & \multirow[t]{2}{*}{$163: 20$} & Upper half & $<924$ & $<968$ & $<946$ \\
\hline S96T005559 & & Lower half & $<977$ & $<973$ & $<975$ \\
\hline S96T004795 & $164: 1$ & Lower half & $<1,020$ & $<1,000$ & $<1,010$ \\
\hline S96T004796 & $164: 13$ & Lower half & $<1,030$ & $<1,040$ & $<1,035$ \\
\hline S96T005035 & \multirow[t]{2}{*}{$164: 14$} & Upper half & $<1,020$ & $<1,030$ & $<1,025$ \\
\hline S96T005030 & & Lower half & $<1,020$ & $<1,020$ & $<1,020$ \\
\hline$\$ 96 \mathrm{~T} 005036$ & \multirow[t]{2}{*}{$164: 15$} & Upper half & $<1,000$ & $<983$ & $<991.5$ \\
\hline S96T005032 & & Lower half & $<987$ & $<1,000$ & $<993.5$ \\
\hline \$96T005037 & \multirow[t]{2}{*}{$164: 16$} & Upper half & $<961$ & $<915$ & $<938$ \\
\hline S96T005033 & & Lower half & $<957$ & $<933$ & $<945$ \\
\hline S96T005038 & \multirow[t]{2}{*}{$164: 17$} & Upper half & $<966$ & $<949$ & $<957.5$ \\
\hline S96T005031 & & Lower half & $<1,090$ & $<1,080$ & $<1,085$ \\
\hline S96T005039 & \multirow[t]{2}{*}{$164: 19$} & Upper half & $<972$ & $<1,000$ & $<986$ \\
\hline S96T005034 & & Lower half & $<1,070$ & $<1,090$ & $<1,080$ \\
\hline S96T005281 & \multirow[t]{2}{*}{$164: 20$} & Upper half & $<860$ & $<925$ & $<892.5$ \\
\hline \$96T005278 & & Lower half & $<943$ & $<966$ & $<954.5$ \\
\hline
\end{tabular}


Table B2-13. Tank 241-AN-104 Analytical Results: Boron (ICP). (3 sheets)

\begin{tabular}{|c|c|c|c|c|c|}
\hline $\begin{array}{l}\text { Somple } \\
\text { vinulier }\end{array}$ & Sange & $\begin{array}{l}\text { Gamingle } \\
\text { hortion }\end{array}$ & Resin: & 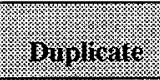 & mean \\
\hline Coginas: & 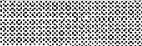 & (3) & 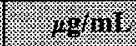 & (n) & (3) \\
\hline S96T005256 & $163: 2$ & Liquid & 65.8 & 68.6 & 67.2 \\
\hline S96T005260 & $163: 4$ & Liquid & 70.1 & 63.4 & 66.75 \\
\hline S96T005527 & $163: 5$ & Liquid & 61.2 & 58.2 & 59.7 \\
\hline S96T005554 & $163: 6$ & Liquid & 64 & 61.9 & 62.95 \\
\hline S96T005255 & $163: 7$ & Liquid & 67 & 71.3 & 69.15 \\
\hline S96T005257 & $163: 8$ & Liquid & 66.1 & 66.1 & 66.1 \\
\hline S96T005258 & $163: 9$ & Liquid & 67 & 65.2 & 66.1 \\
\hline S96T005528 & $163: 10$ & Liquid & 60.9 & 63.4 & 62.15 \\
\hline S96T005739 & $163: 11$ & Liquid & 63.7 & 60.9 & 62.3 \\
\hline S96T005766 & $163: 12$ & Liquid & 59.1 & 64 & 61.55 \\
\hline S96T005741 & $163: 14$ & Liquid & 64 & 62.8 & 63.4 \\
\hline S96T004774 & $164: 1$ & Liquid & 67.8 & 67.9 & 67.85 \\
\hline S96T004778 & $164: 2$ & Liquid & $<60.1$ & 65.1 & $<62.6$ \\
\hline S96T004779 & $164: 3$ & Liquid & 68.6 & 64.2 & 66.4 \\
\hline S96T004780 & $164: 4$ & Liquid & 65.4 & 65.1 & 65.25 \\
\hline S96T004976 & $164: 5$ & Liquid & 67.7 & 65.7 & 66.7 \\
\hline S96T004781 & $164: 7$ & Liquid & 65.4 & 67.1 & 66.25 \\
\hline S96T004977 & $164: 8$ & Liquid & 70.1 & 62.2 & 66.15 \\
\hline S96T004978 & $164: 9$ & Liquid & 68 & 55.2 & $61.6^{\mathrm{QC:c}}$ \\
\hline S96T004979 & $164: 10$ & Liquid & 64.8 & 63.6 & 64.2 \\
\hline S96T004782 & $164: 11$ & Liquid & 63.9 & 66.2 & 65.05 \\
\hline S96T004783 & $164: 12$ & Liquid & 67.1 & 69.2 & 68.15 \\
\hline S96T004784 & $164: 13$ & Liquid & 61.3 & 65.9 & 63.6 \\
\hline S96T005979 & Core 164 & Composite & $<60.1$ & $<60.1$ & $<60.1$ \\
\hline
\end{tabular}


HNF-SD-WM-ER-690 Rev. 0

Table B2-14. Tank 241-AN-104 Analytical Results: Cadmium (ICP). (3 sheets)

\begin{tabular}{|c|c|c|c|c|c|}
\hline $\begin{array}{l}\text { sample } \\
\text { Thillo: }\end{array}$ & Sirnple & U Saraple: & rasurll: & 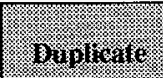 & 119.2) \\
\hline \multicolumn{2}{|c|}{ 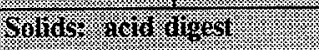 } & (5) & $102 / 8$ & (2. & fly \\
\hline S96T005287 & $163: 1$ & Lower half & 7.21 & 7.39 & 7.3 \\
\hline S96T005288 & $163: 2$ & Lower half & 3.61 & 4.27 & 3.94 \\
\hline \$96T005782 & $163: 12$ & Lower half & 8.01 & 7.97 & 7.99 \\
\hline S96T005749 & \multirow[t]{2}{*}{$163: 14$} & Upper half & 7.14 & 7.06 & 7.1 \\
\hline S96T005750 & & Lower half & 8.41 & 7.92 & 8.165 \\
\hline \$96T005294 & \multirow[t]{2}{*}{$163: 16$} & Upper half & 14.7 & 11.3 & $13^{\mathrm{QC:c}}$ \\
\hline S96T005289 & & Lower half & 12.6 & 9.98 & $11.29^{\mathrm{QC:c}}$ \\
\hline S96T005778 & \multirow[t]{2}{*}{$163: 18$} & Upper half & 6.47 & 7.24 & 6.855 \\
\hline S96T005783 & & Lower half & 6.13 & 6.48 & 6.305 \\
\hline S96T005557 & \multirow[t]{2}{*}{$163: 20$} & Upper half & 10.6 & 10.5 & 10.55 \\
\hline S96T005560 & & Lower half & 11 & 9.74 & 10.37 \\
\hline S96T004797 & $164: 1$ & Lower half & 4.34 & 4.69 & 4.515 \\
\hline S96T004798 & $164: 13$ & Lower half & 24 & 25.5 & 24.75 \\
\hline S96T005042 & \multirow[t]{2}{*}{$164: 14$} & Upper half & 11.7 & 11.8 & 11.75 \\
\hline S96T005040 & & Lower half & 6.52 & 6.52 & 6.52 \\
\hline S96T005061 & \multirow[t]{2}{*}{$164: 15$} & Upper half & 9.28 & 8.68 & 8.98 \\
\hline S96T005043 & & Lower half & 15.2 & 15.1 & 15.15 \\
\hline S96T005062 & \multirow[t]{2}{*}{$164: 16$} & Upper half & $<7.53$ & $<7.47$ & $<7.5$ \\
\hline S96T005044 & & Lower half & $<7.37$ & 7.87 & $<7.62$ \\
\hline S96T005063 & \multirow[t]{2}{*}{$164: 17$} & Upper half & 9.61 & 8.82 & 9.215 \\
\hline S96T005041 & & Lower half & 13.6 & 11.8 & 12.7 \\
\hline S96T005064 & \multirow[t]{2}{*}{$164: 19$} & Upper half & 9.64 & 9.6 & 9.62 \\
\hline S96T005045 & & Lower half & 11 & 11.6 & 11.3 \\
\hline S96T005282 & \multirow[t]{2}{*}{$164: 20$} & Upper half & 15.6 & 12.4 & $14^{\mathrm{QC}: \mathrm{c}}$ \\
\hline S96T005279 & & Lower half & 12.3 & 7.83 & $10.065^{\mathrm{QC:c}}$ \\
\hline S96T005976 & Core 164 & Composite & 13.2 & 12.6 & $12.9^{\mathrm{QC:A}}$ \\
\hline
\end{tabular}




\begin{tabular}{|c|c|c|c|c|c|}
\hline$s+56>$ & $9^{\circ} 96>$ & $\varepsilon \neq 6>$ & ¥еч Іәмо & & 8LZS00L96S \\
\hline$s z \cdot 68>$ & $s \cdot 26>$ & $98>$ & ғГеч 工әdd $_{\Omega}$ & $07: \bullet 9 \mathrm{I}$ & I8ZS00L96S \\
\hline $80 I>$ & $60 I>$ & $\angle O I>$ & fley IәMOT & \multirow[b]{2}{*}{$6 I:+9 I$} & $\downarrow \varepsilon 0 \subseteq 00 \mathrm{~L} 96 \mathrm{~S}$ \\
\hline $9.86>$ & $00 \mathrm{I}>$ & $\tau: L 6>$ & fleY Iəddn & & $680500 \mathrm{~L} 96 \mathrm{~S}$ \\
\hline$S: 80 I>$ & $80 \mathrm{I}>$ & $60 \mathrm{I}>$ & Jleप IәмоT & \multirow[b]{2}{*}{$\angle I: \$ 9 I$} & IEOSO0L96S \\
\hline$S L S 6>$ & $6 \div 6>$ & $9 \cdot 96>$ & I[Eप Iədd $\Omega$ & & $8 \mathcal{E} 0500 \mathrm{~L} 96 \mathrm{~S}$ \\
\hline$\varsigma^{\prime}+6>$ & $\varepsilon \cdot \varepsilon 6>$ & $L S 6>$ & J[एч دәмот] & \multirow[b]{2}{*}{$9 I: \neq 9 I$} & EEOSO0I96S \\
\hline $8^{\circ} \mathcal{E} 6>$ & $\xi_{16>}$ & $I .96>$ & $\pm[E 4$ rədd $\Omega$ & & LEOSOOL96S \\
\hline $\mathcal{S E} 66>$ & $00 \mathrm{I}>$ & $\angle 86>$ & flеч Іәмот & \multirow[b]{2}{*}{$S I:+9 I$} & zE0S00L96S \\
\hline SI $66>$ & $\varepsilon \cdot 86>$ & $00 I>$ & ક્રе .radd $\cap$ & & $9 \mathcal{E} 0 S 00 \mathrm{~L} 96 \mathrm{~S}$ \\
\hline$z 01>$ & $20 \mathrm{I}>$ & $201>$ & IIЕบ Iәмот & \multirow[b]{2}{*}{$\nabla I: t 9 I$} & $0 \varepsilon 0 \$ 00 \mathrm{~L} 96 \mathrm{~S}$ \\
\hline$\varsigma 20 \mathrm{I}>$ & $\varepsilon 0 I>$ & $201>$ & Ifएप Iədd $\cap$ & & SE0\$00L.96S \\
\hline $\mathcal{S}^{\prime} \mathcal{E} O I>$ & t0I $>$ & $\varepsilon 0 I>$ & IโЕบ IәMOT & $\varepsilon I: \neq 9 I$ & $96 \angle 700 L 96 \mathrm{~S}$ \\
\hline IOI $>$ & $00 \mathrm{I}>$ & $201>$ & J[Еप IOMOT & I:t9I & S6Lt00L96S \\
\hline$S \angle 6>$ & $\varepsilon \angle 6>$ & $\angle L L 6>$ & Iтеч Іәмоา & \multirow[b]{2}{*}{ OZ:E9I } & 6S\$\$00L96S \\
\hline $9 \div 6>$ & $8 \cdot 96>$ & $\nabla \cdot 26>$ & IleY Iədd $\Omega$ & & 9ऽ\$S00L96S \\
\hline$S^{\prime} 90 I>$ & $\angle O I>$ & $90 \mathrm{I}>$ & Ј[ец Іәмот] & \multirow[b]{2}{*}{$8 I: \varepsilon 9 T$} & I8LS00L96S \\
\hline $\mathrm{I} \cdot \mathrm{IOI}>$ & $\tau \cdot 66>$ & $\varepsilon 0 \mathrm{I}>$ & fle्प . Iədd $\cap$ & & LLLS00L96S \\
\hline$\varsigma^{\prime} \varsigma_{6}>$ & $L^{\prime} \mathrm{I} 6>$ & $\varepsilon \cdot 66>$ & ғ[еч Іәмот] & \multirow[b]{2}{*}{$9[: \varepsilon 9 I$} & 98Z500L96S \\
\hline $8 \cdot 16>$ & $6 \cdot 76>$ & $L 88>$ & I[eप xədd $_{\bigcap}$ & & E6ZS00L96S \\
\hline$\varsigma 8^{\circ}+6>$ & $\varepsilon 96>$ & $\nabla^{\circ} \varepsilon 6>$ & IFеप хәмот & \multirow[b]{2}{*}{$\downarrow[: \varepsilon 9 I$} & $87 L S 00 L 96 \mathrm{~S}$ \\
\hline$S^{*}$ III $>$ & $\varepsilon I I>$ & ZII $>$ & I[एч ıədd $\Omega$ & & $\angle D L S 00 L 96 \mathrm{~S}$ \\
\hline$s+6>$ & $\$ 6>$ & $\nabla 6>$ & IlЕप IәмоT & $\tau I: \varepsilon 9 I$ & 08LS00L96S \\
\hline$s^{2} 26>$ & $9^{\circ} 56>$ & $t^{*} 68>$ & I[Eप IәMOT & $\tau: \varepsilon 9 \mathrm{I}$ & S82S00.L96S \\
\hline$\$ 9^{\circ} \mathcal{E} 6>$ & $9^{\cdot} \varepsilon 6>$ & $L \cdot \mathcal{E} 6>$ & I[ЕЧ Іәмо:] & $\mathrm{I}: \varepsilon 9 \mathrm{I}$ & t8ZS00L $96 \mathrm{~S}$ \\
\hline \%) & 6 & (3) & & \multicolumn{2}{|r|}{ 104513. } \\
\hline 196 & 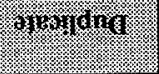 & 1114 & 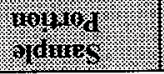 & 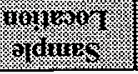 & fartings \\
\hline
\end{tabular}

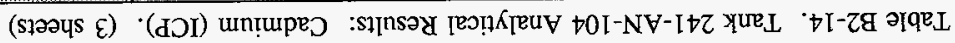


Table B2-14. Tank 241-AN-104 Analytical Results: Cadmium (ICP). (3 sheets)

\begin{tabular}{|c|c|c|c|c|c|}
\hline $\begin{array}{l}\text { Sinivle } \\
\text { Numily }\end{array}$ & $\begin{array}{l}\text { Sarnole } \\
\text { Uvaritio }\end{array}$ & $\begin{array}{l}\text { Sample } \\
\text { Portion }\end{array}$ & I. & Buplucarc & inesu \\
\hline Miguins & & & I. & . $138 \mathrm{~g} / \mathrm{mil}$ & g. \\
\hline S96T005256 & $163: 2$ & Liquid & $<3$ & $<3$ & $<3$ \\
\hline S96T005260 & $163: 4$ & Liquid & $<3$ & $<3$ & $<3$ \\
\hline S96T005527 & $163: 5$ & Liquid & $<3$ & $<3$ & $<3$ \\
\hline S96T005554 & $163: 6$ & Liquid & $<3$ & $<3$ & $<3$ \\
\hline S96T005255 & $163: 7$ & Liquid & $<3$ & $<3$ & $<3$ \\
\hline S96T005257 & $163: 8$ & Liquid & $<3$ & $<3$ & $<3$ \\
\hline S96T005258 & $163: 9$ & Liquid & $<3$ & $<3$ & $<3$ \\
\hline S96T005528 & $163: 10$ & Liquid & $<3$ & $<3$ & $<3$ \\
\hline S96T005739 & $163: 11$ & Liquid & $<3$ & $<3$ & $<3$ \\
\hline S96T005766 & $163: 12$ & Liquid & $<3$ & $<3$ & $<3$ \\
\hline S96T005741 & $163: 14$ & Liquid & $<3$ & $<3$ & $<3$ \\
\hline S96T004774 & $164: 1$ & Liquid & $<6$ & $<6$ & $<6$ \\
\hline S96T004778 & $164: 2$ & Liquid & $<6$ & $<6$ & $<6$ \\
\hline S96T004779 & $164: 3$ & Liquid & $<6$ & $<6$ & $<6$ \\
\hline S96T004780 & $164: 4$ & Liquid & $<3$ & $<3$ & $<3$ \\
\hline S96T004976 & $164: 5$ & Liquid & $<3$ & $<3$ & $<3$ \\
\hline S96T004781 & $164: 7$ & Liquid & $<3$ & $<3$ & $<3$ \\
\hline S96T004977 & $164: 8$ & Liquid & $<3$ & $<3$ & $<3$ \\
\hline S96T004978 & $164: 9$ & Liquid & $<3$ & $<3$ & $<3$ \\
\hline S96T004979 & $164: 10$ & Liquid & $<3$ & $<3$ & $<3$ \\
\hline S96T004782 & $164: 11$ & Liquid & $<3$ & $<3$ & $<3$ \\
\hline S96T004783 & $164: 12$ & Liquid & $<3$ & $<3$ & $<3$ \\
\hline S96T004784 & $164: 13$ & Liquid & $<3$ & $<3$ & $<3$ \\
\hline S96T005979 & Core 164 & Composite & $<6$ & $<6$ & $<6$ \\
\hline
\end{tabular}


Table B2-15. Tank 241-AN-104 Analytical Results: Calcium (ICP) (3 sheets)

\begin{tabular}{|c|c|c|c|c|c|}
\hline 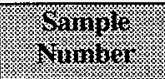 & \% & and & resur & 3) & Matil \\
\hline \multicolumn{2}{|c|}{ 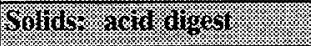 } & (10. & 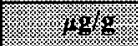 & (3) & (6) \\
\hline S96T005287 & $163: 1$ & Lower half & 170 & 161 & 165.5 \\
\hline S96T005288 & $163: 2$ & Lower half & 255 & 101 & $178^{\mathrm{QC:e}}$ \\
\hline S96T005782 & $163: 12$ & Lower half & 133 & 125 & 129 \\
\hline S96T005749 & \multirow[t]{2}{*}{$163: 14$} & Upper half & 133 & 135 & 134 \\
\hline S96T005750 & & Lower half & 151 & 146 & 148.5 \\
\hline S96T005294 & \multirow[t]{2}{*}{$163: 16$} & Upper half & 257 & 218 & 237.5 \\
\hline S96T005289 & & Lower half & 244 & 197 & $220.5^{\mathrm{QC}: \mathrm{c}}$ \\
\hline S96T005778 & \multirow[t]{2}{*}{$163: 18$} & Upper half & 271 & 273 & 272 \\
\hline S96T005783 & & Lower half & 288 & 286 . & 287 \\
\hline S96T005557 & \multirow[t]{2}{*}{$163: 20$} & Upper half & 359 & 343 & 351 \\
\hline S96T005560 & & Lower half & 296 & 244 & 270 \\
\hline S96T004797 & 164:1 & Lower half & 143 & 132 & 137.5 \\
\hline S96T004798 & $164: 13$ & Lower half & 278 & 288 & 283 \\
\hline S96T005042 & \multirow[t]{2}{*}{$164: 14$} & Upper half & 188 & 197 & 192.5 \\
\hline S96T005040 & & Lower half & 165 & 149 & 157 \\
\hline S96T005061 & \multirow[t]{2}{*}{$164: 15$} & Upper half & 170 & 179 & 174.5 \\
\hline S96T005043 & & Lower half & 238 & 230 & 234 \\
\hline S96T005062 & \multirow[t]{2}{*}{$164: 16$} & Upper half & $<151$ & 166 & $<158.5$ \\
\hline S96T005044 & & Lower half & 172 & 161 & 166.5 \\
\hline S96T005063 & \multirow[t]{2}{*}{$164: 17$} & Upper half & 162 & 181 & 171.5 \\
\hline S96T005041 & & Lower half & 217 & 205 & 211 \\
\hline S96T005064 & \multirow[t]{2}{*}{$164: 19$} & Upper half & 224 & 263 & 243.5 \\
\hline S96T005045 & & Lower half & 178 & 204 & 191 \\
\hline S96T005282 & \multirow[t]{2}{*}{$164: 20$} & Upper half & 499 & 286 & $392.5^{\mathrm{QC}: \mathrm{e}}$ \\
\hline \begin{tabular}{|l|} 
S96T005279 \\
\end{tabular} & & Lower half & 385 & 329 & 357 \\
\hline S96T005976 & Core 164 & Composite & 269 & 218 & $243.5^{\mathrm{QC}: a, \mathrm{e}}$ \\
\hline
\end{tabular}


Table B2-15. Tank 241-AN-104 Analytical Results: Caicium (ICP) (3 sheets)

\begin{tabular}{|c|c|c|c|c|c|}
\hline Gample & waing & $\begin{array}{l}\text { 9. } \\
\text { 16) }\end{array}$ & 7. & 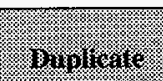 & 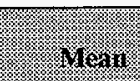 \\
\hline \multicolumn{2}{|c|}{ 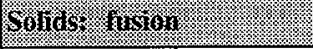 } & 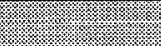 & 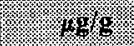 & 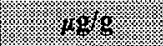 & j/. \\
\hline S96T005284 & $163: 1$ & Lower half & $<1,870$ & $<1,870$ & $<1,870$ \\
\hline S96T005285 & $163: 2$ & Lower half & $<1,790$ & $<1,910$ & $<1,850$ \\
\hline S96T005780 & $163: 12$ & Lower half & $<1,880$ & $<1,900$ & $<1,890$ \\
\hline S96T005747 & \multirow[t]{2}{*}{$163: 14$} & Upper half & $<2,240$ & $<2,250$ & $<2,245$ \\
\hline \$96T005748 & & Lower half & $<1,870$ & $<1,930$ & $<1,900$ \\
\hline S96T005293 & \multirow[t]{2}{*}{$163: 16$} & Upper half & $<1,770$ & $<1,900$ & $<1,835$ \\
\hline S96T005286 & & Lower half & $<1,990$ & $<1,830$ & $<1,910$ \\
\hline S96T005777 & \multirow[t]{2}{*}{$163: 18$} & Upper half & $<2,050$ & $<1,980$ & $<2,015$ \\
\hline S96T005781 & & Lower half & $<2,130$ & $<2,140$ & $<2,135$ \\
\hline S96T005556 & \multirow[t]{2}{*}{$163: 20$} & Upper half & $<1,850$ & $<1,940$ & $<1,895$ \\
\hline S96T005559 & & Lower half & $<1,950$ & $<1,950$ & $<1,950$ \\
\hline \$96T004795 & $164: 1$ & Lower half & $<2,040$ & $<2,010$ & $<2,025$ \\
\hline \$96T004796 & $164: 13$ & Lower half & $<2,060$ & $<2,070$ & $<2,065$ \\
\hline S96T005035 & \multirow[t]{2}{*}{$164: 14$} & Upper half & $<2,040$ & $<2,060$ & $<2,050$ \\
\hline \$96T005030 & & Lower half & $<2,050$ & $<2,040$ & $<2,045$ \\
\hline S96T005036 & \multirow[t]{2}{*}{$164: 15$} & Upper half & $<2,010$ & $<1,970$ & $<1,990$ \\
\hline S96T005032 & & Lower half & $<1,970$ & $<2,000$ & $<1,985$ \\
\hline S96T005037 & \multirow[t]{2}{*}{$164: 16$} & Upper half & $<1,920$ & $<1,830$ & $<1,875$ \\
\hline S96T005033 & & Lower half & $<1,910$ & $<1,870$ & $<1,890$ \\
\hline S96T005038 & \multirow[t]{2}{*}{$164: 17$} & Upper half & $<1,930$ & $<1,900$ & $<1,915$ \\
\hline S96T005031 & & Lower half & $<2,180$ & $<2,170$ & $<2,175$ \\
\hline S96T005039 & \multirow[t]{2}{*}{$164: 19$} & Upper half & $<1,940$ & $<2,000$ & $<1,970$ \\
\hline S96T005034 & & Lower half & $<2,140$ & $<2,170$ & $<2,155$ \\
\hline S96T005281 & \multirow[t]{2}{*}{$164: 20$} & Upper half & $<1,720$ & $<1,850$ & $<1,785$ \\
\hline S96T005278 & & Lower half & $<1,890$ & $<1,930$ & $<1,910$ \\
\hline
\end{tabular}


Table B2-15. Tank 241-AN-104 Analytical Results: Calcium (ICP) (3 sheets)

\begin{tabular}{|c|c|c|c|c|c|}
\hline 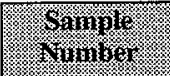 & 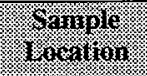 & $f_{1}$ & 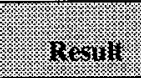 & Surplicate & (1911) \\
\hline 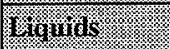 & (6. & & 49.0101 & $(49)$ & (3) \\
\hline S96T005256 & $163: 2$ & Liquid & $<60.1$ & $<60.1$ & $<60.1$ \\
\hline S96T005260 & $163: 4$ & Liquid & $<60.1$ & $<60.1$ & $<60.1$ \\
\hline S96T005527 & $163: 5$ & Liquid & $<60.1$ & $<60.1$ & $<60.1$ \\
\hline S96T005554 & $163: 6$ & Liquid & $<60.1$ & $<60.1$ & $<60.1$ \\
\hline S96T005255 & $163: 7$ & Liquid & $<60.1$ & $<60.1$ & $<60.1$ \\
\hline S96T005257 & $163: 8$ & Liquid & $<60.1$ & $<60.1$ & $<60.1$ \\
\hline S96T005258 & $163: 9$ & Liquid & $<60.1$ & $<60.1$ & $<60.1$ \\
\hline S96T005528 & $163: 10$ & Liquid & $<60.1$ & $<60.1$ & $<60.1$ \\
\hline S96T005739 & $163: 11$ & Liquid & $<60.1$ & $<60.1$ & $<60.1$ \\
\hline S96T005766 & $163: 12$ & Liquid & $<60.1$ & $<60.1$ & $<60.1$ \\
\hline S96T005741 & $163: 14$ & Liquid & $<60.1$ & $<60.1$ & $<60.1$ \\
\hline S96T004774 & $164: 1$ & Liquid & $<120$ & $<120$ & $<120$ \\
\hline S96T004778 & $164: 2$ & Liquid & $<120$ & $<120$ & $<120$ \\
\hline S96T004779 & $164: 3$ & Liquid & $<120$ & $<120$ & $<120$ \\
\hline S96T004780 & $164: 4$ & Liquid & $<60.1$ & $<60.1$ & $<60.1$ \\
\hline S96T004976 & $164: 5$ & Liquid & $<60.1$ & $<60.1$ & $<60.1$ \\
\hline S96T004781 & $164: 7$ & Liquid & $<60.1$ & $<60.1$ & $<60.1$ \\
\hline S96T004977 & $164: 8$ & Liquid & $<60.1$ & $<60.1$ & $<60.1$ \\
\hline S96T004978 & $164: 9$ & Liquid & $<60.1$ & $<60.1$ & $<60.1$ \\
\hline S96T004979 & $164: 10$ & Liquid & $<60.1$ & $<60.1$ & $<60.1$ \\
\hline S96T004782 & $164: 11$ & Liquid & $<60.1$ & $<60.1$ & $<60.1$ \\
\hline S96T004783 & $164: 12$ & Liquid & $<60.1$ & $<60.1$ & $<60.1$ \\
\hline S96T004784 & $164: 13$ & Liquid & $<60.1$ & $<60.1$ & $<60.1$ \\
\hline S96T005979 & Core 164 & Composite & $<120$ & $<120$ & $<120$ \\
\hline
\end{tabular}


Table B2-16. Tank 241-AN-104 Analytical Results: Cerium (ICP). (3 sheets)

\begin{tabular}{|c|c|c|c|c|c|}
\hline 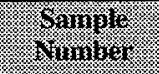 & S Simple: & $\begin{array}{l}\text { Somple } \\
\text { Porrtor }\end{array}$ & R-will: & Bupligrie: & (3) \\
\hline \multicolumn{2}{|c|}{ 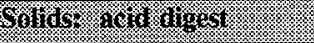 } & 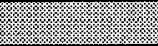 & 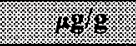 & 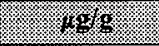 & 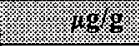 \\
\hline S96T005287 & $163: 1$ & Lower half & $<37.9$ & $<39.3$ & $<38.6$ \\
\hline S96T005288 & $163: 2$ & Lower half & $<44.3$ & $<37.5$ & $<40.9$ \\
\hline S96T005782 & $163: 12$ & Lower half & $<45.7$ & $<46.1$ & $<45.9$ \\
\hline S96T005749 & \multirow[t]{2}{*}{$163: 14$} & Upper half & $<44.8$ & $<43.6$ & $<44.2$ \\
\hline S96T005750 & & Lower half & $<45.9$ & $<45.2$ & $<45.55$ \\
\hline S96T005294 & \multirow[t]{2}{*}{$163: 16$} & Upper half & $<41.3$ & $<37$ & $<39.15$ \\
\hline S96T005289 & & Lower half & $<46.4$ & $<42.6$ & $<44.5$ \\
\hline S96T005778 & \multirow[t]{2}{*}{$163: 18$} & Upper half & $<48.1$ & $<45.4$ & $<46.75$ \\
\hline S96T005783 & & Lower half & $<47.4$ & $<47.2$ & $<47.3$ \\
\hline S96T005557 & \multirow[t]{2}{*}{$163: 20$} & Upper half & $<38$ & $<40.1$ & $<39.05$ \\
\hline S96T005560 & & Lower half & $<44.2$ & $<37.3$ & $<40.75$ \\
\hline S96T004797 & $164: 1$ & Lower half & $<46.9$ & $<47.1$ & $<47$ \\
\hline S96T004798 & $164: 13$ & Lower half & $<48.4$ & $<47.7$ & $<48.05$ \\
\hline S96T005042 & \multirow[t]{2}{*}{$164: 14$} & Upper half & $<47.4$ & $<48$ & $<47.7$ \\
\hline S96T005040 & & Lower half & $<47.7$ & $<47$ & $<47.35$ \\
\hline S96T005061 & \multirow[t]{2}{*}{$164: 15$} & Upper half & $<48.3$ & $<48$ & $<48.15$ \\
\hline S96T005043 & & Lower half & $<46.4$ & $<47.7$ & $<47.05$ \\
\hline S96T005062 & \multirow[t]{2}{*}{$164: 16$} & Upper half & $<151$ & $<149$ & $<150$ \\
\hline S96T005044 & & Lower half & $<147$ & $<150$ & $<148.5$ \\
\hline S96T005063 & \multirow[t]{2}{*}{$164: 17$} & Upper half & $<139$ & $<143$ & $<141$ \\
\hline S96T005041 & & Lower half & $<152$ & $<151$ & $<151.5$ \\
\hline S96T005064 & \multirow[t]{2}{*}{$164: 19$} & Upper half & $<151$ & $<153$ & $<152$ \\
\hline S96T005045 & & Lower half & $<145$ & $<144$ & $<144.5$ \\
\hline S96T005282 & \multirow[t]{2}{*}{$164: 20$} & Upper half & $<39.3$ & $<36.1$ & $<37.7$ \\
\hline S96T005279 & & Lower half & $<45.4$ & $<37.8$ & $<41.6$ \\
\hline S96T005976 & Core 164 & Composite & $<39.8$ & $<39.8$ & $<39.8^{\mathrm{QC:a}}$ \\
\hline
\end{tabular}


Table B2-16. Tank 241-AN-104 Analytical Results: Cerium (ICP). (3 sheets)

\begin{tabular}{|c|c|c|c|c|c|}
\hline $\begin{array}{l}\text { Sample } \\
\text { Auminici }\end{array}$ & $\begin{array}{l}\text { Sample: } \\
\text { Inaritio }\end{array}$ & $\begin{array}{l}\text { Sampic } \\
\text { Portion }\end{array}$ & Resnil & Dinicate & Wrean \\
\hline Solide nosiso & (3: & 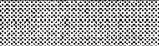 & 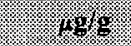 & 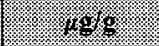 & 1898 \\
\hline S96T005284 & $163: 1$ & Lower half & $<1,870$ & $<1,870$ & $<1,870$ \\
\hline S96T005285 & $163: 2$ & Lower half & $<1,790$ & $<1,910$ & $<1,850$ \\
\hline S96T005780 & $163: 12$ & Lower half & $<1,880$ & $<1,900$ & $<1,890$ \\
\hline S96T005747 & \multirow[t]{2}{*}{$163: 14$} & Upper half & $<2,240$ & $<2,250$ & $<2,245$ \\
\hline S96T005748 & & Lower half & $<1,870$ & $<1,930$ & $<1,900$ \\
\hline S96T005293 & \multirow[t]{2}{*}{$163: 16$} & Upper half & $<1,770$ & $<1,900$ & $<1,835$ \\
\hline S96T005286 & & Lower half & $<1,990$ & $<1,830$ & $<1,910$ \\
\hline S96T005777 & \multirow[t]{2}{*}{$163: 18$} & Upper half & $<2,050$ & $<1,980$ & $<2,015$ \\
\hline S96T005781 & & Lower half & $<2,130$ & $<2,140$ & $<2,135$ \\
\hline S96T005556 & \multirow[t]{2}{*}{$163: 20$} & Upper half & $<1,850$ & $<1,940$ & $<1,895$ \\
\hline S96T005559 & & Lower half & $<1,950$ & $<1,950$ & $<1,950$ \\
\hline S96T004795 & $164: 1$ & Lower half & $<2,040$ & $<2,010$ & $<2,025$ \\
\hline S96T004796 & $164: 13$ & Lower half & $<2,060$ & $<2,070$ & $<2,065$ \\
\hline S96T005035 & \multirow[t]{2}{*}{$164: 14$} & Upper half & $<2,040$ & $<2,060$ & $<2,050$ \\
\hline S96T005030 & & Lower half & $<2,050$ & $<2,040$ & $<2,045$ \\
\hline S96T005036 & \multirow[t]{2}{*}{$164: 15$} & Upper half & $<2,010$ & $<1,970$ & $<1,990$ \\
\hline S96T005032 & & Lower half & $<1,970$ & $<2,000$ & $<1,985$ \\
\hline S96T005037 & \multirow[t]{2}{*}{$164: 16$} & Upper half & $<1,920$ & $<1,830$ & $<1,875$ \\
\hline S96T005033 & & Lower half & $<1,910$ & $<1,870$ & $<1,890$ \\
\hline S96T005038 & \multirow[t]{2}{*}{$164: 17$} & Upper half & $<1,930$ & $<1,900$ & $<1,915$ \\
\hline S96T005031 & & Lower half & $<2,180$ & $<2,170$ & $<2,175$ \\
\hline S96T005039 & \multirow[t]{2}{*}{$164: 19$} & Upper half & $<1,940$ & $<2,000$ & $<1,970$ \\
\hline S96T005034 & & Lower half & $<2,140$ & $<2,170$ & $<2,155$ \\
\hline S96T005281 & \multirow[t]{2}{*}{$164: 20$} & Upper half & $<1,720$ & $<1,850$ & $<1,785$ \\
\hline S96T005278 & & Lower half & $<1,890$ & $<1,930$ & $<1,910$ \\
\hline
\end{tabular}


Table B2-16. Tank 241-AN-104 Analytical Results: Cerium (ICP). (3 sheets)

\begin{tabular}{|c|c|c|c|c|c|}
\hline $\begin{array}{l}\text { Sample } \\
\text { Aliniber }\end{array}$ & $\begin{array}{l}\text { Sampice } \\
\text { I encriton }\end{array}$ & $\begin{array}{l}\text { Sariplo } \\
\text { Iortion }\end{array}$ & Resull & Buplicatre & Mera: \\
\hline Fainis & 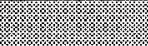 & & 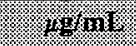 & 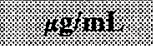 & 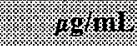 \\
\hline S96T005256 & $163: 2$ & Liquid & $<60.1$ & $<60.1$ & $<60.1$ \\
\hline S96T005260 & $163: 4$ & Liquid & $<60.1$ & $<60.1$ & $<60.1$ \\
\hline S96T005527 & $163: 5$ & Liquid & $<60.1$ & $<60.1$ & $<60.1$ \\
\hline S96T005554 & $163: 6$ & Liquid & $<60.1$ & $<60.1$ & $<60.1$ \\
\hline S96T005255 & $163: 7$ & Liquid & $<60.1$ & $<60.1$ & $<60.1$ \\
\hline S96T005257 & $163: 8$ & Liquid & $<60.1$ & $<60.1$ & $<60.1$ \\
\hline S96T005258 & $163: 9$ & Liquid & $<60.1$ & $<60.1$ & $<60.1$ \\
\hline S96T005528 & $163: 10$ & Liquid & $<60.1$ & $<60.1$ & $<60.1$ \\
\hline S96T005739 & $163: 11$ & Liquid & $<60.1$ & $<60.1$ & $<60.1$ \\
\hline S96T005766 & $163: 12$ & Liquid & $<60.1$ & $<60.1$ & $<60.1$ \\
\hline S96T005741 & $163: 14$ & Liquid & $<60.1$ & $<60.1$ & $<60.1$ \\
\hline S96T004774 & $164: 1$ & Liquid & $<120$ & $<120$ & $<120$ \\
\hline S96T004778 & $164: 2$ & Liquid & $<120$ & $<120$ & $<120$ \\
\hline S96T004779 & $164: 3$ & Liquid & $<120$ & $<120$ & $<120$ \\
\hline S96T004780 & $164: 4$ & Liquid & $<60.1$ & $<60.1$ & $<60.1$ \\
\hline S96T004976 & $164: 5$ & Liquid & $<60.1$ & $<60.1$ & $<60.1$ \\
\hline S96T004781 & $164: 7$ & Liquid & $<60.1$ & $<60.1$ & $<60.1$ \\
\hline S96T004977 & $164: 8$ & Liquid & $<60.1$ & $<60.1$ & $<60.1$ \\
\hline S96T004978 & $164: 9$ & Liquid & $<60.1$ & $<60.1$ & $<60.1$ \\
\hline S96T004979 & $164: 10$ & Liquid & $<60.1$ & $<60.1$ & $<60.1$ \\
\hline S96T004782 & $164: 11$ & Liquid & $<60.1$ & $<60.1$ & $<60.1$ \\
\hline S96T004783 & $164: 12$ & Liquid & $<60.1$ & $<60.1$ & $<60.1$ \\
\hline S96T004784 & $164: 13$ & Liquid & $<60.1$ & $<60.1$ & $<60.1$ \\
\hline S96T005979 & Core 164 & Composite & $<120$ & $<120$ & $<120$ \\
\hline
\end{tabular}


Table B2-17. Tank 241-AN-104 Analytical Results: Chromium (ICP). (3 sheets)

\begin{tabular}{|c|c|c|c|c|c|}
\hline 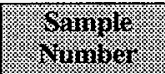 & Grimple & 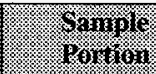 & (6) & 3.1.01464 & 44 \\
\hline \multicolumn{2}{|c|}{ 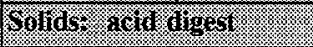 } & & f. & 1. & $\mathrm{n}_{2}$ \\
\hline S96T005287 & $163: 1$ & Lower half & 1,200 & 1,150 & 1,175 \\
\hline S96T005288 & $163: 2$ & Lower half & 728 & 716 & 722 \\
\hline S96T005782 & $163: 12$ & Lower half & 1,220 & 1,180 & 1,200 \\
\hline S96T005749 & \multirow[t]{2}{*}{$163: 14$} & Upper half & 1,050 & 1,080 & 1,065 \\
\hline S96T005750 & & Lower half & 1,260 & 1,240 & 1,250 \\
\hline S96T005294 & \multirow[t]{2}{*}{$163: 16$} & Upper half & 2,270 & 1,860 & 2,065 \\
\hline S96T005289 & & Lower half & 1,970 & 1,630 & 1,800 \\
\hline S96T005778 & \multirow[t]{2}{*}{$163: 18$} & Upper half & 987 & 1,010 & 998.5 \\
\hline S96T005783 & & Lower half & 1,020 & 1,010 & 1,015 \\
\hline S96T005557 & \multirow[t]{2}{*}{$163: 20$} & Upper half & 1,470 & 1,450 & 1,460 \\
\hline S96T005560 & & Lower half & 1,390 & 1,120 & $1,255^{\mathrm{QC}: \mathrm{c}, \mathrm{c}}$ \\
\hline S96T004797 & $164: 1$ & Lower half & 778 & 783 & 780.5 \\
\hline S96T004798 & $164: 13$ & Lower half & 3,490 & 3,680 & 3,585 \\
\hline S96T005042 & \multirow[t]{2}{*}{$164: 14$} & Upper half & 1,790 & 1,770 & 1,780 \\
\hline S96T005040 & & Lower half & 1,040 & 1,040 & 1,040 \\
\hline S96T005061 & \multirow[t]{2}{*}{$164: 15$} & Upper half & 1,360 & 1,390 & 1,375 \\
\hline S96T005043 & & Lower half & 2,340 & 2,260 & 2,300 \\
\hline S96T005062 & \multirow[t]{2}{*}{$164: 16$} & Upper half & 1,240 & 1,370 & 1,305 \\
\hline S96T005044 & & Lower half & 1,310 & 1,290 & 1,300 \\
\hline S96T005063 & \multirow[t]{2}{*}{$164: 17$} & Upper half & 1,490 & 1,620 & 1,555 \\
\hline S96T005041 & & Lower half & 2,120 & 2,150 & 2,135 \\
\hline S96T005064 & \multirow[t]{2}{*}{$164: 19$} & Upper half & 2,030 & 2,090 & 2,060 \\
\hline S96T005045 & & Lower half & 1,860 & 2,120 & 1,990 \\
\hline S96T005282 & \multirow[t]{2}{*}{$164: 20$} & Upper half & 2,380 & 2,000 & 2,190 \\
\hline S96T005279 & & Lower half & 1,770 & 1,170 & $1,470^{\mathrm{QC}: \mathrm{c}}$ \\
\hline S96T005976 & Core 164 & Composite & 2,100 & 1,890 & $1,995^{\mathrm{QC:a}}$ \\
\hline
\end{tabular}


Table B2-17. Tank 241-AN-104 Analytical Results: Chromium (ICP). (3 sheets)

\begin{tabular}{|c|c|c|c|c|c|}
\hline 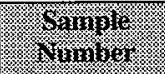 & Sample & 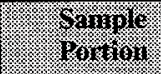 & (2) & B & $\sqrt{1}+12$ \\
\hline \multicolumn{2}{|l|}{ Sridas. Gistisi: } & & 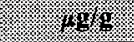 & 68 & 17840 \\
\hline S96T005284 & $163: 1$ & Lower half & 1,440 & 1,270 & 1,355 \\
\hline S96T005285 & $163: 2$ & Lower half & 683 & 606 & 644.5 \\
\hline S96T005780 & $163: 12$ & Lower half & 1,250 & 1,220 & 1,235 \\
\hline \$96T005747 & \multirow[t]{2}{*}{$163: 14$} & Upper half & 1,210 & 1,070 & 1,140 \\
\hline \$96T005748 & & Lower half & 1,480 & 1,220 & 1,350 \\
\hline \$96T005293 & \multirow[t]{2}{*}{$163: 16$} & Upper half & 2,240 & 2,180 & 2,210 \\
\hline S96T005286 & & Lower half & 1,610 & 1,770 & 1,690 \\
\hline S96T005777 & \multirow[t]{2}{*}{$163: 18$} & Upper half & 1,370 & 1,110 & $1,240^{\mathrm{QC}: 0}$ \\
\hline S96T005781 & & Lower half & 1,220 & 1,310 & 1,265 \\
\hline S96T005556 & \multirow[t]{2}{*}{$163: 20$} & Upper half & 3,040 & 1,380 & $2,210^{\mathrm{Qc}: \mathrm{c}}$ \\
\hline \begin{tabular}{|l} 
S96T005559 \\
ST
\end{tabular} & & Lower half & 1,100 & 3,010 & $2,055^{\mathrm{QC}: \mathrm{c}}$ \\
\hline S96T004795 & $164: 1$ & Lower half & 1,870 & 1,780 & 1,825 \\
\hline S96T004796 & $164: 13$ & Lower half & 3,590 & 3,580 & 3,585 \\
\hline S96T005035 & \multirow[t]{2}{*}{$164: 14$} & Upper half & 827 & 776 & 801.5 \\
\hline S96T005030 & & Lower half & 1,020 & 998 & 1,009 \\
\hline S96T005036 & \multirow[t]{2}{*}{$164: 15$} & Upper half & 1,360 & 1,490 & 1,425 \\
\hline S96T005032 & & Lower half & 2,440 & 2,420 & 2,430 \\
\hline S96T005037 & \multirow[t]{2}{*}{$164: 16$} & Upper half & 1,400 & 1,240 & 1,320 \\
\hline S96T005033 & & Lower half & 1,250 & 1,310 & 1,280 \\
\hline S96T005038 & \multirow[t]{2}{*}{$164: 17$} & Upper half & 1,350 & 1,470 & 1,410 \\
\hline S96T005031 & & Lower half & 2,050 & 2,040 & 2,045 \\
\hline S96T005039 & \multirow[t]{2}{*}{$164: 19$} & Upper half & 1,980 & 1,910 & 1,945 \\
\hline S96T005034 & & Lower half & 1,860 & 1,880 & 1,870 \\
\hline S96T005281 & \multirow[t]{2}{*}{$164: 20$} & Upper half & 1,410 & 1,140 & $1,275^{\mathrm{QC}: c}$ \\
\hline S96T005278 & & Lower half & 1,850 & 1,800 & 1,825 \\
\hline
\end{tabular}


Table B2-17. Tank 241-AN-104 Analytical Results: Chromium (ICP). (3 sheets)

\begin{tabular}{|c|c|c|c|c|c|}
\hline Samilite & Saringle & $\begin{array}{l}\text { Sampe } \\
\text { 100rion }\end{array}$ & Trevill: & 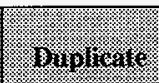 & 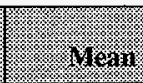 \\
\hline Mrintis: & & צ & $14 \mathrm{~g} / \mathrm{mi}$ & I) & 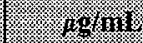 \\
\hline S96T005256 & $163: 2$ & Liquid & 332 & 359 & 345.5 \\
\hline S96T005260 & $163: 4$ & Liquid & 348 & 328 & 338 \\
\hline S96T005527 & $163: 5$ & Liquid & 326 & 321 & 323.5 \\
\hline S96T005554 & $163: 6$ & Liquid & 337 & 336 & 336.5 \\
\hline S96T005255 & $163: 7$ & Liquid & 358 & 377 & 367.5 \\
\hline S96T005257 & $163: 8$ & Liquid & 317 & 327 & 322 \\
\hline S96T005258 & $163: 9$ & Liquid & 341 & 327 & 334 \\
\hline S96T005528 & $163: 10$ & Liquid & 308 & 315 & 311.5 \\
\hline S96T005739 & $163: 11$ & Liquid & 324 & 323 & 323.5 \\
\hline S96T005766 & $163: 12$ & Liquid & 358 & 354 & 356 \\
\hline S96T005741 & $163: 14$ & Liquid & 344 & 343 & 343.5 \\
\hline S96T004774 & $164: 1$ & Liquid & 345 & 349 & 347 \\
\hline S96T004778 & $164: 2$ & Liquid & 291 & 340 & 315.5 \\
\hline S96T004779 & $164: 3$ & Liquid & 341 & 330 & 335.5 \\
\hline S96T004780 & $164: 4$ & Liquid & 333 & 339 & 336 \\
\hline S96T004976 & $164: 5$ & Liquid & 358 & 338 & 348 \\
\hline S96T004781 & $164: 7$ & Liquid & 323 & 334 & 328.5 \\
\hline S96T004977 & $164: 8$ & Liquid & 374 & 322 & 348 \\
\hline S96T004978 & $164: 9$ & Liquid & 349 & 298 & 323.5 \\
\hline S96T004979 & $164: 10$ & Liquid & 332 & 319 & 325.5 \\
\hline S96T004782 & $164: 11$ & Liquid & 321 & 333 & 327 \\
\hline S96T004783 & $164: 12$ & Liquid & 343 & 381 & 362 \\
\hline S96T004784 & $164: 13$ & Liquid & 326 & 341 & 333.5 \\
\hline S96T005979 & Core 164 & Composite & 365 & 357 & 361 \\
\hline
\end{tabular}


Table B2-18. Tank 241-AN-104 Analytical Results: Cobalt (ICP), (3 sheets)

\begin{tabular}{|c|c|c|c|c|c|}
\hline Shimple & (6) & \%on & (y) & 3) & \%) \\
\hline \multicolumn{3}{|c|}{ 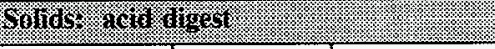 } & \% & $4 \%$ & 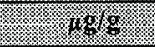 \\
\hline \$96T005287 & $163: 1$ & Lower half & $<7.57$ & $<7.86$ & $<7.715$ \\
\hline \$96T005288 & $163: 2$ & Lower half & $<8.87$ & $<7.49$ & $<8.18$ \\
\hline$\$ 96 \mathrm{~T} 005782$ & $163: 12$ & Lower half & $<9.14$ & $<9.22$ & $<9.18$ \\
\hline S96T005749 & \multirow[t]{2}{*}{$163: 14$} & Upper half & $<8.95$ & $<8.72$ & $<8.835$ \\
\hline S96T005750 & & Lower half & $<9.18$ & $<9.04$ & $<9.11$ \\
\hline S96T005294 & \multirow[t]{2}{*}{$163: 16$} & Upper half & $<8.25$ & $<7.4$ & $<7.825$ \\
\hline S96T005289 & & Lower half & $<9.29$ & $<8.52$ & $<8.905$ \\
\hline S96T005778 & \multirow[t]{2}{*}{$163: 18$} & Upper half & $<9.62$ & $<9.09$ & $<9.355$ \\
\hline S96T005783 & & Lower half & $<9.48$ & $<9.45$ & $<9.465$ \\
\hline S96T005557 & \multirow[t]{2}{*}{$163: 20$} & Upper half & $<7.59$ & $<8.02$ & $<7.805$ \\
\hline \$96T005560 & & Lower half & $<8.85$ & $<7.46$ & $<8.155$ \\
\hline S96T004797 & $164: 1$ & Lower half & $<9.38$ & $<9.42$ & $<9.4$ \\
\hline S96T004798 & $164: 13$ & Lower half & $<9.68$ & $<9.53$ & $<9.605$ \\
\hline \$96T005042 & \multirow[t]{2}{*}{$164: 14$} & Upper half & $<9.49$ & $<9.59$ & $<9.54$ \\
\hline \$96T005040 & & Lower half & $<9.55$ & $<9.4$ & $<9.475$ \\
\hline S96T005061 & \multirow[t]{2}{*}{$164: 15$} & Upper half & $<9.66$ & $<9.6$ & $<9.63$ \\
\hline S96T005043 & & Lower half & $<9.28$ & $<9.55$ & $<9.415$ \\
\hline \$96T005062 & \multirow[t]{2}{*}{$164: 16$} & Upper half & $<30.1$ & 30 & $<30.05$ \\
\hline S96T005044 & & Lower half & 32.7 & 47.3 & $40^{\mathrm{QC:c}}$ \\
\hline S96T005063 & \multirow[t]{2}{*}{$164: 17$} & Upper half & 35.1 & 36.6 & 35.85 \\
\hline S96T005041 & & Lower half & 52.2 & 44.9 & 48.55 \\
\hline S96T005064 & \multirow[t]{2}{*}{$164: 19$} & Upper half & 49.3 & $<30.7$ & $<40^{\mathrm{QC:c}}$ \\
\hline S96T005045 & & Lower half & 47.3 & $<28.8$ & $<38.05^{\mathrm{QC}: \mathrm{c}}$ \\
\hline S96T005282 & \multirow[t]{2}{*}{$164: 20$} & Upper half & $<7.86$ & $<7.21$ & $<7.535$ \\
\hline \$96T005279 & & Lower half & $<9.07$ & $<7.55$ & $<8.31$ \\
\hline S96T005976 & Core 164 & Composite & $<7.95$ & $<7.96$ & $<7.955^{\mathrm{QCa}}$ \\
\hline
\end{tabular}


Table B2-18. Tank 241-AN-104 Analytical Results: Cobalt (ICP), (3 sheets)

\begin{tabular}{|c|c|c|c|c|c|}
\hline S.mole & 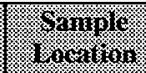 & 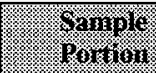 & (2) & 34011/2 atso & (3) \\
\hline \multicolumn{3}{|c|}{ Wodds sysur } & 1\% & 3. & 6\%or \\
\hline S96T005284 & 163:1 & Lower half & $<375$ & $<374$ & $<374.5$ \\
\hline S96T005285 & $163: 2$ & Lower half & $<358$ & $<382$ & $<370$ \\
\hline S96T005780 & $163: 12$ & Lower half & $<376$ & $<380$ & $<378$ \\
\hline S96T005747 & \multirow[t]{2}{*}{$163: 14$} & Upper half & $<448$ & $<451$ & $<449.5$ \\
\hline S96T005748 & & Lower half & $<373$ & $<385$ & $<379$ \\
\hline S96T005293 & \multirow[t]{2}{*}{$163: 16$} & Upper half & $<355$ & $<380$ & $<367.5$ \\
\hline S96T005286 & & Lower half & $<397$ & $<367$ & $<382$ \\
\hline S96T005777 & \multirow[t]{2}{*}{$163: 18$} & Upper half & $<410$ & $<397$ & $<403.5$ \\
\hline S96T005781 & & Lower half & $<426$ & $<428$ & $<427$ \\
\hline S96T005556 & \multirow[t]{2}{*}{$163: 20$} & Upper half & $<370$ & $<387$ & $<378.5$ \\
\hline \$96T005559 & & Lower half & $<391$ & $<389$ & $<390$ \\
\hline S96T004795 & $164: 1$ & Lower half & $<409$ & $<402$ & $<405.5$ \\
\hline S96T004796 & $164: 13$ & Lower half & $<412$ & $<415$ & $<413.5$ \\
\hline S96T005035 & \multirow[t]{2}{*}{$164: 14$} & Upper half & $<408$ & $<412$ & $<410$ \\
\hline S96T005030 & & Lower half & $<410$ & $<407$ & $<408.5$ \\
\hline S96T005036 & \multirow[t]{2}{*}{$164: 15$} & Upper half & $<402$ & $<393$ & $<397.5$ \\
\hline S96T005032 & & Lower half & $<395$ & $<401$ & $<398$ \\
\hline S96T005037 & \multirow[t]{2}{*}{$164: 16$} & Upper half & $<384$ & $<366$ & $<375$ \\
\hline S96T005033 & & Lower half & $<383$ & $<373$ & $<378$ \\
\hline S96T005038 & \multirow[t]{2}{*}{$164: 17$} & Upper half & $<386$ & $<379$ & $<382.5$ \\
\hline S96T005031 & & Lower half & $<437$ & $<434$ & $<435.5$ \\
\hline S96T005039 & \multirow[t]{2}{*}{$164: 19$} & Upper half & $<389$ & $<400$ & $<394.5$ \\
\hline S96T005034 & & Lower half & $<427$ & $<435$ & $<431$ \\
\hline \$96T005281 & \multirow[t]{2}{*}{$164: 20$} & Upper half & $<344$ & $<370$ & $<357$ \\
\hline S96T005278 & & Lower half & $<377$ & $<387$ & $<382$ \\
\hline
\end{tabular}


Table B2-18. Tank 241-AN-104 Analytical Results: Cobalt (ICP), (3 sheets)

\begin{tabular}{|c|c|c|c|c|c|}
\hline 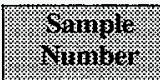 & $\begin{array}{l}\text { Sariple: } \\
\text { f. Indarion: }\end{array}$ & $\begin{array}{l}\text { Sample } \\
\text { Rotion. }\end{array}$ & Iranil. & Buplicate & f \\
\hline Liguras & 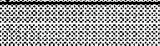 & 6 & $48 \cdot 173:$ & fgrmi & 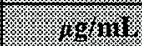 \\
\hline S96T005256 & $163: 2$ & Liquid & $<12$ & $<12$ & $<12$ \\
\hline S96T005260 & $163: 4$ & Liquid & $<12$ & $<12$ & $<12$ \\
\hline S96T005527 & $163: 5$ & Liquid & $<12$ & $<12$ & $<12$ \\
\hline S96T005554 & $163: 6$ & Liquid & $<12$ & $<12$ & $<12$ \\
\hline S96T005255 & $163: 7$ & Liquid & $<12$ & $<12$ & $<12$ \\
\hline S96T005257 & $163: 8$ & Liquid & $<12$ & $<12$ & $<12$ \\
\hline S96T005258 & $163: 9$ & Liquid & $<12$ & $<12$ & $<12$ \\
\hline S96T005528 & $163: 10$ & Liquid & $<12$ & $<12$ & $<12$ \\
\hline S96T005739 & $163: 11$ & Liquid & $<12$ & $<12$ & $<12$ \\
\hline S96T005766 & $163: 12$ & Liquid & $<12$ & $<12$ & $<12$ \\
\hline S96T005741 & $163: 14$ & Liquid & $<12$ & $<12$ & $<12$ \\
\hline S96T004774 & $164: 1$ & Liquid & 65.6 & 62.1 & 63.85 \\
\hline S96T004778 & $164: 2$ & Liquid & 54.8 & 66.5 & 60.65 \\
\hline S96T004779 & $164: 3$ & Liquid & 64.5 & 63.7 & 64.1 \\
\hline S96T004780 & $164: 4$ & Liquid & $<12$ & $<12$ & $<12$ \\
\hline S96T004976 & $164: 5$ & Liquid & $<12$ & $<12$ & $<12$ \\
\hline S96T004781 & $164: 7$ & Liquid & $<12$ & $<12$ & $<12$ \\
\hline S96T004977 & $164: 8$ & Liquid & $<12$ & $<12$ & $<12$ \\
\hline S96T004978 & $164: 9$ & Liquid & $<12$ & $<12$ & $<12$ \\
\hline S96T004979 & $164: 10$ & Liquid & $<12$ & $<12$ & $<12$ \\
\hline S96T004782 & $164: 11$ & Liquid & $<12$ & $<12$ & $<12$ \\
\hline S96T004783 & $164: 12$ & Liquid & $<12$ & $<12$ & $<12$ \\
\hline S96T004784 & $164: 13$ & Liquid & $<12$ & $<12$ & $<12$ \\
\hline S96T005979 & Core 164 & Composite & 75.2 & 67.3 & 71.25 \\
\hline
\end{tabular}


Table B2-19. Tank 241-AN-104 Analytical Results: Copper (ICP). (3 sheets)

\begin{tabular}{|c|c|c|c|c|c|}
\hline 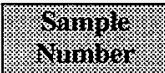 & Gorme & 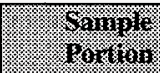 & riseriil & 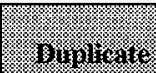 & mentin \\
\hline Gofids & dyest. & & 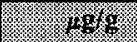 & 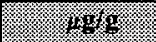 & (6.) \\
\hline S96T005287 & $163: 1$ & Lower half & 4.7 & 4.12 & 4.41 \\
\hline S96T005288 & $163: 2$ & Lower half & $<4.43$ & $<3.75$ & $<4.09$ \\
\hline S96T005782 & $163: 12$ & Lower half & $<4.57$ & $<4.61$ & $<4.59$ \\
\hline S96T005749 & \multirow[t]{2}{*}{$163: 14$} & Upper half & $<4.48$ & $<4.36$ & $<4.42$ \\
\hline S96T005750 & & Lower half & $<4.59$ & $<4.52$ & $<4.555$ \\
\hline S96T005294 & \multirow[t]{2}{*}{$163: 16$} & Upper half & 4.69 & 4.02 & 4.355 \\
\hline S96T005289 & & Lower half & 4.75 & 4.33 & 4.54 \\
\hline S96T005778 & \multirow[t]{2}{*}{$163: 18$} & Upper half & $<4.81$ & $<4.54$ & $<4.675$ \\
\hline S96T005783 & & Lower half & $<4.74$ & $<4.72$ & $<4.73$ \\
\hline S96T005557 & \multirow[t]{2}{*}{$163: 20$} & Upper half & 4.39 & 4.06 & 4.225 \\
\hline S96T005560 & & Lower half & $<4.42$ & 4.38 & $<4.4$ \\
\hline S96T004797 & $164: 1$ & Lower half & 5.34 & 5.79 & 5.565 \\
\hline S96T004798 & $164: 13$ & Lower half & 7.06 & 7.2 & 7.13 \\
\hline S96T005042 & \multirow[t]{2}{*}{$164: 14$} & Upper half & 19 & 5.67 & $12.335^{Q \mathrm{QCe}}$ \\
\hline S96r005040 & & Lower half & 5.12 & 6.01 & 5.565 \\
\hline S96T005061 & \multirow[t]{2}{*}{$164: 15$} & Upper half & 4.98 & 6.02 & 5.5 \\
\hline S96T005043 & & Lower half & 5.6 & 5.6 & 5.6 \\
\hline S96T005062 & \multirow[t]{2}{*}{$164: 16$} & Upper half & $<15.1$ & $<14.9$ & $<15$ \\
\hline S96T005044 & & Lower half & $<14.7$ & $<15$ & $<14.85$ \\
\hline S96T005063 & \multirow[t]{2}{*}{$164: 17$} & Upper half & $<13.9$ & $<14.3$ & $<14.1$ \\
\hline S96T005041 & & Lower half & $<15.2$ & $<15.1$ & $<15.15$ \\
\hline \begin{tabular}{|l|} 
S96T005064 \\
\end{tabular} & \multirow[t]{2}{*}{$164: 19$} & Upper half & $<15.1$ & $<15.3$ & $<15.2$ \\
\hline S96T005045 & & Lower half & $<14.5$ & $<14.4$ & $<14.45$ \\
\hline \begin{tabular}{|l|} 
S96T005282 \\
\end{tabular} & \multirow[t]{2}{*}{$164: 20$} & Upper half & 6.27 & 5.34 & 5.805 \\
\hline S96T005279 & & Lower half & 6.1 & 5.29 & 5.695 \\
\hline S96T005976 & Core 164 & Composite & 5.32 & 5.02 & $5.17^{\mathrm{QC:a}}$ \\
\hline
\end{tabular}


Table B2-19. Tank 241-AN-104 Analytical Results: Copper (ICP). (3 sheets)

\begin{tabular}{|c|c|c|c|c|c|}
\hline $\begin{array}{l}\text { Sumple } \\
\text { I naiber }\end{array}$ & 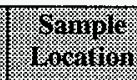 & Sample & resin. & Duplicule & 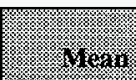 \\
\hline \multicolumn{2}{|c|}{ 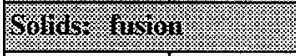 } & & 18.68 & $=1486$ & 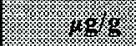 \\
\hline S96T005284 & $163: 1$ & Lower half & $<187$ & $<187$ & $<187$ \\
\hline S96T005285 & $163: 2$ & Lower half & $<179$ & $<191$ & $<185$ \\
\hline S96T005780 & $163: 12$ & Lower half & $<188$ & $<190$ & $<189$ \\
\hline S96T005747 & \multirow[t]{2}{*}{$163: 14$} & Upper half & $<224$ & $<225$ & $<224.5$ \\
\hline S96T005748 & & Lower half & $<187$ & $<193$ & $<190$ \\
\hline S96T005293 & \multirow{2}{*}{$163: 16$} & Upper half & $<177$ & $<190$ & $<183.5$ \\
\hline S96T005286 & & Lower half & $<199$ & $<183$ & $<191$ \\
\hline S96T005777 & \multirow[t]{2}{*}{$163: 18$} & Upper half & $<205$ & $<198$ & $<201.5$ \\
\hline S96T005781 & & Lower half & $<213$ & 339 & $<276^{\mathrm{QC}: \mathrm{e}}$ \\
\hline S96T005556 & \multirow[t]{2}{*}{$163: 20$} & Upper half & $<185$ & $<194$ & $<189.5$ \\
\hline S96T005559 & & Lower half & $<195$ & $<195$ & $<195$ \\
\hline S96T004795 & $164: 1$ & Lower half & $<204$ & $<201$ & $<202.5$ \\
\hline S96T004796 & $164: 13$ & Lower half & $<206$ & $<207$ & $<206.5$ \\
\hline S96T005035 & \multirow[t]{2}{*}{$164: 14$} & Upper half & $<204$ & $<206$ & $<205$ \\
\hline S96T005030 & & Lower half & $<205$ & $<204$ & $<204.5$ \\
\hline S96T005036 & \multirow[t]{2}{*}{$164: 15$} & Upper half & $<201$ & $<197$ & $<199$ \\
\hline S96T005032 & & Lower half & $<197$ & $<200$ & $<198.5$ \\
\hline S96T005037 & \multirow[t]{2}{*}{$164: 16$} & Upper half & $<192$ & $<183$ & $<187.5$ \\
\hline S96T005033 & & Lower half & $<191$ & $<187$ & $<189$ \\
\hline S96T005038 & \multirow[t]{2}{*}{$164: 17$} & Upper half & $<193$ & $<190$ & $<191.5$ \\
\hline S96T005031 & & Lower half & $<218$ & $<217$ & $<217.5$ \\
\hline S96T005039 & \multirow[t]{2}{*}{$164: 19$} & Upper half & $<194$ & $<200$ & $<197$ \\
\hline S96T005034 & & Lower half & $<214$ & $<217$ & $<215.5$ \\
\hline S96T005281 & \multirow[t]{2}{*}{$164: 20$} & Upper half & $<172$ & $<185$ & $<178.5$ \\
\hline S96T005278 & & Lower half & $<189$ & $<193$ & $<191$ \\
\hline
\end{tabular}


HNF-SD-WM-ER-690 Rev. 0

Table B2-19. Tank 241-AN-104 Analytical Results: Copper (ICP). (3 sheets)

\begin{tabular}{|c|c|c|c|c|c|}
\hline $\begin{array}{l}\text { Sarnjle } \\
\text { Nimber }\end{array}$ & $\begin{array}{l}\text { Saraple } \\
\text { I Lecafion }\end{array}$ & $\begin{array}{l}\text { Saripte } \\
\text { Romtion }\end{array}$ & Resulil & Minpiont & $f(18=1$ \\
\hline Iiminis & 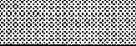 & 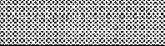 & 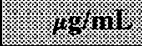 & (1) & 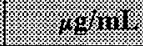 \\
\hline S96T005256 & $163: 2$ & Liquid & $<6.01$ & $<6.01$ & $<6.01$ \\
\hline S96T005260 & $163: 4$ & Liquid & $<6.01$ & $<6.01$ & $<6.01$ \\
\hline S96T005527 & $163: 5$ & Liquid & $<6.01$ & $<6.01$ & $<6.01$ \\
\hline S96T005554 & $163: 6$ & Liquid & $<6.01$ & $<6.01$ & $<6.01$ \\
\hline S96T005255 & $163: 7$ & Liquid & $<6.01$ & $<6.01$ & $<6.01$ \\
\hline S96T005257 & $163: 8$ & Liquid & $<6.01$ & $<6.01$ & $<6.01$ \\
\hline S96T005258 & $163: 9$ & Liquid & $<6.01$ & $<6.01$ & $<6.01$ \\
\hline S96T005528 & $163: 10$ & Liquid & $<6.01$ & $<6.01$ & $<6.01$ \\
\hline S96T005739 & $163: 11$ & Liquid & $<6.01$ & $<6.01$ & $<6.01$ \\
\hline S96T005766 & $163: 12$ & Liquid & $<6.01$ & $<6.01$ & $<6.01$ \\
\hline S96T005741 & $163: 14$ & Liquid & $<6.01$ & $<6.01$ & $<6.01$ \\
\hline S96T004774 & 164:1 & Liquid & $<12$ & $<12$ & $<12$ \\
\hline S96T004778 & $164: 2$ & Liquid & $<12$ & $<12$ & $<12$ \\
\hline S96T004779 & $164: 3$ & Liquid & $<12$ & $<12$ & $<12$ \\
\hline S96T004780 & $164: 4$ & Liquid & $<6.01$ & $<6.01$ & $<6.0 .1$ \\
\hline S96T004976 & $164: 5$ & Liquid & $<6.01$ & $<6.01$ & $<6.01$ \\
\hline S96T004781 & $164: 7$ & Liquid & $<6.01$ & $<6.01$ & $<6.01$ \\
\hline S96T004977 & $164: 8$ & Liquid & $<6.01$ & 9.26 & $<7.635^{\mathrm{QC}: \mathrm{c}}$ \\
\hline S96T004978 & $164: 9$ & Liquid & 9.13 & $<6.01$ & $<7.57^{\mathrm{QC:c}}$ \\
\hline S96T004979 & $164: 10$ & Liquid & $<6.01$ & $<6.01$ & $<6.01$ \\
\hline S96T004782 & $164: 11$ & Liquid & $<6.01$ & $<6.01$ & $<6.01$ \\
\hline S96T004783 & $164: 12$ & Liquid & $<6.01$ & $<6.01$ & $<6.01$ \\
\hline S96T004784 & $164: 13$ & Liquid & $<6.01$ & $<6.01$ & $<6.01$ \\
\hline S96T005979 & Core 164 & Composite & $<12$ & $<12$ & $<12$ \\
\hline
\end{tabular}


Table B2-20. Tank 241-AN-104 Analytical Results: Iron (ICP). (3 sheets)

\begin{tabular}{|c|c|c|c|c|c|}
\hline 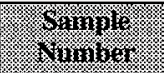 & \% & 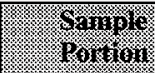 & 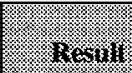 & ( 1401140 & $\mathrm{H}^{2}$ \\
\hline \multicolumn{3}{|c|}{ 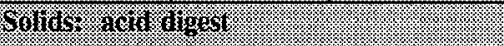 } & $f_{1}$ & 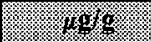 & 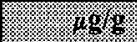 \\
\hline S96T005287 & 163:1 & Lower half & 489 & 570 & 529.5 \\
\hline S96T005288 & $163: 2$ & Lower half & 198 & 181 & 189.5 \\
\hline S96T005782 & $163: 12$ & Lower half & 75.8 & 72.2 & 74 \\
\hline S96T005749 & \multirow[t]{2}{*}{$163: 14$} & Upper half & 91.1 & 100 & 95.55 \\
\hline S96T005750 & & Lower half & 87.1 & 62 & $74.55^{\mathrm{QC}: c}$ \\
\hline S96T005294 & \multirow[t]{2}{*}{$163: 16$} & Upper half & 102 & 84.4 & 93.2 \\
\hline S96T005289 & & Lower half & 87 & 67.6 & $77.3^{\mathrm{QC}: \mathrm{c}}$ \\
\hline S96T005778 & \multirow[t]{2}{*}{$163: 18$} & Upper half & 107 & 154 & $130.5^{Q C: c}$ \\
\hline S96T005783 & & Lower half & 77.8 & 77.7 & 77.75 \\
\hline S96T005557 & \multirow[t]{2}{*}{$163: 20$} & Upper half & 104 & 107 & 105.5 \\
\hline S96T005560 & & Lower half & 171 & 92.3 & $131.65^{Q C i c}$ \\
\hline S96T004797 & $164: 1$ & Lower half & 58.1 & 51.1 & 54.6 \\
\hline S96T004798 & $164: 13$ & Lower half & 188 & 227 & 207.5 \\
\hline S96T005042 & \multirow[t]{2}{*}{$164: 14$} & Upper half & 97.6 & 97.1 & 97.35 \\
\hline S96T005040 & & Lower half & 56.5 & 53.6 & 55.05 \\
\hline S96T005061 & \multirow[t]{2}{*}{$164: 15$} & Upper half & 69.2 & 82.2 & 75.7 \\
\hline S96T005043 & & Lower half & 106 & 108 & 107 \\
\hline S96T005062 & \multirow[t]{2}{*}{$164: 16$} & Upper half & $<75.3$ & $<74.7$ & $<75$ \\
\hline \$96T005044 & & Lower half & $<73.7$ & $<75$ & $<74.35$ \\
\hline \$96T005063 & \multirow[t]{2}{*}{$164: 17$} & Upper half & $<69.7$ & $<71.4$ & $<70.55$ \\
\hline S96T005041 & & Lower half & $<76.2$ & $<75.6$ & $<75.9$ \\
\hline S96T005064 & \multirow{2}{*}{$164: 19$} & Upper half & 80.6 & 133 & $106.8^{Q C: e}$ \\
\hline \$96T005045 & & Lower half & $<72.5$ & $<72$ & $<72.25$ \\
\hline S96T005282 & \multirow[t]{2}{*}{$164: 20$} & Upper half & 126 & 104 & 115 \\
\hline \$96T005279 & & Lower half & 125 & 77.1 & $101.05^{Q C: c}$ \\
\hline S96T005976 & Core 164 & Composite & 104 & 94.9 & $99.45^{\text {QC:a }}$ \\
\hline
\end{tabular}


Table B2-20. Tank 241-AN-104 Analytical Results: Iron (ICP). (3 sheets)

\begin{tabular}{|c|c|c|c|c|c|}
\hline (6) & 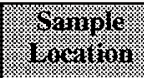 & 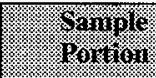 & Resull & Byiglogro & Hean \\
\hline \multicolumn{3}{|l|}{ Golio } & 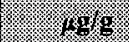 & 40 & $U_{1}$ \\
\hline S96T005284 & $163: 1$ & Lower half & $<937$ & $<936$ & $<936.5$ \\
\hline \$96T005285 & $163: 2$ & Lower half & $<894$ & $<956$ & $<925$ \\
\hline S96T005780 & $163: 12$ & Lower half & $<940$ & $<950$ & $<945$ \\
\hline S96T005747 & \multirow[t]{2}{*}{$163: 14$} & Upper half & $<1,120$ & $<1,130$ & $<1,125$ \\
\hline S96T005748 & & Lower half & $<934$ & $<963$ & $<948.5$ \\
\hline S96T005293 & \multirow[t]{2}{*}{$163: 16$} & Upper half & $<887$ & $<949$ & $<918$ \\
\hline S96T005286 & & Lower half & $<993$ & $<917$ & $<955$ \\
\hline S96T005777 & \multirow[t]{2}{*}{$163: 18$} & Upper half & $<1,030$ & $<992$ & $<1,011$ \\
\hline S96T005781 & & Lower half & 1,890 & 3,620 & $2,755^{\mathrm{QC:c}}$ \\
\hline \$96T005556 & \multirow[t]{2}{*}{$163: 20$} & Upper half & $<924$ & $<968$ & $<946$ \\
\hline S96T005559 & & Lower half & $<977$ & $<973$ & $<975$ \\
\hline S96T004795 & $164: 1$ & Lower half & $<1,020$ & $<1,000$ & $<1,010$ \\
\hline S96T004796 & $164: 13$ & Lower half & $<1,030$ & $<1,040$ & $<1,035$ \\
\hline S96T005035 & \multirow[t]{2}{*}{$164: 14$} & Upper half & $<1,020$ & $<1,030$ & $<1,025$ \\
\hline S96T005030 & & Lower half & $<1,020$ & $<1,020$ & $<1,020$ \\
\hline \$96T005036 & \multirow[t]{2}{*}{$164: 15$} & Upper half & $<1,000$ & $<983$ & $<991.5$ \\
\hline S96T005032 & & Lower half & $<987$ & $<1,000$ & $<993.5$ \\
\hline S96T005037 & \multirow[t]{2}{*}{$164: 16$} & Upper half & $<961$ & $<915$ & $<938$ \\
\hline S96T005033 & & Lower half & $<957$ & $<933$ & $<945$ \\
\hline S96T005038 & \multirow[t]{2}{*}{$164: 17$} & Upper half & $<966$ & $<949$ & $<957.5$ \\
\hline S96T005031 & & Lower half & $<1,090$ & $<1,080$ & $<1,085$ \\
\hline S96T005039 & \multirow[t]{2}{*}{$164: 19$} & Upper half & $<972$ & $<1,000$ & $<986$ \\
\hline S96T005034 & & Lower half & $<1,070$ & $<1,090$ & $<1,080$ \\
\hline S96T005281 & \multirow[t]{2}{*}{$164: 20$} & Upper half & $<860$ & $<925$ & $<892.5$ \\
\hline S96T005278 & & Lower half & $<943$ & $<966$ & $<954.5$ \\
\hline
\end{tabular}


Table B2-20. Tank 241-AN-104 Analytical Results: Iron (ICP). (3 sheets)

\begin{tabular}{|c|c|c|c|c|c|}
\hline 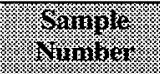 & Shimplo & 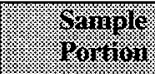 & (nesil) & 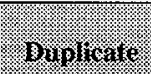 & 314. \\
\hline \%ormis & & & 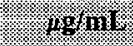 & 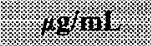 & 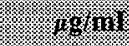 \\
\hline S96T005256 & $163: 2$ & Liquid & $<30.1$ & $<30.1$ & $<30.1$ \\
\hline S96T005260 & $163: 4$ & Liquid & $<30.1$ & $<30.1$ & $<30.1$ \\
\hline S96T005527 & $163: 5$ & Liquid & $<30.1$ & $<30.1$ & $<30.1$ \\
\hline S96T005554 & $163: 6$ & Liquid & $<30.1$ & $<30.1$ & $<30.1$ \\
\hline S96T005255 & $163: 7$ & Liquid & 33.3 & $<30.1$ & $<31.7$ \\
\hline S96T005257 & $163: 8$ & Liquid & $<30.1$ & $<30.1$ & $<30.1$ \\
\hline S96T005258 & $163: 9$ & Liquid & $<30.1$ & $<30.1$ & $<30.1$ \\
\hline S96T005528 & $163: 10$ & Liquid & $<30.1$ & $<30.1$ & $<30.1$ \\
\hline S96T005739 & $163: 11$ & Liquid & $<30.1$ & $<30.1$ & $<30.1$ \\
\hline \$96T005766 & $163: 12$ & Liquid & $<30.1$ & $<30.1$ & $<30.1$ \\
\hline S96T005741 & $163: 14$ & Liquid & $<30.1$ & $<30.1$ & $<30.1$ \\
\hline S96T004774 & $164: 1$ & Liquid & $<60.1$ & $<60.1$ & $<60.1$ \\
\hline S96T004778 & $164: 2$ & Liquid & $<60.1$ & $<60.1$ & $<60.1$ \\
\hline S96T004779 & $164: 3$ & Liquid & $<60.1$ & $<60.1$ & $<60.1$ \\
\hline S96T004780 & $164: 4$ & Liquid & $<30.1$ & $<30.1$ & $<30.1$ \\
\hline S96T004976 & $164: 5$ & Liquid & $<30.1$ & $<30.1$ & $<30.1$ \\
\hline S96T004781 & $164: 7$ & Liquid & $<30.1$ & $<30.1$ & $<30.1$ \\
\hline \$96T004977 & $164: 8$ & Liquid & $<30.1$ & $<30.1$ & $<30.1$ \\
\hline S96T004978 & $164: 9$ & Liquid & $<30.1$ & $<30.1$ & $<30.1$ \\
\hline S96T004979 & $164: 10$ & Liquid & $<30.1$ & $<30.1$ & $<30.1$ \\
\hline S96T004782 & $164: 11$ & Liquid & $<30.1$ & $<30.1$ & $<30.1$ \\
\hline S96T004783 & $164: 12$ & Liquid & $<30.1$ & $<30.1$ & $<30.1$ \\
\hline S96T004784 & $164: 13$ & Liquid & $<30.1$ & $<30.1$ & $<30.1$ \\
\hline S96T005979 & Core 164 & Composite & $<60.1$ & $<60.1$ & $<60.1$ \\
\hline
\end{tabular}


Table B2-21. Tank 241-AN-104 Analytical Results: Lanthanum (ICP). (3 sheets)

\begin{tabular}{|c|c|c|c|c|c|}
\hline 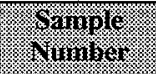 & 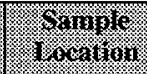 & 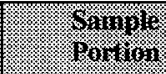 & 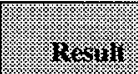 & 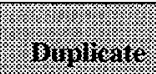 & (1) \\
\hline Womus aro & Wigert & & grgo & (1) & \% \\
\hline S96T005287 & $163: 1$ & Lower half & $<18.9$ & $<19.6$ & $<19.25$ \\
\hline S96T005288 & $163: 2$ & Lower half & $<22.2$ & $<18.7$ & $<20.45$ \\
\hline S96T005782 & $163: 12$ & Lower half & $<22.8$ & $<23$ & $<22.9$ \\
\hline S96T005749 & \multirow[t]{2}{*}{$163: 14$} & Upper half & $<22.4$ & $<21.8$ & $<22.1$ \\
\hline S96T005750 & & Lower half & $<23$ & $<22.6$ & $<22.8$ \\
\hline S96T005294 & \multirow[t]{2}{*}{$163: 16$} & Upper half & $<20.6$ & $<18.5$ & $<19.55$ \\
\hline S96T005289 & & Lower half & $<23.2$ & $<21.3$ & $<22.25$ \\
\hline S96T005778 & \multirow[t]{2}{*}{$163: 18$} & Upper half & $<24.1$ & $<22.7$ & $<23.4$ \\
\hline S96T005783 & & Lower half & $<23.7$ & $<23.6$ & $<23.65$ \\
\hline S96T005557 & \multirow[t]{2}{*}{$163: 20$} & Upper half & $<19$ & $<20.1$ & $<19.55$ \\
\hline S96T005560 & & Lower half & $<22.1$ & $<18.7$ & $<20.4$ \\
\hline S96T004797 & $164: 1$ & Lower half & $<23.4$ & $<23.5$ & $<23.45$ \\
\hline \$96T004798 & $164: 13$ & Lower half & $<24.2$ & $<23.8$ & $<24$ \\
\hline S96T005042 & \multirow[t]{2}{*}{$164: 14$} & Upper half & $<23.7$ & $<24$ & $<23.85$ \\
\hline S96T005040 & & Lower half & $<23.9$ & $<23.5$ & $<23.7$ \\
\hline \$96T005061 & \multirow[t]{2}{*}{$164: 15$} & Upper half & $<24.2$ & $<24$ & $<24.1$ \\
\hline \$96T005043 & & Lower half & $<23.2$ & $<23.9$ & $<23.55$ \\
\hline S96T005062 & \multirow[t]{2}{*}{$164: 16$} & Upper half & $<75.3$ & $<74.7$ & $<75$ \\
\hline \$96T005044 & & Lower half & $<73.7$ & $<75$ & $<74.35$ \\
\hline S96T005063 & \multirow[t]{2}{*}{$164: 17$} & Upper half & $<69.7$ & $<71.4$ & $<70.55$ \\
\hline S96T005041 & & Lower half & $<76.2$ & $<75.6$ & $<75.9$ \\
\hline S96T005064 & \multirow[t]{2}{*}{$164: 19$} & Upper half & $<75.6$ & $<76.7$ & $<76.15$ \\
\hline S96T005045 & & Lower half & $<72.5$ & $<72$ & $<72.25$ \\
\hline S96T005282 & \multirow[t]{2}{*}{$164: 20$} & Upper half & $<19.7$ & $<18$ & $<18.85$ \\
\hline S96T005279 & & Lower half & $<22.7$ & $<18.9$ & $<20.8$ \\
\hline S96T005976 & Core 164 & Composite & $<19.9$ & $<19.9$ & $<19.9^{\mathrm{QC}: \mathrm{a}}$ \\
\hline
\end{tabular}


Table B2-21. Tank 241-AN-104 Analytical Results: Lanthanum (ICP). (3 sheets)

\begin{tabular}{|c|c|c|c|c|c|}
\hline Sonipho & 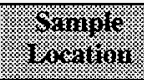 & Womal & (2) & H. & tivis \\
\hline \multicolumn{3}{|c|}{ 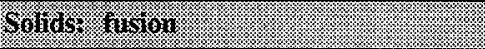 } & 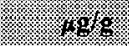 & 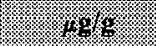 & 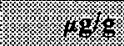 \\
\hline S96T005284 & $163: 1$ & Lower half & $<937$ & $<936$ & $<936.5$ \\
\hline S96T005285 & $163: 2$ & Lower half & $<894$ & $<956$ & $<925$ \\
\hline S96T005780 & $163: 12$ & Lower half & $<940$ & $<950$ & $<945$ \\
\hline S96T005747 & \multirow[t]{2}{*}{$163: 14$} & Upper half & $<1,120$ & $<1,130$ & $<1,125$ \\
\hline S96T005748 & & Lower half & $<934$ & $<963$ & $<948.5$ \\
\hline S96T005293 & \multirow[t]{2}{*}{$163: 16$} & Upper half & $<887$ & $<949$ & $<918$ \\
\hline \$96T005286 & & Lower half & $<993$ & $<917$ & $<955$ \\
\hline S96T005777 & \multirow[t]{2}{*}{$163: 18$} & Upper half & $<1,030$ & $<992$ & $<1,011$ \\
\hline \$96T005781 & & Lower half & $<1,060$ & $<1,070$ & $<1,065$ \\
\hline S96T005556 & \multirow[t]{2}{*}{$163: 20$} & Upper half & $<924$ & $<968$ & $<946$ \\
\hline \$96T005559 & & Lower half & $<977$ & $<973$ & $<975$ \\
\hline S96T004795 & $164: 1$ & Lower half & $<1,020$ & $<1,000$ & $<1,010$ \\
\hline S96T004796 & $164: 13$ & Lower half & $<1,030$ & $<1,040$ & $<1,035$ \\
\hline S96T005035 & \multirow[t]{2}{*}{$164: 14$} & Upper half & $<1,020$ & $<1,030$ & $<1,025$ \\
\hline S96T005030 & & Lower half & $<1,020$ & $<1,020$ & $<1,020$ \\
\hline S96T005036 & \multirow[t]{2}{*}{$164: 15$} & Upper half & $<1,000$ & $<983$ & $<991.5$ \\
\hline S96T005032 & & Lower half & $<987$ & $<1,000$ & $<993.5$ \\
\hline S96T005037 & \multirow[t]{2}{*}{$164: 16$} & Upper half & $<961$ & $<915$ & $<938$ \\
\hline S96T005033 & & Lower half & $<957$ & $<933$ & $<945$ \\
\hline S96T005038 & \multirow[t]{2}{*}{$164: 17$} & Upper half & $<966$ & $<949$ & $<957.5$ \\
\hline S96T005031 & & Lower half & $<1,090$ & $<1,080$ & $<1,085$ \\
\hline \$96T005039 & \multirow[t]{2}{*}{$164: 19$} & Upper half & $<972$ & $<1,000$ & $<986$ \\
\hline S96T005034 & & Lower half & $<1,070$ & $<1,090$ & $<1,080$ \\
\hline S96T005281 & \multirow[t]{2}{*}{$164: 20$} & Upper half & $<860$ & $<925$ & $<892.5$ \\
\hline S96T005278 & & Lower half & $<943$ & $<966$ & $<954.5$ \\
\hline
\end{tabular}


Table B2-21. Tank 241-AN-104 Analytical Results: Lanthanum (ICP). (3 sheets)

\begin{tabular}{|c|c|c|c|c|c|}
\hline Sample & 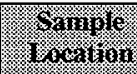 & 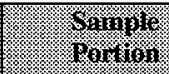 & Rexum & Syiniloge & Yron \\
\hline yining & & & (6) & (6. & (1996m \\
\hline S96T005256 & $163: 2$ & Liquid & $<30.1$ & $<30.1$ & $<30.1$ \\
\hline S96T005260 & $163: 4$ & Liquid & $<30.1$ & $<30.1$ & $<30.1$ \\
\hline S96T005527 & $163: 5$ & Liquid & $<30.1$ & $<30.1$ & $<30.1$ \\
\hline S96T005554 & $163: 6$ & Liquid & $<30.1$ & $<30.1$ & $<30.1$ \\
\hline S96T005255 & $163: 7$ & Liquid & $<30.1$ & $<30.1$ & $<30.1$ \\
\hline S96T005257 & $163: 8$ & Liquid & $<30.1$ & $<30.1$ & $<30.1$ \\
\hline S96T005258 & $163: 9$ & Liquid & $<30.1$ & $<30.1$ & $<30.1$ \\
\hline S96T005528 & $163: 10$ & Liquid & $<30.1$ & $<30.1$ & $<30.1$ \\
\hline S96T005739 & 163:11 & Liquid & $<30.1$ & $<30.1$ & $<30.1$ \\
\hline S96T005766 & $163: 12$ & Liquid & $<30.1$ & $<30.1$ & $<30.1$ \\
\hline S96T005741 & $163: 14$ & Liquid & $<30.1$ & $<30.1$ & $<30.1$ \\
\hline S96T004774 & $164: 1$ & Liquid & $<60.1$ & $<60.1$ & $<60.1$ \\
\hline S96T004778 & $164: 2$ & Liquid & $<60.1$ & $<60.1$ & $<60.1$ \\
\hline S96T004779 & $164: 3$ & Liquid & $<60.1$ & $<60.1$ & $<60.1$ \\
\hline S96T004780 & $164: 4$ & Liquid & $<30.1$ & $<30.1$ & $<30.1$ \\
\hline S96T004976 & $164: 5$ & Liquid & $<30.1$ & $<30.1$ & $<30.1$ \\
\hline S96T004781 & $164: 7$ & Liquid & $<30.1$ & $<30.1$ & $<30.1$ \\
\hline S96T004977 & $164: 8$ & Liquid & $<30.1$ & $<30.1$ & $<30.1$ \\
\hline S96T004978 & $164: 9$ & Liquid & $<30.1$ & $<30.1$ & $<30.1$ \\
\hline S96T004979 & $164: 10$ & Liquid & $<30.1$ & $<30.1$ & $<30.1$ \\
\hline S96T004782 & $164: 11$ & Liquid & $<30.1$ & $<30.1$ & $<30.1$ \\
\hline S96T004783 & $164: 12$ & Liquid & $<30.1$ & $<30.1$ & $<30.1$ \\
\hline S96T004784 & $164: 13$ & Liquid & $<30.1$ & $<30.1$ & $<30.1$ \\
\hline S96T005979 & Core 164 & Composite & $<60.1$ & $<60.1$ & $<60.1$ \\
\hline
\end{tabular}


Table B2-22. Tank 241-AN-104 Analytical Results: Lead (ICP). (3 sheets)

\begin{tabular}{|c|c|c|c|c|c|}
\hline Mongure & 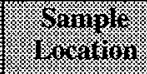 & 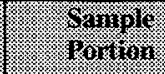 & ravilum & 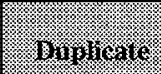 & 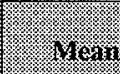 \\
\hline \multicolumn{2}{|c|}{ 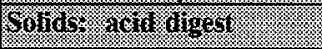 } & 原高 & $19 \%$ & 16. & ryog \\
\hline S96T005287 & $163: 1$ & Lower half & $<37.9$ & $<39.3$ & $<38.6$ \\
\hline S96T005288 & $163: 2$ & Lower half & $<44.3$ & $<37.5$ & $<40.9$ \\
\hline S96T005782 & $163: 12$ & Lower half & $<45.7$ & $<46.1$ & $<45.9$ \\
\hline S96T005749 & \multirow[t]{2}{*}{$163: 14$} & Upper half & $<44.8$ & $<43.6$ & $<44.2$ \\
\hline S96T005750 & & Lower half & $<45.9$ & $<45.2$ & $<45.55$ \\
\hline S96T005294 & \multirow[t]{2}{*}{$163: 16$} & Upper half & $<41.3$ & $<37$ & $<39.15$ \\
\hline \$96T005289 & & Lower half & $<46.4$ & $<42.6$ & $<44.5$ \\
\hline S96T005778 & \multirow[t]{2}{*}{$163: 18$} & Upper half & $<48.1$ & $<45.4$ & $<46.75$ \\
\hline S967005783 & & Lower half & $<47.4$ & $<47.2$ & $<47.3$ \\
\hline S96T005557 & \multirow[t]{2}{*}{$163: 20$} & Upper half & $<38$ & 166 & $<102^{\mathrm{QC}-\mathrm{c}}$ \\
\hline S96T005560 & & Lower half & $<44.2$ & $<37.3$ & $<40.75$ \\
\hline S96T004797 & $164: 1$ & Lower half & $<46.9$ & $<47.1$ & $<47$ \\
\hline S96T004798 & $164: 13$ & Lower half & $<48.4$ & $<47.7$ & $<48.05$ \\
\hline S96T005042 & \multirow[t]{2}{*}{$164: 14$} & Upper half & 434 & 706 & $570^{\mathrm{QC}: \mathrm{c}}$ \\
\hline S96T005040 & & Lower half & $<47.7$ & $<47$ & $<47.35$ \\
\hline S96T005061 & \multirow[t]{2}{*}{$164: 15$} & Upper half & $<48.3$ & $<48$ & $<48.15$ \\
\hline S96T005043 & & Lower half & $<46.4$ & $<47.7$ & $<47.05$ \\
\hline S96T005062 & \multirow[t]{2}{*}{$164: 16$} & Upper half & $<151$ & $<149$ & $<150$ \\
\hline S96T005044 & & Lower half & $<147$ & $<150$ & $<148.5$ \\
\hline \$96T005063 & \multirow[t]{2}{*}{$164: 17$} & Upper half & $<139$ & $<143$ & $<141$ \\
\hline S96T005041 & & Lower half & $<152$ & $<151$ & $<151.5$ \\
\hline S96T005064 & \multirow[t]{2}{*}{$164: 19$} & Upper half & $<151$ & $<153$ & $<152$ \\
\hline S96T005045 & & Lower half & $<145$ & $<144$ & $<144.5$ \\
\hline S96T005282 & \multirow[t]{2}{*}{$164: 20$} & Upper half & 58.9 & 38.9 & $48.9^{\mathrm{QC}: c}$ \\
\hline S96T005279 & & Lower half & $<45.4$ & 45.3 & $<45.35$ \\
\hline S96T005976 & Core 164 & Composite & 42.1 & 41.9 & $42^{Q C: a}$ \\
\hline
\end{tabular}


Table B2-22. Tank 241-AN-104 Analytical Results: Lead (ICP). (3 sheets)

\begin{tabular}{|c|c|c|c|c|c|}
\hline 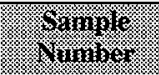 & Syampin & $\begin{array}{l}\text { Signiple } \\
\text { lomoror }\end{array}$ & Rosin: & Buplical & Mara \\
\hline \multicolumn{2}{|c|}{ 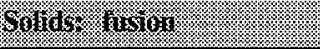 } & 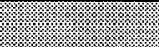 & 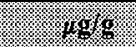 & 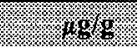 & rises \\
\hline S96T005284 & $163: 1$ & Lower half & $<1,870$ & $<1,870$ & $<1,870$ \\
\hline S96T005285 & $163: 2$ & Lower half & $<1,790$ & $<1,910$ & $<1,850$ \\
\hline S96T005780 & $163: 12$ & Lower half & $<1,880$ & $<1,900$ & $<1,890$ \\
\hline S96T005747 & \multirow[t]{2}{*}{$163: 14$} & Upper half & $<2,240$ & $<2,250$ & $<2,245$ \\
\hline S96T005748 & & Lower half & $<1,870$ & $<1,930$ & $<1,900$ \\
\hline \$96T005293 & \multirow[t]{2}{*}{$163: 16$} & Upper half & $<1,770$ & $<1,900$ & $<1,835$ \\
\hline S96T005286 & & Lower half & $<1,990$ & $<1,830$ & $<1,910$ \\
\hline S96T005777 & \multirow[t]{2}{*}{$163: 18$} & Upper half & $<2,050$ & $<1,980$ & $<2,015$ \\
\hline S96T005781 & & Lower half & $<2,130$ & $<2,140$ & $<2,135$ \\
\hline S96T005556 & \multirow[t]{2}{*}{$163: 20$} & Upper half & $<1,850$ & $<1,940$ & $<1,895$ \\
\hline S96T005559 & & Lower half & $<1,950$ & $<1,950$ & $<1,950$ \\
\hline S96T004795 & $164: 1$ & Lower half & $<2,040$ & $<2,010$ & $<2,025$ \\
\hline S96T004796 & $164: 13$ & Lower half & $<2,060$ & $<2,070$ & $<2,065$ \\
\hline S96T005035 & \multirow[t]{2}{*}{$164: 14$} & Upper half & $<2,040$ & $<2,060$ & $<2,050$ \\
\hline S96T005030 & & Lower half & $<2,050$ & $<2,040$ & $<2,045$ \\
\hline S96T005036 & \multirow[t]{2}{*}{$164: 15$} & Upper half & $<2,010$ & $<1,970$ & $<1,990$ \\
\hline S96T005032 & & Lower half & $<1,970$ & $<2,000$ & $<1,985$ \\
\hline S96T005037 & \multirow[t]{2}{*}{$164: 16$} & Upper half & $<1,920$ & $<1,830$ & $<1,875$ \\
\hline S96T005033 & & Lower half & $<1,910$ & $<1,870$ & $<1,890$ \\
\hline S96T005038 & \multirow[t]{2}{*}{$164: 17$} & Upper half & $<1,930$ & $<1,900$ & $<1,915$ \\
\hline S96T005031 & & Lower half & $<2,180$ & $<2,170$ & $<2,175$ \\
\hline S96T005039 & \multirow[t]{2}{*}{$164: 19$} & Upper half & $<1,940$ & $<2,000$ & $<1,970$ \\
\hline S96T005034 & & Lower half & $<2,140$ & $<2,170$ & $<2,155$ \\
\hline \$96T005281 & \multirow[t]{2}{*}{$164: 20$} & Upper half & $<1,720$ & $<1,850$ & $<1,785$ \\
\hline S96T005278 & & Lower half & $<1,890$ & $<1,930$ & $<1,910$ \\
\hline
\end{tabular}


Table B2-22. Tank 241-AN-104 Analytical Results: Lead (ICP). (3 sheets)

\begin{tabular}{|c|c|c|c|c|c|}
\hline 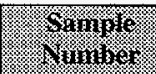 & 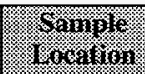 & Mourlo & 16xin & (3) & ing \\
\hline Sinunis & & & $148 \times 171$ & $\sqrt{\text { r }}$ & 3. \\
\hline S96T005256 & $163: 2$ & Liquid & $<60.1$ & 64.3 & $<62.2$ \\
\hline S96T005260 & $163: 4$ & Liquid & 61.5 & 69.5 & 65.5 \\
\hline S96T005527 & $163: 5$ & Liquid & $<60.1$ & $<60.1$ & $<60.1$ \\
\hline S96T005554 & $163: 6$ & Liquid & 60.2 & 60.5 & 60.35 \\
\hline S96T005255 & $163: 7$ & Liquid & 67 & 65.5 & 66.25 \\
\hline S96T005257 & $163: 8$ & Liquid & $<60.1$ & 66.7 & $<63.4$ \\
\hline S96T00S258 & $163: 9$ & Liquid & 60.8 & $<60.1$ & $<60.45$ \\
\hline S96T005528 & $163: 10$ & Liquid & 60.5 & $<60.1$ & $<60.3$ \\
\hline S96T005739 & $163: 11$ & Liquid & 64.7 & 67.1 & 65.9 \\
\hline S96T005766 & $163: 12$ & Liquid & 77.7 & 68.6 & 73.15 \\
\hline S96T005741 & $163: 14$ & Liquid & 60.1 & 66.9 & 63.5 \\
\hline S96T004774 & $164: 1$ & Liquid & $<120$ & $<120$ & $<120$ \\
\hline S96T004778 & $164: 2$ & Liquid & $<120$ & $<120$ & $<120$ \\
\hline S96T004779 & $164: 3$ & Liquid & $<120$ & $<120$ & $<120$ \\
\hline S96T004780 & $164: 4$ & Liquid & $<60.1$ & $<60.1$ & $<60.1$ \\
\hline S96T004976 & $164: 5$ & Liquid & $<60.1$ & $<60.1$ & $<60.1$ \\
\hline S96T004781 & $164: 7$ & Liquid & $<60.1$ & $<60.1$ & $<60.1$ \\
\hline S96T004977 & $164: 8$ & Liquid & 70.7 & $<60.1$ & $<65.4$ \\
\hline \$96T004978 & $164: 9$ & Liquid & $<60.1$ & 76.7 & $<68.4^{\mathrm{QC:c}}$ \\
\hline S96T004979 & $164: 10$ & Liquid & $<60.1$ & $<60.1$ & $<60.1$ \\
\hline S96T004782 & $164: 11$ & Liquid & $<60.1$ & $<60.1$ & $<60.1$ \\
\hline S96T004783 & $164: 12$ & Liquid & $<60.1$ & $<60.1$ & $<60.1$ \\
\hline S96T004784 & $164: 13$ & Liquid & $<60.1$ & $<60.1$ & $<60.1$ \\
\hline S96T005979 & Core 164 & Composite & $<120$ & $<120$ & $<120$ \\
\hline
\end{tabular}


Table B2-23. Tank 241-AN-104 Analytical Results: Lithium (ICP). (3 sheets)

\begin{tabular}{|c|c|c|c|c|c|}
\hline $\begin{array}{l}\text { Sample } \\
\text { Vinnter }\end{array}$ & $\begin{array}{l}\text { Sample. } \\
\text { Griginin. }\end{array}$ & $\begin{array}{l}\text { Sampla } \\
\text { rontion }\end{array}$ & 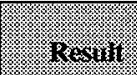 & 8) & Mran \\
\hline \multicolumn{2}{|c|}{ 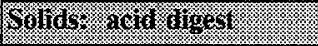 } & $=$ & 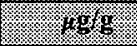 & 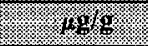 & 4.96 \\
\hline S96T005287 & $163: 1$ & Lower half & $<3.79$ & $<3.93$ & $<3.86$ \\
\hline S96T005288 & $163: 2$ & Lower half & 47 & 45.1 & 46.05 \\
\hline S96T005782 & $163: 12$ & Lower half & 25.4 & 26.2 & 25.8 \\
\hline S96T005749 & \multirow[t]{2}{*}{$163: 14$} & Upper half & 11.9 & 12.6 & 12.25 \\
\hline S96T005750 & & Lower half & 5.28 & 4.99 & 5.135 \\
\hline S96T005294 & \multirow{2}{*}{$163: 16$} & Upper half & 5.36 & 4.99 & 5.175 \\
\hline S96T005289 & & Lower half & $<4.64$ & $<4.26$ & $<4.45$ \\
\hline S96T005778 & \multirow[t]{2}{*}{$163: 18$} & Upper half & 15.1 & 21.5 & $18.3^{\mathrm{ec}: \mathrm{c}}$ \\
\hline S96T005783 & & Lower half & $<4.74$ & 4.82 & $<4.78$ \\
\hline S96T005557 & \multirow[t]{2}{*}{$163: 20$} & Upper half & 7.83 & 8.18 & 8.005 \\
\hline S96T005560 & & Lower half & 8.39 & 6.49 & $7.44^{\mathrm{QC:c}}$ \\
\hline S96T004797 & $164: 1$ & Lower half & $<4.69$ & $<4.71$ & $<4.7$ \\
\hline S96T004798 & $164: 13$ & Lower half & 52.4 & 69 & $60.7^{\mathrm{QC:c}}$ \\
\hline S96T005042 & \multirow[t]{2}{*}{$164: 14$} & Upper half & 22 & 21.9 & 21.95 \\
\hline S96T005040 & & Lower half & 12 & 12.2 & 12.1 \\
\hline S96T005061 & \multirow[t]{2}{*}{$164: 15$} & Upper half & 12.5 & 12.7 & 12.6 \\
\hline S96T005043 & & Lower half & 9.64 & 9.3 & 9.47 \\
\hline S96T005062 & \multirow[t]{2}{*}{$164: 16$} & Upper half & $<15.1$ & $<14.9$ & $<15$ \\
\hline S96T005044 & & Lower half & $<14.7$ & $<15$ & $<14.85$ \\
\hline S96T005063 & \multirow[t]{2}{*}{$164: 17$} & Upper half & $<13.9$ & $<14.3$ & $<14.1$ \\
\hline S96T005041 & & Lower half & $<15.2$ & $<15.1$ & $<15.15$ \\
\hline S96T005064 & \multirow[t]{2}{*}{$164: 19$} & Upper half & 48.9 & 52.2 & 50.55 \\
\hline S96T005045 & & Lower half & $<14.5$ & $<14.4$ & $<14.45$ \\
\hline S96T005282 & \multirow[t]{2}{*}{$164: 20$} & Upper half & 7.46 & 8.82 & 8.14 \\
\hline S96T005279 & & Lower half & 9.85 & 8.56 & 9.205 \\
\hline S96T005976 & Core 164 & Composite & 16.4 & 13.6 & $15^{\text {QC:a }}$ \\
\hline
\end{tabular}


Table B2-23. Tank 241-AN-104 Analytical Results: Lithium (ICP). (3 sheets)

\begin{tabular}{|c|c|c|c|c|c|}
\hline $\begin{array}{l}\text { Sariple } \\
\text { niminer: }\end{array}$ & Somperpe & Sample & Resilit & Bilinilic: & Wran \\
\hline \multicolumn{3}{|c|}{ Solids Irision : } & 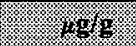 & $14 \mathrm{~g}$ & 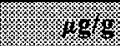 \\
\hline S96T005284 & $163: 1$ & Lower half & $<187$ & $<187$ & $<187$ \\
\hline S96T005285 & $163: 2$ & Lower half & $<179$ & $<191$ & $<185$ \\
\hline \$96T005780 & $163: 12$ & Lower half & $<188$ & $<190$ & $<189$ \\
\hline S96T005747 & \multirow[t]{2}{*}{$163: 14$} & Upper half & $<224$ & $<225$ & $<224.5$ \\
\hline S96T005748 & & Lower half & $<187$ & $<193$ & $<190$ \\
\hline S96T005293 & \multirow[t]{2}{*}{$163: 16$} & Upper half & $<177$ & $<190$ & $<183.5$ \\
\hline S96T005286 & & Lower half & $<199$ & $<183$ & $<191$ \\
\hline S96T005777 & \multirow[t]{2}{*}{$163: 18$} & Upper half & $<205$ & $<198$ & $<201.5$ \\
\hline S96T005781 & & Lower half & $<213$ & $<214$ & $<213.5$ \\
\hline S96T005556 & \multirow[t]{2}{*}{$163: 20$} & Upper half & $<185$ & $<194$ & $<189.5$ \\
\hline S96T005559 & & Lower half & $<195$ & $<195$ & $<195$ \\
\hline$\$ 96 \mathrm{~T} 004795$ & $164: 1$ & Lower half & $<204$ & $<201$ & $<202.5$ \\
\hline S96T004796 & $164: 13$ & Lower half & $<206$ & $<207$ & $<206.5$ \\
\hline S96T005035 & \multirow[t]{2}{*}{$164: 14$} & Upper half & $<204$ & $<206$ & $<205$ \\
\hline S96T005030 & & Lower half & $<205$ & $<204$ & $<204.5$ \\
\hline S96T005036 & \multirow[t]{2}{*}{$164: 15$} & Upper half & $<201$ & $<197$ & $<199$ \\
\hline S96T005032 & & Lower half & $<197$ & $<200$ & $<198.5$ \\
\hline S96T005037 & \multirow[t]{2}{*}{$164: 16$} & Upper half & $<192$ & $<183$ & $<187.5$ \\
\hline S96T005033 & & Lower half & $<191$ & $<187$ & $<189$ \\
\hline S96T005038 & \multirow[t]{2}{*}{$164: 17$} & Upper half & $<193$ & $<190$ & $<191.5$ \\
\hline S96T005031 & & Lower half & $<218$ & $<217$ & $<217.5$ \\
\hline S96T005039 & \multirow[t]{2}{*}{$164: 19$} & Upper half & $<194$ & $<200$ & $<197$ \\
\hline S96T005034 & & Lower half & $<214$ & $<217$ & $<215.5$ \\
\hline S96T005281 & \multirow[t]{2}{*}{$164: 20$} & Upper half & $<172$ & $<185$ & $<178.5$ \\
\hline S96T005278 & & Lower half & $<189$ & $<193$ & $<191$ \\
\hline
\end{tabular}


Table B2-23. Tank 241-AN-104 Analytical Results: Lithium (ICP). (3 sheets)

\begin{tabular}{|c|c|c|c|c|c|}
\hline Winger & Nom & 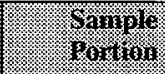 & mesulin & 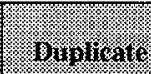 & 1. 2 an. \\
\hline Horum & $4_{10} x^{2}$ & & (4) & $f_{1}$ & 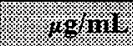 \\
\hline S96T005256 & $163: 2$ & Liquid & 12.5 & 14.7 & 13.6 \\
\hline S96T005260 & $163: 4$ & Liquid & 7.19 & 6.01 & 6.6 \\
\hline S96T005527 & $163: 5$ & Liquid & $<6.01$ & $<6.01$ & $<6.01$ \\
\hline S96T005554 & $163: 6$ & Liquid & $<6.01$ & $<6.01$ & $<6.01$ \\
\hline S96T005255 & $163: 7$ & Liquid & 21.2 & 22.5 & 21.85 \\
\hline S96T005257 & $163: 8$ & Liquid & $<6.01$ & 6.45 & $<6.23$ \\
\hline S96T005258 & $163: 9$ & Liquid & 8.97 & 8.9 & 8.935 \\
\hline \$96T005528 & $163: 10$ & Liquid & $<6.01$ & $<6.01$ & $<6.01$ \\
\hline S96T005739 & $163: 11$ & Liquid & $<6.01$ & $<6.01$ & $<6.01$ \\
\hline S96T005766 & $163: 12$ & Liquid & $<6.01$ & $<6.01$ & $<6.01$ \\
\hline S96T005741 & $163: 14$ & Liquid & $<6.01$ & $<6.01$ & $<6.01$ \\
\hline S96T004774 & $164: 1$ & Liquid & $<12$ & $<12$ & $<12$ \\
\hline S96T004778 & $164: 2$ & Liquid & $<12$ & $<12$ & $<12$ \\
\hline S96T004779 & $164: 3$ & Liquid & $<12$ & $<12$ & $<12$ \\
\hline S96T004780 & $164: 4$ & Liquid & 6.33 & $<6.01$ & $<6.17$ \\
\hline S96T004976 & $164: 5$ & Liquid & 46 & 10.5 & $28.25^{\mathrm{QC}: \mathrm{c}}$ \\
\hline S96T004781 & $164: 7$ & Liquid & 11 & 11.9 & 11.45 \\
\hline S96T004977 & $164: 8$ & Liquid & 18.6 & 28.7 & $23.65^{\mathrm{QC:e}}$ \\
\hline S96T004978 & $164: 9$ & Liquid & 9.56 & 7.3 & $8.43^{\mathrm{QC}: \mathrm{c}}$ \\
\hline S96T004979 & $164: 10$ & Liquid & 15 & 13 & 14 \\
\hline S96T004782 & $164: 11$ & Liquid & 46.9 & 24.5 & $35.7^{\mathrm{Cc} \mathrm{c}}$ \\
\hline S96T004783 & $164: 12$ & Liquid & $<6 . \overline{01}$ & $<6.01$ & $<6.01$ \\
\hline S96T004784 & $164: 13$ & Liquid & 7.43 & 7.8 & 7.615 \\
\hline S96T005979 & Core 164 & Composite & $<12$ & $<12$ & $<12$ \\
\hline
\end{tabular}


Table B2-24. Tank 241-AN-104 Analytical Results: Magnesium (ICP). (3 sheets)

\begin{tabular}{|c|c|c|c|c|c|}
\hline Mannife & 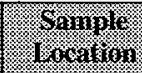 & 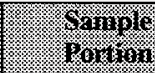 & Rosiln & 861) & Hean. \\
\hline \multicolumn{3}{|c|}{ 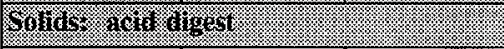 } & 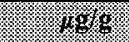 & 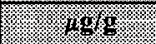 & . \\
\hline S96T005287 & $163: 1$ & Lower half & $<37.9$ & $<39.3$ & $<38.6$ \\
\hline S96T005288 & $163: 2$ & Lower half & $<44.3$ & $<37.5$ & $<40.9$ \\
\hline S96T005782 & $163: 12$ & Lower half & $<45.7$ & $<46.1$ & $<45.9$ \\
\hline S96T005749 & \multirow[t]{2}{*}{$163: 14$} & Upper half & $<44.8$ & $<43.6$ & $<44.2$ \\
\hline S96T005750 & & Lower half & $<45.9$ & $<45.2$ & $<45.55$ \\
\hline \$96T005294 & \multirow[t]{2}{*}{$163: 16$} & Upper half & $<41.3$ & $<37$ & $<39.15$ \\
\hline S96T005289 & & Lower half & $<46.4$ & $<42.6$ & $<44.5$ \\
\hline S96T005778 & \multirow[t]{2}{*}{$163: 18$} & Upper half & $<48.1$ & $<45.4$ & $<46.75$ \\
\hline S96T005783 & & Lower half & $<47.4$ & $<47.2$ & $<47.3$ \\
\hline S96T005557 & \multirow[t]{2}{*}{$163: 20$} & Upper half & $<38$ & $<40.1$ & $<39.05$ \\
\hline S96T005560 & & Lower half & $<44.2$ & $<37.3$ & $<40.75$ \\
\hline S96T004797 & $164: 1$ & Lower half & $<46.9$ & $<47.1$ & $<47$ \\
\hline S96T004798 & $164: 13$ & Lower half & $<48.4$ & $<47.7$ & $<48.05$ \\
\hline S96T005042 & \multirow[t]{2}{*}{$164: 14$} & Upper half & $<47.4$ & $<48$ & $<47.7$ \\
\hline S96T005040 & & Lower half & $<47.7$ & $<47$ & $<47.35$ \\
\hline S96T005061 & \multirow[t]{2}{*}{$164: 15$} & Upper half & $<48.3$ & $<48$ & $<48.15$ \\
\hline S96T005043 & & Lower half & $<46.4$ & $<47.7$ & $<47.05$ \\
\hline \$96T005062 & \multirow[t]{2}{*}{$164: 16$} & Upper half & $<151$ & $<149$ & $<150$ \\
\hline \$96T005044 & & Lower half & $<147$ & $<150$ & $<148.5$ \\
\hline S96T005063 & \multirow[t]{2}{*}{$164: 17$} & Upper half & $<139$ & $<143$ & $<141$ \\
\hline S96T005041 & & Lower half & $<152$ & $<151$ & $<151.5$ \\
\hline S96T005064 & \multirow[t]{2}{*}{$164: 19$} & Upper half & $<151$ & $<153$ & $<152$ \\
\hline S96T005045 & & Lower half & $<145$ & $<144$ & $<144.5$ \\
\hline S96T005282 & \multirow[t]{2}{*}{$164: 20$} & Upper half & $<39.3$ & $<36.1$ & $<37.7$ \\
\hline S96T005279 & & Lower half & $<45.4$ & $<37.8$ & $<41.6$ \\
\hline S96T005976 & Core 164 & Composite & $<39.8$ & $<39.8$ & $<39.8^{\mathrm{QC:a}}$ \\
\hline
\end{tabular}


Table B2-24. Tank 241-AN-104 Analytical Results: Magnesium (ICP). (3 sheets)

\begin{tabular}{|c|c|c|c|c|c|}
\hline Wample & (6.14) & \% & Seran & 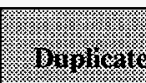 & . \\
\hline 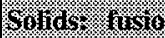 & & & 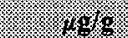 & \% & 16. \\
\hline S96T005284 & $163: 1$ & Lower half & $<1,870$ & $<1,870$ & $<1,870$ \\
\hline S96T005285 & $163: 2$ & Lower half & $<1,790$ & $<1,910$ & $<1,850$ \\
\hline S96T005780 & $163: 12$ & Lower half & $<1,880$ & $<1,900$ & $<1,890$ \\
\hline S96T005747 & \multirow[t]{2}{*}{$163: 14$} & Upper half & $<2,240$ & $<2,250$ & $<2,245$ \\
\hline S96T005748 & & Lower half & $<1,870$ & $<1,930$ & $<1,900$ \\
\hline S96T005293 & \multirow[t]{2}{*}{$163: 16$} & Upper half & $<1,770$ & $<1,900$ & $<1,835$ \\
\hline S96T005286 & & Lower half & $<1,990$ & $<1,830$ & $<1,910$ \\
\hline S96T005777 & \multirow[t]{2}{*}{$163: 18$} & Upper half & $<2,050$ & $<1,980$ & $<2,015$ \\
\hline S96T005781 & & Lower half & $<2,130$ & $<2,140$ & $<2,135$ \\
\hline S96T005556 & \multirow[t]{2}{*}{$163: 20$} & Upper half & $<1,850$ & $<1,940$ & $<1,895$ \\
\hline S96T005559 & & Lower half & $<1,950$ & $<1,950$ & $<1,950$ \\
\hline S96T004795 & $164: 1$ & Lower half & $<2,040$ & $<2,010$ & $<2,025$ \\
\hline S96T004796 & $164: 13$ & Lower half & $<2,060$ & $<2,070$ & $<2,065$ \\
\hline S96T005035 & \multirow[t]{2}{*}{$164: 14$} & Upper half & $<2,040$ & $<2,060$ & $<2,050$ \\
\hline S96T005030 & & Lower half & $<2,050$ & $<2,040$ & $<2,045$ \\
\hline S96T005036 & \multirow[t]{2}{*}{$164: 15$} & Upper half & $<2,010$ & $<1,970$ & $<1,990$ \\
\hline S96T005032 & & Lower half & $<1,970$ & $<2,000$ & $<1,985$ \\
\hline S96T005037 & \multirow[t]{2}{*}{$164: 16$} & Upper half & $<1,920$ & $<1,830$ & $<1,875$ \\
\hline S96T005033 & & Lower half & $<1,910$ & $<1,870$ & $<1,890$ \\
\hline S96T005038 & \multirow[t]{2}{*}{$164: 17$} & Upper half & $<1,930$ & $<1,900$ & $<1,915$ \\
\hline S96T005031 & & Lower half & $<2,180$ & $<2,170$ & $<2,175$ \\
\hline S96T005039 & \multirow[t]{2}{*}{$164: 19$} & Upper half & $<1,940$ & $<2,000$ & $<1,970$ \\
\hline S96T005034 & & Lower half & $<2,140$ & $<2,170$ & $<2,155$ \\
\hline S96T005281 & \multirow[t]{2}{*}{$164: 20$} & Upper half & $<1,720$ & $<1,850$ & $<1,785$ \\
\hline S96T005278 & & Lower half & $<1,890$ & $<1,930$ & $<1,910$ \\
\hline
\end{tabular}


Table B2-24. Tank 241-AN-104 Analytical Results: Magnesium (ICP). (3 sheets)

\begin{tabular}{|c|c|c|c|c|c|}
\hline $\begin{array}{l}\text { Sampole } \\
\text { Number: }\end{array}$ & $\begin{array}{l}\text { Sample: } \\
\text { I } 00 \text { cation }\end{array}$ & $\begin{array}{l}\text { Sampile } \\
\text { Eortion: }\end{array}$ & Iroul & Bipilicater: & 11.20 \\
\hline Tiguids & I. & 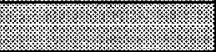 & 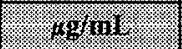 & $18 \% \mathrm{mi}$ & 4.96114 \\
\hline S96T005256 & $163: 2$ & Liquid & $<60.1$ & $<60.1$ & $<60.1$ \\
\hline S96T005260 & $163: 4$ & Liquid & $<60.1$ & $<60.1$ & $<60.1$ \\
\hline S96T005527 & $163: 5$ & Liquid & $<60.1$ & $<60.1$ & $<60.1$ \\
\hline S96T005554 & $163: 6$ & Liquid & $<60.1$ & $<60.1$ & $<60.1$ \\
\hline S96T005255 & $163: 7$ & Liquid & $<60.1$ & $<60.1$ & $<60.1$ \\
\hline S96T005257 & $163: 8$ & Liquid & $<60.1$ & $<60.1$ & $<60.1$ \\
\hline S96T005258 & $163: 9$ & Liquid & $<60.1$ & $<60.1$ & $<60.1$ \\
\hline S96T005528 & $163: 10$ & Liquid & $<60.1$ & $<60.1$ & $<60.1$ \\
\hline S96T005739 & $163: 11$ & Liquid & $<60.1$ & $<60.1$ & $<60.1$ \\
\hline S96T005766 & $163: 12$ & Liquid & $<60.1$ & $<60.1$ & $<60.1$ \\
\hline S96T005741 & $163: 14$ & Liquid & $<60.1$ & $<60.1$ & $<60.1$ \\
\hline S96T004774 & 164:1 & Liquid & $<120$ & $<120$ & $<120$ \\
\hline S96T004778 & $164: 2$ & Liquid & $<120$ & $<120$ & $<120$ \\
\hline S96T004779 & $164: 3$ & Liquid & $<120$ & $<120$ & $<120$ \\
\hline S96T004780 & $164: 4$ & Liquid & $<60.1$ & $<60.1$ & $<60.1$ \\
\hline S96T004976 & $164: 5$ & Liquid & $<60.1$ & $<60.1$ & $<60.1$ \\
\hline S96T004781 & $164: 7$ & Liquid & $<60.1$ & $<60.1$ & $<60.1$ \\
\hline S96T004977 & $164: 8$ & Liquid & $<60.1$ & $<60.1$ & $<60.1$ \\
\hline S96T004978 & $164: 9$ & Liquid & $<60.1$ & $<60.1$ & $<60.1$ \\
\hline S96T004979 & $164: 10$ & Liquid & $<60.1$ & $<60.1$ & $<60.1$ \\
\hline S96T004782 & $164: 11$ & Liquid & $<60.1$ & $<60.1$ & $<60.1$ \\
\hline S96T004783 & $164: 12$ & Liquid & $<60.1$ & $<60.1$ & $<60.1$ \\
\hline S96T004784 & $164: 13$ & Liquid & $<60.1$ & $<60.1$ & $<60.1$ \\
\hline S96T005979 & Core 164 & Composite & $<120$ & $<120$ & $<120$ \\
\hline
\end{tabular}


Table B2-25. Tank 241-AN-104 Analytical Results: Manganese (ICP). (3 sheets)

\begin{tabular}{|c|c|c|c|c|c|}
\hline 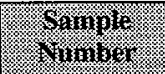 & 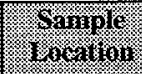 & 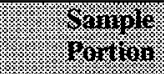 & Resurit & 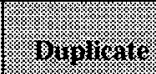 & 4 \\
\hline \multicolumn{3}{|c|}{ 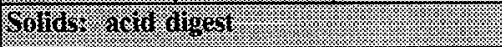 } & 6 & 18. & f. \\
\hline S96T005287 & $163: 1$ & Lower half & 18 & 18.2 & 18.1 \\
\hline S96T005288 & $163: 2$ & Lower half & 9.7 & 9.38 & 9.54 \\
\hline S96T005782 & $163: 12$ & Lower half & 13 & 12.6 & 12.8 \\
\hline S96T005749 & \multirow[t]{2}{*}{$163: 14$} & Upper half & 11.5 & 11.8 & 11.65 \\
\hline S96T005750 & & Lower half & 13.8 & 13.4 & 13.6 \\
\hline S96T005294 & \multirow[t]{2}{*}{$163: 16$} & Upper half & 16.8 & 13.3 & $15.05^{\mathrm{QC}: \mathrm{e}}$ \\
\hline S96T005289 & & Lower half & 16.5 & 13.2 & $14.85^{\mathrm{QC}: \mathrm{c}}$ \\
\hline S96T005778 & \multirow[t]{2}{*}{$163: 18$} & Upper half & 13.4 & 14.1 & 13.75 \\
\hline S96T005783 & & Lower half & 12.9 & 12.9 & 12.9 \\
\hline S96T005557 & \multirow[t]{2}{*}{$163: 20$} & Upper half & 15.3 & 15.1 & 15.2 \\
\hline S96T005560 & & Lower half & 26.1 & 20.7 & $23.4^{\mathrm{ec}: c}$ \\
\hline S96T004797 & $164: 1$ & Lower half & 8.22 & 8.3 & 8.26 \\
\hline S96T004798 & $164: 13$ & Lower half & 39 & 41.1 & 40.05 \\
\hline S96T005042 & \multirow[t]{2}{*}{$164: 14$} & Upper half & 19 & 18.8 & 18.9 \\
\hline S96T005040 & & Lower half & 11.1 & 10.9 & 11 \\
\hline$\$ 96 T 005061$ & \multirow[t]{2}{*}{$164: 15$} & Upper half & 15 & 15.3 & 15.15 \\
\hline S96T005043 & & Lower half & 27.1 & 26.2 & 26.65 \\
\hline \$96T005062 & \multirow[t]{2}{*}{$164: 16$} & Upper half & $<15.1$ & $<14.9$ & $<15$ \\
\hline S96T005044 & & Lower half & 15.8 & 15.6 & 15.7 \\
\hline S96T005063 & \multirow[t]{2}{*}{$164: 17$} & Upper half & 14.5 & 15.3 & 14.9 \\
\hline S96T005041 & & Lower half & 21.2 & 21.6 & 21.4 \\
\hline S96T005064 & \multirow[t]{2}{*}{$164: 19$} & Upper half & 27.3 & 28.1 & 27.7 \\
\hline S96T005045 & & Lower half & 21 & 23.9 & 22.45 \\
\hline \$96T005282 & \multirow[t]{2}{*}{$164: 20$} & Upper half & 17.8 & 14.7 & 16.25 \\
\hline S96T005279 & & Lower half & 17.5 & 11 & $14.25^{\mathrm{QC}: \mathrm{c}}$ \\
\hline S96T005976 & Core 164 & Composite & 22.1 & 19.6 & $20.85^{\mathrm{QC:a}}$ \\
\hline
\end{tabular}


Table B2-25. Tank 241-AN-104 Analytical Results: Manganese (ICP). (3 sheets)

\begin{tabular}{|c|c|c|c|c|c|}
\hline Maninge & Sorvoles & f. & 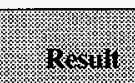 & . & 19.1\% \\
\hline \multicolumn{2}{|c|}{ 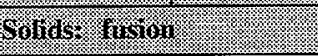 } & & 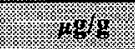 & (18: & 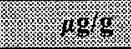 \\
\hline S96T005284 & $163: 1$ & Lower half & $<187$ & $<187$ & $<187$ \\
\hline S96T005285 & $163: 2$ & Lower half & $<179$ & $<191$ & $<185$ \\
\hline S96T005780 & $163: 12$ & Lower half & $<188$ & $<190$ & $<189$ \\
\hline S96T005747 & \multirow[t]{2}{*}{ 163:14 } & Upper half & $<224$ & $<225$ & $<224.5$ \\
\hline S96T005748 & & Lower half & $<187$ & $<193$ & $<190$ \\
\hline S96T005293 & \multirow[t]{2}{*}{$163: 16$} & Upper half & $<177$ & $<190$ & $<183.5$ \\
\hline S96T005286 & & Lower half & $<199$ & $<183$ & $<191$ \\
\hline$\$ 96 \mathrm{~T} 005777$ & \multirow[t]{2}{*}{$163: 18$} & Upper half & $<205$ & 325 & $<265^{\mathrm{QC:c}}$ \\
\hline S96T005781 & & Lower half & $<213$ & $<214$ & $<213.5$ \\
\hline S96T005556 & \multirow[t]{2}{*}{$163: 20$} & Upper half & $<185$ & $<194$ & $<189.5$ \\
\hline S96T005559 & & Lower half & $<195$ & $<195$ & $<195$ \\
\hline S96T004795 & $164: 1$ & Lower half & $<204$ & $<201$ & $<202.5$ \\
\hline S96T004796 & $164: 13$ & Lower half & $<206$ & $<207$ & $<206.5$ \\
\hline S96T005035 & \multirow[t]{2}{*}{$164: 14$} & Upper half & $<204$ & $<206$ & $<205$ \\
\hline \$96T005030 & & Lower half & $<205$ & $<204$ & $<204.5$ \\
\hline S96T005036 & \multirow[t]{2}{*}{$164: 15$} & Upper half & $<201$ & $<197$ & $<199$ \\
\hline S96T005032 & & Lower half & $<197$ & $<200$ & $<198.5$ \\
\hline S96T005037 & \multirow[t]{2}{*}{$164: 16$} & Upper half & $<192$ & $<183$ & $<187.5$ \\
\hline S96T005033 & & Lower half & $<191$ & $<187$ & $<189$ \\
\hline S96T005038 & \multirow[t]{2}{*}{$164: 17$} & Upper half & $<193$ & $<190$ & $<191.5$ \\
\hline S96T005031 & & Lower half & $<218$ & $<217$ & $<217.5$ \\
\hline S96T005039 & \multirow[t]{2}{*}{$164: 19$} & Upper half & $<194$ & $<200$ & $<197$ \\
\hline S96T005034 & & Lower half & $<214$ & $<217$ & $<215.5$ \\
\hline \$96T005281 & \multirow[t]{2}{*}{$164: 20$} & Upper half & $<172$ & $<185$ & $<178.5$ \\
\hline \$96T005278 & & Lower half & $<189$ & $<193$ & $<191$ \\
\hline
\end{tabular}


Table B2-25. Tank 241-AN-104 Analytical Results: Manganese (ICP). (3 sheets)

\begin{tabular}{|c|c|c|c|c|c|}
\hline 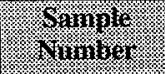 & 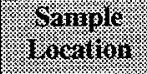 & 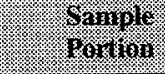 & $\mathrm{Resen}$ & (3) & Hin \\
\hline \% & & 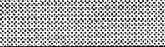 & 1084111 & 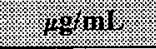 & (6.) \\
\hline S96T005256 & $163: 2$ & Liquid & $<6.01$ & $<6.01$ & $<6.01$ \\
\hline S96T005260 & $163: 4$ & Liquid & $<6.01$ & $<6.01$ & $<6.01$ \\
\hline S96T005527 & $163: 5$ & Liquid & $<6.01$ & $<6.01$ & $<6.01$ \\
\hline S96T005554 & $163: 6$ & Liquid & $<6.01$ & $<6.01$ & $<6.01$ \\
\hline S96T005255 & $163: 7$ & Liquid & $<6.01$ & $<6.01$ & $<6.01$ \\
\hline S96T005257 & $163: 8$ & Liquid & $<6.01$ & $<6.01$ & $<6.01$ \\
\hline S96T005258 & $163: 9$ & Liquid & $<6.01$ & $<6.01$ & $<6.01$ \\
\hline S96T005528 & $163: 10$ & Liquid & $<6.01$ & $<6.01$ & $<6.01$ \\
\hline \$96T005739 & $163: 11$ & Liquid & $<6.01$ & $<6.01$ & $<6.01$ \\
\hline S96T005766 & $163: 12$ & Liquid & $<6.01$ & $<6.01$ & $<6.01$ \\
\hline S96T005741 & $163: 14$ & Liquid & $<6.01$ & $<6.01$ & $<6.01$ \\
\hline S96T004774 & $164: 1$ & Liquid & $<12$ & $<12$ & $<12$ \\
\hline S96T004778 & $164: 2$ & Liquid & $<12$ & $<12$ & $<12$ \\
\hline S96T004779 & $164: 3$ & Liquid & $<12$ & $<12$ & $<12$ \\
\hline S96T004780 & $164: 4$ & Liquid & $<6.01$ & $<6.01$ & $<6.01$ \\
\hline S96T004976 & $164: 5$ & Liquid & $<6.01$ & $<6.01$ & $<6.01$ \\
\hline S96T004781 & $164: 7$ & Liquid & $<6.01$ & $<6.01$ & $<6.01$ \\
\hline S96T004977 & $164: 8$ & Liquid & $<6.01$ & $<6.01$ & $<6.01$ \\
\hline S96T004978 & $164: 9$ & Liquid & $<6.01$ & $<6.01$ & $<6.01$ \\
\hline S96T004979 & $164: 10$ & Liquid & $<6.01$ & $<6.01$ & $<6.01$ \\
\hline S96T004782 & $164: 11$ & Liquid & $<6.01$ & $<6.01$ & $<6.01$ \\
\hline S96T004783 & $164: 12$ & Liquid & $<6.01$ & $<6.01$ & $<6.01$ \\
\hline S96T004784 & $164: 13$ & Liquid & $<6.01$ & $<6.01$ & $<6.01$ \\
\hline S96T005979 & Core 164 & Composite & $<12$ & $<12$ & $<12$ \\
\hline
\end{tabular}


HNF-SD-WM-ER-690 Rev. 0

Table B2-26. Tank 241-AN-104 Analytical Results: Molybdenum (ICP). (3 sheets)

\begin{tabular}{|c|c|c|c|c|c|}
\hline STinolo & Samportiou & 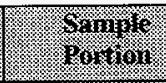 & n.rill & Minlirate & 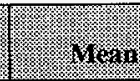 \\
\hline \multicolumn{2}{|c|}{ Soldas idil aigess } & (3) & 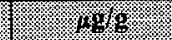 & 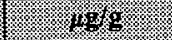 & (3) \\
\hline S96T005287 & $163: 1$ & Lower half & 44.1 & 43.2 & 43.65 \\
\hline S96T005288 & $163: 2$ & Lower half & 37.4 & 36.8 & 37.1 \\
\hline S96T005782 & $163: 12$ & Lower half & 45.2 & 42.6 & 43.9 \\
\hline S96T005749 & \multirow[t]{2}{*}{$163: 14$} & Upper half & 42 & 44.1 & 43.05 \\
\hline S96T005750 & & Lower half & 45.2 & 45.4 & 45.3 \\
\hline S96T005294 & \multirow[t]{2}{*}{$163: 16$} & Upper half & 41.6 & 41.7 & 41.65 \\
\hline S96T005289 & & Lower half & 42.2 & 44.7 & 43.45 \\
\hline S96T005778 & \multirow[t]{2}{*}{$163: 18$} & Upper half & 36.8 & 38.4 & 37.6 \\
\hline S96T005783 & & Lower half & 38 & 37.5 & 37.75 \\
\hline S96T005557 & \multirow[t]{2}{*}{$163: 20$} & Upper half & 37.3 & 38.5 & 37.9 \\
\hline S96T005560 & & Lower half & 40.6 & 33.1 & $36.85^{\mathrm{QC}: c}$ \\
\hline \$96T004797 & $164: 1$ & Lower half & 42.4 & 43.7 & 43.05 \\
\hline S96T004798 & $164: 13$ & Lower half & 44.7 & 48.5 & 46.6 \\
\hline S96T005042 & \multirow[t]{2}{*}{$164: 14$} & Upper half & 45 & 43.9 & 44.45 \\
\hline S96T005040 & & Lower half & 46.9 & 49.4 & 48.15 \\
\hline S96T005061 & \multirow[t]{2}{*}{$164: 15$} & Upper half & 42.9 & 45.1 & 44 \\
\hline S96T005043 & & Lower half & 44.1 & 44.2 & 44.15 \\
\hline S96T005062 & \multirow[t]{2}{*}{$164: 16$} & Upper half & $<75.3$ & $<74.7$ & $<75$ \\
\hline S96T005044 & & Lower half & $<73.7$ & $<75$ & $<74.35$ \\
\hline \$96T005063 & \multirow[t]{2}{*}{$164: 17$} & Upper half & $<69.7$ & $<71.4$ & $<70.55$ \\
\hline S96T005041 & & Lower half & $<76.2$ & $<75.6$ & $<75.9$ \\
\hline S96T005064 & \multirow[t]{2}{*}{$164: 19$} & Upper half & $<75.6$ & $<76.7$ & $<76.15$ \\
\hline S96T005045 & & Lower half & $<72.5$ & $<72$ & $<72.25$ \\
\hline \begin{tabular}{|l|} 
S96T005282 \\
\end{tabular} & \multirow[t]{2}{*}{$164: 20$} & Upper half & 44.7 & 43.8 & 44.25 \\
\hline S96T005279 & & Lower half & 40.5 & 43.3 & 41.9 \\
\hline S96T005976 & Core 164 & Composite & 43.3 & 43.5 & 43.4 \\
\hline
\end{tabular}




\begin{tabular}{|c|c|c|c|c|c|}
\hline$s^{\circ}+56>$ & $996>$ & $\varepsilon \pm 6>$ & I[EY IәMOT] & & $8 L Z 500 L 96 S$ \\
\hline$s^{2} 268>$ & $\$ 26>$ & $098>$ & fley Iədd $\Pi$ & $0 z: \nabla 9 I$ & I8ZS00L96S \\
\hline $080^{6} I>$ & $060^{\circ} \mathrm{I}>$ & $0 \angle 0^{\circ} I>$ & J[ЕU Iәмот & \multirow[b]{2}{*}{$6 I: 79 I$} & $\nabla \varepsilon 0 S 00 \mathrm{~L} 96 \mathrm{~S}$ \\
\hline $986>$ & $000^{\circ} I>$ & $\tau L 6>$ & J[eч radd $\Omega$ & & $6 \mathcal{E} 0 S 00 \mathrm{~L} 96 \mathrm{~S}$ \\
\hline$\$ 80^{\circ} \mathrm{I}>$ & $080^{\circ} \mathrm{I}>$ & $060^{\circ} I>$ & I[Еप ІәмоТ] & \multirow[b]{2}{*}{$L I: \nabla 9 I$} & IEOS00L96S \\
\hline$S \angle S 6>$ & $6 t 6>$ & $996>$ & $\pm[$ சu Iədd $\Omega$ & & $8 \mathcal{E} 0500 L 96 \mathrm{~S}$ \\
\hline$s+6>$ & $\varepsilon \varepsilon 6>$ & $\angle S 6>$ & IIRप Iәмот & \multirow[b]{2}{*}{$9 I: \neq 9 I$} & EEOSO0L96S \\
\hline $8 \mathcal{E} 6>$ & SI6> & $196>$ & $f^{e \varphi}$ دədd $\Omega$ & & $\angle \varepsilon 0 S 00 \mathrm{~L} 96 \mathrm{~S}$ \\
\hline$\varsigma^{\cdot} \varepsilon 66>$ & $000^{6} I>$ & $\angle 86>$ & IlEप Iәмот & \multirow[b]{2}{*}{$S I: t 9 I$} & ZE0S00L96S \\
\hline $5.166>$ & $\varepsilon 86>$ & $000^{\prime} I>$ & 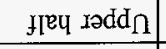 & & $9 \varepsilon 0500 L 96 \mathrm{~S}$ \\
\hline $020^{\prime} I>$ & $0 Z 0^{\circ} \mathrm{I}>$ & $020^{\circ} 1>$ & fleप дамот & \multirow[b]{2}{*}{$t I: t 9 I$} & OE0SO0L96S \\
\hline$S 20^{\circ} \mathrm{I}>$ & $0 \varepsilon 0^{6} \mathrm{I}>$ & $020^{\circ} \mathrm{I}>$ & 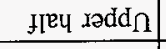 & & $\subseteq \varepsilon 0 \subseteq 00 L 96 \mathrm{~S}$ \\
\hline$\$ \varepsilon 0^{6} \mathrm{I}>$ & $0+0^{6} I>$ & $0 \varepsilon 0^{\circ} I>$ & 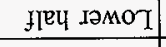 & $\varepsilon I: \neq 9 I$ & $96 \angle t 00 \mathrm{~L} 96 \mathrm{~S}$ \\
\hline $0 \mathrm{IO}^{6} \mathrm{I}>$ & $000^{\circ} \mathrm{I}>$ & $020^{\circ} \mathrm{I}>$ & I[Еप IәMOT] & $I: t 9 I$ & $\subseteq 6 \angle t 00 L 96 \mathrm{~S}$ \\
\hline SL6> & $\varepsilon L 6>$ & $\angle L 6>$ & I'еч Iәмот & \multirow[b]{2}{*}{ 0Z:ع9I } & $6 \$ \subseteq \$ 00 L 96 \mathrm{~S}$ \\
\hline $976>$ & $896>$ & $\nabla 26>$ & f[вч Iədd $\Omega$ & & 9\$\$S00L96S \\
\hline$\$ 90^{\circ} I>$ & $0 \angle 0^{\circ} T>$ & $090^{\prime} I>$ & J[Еप دәмо I & \multirow[b]{2}{*}{$8 I: \varepsilon 9 I$} & I8LSO0L96S \\
\hline I I $0^{\prime} I>$ & $266>$ & $0 \varepsilon 0^{\circ} \mathrm{I}>$ & I[Eप Iədd $\Omega$ & & LLLSO0L96S \\
\hline$\$ \$ 6>$ & $\angle \mathrm{I} 6>$ & $\varepsilon 66>$ & fleप гәмот & \multirow[b]{2}{*}{ 9I:\&9I } & 98ZS00L.96S \\
\hline $8 \mathrm{I} 6>$ & $6+6>$ & $\angle 88>$ & Iొе4 ıәdd & & E62\$00L96S \\
\hline$s \cdot 8 t 6>$ & $\varepsilon 96>$ & $\nabla \varepsilon 6>$ & J[Еप IәMOT & \multirow[b]{2}{*}{$\nabla I: E 9 I$} & $87 \angle S 00 L 96 \mathrm{~S}$ \\
\hline$S Z I^{\prime} I>$ & $0 E I^{\prime} I>$ & $0 Z I^{6} \mathrm{I}>$ & fleप Iədd $\cap$ & & $\angle t \angle S 00 L 96 \mathrm{~S}$ \\
\hline$S+6>$ & $0 \leq 6>$ & $0 \pm 6>$ & J[Eप IOMOT & ZI:E9I & 08LS00L96S \\
\hline$\$ 26>$ & $9 \$ 6>$ & $\nabla 68>$ & I[еप Iәмот & $z: \varepsilon 9 \mathrm{I}$ & S8ZS00L96S \\
\hline$s \cdot 9 \mathcal{E} 6>$ & $9 \varepsilon 6>$ & $\angle E G>$ & I[EY IәMOT & $\mathrm{I}: \varepsilon 9 \mathrm{I}$ & $782500 \mathrm{~L} 96 \mathrm{~S}$ \\
\hline 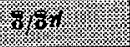 & \% & 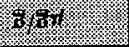 & & & mosib: \\
\hline 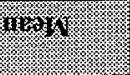 & (4) & 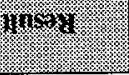 & 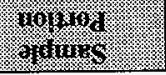 & mon & \%). \\
\hline
\end{tabular}

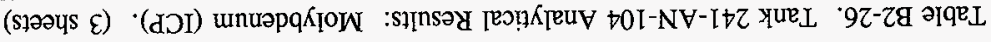


Table B2-26. Tank 241-AN-104 Analytical Results: Molybdenum (ICP). (3 sheets)

\begin{tabular}{|c|c|c|c|c|c|}
\hline 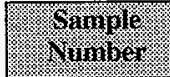 & Som & (1) & 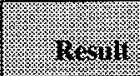 & 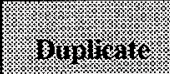 & 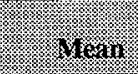 \\
\hline Hoins. & & (3. & 4 & 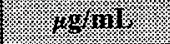 & 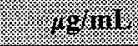 \\
\hline S96T005256 & $163: 2$ & Liquid & 77 & 85.4 & 81.2 \\
\hline S96T005260 & $163: 4$ & Liquid & 83.2 & 77.5 & 80.35 \\
\hline S96T005527 & $163: 5$ & Liquid & 77.5 & 76.6 & 77.05 \\
\hline S96T005554 & $163: 6$ & Liquid & 80.5 & 79.7 & 80.1 \\
\hline S96T005255 & $163: 7$ & Liquid & 84.3 & 89.6 & 86.95 \\
\hline S96T005257 & $163: 8$ & Liquid & 73 & 78.6 & 75.8 \\
\hline S96T005258 & $163: 9$ & Liquid & 80.4 & 77.9 & 79.15 \\
\hline S96T005528 & 163:10 & Liquid & 74.4 & 75.3 & 74.85 \\
\hline S96T005739 & $163: 11$ & Liquid & 77.3 & 79.6 & 78.45 \\
\hline S96T005766 & $163: 12$ & Liquid & 85.4 & 84.5 & 84.95 \\
\hline S96T005741 & $163: 14$ & Liquid & 75.4 & 79.4 & 77.4 \\
\hline \$96T004774 & $164: 1$ & Liquid & 80 & 82.8 & 81.4 \\
\hline S96T004778 & $164: 2$ & Liquid & 69.2 & 81.6 & 75.4 \\
\hline S96T004779 & $164: 3$ & Liquid & 80.3 & 76.4 & 78.35 \\
\hline$\$ 96 \mathrm{~T} 004780$ & $164: 4$ & Liquid & 80.2 & 82.9 & 81.55 \\
\hline \$96T004976 & $164: 5$ & Liquid & 87.4 & 82.7 & 85.05 \\
\hline S96T004781 & $164: 7$ & Liquid & 76.9 & 81.4 & 79.15 \\
\hline S96T004977 & $164: 8$ & Liquid & 91.9 & 78.1 & 85 \\
\hline S96T004978 & $164: 9$ & Liquid & 84.3 & 72.5 & 78.4 \\
\hline \$96T004979 & $164: 10$ & Liquid & 79.9 & 77.4 & 78.65 \\
\hline S96T004782 & 164:11 & Liquid & 78 & 81.1 & 79.55 \\
\hline S96T004783 & $164: 12$ & Liquid & 83.4 & 89.1 & 86.25 \\
\hline S96T004784 & $164: 13$ & Liquid & 79.2 & 83.2 & 81.2 \\
\hline S96T005979 & Core 164 & Composite & 88.9 & 85 & 86.95 \\
\hline
\end{tabular}


Table B2-27. Tank 241-AN-104 Analytical Results: Neodymium (ICP). (3 sheets)

\begin{tabular}{|c|c|c|c|c|c|}
\hline Humer & \% & 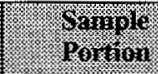 & 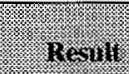 & 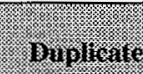 & (1) \\
\hline \multicolumn{2}{|c|}{ 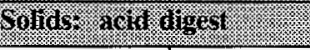 } & (3) & m. & $268 \%$ & 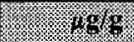 \\
\hline \$96T005287 & $163: 1$ & Lower half & $<37.9$ & $<39.3$ & $<38.6$ \\
\hline S96T005288 & $163: 2$ & Lower half & $<44.3$ & $<37.5$ & $<40.9$ \\
\hline S96T005782 & $163: 12$ & Lower half & $<45.7$ & $<46.1$ & $<45.9$ \\
\hline S96T005749 & \multirow[t]{2}{*}{$163: 14$} & Upper half & $<44.8$ & $<43.6$ & $<44.2$ \\
\hline S96T005750 & & Lower half & $<45.9$ & $<45.2$ & $<45.55$ \\
\hline \$96T005294 & \multirow[t]{2}{*}{$163: 16$} & Upper half & $<\overline{41.3}$ & $<37$ & $<39.15$ \\
\hline S96T005289 & & Lower half & $<46.4$ & $<42.6$ & $<44.5$ \\
\hline S96T005778 & \multirow[t]{2}{*}{$163: 18$} & Upper half & $<48.1$ & $<45.4$ & $<46.75$ \\
\hline \$96T005783 & & Lower half & $<47.4$ & $<47.2$ & $<47.3$ \\
\hline S96T005557 & \multirow[t]{2}{*}{$163: 20$} & Upper half & $<38$ & $<40.1$ & $<39.05$ \\
\hline S96T005560 & & Lower half & $<44.2$ & $<37.3$ & $<40.75$ \\
\hline S96T004797 & $164: 1$ & Lower half & $<46.9$ & $<47.1$ & $<47$ \\
\hline S96T004798 & $164: 13$ & Lower half & $<48.4$ & $<47.7$ & $<48.05$ \\
\hline S96T005042 & \multirow[t]{2}{*}{$164: 14$} & Upper half & $<47.4$ & $<48$ & $<47.7$ \\
\hline S96T005040 & & Lower half & $<47.7$ & $<47$ & $<47 . \overline{35}$ \\
\hline S96T005061 & \multirow[t]{2}{*}{$164: 15$} & Upper half & $<48.3$ & $<\overline{48}$ & $<48.15$ \\
\hline S96T005043 & & Lower half & $<46.4$ & $<47.7$ & $<47.05$ \\
\hline S96T005062 & \multirow[t]{2}{*}{$164: 16$} & Upper half & $<151$ & $<149$ & $<150$ \\
\hline S96T005044 & & Lower half & $<147$ & $<150$ & $<148.5$ \\
\hline S96T005063 & \multirow[t]{2}{*}{$164: 17$} & Upper half & $<139$ & $<143$ & $<141$ \\
\hline S96T005041 & & Lower half & $<152$ & $<151$ & $<151.5$ \\
\hline S96T005064 & \multirow[t]{2}{*}{$164: 19$} & Upper half & $<151$ & $<153$ & $<152$ \\
\hline \$96T005045 & & Lower half & $<145$ & $<144$ & $<144.5$ \\
\hline S96T005282 & \multirow[t]{2}{*}{$164: 20$} & Upper half & $<39.3$ & $<36.1$ & $<37.7$ \\
\hline S96T005279 & & Lower half & $<45.4$ & $<37.8$ & $<41.6$ \\
\hline S96T005976 & Core 164 & Composite & $<39.8$ & $<39.8$ & $<39.8^{\mathrm{QC}: a}$ \\
\hline
\end{tabular}


Table B2-27. Tank 241-AN-104 Analytical Results: Neodymium (ICP). (3 sheets)

\begin{tabular}{|c|c|c|c|c|c|}
\hline Shimple & 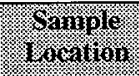 & (6) & (1) & 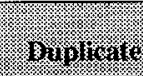 & 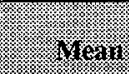 \\
\hline \multicolumn{3}{|c|}{ Soldigy MuSion } & 428 & 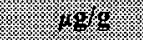 & 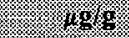 \\
\hline S96T005284 & $163: 1$ & Lower half & $<1,870$ & $<1,870$ & $<1,870$ \\
\hline S96T005285 & $163: 2$ & Lower half & $<1,790$ & $<1,910$ & $<1,850$ \\
\hline S96T005780 & $163: 12$ & Lower half & $<1,880$ & $<1,900$ & $<1,890$ \\
\hline \$96T005747 & \multirow[t]{2}{*}{$163: 14$} & Upper half & $<2,240$ & $<2,250$ & $<2,245$ \\
\hline S96T005748 & & Lower half & $<1,870$ & $<1,930$ & $<1,900$ \\
\hline S96T005293 & \multirow[t]{2}{*}{$163: 16$} & Upper half & $<1,770$ & $<1,900$ & $<1,835$ \\
\hline S96T005286 & & Lower half & $<1,990$ & $<1,830$ & $<1,910$ \\
\hline S96T005777 & \multirow[t]{2}{*}{$163: 18$} & Upper half & $<2,050$ & $<1,980$ & $<2,015$ \\
\hline S96T005781 & & Lower half & $<2,130$ & $<2,140$ & $<2,135$ \\
\hline S96T005556 & \multirow[t]{2}{*}{$163: 20$} & Upper half & $<1,850$ & $<1,940$ & $<1,895$ \\
\hline S96T005559 & & Lower half & $<1,950$ & $<1,950$ & $<1,950$ \\
\hline S96T004795 & $164: 1$ & Lower half & $<2,040$ & $<2,010$ & $<2,025$ \\
\hline \$96T004796 & $164: 13$ & Lower half & $<2,060$ & $<2,070$ & $<2,065$ \\
\hline \$96T005035 & \multirow[t]{2}{*}{$164: 14$} & Upper half & $<2,040$ & $<2,060$ & $<2,050$ \\
\hline S96T005030 & & Lower half & $<2,050$ & $<2,040$ & $<2,045$ \\
\hline S96T005036 & \multirow[t]{2}{*}{$164: 15$} & Upper half & $<2,010$ & $<1,970$ & $<1,990$ \\
\hline S96T005032 & & Lower half & $<1,970$ & $<2,000$ & $<1,985$ \\
\hline S96T005037 & \multirow[t]{2}{*}{$164: 16$} & Upper half & $<1,920$ & $<1,830$ & $<1,875$ \\
\hline S96T005033 & & Lower half & $<1,910$ & $<1,870$ & $<1,890$ \\
\hline S96T005038 & \multirow[t]{2}{*}{$164: 17$} & Upper half & $<1,930$ & $<1,900$ & $<1,915$ \\
\hline S96T005031 & & Lower half & $<2,180$ & $<2,170$ & $<2,175$ \\
\hline S96T005039 & \multirow[t]{2}{*}{$164: 19$} & Upper half & $<1,940$ & $<2,000$ & $<1,970$ \\
\hline S96T005034 & & Lower half & $<2,140$ & $<2,170$ & $<2,155$ \\
\hline S96T005281 & \multirow[t]{2}{*}{$164: 20$} & Upper half & $<1,720$ & $<1,850$ & $<1,785$ \\
\hline S96T005278 & & Lower half & $<1,890$ & $<1,930$ & $<1,910$ \\
\hline
\end{tabular}


HNF-SD-WM-ER-690 Rev. 0

Table B2-27. Tank 241-AN-104 Analytical Results: Neodymium (ICP). (3 sheets)

\begin{tabular}{|c|c|c|c|c|c|}
\hline 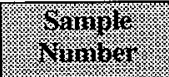 & Sorriple & ( & W & 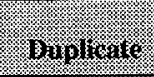 & 4 erain \\
\hline Wuras & & & 19610. & 897m! & (6) \\
\hline S96T005256 & $163: 2$ & Liquid & $<60.1$ & $<60.1$ & $<60.1$ \\
\hline \$96T005260 & $163: 4$ & Liquid & $<60.1$ & $<60.1$ & $<60.1$ \\
\hline \$96T005527 & $163: 5$ & Liquid & $<60.1$ & $<60.1$ & $<60.1$ \\
\hline S96T005554 & $163: 6$ & Liquid & $<60.1$ & $<60.1$ & $<60.1$ \\
\hline S96T005255 & $163: 7$ & Liquid & $<60.1$ & $<60.1$ & $<60.1$ \\
\hline S96T005257 & $163: 8$ & Liquid & $<60.1$ & $<60.1$ & $<60.1$ \\
\hline S96T005258 & $163: 9$ & Liquid & $<60.1$ & $<60.1$ & $<60.1$ \\
\hline S96T005528 & $163: 10$ & Liquid & $<60.1$ & $<60.1$ & $<60.1$ \\
\hline S96T005739 & $163: 11$ & Liquid & $<60.1$ & $<60.1$ & $<60.1$ \\
\hline S96T005766 & $163: 12$ & Liquid & $<60.1$ & $<60.1$ & $<60.1$ \\
\hline S96T005741 & $163: 14$ & Liquid & $<60.1$ & $<60.1$ & $<60.1$ \\
\hline S96T004774 & $164: 1$ & Liquid & $<120$ & $<120$ & $<120$ \\
\hline \$96T004778 & $164: 2$ & Liquid & $<120$ & $<120$ & $<120$ \\
\hline S96T004779 & $164: 3$ & Liquid & $<120$ & $<120$ & $<120$ \\
\hline S96T004780 & $164: 4$ & Liquid & $<60.1$ & $<60.1$ & $<60.1$ \\
\hline S96T004976 & $164: 5$ & Liquid & $<60.1$ & $<60.1$ & $<60.1$ \\
\hline S96T004781 & $164: 7$ & Liquid & $<60.1$ & $<60.1$ & $<60.1$ \\
\hline S96T004977 & $164: 8$ & Liquid & $<60.1$ & $<60.1$ & $<60.1$ \\
\hline S96T004978 & $164: 9$ & Liquid & $<60.1$ & $<60.1$ & $<60.1$ \\
\hline S96T004979 & $164: 10$ & Liquid & $<60.1$ & $<60.1$ & $<60.1$ \\
\hline S96T004782 & $164: 11$ & Liquid & $<60.1$ & $<60.1$ & $<60.1$ \\
\hline S96T004783 & $164: 12$ & Liquid & $<60.1$ & $<60.1$ & $<60.1$ \\
\hline S96T004784 & $164: 13$ & Liquid & $<60.1$ & $<60.1$ & $<60.1$ \\
\hline S96T005979 & Core 164 & Composite & $<120$ & $<120$ & $<120$ \\
\hline
\end{tabular}


Table B2-28. Tank 241-AN-104 Analytical Results: Nickel (ICP). (3 sheets)

\begin{tabular}{|c|c|c|c|c|c|}
\hline 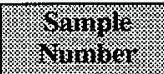 & 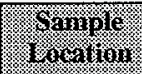 & 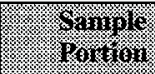 & Resura & 36. & Mean \\
\hline \multicolumn{2}{|c|}{ 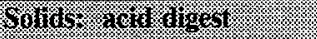 } & (3) & 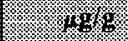 & \% & 48 \\
\hline S96T005287 & $163: 1$ & Lower half & 42.8 & 40.8 & 41.8 \\
\hline$\$ 96 T 005288$ & $163: 2$ & Lower half & 23.7 & 24 & 23.85 \\
\hline S96T005782 & $163: 12$ & Lower half & 43.5 & 44.6 & 44.05 \\
\hline S96T005749 & \multirow[t]{2}{*}{$163: 14$} & Upper half & 41 & 38.1 & 39.55 \\
\hline S96T005750 & & Lower half & 46.5 & 44.6 & 45.55 \\
\hline S96T005294 & \multirow[t]{2}{*}{$163: 16$} & Upper half & 89.3 & 67.3 & $78.3^{\mathrm{QC}: \mathrm{c}}$ \\
\hline S96T005289 & & Lower half & 74.8 & 63.2 & 69 \\
\hline S96T005778 & \multirow[t]{2}{*}{$163: 18$} & Upper half & 39.7 & 43.8 & 41.75 \\
\hline S96T005783 & & Lower half & 36.8 & 38.7 & 37.75 \\
\hline S96T005557 & \multirow[t]{2}{*}{$163: 20$} & Upper half & 62.2 & 58.3 & 60.25 \\
\hline \$96T005560 & & Lower half & 59.8 & 45.2 & $52.5^{\mathrm{QC}: \mathrm{c}}$ \\
\hline S96T004797 & $164: 1$ & Lower half & 30.7 & 27.3 & 29 \\
\hline S96T004798 & $164: 13$ & Lower half & 148 & 159 & 153.5 \\
\hline S96T005042 & \multirow[t]{2}{*}{$164: 14$} & Upper half & 72.9 & 70.7 & 71.8 \\
\hline S96T005040 & & Lower half & 39.2 & 40.6 & 39.9 \\
\hline S96T005061 & \multirow[t]{2}{*}{$164: 15$} & Upper half & 52.3 & 55.5 & 53.9 \\
\hline \$96T005043 & & Lower half & 89.8 & 89.9 & 89.85 \\
\hline S96T005062 & \multirow[t]{2}{*}{$164: 16$} & Upper half & 48 & 54.6 & 51.3 \\
\hline S96T005044 & & Lower half & 51 & 44.3 & 47.65 \\
\hline S96T005063 & \multirow[t]{2}{*}{$164: 17$} & Upper half & 52 & 63.3 & 57.65 \\
\hline S96T005041 & & Lower half & 84.3 & 88.3 & 86.3 \\
\hline S96T005064 & \multirow[t]{2}{*}{$164: 19$} & Upper half & 82.2 & 79.5 & 80.85 \\
\hline S96T005045 & & Lower half & 66.3 & 75.8 & 71.05 \\
\hline S96T005282 & \multirow[t]{2}{*}{$164: 20$} & Upper half & 93.7 & 73 & $83.35^{\mathrm{QC}: \mathrm{c}}$ \\
\hline S96T005279 & & Lower half & 73.3 & 44.3 & $58.8^{Q C \cdot c}$ \\
\hline S96T005976 & Core 164 & Composite & 79.5 & 70.5 & $75^{\mathrm{QC}: \mathrm{a}}$ \\
\hline
\end{tabular}


Table B2-28. Tank 241-AN-104 Analytical Results: Nickel (ICP). (3 sheets)

\begin{tabular}{|c|c|c|c|c|c|}
\hline Sornule & 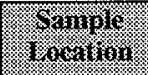 & Pow & mesuil: & Hiluligate & 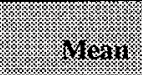 \\
\hline Soldis rivioi & 4.8 & (5.). & 40.8 & 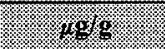 & $1.8 \mathrm{~s}$ \\
\hline S96T005284 & $163: 1$ & Lower half & 1,090 & 4,080 & $2,585^{\mathrm{QC}: \mathrm{e}}$ \\
\hline S96T005285 & $163: 2$ & Lower half & 2,750 & 2,930 & 2,840 \\
\hline S96T005780 & $163: 12$ & Lower half & 1,720 & 3,810 & $2,765^{\mathrm{QC}: \mathrm{c}}$ \\
\hline S96T005747 & \multirow[t]{2}{*}{$163: 14$} & Upper half & 3,630 & 5,410 & $4,520^{\mathrm{QC}: \mathrm{e}}$ \\
\hline S96T005748 & & Lower half & 4,620 & 3,120 & $3,870^{\mathrm{QC}: \mathrm{c}}$ \\
\hline S96T005293 & \multirow[t]{2}{*}{$163: 16$} & Upper half & 2,380 & 5,020 & $3,700^{\mathrm{QC}: e}$ \\
\hline S96T005286 & & Lower half & 2,250 & 2,670 & 2,460 \\
\hline S96T005777 & \multirow[t]{2}{*}{$163: 18$} & Upper half & 23,600 & $1.200 \mathrm{E}+05$ & $71,800^{\mathrm{QC:c}}$ \\
\hline S96T005781 & & Lower half & 8,600 & 2,880 & $5,740^{\mathrm{QC}: \mathrm{c}}$ \\
\hline \$96T005556 & \multirow[t]{2}{*}{$163: 20$} & Upper half & 2,020 & 4,270 & $3,145^{\mathrm{QC:c}}$ \\
\hline S96T005559 & & Lower half & 1,470 & 3,220 & $2,345^{\mathrm{QC}: \mathrm{c}}$ \\
\hline S96T004795 & $164: 1$ & Lower half & 5,820 & 4,300 & $5,060^{\mathrm{QC}: \mathrm{e}}$ \\
\hline S96T004796 & $164: 13$ & Lower half & 6,180 & 4,480 & $5,330^{\mathrm{QC}: \mathrm{c}}$ \\
\hline S96T005035 & \multirow[t]{2}{*}{$164: 14$} & Upper half & 1,600 & 5,870 & $3,735^{Q C: c}$ \\
\hline S96T005030 & & Lower half & 6,060 & 2,920 & $4,490^{\mathrm{QC}: \mathrm{c}}$ \\
\hline S96T005036 & \multirow[t]{2}{*}{$164: 15$} & Upper half & 4,920 & 3,230 & $4,075^{\mathrm{QC:c}}$ \\
\hline S96T005032 & & Lower half & 5,450 & 1,470 & $3,460^{\mathrm{QC}: \mathrm{c}}$ \\
\hline S96T005037 & \multirow[t]{2}{*}{$164: 16$} & Upper half & 779 & 486 & $632.5^{\mathrm{QC}: \mathrm{c}}$ \\
\hline S96T005033 & & Lower half & 2,500 & 1,840 & $2,170^{\mathrm{QC}: \mathrm{c}}$ \\
\hline S96T005038 & \multirow[t]{2}{*}{$164: 17$} & Upper half & 1,100 & 506 & $803^{\mathrm{QC}: c}$ \\
\hline S96T005031 & & Lower half & 553 & 2,670 & $1,611.5^{\mathrm{QC}: \mathrm{c}}$ \\
\hline S96T005039 & \multirow[t]{2}{*}{$164: 19$} & Upper half & 467 & 1,980 & $1,223.5^{\mathrm{QC}: \mathrm{c}}$ \\
\hline S96T005034 & & Lower half & $<427$ & 558 & $<492.5^{\mathrm{QC}: \mathrm{c}}$ \\
\hline S96T005281 & \multirow[t]{2}{*}{$164: 20$} & Upper half & 2,100 & 1,280 & $1,690^{\mathrm{QC}: c}$ \\
\hline S96T005278 & & Lower half & 1,920 & 2,820 & $2,370^{\mathrm{QC}: \mathrm{c}}$ \\
\hline
\end{tabular}


HNF-SD-WM-ER-690 Rev. 0

Table B2-28. Tank 241-AN-104 Analytical Results: Nickel (ICP). (3 sheets)

\begin{tabular}{|c|c|c|c|c|c|}
\hline 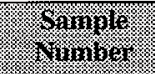 & . & for & r. & 84ingarit & (1) \\
\hline (91811\% & & (1) & . & hertials & 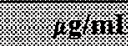 \\
\hline S96T005256 & $163: 2$ & Liquid & $<12$ & $<12$ & $<12$ \\
\hline S96T005260 & $163: 4$ & Liquid & $<12$ & $<12$ & $<12$ \\
\hline \$96T005527 & $163: 5$ & Liquid & $<12$ & $<12$ & $<12$ \\
\hline S96T005554 & $163: 6$ & Liquid & $<12$ & $<12$ & $<12$ \\
\hline \$96T005255 & $163: 7$ & Liquid & $<12$ & $<12$ & $<12$ \\
\hline S96T005257 & $163: 8$ & Liquid & $<12$ & $<12$ & $<12$ \\
\hline \$96T005258 & $163: 9$ & Liquid & $<12$ & $<12$ & $<12$ \\
\hline S96T005528 & $163: 10$ & Liquid & $<12$ & $<12$ & $<12$ \\
\hline S96T005739 & $163: 11$ & Liquid & $<12$ & $<12$ & $<12$ \\
\hline S96T005766 & $163: 12$ & Liquid & $<12$ & $<12$ & $<12$ \\
\hline S96T005741 & $163: 14$ & Liquid & $<12$ & $<12$ & $<12$ \\
\hline \$96T004774 & $164: 1$ & Liquid & $<24$ & $<24$ & $<24$ \\
\hline S96T004778 & $164: 2$ & Liquid & $<24$ & $<24$ & $<24$ \\
\hline S96T004779 & $164: 3$ & Liquid & $<24$ & $<24$ & $<24$ \\
\hline S96T004780 & $164: 4$ & Liquid & $<12$ & $<12$ & $<12$ \\
\hline S96T004976 & $164: 5$ & Liquid & $<12$ & $<12$ & $<12$ \\
\hline S96T004781 & $164: 7$ & Liquid & $<12$ & $<12$ & $<12$ \\
\hline S96T004977 & $164: 8$ & Liquid & $<12$ & $<12$ & $<12$ \\
\hline S96T004978 & $164: 9$ & Liquid & $<12$ & $<12$ & $<12$ \\
\hline S96T004979 & $164: 10$ & Liquid & $<12$ & $<12$ & $<12$ \\
\hline S96T004782 & 164:11 & Liquid & $<12$ & $<12$ & $<12$ \\
\hline S96T004783 & $164: 12$ & Liquid & $<12$ & $<12$ & $<12$ \\
\hline S96T004784 & $164: 13$ & Liquid & $<12$ & $<12$ & $<12$ \\
\hline S96T005979 & Core 164 & Composite & $<24$ & $<24$ & $<24$ \\
\hline
\end{tabular}


Table B2-29. Tank 241-AN-104 Analytical Results: Phosphorus (ICP). (3 sheets)

\begin{tabular}{|c|c|c|c|c|c|}
\hline $\begin{array}{l}\text { Saminle } \\
\text { Siminer }\end{array}$ & Sogripur & $\begin{array}{l}\text { Sampla } \\
\text { Imarima }\end{array}$ & marill & Binilingte: & 1. Mran \\
\hline \multicolumn{3}{|c|}{ Sotrds weil aygest } & (1) & 箦: & (1) \\
\hline S96T005287 & $163: 1$ & Lower half & 1,120 & 866 & $993^{\mathrm{CC:c,c}}$ \\
\hline S96T005288 & $163: 2$ & Lower half & 1,400 & 1,390 & 1,395 \\
\hline S96T005782 & $163: 12$ & Lower half & 1,600 & 2,250 & $1,925^{\mathrm{QC}: \mathrm{c}}$ \\
\hline \begin{tabular}{|l|} 
S96T005749 \\
\end{tabular} & \multirow[t]{2}{*}{$163: 14$} & Upper half & 809 & 711 & 760 \\
\hline S96T005750 & & Lower half & 957 & 626 & $791.5^{\mathrm{QC:c}}$ \\
\hline S96T005294 & \multirow[t]{2}{*}{$163: 16$} & Upper half & 1,180 & 935 & $1,057.5^{\mathrm{QC}: \mathrm{c}}$ \\
\hline S96T005289 & & Lower half & 1,150 & 858 & $1,004^{\mathrm{QC:0}}$ \\
\hline S96T005778 & \multirow[t]{2}{*}{$163: 18$} & Upper half & 1,270 & 1,260 & 1,265 \\
\hline S96T005783 & & Lower half & 1,220 & 1,330 & 1,275 \\
\hline S96T005557 & \multirow[t]{2}{*}{$163: 20$} & Upper half & 1,400 & 1,610 & 1,505 \\
\hline S96T005560 & & Lower half & 6,210 & 5,380 & $5,795^{\mathrm{QC}: c}$ \\
\hline S96T004797 & $164: 1$ & Lower half & 2,070 & 1,770 & $1,920^{\mathrm{QC:c}}$ \\
\hline \begin{tabular}{|l|} 
S96T004798 \\
\end{tabular} & $164: 13$ & Lower half & 1,990 & 1,970 & $1,980^{\mathrm{OC:c}}$ \\
\hline S96T005042 & \multirow[t]{2}{*}{$164: 14$} & Upper half & 991 & 1,170 & $1,080.5$ \\
\hline S96T005040 & & Lower half & 797 & 688 & 742.5 \\
\hline S96T005061 & \multirow[t]{2}{*}{$164: 15$} & Upper half & 961 & 1,190 & $1,075.5^{\mathrm{QC:c}}$ \\
\hline S96T005043 & & Lower half & 798 & 813 & 805.5 \\
\hline S96T005062 & \multirow[t]{2}{*}{$164: 16$} & Upper half & 692 & 710 & 701 \\
\hline \begin{tabular}{|l|} 
S96T005044 \\
\end{tabular} & & Lower half & 1,090 & 1,110 & 1,100 \\
\hline S96T005063 & \multirow[t]{2}{*}{$164: 17$} & Upper half & 1,470 & 861 & $1,165.5^{\mathrm{QC}: \mathrm{c}}$ \\
\hline S96T005041 & & Lower half & 1,430 & 1,050 & $1,240^{\mathrm{QC}: \mathrm{c}}$ \\
\hline S96T005064 & \multirow[t]{2}{*}{$164: 19$} & Upper half & 1,860 & 1,440 & $1,650^{\mathrm{QC}: \mathrm{c}}$ \\
\hline S96T005045 & & Lower half & 863 & 815 & 839 \\
\hline S96T005282 & \multirow[t]{2}{*}{$164: 20$} & Upper half & 1,050 & 951 & $1,000.5$ \\
\hline S96T005279 & & Lower half & 1,590 & 966 & $1,278^{\mathrm{QC}: \mathrm{d}, \mathrm{c}}$ \\
\hline S96T005976 & Core 164 & Composite & 1,000 & 790 & $895^{\mathrm{QC}: c}$ \\
\hline
\end{tabular}


Table B2-29. Tank 241-AN-104 Analytical Results: Phosphorus (ICP). (3 sheets)

\begin{tabular}{|c|c|c|c|c|c|}
\hline 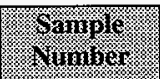 & 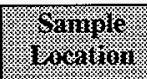 & 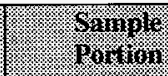 & Hesuli. & 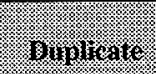 & mengen. \\
\hline \multicolumn{2}{|c|}{ gollas } & & (3) & was & 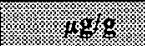 \\
\hline S96T005284 & $163: 1$ & Lower half & $<3,750$ & $<3,740$ & $<3,745$ \\
\hline S96T005285 & $163: 2$ & Lower half & $<3,580$ & $<3,820$ & $<3,700$ \\
\hline S96T005780 & $163: 12$ & Lower half & $<3,760$ & $<3,800$ & $<3,780$ \\
\hline S96T005747 & \multirow[t]{2}{*}{$163: 14$} & Upper half & $<4,480$ & $<4,510$ & $<4,495$ \\
\hline S96T005748 & & Lower half & $<3,730$ & $<3,850$ & $<3,790$ \\
\hline S96T005293 & \multirow[t]{2}{*}{$163: 16$} & Upper half & $<3,550$ & $<3,800$ & $<3,675$ \\
\hline S96T005286 & & Lower half & $<3,970$ & $<3,670$ & $<3,820$ \\
\hline S96T005777 & \multirow[t]{2}{*}{$163: 18$} & Upper half & $<4,100$ & $<3,970$ & $<4,035$ \\
\hline S96T005781 & & Lower half & $<4,260$ & $<4,280$ & $<4,270$ \\
\hline S96T005556 & \multirow[t]{2}{*}{$163: 20$} & Upper half & $<3,700$ & $<3,870$ & $<3,785$ \\
\hline S96T005559 & & Lower half & 5,360 & $<3,890$ & $<4,625^{\mathrm{QC}: \mathrm{c}}$ \\
\hline S96T004795 & $164: 1$ & Lower half & $<4,090$ & $<4,020$ & $<4,055$ \\
\hline S96T004796 & $164: 13$ & Lower half & $<4,120$ & $<4,150$ & $<4,135$ \\
\hline S96T005035 & \multirow[t]{2}{*}{$164: 14$} & Upper half & $<4,080$ & $<4,120$ & $<4,100$ \\
\hline S96T005030 & & Lower half & $<4,100$ & $<4,070$ & $<4,085$ \\
\hline S96T005036 & \multirow[t]{2}{*}{$164: 15$} & Upper half & $<4,020$ & $<3,930$ & $<3,975$ \\
\hline S96T005032 & & Lower half & $<3,950$ & $<4,010$ & $<3,980$ \\
\hline \$96T005037 & \multirow[t]{2}{*}{$164: 16$} & Upper half & $<3,840$ & $<3,660$ & $<3,750$ \\
\hline S96T005033 & & Lower half & $<3,830$ & $<3,730$ & $<3,780$ \\
\hline S96T005038 & \multirow[t]{2}{*}{$164: 17$} & Upper half & $<3,860$ & $<3,790$ & $<3,825$ \\
\hline S96T005031 & & Lower half & $<4,370$ & $<4,340$ & $<4,355$ \\
\hline S96T005039 & \multirow[t]{2}{*}{$164: 19$} & Upper half & $<3,890$ & $<4,000$ & $<3,945$ \\
\hline \begin{tabular}{|l|} 
S96T005034 \\
\end{tabular} & & Lower half & $<4,270$ & $<4,350$ & $<4,310$ \\
\hline S96T005281 & \multirow[t]{2}{*}{$164: 20$} & Upper half & $<3,440$ & 5,400 & $<4,420^{\mathrm{QC:c}}$ \\
\hline S96T005278 & & Lower half & $<3,770$ & $<3,870$ & $<3,820$ \\
\hline
\end{tabular}


Table B2-29. Tank 241-AN-104 Analytical Results: Phosphorus (ICP). (3 sheets)

\begin{tabular}{|c|c|c|c|c|c|}
\hline $\begin{array}{l}\text { Samople } \\
\text { Nuniner }\end{array}$ & Sirmole & $\begin{array}{l}\text { Sample } \\
\text { Poritim }\end{array}$ & 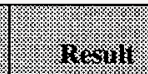 & 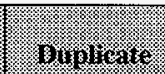 & f \\
\hline himinds & (3) & 8 & 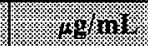 & $49 . \mathrm{mi}$ & (1) \\
\hline S96T005256 & 163:2 & Liquid & 862 & 954 & 908 \\
\hline S96T005260 & $163: 4$ & Liquid & 1,000 & 938 & 969 \\
\hline S96T005527 & $163: 5$ & Liquid & 960 & 943 & 951.5 \\
\hline S96T005554 & $163: 6$ & Liquid & 992 & 964 & 978 \\
\hline \begin{tabular}{|l|} 
S9T005255 \\
\end{tabular} & $163: 7$ & \begin{tabular}{|l|} 
Liquid \\
\end{tabular} & 1,030 & 1,070 & 1,050 \\
\hline S96T005257 & $163: 8$ & Liquid & 835 & 850 & 842.5 \\
\hline S96T005258 & $163: 9$ & Liquid & 876 & 860 & 868 \\
\hline S96T005528 & $163: 10$ & Liquid & 785 & 804 & 794.5 \\
\hline \begin{tabular}{|l|l} 
T905739 \\
\end{tabular} & $163: 11$ & Liquid & 729 & 736 & 732.5 \\
\hline S96T005766 & $163: 12$ & Liquid & 554 & 529 & 541.5 \\
\hline S96T005741 & $163: 14$ & Liquid & 559 & 561 & 560 \\
\hline S96T004774 & $164: 1$ & Liquid & 908 & 895 & 901.5 \\
\hline S96T004778 & $164: 2$ & Liquid & 847 & 973 & 910 \\
\hline S96T004779 & $164: 3$ & Liquid & 954 & 909 & 931.5 \\
\hline S96T004780 & $164: 4$ & Liquid & 908 & 877 & 892.5 \\
\hline S96T004976 & $164: 5$ & Liquid & 1,150 & 886 & $1,018^{\mathrm{QC}: c, c}$ \\
\hline S96T004781 & $164: 7$ & Liquid & 860 & 878 & 869 \\
\hline S96T004977 & $164: 8$ & Liquid & 1,130 & 937 & $1,033.5$ \\
\hline S96T004978 & $164: 9$ & Liquid & 1,020 & 860 & 940 \\
\hline S96T004979 & $164: 10$ & Liquid & 959 & 888 & 923.5 \\
\hline S96T004782 & $164: 11$ & Liquid & 900 & 899 & 899.5 \\
\hline S96T004783 & $164: 12$ & Liquid & 883 & 1,050 & 966.5 \\
\hline S96T004784 & $164: 13$ & Liquid & 804 & 837 & 820.5 \\
\hline S96T005979 & Core 164 & Composite & 1,060 & 995 & $1,027.5$ \\
\hline
\end{tabular}


Table B2-30. Tank 241-AN-104 Analytical Results: Potassium (ICP). (2 sheets)

\begin{tabular}{|c|c|c|c|c|c|}
\hline 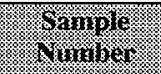 & 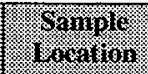 & 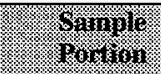 & (3c) & Sintiruer. & (2. \\
\hline \multicolumn{3}{|c|}{ 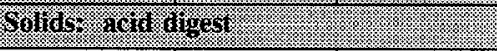 } & fing & (3. & 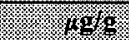 \\
\hline S96T005287 & $163: 1$ & Lower half & 3,590 & 3,510 & 3,550 \\
\hline S96T005288 & $163: 2$ & Lower half & 3,050 & 3,200 & $3,125^{\mathrm{QC:d}}$ \\
\hline S96T005782 & $163: 12$ & Lower half & 3,660 & 3,460 & 3,560 \\
\hline S96T005749 & \multirow[t]{2}{*}{$163: 14$} & Upper half & 3,450 & 3,590 & 3,520 \\
\hline S96T005750 & & Lower half & 3,650 & 3,770 & 3,710 \\
\hline S96T005294 & \multirow[t]{2}{*}{$163: 16$} & Upper half & 3,330 & 3,410 & 3,370 \\
\hline S96T005289 & & Lower half & 3,450 & 3,720 & $3,585^{\mathrm{QC:d}}$ \\
\hline S96T005778 & \multirow[t]{2}{*}{$163: 18$} & Upper half & 3,150 & 3,150 & 3,150 \\
\hline S96T005783 & & Lower half & 3,200 & 3,060 & 3,130 \\
\hline S96T005557 & \multirow[t]{2}{*}{$163: 20$} & Upper half & 3,070 & 3,220 & 3,145 \\
\hline S96T005560 & & Lower half & 3,350 & 2,680 & $3,015^{\mathrm{QC}: c, \mathrm{c}}$ \\
\hline S96T004797 & $164: 1$ & Lower half & 3,690 & 3,720 & 3,705 \\
\hline S96T004798 & $164: 13$ & Lower half & 3,820 & 4,020 & $3,920^{\mathrm{QC}: \mathrm{d}}$ \\
\hline S96T005042 & \multirow[t]{2}{*}{$164: 14$} & Upper half & 3,820 & 3,880 & 3,850 \\
\hline S96T005040 & & Lower half & 3,940 & 4,070 & 4,005 \\
\hline S96T005061 & \multirow[t]{2}{*}{$164: 15$} & Upper half & 3,750 & 3,860 & 3,805 \\
\hline S96T005043 & & Lower half & 3,740 & 3,700 & 3,720 \\
\hline S96T005062 & \multirow[t]{2}{*}{$164: 16$} & Upper half & 3,830 & 3,820 & 3,825 \\
\hline S96T005044 & & Lower half & 3,530 & 3,690 & 3,610 \\
\hline S96T005063 & \multirow[t]{2}{*}{$164: 17$} & Upper half & 3,810 & 4,060 & 3,935 \\
\hline S96T005041 & & Lower half & 3,490 & 3,800 & 3,645 \\
\hline S96T005064 & \multirow[t]{2}{*}{$164: 19$} & Upper half & 3,460 & 3,570 & 3,515 \\
\hline S96T005045 & & Lower half & 3,980 & 3,810 & 3,895 \\
\hline S96T005282 & \multirow[t]{2}{*}{$164: 20$} & Upper half & 3,590 & 3,780 & 3,685 \\
\hline S96T005279 & & Lower half & 3,330 & 3,840 & 3,585 \\
\hline S96T005976 & Core 164 & Composite & 3,630 & 3,810 & $3,720^{\mathrm{QC}: \mathrm{a}}$ \\
\hline
\end{tabular}




\begin{tabular}{|c|c|c|c|c|c|}
\hline$\$ 68^{6} 9$ & $0 \angle L ' 9$ & $020^{\circ} \mathrm{L}$ & əIIsoduro & t9ז & $6 L 6 \mathrm{~S} 00 \mathrm{~L} 96 \mathrm{~S}$ \\
\hline$p: 00062^{\prime} 9$ & $00 \varsigma^{6} 9$ & $080^{\circ} 9$ & prnbry & $\varepsilon I: t 9 I$ & $t 8 L t 00 L 96 \mathrm{~S}$ \\
\hline$=00058^{\circ} 9$ & $09 I^{\circ} \mathrm{L}$ & $0+5^{\prime} 9$ & p!̣b!̣ & $Z I: t 9 I$ & $\varepsilon 8 \angle t 00 \mathrm{~L} 96 \mathrm{~S}$ \\
\hline $\mathrm{p}: 00062^{\prime} 9$ & $0 \angle \nabla^{\circ} 9$ & OI'9 & pinbĩ & {$[I: t 9 I$} & $28 \angle t 00 L 96 \mathrm{~S}$ \\
\hline $0 z t^{6} 9$ & $0 \varepsilon \varepsilon^{\prime} 9$ & ors'9 & pinb!̣ & $0 \mathrm{I}:+9 \mathrm{I}$ & $6 L 6+00 \mathrm{~L} 96 \mathrm{~S}$ \\
\hline OES 9 & $086^{\circ} \mathrm{S}$ & $080^{\circ} L$ & p!nb! & $6:+9 \mathrm{I}$ & $8 L 6+00 \mathrm{~L} 96 \mathrm{~S}$ \\
\hline $0: 00 \$ 98^{\circ} 9$ & $06 \tau^{\circ} 9$ & $00 t^{\circ} \mathrm{L}$ & p!̣nb!x & $8: 79 \mathrm{I}$ & $\angle L 6+00 L 96 \mathrm{~S}$ \\
\hline$p: 0006 z^{6} 9$ & OIt'9 & $0 L I^{\prime} 9$ & pı̣nb!̃ & $L: t 9 I$ & $18 \angle t 00 L 96 \mathrm{~S}$ \\
\hline $00088 \nabla^{\prime} 9$ & $0 \varepsilon \varepsilon^{\prime} 9$ & $0+9 \times 9$ & p!̣b!̣ & $s: t 9 I$ & $9 L 6 \mathrm{t} 00 \mathrm{~L} 96 \mathrm{~S}$ \\
\hline $\mathrm{p}: \supset 00<t^{\prime} 9$ & $009^{\prime} 9$ & $O+\varepsilon^{\prime} 9$ & p!nbịt & $t: t 91$ & $08 \angle t 00 L 96 \mathrm{~S}$ \\
\hline $0 t L^{\prime} 9$ & $029^{\circ} 9$ & $098^{\circ} 9$ & p!nb!̣ & $\varepsilon: t 9 \mathrm{I}$ & $6 L L+00 L 196 \mathrm{~S}$ \\
\hline $0 \varepsilon t^{\prime} 9$ & $056^{6} 9$ & $016^{\circ} \mathrm{s}$ & pı̣b!̣ & $\tau: t 9 \mathrm{I}$ & $8 L L+00 \perp 96 S$ \\
\hline $068^{\prime} 9$ & $096^{6} 9$ & $028^{\circ} 9$ & pinb!̣ & $I: \neq 9 I$ & $\nabla L L+00 \mathrm{~L} 96 \mathrm{~S}$ \\
\hline OIL'9 & $099^{\prime} 9$ & $09 L^{\circ} 9$ & pụb!̣t & $t I: \varepsilon 9 I$ & {$[+\angle S 00 L 96 \mathrm{~S}$} \\
\hline $0 z \tau^{\prime} L$ & $080^{\circ} \mathrm{L}$ & $09 \varepsilon^{\prime} L$ & p!̣b!r & ZI:દ9I & $99 \angle S 00 L 96 S$ \\
\hline $059^{\circ} 9$ & $0 \angle 9^{\circ} 9$ & Oع9“9 & pı̣b!̣ & $I I: \varepsilon 9 I$ & $6 \varepsilon \angle S 00 \mathrm{~L} 96 \mathrm{~S}$ \\
\hline OSt 9 & $0 \angle t^{c} 9$ & OEt‘9 & p!nb! & or: $: 9 \mathrm{I}$ & $825 S 00 \mathrm{~L} 96 \mathrm{~S}$ \\
\hline$\$ 98^{\circ} 9$ & $06 S^{\circ} 9$ & $0+r^{\circ} \angle$ & p!nb? & $6: \varepsilon 9 \mathrm{I}$ & $8 S z S 00 L 96 S$ \\
\hline $0 E L^{\prime} 9$ & $0 z 8^{\circ} 9$ & $0+9^{\circ} 9$ & p!nb!̣ & $8: \varepsilon 9 I$ & $\angle S 2 S 00 \mathrm{~L} 96 \mathrm{~S}$ \\
\hline $\mathrm{p}: 00 S \angle D^{\circ} L$ & $0 L^{\prime} L$ & $0 \pm \tau^{6} L$ & p!̣b! & $L: \varepsilon 9 \mathrm{I}$ & SSZS00L96S \\
\hline $09 \angle 9$ & $0 \angle 9^{6} 9$ & $058^{\circ} 9$ & p!nb!r & $9: \varepsilon 9 I$ & $t S S S 00 L 96 S$ \\
\hline$S \angle S^{\prime} 9$ & $0 \varepsilon 9^{\circ} 9$ & $02 s^{\prime} 9$ & pinb!r & s:E9I & $\angle z \varsigma S 00 \mathrm{~L} 96 \mathrm{~S}$ \\
\hline$: 00 S t 6^{\circ} 9$ & $069^{\circ} 9$ & $00 z^{\prime} L$ & p!nb! & $\nabla: \varepsilon 9 I$ & $092500 . \mathrm{L} 96 \mathrm{~S}$ \\
\hline$\$ \& 6^{\circ} 9$ & $06 \mathrm{I}^{\prime} \mathrm{L}$ & $089^{\circ} 9$ & p!̣nb!̣ & 乙:६9I & $9 \$ 2 \$ 00 \mathrm{~L} 96 \mathrm{~S}$ \\
\hline ringar & mars? & yingrt & & & spiniming \\
\hline 18010 & ongoning & 1950\% & $\begin{array}{l}\text { noviror } \\
\text { oranives }\end{array}$ & 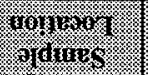 & 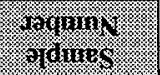 \\
\hline
\end{tabular}

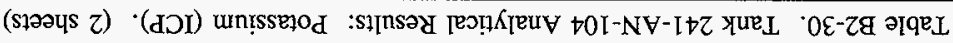


Table B2-31. Tank 241-AN-104 Analytical Results: Samarium (ICP). (3 sheets)

\begin{tabular}{|c|c|c|c|c|c|}
\hline Sirnple: & $\begin{array}{l}\text { Sample: } \\
\text { Irortion: }\end{array}$ & 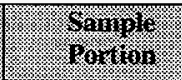 & Rrevilis & 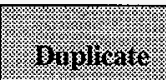 & rilezin \\
\hline \multicolumn{3}{|c|}{ Soflds achi: inges : } & 1. & 1.96 & . \\
\hline S96T005287 & $163: 1$ & Lower half & $<37.9$ & $<39.3$ & $<38.6$ \\
\hline S96T005288 & $163: 2$ & Lower half & $<44.3$ & $<37.5$ & $<40.9$ \\
\hline S96T005782 & $163: 12$ & Lower half & $<45.7$ & $<46.1$ & $<45.9$ \\
\hline S96T005749 & \multirow[t]{2}{*}{$163: 14$} & Upper half & $<44.8$ & $<43.6$ & $<44.2$ \\
\hline S96T005750 & & Lower half & $<45.9$ & $<45.2$ & $<45.55$ \\
\hline S96T005294 & \multirow[t]{2}{*}{$163: 16$} & Upper half & $<41.3$ & $<37$ & $<39.15$ \\
\hline S96T005289 & & Lower half & $<46.4$ & $<42.6$ & $<44.5$ \\
\hline S96T005778 & \multirow[t]{2}{*}{$163: 18$} & Upper half & $<48.1$ & $<45.4$ & $<46.75$ \\
\hline S96T005783 & & Lower half & $<47.4$ & $<47.2$ & $<47.3$ \\
\hline S96T005557 & \multirow[t]{2}{*}{$163: 20$} & Upper half & $<38$ & $<40.1$ & $<39.05$ \\
\hline S96T005560 & & Lower half & $<44.2$ & $<37.3$ & $<40.75$ \\
\hline S96T004797 & $164: 1$ & Lower half & $<46.9$ & $<47.1$ & $<47$ \\
\hline S96T004798 & $164: 13$ & Lower half & $<48.4$ & $<47.7$ & $<48.05$ \\
\hline S96T005042 & \multirow[t]{2}{*}{$164: 14$} & Upper half & $<47.4$ & $<48$ & $<47.7$ \\
\hline S96T005040 & & Lower half & $<47.7$ & $<47$ & $<47.35$ \\
\hline S96T005061 & \multirow[t]{2}{*}{$164: 15$} & Upper half & $<48.3$ & $<48$ & $<48.15$ \\
\hline S96T005043 & & Lower half & $<46.4$ & $<47.7$ & $<47.05$ \\
\hline S96T005062 & \multirow[t]{2}{*}{$164: 16$} & Upper half & $<151$ & $<149$ & $<150$ \\
\hline S96T005044 & & Lower half & $<147$ & $<150$ & $<148.5$ \\
\hline S96T005063 & \multirow[t]{2}{*}{$164: 17$} & Upper half & $<139$ & $<143$ & $<141$ \\
\hline S96T005041 & & Lower half & $<152$ & $<151$ & $<151.5$ \\
\hline S96T005064 & \multirow[t]{2}{*}{$164: 19$} & Upper half & $<151$ & $<153$ & $<152$ \\
\hline S96T005045 & & Lower half & $<145$ & $<144$ & $<144.5$ \\
\hline S96T005282 & \multirow[t]{2}{*}{$164: 20$} & Upper half & $<39.3$ & $<36.1$ & $<37.7$ \\
\hline S96T005279 & & \begin{tabular}{|l|} 
Lower half \\
\end{tabular} & $<45.4$ & $<37.8$ & $<41.6$ \\
\hline S96T005976 & Core 164 & Composite & $<39.8$ & $<39.8$ & $<39.8^{\mathrm{QCa} a}$ \\
\hline
\end{tabular}


Table B2-31. Tank 241-AN-104 Analytical Results: Samarium (ICP). (3 sheets)

\begin{tabular}{|c|c|c|c|c|c|}
\hline (6) & 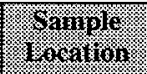 & 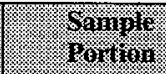 & Resum & Mingliear & ing \\
\hline \multicolumn{2}{|c|}{ 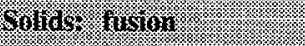 } & (3) & (1) & 689 & 13y \\
\hline S96T005284 & $163: 1$ & Lower half & $<1,870$ & $<1,870$ & $<1,870$ \\
\hline S96T005285 & $163: 2$ & Lower half & $<1,790$ & $<1,910$ & $<1,850$ \\
\hline S96T005780 & $163: 12$ & Lower half & $<1,880$ & $<1,900$ & $<1,890$ \\
\hline S96T005747 & \multirow[t]{2}{*}{$163: 14$} & Upper half & $<2,240$ & $<2,250$ & $<2,245$ \\
\hline S96T005748 & & Lower half & $<1,870$ & $<1,930$ & $<1,900$ \\
\hline S96T005293 & \multirow[t]{2}{*}{$163: 16$} & Upper half & $<1,770$ & $<1,900$ & $<1,835$ \\
\hline S96T005286 & & Lower half & $<1,990$ & $<1,830$ & $<1,910$ \\
\hline S96T005777 & \multirow[t]{2}{*}{$163: 18$} & Upper half & $<2,050$ & $<1,980$ & $<2,015$ \\
\hline S96T005781 & & Lower half & $<2,130$ & $<2,140$ & $<2,135$ \\
\hline S96T005556 & \multirow[t]{2}{*}{$163: 20$} & Upper half & $<1,850$ & $<1,940$ & $<1,895$ \\
\hline S96T005559 & & Lower half & $<1,950$ & $<1,950$ & $<1,950$ \\
\hline S96T004795 & $164: 1$ & Lower half & $<2,040$ & $<2,010$ & $<2,025$ \\
\hline S96T004796 & $164: 13$ & Lower half & $<2,060$ & $<2,070$ & $<2,065$ \\
\hline S96T005035 & \multirow[t]{2}{*}{$164: 14$} & Upper half & $<2,040$ & $<2,060$ & $<2,050$ \\
\hline S96T005030 & & Lower half & $<2,050$ & $<2,040$ & $<2,045$ \\
\hline S96T005036 & \multirow[t]{2}{*}{$164: 15$} & Upper half & $<2,010$ & $<1,970$ & $<1,990$ \\
\hline S96T005032 & & Lower half & $<1,970$ & $<2,000$ & $<1,985$ \\
\hline S96T005037 & \multirow[t]{2}{*}{$164: 16$} & Upper half & $<1,920$ & $<1,830$ & $<1,875$ \\
\hline S96T005033 & & Lower half & $<1,910$ & $<1,870$ & $<1,890$ \\
\hline S96T005038 & \multirow[t]{2}{*}{$164: 17$} & Upper half & $<1,930$ & $<1,900$ & $<1,915$ \\
\hline S96T005031 & & Lower half & $<2,180$ & $<2,170$ & $<2,175$ \\
\hline S96T005039 & \multirow[t]{2}{*}{$164: 19$} & Upper half & $<1,940$ & $<2,000$ & $<1,970$ \\
\hline S96T005034 & & Lower half & $<2,140$ & $<2,170$ & $<2,155$ \\
\hline S96T005281 & \multirow[t]{2}{*}{$164: 20$} & Upper half & $<1,720$ & $<1,850$ & $<1,785$ \\
\hline S96T005278 & & Lower half & $<1,890$ & $<1,930$ & $<1,910$ \\
\hline
\end{tabular}


Table B2-31. Tank 241-AN-104 Analytical Results: Samarium (ICP). (3 sheets)

\begin{tabular}{|c|c|c|c|c|c|}
\hline Similo & Stimpice & $\begin{array}{l}\text { Saruplo } \\
\text { Portion }\end{array}$ & Resull. & ( Gopitiente & (1) \\
\hline Vivinitis: & 8 & 8 & ( $84 \mathrm{ml}$ & (1) & (2. \\
\hline S96T005256 & $163: 2$ & Liquid & $<60.1$ & $<60.1$ & $<60.1$ \\
\hline S96T005260 & $163: 4$ & Liquid & $<60.1$ & $<60.1$ & $<60.1$ \\
\hline S96T005527 & $163: 5$ & Liquid & $<60.1$ & $<60.1$ & $<60.1$ \\
\hline S96T005554 & $163: 6$ & Liquid & $<60.1$ & $<60.1$ & $<60.1$ \\
\hline S96T005255 & $163: 7$ & Liquid & $<60.1$ & $<60.1$ & $<60.1$ \\
\hline \begin{tabular}{|l|} 
S96T005257 \\
\end{tabular} & $163: 8$ & Liquid & $<60.1$ & $<60.1$ & $<60.1$ \\
\hline S96T005258 & $163: 9$ & Liquid & $<60.1$ & $<60.1$ & $<60.1$ \\
\hline S96T005528 & $163: 10$ & Liquid & $<60.1$ & $<60.1$ & $<60.1$ \\
\hline \begin{tabular}{|l|} 
S96T005739 \\
\end{tabular} & 163:11 & Liquid & $<60.1$ & $<60.1$ & $<60.1$ \\
\hline S96r005766 & $163: 12$ & Liquid & $<60.1$ & $<60.1$ & $<60.1$ \\
\hline S96T005741 & $163: 14$ & Liquid & $<60.1$ & $<60.1$ & $<60.1$ \\
\hline S96T004774 & $164: 1$ & Liquid & $<120$ & $<120$ & $<120$ \\
\hline S96T004778 & $164: 2$ & Liquid & $<120$ & $<120$ & $<120$ \\
\hline S96T004779 & $164: 3$ & Liquid & $<120$ & $<120$ & $<120$ \\
\hline S96T004780 & $164: 4$ & Liquid & $<60.1$ & $<60.1$ & $<60.1$ \\
\hline S96T004976 & $164: 5$ & Liquid & $<60.1$ & $<60.1$ & $<60.1$ \\
\hline S96T004781 & $164: 7$ & Liquid & $<60.1$ & $<60.1$ & $<60.1$ \\
\hline S96T004977 & $164: 8$ & Liquid & $<60.1$ & $<60.1$ & $<60.1$ \\
\hline S96T004978 & $164: 9$ & Liquid & $<60.1$ & $<60.1$ & $<60.1$ \\
\hline S96T004979 & $164: 10$ & Liquid & $<60.1$ & $<60.1$ & $<60.1$ \\
\hline S96T004782 & $164: 11$ & Liquid & $<60.1$ & $<60.1$ & $<60.1$ \\
\hline S96T004783 & $164: 12$ & Liquid & $<60.1$ & $<60.1$ & $<60.1$ \\
\hline S96T004784 & $164: 13$ & Liquid & $<60.1$ & $<60.1$ & $<60.1$ \\
\hline S96T005979 & Core 164 & Composite & $<120$ & $<120$ & $<120$ \\
\hline
\end{tabular}


Table B2-32. Tank 241-AN-104 Analytical Results: Selenium (ICP). (3 sheets)

\begin{tabular}{|c|c|c|c|c|c|}
\hline 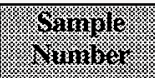 & 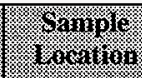 & f & m w will & (6)inligare & (y) \\
\hline 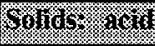 & dingesto & 8) & $49 \%$ & \%g & F. \\
\hline S96T005287 & $163: 1$ & Lower half & $<37.9$ & $<39.3$ & $<38.6$ \\
\hline \$96T005288 & $163: 2$ & Lower half & $<44.3$ & $<37.5$ & $<40.9$ \\
\hline S96T005782 & $163: 12$ & Lower half & $<45.7$ & $<46.1$ & $<45.9$ \\
\hline S96T005749 & \multirow[t]{2}{*}{$163: 14$} & Upper half & $<44.8$ & $<43.6$ & $<44.2$ \\
\hline S96T005750 & & Lower half & $<45.9$ & $<45.2$ & $<45.55$ \\
\hline S96T005294 & \multirow[t]{2}{*}{$163: 16$} & Upper half & $<41.3$ & $<37$ & $<39.15$ \\
\hline S96T005289 & & Lower half & $<46.4$ & $<42.6$ & $<44.5$ \\
\hline S96T005778 & \multirow[t]{2}{*}{$163: 18$} & Upper half & $<48.1$ & $<45.4$ & $<46.75$ \\
\hline S96T005783 & & Lower half & $<47.4$ & $<47.2$ & $<47.3$ \\
\hline S96T005557 & \multirow[t]{2}{*}{$163: 20$} & Upper half & $<38$ & $<40.1$ & $<39.05$ \\
\hline S96T005560 & & Lower half & $<44.2$ & $<37.3$ & $<40.75$ \\
\hline S96T004797 & $164: 1$ & Lower half & $<46.9$ & $<47.1$ & $<47$ \\
\hline S96T004798 & $164: 13$ & Lower half & $<48.4$ & $<47.7$ & $<48.05$ \\
\hline S96T005042 & \multirow[t]{2}{*}{$164: 14$} & Upper half & $<47.4$ & $<48$ & $<47.7$ \\
\hline S96T005040 & & Lower half & $<47.7$ & $<47$ & $<47.35$ \\
\hline S96T005061 & \multirow[t]{2}{*}{$164: 15$} & Upper half & $<48.3$ & $<48$ & $<48.15$ \\
\hline S96T005043 & & Lower half & $<46.4$ & $<47.7$ & $<47.05$ \\
\hline S96T005062 & \multirow[t]{2}{*}{$164: 16$} & Upper half & 194 & $<149$ & $<171.5^{\mathrm{QC}: \mathrm{e}}$ \\
\hline S96T005044 & & Lower half & $<147$ & 185 & $<166^{\mathrm{QC}: \mathrm{c}}$ \\
\hline S96T005063 & \multirow[t]{2}{*}{$164: 17$} & Upper half & 157 & 182 & 169.5 \\
\hline S96T005041 & & Lower half & 213 & 279 & $246^{\mathrm{QC}: \mathrm{c}}$ \\
\hline S96T005064 & \multirow[t]{2}{*}{$164: 19$} & Upper half & 244 & 221 & 232.5 \\
\hline S96T005045 & & Lower half & 237 & 216 & 226.5 \\
\hline \$96T005282 & \multirow[t]{2}{*}{$164: 20$} & Upper half & $<39.3$ & $<36.1$ & $<37.7$ \\
\hline S96T005279 & & Lower half & $<45.4$ & $<37.8$ & $<41.6$ \\
\hline \$96T005976 & Core 164 & Composite & $<39.8$ & $<39.8$ & $<39.8^{\text {QC:a }}$ \\
\hline
\end{tabular}


Table B2-32. Tank 241-AN-104 Analytical Results: Selenium (ICP). (3 sheets)

\begin{tabular}{|c|c|c|c|c|c|}
\hline $\begin{array}{l}\text { Somule } \\
\text { Ninimer }\end{array}$ & 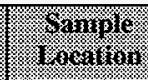 & $\begin{array}{l}\text { Simple } \\
\text { S rorition }\end{array}$ & raxinil & 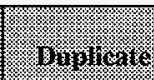 & Mresi \\
\hline sotas inge & 11 & 1 & 498 & (1) $/ 1,5 \mathrm{~g}$ & 1,89 \\
\hline S96T005284 & $163: 1$ & Lower half & $<1,870$ & $<1,870$ & $<1,870$ \\
\hline S96T005285 & $163: 2$ & Lower half & $<1,790$ & $<1,910$ & $<1,850$ \\
\hline S96T005780 & $163: 12$ & Lower half & $<1,880$ & $<1,900$ & $<1,890$ \\
\hline S96T005747 & \multirow[t]{2}{*}{$163: 14$} & Upper half & $<2,240$ & $<2,250$ & $<2,245$ \\
\hline S96T005748 & & Lower half & $<1,870$ & $<1,930$ & $<1,900$ \\
\hline S96T005293 & \multirow[t]{2}{*}{$163: 16$} & Upper half & $<1,770$ & $<1,900$ & $<1,835$ \\
\hline S96T005286 & & Lower half & $<1,990$ & $<1,830$ & $<1,910$ \\
\hline S96T005777 & \multirow[t]{2}{*}{$163: 18$} & Upper half & $<2,050$ & $<1,980$ & $<2,015$ \\
\hline S96T005781 & & Lower half & $<2,130$ & $<2,140$ & $<2,135$ \\
\hline S96T005556 & \multirow[t]{2}{*}{$163: 20$} & Upper half & $<1,850$ & $<1,940$ & $<1,895$ \\
\hline S96T005559 & & Lower half & $<1,950$ & $<1,950$ & $<1,950$ \\
\hline S96T004795 & $164: 1$ & Lower half & $<2,040$ & $<2,010$ & $<2,025$ \\
\hline S96T004796 & $164: 13$ & Lower half & $<2,060$ & $<2,070$ & $<2,065$ \\
\hline S96T005035 & \multirow[t]{2}{*}{$164: 14$} & Upper half & $<2,040$ & $<2,060$ & $<2,050$ \\
\hline S96T005030 & & Lower half & $<2,050$ & $<2,040$ & $<2,045$ \\
\hline S96T005036 & \multirow[t]{2}{*}{$164: 15$} & Upper half & $<2,010$ & $<1,970$ & $<1,990$ \\
\hline S96T005032 & & Lower half & $<1,970$ & $<2,000$ & $<1,985$ \\
\hline S96T005037 & \multirow[t]{2}{*}{$164: 16$} & Upper half & $<1,920$ & $<1,830$ & $<1,875$ \\
\hline S96T005033 & & Lower half & $<1,910$ & $<1,870$ & $<1,890$ \\
\hline S96T005038 & \multirow[t]{2}{*}{$164: 17$} & Upper half & $<1,930$ & $<1,900$ & $<1,915$ \\
\hline S96T005031 & & Lower half & $<2,180$ & $<2,170$ & $<2,175$ \\
\hline S96T005039 & \multirow[t]{2}{*}{$164: 19$} & Upper half & $<1,940$ & $<2,000$ & $<1,970$ \\
\hline S96T005034 & & Lower half & $<2,140$ & $<2,170$ & $<2,155$ \\
\hline S96T005281 & \multirow[t]{2}{*}{$164: 20$} & Upper half & $<1,720$ & $<1,850$ & $<1,785$ \\
\hline S96T005278 & & Lower half & $<1,890$ & $<1,930$ & $<1,910$ \\
\hline
\end{tabular}


Table B2-32. Tank 241-AN-104 Analytical Results: Selenium (ICP). (3 sheets)

\begin{tabular}{|c|c|c|c|c|c|}
\hline 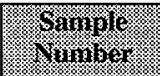 & 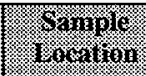 & f & atam & 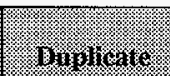 & youris \\
\hline Wuirs & 4 & & 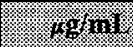 & 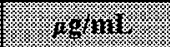 & 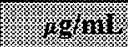 \\
\hline S96T005256 & $163: 2$ & Liquid & $<60.1$ & $<60.1$ & $<60.1$ \\
\hline S96T005260 & $163: 4$ & Liquid & $<60.1$ & $<60.1$ & $<60.1$ \\
\hline S96T005527 & $163: 5$ & Liquid & $<60.1$ & $<60.1$ & $<60.1$ \\
\hline S96T005554 & $163: 6$ & Liquid & $<60.1$ & $<60.1$ & $<60.1$ \\
\hline S96T005255 & $163: 7$ & Liquid & $<60.1$ & $<60.1$ & $<60.1$ \\
\hline S96T005257 & $163: 8$ & Liquid & $<60.1$ & $<60.1$ & $<60.1$ \\
\hline S96T005258 & $163: 9$ & Liquid & $<60.1$ & $<60.1$ & $<60.1$ \\
\hline S96T005528 & $163: 10$ & Liquid & $<60.1$ & $<60.1$ & $<60.1$ \\
\hline \$96T005739 & 163:11 & Liquid & $<60.1$ & $<60.1$ & $<60.1$ \\
\hline S96T005766 & $163: 12$ & Liquid & $<60.1$ & $<60.1$ & $<60.1$ \\
\hline \$96T005741 & $163: 14$ & Liquid & $<60.1$ & $<60.1$ & $<60.1$ \\
\hline S96T004774 & $164: 1$ & Liquid & $<120$ & $<120$ & $<120$ \\
\hline S96T004778 & $164: 2$ & Liquid & $<120$ & $<120$ & $<120$ \\
\hline \begin{tabular}{|l|} 
S96T004779 \\
\end{tabular} & $164: 3$ & Liquid & $<120$ & $<120$ & $<120$ \\
\hline \$96T004780 & $164: 4$ & Liquid & $<60.1$ & $<60.1$ & $<60.1$ \\
\hline S96T004976 & $164: 5$ & Liquid & $<60.1$ & $<60.1$ & $<60.1$ \\
\hline S96T004781 & $164: 7$ & Liquid & $<60.1$ & $<60.1$ & $<60.1$ \\
\hline S96T004977 & $164: 8$ & Liquid & $<60.1$ & $<60.1$ & $<60.1$ \\
\hline S96T004978 & $164: 9$ & Liquid & $<60.1$ & $<60.1$ & $<60.1$ \\
\hline \begin{tabular}{|l|} 
S96T004979 \\
\end{tabular} & $164: 10$ & Liquid & $<60.1$ & $<60.1$ & $<60.1$ \\
\hline S96T004782 & $164: 11$ & Liquid & $<60.1$ & $<60.1$ & $<60.1$ \\
\hline S96T004783 & $164: 12$ & Liquid & $<60.1$ & $<60.1$ & $<60.1$ \\
\hline S96T004784 & $164: 13$ & Liquid & $<60.1$ & $<60.1$ & $<60.1$ \\
\hline \begin{tabular}{|l|} 
S96T005979 \\
\end{tabular} & Core 164 & Composite & $<120$ & $<120$ & $<120$ \\
\hline
\end{tabular}


Table B2-33. Tank 241-AN-104 Analytical Results:- Silicon (ICP). (3 sheets)

\begin{tabular}{|c|c|c|c|c|c|}
\hline Simingle & Simingle & S Sample & resill & Simainiculs & (1) Vum \\
\hline \multicolumn{3}{|c|}{ Sorids a ovi inigest } & kPt? & /g9.9: & $\sqrt{16 \% 8}$ \\
\hline S96T005287 & $163: 1$ & Lower half & 803 & 881 & $842^{\text {QC:b }}$ \\
\hline S96T005288 & $163: 2$ & Lower half & 952 & 872 & $912^{\mathrm{QC}: \mathrm{b}, \mathrm{c}}$ \\
\hline S96T005782 & $163: 12$ & Lower half & 507 & 507 & $507^{\mathrm{QC:b}}$ \\
\hline S96T005749 & \multirow[t]{2}{*}{$163: 14$} & Upper half & 193 & 191 & $192^{\mathrm{QC}: \mathrm{b}}$ \\
\hline S96T005750 & & Lower half & 579 & 530 & $554.5^{\mathrm{QC}: \mathrm{b}}$ \\
\hline S96T005294 & \multirow[t]{2}{*}{$163: 16$} & Upper half & 780 & 644 & $712^{\mathrm{QC}: \mathrm{b}}$ \\
\hline S96T005289 & & Lower half & 1,030 & 721 & $875.5^{\mathrm{QC}: \mathrm{b}, \mathrm{c}}$ \\
\hline S96T005778 & \multirow[t]{2}{*}{$163: 18$} & Upper half & 377 & 292 & $334.5^{\mathrm{QC}: b, c}$ \\
\hline S96T005783 & & Lower half & 675 & 652 & $663.5^{Q C: b}$ \\
\hline S96T005557 & \multirow{2}{*}{$163: 20$} & Upper half & 742 & 625 & $683.5^{\mathrm{QC:b}}$ \\
\hline S96T005560 & & Lower half & 882 & 770 & $826^{\mathrm{QC:b,c}}$ \\
\hline \begin{tabular}{|l|} 
S96T004797 \\
\end{tabular} & 164:1 & Lower half & 887 & 897 & $892^{\text {QC:b }}$ \\
\hline S96T004798 & $164: 13$ & Lower half & 1,040 & 949 & $994.5^{\mathrm{QC}: \mathrm{b}, \mathrm{c}}$ \\
\hline S96T005042 & \multirow[t]{2}{*}{$164: 14$} & Upper half & 401 & 547 & $474^{\mathrm{QC:b,c}}$ \\
\hline S96T005040 & & Lower half & 776 & 1,060 & $918^{Q C: b, e}$ \\
\hline S96T005061 & \multirow[t]{2}{*}{$164: 15$} & Upper half & 567 & 540 & $553.5^{\mathrm{QC}: \mathrm{b}}$ \\
\hline S96T005043 & & Lower half & 717 & 652 & $684.5^{\mathrm{QC}: \mathrm{b}}$ \\
\hline S96T005062 & \multirow[t]{2}{*}{$164: 16$} & Upper half & 477 & 524 & $500.5^{\mathrm{QC}: \mathrm{b}}$ \\
\hline S96T005044 & & Lower half & 682 & 615 & $648.5^{\mathrm{QC:b}}$ \\
\hline S96T005063 & \multirow[t]{2}{*}{ 164:17 } & Upper half & 317 & 309 & $313^{Q C: b}$ \\
\hline S96T005041 & & Lower half & 567 & 615 & $591^{\text {QC:b }}$ \\
\hline S96T005064 & \multirow[t]{2}{*}{$164: 19$} & Upper half & 630 & 519 & $574.5^{\mathrm{QC}: \mathrm{b}}$ \\
\hline S96T005045 & & Lower half & 614 & 663 & $638.5^{\mathrm{QC}: \mathrm{b}}$ \\
\hline S96T005282 & \multirow[t]{2}{*}{$164: 20$} & Upper half & 355 & 592 & $473.5^{\mathrm{QC}: \mathrm{b}, \mathrm{c}}$ \\
\hline S96T005279 & & Lower half & 671 & 685 & $678^{\text {QC:b }}$ \\
\hline S96T005976 & Core 164 & Composite & 688 & 835 & $761.5^{\mathrm{QC}: \mathrm{b}}$ \\
\hline
\end{tabular}


Table B2-33. Tank 241-AN-104 Analytical Results: Silicon (ICP). (3 sheets)

\begin{tabular}{|c|c|c|c|c|c|}
\hline W & H.1\% & 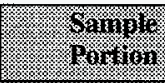 & $\mathrm{R} \times 2$ & 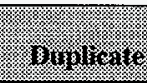 & (3) \\
\hline \multicolumn{3}{|c|}{ 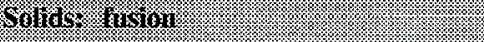 } & 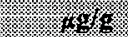 & (4) & 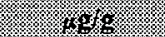 \\
\hline S96T005284 & $163: 1$ & Lower half & $<937$ & 1,010 & $<973.5$ \\
\hline S96T005285 & $163: 2$ & Lower half & 1,180 & $<956$ & $<1,068^{\mathrm{QC:e}}$ \\
\hline S96T005780 & $163: 12$ & Lower half & $<940$ & 1,130 & $<1,035$ \\
\hline S96T005747 & \multirow[t]{2}{*}{$163: 14$} & Upper half & $<1,120$ & $<1,130$ & $<1,125$ \\
\hline S96T005748 & & Lower half & 938 & 1,140 & 1,039 \\
\hline S96T005293 & \multirow[t]{2}{*}{$163: 16$} & Upper half & $<887$ & $<949$ & $<918$ \\
\hline S96T005286 & & Lower half & $<993$ & $<917$ & $<955$ \\
\hline S96T005777 & \multirow[t]{2}{*}{$163: 18$} & Upper half & $<1,030$ & $<992$ & $<1,011$ \\
\hline S96T005781 & & Lower half & 1,260 & 1,660 & $1,460^{\mathrm{QC:c}}$ \\
\hline S96T005556 & \multirow[t]{2}{*}{$163: 20$} & Upper half & $<924$ & $<968$ & $<946$ \\
\hline \$96T005559 & & Lower half & $<977$ & $<973$ & $<975$ \\
\hline S96T004795 & $164: 1$ & Lower half & $<1,020$ & $<1,000$ & $<1,010$ \\
\hline S96T004796 & $164: 13$ & Lower half & 1,090 & $<1,040$ & $<1,065$ \\
\hline S96T005035 & \multirow[t]{2}{*}{$164: 14$} & Upper half & 1,300 & 1,240 & 1,270 \\
\hline S96T005030 & & Lower half & 1,750 & $<1,020$ & $<1,385^{\mathrm{QC}: \mathrm{c}}$ \\
\hline \$96T005036 & \multirow[t]{2}{*}{$164: 15$} & Upper half & $<1,000$ & $<983$ & $<991.5$ \\
\hline S96T005032 & & Lower half & $<987$ & $<1,000$ & $<993.5$ \\
\hline S96T005037 & \multirow[t]{2}{*}{ 164:16 } & Upper half & $<961$ & $<915$ & $<938$ \\
\hline S96T005033 & & Lower half & 987 & $<933$ & $<960$ \\
\hline S96T005038 & \multirow[t]{2}{*}{$164: 17$} & Upper half & $<966$ & $<949$ & $<957.5$ \\
\hline S96T005031 & & Lower half & $<1,090$ & 1,110 & $<1,100$ \\
\hline S96T005039 & \multirow[t]{2}{*}{$164: 19$} & Upper half & $<972$ & $<1,000$ & $<986$ \\
\hline S96T005034 & & Lower half & $<1,070$ & $<1,090$ & $<1,080$ \\
\hline S96T005281 & \multirow[t]{2}{*}{$164: 20$} & Upper half & 2,060 & $<925$ & $<1,492.5^{\mathrm{QC}: \mathrm{c}}$ \\
\hline S96T005278 & & Lower half & $<943$ & $<966$ & $<954.5$ \\
\hline
\end{tabular}


Table B2-33. Tank 241-AN-104 Analytical Results: Silicon (ICP). (3 sheets)

\begin{tabular}{|c|c|c|c|c|c|}
\hline 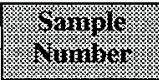 & Sirmple: & $\begin{array}{l}\text { Sariple } \\
\text { Portion }\end{array}$ & Rest1: & Bigligure & Mear \\
\hline Iiquids & (s) & & (48. & 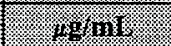 & 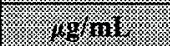 \\
\hline S96T005256 & $163: 2$ & Liquid & 202 & 220 & 211 \\
\hline S96T005260 & $163: 4$ & Liquid & 234 & 222 & 228 \\
\hline S96T005527 & $163: 5$ & Liquid & 173 & 173 & 173 \\
\hline S96T005554 & $163: 6$ & Liquid & 200 & 200 & 200 \\
\hline S96T005255 & $163: 7$ & Liquid & 230 & 242 & 236 \\
\hline S96T005257 & $163: 8$ & Liquid & 236 & 248 & 242 \\
\hline S96T005258 & $163: 9$ & Liquid & 220 & 217 & 218.5 \\
\hline S96T005528 & $163: 10$ & Liquid & 204 & 208 & 206 \\
\hline S96T005739 & $163: 11$ & Liquid & 245 & 243 & 244 \\
\hline S96T005766 & $163: 12$ & Liquid & 158 & 155 & 156.5 \\
\hline S96T005741 & $163: 14$ & Liquid & 220 & 223 & 221.5 \\
\hline \begin{tabular}{|l|} 
S96T004774 \\
\end{tabular} & $164: 1$ & Liquid & 478 & 507 & 492.5 \\
\hline S96T004778 & $164: 2$ & Liquid & 170 & 205 & 187.5 \\
\hline S96T004779 & $164: 3$ & Liquid & 203 & 192 & 197.5 \\
\hline S96T004780 & $164: 4$ & Liquid & 224 & 216 & 220 \\
\hline S96T004976 & $164: 5$ & Liquid & 390 & 249 & $319.5^{\mathrm{QC}: \mathrm{c}}$ \\
\hline S96T004781 & $164: 7$ & Liquid & 216 & 221 & 218.5 \\
\hline S96T004977 & $164: 8$ & Liquid & 358 & 317 & 337.5 \\
\hline S96T004978 & $164: 9$ & Liquid & 363 & 263 & $313^{\mathrm{QC:c}}$ \\
\hline S96T004979 & $164: 10$ & Liquid & 245 & 219 & 232 \\
\hline S96T004782 & $164: 11$ & Liquid & 279 & 269 & 274 \\
\hline S96T004783 & $164: 12$ & Liquid & 260 & 288 & 274 \\
\hline S96T004784 & $164: 13$ & Liquid & 213 & 206 & 209.5 \\
\hline S96T005979 & Core 164 & Composite & 411 & 347 & 379 \\
\hline
\end{tabular}


HNF-SD-WM-ER-690 Rev. 0

Table B2-34. Tank 241-AN-104 Analytical Results: Silver (ICP). (3 sheets)

\begin{tabular}{|c|c|c|c|c|c|}
\hline Mrano & 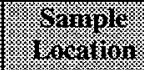 & The & risuril & (1) & 1) \\
\hline \multicolumn{2}{|c|}{ 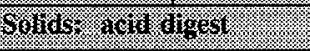 } & (l) & $f_{1}$ & (3) & (3) \\
\hline S96T005287 & $163: 1$ & Lower half & 14.1 & 13.8 & $13.95^{\mathrm{QCa}, \mathrm{c}}$ \\
\hline S96T005288 & $163: 2$ & Lower half & 16.9 & 16.2 & $16.55^{\mathrm{QC:a}, \mathrm{c}}$ \\
\hline S96T005782 & $163: 12$ & Lower half & 12.3 & 12.3 & 12.3 \\
\hline S96T005749 & \multirow[t]{2}{*}{$163: 14$} & Upper half & 13.8 & 13.7 & 13.75 \\
\hline S96T005750 & & Lower half & 13 & $13: 1$ & 13.05 \\
\hline S96T005294 & \multirow[t]{2}{*}{$163: 16$} & Upper half & 38.6 & 27.3 & $32.95^{\mathrm{QC}: \mathrm{a}, \mathrm{e}}$ \\
\hline S96T005289 & & Lower half & 15 & 14.4 & $14.7^{\mathrm{QC}: \mathrm{a}, \mathrm{c}}$ \\
\hline S96T005778 & \multirow[t]{2}{*}{$163: 18$} & Upper half & 14.4 & 14.9 & 14.65 \\
\hline S96T005783 & & Lower half & 15 & 14.7 & 14.85 \\
\hline S96T005557 & \multirow[t]{2}{*}{$163: 20$} & Upper half & 15.7 & 15.4 & $15.55^{\mathrm{QC}: \mathrm{a}}$ \\
\hline S96T005560 & & Lower half & 17.7 & 14.8 & $16.25^{\mathrm{QC:a}, \mathrm{c}}$ \\
\hline \$96T004797 & $164: 1$ & Lower half & 13.7 & 14.2 & 13.95 \\
\hline S96T004798 & $164: 13$ & Lower half & 14.5 & 14.3 & 14.4 \\
\hline S96T005042 & \multirow[t]{2}{*}{$164: 14$} & Upper half & 13.7 & 14.4 & 14.05 \\
\hline \$96T005040 & & Lower half & 14.6 & 14.1 & 14.35 \\
\hline S96T005061 & \multirow[t]{2}{*}{$164: 15$} & Upper half & 14.5 & 14.8 & 14.65 \\
\hline S96T005043 & & Lower half & 14.3 & 14.5 & 14.4 \\
\hline S96T005062 & \multirow[t]{2}{*}{$164: 16$} & Upper half & $<15.1$ & $<14.9$ & $<15$ \\
\hline S96T005044 & & Lower half & $<14.7$ & $<15$ & $<14.85$ \\
\hline S96T005063 & \multirow[t]{2}{*}{$164: 17$} & Upper half & $<13.9$ & $<14.3$ & $<14.1$ \\
\hline S96T005041 & & Lower half & $<15.2$ & $<15.1$ & $<15.15$ \\
\hline S96T005064 & \multirow[t]{2}{*}{$164: 19$} & Upper half & $<15.1$ & $<15.3$ & $<15.2$ \\
\hline S96T005045 & & Lower half & $<14.5$ & $<14.4$ & $<14.45$ \\
\hline S96T005282 & \multirow[t]{2}{*}{$164: 20$} & Upper half & 26.4 & 21.2 & $23.8^{\mathrm{QC}: \mathrm{c}}$ \\
\hline S96T005279 & & Lower half & 15.9 & 14 & 14.95 \\
\hline S96T005976 & Core 164 & Composite & 14.2 & 13.5 & $13.85^{\mathrm{QC}: \mathrm{a}}$ \\
\hline
\end{tabular}


Table B2-34. Tank 241-AN-104 Analytical Results: Silver (ICP). (3 sheets)

\begin{tabular}{|c|c|c|c|c|c|}
\hline $\begin{array}{l}\text { Sample } \\
\text { Trnibis: }\end{array}$ & 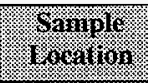 & $\begin{array}{l}\text { Samile } \\
\text { Portion }\end{array}$ & 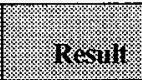 & Dinglictio: & 19rin \\
\hline \multicolumn{3}{|c|}{ Solidst hision : } & 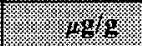 & 1089 & 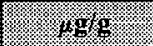 \\
\hline S96T005284 & $163: 1$ & Lower half & $<187$ & $<187$ & $<187^{\mathrm{QC:c}}$ \\
\hline S96T005285 & $163: 2$ & Lower half & $<179$ & $<191$ & $<185^{\mathrm{QC:c}}$ \\
\hline S96T005780 & $163: 12$ & Lower half & $<188$ & $<190$ & $<189$ \\
\hline S96T005747 & \multirow[t]{2}{*}{$163: 14$} & Upper half & $<224$ & $<225$ & $<224.5$ \\
\hline S96T005748 & & Lower half & $<187$ & $<193$ & $<190$ \\
\hline S96T005293 & \multirow[t]{2}{*}{$163: 16$} & Upper half & $<177$ & $<190$ & $<183.5$ \\
\hline S96T005286 & & Lower half & $<199$ & $<183$ & $<191^{\text {QC:c }}$ \\
\hline S96T005777 & \multirow[t]{2}{*}{$163: 18$} & Upper half & $<205$ & $<198$ & $<201.5$ \\
\hline S96T005781 & & Lower half & $<213$ & $<214$ & $<213.5^{\mathrm{QC}: \mathrm{c}}$ \\
\hline S96T005556 & \multirow[t]{2}{*}{$163: 20$} & Upper half & $<185$ & $<194$ & $<189.5$ \\
\hline S96T005559 & & Lower half & $<195$ & $<195$ & $<195^{\mathrm{QC}: \mathrm{C}}$ \\
\hline S96T004795 & $164: 1$ & Lower half & $<204$ & $<201$ & $<202.5$ \\
\hline S96T004796 & $164: 13$ & Lower half & $<206$ & $<207$ & $<206.5$ \\
\hline S96T005035 & \multirow[t]{2}{*}{$164: 14$} & Upper half & $<204$ & $<206$ & $<205$ \\
\hline S96T005030 & & Lower half & $<205$ & $<204$ & $<204.5$ \\
\hline S96T005036 & \multirow[t]{2}{*}{$164: 15$} & Upper half & $<201$ & $<197$ & $<199$ \\
\hline S96T005032 & & Lower half & $<197$ & $<200$ & $<198.5$ \\
\hline S96T005037 & \multirow[t]{2}{*}{$164: 16$} & Upper half & $<192$ & $<183$ & $<187.5$ \\
\hline S96T005033 & & Lower half & $<191$ & $<187$ & $<189$ \\
\hline S96T005038 & \multirow[t]{2}{*}{$164: 17$} & Upper half & $<193$ & $<190$ & $<191.5$ \\
\hline S96T005031 & & Lower half & $<218$ & $<217$ & $<217.5$ \\
\hline S96T005039 & \multirow[t]{2}{*}{$164: 19$} & Upper half & $<194$ & $<200$ & $<197$ \\
\hline S96T005034 & & Lower half & $<214$ & $<217$ & $<215.5$ \\
\hline S96T005281 & \multirow[t]{2}{*}{$164: 20$} & Upper half & $<172$ & $<185$ & $<178.5$ \\
\hline S96T005278 & & Lower half & $<189$ & $<193$ & $<191^{Q C: c}$ \\
\hline
\end{tabular}


Table B2-34. Tank 241-AN-104 Analytical Results: Silver (ICP). (3 sheets)

\begin{tabular}{|c|c|c|c|c|c|}
\hline $\begin{array}{l}\text { Sample } \\
\text { Symbir }\end{array}$ & 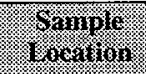 & 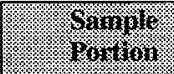 & foriti. & 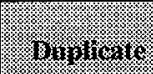 & Hean \\
\hline binums & & 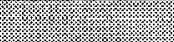 & 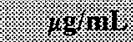 & 1094114 & $186.101 \%$ \\
\hline S96T005256 & $163: 2$ & Liquid & 19.1 & 19.9 & 19.5 \\
\hline S96T005260 & $163: 4$ & Liquid & 19.2 & 17.8 & 18.5 \\
\hline S96T005527 & $163: 5$ & Liquid & 18 & 18.1 & 18.05 \\
\hline S96T005554 & $163: 6$ & Liquid & 18.6 & 17.1 & 17.85 \\
\hline S96T005255 & $163: 7$ & Liquid & 19.3 & 20.2 & 19.75 \\
\hline S96T005257 & $163: 8$ & Liquid & 17.7 & 18.4 & 18.05 \\
\hline S96T005258 & $163: 9$ & Liquid & 19.6 & 17.9 & 18.75 \\
\hline$\$ 96 \mathrm{~T} 005528$ & $163: 10$ & Liquid & 17 & 17.4 & 17.2 \\
\hline S96T005739 & $163: 11$ & Liquid & 17.2 & 16.9 & 17.05 \\
\hline S96T005766 & $163: 12$ & Liquid & 18.3 & 19 & 18.65 \\
\hline S96T005741 & $163: 14$ & Liquid & 18.1 & 18.6 & 18.35 \\
\hline S96T004774 & $164: 1$ & Liquid & $<12$ & $<12$ & $<12^{\mathrm{QC}: \mathrm{c}}$ \\
\hline S96T004778 & $164: 2$ & Liquid & $<12$ & $<12$ & $<12$ \\
\hline S96T004779 & $164: 3$ & Liquid & $<12$ & $<12$ & $<12$ \\
\hline S96T004780 & $164: 4$ & Liquid & 18.5 & 18.8 & 18.65 \\
\hline S96T004976 & $164: 5$ & Liquid & 19.8 & 18.7 & 19.25 \\
\hline S96T004781 & $164: 7$ & Liquid & 18 & 18.9 & 18.45 \\
\hline S96T004977 & $164: 8$ & Liquid & 20.8 & 17.3 & 19.05 \\
\hline S96T004978 & $164: 9$ & Liquid & 20 & 17.3 & 18.65 \\
\hline S96T004979 & $164: 10$ & Liquid & 18.6 & 18.2 & 18.4 \\
\hline S96T004782. & $164: 11$ & Liquid & 18 & 19.5 & 18.75 \\
\hline S96T004783 & $164: 12$ & Liquid & 19.2 & 20.4 & 19.8 \\
\hline S96T004784 & $164: 13$ & Liquid & 17.9 & 18.8 & 18.35 \\
\hline S96T005979 & Core 164 & Composite & $<12$ & $<12$ & $<12$ \\
\hline
\end{tabular}


Table B2-35. Tank 241-AN-104 Analytical Results: Sodium (ICP). (3 sheets)

\begin{tabular}{|c|c|c|c|c|c|}
\hline Somple & Samiole & Shom & resail & 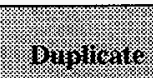 & 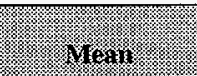 \\
\hline 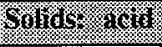 & 618otw & (3) & 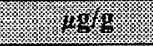 & \% & 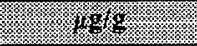 \\
\hline \$96T005287 & $163: 1$ & Lower half & $1.910 \mathrm{E}+05$ & $1.920 \mathrm{E}+05$ & $1.915 E+05^{Q C: d}$ \\
\hline \$96T005288 & $163: 2$ & Lower half & $1.960 \mathrm{E}+05$ & $1.970 \mathrm{E}+05$ & $1.965 \mathrm{E}+05^{\mathrm{QC:d}}$ \\
\hline S96T005782 & $163: 12$ & Lower half & $1.730 \mathrm{E}+05$ & $1.680 \mathrm{E}+05$ & $1.705 \mathrm{E}+05$ \\
\hline S96T005749 & \multirow[t]{2}{*}{$163: 14$} & Upper half & $1.900 \mathrm{E}+05$ & $1.840 \mathrm{E}+05$ & $1.870 \mathrm{E}+05$ \\
\hline S96T005750 & & Lower half & $1.830 \mathrm{E}+05$ & $1.800 \mathrm{E}+05$ & $1.815 \mathrm{E}+05$ \\
\hline S96T005294 & \multirow[t]{2}{*}{$163: 16$} & Upper half & $1.930 E+05$ & $1.850 \mathrm{E}+05$ & $1.890 \mathrm{E}+05$ \\
\hline S96T005289 & & Lower half & $1.910 \mathrm{E}+05$ & $1.830 \mathrm{E}+05$ & $1.870 \mathrm{E}+05^{\mathrm{QC}: \mathrm{d}}$ \\
\hline S96T005778 & \multirow[t]{2}{*}{$163: 18$} & Upper half & $1.920 \mathrm{E}+05$ & $1.940 \mathrm{E}+05$ & $1.930 \mathrm{E}+05$ \\
\hline S96T005783 & & Lower half & $1.960 \mathrm{E}+05$ & $1.970 \mathrm{E}+05$ & $1.965 \mathrm{E}+05$ \\
\hline S96T005557 & \multirow[t]{2}{*}{$163: 20$} & Upper half & $2.020 \mathrm{E}+05$ & $2.020 \mathrm{E}+05$ & $2.020 \mathrm{E}+05$ \\
\hline$\$ 96 \mathrm{~T} 005560$ & & Lower half & $2.310 \mathrm{E}+05$ & $1.830 \mathrm{E}+05$ & $2.070 \mathrm{E}+05^{\mathrm{QC}: c, \mathrm{c}}$ \\
\hline S96T004797 & $164: 1$ & Lower half & $1.920 \mathrm{E}+05$ & $1.950 \mathrm{E}+05$ & $1.935 \mathrm{E}+05^{\mathrm{QC} \mathrm{c}}$ \\
\hline S96T004798 & $164: 13$ & Lower half & $1.870 \mathrm{E}+05$ & $1.880 \mathrm{E}+05$ & $1.875 \mathrm{E}+05$ \\
\hline S96T005042 & \multirow[t]{2}{*}{$164: 14$} & Upper half & $1.910 \mathrm{E}+05$ & $1.890 \mathrm{E}+05$ & $1.900 \mathrm{E}+05$ \\
\hline$\$ 96 \mathrm{~T} 005040$ & & Lower half & $1.880 \mathrm{E}+05$ & $1.900 E+05$ & $1.890 E+05$ \\
\hline S96T005061 & \multirow[t]{2}{*}{$164: 15$} & Upper half & $1.940 \mathrm{E}+05$ & $1.980 \mathrm{E}+05$ & $1.960 \mathrm{E}+05$ \\
\hline \$96T005043 & & Lower half & $1.970 \mathrm{E}+05$ & $1.940 \mathrm{E}+05$ & $1.955 \mathrm{E}+05$ \\
\hline S96T005062 & \multirow[t]{2}{*}{$164: 16$} & Upper half & $1.950 \mathrm{E}+05$ & $2.050 \mathrm{E}+05$ & $2.000 \mathrm{E}+05$ \\
\hline \$96T005044 & & Lower half & $1.990 \mathrm{E}+05$ & $1.950 \mathrm{E}+05$ & $1.970 \mathrm{E}+05$ \\
\hline \$96T005063 & \multirow[t]{2}{*}{$164: 17$} & Upper half & $1.900 \mathrm{E}+05$ & $1.960 \mathrm{E}+05$ & $1.930 \mathrm{E}+05$ \\
\hline S96T005041 & & Lower half & $1.990 \mathrm{E}+05$ & $1.940 \mathrm{E}+05$ & $1.965 \mathrm{E}+05^{\mathrm{QC}: \mathrm{d}}$ \\
\hline \$96T005064 & \multirow[t]{2}{*}{$164: 19$} & Upper half & $1.960 \mathrm{E}+05$ & $2.080 \mathrm{E}+05$ & $2.020 \mathrm{E}+05$ \\
\hline S96T005045 & & Lower half & $1.870 \mathrm{E}+05$ & $1.960 \mathrm{E}+05$ & $1.915 \mathrm{E}+05$ \\
\hline S96T005282 & \multirow[t]{2}{*}{$164: 20$} & Upper half & $1.900 \mathrm{E}+05$ & $1.850 \mathrm{E}+05$ & $1.875 \mathrm{E}+05$ \\
\hline S96T005279 & & Lower half & $2.130 \mathrm{E}+05$ & $1.920 \mathrm{E}+05$ & $2.025 \mathrm{E}+05^{\mathrm{QC}: \mathrm{d}}$ \\
\hline S96T005976 & Core 164 & Composite & $1.950 \mathrm{E}+05$ & $1.880 \mathrm{E}+05$ & $1.915 \mathrm{E}+05$ \\
\hline
\end{tabular}


Table B2-35. Tank 241-AN-104 Analytical Results: Sodium (ICP). (3 sheets)

\begin{tabular}{|c|c|c|c|c|c|}
\hline $\begin{array}{l}\text { ShMulo } \\
\text { MHinev }\end{array}$ & Whimple & 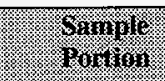 & Resilil & 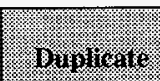 & 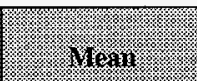 \\
\hline \multicolumn{3}{|c|}{ 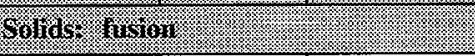 } & 1698 & 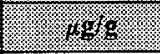 & 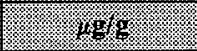 \\
\hline \$96T005284 & $163: 1$ & Lower half & $2.310 \mathrm{E}+05$ & $2.390 \mathrm{E}+05$ & $2.350 \mathrm{E}+05^{\mathrm{QC:c}}$ \\
\hline \$96T005285 & $163: 2$ & Lower half & $2.200 \mathrm{E}+05$ & $2.300 \mathrm{E}+05$ & $2.250 \mathrm{E}+05^{\mathrm{QC:d}}$ \\
\hline S96T005780 & $163: 12$ & Lower half & $2.180 \mathrm{E}+05$ & $2.180 \mathrm{E}+05$ & $2.180 \mathrm{E}+05$ \\
\hline S96T005747 & \multirow[t]{2}{*}{$163: 14$} & Upper half & $2.240 \mathrm{E}+05$ & $2.330 \mathrm{E}+05$ & $2.285 \mathrm{E}+05$ \\
\hline S96T005748 & & Lower half & $2.200 \mathrm{E}+05$ & $2.150 \mathrm{E}+05$ & $2.175 \mathrm{E}+05$ \\
\hline S96T005293 & \multirow[t]{2}{*}{$163: 16$} & Upper half & $2.320 \mathrm{E}+05$ & $2.380 \mathrm{E}+05$ & $2.350 \mathrm{E}+05$ \\
\hline S96T005286 & & Lower half & $2.380 \mathrm{E}+05$ & $2.210 \mathrm{E}+05$ & $2.295 \mathrm{E}+05$ \\
\hline S96T005777 & \multirow[t]{2}{*}{$163: 18$} & Upper half & $3.140 \mathrm{E}+05$ & $2.760 \mathrm{E}+05$ & $2.950 \mathrm{E}+05$ \\
\hline S96T005781 & & Lower half & $3.180 \mathrm{E}+05$ & $3.120 \mathrm{E}+05$ & $3.150 \mathrm{E}+05^{\mathrm{QC} c \mathrm{c}}$ \\
\hline S96T005556 & \multirow[t]{2}{*}{$163: 20$} & Upper half & $2.560 \mathrm{E}+05$ & $2.430 \mathrm{E}+05$ & $2.495 \mathrm{E}+05$ \\
\hline S96T005559 & & Lower half & $2.410 \mathrm{E}+05$ & $2.480 \mathrm{E}+05$ & $2.445 \mathrm{E}+05$ \\
\hline S96T004795 & $164: 1$ & Lower half & $2.480 \mathrm{E}+05$ & $2.400 \mathrm{E}+05$ & $2.440 \mathrm{E}+05$ \\
\hline S96T004796 & $164: 13$ & Lower half & $2.320 \mathrm{E}+05$ & $2.270 \mathrm{E}+05$ & $2.295 \mathrm{E}+05^{\mathrm{QC}: \mathrm{c}}$ \\
\hline S96T005035 & \multirow[t]{2}{*}{$164: 14$} & Upper half & $2.380 \mathrm{E}+05$ & $2.380 \mathrm{E}+05$ & $2.380 \mathrm{E}+05$ \\
\hline S96T005030 & & Lower half & $2.340 \mathrm{E}+05$ & $2.330 \mathrm{E}+05$ & $2.335 \mathrm{E}+05$ \\
\hline \$96T005036 & \multirow[t]{2}{*}{$164: 15$} & Upper half & $2.390 \mathrm{E}+05$ & $2.320 \mathrm{E}+05$ & $2.355 \mathrm{E}+05$ \\
\hline S96T005032 & & Lower half & $2.400 \mathrm{E}+05$ & $2.240 \mathrm{E}+05$ & $2.320 \mathrm{E}+05^{\mathrm{QC}: \mathrm{d}}$ \\
\hline S96T005037 & \multirow[t]{2}{*}{$164: 16$} & Upper half & $2.140 \mathrm{E}+05$ & $2.180 \mathrm{E}+05$ & $2.160 \mathrm{E}+05$ \\
\hline \$96T005033 & & Lower half & $2.290 \mathrm{E}+05$ & $2.370 \mathrm{E}+05$ & $2.330 \mathrm{E}+05$ \\
\hline S96T005038 & \multirow[t]{2}{*}{$164: 17$} & Upper half & $2.080 \mathrm{E}+05$ & $2.100 \mathrm{E}+05$ & $2.090 \mathrm{E}+05$ \\
\hline S96T005031 & & Lower half & $2.180 \mathrm{E}+05$ & $2.300 \mathrm{E}+05$ & $2.240 \mathrm{E}+05$ \\
\hline \begin{tabular}{|l|} 
S96T005039 \\
\end{tabular} & \multirow[t]{2}{*}{$164: 19$} & Upper half & $2.090 \mathrm{E}+05$ & $2.200 \mathrm{E}+05$ & $2.145 \mathrm{E}+05$ \\
\hline S96T005034 & & Lower half & $2.050 \mathrm{E}+05$ & $2.180 \mathrm{E}+05$ & $2.115 \mathrm{E}+05$ \\
\hline S96T005281 & \multirow[t]{2}{*}{$164: 20$} & Upper half & $2.450 \mathrm{E}+05$ & $2.420 \mathrm{E}+05$ & $2.435 \mathrm{E}+05$ \\
\hline S96T005278 & & Lower half & $2.490 \mathrm{E}+05$ & $2.530 \mathrm{E}+05$ & $2.510 \mathrm{E}+05^{\mathrm{QC:c}}$ \\
\hline
\end{tabular}


Table B2-35. Tank 241-AN-104 Analytical Results: Sodium (ICP). (3 sheets)

\begin{tabular}{|c|c|c|c|c|c|}
\hline 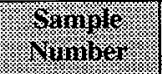 & Yoroules & 6romole & Resin & Bivingar & (1) \\
\hline Sinumis & & & 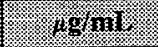 & 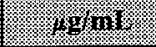 & \%8. \\
\hline \$96T005256 & $163: 2$ & Liquid & $2.480 \mathrm{E}+05$ & $2.680 \mathrm{E}+05$ & $2.580 E+05$ \\
\hline S96T005260 & $163: 4$ & Liquid & $2.710 \mathrm{E}+05$ & $2.520 \mathrm{E}+05$ & $2.615 \mathrm{E}+05^{\alpha \mathrm{C}: c}$ \\
\hline S96T005527 & $163: 5$ & Liquid & $2.510 E+05$ & $2.480 \mathrm{E}+05$ & $2.495 \mathrm{E}+05^{\mathrm{QC}: \mathrm{c}}$ \\
\hline S96T005554 & $163: 6$ & Liquid & $2.560 \mathrm{E}+05$ & $2.530 \mathrm{E}+05$ & $2.545 \mathrm{E}+05$ \\
\hline \$96T005255 & $163: 7$ & Liquid & $2.730 \mathrm{E}+05$ & $2.890 \mathrm{E}+05$ & $2.810 \mathrm{E}+05^{\mathrm{QC}: \mathrm{d}}$ \\
\hline S96T005257 & $163: 8$ & Liquid & $2.470 E+05$ & $2.520 \mathrm{E}+05$ & $2.495 \mathrm{E}+05$ \\
\hline S96T005258 & $163: 9$ & Liquid & $2.710 E+05$ & $2.550 \mathrm{E}+05$ & $2.630 \mathrm{E}+05$ \\
\hline S96T005528 & $163: 10$ & Liquid & $2.430 \mathrm{E}+05$ & $2.450 \mathrm{E}+05$ & $2.440 \mathrm{E}+05$ \\
\hline S96T005739 & $163: 11$ & Liquid & $2.460 \mathrm{E}+05$ & $2.450 \mathrm{E}+05$ & $2.455 \mathrm{E}+05$ \\
\hline S96T005766 & $163: 12$ & Liquid & $2.630 \mathrm{E}+05$ & $2.600 \mathrm{E}+05$ & $2.615 E+05$ \\
\hline S96T005741 & $163: 14$ & Liquid & $2.470 \mathrm{E}+05$ & $2.460 E+05$ & $2.465 \mathrm{E}+05$ \\
\hline S96T004774 & $164: 1$ & Liquid & $2.540 \mathrm{E}+05$ & $2.610 E+05$ & $2.575 \mathrm{E}+05^{\mathrm{QC:d}}$ \\
\hline S96T004778 & $164: 2$ & Liquid & $2.290 \mathrm{E}+05$ & $2.710 E+05$ & $2.500 \mathrm{E}+05$ \\
\hline S96T004779 & $164: 3$ & Liquid & $2.580 \mathrm{E}+05$ & $2.560 \mathrm{E}+05$ & $2.570 E+05$ \\
\hline S96T004780 & $164: 4$ & Liquid & $2.490 \mathrm{E}+05$ & $2.580 \mathrm{E}+05$ & $2.535 \mathrm{E}+05^{\mathrm{QC:d}}$ \\
\hline S96T004976 & $164: 5$ & Liquid & $2.650 \mathrm{E}+05$ & $2.520 \mathrm{E}+05$ & $2.585 \mathrm{E}+05^{\mathrm{QC}: \mathrm{c}}$ \\
\hline S96T004781 & $164: 7$ & Liquid & $2.440 \mathrm{E}+05$ & $2.510 \mathrm{E}+05$ & $2.475 \mathrm{E}+05^{\mathrm{QC}: \mathrm{d}}$ \\
\hline \$96T004977 & $164: 8$ & Liquid & $2.880 \mathrm{E}+05$ & $2.440 \mathrm{E}+05$ & $2.660 \mathrm{E}+05^{\mathrm{QC:c}}$ \\
\hline S96T004978 & $164: 9$ & Liquid & $2.810 \mathrm{E}+05$ & $2.360 \mathrm{E}+05$ & $2.585 E+05$ \\
\hline S96T004979 & $164: 10$ & Liquid & $2.570 \mathrm{E}+05$ & $2.470 \mathrm{E}+05$ & $2.520 \mathrm{E}+05$ \\
\hline S96T004782 & $164: 11$ & Liquid & $2.430 \mathrm{E}+05$ & $2.570 \mathrm{E}+05$ & $2.500 \mathrm{E}+05^{\mathrm{QC:d}}$ \\
\hline S96T004783 & $164: 12$ & Liquid & $2.580 \mathrm{E}+05$ & $2.820 \mathrm{E}+05$ & $2.700 \mathrm{E}+05^{\mathrm{QC}: \mathrm{c}}$ \\
\hline S96T004784 & $164: 13$ & Liquid & $2.410 \mathrm{E}+05$ & $2.540 \mathrm{E}+05$ & $2.475 \mathrm{E}+05^{\mathrm{QC}: d}$ \\
\hline S96T005979 & Core 164 & Composite & $2.670 \mathrm{E}+05$ & $2.550 \mathrm{E}+05$ & $2.610 \mathrm{E}+05^{\mathrm{QC}: \mathrm{c}}$ \\
\hline
\end{tabular}


Table B2-36. Tank 241-AN-104 Analytical Results: Strontium (ICP). (3 sheets)

\begin{tabular}{|c|c|c|c|c|c|}
\hline ofingle & 6yole & 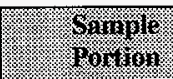 & (1) & k & 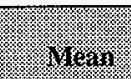 \\
\hline wotid wor & 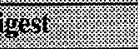 & (1) & 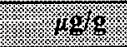 & it. & 16.6. \\
\hline S96T005287 & $163: 1$ & Lower half & $<3.79$ & $<3.93$ & $<3.86$ \\
\hline S96T005288 & $163: 2$ & Lower half & $<4.43$ & $<3.75$ & $<4.09$ \\
\hline S96T005782 & $163: 12$ & Lower half & $<4.57$ & $<4.61$ & $<4.59$ \\
\hline S96T005749 & \multirow[t]{2}{*}{$163: 14$} & Upper half & $<4.48$ & $<4.36$ & $<4.42$ \\
\hline S96T005750 & & Lower half & $<4.59$ & $<4.52$ & $<4.555$ \\
\hline S96T005294 & \multirow[t]{2}{*}{$163: 16$} & Upper half & $<4.13$ & $<3.7$ & $<3.915$ \\
\hline S96T005289 & & Lower half & $<4.64$ & $<4.26$ & $<4.45$ \\
\hline S96T005778 & \multirow[t]{2}{*}{$163: 18$} & Upper half & $<4.81$ & $<4.54$ & $<4.675$ \\
\hline S96T005783 & & Lower half & $<4.74$ & $<4.72$ & $<4.73$ \\
\hline S96T005557 & \multirow[t]{2}{*}{$163: 20$} & Upper half & $<3.8$ & $<4.01$ & $<3.905$ \\
\hline S96T005560 & & Lower half & $<4.42$ & $<3.73$ & $<4.075$ \\
\hline S96T004797 & $164: 1$ & Lower half & $<4.69$ & $<4.71$ & $<4.7$ \\
\hline \$96T004798 & $164: 13$ & Lower half & $<4.84$ & $<4.77$ & $<4.805$ \\
\hline S96T005042 & \multirow[t]{2}{*}{$164: 14$} & Upper half & $<4.74$ & $<4.8$ & $<4.77$ \\
\hline S96T005040 & & Lower half & $<4.77$ & $<4.7$ & $<4.735$ \\
\hline S96T005061 & \multirow[t]{2}{*}{$164: 15$} & Upper half & $<4.83$ & $<4.8$ & $<4.815$ \\
\hline S96T005043 & & Lower half & $<4.64$ & $<4.77$ & $<4.705$ \\
\hline S96T005062 & \multirow[t]{2}{*}{$164: 16$} & Upper half & $<15.1$ & $<14.9$ & $<15$ \\
\hline S96T005044 & & Lower half & $<14.7$ & $<15$ & $<14.85$ \\
\hline S96T005063 & \multirow[t]{2}{*}{$164: 17$} & Upper half & $<13.9$ & $<14.3$ & $<14.1$ \\
\hline S96T005041 & & Lower half & $<15.2$ & $<15.1$ & $<15.15$ \\
\hline S96T005064 & \multirow[t]{2}{*}{$164: 19$} & Upper half & $<15.1$ & $<15.3$ & $<15.2$ \\
\hline S96T005045 & & Lower half & $<14.5$ & $<14.4$ & $<14.45$ \\
\hline S96T005282 & \multirow[t]{2}{*}{$164: 20$} & Upper half & $<3.93$ & $<3.61$ & $<3.77$ \\
\hline S96T005279 & & Lower half & $<4.54$ & $<3.78$ & $<4.16$ \\
\hline S96T005976 & Core 164 & Composite & $<3.98$ & $<3.98$ & $<3.98^{\mathrm{QC}: \mathrm{a}}$ \\
\hline
\end{tabular}


HNF-SD-WM-ER-690 Rev. 0

Table B2-36. Tank 241-AN-104 Analytical Results: Strontium (ICP). (3 sheets)

\begin{tabular}{|c|c|c|c|c|c|}
\hline Whanger & ogruple & ogyogio & ryour & Huingin & Megin \\
\hline Solivis vinioi & 1\%: & & . & 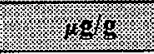 & 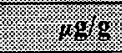 \\
\hline S96T005284 & $163: 1$ & Lower half & $<187$ & $<187$ & $<187$ \\
\hline S96T005285 & $163: 2$ & Lower half & $<179$ & $<191$ & $<185$ \\
\hline S96T005780 & $163: 12$ & Lower half & $<188$ & $<190$ & $<189$ \\
\hline S96T005747 & \multirow[t]{2}{*}{$163: 14$} & Upper half & $<224$ & $<225$ & $<224.5$ \\
\hline S96T005748 & & Lower half & $<187$ & $<193$ & $<190$ \\
\hline S96T005293 & \multirow[t]{2}{*}{$163: 16$} & Upper half & $<177$ & $<190$ & $<183.5$ \\
\hline S96T005286 & & Lower half & $<199$ & $<183$ & $<191$ \\
\hline S96T005777 & \multirow[t]{2}{*}{$163: 18$} & Upper half & $<205$ & $<198$ & $<201.5$ \\
\hline S96T005781 & & Lower half & $<213$ & $<214$ & $<213.5$ \\
\hline S96T005556 & \multirow[t]{2}{*}{$163: 20$} & Upper half & $<185$ & $<194$ & $<189.5$ \\
\hline S96T005559 & & Lower half & $<195$ & $<195$ & $<195$ \\
\hline S96T004795 & $164: 1$ & Lower half & $<204$ & $<201$ & $<202.5$ \\
\hline S96T004796 & $164: 13$ & Lower half & $<206$ & $<207$ & $<206.5$ \\
\hline S96T005035 & \multirow[t]{2}{*}{$164: 14$} & Upper half & $<204$ & $<206$ & $<205$ \\
\hline S96T005030 & & Lower half & $<205$ & $<204$ & $<204.5$ \\
\hline S96T005036 & \multirow[t]{2}{*}{$164: 15$} & Upper half & $<201$ & $<197$ & $<199$ \\
\hline S96T005032 & & Lower half & $<197$ & $<200$ & $<198.5$ \\
\hline S96T005037 & \multirow[t]{2}{*}{$164: 16$} & Upper half & $<192$ & $<183$ & $<187.5$ \\
\hline S96T005033 & & Lower half & $<191$ & $<187$ & $<189$ \\
\hline S96T005038 & \multirow[t]{2}{*}{$164: 17$} & Upper half & $<193$ & $<190$ & $<191.5$ \\
\hline S96T005031 & & Lower half & $<218$ & $<217$ & $<217.5$ \\
\hline \$96T005039 & \multirow[t]{2}{*}{$164: 19$} & Upper half & $<194$ & $<200$ & $<197$ \\
\hline S96T005034 & & Lower half & $<214$ & $<217$ & $<215.5$ \\
\hline S96T005281 & \multirow[t]{2}{*}{$164: 20$} & Upper half & $<172$ & $<185$ & $<178.5$ \\
\hline S96T005278 & & Lower half & $<189$ & $<193$ & $<191$ \\
\hline
\end{tabular}


Table B2-36. Tank 241-AN-104 Analytical Results: Strontium (ICP). (3 sheets)

\begin{tabular}{|c|c|c|c|c|c|}
\hline WMir & Oranger & 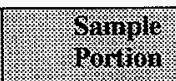 & 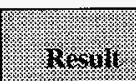 & 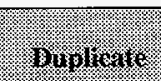 & (1) \\
\hline yiging & . & (3.: & 1894n! & \% & (6) \\
\hline \$96T005256 & $163: 2$ & Liquid & $<6.01$ & $<6.01$ & $<6.01$ \\
\hline S96T005260 & $163: 4$ & Liquid & $<6.01$ & $<6.01$ & $<6.01$ \\
\hline \$96T005527 & $163: 5$ & Liquid & $<6.01$ & $<6.01$ & $<6.01$ \\
\hline S96T005554 & $163: 6$ & Liquid & $<6.01$ & $<6.01$ & $<6.01$ \\
\hline \$96T005255 & $163: 7$ & Liquid & $<6.01$ & $<6.01$ & $<6.01$ \\
\hline S96T005257 & $163: 8$ & Liquid & $<6.01$ & $<6.01$ & $<6.01$ \\
\hline S96T005258 & $163: 9$ & Liquid & $<6.01$ & $<6.01$ & $<6.01$ \\
\hline S96T005528 & $163: 10$ & Liquid & $<6.01$ & $<6.01$ & $<6.01$ \\
\hline S96T005739 & $163: 11$ & Liquid & $<6.01$ & $<6.01$ & $<6.01$ \\
\hline S96T005766 & $163: 12$ & Liquid & $<6.01$ & $<6.01$ & $<6.01$ \\
\hline \$96T005741 & $163: 14$ & Liquid & $<6.01$ & $<6.01$ & $<6.01$ \\
\hline S96T004774 & $164: 1$ & Liquid & $<12$ & $<12$ & $<12$ \\
\hline S96T004778 & $164: 2$ & Liquid & $<12$ & $<12$ & $<12$ \\
\hline S96T004779 & $164: 3$ & Liquid & $<12$ & $<12$ & $<12$ \\
\hline S96T004780 & $164: 4$ & Liquid & $<6.01$ & $<6.01$ & $<6.01$ \\
\hline \$96T004976 & $164: 5$ & Liquid & $<6.01$ & $<6.01$ & $<6.01$ \\
\hline S96T004781 & $164: 7$ & Liquid & $<6.01$ & $<6.01$ & $<6.01$ \\
\hline S96T004977 & $164: 8$ & Liquid & $<6.01$ & $<6.01$ & $<6.01$ \\
\hline S96T004978 & $164: 9$ & Liquid & $<6.01$ & $<6.01$ & $<6.01$ \\
\hline S96T004979 & $164: 10$ & Liquid & $<6.01$ & $<6.01$ & $<6.01$ \\
\hline S96T004782 & $164: 11$ & Liquid & $<6.01$ & $<6.01$ & $<6.01$ \\
\hline S96T004783 & $164: 12$ & Liquid & $<6.01$ & $<6.01$ & $<6.01$ \\
\hline S96T004784 & $164: 13$ & Liquid & $<6.01$ & $<6.01$ & $<6.01$ \\
\hline S96T005979 & Core 164 & Composite & $<12$ & $<12$ & $<12$ \\
\hline
\end{tabular}


HNF-SD-WM-ER-690 Rev. 0

Table B2-37. Tank 241-AN-104 Analytical Results: Sulfur (ICP). (3 sheets)

\begin{tabular}{|c|c|c|c|c|c|}
\hline 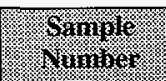 & ormole & 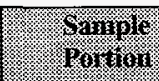 & (2) & 18) & (3) \\
\hline \multicolumn{2}{|c|}{ 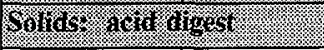 } & l. & 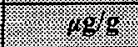 & 1. & ma \\
\hline S96T005287 & $163: 1$ & Lower half & 3,510 & 3,420 & 3,465 \\
\hline S96T005288 & $163: 2$ & Lower half & 1,870 & 1,940 & $1,905^{\mathrm{QC}: d}$ \\
\hline S96T005782 & $163: 12$ & Lower half & 2,550 & 2,470 & 2,510 \\
\hline S96T005749 & \multirow[t]{2}{*}{$163: 14$} & Upper half & 2,990 & 3,030 & 3,010 \\
\hline S96T005750 & & Lower half & 3,340 & 3,310 & 3,325 \\
\hline \$96T005294 & \multirow[t]{2}{*}{$163: 16$} & Upper half & 4,910 & 4,090 & 4,500 \\
\hline S96T005289 & & Lower half & 4,550 & 3,840 & 4,195 \\
\hline S96T005778 & \multirow[t]{2}{*}{$163: 18$} & Upper half & 5,430 & 5,490 & 5,460 \\
\hline S96T005783 & & Lower half & 5,590 & $5 ; 570$ & 5,580 \\
\hline$\$ 96 T 005557$ & \multirow[t]{2}{*}{$163: 20$} & Upper half & 5,160 & 5,030 & 5,095 \\
\hline \$96T005560 & & Lower half & 4,330 & 3,520 & $3,925^{\mathrm{QC:c,c}}$ \\
\hline \$96T004797 & $164: 1$ & Lower half & 2,500 & 2,530 & 2,515 \\
\hline S96T004798 & $164: 13$ & Lower half & 2,780 & 2,860 & 2,820 \\
\hline \$96T005042 & \multirow[t]{2}{*}{$164: 14$} & Upper half & 3,260 & 3,270 & 3,265 \\
\hline \$96T005040 & & Lower half & 3,210 & 3,160 & 3,185 \\
\hline S96T005061 & \multirow[t]{2}{*}{$164: 15$} & Upper half & 3,390 & 3,460 & 3,425 \\
\hline$\$ 96 \mathrm{T005043}$ & & Lower half & 3,970 & 3,780 & 3,875 \\
\hline \$96T005062 & \multirow[t]{2}{*}{$164: 16$} & Upper half & 3,270 & 3,670 & 3,470 \\
\hline \$96T005044 & & Lower half & 3,620 & 3,600 & 3,610 \\
\hline S96T005063 & \multirow[t]{2}{*}{$164: 17$} & Upper half & 3,390 & 3,520 & 3,455 \\
\hline \$96T005041 & & Lower half & 4,050 & 4,080 & 4,065 \\
\hline S96T005064 & \multirow[t]{2}{*}{$164: 19$} & Upper half & 4,100 & 4,360 & 4,230 \\
\hline S96T005045 & & Lower half & 3,520 & 4,020 & 3,770 \\
\hline S96T005282 & \multirow[t]{2}{*}{$164: 20$} & Upper half & 4,150 & 3,730 & 3,940 \\
\hline \$96T005279 & & Lower half & 5,550 & 3,950 & $4,750^{\mathrm{QC:d,c}}$ \\
\hline S96T005976 & Core 164 & Composite & 4,220 & 3,740 & 3,980 \\
\hline
\end{tabular}


Table B2-37. Tank 241-AN-104 Analytical Results: Sulfur (ICP). (3 sheets)

\begin{tabular}{|c|c|c|c|c|c|}
\hline 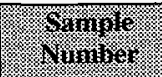 & Whande & (1939) & rex & 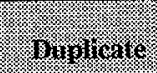 & Her \\
\hline \multicolumn{2}{|c|}{ 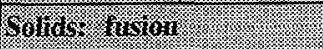 } & (3) & . & (3. & (3) \\
\hline S96T005284 & $163: 1$ & Lower half & 4,500 & 4,000 & 4,250 \\
\hline S96T005285 & $163: 2$ & Lower half & $<1,790$ & $<1,910$ & $<1,850$ \\
\hline S96T005780 & $163: 12$ & Lower half & 2,760 & 2,820 & 2,790 \\
\hline S96T005747 & \multirow[t]{2}{*}{$163: 14$} & Upper half & 3,400 & 3,100 & 3,250 \\
\hline S96T005748 & & Lower half & 3,920 & 3,370 & 3,645 \\
\hline S96T005293 & \multirow[t]{2}{*}{$163: 16$} & Upper half & 5,010 & 5,010 & 5,010 \\
\hline S96T005286 & & Lower half & 4,180 & 4,390 & 4,285 \\
\hline S96T005777 & \multirow[t]{2}{*}{$163: 18$} & Upper half & 7,840 & 6,450 & 7,145 \\
\hline S96T005781 & & Lower half & 6,880 & 7,310 & 7,095 \\
\hline S96T005556 & \multirow[t]{2}{*}{$163: 20$} & Upper half & 5,560 & 5,470 & 5,515 \\
\hline \$96T005559 & & Lower half & 3,970 & 5,520 & $4,745^{\mathrm{QC}: \mathrm{c}}$ \\
\hline S96T004795 & $164: 1$ & Lower half & 3,480 & 3,140 & 3,310 \\
\hline S96T004796 & $164: 13$ & Lower half & 2,720 & 3,120 & 2,920 \\
\hline S96T005035 & \multirow[t]{2}{*}{$164: 14$} & Upper half & 2,690 & 2,750 & 2,720 \\
\hline S96T005030 & & Lower half & 3,300 & 3,410 & 3,355 \\
\hline S96T005036 & \multirow[t]{2}{*}{$164: 15$} & Upper half & 3,580 & 3,460 & 3,520 \\
\hline S96T005032 & & Lower half & 3,910 & 4,030 & 3,970 \\
\hline \$96T005037 & \multirow[t]{2}{*}{$164: 16$} & Upper half & 3,400 & 3,360 & 3,380 \\
\hline \$96T005033 & & Lower half & 3,580 & 3,550 & 3,565 \\
\hline \$96T005038 & \multirow[t]{2}{*}{$164: 17$} & Upper half & 2,790 & 3,360 & 3,075 \\
\hline S96T005031 & & Lower half & 4,070 & 4,100 & 4,085 \\
\hline S96T005039 & \multirow[t]{2}{*}{$164: 19$} & Upper half & 4,070 & 3,800 & 3,935 \\
\hline$\longdiv { \$ 9 6 T 0 0 5 0 3 4 }$ & & Lower half & 3,430 & 3,630 & 3,530 \\
\hline \$96T005281 & \multirow[t]{2}{*}{$164: 20$} & Upper half & 5,110 & 3,760 & $4,435^{\mathrm{QC:c}}$ \\
\hline S96T005278 & & Lower half & 5,700 & 5,600 & 5,650 \\
\hline
\end{tabular}


HNF-SD-WM-ER-690 Rev. 0

Table B2-37. Tank 241-AN-104 Analytical Results: Sulfur (ICP). (3 sheets)

\begin{tabular}{|c|c|c|c|c|c|}
\hline Wamal & 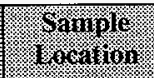 & $\begin{array}{l}\text { Ganple } \\
\text { Pyingur }\end{array}$ & (1) & 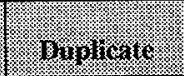 & $4 \log 1$ \\
\hline finumis & & & 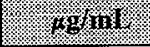 & \%glain: & \% \\
\hline \$96T005256 & $163: 2$ & Liquid & 1,210 & 1,310 & 1,260 \\
\hline S96T005260 & $163: 4$ & Liquid & 1,200 & 1,120 & 1,160 \\
\hline \$96T005527 & $163: 5$ & Liquid & 1,120 & 1,080 & 1,100 \\
\hline S96T005554 & $163: 6$ & Liquid & 1,140 & 1,140 & 1,140 \\
\hline S96T005255 & $163: 7$ & Liquid & 1,220 & 1,280 & 1,250 \\
\hline \$96T005257 & $163: 8$ & Liquid & 1,110 & 1,130 & 1,120 \\
\hline$\$ 96 \mathrm{~T} 005258$ & $163: 9$ & Liquid & 1,180 & 1,130 & 1,155 \\
\hline S96T005528 & $163: 10$ & Liquid & 1,050 & 1,090 & 1,070 \\
\hline \$96T005739 & $163: 11$ & Liquid & 1,120 & 1,120 & 1,120 \\
\hline S96T005766 & $163: 12$ & Liquid & 1,390 & 1,380 & 1,385 \\
\hline S96T005741 & $163: 14$ & Liquid & 1,470 & 1,440 & 1,455 \\
\hline S96T004774 & $164: 1$ & Liquid & 1,200 & 1,220 & 1,210 \\
\hline \$96T004778 & $164: 2$ & Liquid & 1,010 & 1,160 & 1,085 \\
\hline S96T004779 & $164: 3$ & Liquid & 1,160 & 1,130 & 1,145 \\
\hline \$96T004780 & $164: 4$ & Liquid & 1,100 & 1,110 & 1,105 \\
\hline S96T004976 & $164: 5$ & Liquid & 1,210 & 1,140 & 1,175 \\
\hline S96T004781 & $164: 7$ & Liquid & 1,100 & 1,170 & 1,135 \\
\hline S96T004977 & $164: 8$ & Liquid & 1,310 & 1,100 & 1,205 \\
\hline S96T004978 & $164: 9$ & Liquid & 1,210 & 1,040 & 1,125 \\
\hline S96T004979 & $164: 10$ & Liquid & 1,140 & 1,100 & 1,120 \\
\hline S96T004782 & 164:11 & Liquid & 1,110 & 1,160 & 1,135 \\
\hline S96T004783 & $164: 12$ & Liquid & 1,140 & 1,290 & 1,215 \\
\hline S96T004784 & $164: 13$ & Liquid & 1,150 & 1,220 & $1,185^{\text {QC:d }}$ \\
\hline S96T005979 & Core 164 & Composite & 1,220 & 1,180 & 1,200 \\
\hline
\end{tabular}


Table B2-38. Tank 241-AN-104 Analytical Results: Thallium (ICP). (3 sheets)

\begin{tabular}{|c|c|c|c|c|c|}
\hline (1) & Oraple & 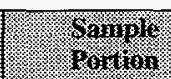 & (3) & 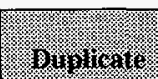 & (1) \\
\hline \multicolumn{3}{|c|}{ 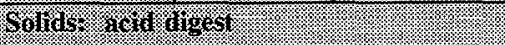 } & $x_{3}$ & $4 \%$ & (3) \\
\hline S96T005287 & $163: 1$ & Lower half & $<75.7$ & $<78.6$ & $<77.15$ \\
\hline S96T005288 & $163: 2$ & Lower half & $<88.7$ & $<74.9$ & $<81.8$ \\
\hline S96T005782 & $163: 12$ & Lower half & $<91.4$ & $<92.2$ & $<91.8$ \\
\hline S96T005749 & \multirow[t]{2}{*}{$163: 14$} & Upper half & $<89.5$ & $<87.2$ & $<88.35$ \\
\hline S96T005750 & & Lower half & $<91.8$ & $<90.4$ & $<91.1$ \\
\hline$\$ 96 \mathrm{~T} 005294$ & \multirow[t]{2}{*}{$163: 16$} & Upper half & $<82.5$ & $<74$ & $<78.25$ \\
\hline S96T005289 & & Lower half & $<92.9$ & $<85.2$ & $<89.05$ \\
\hline \begin{tabular}{|l|} 
S96T005778 \\
\end{tabular} & \multirow[t]{2}{*}{$163: 18$} & Upper half & $<96.2$ & $<90.9$ & $<93.55$ \\
\hline S96T005783 & & Lower half & $<94.8$ & $<94.5$ & $<94.65$ \\
\hline \$96T005557 & \multirow[t]{2}{*}{$163: 20$} & Upper half & $<75.9$ & $<80.2$ & $<78.05$ \\
\hline S96T005560 & & Lower half & $<88.5$ & $<74.6$ & $<81.55$ \\
\hline$\$ 96 \mathrm{T004797}$ & $164: 1$ & Lower half & $<93.8$ & $<94.2$ & $<94$ \\
\hline \$96T004798 & $164: 13$ & Lower half & $<96.8$ & $<95.3$ & $<96.05$ \\
\hline \$96T005042 & \multirow[t]{2}{*}{$164: 14$} & Upper half & $<94.9$ & $<95.9$ & $<95.4$ \\
\hline S96T005040 & & Lower half & $<95.5$ & $<94$ & $<94.75$ \\
\hline S96T005061 & \multirow[t]{2}{*}{$164: 15$} & Upper half & $<96.6$ & $<96$ & $<96.3$ \\
\hline S96T005043 & & Lower half & $<92.8$ & $<95.5$ & $<94.15$ \\
\hline S96T005062 & \multirow[t]{2}{*}{$164: 16$} & Upper half & $<301$ & $<299$ & $<300$ \\
\hline S96T005044 & & Lower half & $<295$ & $<300$ & $<297.5$ \\
\hline S96T005063 & \multirow[t]{2}{*}{$164: 17$} & Upper half & $<279$ & $<286$ & $<282.5$ \\
\hline S96T005041 & & Lower half & $<305$ & $<302$ & $<303.5$ \\
\hline S96T005064 & \multirow[t]{2}{*}{$164: 19$} & Upper half & $<303$ & $<307$ & $<305$ \\
\hline S96T005045 & & Lower half & $<290$ & $<288$ & $<289$ \\
\hline \begin{tabular}{|l|} 
S96T005282 \\
\end{tabular} & \multirow[t]{2}{*}{$164: 20$} & Upper half & $<78.6$ & $<72.1$ & $<75.35$ \\
\hline \$96T005279 & & Lower half & $<90.7$ & $<75.5$ & $<83.1$ \\
\hline S96T005976 & Core 164 & Composite & $<79.5$ & $<79.6$ & $<79.55^{\mathrm{QC}: \mathrm{a}}$ \\
\hline
\end{tabular}


HNF-SD-WM-ER-690 Rev. 0

Table B2-38. Tank 241-AN-104 Analytical Results: Thallium (ICP). (3 sheets)

\begin{tabular}{|c|c|c|c|c|c|}
\hline Siminge & \%oringur & 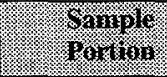 & Resuin & Biulumate & (6) \\
\hline \multicolumn{2}{|c|}{ 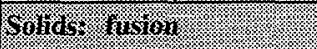 } & & 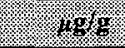 & 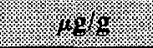 & (1) \\
\hline S96T005284 & $163: 1$ & Lower half & $<3,750$ & $<3,740$ & $<3,745$ \\
\hline \$96T005285 & $163: 2$ & Lower half & $<3,580$ & $<3,820$ & $<3,700$ \\
\hline S96T005780 & $163: 12$ & Lower half & $<3,760$ & $<3,800$ & $<3,780$ \\
\hline \$96T005747 & \multirow[t]{2}{*}{$163: 14$} & Upper half & $<4,480$ & $<4,510$ & $<4,495$ \\
\hline S96T005748 & & Lower half & $<3,730$ & $<3,850$ & $<3,790$ \\
\hline S96T005293 & \multirow[t]{2}{*}{$163: 16$} & Upper half & $<3,550$ & $<3,800$ & $<3,675$ \\
\hline S96T005286 & & Lower half & $<3,970$ & $<3,670$ & $<3,820$ \\
\hline S96T005777 & \multirow[t]{2}{*}{$163: 18$} & Upper half & $<4,100$ & $<3,970$ & $<4,035$ \\
\hline \$96T005781 & & Lower half & $<4,260$ & $<4,280$ & $<4,270$ \\
\hline S96T005556 & \multirow[t]{2}{*}{$163: 20$} & Upper half & $<3,700$ & $<3,870$ & $<3,785$ \\
\hline \$96T005559 & & Lower half & $<3,910$ & $<3,890$ & $<3,900$ \\
\hline S96T004795 & $164: 1$ & Lower half & $<4,090$ & $<4,020$ & $<4,055$ \\
\hline S96T004796 & $164: 13$ & Lower half & $<4,120$ & $<4,150$ & $<4,135$ \\
\hline S96T005035 & \multirow[t]{2}{*}{$164: 14$} & Upper half & $<4,080$ & $<4,120$ & $<4,100$ \\
\hline S96T005030 & & Lower half & $<4,100$ & $<4,070$ & $<4,085$ \\
\hline$\$ 96 T 005036$ & \multirow[t]{2}{*}{$164: 15$} & Upper half & $<4,020$ & $<3,930$ & $<3,975$ \\
\hline S96T005032 & & Lower half & $<3,950$ & $<4,010$ & $<3,980$ \\
\hline \$96T005037 & \multirow[t]{2}{*}{$164: 16$} & Upper half & $<3,840$ & $<3,660$ & $<3,750$ \\
\hline$\$ 96 \mathrm{~T} 005033$ & & Lower half & $<3,830$ & $<3,730$ & $<3,780$ \\
\hline S96T005038 & \multirow[t]{2}{*}{$164: 17$} & Upper half & $<3,860$ & $<3,790$ & $<3,825$ \\
\hline S96T005031 & & Lower half & $<4,370$ & $<4,340$ & $<4,355$ \\
\hline S96T005039 & \multirow[t]{2}{*}{$164: 19$} & Upper half & $<3,890$ & $<4,000$ & $<3,945$ \\
\hline S96T005034 & & Lower half & $<4,270$ & $<4,350$ & $<4,310$ \\
\hline S96T005281 & \multirow[t]{2}{*}{$164: 20$} & Upper half & $<3,440$ & $<3,700$ & $<3,570$ \\
\hline S96T005278 & & Lower half & $<3,770$ & $<3,870$ & $<3,820$ \\
\hline
\end{tabular}


Table B2-38. Tank 241-AN-104 Analytical Results: Thallium (ICP). (3 sheets)

\begin{tabular}{|c|c|c|c|c|c|}
\hline $\begin{array}{l}\text { Sample } \\
\text { Ninnili- }\end{array}$ & Sarnole & $\begin{array}{l}\text { Sampro } \\
\text { fortion }\end{array}$ & Resurt & 8yiligate & 8. n1: \\
\hline Mininis: & 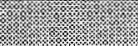 & : & 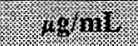 & ( 169111 & 1898910 \\
\hline S96T005256 & $163: 2$ & Liquid & $<120$ & $<120$ & $<120$ \\
\hline S96T005260 & $163: 4$ & Liquid & $<120$ & $<120$ & $<120$ \\
\hline S96T005527 & $163: 5$ & Liquid & $<120$ & $<120$ & $<120$ \\
\hline S96T005554 & $163: 6$ & Liquid & $<120$ & $<\overline{120}$ & $<120$ \\
\hline S96T005255 & $163: 7$ & Liquid & $<120$ & $<120$ & $<120$ \\
\hline S96T005257 & $163: 8$ & Liquid & $<120$ & $<120$ & $<120$ \\
\hline S96T005258 & $163: 9$ & Liquid & $<120$ & $<120$ & $<120$ \\
\hline S96T005528 & $163: 10$ & Liquid & $<120$ & $<120$ & $<120$ \\
\hline S96T005739 & $163: 11$ & Liquid & $<120$ & $<120$ & $<120$ \\
\hline S96r005766 & $163: 12$ & Liquid & $<120$ & $<120$ & $<120$ \\
\hline S96T005741 & $163: 14$ & Liquid & $<120$ & $<120$ & $<120$ \\
\hline S96T004774 & $164: 1$ & Liquid & $<240$ & $<240$ & $<240$ \\
\hline S96T004778 & $164: 2$ & Liquid & $<240$ & $<240$ & $<240$ \\
\hline S96T004779 & $164: \overline{3}$ & Liquid & $<240$ & $<240$ & $<240$ \\
\hline S96T004780 & $164: 4$ & Liquid & $<120$ & $<120$ & $<120$ \\
\hline S96T004976 & $164: 5$ & Liquid & $<120$ & $<120$ & $<120$ \\
\hline S96T004781 & $164: 7$ & Liquid & $<120$ & $<120$ & $<120$ \\
\hline S96T004977 & $164: 8$ & Liquid & $<120$ & $<120$ & $<120$ \\
\hline S96T004978 & $164: 9$ & Liquid & $<120$ & $<120$ & $<120$ \\
\hline S96T004979 & $164: 10$ & Liquid & $<120$ & $<120$ & $<120$ \\
\hline S96T004782 & $164: 11$ & Liquid & $<120$ & $<120$ & $<120$ \\
\hline S96T004783 & $164: 12$ & Liquid & $<120$ & $<120$ & $<120$ \\
\hline S96T004784 & $164: 13$ & Liquid & $<120$ & $<120$ & $<120$ \\
\hline S96T005979 & Core 164 & Composite & $<240$ & $<240$ & $<240$ \\
\hline
\end{tabular}


Table B2-39. Tank 241-AN-104 Analytical Results: Titanium (ICP). (3 sheets)

\begin{tabular}{|c|c|c|c|c|c|}
\hline Shanie & 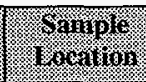 & \%quning & Rosinit & (3) & 109. \\
\hline \multicolumn{3}{|c|}{ 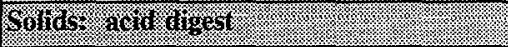 } & $368 \%$ & 686 & 45 \\
\hline S96T005287 & $163: 1$ & Lower half & $<3.79$ & $<3.93$ & $<3.86$ \\
\hline \$96T005288 & $163: 2$ & Lower half & $<4.43$ & $<3.75$ & $<4.09$ \\
\hline S96T005782 & $163: 12$ & Lower half & $<4.57$ & $<4.61$ & $<4.59^{\mathrm{QC}: \mathrm{a}}$ \\
\hline S96T005749 & \multirow[t]{2}{*}{$163: 14$} & Upper half & $<4.48$ & $<4.36$ & $<4.42^{\mathrm{QC:a}}$ \\
\hline$\$ 96 \mathrm{~T} 005750$ & & Lower half & $<4.59$ & $<4.52$ & $<4.555^{\mathrm{QC:a}}$ \\
\hline \$96T005294 & \multirow[t]{2}{*}{$163: 16$} & Upper half & $<4.13$ & $<3.7$ & $<3.915$ \\
\hline \$96T005289 & & Lower half & $<4.64$ & $<4.26$ & $<4.45$ \\
\hline S96T005778 & \multirow[t]{2}{*}{$163: 18$} & Upper half & $<4.81$ & $<4.54$ & $<4.675^{\mathrm{QC:a}}$ \\
\hline \$96T005783 & & Lower half & $<4.74$ & $<4.72$ & $<4.73^{\mathrm{QC}: \mathrm{a}}$ \\
\hline S96T005557 & \multirow[t]{2}{*}{$163: 20$} & Upper half & $<3.8$ & $<4.01$ & $<3.905$ \\
\hline$\overline{\text { S96T005560 }}$ & & Lower half & $<4.42$ & $<3.73$ & $<4.075$ \\
\hline S96T004797 & $164: 1$ & Lower half & $<4.69$ & $<4.71$ & $<4.7$ \\
\hline S96T004798 & $164: 13$ & Lower half & $<4.84$ & $<4.77$ & $<4.805$ \\
\hline 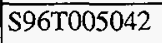 & \multirow[t]{2}{*}{$164: 14$} & Upper half & $<4.74$ & $<4.8$ & $<4.77$ \\
\hline S96T005040 & & Lower half & $<4.77$ & $<4.7$ & $<4.735$ \\
\hline S96T005061 & \multirow[t]{2}{*}{$164: 15$} & Upper half & $<4.83$ & $<4.8$ & $<4.815$ \\
\hline \begin{tabular}{|l} 
S96T005043 \\
\end{tabular} & & Lower half & $<4.64$ & $<4.77$ & $<4.705$ \\
\hline S96T005062 & \multirow[t]{2}{*}{$164: 16$} & Upper half & $<15.1$ & $<14.9$ & $<15$ \\
\hline S96T005044 & & Lower half & $<14.7$ & $<15$ & $<14.85$ \\
\hline \$96T005063 & \multirow[t]{2}{*}{$164: 17$} & Upper half & $<13.9$ & $<14.3$ & $<14.1$ \\
\hline S96T005041 & & Lower half & $<15.2$ & $<15.1$ & $<15.15$ \\
\hline S96T005064 & \multirow[t]{2}{*}{$164: 19$} & Upper half & $<15.1$ & $<15.3$ & $<\overline{15.2}$ \\
\hline S96T005045 & & Lower half & $<14.5$ & $<14.4$ & $<14.45$ \\
\hline \$96T005282 & \multirow[t]{2}{*}{$164: 20$} & Upper half & $<3.93$ & $<3.61$ & $<3.77$ \\
\hline S96T005279 & & Lower half & $<4.54$ & $<3.78$ & $<4.16$ \\
\hline S96T005976 & Core 164 & Composite & $<3.98$ & $<3.98$ & $<3.98^{\mathrm{QC}: \mathrm{a}}$ \\
\hline
\end{tabular}


Table B2-39. Tank 241-AN-104 Analytical Results: Titanium (ICP). (3 sheets)

\begin{tabular}{|c|c|c|c|c|c|}
\hline 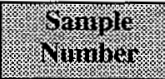 & thar & 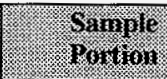 & . & 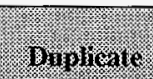 & 10ain \\
\hline S618. & & 8 & . & $4 \%$ & (1) \\
\hline S96T005284 & $163: 1$ & Lower half & $<187$ & $<187$ & $<187$ \\
\hline S96T005285 & $163: 2$ & Lower half & $<179$ & $<191$ & $<185$ \\
\hline S96T005780 & $163: 12$ & Lower half & $<188$ & $<190$ & $<189$ \\
\hline S96T005747 & \multirow[t]{2}{*}{$163: \overline{14}$} & Upper half & $<224$ & $<225$ & $<224.5$ \\
\hline S96T005748 & & Lower half & $<187$ & $<193$ & $<190$ \\
\hline S96T005293 & \multirow[t]{2}{*}{$163: 16$} & Upper half & $<177$ & $<190$ & $<183.5$ \\
\hline$\$ 96 \mathrm{T005286}$ & & Lower half & $<199$ & $<183$ & $<191$ \\
\hline \$96T005777 & \multirow[t]{2}{*}{$163: 18$} & Upper half & $<205$ & $<198$ & $<201.5$ \\
\hline S96T005781 & & Lower half & $<213$ & $<214$ & $<213.5$ \\
\hline S96T005556 & \multirow[t]{2}{*}{$163: 20$} & Upper half & $<185$ & $<194$ & $<189.5$ \\
\hline \$96T005559 & & Lower half & $<195$ & $<195$ & $<195$ \\
\hline \$96T004795 & $164: 1$ & Lower half & $<204$ & $<201$ & $<202.5$ \\
\hline S96T004796 & $164: 13$ & Lower half & $<206$ & $<207$ & $<206.5$ \\
\hline S96T005035 & \multirow[t]{2}{*}{$164: 14$} & Upper half & $<204$ & $<206$ & $<205$ \\
\hline S96Г005030 & & Lower half & $<205$ & $<204$ & $<204.5$ \\
\hline S96T005036 & \multirow[t]{2}{*}{$164: 15$} & Upper half & $<201$ & $<197$ & $<199$ \\
\hline \$96T005032 & & Lower half & $<197$ & $<200$ & $<198.5$ \\
\hline S96T005037 & \multirow[t]{2}{*}{$164: 16$} & Upper half & $<\overline{192}$ & $<183$ & $<187.5$ \\
\hline S96T005033 & & Lower half & $<191$ & $<187$ & $<189$ \\
\hline S96T005038 & \multirow[t]{2}{*}{$164: 17$} & Upper half & $<193$ & $<190$ & $<191.5$ \\
\hline$\overline{\$ 96 \mathrm{~T} 005031}$ & & Lower half & $<218$ & $<217$ & $<217.5$ \\
\hline S96T005039 & \multirow[t]{2}{*}{$164: 19$} & Upper half & $<194$ & $<200$ & $<197$ \\
\hline \$96T005034 & & Lower half & $<214$ & $<217$ & $<215.5$ \\
\hline S96T005281 & \multirow[t]{2}{*}{$164: 20$} & Upper half & $<172$ & $<185$ & $<178.5$ \\
\hline S96T005278 & & Lower half & $<189$ & $<193$ & $<191$ \\
\hline
\end{tabular}


HNF-SD-WM-ER-690 Rev. 0

Table B2-39. Tank 241-AN-104 Analytical Results: Titanium (ICP). (3 sheets)

\begin{tabular}{|c|c|c|c|c|c|}
\hline Sumplo & Somolom & Gomole & Resint & 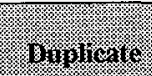 & (16) \\
\hline Hilnis & & 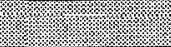 & 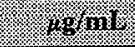 & 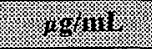 & 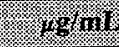 \\
\hline S96T005256 & $163: 2$ & Liquid & $<6.01$ & $<6.01$ & $<6.01$ \\
\hline$\$ 96 T 005260$ & $163: 4$ & Liquid & $<6.01$ & $<6.01$ & $<6.01$ \\
\hline \$96T005527 & $163: 5$ & Liquid & $<6.01$ & $<6.01$ & $<6.01$ \\
\hline \$96T005554 & $163: 6$ & Liquid & $<6.01$ & $<6.01$ & $<6.01$ \\
\hline S96T005255 & $163: 7$ & Liquid & $<6.01$ & $<6.01$ & $<6.01$ \\
\hline S96T005257 & $163: 8$ & Liquid & $<6.01$ & $<6.01$ & $<6.01$ \\
\hline \$96T005258 & $163: 9$ & Liquid & $<6.01$ & $<6.01$ & $<6.01$ \\
\hline S96T005528 & $163: 10$ & Liquid & $<6.01$ & $<6.01$ & $<6.01$ \\
\hline S96T005739 & $163: 11$ & Liquid & $<6.01$ & $<6.01$ & $<6.01$ \\
\hline \$96T005766 & $163: 12$ & Liquid & $<6.01$ & $<6.01$ & $<6.01$ \\
\hline S96T005741 & $163: 14$ & Liquid & $<6.01$ & $<6.01$ & $<6.01$ \\
\hline S96T004774 & $164: 1$ & Liquid & $<12$ & $<12$ & $<12$ \\
\hline \$96T004778 & $164: 2$ & Liquid & $<12$ & $<12$ & $<12$ \\
\hline \$96T004779 & $164: 3$ & Liquid & $<12$ & $<12$ & $<12$ \\
\hline S96T004780 & $164: 4$ & Liquid & $<6.01$ & $<6.01$ & $<6.01$ \\
\hline S96T004976 & $164: 5$ & Liquid & $<6.01$ & $<6.01$ & $<6.01$ \\
\hline \$96T004781 & $164: 7$ & Liquid & $<6.01$ & $<6.01$ & $<6.01$ \\
\hline \$96T004977 & $164: 8$ & Liquid & $<6.01$ & $<6.01$ & $<6.01$ \\
\hline S96T004978 & $164: 9$ & Liquid & $<6.01$ & $<6.01$ & $<6.01$ \\
\hline S96T004979 & $164: 10$ & Liquid & $<6.01$ & $<6.01$ & $<6.01$ \\
\hline S96T004782 & $164: 11$ & Liquid & $<6.01$ & $<6.01$ & $<6.01$ \\
\hline S96T004783 & $164: 12$ & Liquid & $<6.01$ & $<6.01$ & $<6.01$ \\
\hline S96T004784 & $164: 13$ & Liquid & $<6.01$ & $<6.01$ & $<6.01$ \\
\hline S96T005979 & Core 164 & Composite & $<12$ & $<12$ & $<12$ \\
\hline
\end{tabular}


HNF-SD-WM-ER-690 Rev. 0

Table B2-40. Tank 241-AN-104 Analytical Results: Total Uranium (ICP). (3 sheets)

\begin{tabular}{|c|c|c|c|c|c|}
\hline $\begin{array}{l}\text { Sarnule } \\
\text { Sminter }\end{array}$ & Sogringe & 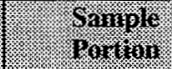 & Rersin: & Puplisalt & Neran \\
\hline \multicolumn{2}{|c|}{ Srolids yelo digers } & 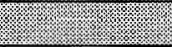 & 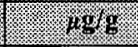 & 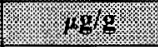 & 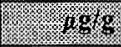 \\
\hline S96T005287 & $163: 1$ & Lower half & $<189$ & $<196$ & $<192.5$ \\
\hline S96T005288 & $163: 2$ & Lower half & $<222$ & $<187$ & $<204.5$ \\
\hline S96T005782 & $163: 12$ & Lower half & $<228$ & $<\overline{230}$ & $<229$ \\
\hline \$96T005749 & \multirow{2}{*}{$163: 14$} & Upper half & $<224$ & $<218$ & $<221$ \\
\hline S96T005750 & & Lower half & $<230$ & $<226$ & $<228$ \\
\hline S96T005294 & \multirow[t]{2}{*}{$163: 16$} & Upper half & 230 & 196 & 213 \\
\hline S96T005289 & & Lower half & $<232$ & $<213$ & $<222.5$ \\
\hline S96T005778 & \multirow[t]{2}{*}{$163: 18$} & Upper half & 264 & 282 & 273 \\
\hline S96T005783 & & Lower half & 268 & 269 & 268.5 \\
\hline S96T005557 & \multirow[t]{2}{*}{$163: 20$} & Upper half & 237 & 235 & 236 \\
\hline S96T005560 & & Lower half & 353 & 283 & $318^{Q C: c}$ \\
\hline S96T004797. & $164: 1$ & Lower half & $<234$ & $<235$ & $<234.5$ \\
\hline S96T004798 & $164: 13$ & Lower half & $<242$ & $<238$ & $<240$ \\
\hline S96T005042 & \multirow[t]{2}{*}{$164: 14$} & Upper half & $<237$ & $<240$ & $<238.5$ \\
\hline S96T005040 & & Lower half & $<239$ & $<235$ & $<237$ \\
\hline S96T005061 & \multirow[t]{2}{*}{$164: 15$} & Upper half & $<242$ & $<240$ & $<241$ \\
\hline S96T005043 & & Lower half & $<232$ & $<239$ & $<235.5$ \\
\hline S96T005062 & \multirow[t]{2}{*}{$164: 16$} & Upper half & $<753$ & $<747$ & $<750$ \\
\hline \$96T005044 & & Lower half & $<737$ & $<750$ & $<743.5$ \\
\hline S96T005063 & \multirow[t]{2}{*}{$164: 17$} & Upper half & $<697$ & $<714$ & $<705.5$ \\
\hline S96T005041 & & Lower half & $<762$ & $<756$ & $<759$ \\
\hline S96T005064 & \multirow[t]{2}{*}{$164: 19$} & Upper half & $<756$ & $<767$ & $<761.5$ \\
\hline S96T005045 & & Lower half & $<725$ & $<720$ & $<722.5$ \\
\hline S96T005282 & \multirow[t]{2}{*}{$164: 20$} & Upper half & $<197$ & $<180$ & $<188.5$ \\
\hline S96T005279 & & Lower half & $<227$ & $<189$ & $<208$ \\
\hline S96T005976 & Core 164 & Composite & $<199$ & $<199$ & $<199^{Q \mathrm{QC}: a}$ \\
\hline
\end{tabular}


HNF-SD-WM-ER-690 Rev. 0

Table B2-40. Tank 241-AN-104 Analytical Results: Total Uranium (ICP). (3 sheets)

\begin{tabular}{|c|c|c|c|c|c|}
\hline 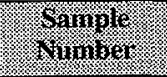 & mol & (6) & 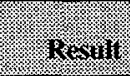 & \%) & (6) \\
\hline 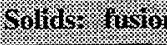 & & (3. & 689 & 3. & \%got \\
\hline S96T005284 & $163: 1$ & Lower half & $<9,370$ & $<9,360$ & $<9,365$ \\
\hline S96T005285 & $163: 2$ & Lower half & $<8,940$ & $<9,560$ & $<9,250$ \\
\hline S96T005780 & $163: 12$ & Lower half & $<9,400$ & $<9,500$ & $<9,450$ \\
\hline S96T005747 & \multirow[t]{2}{*}{$163: 14$} & Upper half & $<11,200$ & $<11,300$ & $<11,250$ \\
\hline$\$ 96 \mathrm{~T} 005748$ & & Lower half & $<9,340$ & $<9,630$ & $<9,485$ \\
\hline \$96T005293 & \multirow[t]{2}{*}{$163: 16$} & Upper half & $<8,870$ & $<9,490$ & $<9,180$ \\
\hline S96T005286 & & Lower half & $<9,930$ & $<9,170$ & $<9,550$ \\
\hline \$96T005777 & \multirow[t]{2}{*}{$163: 18$} & Upper half & $<10,300$ & $<9,920$ & $<10,110$ \\
\hline S96T005781 & & Lower half & $<10,600$ & $<10,700$ & $<10,650$ \\
\hline S96T005556 & \multirow[t]{2}{*}{$163: 20$} & Upper half & $<9,240$ & $<9,680$ & $<9,460$ \\
\hline S96T005559 & & Lower half & $<9, \overline{770}$ & $<9,730$ & $<9,750$ \\
\hline S96T004795 & $164: 1$ & Lower half & $<10,200$ & $<10,000$ & $<10,100$ \\
\hline \$96T004796 & $164: 13$ & Lower half & $<10,300$ & $<10,400$ & $<10,350$ \\
\hline S96T005035 & \multirow[t]{2}{*}{$164: 14$} & Upper half & $<10,200$ & $<10,300$ & $<10,250$ \\
\hline S96T005030 & & Lower half & $<10,200$ & $<10,200$ & $<10,200$ \\
\hline S96T005036 & \multirow[t]{2}{*}{$164: 15$} & Upper half & $<10,000$ & $<9,830$ & $<9,915$ \\
\hline S96T005032 & & Lower half & $<9,870$ & $<10,000$ & $<9,935$ \\
\hline S96T005037 & \multirow[t]{2}{*}{$164: 16$} & Upper half & $<9,610$ & $<9,150$ & $<9,380$ \\
\hline S96T005033 & & Lower half & $<9,570$ & $<9,330$ & $<9,450$ \\
\hline S96T005038 & \multirow[t]{2}{*}{$164: 17$} & Upper half & $<9,660$ & $<\overline{9}, 490$ & $<9,575$ \\
\hline S96T005031 & & Lower half & $<10,900$ & $<10,800$ & $<10,850$ \\
\hline S96T005039 & \multirow[t]{2}{*}{$164: 19$} & Upper half & $<9,720$ & $<\overline{10,000}$ & $<9,860$ \\
\hline S96T005034 & & Lower half & $<10,700$ & $<10,900$ & $<10,800$ \\
\hline S96T005281 & \multirow[t]{2}{*}{$164: 20$} & Upper half & $<8,600$ & $<9,250$ & $<8,925$ \\
\hline S96T005278 & & Lower half & $<9,430$ & $<9,660$ & $<9,545$ \\
\hline
\end{tabular}


HNF-SD-WM-ER-690 Rev. 0

Table B2-40. Tank 241-AN-104 Analytical Results: Total Uranium (ICP). (3 sheets)

\begin{tabular}{|c|c|c|c|c|c|}
\hline 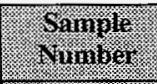 & Mamp & 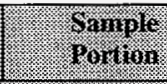 & Resing & (9u1) & Wor \\
\hline Haud & $1 \%$ \% & (1) & K & . & mown \\
\hline \$96T005256 & $163: 2$ & Liquid & $<300$ & $<300$ & $<300$ \\
\hline S96T005260 & $163: 4$ & Liquid & $<300$ & $<300$ & $<300$ \\
\hline \$96T005527 & $163: 5$ & Liquid & $<300$ & $<300$ & $<300$ \\
\hline S96T005554 & $163: 6$ & Liquid & $<300$ & $<300$ & $<300$ \\
\hline S96T005255 & $163: 7$ & Liquid & $<300$ & $<300$ & $<300$ \\
\hline \$96T005257 & $163: 8$ & Liquid & $<300$ & $<300$ & $<300$ \\
\hline S96T005258 & $163: 9$ & Liquid & $<300$ & $<300$ & $<300$ \\
\hline S96T005528 & $163: 10$ & Liquid & $<300$ & $<300$ & $<300$ \\
\hline S96T005739 & $163: 11$ & Liquid & $<300$ & $<300$ & $<300$ \\
\hline S96T005766 & $163: 12$ & Liquid & $<300$ & $<300$ & $<300$ \\
\hline S96T005741 & $163: 14$ & Liquid & $<300$ & $<300$ & $<300$ \\
\hline$\$ 96 \mathrm{~T} 004774$ & $164: 1$ & Liquid & $<600$ & $<600$ & $<600$ \\
\hline S96T004778 & $164: 2$ & Liquid & $<600$ & $<600$ & $<\overline{600}$ \\
\hline S96T004779 & $164: 3$ & Liquid & $<600$ & $<600$ & $<600$ \\
\hline S96T004780 & $164: 4$ & Liquid & $<300$ & $<300$ & $<300$ \\
\hline S96T004976 & $164: 5$ & Liquid & $<300$ & $<300$ & $<\overline{300}$ \\
\hline S96T004781 & $164: 7$ & Liquid & $<300$ & $<300$ & $<300$ \\
\hline S96T004977 & $164: 8$ & Liquid & $<300$ & $<300$ & $<300$ \\
\hline S96T004978 & $164: 9$ & Liquid & $<300$ & $<300$ & $<300$ \\
\hline S96T004979 & $164: 10$ & Liquid & $<300$ & $<300$ & $<300$ \\
\hline S96T004782 & $164: 11$ & Liquid & $<300$ & $<300$ & $<300$ \\
\hline S96T004783 & $164: 12$ & Liquid & $<300$ & $<300$ & $<300$ \\
\hline S96T004784 & $164: 13$ & Liquid & $<300$ & $<300$ & $<300$ \\
\hline S96T005979 & Core 164 & Composite & $<600$ & $<600$ & $<600$ \\
\hline
\end{tabular}


HNF-SD-WM-ER-690 Rev. 0

Table B2-41. Tank 241-AN-104 Analytical Results: Vanadium (ICP). (3 sheets)

\begin{tabular}{|c|c|c|c|c|c|}
\hline wample & Sompingie & . $140 \%$ & 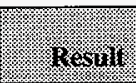 & O) & (2) \\
\hline \multicolumn{2}{|c|}{ 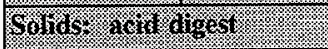 } & (3. & 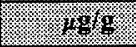 & 160\% & (6) \\
\hline S96T005287 & $163: 1$ & Lower half & $<18.9$ & $<19.6$ & $<19.25$ \\
\hline S96T005288 & $163: 2$ & Lower half & $<22.2$ & $<18.7$ & $<20.45$ \\
\hline S96T005782 & $163: 12$ & Lower half & $<22.8$ & $<23$ & $<22.9$ \\
\hline S96T005749 & \multirow[t]{2}{*}{$163: 14$} & Upper half & $<22.4$ & $<21.8$ & $<22.1$ \\
\hline S96T005750 & & Lower half & $<23$ & $<22.6$ & $<22.8$ \\
\hline \$96T005294 & \multirow[t]{2}{*}{$163: 16$} & Upper half & $<20.6$ & $<18.5$ & $<19.55$ \\
\hline S96T005289 & & Lower half & $<23.2$ & $<21.3$ & $<22.25$ \\
\hline \$96T005778 & \multirow[t]{2}{*}{$163: 18$} & Upper half & $<24.1$ & $<22.7$ & $<23.4$ \\
\hline$\$ 96 \mathrm{~T} 005783$ & & Lower half & $<23.7$ & $<23.6$ & $<23.65$ \\
\hline \$96T005557 & \multirow[t]{2}{*}{$163: 20$} & Upper half & $<19$ & $<20.1$ & $<19.55$ \\
\hline S96T005560 & & Lower half & $<22.1$ & $<18.7$ & $<20.4$ \\
\hline \$96T004797 & $164: 1$ & Lower half & $<23.4$ & $<23.5$ & $<23.45$ \\
\hline S96T004798 & $164: 13$ & Lower half & $<24.2$ & $<23.8$ & $<24$ \\
\hline S96T005042 & \multirow[t]{2}{*}{$164: 14$} & Upper half & $<23.7$ & $<24$ & $<23.85$ \\
\hline S96T005040 & & Lower half & $<23.9$ & $<23.5$ & $<23.7$ \\
\hline S96T005061 & \multirow[t]{2}{*}{$164: 15$} & Upper half & $<24.2$ & $<24$ & $<24.1$ \\
\hline S96T005043 & & Lower half & $<23.2$ & $<23.9$ & $<23.55$ \\
\hline S96T005062 & \multirow[t]{2}{*}{$164: 16$} & Upper half & $<75.3$ & $<74.7$ & $<75$ \\
\hline S96T005044 & & Lower half & $<73.7$ & $<75$ & $<74.35$ \\
\hline S96T005063 & \multirow[t]{2}{*}{$164: 17$} & Upper half & $<69.7$ & $<71.4$ & $<70.55$ \\
\hline S96T005041 & & Lower half & $<76.2$ & $<75.6$ & $<75.9$ \\
\hline \$96T005064 & \multirow[t]{2}{*}{$164: 19$} & Upper half & $<75.6$ & $<76.7$ & $<76.15$ \\
\hline S96T005045 & & Lower half & $<72.5$ & $<72$ & $<72.25$ \\
\hline S96T005282 & \multirow[t]{2}{*}{$164: 20$} & Upper half & $<19.7$ & $<18$ & $<18.85$ \\
\hline$\$ 96 \mathrm{~T} 005279$ & & Lower half & $<22.7$ & $<18.9$ & $<20.8$ \\
\hline \$96T005976 & Core 164 & Composite & $<19.9$ & $<19.9$ & $<19.9^{\mathrm{QC:a}}$ \\
\hline
\end{tabular}


Table B2-41. Tank 241-AN-104 Analytical Results: Vanadium (ICP). (3 sheets)

\begin{tabular}{|c|c|c|c|c|c|}
\hline Sample & 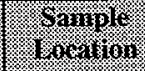 & 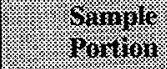 & pesinit & Bimplica fes & 16ru \\
\hline \multicolumn{3}{|c|}{ 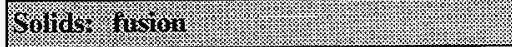 } & (3. & 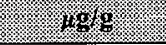 & 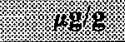 \\
\hline S96T005284 & $163: 1$ & Lower half & $<937$ & $<936$ & $<936.5$ \\
\hline S96T005285 & $163: 2$ & Lower half & $<894$ & $<956$ & $<925$ \\
\hline S96T005780 & $163: 12$ & Lower half & $<940$ & $<950$ & $<945$ \\
\hline S96T005747 & \multirow[t]{2}{*}{$163: 14$} & Upper half & $<1,120$ & $<1,130$ & $<1,125$ \\
\hline S96T005748 & & Lower half & $<934$ & $<963$ & $<948.5$ \\
\hline S96T005293 & \multirow[t]{2}{*}{$163: 16$} & Upper half & $<887$ & $<949$ & $<918$ \\
\hline S96T005286 & & Lower half & $<993$ & $<917$ & $<955$ \\
\hline S96T005777 & \multirow[t]{2}{*}{$163: 18$} & Upper half & $<1,030$ & $<992$ & $<1,011$ \\
\hline S96T005781 & & Lower half & $<1,060$ & $<1,070$ & $<1,065$ \\
\hline \$96T005556 & \multirow[t]{2}{*}{$163: 20$} & Upper half & $<924$ & $<968$ & $<946$ \\
\hline S96T005559 & & Lower half & $<977$ & $<973$ & $<975$ \\
\hline S96T004795 & $164: 1$ & Lower half & $<1,020$ & $<1,000$ & $<1,010$ \\
\hline S96T004796 & $164: 13$ & Lower half & $<1,030$ & $<1,040$ & $<1,035$ \\
\hline S96T005035 & \multirow[t]{2}{*}{$164: 14$} & Upper half & $<1,020$ & $<1,030$ & $<1,025$ \\
\hline S96T005030 & & Lower half & $<1,020$ & $<1,020$ & $<1,020$ \\
\hline S96T005036 & \multirow[t]{2}{*}{$164: 15$} & Upper half & $<1,000$ & $<983$ & $<991.5$ \\
\hline S96T005032 & & Lower half & $<987$ & $<1,000$ & $<993.5$ \\
\hline S96T005037 & \multirow[t]{2}{*}{$164: 16$} & Upper half & $<961$ & $<915$ & $<938$ \\
\hline S96T005033 & & Lower half & $<957$ & $<933$ & $<945$ \\
\hline S96T005038 & \multirow[t]{2}{*}{$164: 17$} & Upper half & $<966$ & $<949$ & $<957.5$ \\
\hline S96T005031 & & Lower half & $<1,090$ & $<1,080$ & $<1,085$ \\
\hline S96T005039 & \multirow[t]{2}{*}{$164: 19$} & Upper haif & $<972$ & $<1,000$ & $<986$ \\
\hline S96T005034 & & Lower half & $<1,070$ & $<1,090$ & $<1,080$ \\
\hline S96T005281 & \multirow[t]{2}{*}{$164: 20$} & Upper half & $<860$ & $<925$ & $<892.5$ \\
\hline S96T005278 & & Lower half & $<943$ & $<966$ & $<954.5$ \\
\hline
\end{tabular}


Table B2-41. Tank 241-AN-104 Analytical Results: Vanadium (ICP). (3 sheets)

\begin{tabular}{|c|c|c|c|c|c|}
\hline grample & \%amplis. & (3) & (1) & Holesis. & (1) \\
\hline Hainis. & & & 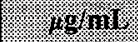 & 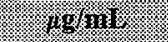 & (x) \\
\hline S96T005256 & $163: 2$ & Liquid & $<30.1$ & $<30.1$ & $<30.1$ \\
\hline S96T005260 & $163: 4$ & Liquid & $<30.1$ & $<30.1$ & $<30.1$ \\
\hline S96T005527 & $163: 5$ & Liquid & $<30.1$ & $<30.1$ & $<30.1$ \\
\hline S96T005554 & $163: 6$ & Liquid & $<30.1$ & $<30.1$ & $<30.1$ \\
\hline S96T005255 & $163: 7$ & Liquid & $<30.1$ & $<30.1$ & $<30.1$ \\
\hline S96T005257 & $163: 8$ & Liquid & $<30.1$ & $<30.1$ & $<30.1$ \\
\hline S96T005258 & $163: 9$ & Liquid & $<30.1$ & $<30.1$ & $<30.1$ \\
\hline S96T005528 & $163: 10$ & Liquid & $<30.1$ & $<30.1$ & $<30.1$ \\
\hline S96T005739 & $163: 11$ & Liquid & $<30.1$ & $<30.1$ & $<30.1$ \\
\hline S96T005766 & $163: 12$ & Liquid & $<30.1$ & $<30.1$ & $<30.1$ \\
\hline \$96T005741 & $163: 14$ & Liquid & $<30.1$ & $<30.1$ & $<30.1$ \\
\hline S96T004774 & $164: 1$ & Liquid & $<60.1$ & $<60.1$ & $<60.1$ \\
\hline \$96T004778 & $164: 2$ & Liquid & $<60.1$ & $<60.1$ & $<60.1$ \\
\hline S96T004779 & $164: 3$ & Liquid & $<60.1$ & $<60.1$ & $<60.1$ \\
\hline S96T004780 & $164: 4$ & Liquid & $<30.1$ & $<30.1$ & $<30.1$ \\
\hline S96T004976 & $164: 5$ & Liquid & $<30.1$ & $<30.1$ & $<30.1$ \\
\hline S96T004781 & $164: 7$ & Liquid & $<30.1$ & $<30.1$ & $<30.1$ \\
\hline S96T004977 & $164: 8$ & Liquid & $<30.1$ & $<30.1$ & $<30.1$ \\
\hline S96T004978 & $164: 9$ & Liquid & $<30.1$ & $<30.1$ & $<30.1$ \\
\hline S96T004979 & $164: 10$ & Liquid & $<30.1$ & $<30.1$ & $<30.1$ \\
\hline S96T004782 & $164: 11$ & Liquid & $<30.1$ & $<30.1$ & $<30.1$ \\
\hline S96T004783 & $164: 12$ & Liquid & $<30.1$ & $<30.1$ & $<30.1$ \\
\hline S96T004784 & $164: 13$ & Liquid & $<30.1$ & $<30.1$ & $<30.1$ \\
\hline S96T005979 & Core 164 & Composite & $<60.1$ & $<60.1$ & $<60.1$ \\
\hline
\end{tabular}


Table B2-42. Tank 241-AN-104 Analytical Results: Zinc (ICP). (3 sheets)

\begin{tabular}{|c|c|c|c|c|c|}
\hline $\begin{array}{l}\text { Sample } \\
\text { Armber- }\end{array}$ & $\begin{array}{l}\text { Sarivole: } \\
\text { I ocrifon }\end{array}$ & 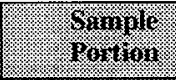 & Resin & Burilsate & Mear \\
\hline \multicolumn{2}{|c|}{ Solids o div Gigest } & 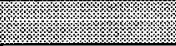 & 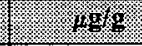 & 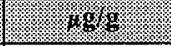 & 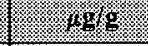 \\
\hline S96T005287 & $163: 1$ & Lower half & 26.2 & 25.2 & 25.7 \\
\hline S96T005288 & $163: 2$ & Lower half & 26.8 & 15.7 & $21.25^{\mathrm{QC}: \mathrm{c}}$ \\
\hline S96T005782 & $163: 12$ & Lower half & 10.2 & 9.99 & 10.095 \\
\hline S96T005749 & \multirow[t]{2}{*}{$163: 14$} & Upper half & 9.61 & 9.6 & 9.605 \\
\hline S96T005750 & & Lower half & 7.68 & 8.11 & 7.895 \\
\hline S96T005294 & \multirow[t]{2}{*}{$163: 16$} & Upper half & 17.8 & 16.5 & 17.15 \\
\hline S96T005289 & & Lower half & 16.9 & 14.5 & 15.7 \\
\hline S96T005778 & \multirow[t]{2}{*}{$163: 18$} & Upper half & 8.5 & 9.96 & 9.23 \\
\hline S96T005783 & & Lower half & 8.51 & 8.53 & 8.52 \\
\hline S96T005557 & \multirow[t]{2}{*}{$163: 20$} & Upper half & 24.1 & 25.8 & 24.95 \\
\hline S96T005560 & & Lower half & 27.2 & 22.8 & 25 \\
\hline S96T004797 & $164: 1$ & Lower half & 29.5 & 29.5 & 29.5 \\
\hline S96T004798 & $164: 13$ & Lower half & 66 & 39 & $52.5^{\mathrm{Qc:c}}$ \\
\hline S96T005042 & \multirow[t]{2}{*}{$164: 14$} & Upper half & 37 & 32.1 & 34.55 \\
\hline S96T005040 & & Lower half & 29.4 & 21.1 & $25.25^{\mathrm{QC}: \mathrm{c}}$ \\
\hline S96T005061 & \multirow[t]{2}{*}{$164: 15$} & Upper half & 17.9 & 33.1 & $25.5^{\mathrm{QC:c}}$ \\
\hline S96T005043 & & Lower half & 22.1 & 30.7 & $26.4^{\mathrm{QC}: \mathrm{c}}$ \\
\hline S96T005062 & \multirow[t]{2}{*}{$164: 16$} & Upper half & $<15.1$ & $<14.9$ & $<15$ \\
\hline S96T005044 & & Lower half & 36.4 & $<15$ & $<25.7^{\mathrm{QC:C}}$ \\
\hline S96T005063 & \multirow[t]{2}{*}{$164: 17$} & Upper half & 14.4 & 14.4 & 14.4 \\
\hline S96T005041 & & Lower half & 31.6 & 17.7 & $24.65^{\mathrm{QC}: \mathrm{c}}$ \\
\hline S96T005064 & \multirow[t]{2}{*}{$164: 19$} & Upper half & 23.5 & 24 & 23.75 \\
\hline \begin{tabular}{|l|} 
S96T005045 \\
\end{tabular} & & Lower half & 21 & 20 & 20.5 \\
\hline S96T005282 & \multirow[t]{2}{*}{$164: 20$} & Upper half & 18.8 & 15.8 & 17.3 \\
\hline S96T005279 & & Lower half & 12.9 & 11.5 & 12.2 \\
\hline S96T005976 & Core 164 & Composite & 19.2 & 14.4 & $16.8^{\mathrm{QC:a,c}}$ \\
\hline
\end{tabular}


Table B2-42. Tank 241-AN-104 Analytical Results: Zinc (ICP). (3 sheets)

\begin{tabular}{|c|c|c|c|c|c|}
\hline Manph & Sominger & 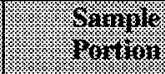 & metrin & 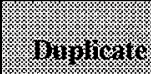 & (1) \\
\hline Sinds & VIr & (3) & (1) & (1) & 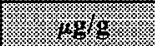 \\
\hline \$96T005284 & $163: 1$ & Lower half & 466 & $<187$ & $<326.5^{\mathrm{QC}: \mathrm{e}}$ \\
\hline S96T005285 & $163: 2$ & Lower half & $<179$ & $<191$ & $<185$ \\
\hline S96T005780 & $163: 12$ & Lower half & $<188$ & $<190$ & $<189$ \\
\hline S96T005747 & \multirow[t]{2}{*}{$163: 14$} & Upper half & $<224$ & $<225$ & $<224.5$ \\
\hline S96T005748 & & Lower half & $<187$ & $<193$ & $<190$ \\
\hline \$96T005293 & \multirow[t]{2}{*}{$163: 16$} & Upper half & 253 & $<190$ & $<221.5^{\mathrm{QC}: \mathrm{e}}$ \\
\hline$\$ 96 T 005286$ & & Lower half & $<199$ & $<183$ & $<191$ \\
\hline S96T005777 & \multirow[t]{2}{*}{$163: 18$} & Upper half & $<205$ & 445 & $<325^{\mathrm{QC}: \mathrm{c}}$ \\
\hline S96T005781 & & Lower half & $<213$ & 262 & $<237.5^{\mathrm{QC}: \mathrm{e}}$ \\
\hline S96T005556 & \multirow[t]{2}{*}{$163: 20$} & Upper half & $<185$ & $<194$ & $<189.5$ \\
\hline \$96T005559 & & Lower half & $<195$ & $<195$ & $<195$ \\
\hline$\$ 96 \mathrm{T004795}$ & 164:I & Lower half & $<204$ & $<201$ & $<202.5$ \\
\hline S96T004796 & $164: 13$ & Lower half & $<206$ & $<207$ & $<206.5$ \\
\hline S96T005035 & \multirow[t]{2}{*}{$164: 14$} & Upper half & $<204$ & $<206$ & $<205$ \\
\hline S96T005030 & & Lower half & $<205$ & $<204$ & $<204.5$ \\
\hline S96T005036 & \multirow[t]{2}{*}{$164: 15$} & Upper half & 430 & 493 & 461.5 \\
\hline S96T005032 & & Lower half & 316 & 429 & $372.5^{\mathrm{QC}: \mathrm{c}}$ \\
\hline S96T005037 & \multirow[t]{2}{*}{$164: 16$} & Upper half & 238 & 635 & $436.5^{\mathrm{QC:c}}$ \\
\hline S96T005033 & & Lower half & 344 & $<187$ & $<265.5^{\mathrm{QC}: \mathrm{c}}$ \\
\hline S96T005038 & \multirow[t]{2}{*}{$164: 17$} & Upper half & $<193$ & $<190$ & $<191.5$ \\
\hline S96T005031 & & Lower half & $<218$ & $<217$ & $<217.5$ \\
\hline S96T005039 & \multirow[t]{2}{*}{ 164:19 } & Upper half & $<194$ & $<200$ & $<197$ \\
\hline$\$ 96 T 005034$ & & Lower half & $<214$ & $<217$ & $<215.5$ \\
\hline \$96T005281 & \multirow[t]{2}{*}{$164: 20$} & Upper half & $<172$ & $<185$ & $<178.5$ \\
\hline S96T005278 & & Lower half & 196 & $<193$ & $<194.5$ \\
\hline
\end{tabular}


Table B2-42. Tank 241-AN-104 Analytical Results: Zinc (ICP). (3 sheets)

\begin{tabular}{|c|c|c|c|c|c|}
\hline Sample & $\begin{array}{l}\text { Sample } \\
\text { Iracition }\end{array}$ & $\begin{array}{l}\text { Sample } \\
\text { rowirn }\end{array}$ & Resin: & 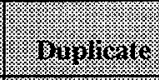 & Mear \\
\hline Miguins & 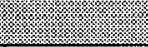 & l: & $896 / \mathrm{ml}$ & 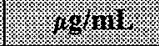 & 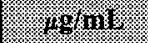 \\
\hline S96T005256 & $163: 2$ & Liquid & 9 & 9.9 & 9.45 \\
\hline S96T005260 & $163: 4$ & Liquid & 7.74 & 7.25 & 7.495 \\
\hline S96T005527 & $163: 5$ & Liquid & $<6.01$ & $<6.01$ & $<6.01$ \\
\hline S96T005554 & $163: 6$ & Liquid & $<6.01$ & $<6.01$ & $<6.01$ \\
\hline S96T005255 & $163: 7$ & Liquid & $<6.01$ & $<6.01$ & $<6.01$ \\
\hline S96T005257 & $163: 8$ & Liquid & 7.75 & 8.75 & 8.25 \\
\hline S96T005258 & $163: 9$ & Liquid & 7.66 & 7.38 & 7.52 \\
\hline S96T005528 & $163: 10$ & Liquid & $<6.01$ & $<6.01$ & $<6.01$ \\
\hline S96T005739 & $163: 11$ & Liquid & $<6.01$ & $<6.01$ & $<6.01$ \\
\hline S96T005766 & $163: 12$ & Liquid & $<6.01$ & $<6.01$ & $<6.01$ \\
\hline S96T005741 & $163: 14$ & Liquid & $<6.01$ & $<6.01$ & $<6.01$ \\
\hline S96r004774 & $164: 1$ & Liquid & 12.2 & 14.7 & 13.45 \\
\hline S96T004778 & $164: 2$ & Liquid & $<12$ & $<12$ & $<12$ \\
\hline S96T004779 & $164: 3$ & Liquid & 17.5 & 15.7 & 16.6 \\
\hline S96T004780 & $164: 4$ & Liquid & 24.2 & 26.4 & 25.3 \\
\hline S96T004976 & $164: 5$ & Liquid & 24.9 & 22.4 & 23.65 \\
\hline S96T004781 & $164: 7$ & Liquid & 24.8 & 25 & 24.9 \\
\hline S96T004977 & $164: 8$ & Liquid & 24.1 & 24 & 24.05 \\
\hline S96T004978 & $164: 9$ & Liquid & 25.7 & 20 & $22.85^{\mathrm{QC}: \mathrm{c}}$ \\
\hline S96T004979 & $164: 10$ & Liquid & 20.8 & 21.8 & 21.3 \\
\hline S96T004782 & $164: 11$ & Liquid & 24 & 24.8 & 24.4 \\
\hline S96T004783 & $164: 12$ & Liquid & 22.5 & 25.4 & 23.95 \\
\hline S96T004784 & $164: 13$ & Liquid & 27.8 & 22.6 & $25.2^{\mathrm{QC:c}}$ \\
\hline S96T005979 & Core 164 & Composite & $<12$ & $<12$ & $<12$ \\
\hline
\end{tabular}


Table B2-43. Tank 241-AN-104 Analytical Results: Zirconium (ICP). (3 sheets)

\begin{tabular}{|c|c|c|c|c|c|}
\hline 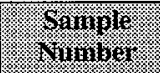 & 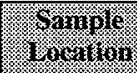 & 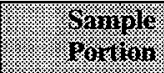 & resring & 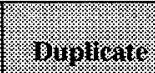 & l. \\
\hline \multicolumn{2}{|c|}{ 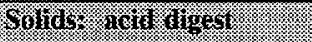 } & & 68 & 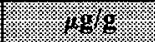 & 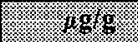 \\
\hline S96T005287 & $163: 1$ & Lower half & 13.9 & 13.1 & 13.5 \\
\hline S96T005288 & $163: 2$ & Lower half & 9.09 & 9.13 & 9.11 \\
\hline S96T005782 & $163: 12$ & Lower half & 20.1 & 17.8 & 18.95 \\
\hline S96T005749 & \multirow[t]{2}{*}{$163: 14$} & Upper half & 11.8 & 11.9 & 11.85 \\
\hline S96T005750 & & Lower half & 12.9 & 13.6 & 13.25 \\
\hline S96T005294 & \multirow[t]{2}{*}{$163: 16$} & Upper half & 19.5 & 16.8 & 18.15 \\
\hline S96T005289 & & Lower half & 19.2 & 16.4 & 17.8 \\
\hline S96T005778 & \multirow[t]{2}{*}{$163: 18$} & Upper half & 20.4 & 20.7 & 20.55 \\
\hline S96T005783 & & Lower half & 19.9 & 19.8 & 19.85 \\
\hline S96T005557 & \multirow[t]{2}{*}{$163: 20$} & Upper half & 19.3 & 19.2 & 19.25 \\
\hline S96T005560 & & Lower half & 24.2 & 19.6 & $21.9^{\mathrm{QC} c \mathrm{c}}$ \\
\hline S96T004797 & $164: 1$ & Lower half & 9.94 & 9.52 & 9.73 \\
\hline S96T004798 & $164: 13$ & Lower half & 21.7 & 22.4 & 22.05 \\
\hline S96T005042 & \multirow[t]{2}{*}{$164: 14$} & Upper half & 15.5 & 15.7 & 15.6 \\
\hline S96T005040 & & Lower half & 12.5 & 12.5 & 12.5 \\
\hline S96T005061 & \multirow[t]{2}{*}{$164: 15$} & Upper half & 13.7 & 13.9 & 13.8 \\
\hline S96T005043 & & Lower half & 17.6 & 16.7 & 17.15 \\
\hline S96T005062 & \multirow[t]{2}{*}{$164: 16$} & Upper half & 21.6 & $<14.9$ & $<18.25^{\mathrm{QC} c}$ \\
\hline S96T005044 & & Lower half & 17.5 & $<15$ & $<16.25$ \\
\hline S96T005063 & \multirow[t]{2}{*}{$164: 17$} & Upper half & $<13.9$ & 25 & $<19.45^{\mathrm{QC}: \mathrm{O}}$ \\
\hline S96T005041 & & Lower half & 17.6 & 21.2 & 19.4 \\
\hline S96T005064 & \multirow[t]{2}{*}{$164: 19$} & Upper half & 19.7 & 23.3 & 21.5 \\
\hline S96T005045 & & Lower half & 16.5 & 22.4 & $19.45^{\mathrm{QC}: \mathrm{c}}$ \\
\hline S96T005282 & \multirow[t]{2}{*}{$164: 20$} & Upper half & 17.3 & 15.6 & 16.45 \\
\hline S96T005279 & & Lower half & 18.8 & 13.7 & $16.25^{\mathrm{QC}: \mathrm{c}}$ \\
\hline S96T005976 & Core 164 & Composite & 17.6 & 16.1 & 16.85 \\
\hline
\end{tabular}


Table B2-43. Tank 241-AN-104 Analytical Results: Zirconium (ICP). (3 sheets)

\begin{tabular}{|c|c|c|c|c|c|}
\hline 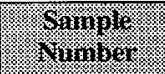 & Fringlo & (1) & Resing & 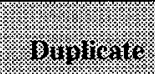 & 4\%an \\
\hline \multicolumn{2}{|c|}{ 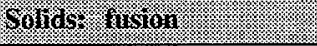 } & & \% & (1) & . \\
\hline S96T005284 & $163: 1$ & Lower half & $<187$ & $<187$ & $<187$ \\
\hline S96T005285 & $163: 2$ & Lower half & $<179$ & $<191$ & $<185$ \\
\hline S96T005780 & $163: 12$ & Lower half & $<188$ & $<190$ & $<189$ \\
\hline S96T005747 & \multirow[t]{2}{*}{$163: 14$} & Upper half & $<224$ & $<225$ & $<224.5$ \\
\hline S96T005748 & & Lower half & $<187$ & $<193$ & $<190$ \\
\hline S96T005293 & \multirow[t]{2}{*}{$163: 16$} & Upper half & $<177$ & $<190$ & $<183.5$ \\
\hline S96T005286 & & Lower half & $<199$ & $<183$ & $<191$ \\
\hline S96T005777 & \multirow[t]{2}{*}{$163: 18$} & Upper half & $<205$ & $<198$ & $<201.5$ \\
\hline S96T005781 & & Lower half & $<213$ & $<214$ & $<213.5$ \\
\hline S96T005556 & \multirow[t]{2}{*}{$163: 20$} & Upper half & $<185$ & $<194$ & $<189.5$ \\
\hline S96T005559 & & Lower half & $<195$ & $<195$ & $<195$ \\
\hline S96T004795 & $164: 1$ & Lower half & $<204$ & $<201$ & $<202.5$ \\
\hline S96T004796 & $164: 13$ & Lower half & $<206$ & $<207$ & $<206.5$ \\
\hline S96T005035 & \multirow[t]{2}{*}{$164: 14$} & Upper half & $<204$ & $<206$ & $<205$ \\
\hline S96T005030 & & Lower half & $<205$ & $<204$ & $<204.5$ \\
\hline S96T005036 & \multirow[t]{2}{*}{$164: 15$} & Upper half & $<201$ & $<197$ & $<199$ \\
\hline S96T005032 & & Lower half & $<197$ & $<200$ & $<198.5$ \\
\hline S96T005037 & \multirow[t]{2}{*}{$164: 16$} & Upper half & $<192$ & $<183$ & $<187.5$ \\
\hline S96T005033 & & Lower half & $<191$ & $<187$ & $<189$ \\
\hline \$96T005038 & \multirow[t]{2}{*}{$164: 17$} & Upper half & $<193$ & $<190$ & $<191.5$ \\
\hline S96T005031 & & Lower half & $<218$ & $<217$ & $<217.5$ \\
\hline S96T005039 & \multirow[t]{2}{*}{ 164:19 } & Upper half & $<194$ & $<200$ & $<197$ \\
\hline S96T005034 & & Lower half & $<214$ & $<217$ & $<215.5$ \\
\hline S96T005281 & \multirow[t]{2}{*}{$164: 20$} & Upper half & $<172$ & $<185$ & $<178.5$ \\
\hline S96T005278 & & Lower half & $<189$ & $<193$ & $<191$ \\
\hline
\end{tabular}


HNF-SD-WM-ER-690 Rev. 0

Table B2-43. Tank 241-AN-104 Analytical Results: Zirconium (ICP). (3 sheets)

\begin{tabular}{|c|c|c|c|c|c|}
\hline $\begin{array}{l}\text { Sample: } \\
\text { Niminger: }\end{array}$ & $\begin{array}{l}\text { Saringle } \\
\text { Laction. }\end{array}$ & 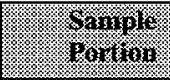 & Resurit & Duriliger & 18601 \\
\hline Migrimas & & 4. & 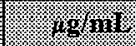 & 4agnir & $\sqrt{4} \cdot 1.96111$ \\
\hline S96T005256 & $163: 2$ & Liquid & 7.98 & 8.31 & 8.145 \\
\hline S96T005260 & $163: 4$ & Liquid & 6.78 & 6.17 & 6.475 \\
\hline S96T005527 & $163: 5$ & Liquid & 6.93 & 6.43 & 6.68 \\
\hline S96T005554 & $163: 6$ & Liquid & 7.53 & 8.4 & 7.965 \\
\hline S96T005255 & $163: 7$ & Liquid & 8.01 & 8.13 & 8.07 \\
\hline S96T005257 & $163: 8$ & Liquid & $<6.01$ & $<6.01$ & $<6.01$ \\
\hline \begin{tabular}{|l} 
S96T005258 \\
\end{tabular} & $163: 9$ & Liquid & $<6.01$ & $<6.01$ & $<6.01$ \\
\hline S96T005528 & $163: 10$ & Liquid & $<6.01$ & $<6.01$ & $<6.01$ \\
\hline S96T005739 & $163: 11$ & Liquid & $<6.01$ & $<6.01$ & $<6.01$ \\
\hline S96T005766 & $163: 12$ & Liquid & $<6.01$ & $<6.01$ & $<6.01$ \\
\hline S96T005741 & $163: 14$ & Liquid & 8.73 & 8.28 & 8.505 \\
\hline S96T004774 & $164: 1$ & Liquid & $<12$ & $<12$ & $<12$ \\
\hline S96T004778 & $164: 2$ & Liquid & $<12$ & $<12$ & $<12$ \\
\hline \begin{tabular}{|l|l} 
S96T004779 \\
\end{tabular} & $164: 3$ & Liquid & $<12$ & $<12$ & $<12$ \\
\hline S96T004780 & $164: 4$ & Liquid & 7.51 & 6.73 & 7.12 \\
\hline S96T004976 & $164: 5$ & Liquid & 8.72 & 7.77 & 8.245 \\
\hline S96T004781 & $164: 7$ & Liquid & 6.49 & 6.95 & 6.72 \\
\hline S96T004977 & $164: 8$ & Liquid & 11.7 & 11.1 & 11.4 \\
\hline S96T004978 & $164: 9$ & Liquid & $<6.01$ & $<6.01$ & $<6.01$ \\
\hline S96r004979 & $164: 10$ & Liquid & $<6.01$ & $<6.01$ & $<6.01$ \\
\hline S96T004782 & $164: 11$ & Liquid & 7.34 & 6.04 & 6.69 \\
\hline S96T004783 & $164: 12$ & Liquid & 8.17 & 8.59 & 8.38 \\
\hline S96T004784 & $164: 13$ & Liquid & 7.78 & 7.82 & 7.8 \\
\hline S96T005979 & Core 164 & Composite & $<12$ & $<12$ & $<12$ \\
\hline
\end{tabular}


HNF-SD-WM-ER-690 Rev. 0

Table B2-44. Tank 241-AN-104 Analytical Results: Bromide (IC). (2 sheets)

\begin{tabular}{|c|c|c|c|c|c|}
\hline 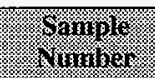 & Wompror & orimo & oferill & 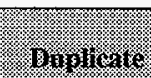 & (3) \\
\hline gindr. & thestst & (3. & (3) & \% & (4: \\
\hline S96T005290 & $163: 1$ & Lower half & $<498.5$ & $<476$ & $<487.25$ \\
\hline S96T005291 & $163: 2$ & Lower half & $<934.2$ & $<904$ & $<919.1$ \\
\hline S96T005784 & $163: 12$ & Lower half & 1,038 & 1,020 & 1,029 \\
\hline S96T005751 & \multirow[t]{2}{*}{$163: 14$} & Upper half & $<1,024$ & $<1,030$ & $<1,027$ \\
\hline S96T005752 & & Lower half & $<1,107$ & $<1,100$ & $<1,103.5$ \\
\hline S96T005295 & \multirow[t]{2}{*}{$163: 16$} & Upper half & $<594$ & $<579$ & $<586.5$ \\
\hline S96T005292 & & Lower half & $<881.3$ & $<941$ & $<911.15$ \\
\hline S96T005779 & \multirow[t]{2}{*}{$163: 18$} & Upper half & 667.9 & 635 & 651.45 \\
\hline S96T005785 & & Lower half & $<563.1$ & $<521$ & $<542.05$ \\
\hline \$96T005558 & \multirow[t]{2}{*}{$163: 20$} & Upper half & $<512.6$ & $<494$ & $<503.3$ \\
\hline S96T005561 & & Lower half & $<477.4$ & $<491$ & $<484.2$ \\
\hline S96T004799 & $164: 1$ & Lower half & $<1,007$ & $<1,020$ & $<1,013.5$ \\
\hline$\$ 96 \mathrm{~T} 004800$ & $164: 13$ & Lower half & $<1,038$ & $<1,020$ & $<1,029$ \\
\hline S96T005067 & \multirow[t]{2}{*}{$164: 14$} & Upper half & 1,158 & 1,160 & 1,159 \\
\hline S96T005065 & & Lower half & 389.6 & 513 & $451.3^{\mathrm{QC}: \mathrm{c}}$ \\
\hline \$96T005071 & \multirow[t]{2}{*}{$164: 15$} & Upper half & 1,091 & $<1,040$ & $<1,065.5$ \\
\hline S96T005068 & & Lower half & $<1,031$ & $<1,000$ & $<1,015.5$ \\
\hline S96T005072 & \multirow[t]{2}{*}{$1.64: 16$} & Upper half & $<519.5$ & $<507$ & $<513.25$ \\
\hline S96T005069 & & Lower half & $<1,060$ & $<1,060$ & $<1,060$ \\
\hline S96T005073 & \multirow[t]{2}{*}{$164: 17$} & Upper half & $<577.3$ & $<579$ & $<578.15$ \\
\hline S96T005066 & & Lower half & $<948.7$ & $<954$ & $<951.35$ \\
\hline S96T005074 & \multirow[t]{2}{*}{$164: 19$} & Upper half & 1,165 & 1,160 & $1,162.5$ \\
\hline S96T005070 & & Lower half & 956.5 & $<939$ & $<947.75$ \\
\hline S96T005283 & \multirow[t]{2}{*}{$164: 20$} & Upper half & $<515.9$ & $<520$ & $<517.95$ \\
\hline S96T005280 & & Lower half & $<506.2$ & $<510$ & $<508.1$ \\
\hline S96T005977 & Core 164 & Composite & 1,145 & $<976$ & $<1,060.5$ \\
\hline
\end{tabular}


Table B2-44. Tank 241-AN-104 Analytical Results: Bromide (IC). (2 sheets)

\begin{tabular}{|c|c|c|c|c|c|}
\hline 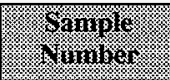 & $\begin{array}{l}\text { Samples } \\
\text { Iorationt }\end{array}$ & 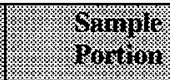 & Proning & 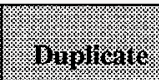 & I. 10.10 \\
\hline Hiranis: & : & 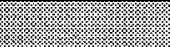 & 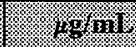 & 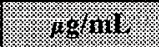 & 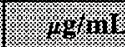 \\
\hline S96T005256 & $163: 2$ & Liquid & $<1,275$ & $<1,280$ & $<1,277.5$ \\
\hline S96T005260 & $163: 4$ & Liquid & 916.9 & 950 & 933.45 \\
\hline S96T005527 & $163: 5$ & Liquid & 815.8 & 893 & 854.4 \\
\hline S96T005554 & $163: 6$ & Liquid & 736.3 & 748 & 742.15 \\
\hline S96T005255 & $163: 7$ & Liquid & 654.9 & 650 & 652.45 \\
\hline S96T005257 & $163: 8$ & Liquid & 731.3 & 749 & 740.15 \\
\hline S96T005258 & $163: 9$ & Liquid & 1,104 & 1,050 & 1,077 \\
\hline S96T005528 & $163: 10$ & Liquid & 1,107 & 1,080 & $1,093.5$ \\
\hline S96T005739 & $163: 11$ & Liquid & $<643.9$ & $<644$ & $<643.95$ \\
\hline S96T005766 & $163: 12$ & Liquid & 1,128 & 1,150 & 1,139 \\
\hline S96T005741 & $163: 14$ & Liquid & 757.2 & 759 & 758.1 \\
\hline S96T004774 & $164: 1$ & Liquid & $<643.9$ & $<644$ & $<643.95$ \\
\hline S96T004778 & $164: 2$ & Liquid & 1,167 & 1,120 & $1,143.5$ \\
\hline S96T004779 & $164: 3$ & Liquid & 1,756 & 1,740 & 1,748 \\
\hline S96T004780 & $164: 4$ & Liquid & 1,738 & 1,740 & 1,739 \\
\hline S96T004976 & $164: 5$ & Liquid & $<1,275$ & $<1,280$ & $<1,277.5$ \\
\hline S96T004781 & $164: 7$ & Liquid & $<1,275$ & $<1,280$ & $<1,277.5$ \\
\hline S96T004977 & $164: 8$ & Liquid & $<1,275$ & $<1,280$ & $<1,277.5$ \\
\hline S96T004978 & $164: 9$ & Liquid & 848.7 & 844 & 846.35 \\
\hline S96T004979 & $164: 10$ & Liquid & 825.2 & 810 & 817.6 \\
\hline S96T004782 & $164: 11$ & Liquid & 1,707 & 1,750 & $1,728.5$ \\
\hline S96T004783 & $164: 12$ & Liquid & $<1,275$ & $<1,280$ & $<1,277.5$ \\
\hline S96T004784 & $164: 13$ & Liquid & 902.5 & 887 & 894.75 \\
\hline S96T005979 & Core 164 & Composite & 1,520 & 1,580 & 1,550 \\
\hline
\end{tabular}


Table B2-45. Tank 241-AN-104 Analytical Results: Chloride (IC). (2 sheets)

\begin{tabular}{|c|c|c|c|c|c|}
\hline $\begin{array}{l}\text { Gample } \\
\text { Amming }\end{array}$ & 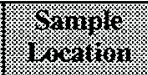 & $\begin{array}{l}\text { Sample } \\
\text { Tomilit }\end{array}$ & Rerair & Dounicate. & $\int$ \\
\hline \multicolumn{3}{|c|}{ 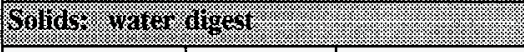 } & lig.g. & $\sqrt{1.8 .6396}$ & 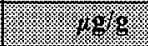 \\
\hline S96T005290 & $163: 1$ & Lower half & 4,626 & 4,450 & $4,538^{Q C: d}$ \\
\hline S96T005291 & $163: 2$ & Lower half & 4,471 & 3,690 & $4,080.5$ \\
\hline S96T005784 & $163: 12$ & Lower half & 4,688 & 4,830 & 4,759 \\
\hline \begin{tabular}{|l} 
S96T005751 \\
\end{tabular} & \multirow[t]{2}{*}{$163: 14$} & Upper half & 4,688 & 4,810 & 4,749 \\
\hline S96T005752 & & Lower half & 4,849 & 5,110 & $4,979.5$ \\
\hline$\$ 96 \mathrm{~T} 005295$ & \multirow[t]{2}{*}{$163: 16$} & Upper half & 4,329 & 4,170 & $4,249.5$ \\
\hline S96T005292 & & Lower half & 4,754 & 4,760 & 4,757 \\
\hline S96T005779 & \multirow[t]{2}{*}{$163: 18$} & Upper half & 3,864 & 4,240 & 4,052 \\
\hline S96r005785 & & Lower half & 3,829 & 4,170 & $3,999.5$ \\
\hline \begin{tabular}{|l|} 
S96T005558 \\
\end{tabular} & \multirow[t]{2}{*}{$163: 20$} & Upper half & 3,848 & 4,000 & 3,924 \\
\hline S96T005561 & & Lower half & 3,711 & 3,610 & $3,660.5$ \\
\hline S96T004799 & $164: 1$ & Lower half & 4,824 & 4,910 & 4,867 \\
\hline S96T004800 & $164: 13$ & Lower half & 3,148 & 3,180 & 3,164 \\
\hline S96r005067 & \multirow[t]{2}{*}{$164: 14$} & Upper half & 5,027 & 5,030 & $5,028.5$ \\
\hline S96T005065 & & Lower half & 4,980 & 4,990 & 4,985 \\
\hline S96T005071 & \multirow[t]{2}{*}{$164: 15$} & Upper half & 4,842 & 4,750 & 4,796 \\
\hline S96T005068 & & Lower half & 4,551 & 4,410 & $4,480.5$ \\
\hline S96T005072 & \multirow[t]{2}{*}{$164: 16$} & Upper half & 4,760 & 4,630 & 4,695 \\
\hline S96T005069 & & Lower half & 4,959 & 4,740 & $4,849.5$ \\
\hline S96T005073 & \multirow[t]{2}{*}{$164: 17$} & Upper half & 4,440 & 4,720 & 4,580 \\
\hline S96T005066 & & Lower half & 4,585 & 4,430 & $4,507.5$ \\
\hline S96T005074 & \multirow[t]{2}{*}{$164: 19$} & Upper half & 4,483 & 4,480 & $4,481.5$ \\
\hline S96T005070 & & Lower half & 4,627 & 4,580 & $4,603.5$ \\
\hline S96T005283 & \multirow[t]{2}{*}{$164: 20$} & Upper half & 4,453 & 4,490 & $4,471.5$ \\
\hline S96T005280 & & Lower half & 4,345 & 4,360 & $4,352.5$ \\
\hline S96T005977 & Core 164 & Composite & 4,016 & 4,150 & 4,083 \\
\hline
\end{tabular}


Table B2-45. Tank 241-AN-104 Analytical Results: Chloride (IC). (2 sheets)

\begin{tabular}{|c|c|c|c|c|c|}
\hline Simple: & $\begin{array}{l}\text { Sirnule } \\
\text { incuiror }\end{array}$ & $\begin{array}{l}\text { Tanjof } \\
\text { fortion }\end{array}$ & Revilin & Binjulisate. & is: \\
\hline \multicolumn{3}{|c|}{ (1) } & ogkmL & 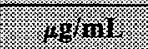 & I) \\
\hline S96T005256 & $163: 2$ & Liquid & 15,330 & 15,100 & 15,215 \\
\hline S96T005260 & $163: 4$ & Liquid & 8,157 & 8,080 & $8,118.5$ \\
\hline S96T005527 & $163: 5$ & Liquid & 7,596 & 7,520 & 7,558 \\
\hline S96T005554 & $163: 6$ & Liquid & 8,000 & 7,970 & 7,985 \\
\hline S96T005255 & $163: 7$ & Liquid & 3,655 & 3,630 & $3,642.5$ \\
\hline S96T005257 & $163: 8$ & Liquid & 7,988 & 7,970 & 7,979 \\
\hline S96T005258 & $163: 9$ & Liquid & 7,611 & 7,740 & $7,675.5$ \\
\hline S96T005528 & $163: 10$ & Liquid & 7,809 & 7,840 & $7,824.5$ \\
\hline S96T005739 & $163: 11$ & Liquid & 7,647 & 7,510 & $7,578.5$ \\
\hline S96T005766 & $163: 12$ & Liquid & 7,595 & 7,550 & $7,572.5$ \\
\hline S96T005741 & $163: 14$ & Liquid & 8,152 & 8,120 & 8,136 \\
\hline S96T004774 & $164: 1$ & Liquid & 8,285 & 8,080 & $8,182.5$ \\
\hline S96T004778 & $164: 2$ & Liquid & 8,376 & 8,360 & 8,368 \\
\hline S96T004779 & $164: 3$ & Liquid & 8,019 & 8,110 & $8,064.5$ \\
\hline S96T004780 & $164: 4$ & Liquid & 7,843 & 8,140 & $7,991.5$ \\
\hline S96T004976 & $164: 5$ & Liquid & 6,826 & 6,810 & 6,818 \\
\hline S96T004781 & $164: 7$ & Liquid & 7,215 & 7,340 & $7,277.5$ \\
\hline S96T004977 & $164: 8$ & Liquid & 8,932 & 8,650 & 8,791 \\
\hline S96T004978 & $164: 9$ & Liquid & 8,091 & 9,170 & $8,630.5$ \\
\hline S96T004979 & $164: 10$ & Liquid & 8,108 & 8,120 & 8,114 \\
\hline S96T004782 & $164: 11$ & Liquid & 7,868 & 7,910 & 7,889 \\
\hline S96T004783 & $164: 12$ & Liquid & 8,429 & 8,600 & $8,514.5$ \\
\hline S96T004784 & $164: 13$ & Liquid & 8,239 & 7,890 & $8,064.5$ \\
\hline S96T005979 & Core 164 & Composite & 9,617 & 9,300 & $9,458.5$ \\
\hline
\end{tabular}


HNF-SD-WM-ER-690 Rev. 0

Table B2-46. Tank 241-AN-104 Analytical Results: Fluoride (IC). (2 sheets)

\begin{tabular}{|c|c|c|c|c|c|}
\hline Sample & 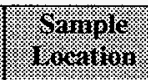 & $\begin{array}{l}\text { Sinipiti } \\
\text { Dorition }\end{array}$ & ingsill & 8uplunte & W610 \\
\hline Solids : yate & trigest & (3. & 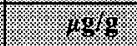 & $\sqrt{3}=8.8$ & $\sqrt{1.68}$ \\
\hline S96T005290 & $163: 1$ & Lower half & 304.8 & 290 & 297.4 \\
\hline S96T005291 & $163: 2$ & Lower half & 411.2 & 552 & $481.6^{\mathrm{QC} e \mathrm{c}}$ \\
\hline S96T005784 & $163: 12$ & Lower half & 583.1 & 593 & $588.05^{\mathrm{QC}: \mathrm{c}}$ \\
\hline \begin{tabular}{|l} 
S96T005751 \\
\end{tabular} & \multirow[t]{2}{*}{$163: 14$} & Upper half & 618.9 & 616 & 617.45 \\
\hline S96T005752 & & Lower half & 677 & 638 & 657.5 \\
\hline \begin{tabular}{|l} 
S96T005295 \\
\end{tabular} & \multirow[t]{2}{*}{$163: 16$} & Upper half & 806.1 & 657 & $731.55^{\mathrm{QC:c}}$ \\
\hline S96T005292 & & Lower half & 1,021 & 1,130 & $1,075.5$ \\
\hline \begin{tabular}{|l|} 
S96T005779 \\
\end{tabular} & \multirow[t]{2}{*}{$163: 18$} & Upper half & 651.8 & 669 & 660.4 \\
\hline \begin{tabular}{|l} 
S96T005785 \\
\end{tabular} & & Lower half & 675 & 626 & 650.5 \\
\hline S96T005558 & \multirow[t]{2}{*}{$163: 20$} & Upper half & 893.2 & 985 & $939.1^{\mathrm{QC}: \mathrm{d}}$ \\
\hline S96T005561 & & Lower half & 3,301 & 3,140 & $3,220.5$ \\
\hline \begin{tabular}{|l|l} 
S96T004799 &
\end{tabular} & $164: 1$ & Lower half & 850.7 & 825 & 837.85 \\
\hline S96T004800 & $164: 13$ & Lower half & 643.5 & 622 & 632.75 \\
\hline S96T005067 & \multirow[t]{2}{*}{$164: 14$} & Upper half & 636.8 & 635 & 635.9 \\
\hline S96T005065 & & Lower half & 562.6 & 645 & $603.8^{\mathrm{QC}: c}$ \\
\hline S96T005071 & \multirow[t]{2}{*}{$164: 15$} & Upper half & 427.7 & 482 & 454.85 \\
\hline \begin{tabular}{|l|} 
S96T005068 \\
\end{tabular} & & Lower half & 769.6 & 709 & 739.3 \\
\hline \begin{tabular}{|l|} 
S96T005072 \\
\end{tabular} & \multirow[t]{2}{*}{$164: 16$} & Upper half & 405.5 & 422 & 413.75 \\
\hline S96T005069 & & Lower half & 468.4 & 461 & 464.7 \\
\hline S96T005073 & \multirow[t]{2}{*}{$164: 17$} & Upper half & 449.5 & 426 & $437.75^{\mathrm{QC} d}$ \\
\hline S96T005066 & & Lower half & 692.3 & 674 & 683.15 \\
\hline S96T005074 & \multirow[t]{2}{*}{$164: 19$} & Upper half & 680.7 & 701 & 690.85 \\
\hline S96T005070 & & Lower half & 651.1 & 644 & $647.55^{\mathrm{QC:d}}$ \\
\hline S96T005283 & \multirow[t]{2}{*}{$164: 20$} & Upper half & 836.7 & 854 & $845.35^{\mathrm{QC:d}}$ \\
\hline S96T005280 & & Lower half & 2,109 & 2,050 & $2,079.5$ \\
\hline S96T005977 & Core 164 & Composite & 532.4 & 784 & $658.2^{\mathrm{QC}: \mathrm{e}}$ \\
\hline
\end{tabular}


Table B2-46. Tank 241-AN-104 Analytical Results: Fluoride (IC). (2 sheets)

\begin{tabular}{|c|c|c|c|c|c|}
\hline 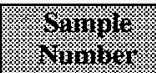 & 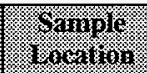 & 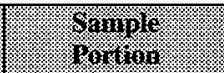 & 3escil & Burtinat & \% \\
\hline Hoving & 原 & l. & 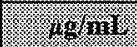 & $\sqrt{1.4963}$ & 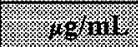 \\
\hline S96T005256 & $163: 2$ & Liquid & $<122.4$ & $<122$ & $<122.2$ \\
\hline S96T005260 & $163: 4$ & Liquid & $<49.69$ & $<49.7$ & $<49.695$ \\
\hline S96T005527 & $163: 5$ & Liquid & $<61.81$ & $<61.8$ & $<61.805$ \\
\hline S96T005554 & $163: 6$ & Liquid & $<61.81$ & $<61.8$ & $<61.805$ \\
\hline S96T005255 & $163: 7$ & Liquid & $<49.69$ & $<49.7$ & $<49.695$ \\
\hline S96T005257 & $163: 8$ & Liquid & $<61.81$ & $<61.8$ & $<61.805$ \\
\hline S96T005258 & $163: 9$ & Liquid & $<49.69$ & $<49.7$ & $<49.695$ \\
\hline S96T005528 & $163: 10$ & Liquid & $<61.81$ & $<61.8$ & $<61.805$ \\
\hline S96T005739 & 163:11 & Liquid & $<61.81$ & $<61.8$ & $<61.805$ \\
\hline S96T005766 & $163: 12$ & Liquid & $<61.81$ & $<61.8$ & $<61.805$ \\
\hline S96T005741 & $163: 14$ & Liquid & $<61.81$ & $<61.8$ & $<61.805$ \\
\hline S96T004774 & $164: 1$ & Liquid & $<61.81$ & $<61.8$ & $<61.805$ \\
\hline S96T004778 & $164: 2$ & Liquid & $<61.81$ & $<61.8$ & $<61.805$ \\
\hline \begin{tabular}{|l|} 
S96T004779 \\
\end{tabular} & $164: 3$ & Liquid & $<122.4$ & $<122$ & $<122.2$ \\
\hline S96T004780 & $164: 4$ & Liquid & $<122.4$ & $<122$ & $<122.2$ \\
\hline S96T004976 & $164: 5$ & Liquid & 273.4 & $<122$ & $<197.7^{\mathrm{QCe}}$ \\
\hline S96T004781 & $164: 7$ & Liquid & $<122.4$ & $<122$ & $<122.2$ \\
\hline S96T004977 & $164: 8$ & Liquid & 357.5 & 333 & 345.25 \\
\hline S96T004978 & $164: 9$ & Liquid & $<49.69$ & $<49.7$ & $<49.695$ \\
\hline \begin{tabular}{|l|} 
S96T004979 \\
\end{tabular} & $164: 10$ & Liquid & $<49.69$ & $<49.7$ & $<49.695$ \\
\hline S96T004782 & $164: 11$ & Liquid & $<122.4$ & $<122$ & $<122.2$ \\
\hline S96T004783 & $164: 12$ & Liquid & $<122.4$ & $<122$ & $<122.2$ \\
\hline S96T004784 & $164: 13$ & Liquid & $<61.81$ & $<61.8$ & $<61.805$ \\
\hline S96T005979 & Core 164 & Composite & $<122.4$ & $<122$ & $<122.2$ \\
\hline
\end{tabular}


Table B2-47. Tank 241-AN-104 Analytical Results: Formate (IC). (2 sheets)

\begin{tabular}{|c|c|c|c|c|c|}
\hline Sumple & Saminle & T. Simpling & Resulin & 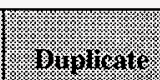 & U: \\
\hline \multicolumn{3}{|c|}{ Siblias yarer argest } & 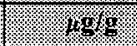 & $\sqrt{168}$ & $\sqrt{3.7698}$ \\
\hline S96T005290 & $163: 1$ & Lower half & 707.6 & 711 & 709.3 \\
\hline S96T005291 & $163: 2$ & Lower half & 645.1 & 531 & 588.05 \\
\hline S96T005784 & $163: 12$ & Lower half & 650.9 & 635 & 642.95 \\
\hline S96T005751 & \multirow[t]{2}{*}{$163: 14$} & Upper half & 633.2 & 620 & 626.6 \\
\hline S96T005752 & & Lower half & 624.9 & 630 & 627.45 \\
\hline S96T005295 & \multirow[t]{2}{*}{$163: 16$} & Upper half & 915.4 & 910 & 912.7 \\
\hline S96T005292 & & Lower half & 1,417 & 1,580 & $1,498.5$ \\
\hline S96T005779 & \multirow[t]{2}{*}{$163: 18$} & Upper half & 879.8 & 841 & 860.4 \\
\hline S96T005785 & & Lower half & 850.7 & 799 & 824.85 \\
\hline S96T005558 & \multirow[t]{2}{*}{$163: 20$} & Upper half & 690.9 & 738 & 714.45 \\
\hline S96T005561 & & Lower half & 636.3 & 603 & 619.65 \\
\hline S97T000648 & 164:1 & Lower half & 569.2 & 601 & 585.1 \\
\hline S97T000649 & $164: 13$ & Lower half & 945.6 & 832 & 888.8 \\
\hline S96T005067 & \multirow[t]{2}{*}{$164: 14$} & Upper half & 853.2 & 850 & 851.6 \\
\hline S96T005065 & & Lower half & 742 & 747 & 744.5 \\
\hline S96T005071 & \multirow[t]{2}{*}{$164: 15$} & Upper half & 724.2 & 695 & 709.6 \\
\hline S96T005068 & & Lower half & 741.2 & 694 & 717.6 \\
\hline S96T005072 & \multirow[t]{2}{*}{$164: 16$} & Upper half & 612.3 & 652 & 632.15 \\
\hline S96T005069 & & Lower half & 695.4 & 701 & 698.2 \\
\hline S96T005073 & \multirow[t]{2}{*}{$164: 17$} & Upper half & 656.9 & 625 & 640.95 \\
\hline S96T005066 & & Lower half & 742.5 & 737 & 739.75 \\
\hline S96T005074 & \multirow[t]{2}{*}{$164: 19$} & Upper half & 749.5 & 716 & 732.75 \\
\hline S96T005070 & & Lower half & 782.5 & 762 & 772.25 \\
\hline S96T005283 & \multirow[t]{2}{*}{$164: 20$} & Upper half & 777.1 & 833 & 805.05 \\
\hline S96T005280 & & Lower half & 790.4 & 875 & 832.7 \\
\hline S96T005977 & Core 164 & Composite & 762.6 & 702 & 732.3 \\
\hline
\end{tabular}


HNF-SD-WM-ER-690 Rev. 0

Table B2-47. Tank 241-AN-104 Analytical Results: Formate (IC). (2 sheets)

\begin{tabular}{|c|c|c|c|c|c|}
\hline (6) & 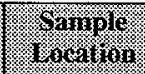 & 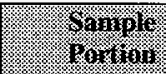 & Ressin) & 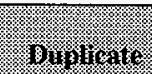 & $21(2,1):=$ \\
\hline Havins & (1. & & 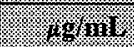 & 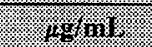 & 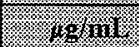 \\
\hline S96T005256 & $163: 2$ & Liquid & 1,249 & 1,360 & $1,304.5$ \\
\hline \$96T005260 & $163: 4$ & Liquid & 1,112 & 1,050 & 1,081 \\
\hline S96T005527 & $163: 5$ & Liquid & 459.5 & 480 & 469.75 \\
\hline S96T005554 & $163: 6$ & Liquid & 491 & 489 & 490 \\
\hline S96T005255 & $163: 7$ & Liquid & 1,070 & 1,070 & 1,070 \\
\hline S96T005257 & $163: 8$ & Liquid & $<426.3$ & $<426$ & $<426.15$ \\
\hline S96T005258 & $163: 9$ & Liquid & 1,019 & 1,050 & $1,034.5$ \\
\hline S96T005528 & $163: 10$ & Liquid & 418.6 & 435 & 426.8 \\
\hline S96T005739 & 163:11 & Liquid & 1,024 & 1,070 & 1,047 \\
\hline S96T005766 & $163: 12$ & Liquid & 1,168 & 1,130 & 1,149 \\
\hline S96T005741 & $163: 14$ & Liquid & 1,089 & 1,120 & $1,104.5$ \\
\hline S96T004774 & $164: 1$ & Liquid & 1,138 & 1,140 & 1,139 \\
\hline S96T004778 & $164: 2$ & Liquid & 750.3 & 873 & 811.65 \\
\hline S96T004779 & $164: 3$ & Liquid & 1,033 & 1,050 & $1,041.5$ \\
\hline S96T004780 & $164: 4$ & Liquid & 1,009 & 1,020 & $1,014.5$ \\
\hline S96T004976 & $164: 5$ & Liquid & 1,003 & 972 & 987.5 \\
\hline S96T004781 & $164: 7$ & Liquid & 1,098 & 1,020 & 1,059 \\
\hline S96T004977 & $164: 8$ & Liquid & 1,373 & 1,070 & $1,221.5^{\mathrm{QC:c}}$ \\
\hline S96T004978 & $164: 9$ & Liquid & 1,103 & 1,040 & $1,071.5$ \\
\hline S96T004979 & $164: 10$ & Liquid & 1,067 & 1,040 & $1,053.5$ \\
\hline S96T004782 & $164: 11$ & Liquid & 1,073 & 1,060 & $1,066.5$ \\
\hline S96T004783 & $164: 12$ & Liquid & 1,061 & 1,060 & $1,060.5$ \\
\hline S96T004784 & $164: 13$ & Liquid & 994.6 & 971 & 982.8 \\
\hline S96T005979 & Core 164 & Composite & 3,447 & 3,430 & $3,438.5$ \\
\hline
\end{tabular}


Table B2-48. Tank 241-AN-104 Analytical Results: Nitrate (IC). (2 sheets)

\begin{tabular}{|c|c|c|c|c|c|}
\hline Whinger & Sampie & 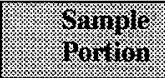 & Rovin & 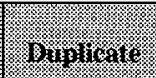 & 14yorit \\
\hline \multicolumn{2}{|c|}{ 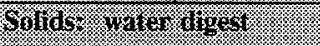 } & & \%glo & 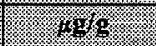 & $\int_{2}=1 \%$ \\
\hline S96T005290 & $163: 1$ & Lower half & $1.424 E+05$ & $1.360 \mathrm{E}+05$ & $1.392 \mathrm{E}+05$ \\
\hline S96T005291 & $163: 2$ & Lower half & $2.218 \mathrm{E}+05$ & $2.760 \mathrm{E}+05$ & $2.489 \mathrm{E}+05^{\mathrm{QC}: c}$ \\
\hline S96T005784 & $163: 12$ & Lower half & $1.114 \mathrm{E}+05$ & $1.120 \mathrm{E}+05$ & $1.117 \mathrm{E}+05$ \\
\hline S96T005751 & \multirow[t]{2}{*}{$163: 14$} & Upper half & 96,210 & 97,500 & 96,855 \\
\hline S96T005752 & & Lower half & 97,720 & 95,800 & 96,760 \\
\hline S96T005295 & \multirow[t]{2}{*}{$163: 16$} & Upper half & $1.003 \mathrm{E}+05$ & 96,900 & 98,600 \\
\hline S96T005292 & & Lower half & $1.041 \mathrm{E}+05$ & $1.020 \mathrm{E}+05$ & $1.031 \mathrm{E}+05$ \\
\hline \$96T005779 & \multirow[t]{2}{*}{$163: 18$} & Upper half & $1.156 \mathrm{E}+05$ & $1.520 \mathrm{E}+05$ & $1.338 \mathrm{E}+05^{\mathrm{QC}: c}$ \\
\hline S96T005785 & & Lower half & $1.042 \mathrm{E}+05$ & $1.020 \mathrm{E}+05$ & $1.031 \mathrm{E}+05$ \\
\hline S96T005558 & \multirow[t]{2}{*}{$163: 20$} & Upper half & $1.395 \mathrm{E}+05$ & $1.220 \mathrm{E}+05$ & $1.308 \mathrm{E}+05$ \\
\hline S96T005561 & & Lower half & $1.611 \mathrm{E}+05$ & $1.730 \mathrm{E}+05$ & $1.671 \mathrm{E}+05^{\mathrm{QC}: \mathrm{c}}$ \\
\hline S96T004799 & $164: 1$ & Lower half & $1.461 \mathrm{E}+05$ & $1.470 \mathrm{E}+05$ & $1.466 \mathrm{E}+05$ \\
\hline S96T004800 & $164: 13$ & Lower half & $1.608 \mathrm{E}+05$ & $1.590 \mathrm{E}+05$ & $1.599 \mathrm{E}+05$ \\
\hline S96T005067 & \multirow[t]{2}{*}{$164: 14$} & Upper half & $1.036 \mathrm{E}+05$ & $1.050 \mathrm{E}+05$ & $1.043 \mathrm{E}+05$ \\
\hline \$96T005065 & & Lower half & 98,000 & $1.010 E+05$ & 99,500 \\
\hline S96T005071 & \multirow[t]{2}{*}{$164: 15$} & Upper half & $1.056 \mathrm{E}+05$ & $1.030 \mathrm{E}+05$ & $1.043 E+05$ \\
\hline S96T005068 & & Lower half & $1.030 \mathrm{E}+05$ & 98,800 & $1.009 \mathrm{E}+05$ \\
\hline S96T005072 & \multirow[t]{2}{*}{$164: 16$} & Upper half & $1.004 E+05$ & $1.010 \mathrm{E}+05$ & $1.007 \mathrm{E}+05$ \\
\hline S96T005069 & & Lower half & $1.027 \mathrm{E}+05$ & $1.050 \mathrm{E}+05$ & $1.039 E+05$ \\
\hline S96T005073 & \multirow[t]{2}{*}{$164: 17$} & Upper half & $1.098 \mathrm{E}+05$ & $1.020 \mathrm{E}+05$ & $1.059 \mathrm{E}+05$ \\
\hline S96T005066 & & Lower half & $1.029 \mathrm{E}+05$ & $1.020 \mathrm{E}+05$ & $1.025 \mathrm{E}+05$ \\
\hline S96T005074 & \multirow[t]{2}{*}{$164: 19$} & Upper half & $1.111 E+05$ & $1.120 \mathrm{E}+05$ & $1.116 \mathrm{E}+05$ \\
\hline S96T005070 & & Lower half & $1.035 \mathrm{E}+05$ & $1.050 \mathrm{E}+05$ & $1.043 \mathrm{E}+05$ \\
\hline S96T005283 & \multirow[t]{2}{*}{$164: 20$} & Upper half & $1.046 \mathrm{E}+05$ & $1.040 \mathrm{E}+05$ & $1.043 \mathrm{E}+05$ \\
\hline S96T005280 & & Lower half & 95,590 & 96,700 & 96,145 \\
\hline S96T005977 & Core 164 & Composite & $1.018 \mathrm{E}+05$ & $1.0 \mathrm{E}+05$ & $1.009 E+05$ \\
\hline
\end{tabular}




\begin{tabular}{|c|c|c|c|c|c|}
\hline $\mathrm{SO}+\mathrm{B} 990^{\circ} \mathrm{Z}$ & $50+3090 \cdot z$ & $S 0+\exists z \angle 0^{\circ} z$ & วા!Soduo & 79 & 6L6\$00L96S \\
\hline$S 0+389 L^{\circ} \mathrm{I}$ & $S 0+30 Z L I$ & $50+\exists 918^{\circ} \mathrm{I}$ & pı̣nbrt & $\varepsilon I: \neq 9 I$ & $\triangleright 8 L t 00 \mathrm{~L} 96 \mathrm{~S}$ \\
\hline $\mathrm{SO}+\mathrm{B}+8 L^{\circ} \mathrm{I}$ & $\mathrm{SO}+\exists 0 L L \cdot \mathrm{L}$ & $S 0+\exists L 6 L I I$ & ptnby & $z I: \neq 9 I$ & $\varepsilon 8 L t 00 L 96 \mathrm{~S}$ \\
\hline $\mathrm{SO}+\exists \varepsilon 99^{\circ} \mathrm{I}$ & $50+\exists 0 \angle 9^{\circ} \mathrm{I}$ & $50+\exists 959^{\circ} \mathrm{I}$ & p!nb!̣ & $I I:+9 I$ & $z 8 \angle t 00 \mathrm{~L} 96 \mathrm{~S}$ \\
\hline$S 0+38 Z L I$ & $S O+\exists O E L \cdot I$ & $S O+\exists S Z L \cdot I$ & p!nbr: & $0 I:+9 I$ & $6 L 6+00 L 96 S$ \\
\hline$S 0+\exists S 8 L^{\circ} I$ & $\mathrm{SO}+308 L^{\circ} \mathrm{I}$ & $S 0+\exists 06 L^{: I}$ & p!nbrา & $6: t 9 I$ & $8 L 6700196 \mathrm{~S}$ \\
\hline$S 0+\exists L S L I$ & $\mathrm{SO}+309 L^{\circ} \mathrm{I}$ & $S O+\exists t S L \cdot I$ & p!nbIT & $8: t 9 I$ & LL6t00I96S \\
\hline S0+3889.' & $\mathrm{S} 0+30 \angle 9^{\circ} \mathrm{I}$ & $50+3569^{\circ} \mathrm{I}$ & p!nb!l & $L:+9 I$ & L8L700L96S \\
\hline $\mathrm{S} 0+\exists 8 \angle \nabla^{\circ} \mathrm{I}$ & so+ $306 t^{\circ} !$ & $50+\exists 990^{\circ} 1$ & p!nb!̣ & $s: t 9 I$ & $9 \angle 6700 \mathrm{~L} 96 \mathrm{~S}$ \\
\hline $50+3669^{\circ} \mathrm{I}$ & $\mathrm{SO}+3089^{\circ} \mathrm{I}$ & $S O+\exists L L L^{\circ} \mathrm{I}$ & pInb!T & $t: t 9 I$ & $08 \angle t 00 L 96 \mathrm{~S}$ \\
\hline $\mathrm{SO}+30 \angle 9^{\circ} \mathrm{I}$ & $\mathrm{SO}+7099^{\circ} \mathrm{I}$ & $S 0+\exists 6 L 9^{\circ} \mathrm{I}$ & p!nbre & $\varepsilon:+9 I$ & 6LLt00L L $6 \mathrm{~S}$ \\
\hline $\mathrm{SO}+\mathrm{GS} \angle 8^{\circ} \mathrm{I}$ & $\mathrm{SO}+\mathrm{B} 098^{\circ} \mathrm{I}$ & $50+\exists 688^{\prime} \mathrm{I}$ & p!nb!) & $z: \neq 9 I$ & $8 \angle L t 00 L 96 \mathrm{~S}$ \\
\hline S0+BE $09^{\circ} \mathrm{I}$ & $50+\exists 009^{\circ} \mathrm{I}$ & $S 0+\exists S 09^{\circ} \mathrm{I}$ & p!nby 1 & $I: t 9 I$ & tLLt00L96S \\
\hline $50+3 r s 9^{\circ} \mathrm{I}$ & $50+3079^{\circ} \mathrm{I}$ & $50+\exists I 99^{\circ} \mathrm{I}$ & pı̣b!e & $\nabla[: \varepsilon 9 I$ & I $\angle S 00 . \mathrm{L96S}$ \\
\hline so+30Zt'I & so+ $30 z t^{\prime} \mathrm{I}$ & $s 0+\exists 6 I^{\prime} I$ & p!̣nb! 1 & $Z I: \varepsilon 9 I$ & $99 L S 00 L 96 \mathrm{~S}$ \\
\hline SO+3925 I & $\varsigma 0+\exists 0 \mathcal{S} \varsigma^{\prime} \mathrm{I}$ & $s_{0}+3 z z s^{\circ} 1$ & p!nb! I & I I:E9I & $6 \mathcal{E L S 0 0 L 9 6 S}$ \\
\hline$S 0+\exists \angle 8 L^{\circ} \mathrm{I}$ & $50+306 L^{\circ} \mathrm{I}$ & $50+\exists \varepsilon 8 L^{\circ} \mathrm{I}$ & pInbrT & $0\lfloor: \varepsilon 9 I$ & 8ZS\$00L96S \\
\hline SO+BIt L'I & $S O+\exists O S L I$ & $S 0+\exists I E L \cdot I$ & p!nbrẹ & $6: \varepsilon 9 I$ & 8SZS00L96S \\
\hline $50+3099^{\circ} \mathrm{I}$ & $50+3099^{\circ} \mathrm{I}$ & $50+3099^{\circ} \mathrm{I}$ & p!nb!x & 8:E9I & LSZS00L96S \\
\hline $0 \varepsilon \mathcal{Z}^{\prime} 6 L$ & $000^{\circ} 08$ & $09 t^{\circ} 8 L$ & p!nbr. & $L: \varepsilon 9 I$ & SSZS00L96S \\
\hline $50+\exists \triangleright\left[9^{\circ} \mathrm{I}\right.$ & $50+30 E 9^{\circ} \mathrm{I}$ & $S 0+\exists \angle 6 S^{\circ} \mathrm{I}$ & pinbrt & $9: \varepsilon 9 I$ & tSSSO0L96S \\
\hline SO+GE8S: I & $\mathrm{SO}_{0}+\exists 06 \mathrm{~S}^{\prime} \mathrm{I}$ & $S 0+\exists S L S^{\circ} \mathrm{I}$ & p!nbị & $\$: \varepsilon 9 I$ & LZSS00L96S \\
\hline$S 0+39 D L^{\prime} I$ & $90+\exists 09 L \cdot I$ & $S 0+\exists I E L^{\prime} I$ & p!̣nb! & $\nabla: \varepsilon 9 I$ & 097\$00L96S \\
\hline $00 S 0+\exists S 6 I^{\circ} \mathcal{E}$ & $\mathrm{S} 0+\exists 06 \mathrm{I}^{\circ} \varepsilon$ & $50+\exists 66 I^{\circ} \varepsilon$ & p!nb!x & $\tau: \varepsilon 9 \mathrm{I}$ & 9SZS00L96S \\
\hline \%) & (1) & gling & & 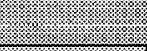 & . \\
\hline mesir) & 96014) & 6) & 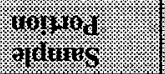 & 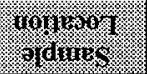 & 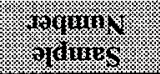 \\
\hline
\end{tabular}

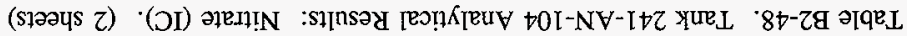


Table B2-49. Tank 241-AN-104 Analytical Results: Nitrite (IC). (2 sheets)

\begin{tabular}{|c|c|c|c|c|c|}
\hline Haingla & Mample & 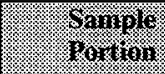 & Rerinitis & 8) & (1) \\
\hline \multicolumn{3}{|c|}{ 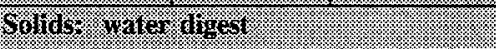 } & $\operatorname{lgg}$ & gyo & 10 \\
\hline S96T005290 & $163: 1$ & Lower half & 69,320 & 66,300 & $67,810^{\mathrm{QC:}: \mathrm{d}}$ \\
\hline S96T005291 & $163: 2$ & Lower half & 69,010 & 66,900 & 67,955 \\
\hline S96T005784 & $163: 12$ & Lower half & 63,810 & 64,400 & 64,105 \\
\hline S96T005751 & \multirow[t]{2}{*}{$163: 14$} & Upper half & 64,460 & 65,300 & 64,880 \\
\hline S96T005752 & & Lower half & 65,830 & 66,300 & 66,065 \\
\hline S96T005295 & \multirow[t]{2}{*}{$163: 16$} & Upper half & 56,690 & 54,800 & 55,745 \\
\hline S96T005292 & & Lower half & 62,630 & 65,100 & 63,865 \\
\hline S96T005779 & \multirow[t]{2}{*}{$163: 18$} & Upper half & 61,740 & 59,400 & 60,570 \\
\hline S96T005785 & & Lower half & 60,240 & 63,900 & 62,070 \\
\hline S96T005558 & \multirow[t]{2}{*}{$163: 20$} & Upper half & 60,230 & 62,200 & 61,215 \\
\hline S96T005561 & & Lower half & 56,880 & 56,100 & 56,490 \\
\hline S96T004799 & $164: 1$ & Lower half & 63,340 & 63,700 & 63,520 \\
\hline S96T004800 & $164: 13$ & Lower half & 57,810 & 57,200 & 57,505 \\
\hline S96T005067 & \multirow[t]{2}{*}{$164: 14$} & Upper half & 65,150 & 64,800 & 64,975 \\
\hline S96T005065 & & Lower half & 67,630 & 68,100 & 67,865 \\
\hline \$96T005071 & \multirow[t]{2}{*}{$164: 15$} & Upper half & 65,920 & 64,200 & 65,060 \\
\hline S96T005068 & & Lower half & 60,700 & 59,000 & 59,850 \\
\hline S96T005072 & \multirow[t]{2}{*}{$164: 16$} & Upper half & 64,690 & 62,400 & 63,545 \\
\hline S96T005069 & & Lower half & 74,380 & 72,200 & 73,290 \\
\hline \$96T005073 & \multirow[t]{2}{*}{$164: 17$} & Upper half & 60,520 & 63,400 & 61,960 \\
\hline S96T005066 & & Lower half & 59,300 & 59,000 & 59,150 \\
\hline S96T005074 & \multirow[t]{2}{*}{$164: 19$} & Upper half & 58,760 & 60,100 & 59,430 \\
\hline S96T005070 & & Lower half & 62,010 & 61,800 & 61,905 \\
\hline S96T005283 & \multirow[t]{2}{*}{$164: 20$} & Upper half & 68,590 & 69,300 & 68,945 \\
\hline S96T005280 & & Lower half & 64,170 & 64,100 & 64,135 \\
\hline S96T005977 & Core 164 & Composite & 57,870 & 58,200 & 58,035 \\
\hline
\end{tabular}


Table B2-49. Tank 241-AN-104 Analytical Results: Nitrite (IC). (2 sheets)

\begin{tabular}{|c|c|c|c|c|c|}
\hline (1) & \%amprom & 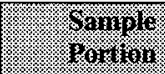 & 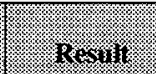 & OHiphate & Tin \\
\hline Framal & & & 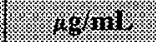 & 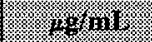 & \% \\
\hline S96T005256 & $163: 2$ & Liquid & $2.271 \mathrm{E}+05$ & $2.230 \mathrm{E}+05$ & $2.251 \mathrm{E}+05^{\mathrm{QC}}$ \\
\hline S96T005260 & $163: 4$ & Liquid & $1.136 \mathrm{E}+05$ & $1.120 \mathrm{E}+05$ & $1.128 \mathrm{E}+05$ \\
\hline S96T005527 & $163: 5$ & Liquid & $1.327 \mathrm{E}+05$ & $1.330 \mathrm{E}+05$ & $1.329 \mathrm{E}+05$ \\
\hline S96T005554 & $163: 6$ & Liquid & $1.191 \mathrm{E}+05$ & $1.260 \mathrm{E}+05$ & $1.226 \mathrm{E}+05$ \\
\hline S96T005255 & $163: 7$ & Liquid & 49,300 & 49,100 & 49,200 \\
\hline S96T005257 & $163: 8$ & Liquid & $1.174 \mathrm{E}+05$ & $1.170 \mathrm{E}+05$ & $1.172 \mathrm{E}+05$ \\
\hline S96T005258 & $163: 9$ & Liquid & $1.077 \mathrm{E}+05$ & $1.060 \mathrm{E}+05$ & $1.069 \mathrm{E}+05$ \\
\hline S96T005528 & $163: 10$ & Liquid & $1.292 \mathrm{E}+05$ & $1.300 \mathrm{E}+05$ & $1.296 \mathrm{E}+05$ \\
\hline S96T005739 & 163:11 & Liquid & 91,010 & 93,600 & 92,305 \\
\hline S96T005766 & 163:12 & Liquid & $1.183 E+05$ & $1.080 \mathrm{E}+05$ & 1.132E+05 \\
\hline S96T005741 & $163: 14$ & Liquid & $3.126 \mathrm{E}+05$ & $3.170 \mathrm{E}+05$ & $3.148 \mathrm{E}+05$ \\
\hline S96T004774 & $164: 1$ & Liquid & $1.140 \mathrm{E}+05$ & $1.140 \mathrm{E}+05$ & $1.140 \mathrm{E}+05$ \\
\hline S96T004778 & $164: 2$ & Liquid & $1.175 \mathrm{E}+05$ & $1.180 \mathrm{E}+05$ & $1.178 \mathrm{E}+05$ \\
\hline \$96T004779 & $164: 3$ & Liquid & $1.091 \mathrm{E}+05$ & $1.090 \mathrm{E}+05$ & $1.091 \mathrm{E}+05$ \\
\hline S96T004780 & $164: 4$ & Liquid & $1.077 \mathrm{E}+05$ & $1.080 \mathrm{E}+05$ & $1.079 \mathrm{E}+05$ \\
\hline \$96T004976 & $164: 5$ & Liquid & 94,260 & 94,000 & 94,130 \\
\hline S96T004781 & $164: 7$ & Liquid & $1.094 \mathrm{E}+05$ & $1.100 \mathrm{E}+05$ & $1.097 \mathrm{E}+05$ \\
\hline S96T004977 & $164: 8$ & Liquid & $1.239 \mathrm{E}+05$ & $1.220 \mathrm{E}+05$ & $1.230 \mathrm{E}+05$ \\
\hline S96T004978 & $164: 9$ & Liquid & $1.137 \mathrm{E}+05$ & $1.160 \mathrm{E}+05$ & $1.149 \mathrm{E}+05$ \\
\hline S96T004979 & $164: 10$ & Liquid & $1.111 E+05$ & $1.120 \mathrm{E}+05$ & $1.116 \mathrm{E}+05$ \\
\hline S96T004782 & $164: 11$ & Liquid & $1.046 \mathrm{E}+05$ & $1.050 \mathrm{E}+05$ & $1.048 \mathrm{E}+05$ \\
\hline S96T004783 & $164: 12$ & Liquid & $1.279 \mathrm{E}+05$ & $1.300 \mathrm{E}+05$ & $1.290 \mathrm{E}+05$ \\
\hline S96T004784 & $164: 13$ & Liquid & $1.188 \mathrm{E}+05$ & $1.140 \mathrm{E}+05$ & $1.164 \mathrm{E}+05$ \\
\hline S96T005979 & Core 164 & Composite & $1.325 \mathrm{E}+05$ & $1.290 \mathrm{E}+05$ & $1.308 \mathrm{E}+05$ \\
\hline
\end{tabular}


HNF-SD-WM-ER-690 Rev. 0

Table B2-50. Tank 241-AN-104 Analytical Results: Phosphate (IC). (2 sheets)

\begin{tabular}{|c|c|c|c|c|c|}
\hline 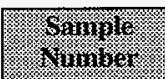 & Mande & forme & \%ickin & 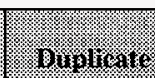 & (1) \\
\hline \multicolumn{2}{|c|}{ Solits. water afiger } & 器 & \%g. & \% & 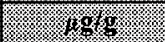 \\
\hline S96T005290 & 163:1 & Lower half & 3,088 & 2,920 & 3,004 \\
\hline S96T005291 & $163: 2$ & Lower half & 3,221 & 3,770 & $3,495.5$ \\
\hline S96T005784 & $163: 12$ & Lower half & 4,823 & 4,300 & $4,561.5$ \\
\hline S96T005751 & \multirow[t]{2}{*}{$163: 14$} & Upper half & 2,284 & 2,130 & 2,207 \\
\hline S96T005752 & & Lower half & 2,603 & 2,620 & $2,611.5$ \\
\hline S96T005295 & \multirow[t]{2}{*}{$163: 16$} & Upper half & 2,692 & 2,410 & 2,551 \\
\hline S96T005292 & & Lower half & 3,500 & 4,170 & 3,835 \\
\hline S96T005779 & \multirow[t]{2}{*}{$163: 18$} & Upper half & 3,379 & 3,220 & $3,299.5$ \\
\hline S96T005785 & & Lower half & 3,496 & 3,430 & 3,463 \\
\hline S96T005558 & \multirow[t]{2}{*}{$163: 20$} & Upper half & 3,704 & 4,070 & 3,887 \\
\hline S96T005561 & & Lower half & 15,920 & 15,300 & 15,610 \\
\hline S96T004799 & $164: 1$ & Lower half & 5,752 & 5,830 & 5,791 \\
\hline S96T004800 & $164: 13$ & Lower half & 1,529 & $<980$ & $<1,254.5^{\mathrm{QC}: \mathrm{c}}$ \\
\hline S96T005067 & \multirow[t]{2}{*}{$164: 14$} & Upper half & 2,968 & 2,930 & 2,949 \\
\hline S96T005065 & & Lower half & 2,240 & 3,010 & $2,625^{\mathrm{QC}: c}$ \\
\hline S96T005071 & \multirow[t]{2}{*}{$164: 15$} & Upper half & 2,234 & 2,350 & 2,292 \\
\hline S96T005068 & & Lower half & 2,103 & 2,270 & $2,186.5$ \\
\hline S96T005072 & \multirow[t]{2}{*}{$164: 16$} & Upper half & 2,689 & 1,880 & $2,284.5^{\mathrm{QC:c}}$ \\
\hline S96T005069 & & Lower half & 1,535 & 1,370 & $1,452.5$ \\
\hline S96T005073 & \multirow[t]{2}{*}{$164: 17$} & Upper half & 3,195 & 2,800 & $2,997.5$ \\
\hline S96T005066 & & Lower half & 4,484 & 4,840 & 4,662 \\
\hline S96T005074 & \multirow[t]{2}{*}{$164: 19$} & Upper half & 4,189 & 4,410 & $4,299.5$ \\
\hline S96T005070 & & Lower half & 3,961 & 4,300 & $4,130.5$ \\
\hline \$96T005283 & \multirow[t]{2}{*}{$164: 20$} & Upper half & 3,622 & 3,450 & 3,536 \\
\hline \$96T005280 & & Lower half & 3,384 & 3,200 & 3,292 \\
\hline S96T005977 & Core 164 & Composite & 3,555 & 3,130 & $3,342.5$ \\
\hline
\end{tabular}


Table B2-50. Tank 241-AN-104 Analytical Results: Phosphate (IC). (2 sheets)

\begin{tabular}{|c|c|c|c|c|c|}
\hline 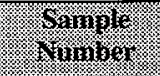 & Somple & 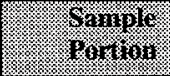 & ingsin: & 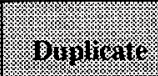 & W \\
\hline \multicolumn{2}{|c|}{ ly } & & (1961) & $\int$ & (49 \\
\hline S96T005256 & $163: 2$ & Liquid & 3,859 & 3,790 & $3,824.5$ \\
\hline S96T005260 & $163: 4$ & Liquid & 2,458 & 2,350 & 2,404 \\
\hline \$96T005527 & $163: 5$ & Liquid & 2,169 & 2,080 & $2,124.5$ \\
\hline S96T005554 & $163: 6$ & Liquid & 2,672 & 2,280 & 2,476 \\
\hline S96T005255 & $163: 7$ & Liquid & 2,615 & 2,570 & $2,592.5$ \\
\hline S96T005257 & $163: 8$ & Liquid & 1,747 & 1,870 & $1,808.5$ \\
\hline S96T005258 & $163: 9$ & Liquid & 2,068 & 2,040 & 2,054 \\
\hline S96T005528 & $163: 10$ & Liquid & 1,839 & 1,870 & $1,854.5$ \\
\hline \$96T005739 & $163: 11$ & Liquid & 1,252 & 1,580 & $1,416^{\mathrm{QC:c}}$ \\
\hline S96T005766 & $163: 12$ & Liquid & 1,039 & 893 & 966 \\
\hline S96T005741 & $163: 14$ & Liquid & 1,027 & 997 & 1,012 \\
\hline S96T004774 & $164: 1$ & Liquid & 2,161 & 2,310 & $2,235.5$ \\
\hline S96T004778 & $164: 2$ & Liquid & 2,665 & 2,750 & $2,707.5$ \\
\hline S96T004779 & $164: 3$ & Liquid & 2,832 & 3,110 & 2,971 \\
\hline S96T004780 & $164: 4$ & Liquid & 2,769 & 3,100 & $2,934.5$ \\
\hline S96T004976 & $164: 5$ & Liquid & 1,679 & $<1,220$ & $<1,449.5^{\mathrm{QC}: \mathrm{c}}$ \\
\hline S96T004781 & $164: 7$ & Liquid & 3,775 & 1,810 & $2,792.5^{\mathrm{QC:c}}$ \\
\hline S96T004977 & $164: 8$ & Liquid & 2,194 & 2,150 & 2,172 \\
\hline S96T004978 & $164: 9$ & Liquid & 2,479 & 2,230 & $2,354.5$ \\
\hline S96T004979 & $164: 10$ & Liquid & 2,171 & 2,180 & $2,175.5$ \\
\hline S96T004782 & 164:11 & Liquid & 3,081 & 2,610 & $2,845.5$ \\
\hline S96T004783 & $164: 12$ & Liquid & 2,648 & 2,710 & 2,679 \\
\hline S96T004784 & $164: 13$ & Liquid & 2,205 & 2,100 & $2,152.5$ \\
\hline S96T005979 & Core 164 & Composite & 3,980 & 3,610 & 3,795 \\
\hline
\end{tabular}


HNF-SD-WM-ER-690 Rev. 0

Table B2-51. Tank 241-AN-104 Analytical Results: Sulfate (IC). (2 sheets)

\begin{tabular}{|c|c|c|c|c|c|}
\hline $\begin{array}{l}\text { Sample: } \\
\text { Sumbir }\end{array}$ & S Simpere & $\begin{array}{l}\text { Samplo } \\
\text { Intiva }\end{array}$ & Resull & Gugition. & Var: \\
\hline \multicolumn{3}{|c|}{ Gritis water digers } & $\sqrt{1 / 2}$ & (2) & 10 \\
\hline S96T005290 & $163: 1$ & Lower half & 11,380 & 11,100 & 11,240 \\
\hline S96T005291 & $163: 2$ & Lower half & 3,653 & 3,900 & $3,776.5$ \\
\hline S96T005784 & $163: 12$ & Lower half & 7,544 & 7,270 & 7,407 \\
\hline S96T005751 & \multirow[t]{2}{*}{$163: 14$} & Upper half & 9,571 & 9,420 & $9,495.5$ \\
\hline S96T005752 & & Lower half & 10,410 & 10,200 & 10,305 \\
\hline S96T005295 & \multirow[t]{2}{*}{$163: 16$} & Upper half & 12,600 & 12,400 & 12,500 \\
\hline S96T005292 & & Lower half & 12,940 & 12,900 & 12,920 \\
\hline S96T005779 & \multirow[t]{2}{*}{$163: 18$} & Upper half & 16,480 & 16,600 & 16,540 \\
\hline S96T005785 & & Lower half & 16,490 & 16,300 & 16,395 \\
\hline S96T005558 & \multirow[t]{2}{*}{$163: 20$} & Upper half & 14,400 & 14,900 & 14,650 \\
\hline S96T005561 & & Lower half & 10,370 & 10,500 & 10,435 \\
\hline S96T004799 & $164: 1$ & Lower half & 8,357 & 8,610 & $8,483.5$ \\
\hline S96T004800 & $164: 13$ & Lower half & 4,386 & 4,370 & 4,378 \\
\hline S96T005067 & \multirow[t]{2}{*}{$164: 14$} & Upper half & 11,020 & 11,000 & 11,010 \\
\hline S96T005065 & & Lower half & 8,954 & 8,730 & 8,842 \\
\hline S96T005071 & \multirow[t]{2}{*}{$164: 15$} & Upper half & 10,250 & 9,880 & 10,065 \\
\hline S96T005068 & & Lower half & 11,650 & 10,800 & 11,225 \\
\hline S96T005072 & \multirow[t]{2}{*}{$164: 16$} & Upper half & 9,477 & 12,000 & $10,738.5^{\mathrm{QC}: c}$ \\
\hline S96T005069 & & Lower half & 10,130 & 9,960 & 10,045 \\
\hline S96T005073 & \multirow[t]{2}{*}{$164: 17$} & Upper half & 10,230 & 9,980 & 10,105 \\
\hline S96T005066 & & Lower half & 12,480 & 12,600 & 12,540 \\
\hline S96T005074 & \multirow[t]{2}{*}{$164: 19$} & Upper half & 11,960 & 13,100 & 12,530 \\
\hline S96T005070 & & Lower half & 12,640 & 12,800 & 12,720 \\
\hline S96T005283 & \multirow[t]{2}{*}{$164: 20$} & Upper half & 13,190 & 13,500 & 13,345 \\
\hline S96T005280 & & Lower half & 16,590 & 17,100 & 16,845 \\
\hline S96T005977 & Core 164 & Composite & 11,710 & 11,500 & 11,605 \\
\hline
\end{tabular}


Table B2-51. Tank 241-AN-104 Analytical Results: Sulfate (IC). (2 sheets)

\begin{tabular}{|c|c|c|c|c|c|}
\hline $\begin{array}{l}\text { Sample } \\
\text { number }\end{array}$ & $\begin{array}{l}\text { Sarmple } \\
\text { ireatin }\end{array}$ & 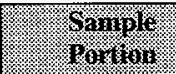 & resul: & Suluilicate & Nran \\
\hline Iigurits. & 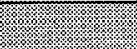 & 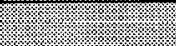 & 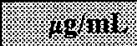 & 1484nin & (2) \\
\hline S96T005256 & $163: 2$ & Liquid & 2,796 & 2,490 & 2,643 \\
\hline S96T005260 & $163: 4$ & Liquid & 1,925 & 1,800 & $1,862.5$ \\
\hline S96T005527 & $163: 5$ & Liquid & 1,036 & 1,090 & 1,063 \\
\hline S96T005554 & $163: 6$ & Liquid & $<710.8$ & 754 & $<732.4$ \\
\hline S96T005255 & $163: 7$ & Liquid & 1,023 & 1,470 & $1,246.5^{\mathrm{QC}: \mathrm{c}}$ \\
\hline S96T005257 & $163: 8$ & Liquid & 1,045 & 1,090 & $1,067.5$ \\
\hline S96T005258 & $163: 9$ & Liquid & 1,484 & 2,080 & $1,782^{\mathrm{QC}: \mathrm{c}}$ \\
\hline S96T005528 & $163: 10$ & Liquid & 1,452 & 1,710 & 1,581 \\
\hline S96T005739 & 163:11 & Liquid & $<710.8$ & $<711$ & $<710.9$ \\
\hline S96T005766 & $163: 12$ & Liquid & 911.3 & $<711$ & $<811.15^{\text {QC:e }}$ \\
\hline S96T005741 & $163: 14$ & Liquid & 1,670 & 1,330 & $1,500^{\mathrm{QC}: \mathrm{c}}$ \\
\hline S96T004774 & $164: 1$ & Liquid & 1,980 & 1,870 & 1,925 \\
\hline S96T004778 & $164: 2$ & Liquid & 2,100 & 1,810 & 1,955 \\
\hline S96T004779 & $164: 3$ & Liquid & 1,997 & 2,020 & $2,008.5$ \\
\hline S96T004780 & $164: 4$ & Liquid & 1,933 & 1,980 & $1,956.5$ \\
\hline S96T004976 & $164: 5$ & Liquid & $<1,408$ & $<1,410$ & $<1,409$ \\
\hline S96T004781 & $164: 7$ & Liquid & 2,725 & 1,500 & $2,112.5^{\mathrm{QC:e}}$ \\
\hline S96T004977 & $164: 8$ & Liquid & $<1,408$ & $<1,410$ & $<1,409$ \\
\hline S96T004978 & $164: 9$ & Liquid & 1,642 & 1,930 & 1,786 \\
\hline \begin{tabular}{|l} 
S96T004979 \\
\end{tabular} & $164: 10$ & Liquid & 1,463 & 1,780 & $1,621.5$ \\
\hline S96T004782 & $164: 11$ & Liquid & 1,963 & 2,110 & $2,036.5$ \\
\hline S96T004783 & $164: 12$ & Liquid & 1,818 & 2,310 & $2,064^{\mathrm{QC} e}$ \\
\hline S96T004784 & $164: 13$ & Liquid & 1,880 & 2,090 & 1,985 \\
\hline S96T005979 & Core 164 & Composite & 2,118 & 2,500 & 2,309 \\
\hline
\end{tabular}


Table B2-52. Tank 241-AN-104 Analytical Results: Acetate (IC). (2 sheets)

\begin{tabular}{|c|c|c|c|c|c|}
\hline 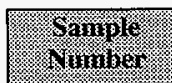 & 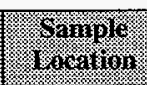 & (3) & resanil & 1) & kn \\
\hline \multicolumn{2}{|c|}{ 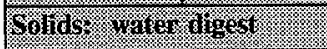 } & & div. & \% & 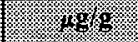 \\
\hline S96T005290 & 163:1 & Lower half & 425.6 & 507 & 466.3 \\
\hline S96T005291 & $163: 2$ & Lower half & 476.5 & 346 & $411.25^{\mathrm{QC}: \mathrm{c}}$ \\
\hline S96T005784 & $163: 12$ & Lower half & 495.9 & 443 & 469.45 \\
\hline S96T005751 & \multirow[t]{2}{*}{ 163:14 } & Upper half & 429.5 & 438 & $433.75^{\mathrm{QC}: \mathrm{d}}$ \\
\hline S96T005752 & & Lower half & 442.5 & 469 & $455.75^{\mathrm{QC}: \mathrm{d}}$ \\
\hline S96T005295 & \multirow[t]{2}{*}{$163: 16$} & Upper half & $<149.9$ & $<146$ & $<147.95$ \\
\hline$\$ 96 \mathrm{~T} 005292$ & & Lower half & 1,019 & 1,090 & $1,054.5$ \\
\hline S96T005779 & \multirow[t]{2}{*}{$163: 18$} & Upper half & 608.3 & 554 & 581.15 \\
\hline S96T005785 & & Lower half & 554.7 & 489 & 521.85 \\
\hline S96T005558 & \multirow[t]{2}{*}{$163: 20$} & Upper half & 483.5 & 493 & 488.25 \\
\hline S96T005561 & & Lower half & 439.5 & 459 & 449.25 \\
\hline S97T000648 & $164: 1$ & Lower half & 332 & 354 & 343 \\
\hline S97T000649 & $164: 13$ & Lower half & 604.9 & 591 & $597.95^{\mathrm{QC:d}}$ \\
\hline S96T005067 & \multirow[t]{2}{*}{$164: 14$} & Upper half & 623.2 & 637 & 630.1 \\
\hline \$96T005065 & & Lower half & 524.7 & 490 & 507.35 \\
\hline S96T005071 & \multirow[t]{2}{*}{$164: 15$} & Upper half & 517.5 & 516 & 516.75 \\
\hline S96T005068 & & Lower half & 508.9 & 470 & 489.45 \\
\hline \$96T005072 & \multirow[t]{2}{*}{$164: 16$} & Upper half & 553.3 & 554 & 553.65 \\
\hline \$96T005069 & & Lower half & 751.7 & 635 & 693.35 \\
\hline S96T005073 & \multirow[t]{2}{*}{$164: 17$} & Upper half & 543.7 & 568 & 555.85 \\
\hline S96T005066 & & Lower half & 529.8 & 511 & 520.4 \\
\hline \$96T005074 & \multirow[t]{2}{*}{$164: 19$} & Upper half & 502.2 & 495 & 498.6 \\
\hline S96T005070 & & Lower half & 618.8 & 562 & 590.4 \\
\hline S96T005283 & \multirow[t]{2}{*}{$164: 20$} & Upper half & 613 & 661 & 637 \\
\hline S96T005280 & & Lower half & 661 & 688 & 674.5 \\
\hline S96T005977 & Core 164 & Composite & 612.7 & 660 & $636.35^{\mathrm{QC}: \mathrm{c}}$ \\
\hline
\end{tabular}


Table B2-52. Tank 241-AN-104 Analytical Results: Acetate (IC). (2 sheets)

\begin{tabular}{|c|c|c|c|c|c|}
\hline $\begin{array}{l}\text { Sanple } \\
\text { Surnber }\end{array}$ & 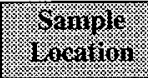 & $\begin{array}{l}\text { Sranjle } \\
\text { Jowtion }\end{array}$ & Resinitir & 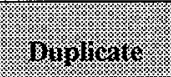 & Man: \\
\hline Migias: & 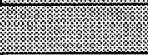 & 8 & $\sqrt{1.4 m m}$ & (6. & 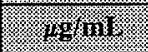 \\
\hline S96T005256 & $163: 2$ & Liquid & 1,917 & 1,150 & $1,533.5^{\mathrm{QC}: \mathrm{e}}$ \\
\hline S96T005260 & $163: 4$ & Liquid & 620.7 & 616 & 618.35 \\
\hline S96T005527 & $163: 5$ & Liquid & 180.9 & 144 & $162.45^{\mathrm{QC}: \mathrm{e}}$ \\
\hline S96T005554 & $163: 6$ & Liquid & 189.5 & 339 & $264.25^{\mathrm{QC}: e}$ \\
\hline S96T005255 & $163: 7$ & Liquid & 550.9 & 645 & 597.95 \\
\hline S96T005257 & $163: 8$ & Liquid & $<406$ & 1,290 & $<848^{\mathrm{QC}: \mathrm{e}}$ \\
\hline S96T005258 & $163: 9$ & Liquid & 564.3 & 565 & 564.65 \\
\hline S96T005528 & $163: 10$ & Liquid & 289.6 & 293 & 291.3 \\
\hline S96T005739 & $163: 11$ & Liquid & 820.4 & 777 & 798.7 \\
\hline S96T005766 & $163: 12$ & Liquid & 788.4 & 788 & 788.2 \\
\hline S96T005741 & $163: 14$ & Liquid & 826 & 864 & 845 \\
\hline S96T004774 & $164: 1$ & Liquid & 515.7 & 532 & 523.85 \\
\hline S96T004778 & $164: 2$ & Liquid & 557.6 & 665 & 611.3 \\
\hline S96T004779 & $164: 3$ & Liquid & 523.3 & 536 & 529.65 \\
\hline S96T004780 & $164: 4$ & Liquid & 552.9 & 567 & 559.95 \\
\hline S96T004976 & $164: 5$ & Liquid & 452.5 & 453 & 452.75 \\
\hline S96T004781 & $164: 7$ & Liquid & 626.2 & 566 & 596.1 \\
\hline S96T004977 & $164: 8$ & Liquid & 685.3 & 506 & $595.65^{\text {QC:e }}$ \\
\hline S96T004978 & $164: 9$ & Liquid & 609.3 & 621 & 615.15 \\
\hline S96T004979 & $164: 10$ & Liquid & 504 & 512 & 508 \\
\hline S96T004782 & $164: 11$ & Liquid & 563.1 & 546 & 554.55 \\
\hline S96T004783 & $164: 12$ & Liquid & 643.4 & 623 & 633.2 \\
\hline S96T004784 & $164: 13$ & Liquid & 598.6 & 578 & 588.3 \\
\hline S96T005979 & Core 164 & Composite & 1,773 & 1,990 & $1,881.5$ \\
\hline
\end{tabular}


HNF-SD-WM-ER-690 Rev. 0

Table B2-53. Tank 241-AN-104 Analytical Results: Oxalate (IC). (2 sheets)

\begin{tabular}{|c|c|c|c|c|c|}
\hline Shaninger & Symere & Sample: & Resilit & Buinimats & Meri: \\
\hline \multicolumn{3}{|c|}{ 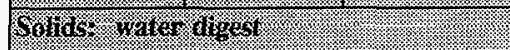 } & 6.98 & 1. & 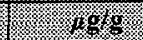 \\
\hline S96T005290 & $163: 1$ & Lower half & 9,520 & 9,290 & 9,405 \\
\hline S96T005291 & $163: 2$ & Lower half & 5,755 & 5,550 & $5,652.5$ \\
\hline S96T005784 & $163: 12$ & Lower half & 7,379 & 7,730 & $7,554.5$ \\
\hline S96T005751 & \multirow[t]{2}{*}{$163: 14$} & Upper half & 9,914 & 10,100 & 10,007 \\
\hline S96T005752 & & Lower half & 10,180 & 9,240 & 9,710 \\
\hline S96T005295 & \multirow[t]{2}{*}{$163: 16$} & Upper half & 9,188 & 9,380 & 9,284 \\
\hline \$96T005292 & & Lower half & 10,400 & 9,770 & 10,085 \\
\hline S96T005779 & \multirow[t]{2}{*}{$163: 18$} & Upper half & 6,127 & 6,140 & $6,133.5$ \\
\hline S96T005785 & & Lower half & 5,936 & 5,560 & 5,748 \\
\hline S96T005558 & \multirow[t]{2}{*}{$163: 20$} & Upper half & 6,205 & 6,800 & $6,502.5$ \\
\hline S96T005561 & & Lower half & 8,438 & 7,780 & 8,109 \\
\hline S96T004799 & 164:1 & Lower half & 7,609 & 7,810 & $7,709.5$ \\
\hline$\$ 96 \mathrm{~T} 004800$ & $164: 13$ & Lower half & 2,105 & 2,110 & $2,107.5$ \\
\hline \$96T005067 & \multirow[t]{2}{*}{$164: 14$} & Upper half & 10,810 & 10,700 & 10,755 \\
\hline$\$ 96 \mathrm{~T} 005065$ & & Lower half & 9,210 & 9,390 & 9,300 \\
\hline S96T005071 & \multirow[t]{2}{*}{$164: 15$} & Upper half & 8,224 & 8,100 & 8,162 \\
\hline \$96T005068 & & Lower half & 8,731 & 8,230 & $8,480.5$ \\
\hline S96T005072 & \multirow[t]{2}{*}{$164: 16$} & Upper half & 8,017 & 10,200 & $9,108.5^{\mathrm{QC:c}}$ \\
\hline S96T005069 & & Lower half & 7,865 & 7,310 & $7,587.5$ \\
\hline \$96T005073 & \multirow[t]{2}{*}{$164: 17$} & Upper half & 9,419 & 8,590 & $9,004.5$ \\
\hline S96T005066 & & Lower half & 12,160 & 11,900 & 12,030 \\
\hline S96T005074 & \multirow[t]{2}{*}{$164: 19$} & Upper half & 11,310 & 10,900 & 11,105 \\
\hline \$96T005070 & & Lower half & 9,839 & 10,500 & $10,169.5$ \\
\hline S96T005283 & \multirow[t]{2}{*}{$164: 20$} & Upper half & 9,832 & 9,650 & 9,741 \\
\hline S96T005280 & & Lower half & 8,172 & 8,220 & 8,196 \\
\hline S96T005977 & Core 164 & Composite & 9,783 & 9,550 & $9,666.5$ \\
\hline
\end{tabular}




\begin{tabular}{|c|c|c|c|c|c|}
\hline $50 \angle 0^{\circ} \mathrm{I}>$ & $0 L 0^{\circ} \mathrm{I}>$ & $I \angle 0^{\circ} I>$ & əి!soduo & t91 ว.0ว & $6 L 6 \mathrm{~S} 00 \mathrm{~L} 96 \mathrm{~S}$ \\
\hline$\varsigma 6.0 t \varsigma>$ & $I t s>$ & $6.0 t s>$ & p!nb!x & $\varepsilon I: t 9 I$ & $\nabla 8 \mathrm{~L}+00 \mathrm{~L} 96 \mathrm{~S}$ \\
\hline$\varsigma^{\circ} 0 L 0^{6} I>$ & $0 \angle 0^{\circ} \mathrm{I}>$ & $I \angle 0^{\circ} I>$ & pẹnb!̣ & $2 I: t 9 I$ & $\varepsilon 8 \angle t 00 \perp 96 \mathrm{~S}$ \\
\hline$\varsigma^{\circ} 0 \angle 0^{\circ} \mathrm{I}>$ & $0 \angle 0^{\circ} I>$ & {$\left[\angle 0^{\prime} I>\right.$} & pinb!n & $I I: t 9 I$ & $z 8 \mathrm{Lt} 00 \mathrm{~L} 96 \mathrm{~S}$ \\
\hline$\therefore: 00 L \subseteq S S$ & 919 & $t \cdot s 6 t$ & pụnb!x & $0 I: t 9 I$ & $6 L 6+00 \mathrm{~L} 96 \mathrm{~S}$ \\
\hline $68 t$ & $z L D$ & $90 \mathrm{~s}$ & punb!r & $6:+9 I$ & $8 L 6+00 L 96 \mathrm{~S}$ \\
\hline$\varsigma 6.0 t \varsigma>$ & Its $>$ & $6.0 t s>$ & pụnbry & $8:+91$ & $\angle L 6+00 L 96 \mathrm{~S}$ \\
\hline $8: 00 Z S \tau^{\prime} I>$ & $0 \angle 0^{\circ} I>$ & $\nabla \varepsilon t^{\prime} I$ & pinb! T & $L: t 9 I$ & {$[8 \mathrm{Lt}+00 \mathrm{~L} 96 \mathrm{~S}$} \\
\hline $56.0 t S>$ & It $S>$ & $6.0 t \varsigma>$ & p!nb!r & s:tol & $9 L 6+00 \mathrm{~L} 96 \mathrm{~S}$ \\
\hline$S^{\circ} 0 \angle 0^{\prime} I>$ & $0 \angle 0^{\prime} I>$ & $I \angle O^{6} I>$ & pụbị & $t:+9$ & $08 \angle+00 \mathrm{~L} 96 \mathrm{~S}$ \\
\hline$\subseteq 0 \angle 0^{6}[>$ & $0 \angle 0^{\circ} I>$ & $I \angle 0^{i} I>$ & pinbỵ & $\varepsilon:+9 I$ & $6 \angle L+00 L 96 \mathrm{~S}$ \\
\hline $0: 00 S \cdot 28 L>$ & $1+s>$ & $\nabla 20^{\prime} \mathrm{I}$ & prnbig & $z:+9 I$ & 8LLt00L $96 \mathrm{~S}$ \\
\hline$L 609$ & 919 & $t \cdot \varepsilon 09$ & p!nb!ר & $I:+9 I$ & $\nabla \angle L+00 \mathrm{~L} 96 \mathrm{~S}$ \\
\hline$\varsigma 6^{\circ} 0 t \varsigma>$ & It $s>$ & $6.005>$ & p!nb!̣ & $\nabla I: \varepsilon 9 \mathrm{I}$ & $\tau+\angle S 00 L 96 \mathrm{~S}$ \\
\hline$\varsigma 6.0 t \varsigma>$ & $I t S\rangle$ & $6.0 t s>$ & pụnbra & ZI:乏9I & $99 \angle \mathrm{S} 00 \mathrm{~L} 96 \mathrm{~S}$ \\
\hline$\varsigma 60 t \varsigma>$ & $I t s>$ & $6.07 \varsigma>$ & p!nb? & II:E9I & $6 \varepsilon \angle S 00 L 96 S$ \\
\hline$\varsigma 6.0 t \varsigma>$ & $I t \varsigma>$ & $6.0 t \varsigma>$ & p!nb!̣ & $0 \mathrm{I}: \varepsilon 9 \mathrm{I}$ & $8 Z S 500 \mathrm{~L} 96 \mathrm{~S}$ \\
\hline $0: 00 \mathrm{S6}^{\circ} 9 \mathrm{IL}$ & $8 z \mathrm{~s}$ & 6.506 & pinb!r & $6: £ 9 I$ & $8 \subseteq 2 \$ 00 \mathrm{~L} 96 \mathrm{~S}$ \\
\hline$\$ 6.6 \varsigma \varsigma>$ & $6 L S$ & $6.0 t s>$ & p!nbit & $8: \varepsilon 9 I$ & $\angle S Z S 00 L 96 S$ \\
\hline $6+\varepsilon t>$ & $\underline{s E t>}$ & $8 \cdot t \varepsilon t>$ & p!nbọn & $L: \varepsilon 9 \mathrm{I}$ & SSZSOOL96S \\
\hline$\varsigma 6.0 t s>$ & It $s>$ & $6.00 \varsigma>$ & punb!̣ & $9: \mathcal{E I}$ & $t S S S 00 L 96 \mathrm{~S}$ \\
\hline$\varsigma 60 t \varsigma>$ & It $S>$ & $6.0+5>$ & prnb!̣ & ૬:ع9I & $\angle Z S S O 0 L 96 \mathrm{~S}$ \\
\hline $0.008<t 9$ & $69 L$ & 9.92s & pinb!r & t:६9I & $092500 \mathrm{~L} 96 \mathrm{~S}$ \\
\hline$\varsigma^{\circ} 0 \angle 0^{\circ} I>$ & $0 \angle 0^{\circ} I>$ & $I \angle O^{6} I>$ & pınb!̣ & $\tau: \varepsilon 9 \mathrm{I}$ & 9SZSOOL $96 \mathrm{~S}$ \\
\hline mor & (1) & 10 & & & semor \\
\hline "atru & गitringrif & 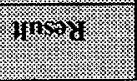 & agrod & 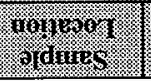 & $\begin{array}{l}\text { magrony } \\
\text { 2itures }\end{array}$ \\
\hline
\end{tabular}

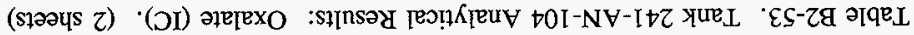


HNF-SD-WM-ER-690 Rev. 0

Table B2-54. Tank 241-AN-104 Analytical Results: Hexavalent Chromium $(\mathrm{Cr}+6)$.

\begin{tabular}{|c|c|c|c|c|c|}
\hline 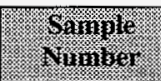 & $\begin{array}{l}\text { Sample } \\
\text { Thration }\end{array}$ & $\begin{array}{l}\text { Samine. } \\
\text { rorition. }\end{array}$ & Resill & 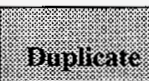 & Meri \\
\hline Wainds: & $\sqrt{-8.8}$ & : & ogrmis. & . $19 \%$ & 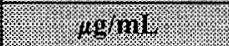 \\
\hline S96T005980 & Core 164 & Composite & 214 & 211 & 212.5 \\
\hline \multicolumn{2}{|c|}{ Gulids: vater tigetr. } & & 7867 & 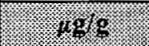 & , $8 \%$ \\
\hline S96T005977 & Core 164 & Composite & 203 & 197 & 200 \\
\hline
\end{tabular}

Table B2-55. Tank 241-AN-104 Analytical Results:

Total Inorganic Carbon/Total Organic Carbon.

\begin{tabular}{|c|c|c|c|c|c|}
\hline $\begin{array}{l}\text { Sample } \\
\text { Number }\end{array}$ & $\begin{array}{l}\text { Simplo } \\
\text { lorcation }\end{array}$ & Sarmile & Kesilil & Bunlicate & 118an \\
\hline se & (1. & Iriviri & 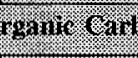 & & \\
\hline Solios & 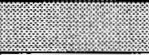 & 2 & 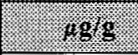 & $\sqrt{4858}$ & 1.80 \\
\hline S96T005972 & Core 164 & Composite & 15,000 & 14,300 & $14,650^{\mathrm{QC}: \mathrm{c}}$ \\
\hline Miguia: & & ? & $149 . \mathrm{ni}$ & fogran. & (1) whint \\
\hline S96T005978 & Core 164 & Composite & 2,280 & 2,050 & 2,165 \\
\hline & & 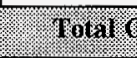 & gavir 9 aro & & \\
\hline Soliks & & & 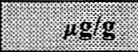 & . & 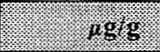 \\
\hline S96T005972 & Core 164 & Composite & 4,650 & 4,600 & 4,625 \\
\hline Miguas & : & & $1,8 . \mathrm{mi}$ & merni. & . $18 \mathrm{~min}$ \\
\hline S96T005978 & Core 164 & Composite & 4,040 & 2,770 & $3,405^{\mathrm{Qc}: \mathrm{c}}$ \\
\hline
\end{tabular}

Table B2-56. Tank 241-AN-104 Analytical Results: Hydroxide (OH Direct).

\begin{tabular}{|c|c|c|c|c|c|}
\hline Simple: & $\begin{array}{l}\text { Sample } \\
\text { Yoration }\end{array}$ & $\begin{array}{l}\text { Sarnote } \\
\text { Portion }\end{array}$ & (result & Buplicate & Meris \\
\hline Soldids: wire & Aigest: & & $1.1 .40 \%$ & 1.1048 & 18969 \\
\hline S96T005977 & Core 164 & Composite & 36,100 & 38,200 & 37,150 \\
\hline Miguids. & & & (1. $198 \mathrm{~nm}$ & 3.9411 & (1) \\
\hline S96T005981 & Core 164 & Composite & 71,400 & 70,400 & 70,900 \\
\hline
\end{tabular}


Table B2-57. Tank 241-AN-104 Analytical Results: Percent Water ${ }^{1}$ (Thermogravimetric Analysis). (2 sheets)

\begin{tabular}{|c|c|c|c|c|c|}
\hline 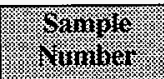 & 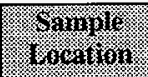 & 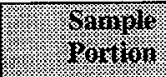 & 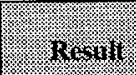 & 9u1ninge. & $4 \operatorname{sen}$ \\
\hline SIIU & & & \%. & $\%$ & 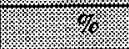 \\
\hline S96T005269 & $163: 1$ & Lower half & 42.53 & 41.69 & 42.11 \\
\hline S96T005270 & $163: 2$ & Lower half & 50.56 & 51.34 & 50.95 \\
\hline \$96T005771 & $163: 12$ & Lower half & 46.83 & 47.15 & 46.99 \\
\hline S96T005743 & \multirow[t]{2}{*}{$163: 14$} & Upper half & 44.54 & 46.36 & 45.45 \\
\hline S96T005744 & & Lower half & 44.4 & 43.19 & 43.80 \\
\hline S96T005271 & \multirow[t]{2}{*}{$163: 16$} & Upper half & 50.23 & 45.36 & 47.80 \\
\hline S96T005272 & & Lower half & 46.27 & 43.9 & 45.09 \\
\hline S96T005772 & \multirow[t]{2}{*}{$163: 18$} & Upper half & 40.95 & 41.57 & 41.26 \\
\hline S96T005773 & & Lower half & 39.90 & 40.85 & 40.38 \\
\hline S96T005548 & \multirow[t]{2}{*}{$163: 20$} & Upper half & 40.80 & 40.98 & 40.89 \\
\hline S96T005549 & & Lower half & 41.27 & 40.91 & 41.09 \\
\hline S96T004772 & $164: 1$ & Lower half & 41.31 & 43.6 & 42.46 \\
\hline$\$ 96 \mathrm{~T} 004773$ & $164: 13$ & Lower half & 47.20 & 45.64 & 46.42 \\
\hline S96T004984 & \multirow[t]{2}{*}{$164: 14$} & Upper half & 46.04 & 45.63 & 45.84 \\
\hline S96T004985 & & Lower half & $46 . \overline{72}$ & 46.60 & 46.66 \\
\hline S96T004986 & \multirow[t]{2}{*}{$164: 15$} & Upper half & 47.48 & 46.86 & 47.17 \\
\hline S96T004987 & & Lower half & 45.45 & 45.42 & 45.44 \\
\hline S96T004988 & \multirow[t]{2}{*}{$164: 16$} & Upper half & 45.09 & 47.25 & 46.17 \\
\hline S96T004989 & & Lower half & 47.18 & 49.33 & 48.26 \\
\hline \$96T004990 & \multirow[t]{2}{*}{$164: 17$} & Upper half & 49.51 & 49.85 & 49.68 \\
\hline \$96T004991 & & Lower half & 47.76 & 48.57 & 48.17 \\
\hline S96T004992 & \multirow[t]{2}{*}{$164: 19$} & Upper half & 46.16 & 45.93 & 46.05 \\
\hline S96T004993 & & Lower half & 46.08 & 44.00 & 45.04 \\
\hline S96T005249 & \multirow[t]{2}{*}{$164: 20$} & Upper half & 50.75 & 48.55 & 49.65 \\
\hline \$96T005250 & & Lower half & 50.51 & 50.05 & 50.28 \\
\hline S96T005972 & Core 164 & Composite & 45.25 & 36.54 & 40.89 \\
\hline
\end{tabular}


Table B2-57. Tank 241-AN-104 Analytical Results: Percent Water ${ }^{1}$ (Thermogravimetric Analysis). (2 sheets)

\begin{tabular}{|c|c|c|c|c|c|}
\hline $\begin{array}{l}\text { Samples: } \\
\text { Nunnor: }\end{array}$ & $\begin{array}{l}\text { Simpinile: } \\
\text { I }\end{array}$ & S Samplo & R. & 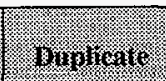 & Mernes \\
\hline Manos & 10 & . & 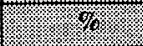 & . & 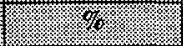 \\
\hline S96T005256 & $163: 2$ & Liquid & 52.1 & 51.87 & 51.99 \\
\hline S96T005260 & $163: 4$ & Liquid & 52.13 & 51.77 & 51.95 \\
\hline S96T005527 & $163: 5$ & Liquid & 52.09 & 52.01 & 52.05 \\
\hline S96T005554 & $163: 6$ & Liquid & 52.41 & 52.27 & 52.34 \\
\hline S96T005255 & $163: 7$ & Liquid & 51.47 & 51.75 & 51.61 \\
\hline S96T005257 & $163: 8$ & Liquid & 52.13 & 51.93 & 52.03 \\
\hline S96T005258 & $163: 9$ & Liquid & 51.92 & 52.35 & 52.14 \\
\hline S96T005528 & $163: 10$ & Liquid & 51.77 & 52.41 & 52.09 \\
\hline S96T005739 & $163: 11$ & Liquid & 50.94 & 50.26 & 50.60 \\
\hline S96T005766 & $163: 12$ & Liquid & 52.44 & 52.71 & 52.58 \\
\hline S96T005741 & $163: 14$ & Liquid & 51.77 & 51.18 & 51.48 \\
\hline S96T004774 & $1.64: 1$ & Liquid & 47.35 & 51.28 & 49.32 \\
\hline S96T004778 & $164: 2$ & Liquid & 52.25 & 49.11 & 50.68 \\
\hline S96T004779 & $164: 3$ & Liquid & 51.03 & 51.09 & 51.06 \\
\hline S96T004780 & $164: 4$ & Liquid & 51.65 & 51.61 & 51.63 \\
\hline S96T004976 & $164: 5$ & Liquid & 51.77 & 49.57 & 50.67 \\
\hline S96T004781 & $164: 7$ & Liquid & 51.82 & 52.23 & 52.03 \\
\hline S96T004977 & $164: 8$ & Liquid & 49.32 & 51.88 & 50.60 \\
\hline S96T004978 & $164: 9$ & Liquid & 52.03 & 51.03 & 51.53 \\
\hline S96T004979 & $164: 10$ & Liquid & 51.56 & 51.84 & 51.70 \\
\hline S96T004782 & $164: 11$ & Liquid & 51.73 & 51.69 & 51.71 \\
\hline $96 \mathrm{~T} 004783$ & $164: 12$ & Liquid & 51.37 & 50.87 & 51.12 \\
\hline S96T004784 & $164: 13$ & Liquid & 51.44 & 51.76 & 51.60 \\
\hline S96T005978 & Core 164 & Composite & 50.86 & 51.37 & 51.12 \\
\hline
\end{tabular}

Note:

Some waste samples were contaminated by hydrostatic head fluid, and the moisture content was corrected. 
HNF-SD-WM-ER-690 Rev. 0

Table B2-58. Tank 241-AN-104 Analytical Results: Exotherm - transition 1 (Differential Scanning Calorimetry). (2 sheets)

\begin{tabular}{|c|c|c|c|c|c|}
\hline $\begin{array}{l}\text { Srangle } \\
\text { Nimolort }\end{array}$ & 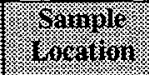 & $\begin{array}{l}\text { Singiot } \\
\text { Porion }\end{array}$ & 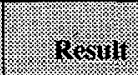 & Blopitivir. & I. 10 \\
\hline \multicolumn{3}{|c|}{ Marins } & 10 & 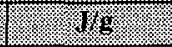 & 等 \\
\hline S96T005256 & $163: 2$ & Liquid & 0.0 & 0.0 & 0.0 \\
\hline S96T005260 & $163: 4$ & Liquid & 14.7 & 0.0 & $7.35^{\mathrm{QC:c}}$ \\
\hline S96T005527 & $163: 5$ & Liquid & 27.7 & 38.4 & $33.05^{\mathrm{QC}: \mathrm{c}}$ \\
\hline S96T005254 & $163: 6$ & Liquid & 0.0 & 0.0 & 0.0 \\
\hline S96T005255 & $163: 7$ & Liquid & 0.0 & 0.0 & 0.0 \\
\hline S96T005257 & $163: 8$ & Liquid & 0.0 & 0.0 & 0.0 \\
\hline S96T005258 & $163: 9$ & Liquid & 0.0 & 14.6 & $7.35^{\mathrm{QC:c}}$ \\
\hline S96T005528 & $163: 10$ & Liquid & 39.3 & 0.0 & $19.65^{Q C: c}$ \\
\hline S96T005739 & $163: 11$ & Liquid & 0.0 & 27.1 & $13.55^{\mathrm{QC}: \mathrm{e}}$ \\
\hline S96T005766 & $163: 12$ & Liquid & 0.0 & 0.0 & 0.0 \\
\hline S96T005741 & $163: 14$ & Liquid & 0.0 & 15.9 & $7.95^{\mathrm{QC:C}}$ \\
\hline S96T004774 & $164: 1$ & Liquid & 0.0 & 0.0 & 0.0 \\
\hline S96T004778 & $164: 2$ & Liquid & 0.0 & 0.0 & 0.0 \\
\hline S96T004779 & $164: 3$ & Liquid & 0.0 & 0.0 & 0.0 \\
\hline S96T004780 & $164: 4$ & Liquid & 0.0 & 15.7 & $7.85^{\mathrm{eC} e \mathrm{e}}$ \\
\hline S96T004976 & $164: 5$ & Liquid & 0.0 & 0.0 & 0.0 \\
\hline S96T004781 & $164: 7$ & Liquid & 0.0 & 0.0 & 0.0 \\
\hline S96T004977 & $164: 8$ & Liquid & 0.0 & 0.0 & 0.0 \\
\hline S96T004978 & $164: 9$ & Liquid & 0.0 & 0.0 & 0.0 \\
\hline S96T004979 & $164: 10$ & Liquid & 0.0 & 0.0 & 0.0 \\
\hline S96T004782 & $164: 11$ & Liquid & 0.0 & 0.0 & 0.0 \\
\hline S96T004783 & $164: 12$ & Liquid & 0.0 & 0.0 & 0.0 \\
\hline S96T004784 & $164: 13$ & Liquid & 0.0 & 0.0 & 0.0 \\
\hline S96T005978 & Core 164 & Composite & 18.5 & 19.4 & 18.95 \\
\hline
\end{tabular}


Table B2-58. Tank 241-AN-104 Analytical Results: Exotherm - transition 1 (Differential Scanning Calorimetry). (2 sheets)

\begin{tabular}{|c|c|c|c|c|c|}
\hline Swingle & Sarvile & Squide & Resulus & Buplicate & Tern \\
\hline Srotids: & 8 & $4=$ & 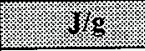 & $1 / \mathrm{g}$ & 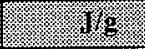 \\
\hline S96T005269 & 163:1 & Lower half & 0.0 & 0.0 & 0.0 \\
\hline S96T005270 & $163: 2$ & Lower half & 0.0 & 0.0 & 0.0 \\
\hline S96T005771 & $163: 12$ & Lower half & 31.6 & 25.9 & 28.75 \\
\hline S96T005743 & $163: 14$ & Upper half & 0.0 & 0.0 & 0.0 \\
\hline S96T005744 & & Lower half & 0.0 & 0.0 & 0.0 \\
\hline S96T005271 & $163: 16$ & Upper half & 106.6 & 32.8 & $69.7^{\mathrm{QC:c}}$ \\
\hline S96T005272 & & Lower half & 31.4 & 24.2 & 27.8 \\
\hline S96T005772 & $163: 18$ & Upper half & 23.6 & 19.5 & 21.55 \\
\hline S96T005773 & & Lower half & 22.1 & 23.6 & 22.85 \\
\hline S96T005548 & $163: 20$ & Upper half & 0.0 & 0.0 & 0.0 \\
\hline S96T005549 & & Lower half & 0.0 & 0.0 & 0.0 \\
\hline S96T004772 & $164: 1$ & Lower half & 0.0 & 0.0 & 0.0 \\
\hline S96T004773 & $164: 13$ & Lower half & 0.0 & 0.0 & 0.0 \\
\hline S96T004984 & $164: 14$ & Upper half & 0.0 & 0.0 & 0.0 \\
\hline S96T004985 & & Lower half & 0.0 & 0.0 & 0.0 \\
\hline S96T004986 & $164: 15$ & Upper half & 35.7 & 19.7 & $27.7^{\mathrm{QC:c}}$ \\
\hline S96T004987 & & Lower half & 25.9 & 33.6 & 29.75 \\
\hline S96T004988 & $164: 16$ & Upper half & 32.6 & 24.9 & 28.75 \\
\hline S96T004989 & & Lower half & 16.5 & 0.0 & $8.25^{\mathrm{QC}: c}$ \\
\hline S96T004990 & $164: 17$ & Upper half & 7 & 0.0 & $3.50^{\mathrm{QC}: \mathrm{c}}$ \\
\hline S96T004991 & & Lower half & 2.2 & 4.7 & $3.45^{\mathrm{QC}: e}$ \\
\hline S96T004992 & $164: 19$ & Upper half & 0.0 & 0.0 & 0.0 \\
\hline S96T004993 & & Lower half & 0.0 & 0.0 & 0.0 \\
\hline S96T005249 & $164: 20$ & Upper half & 0.0 & 0.0 & 0.0 \\
\hline S96T005250 & & Lower half & 0.0 & 0.0 & 0.0 \\
\hline S96T005972 & Core 164 & Composite & 101 & 105 & 103 \\
\hline
\end{tabular}




\begin{tabular}{|c|c|c|c|c|c|}
\hline$\angle S^{\circ} I$ & $\forall / N$ & $\angle S^{\circ} I$ & วl!soduros & 791 aloग & 0L6S00L96S \\
\hline $09 . \mathrm{I}$ & $\forall / N$ & $09^{\circ} \mathrm{I}$ & J[EY IOMOT] & \multirow[b]{2}{*}{$0 \tau:+91$} & $\varepsilon 22 \$ 00 \mathrm{~L} 96 \mathrm{~S}$ \\
\hline $09^{\circ} \mathrm{I}$ & $\mathrm{V} / \mathrm{N}$ & $09 \%$ & fleq $x \partial d d \Omega$ & & zzzS00L96S \\
\hline $8 S^{\circ} I$ & $\forall / N$ & $8 S^{\circ} I$ & IIEบ เәмот & \multirow[b]{2}{*}{$6 \mathrm{I}: 79 \mathrm{I}$} & DL6t00L96S \\
\hline $95^{\circ} \mathrm{I}$ & $\forall / N$ & $95^{\circ} \mathrm{I}$ & Jएप & & $\varepsilon L 6+00 L 96 \mathrm{~S}$ \\
\hline ş' I & $\forall / N$ & SSI & J[EY IәMOT & \multirow[b]{2}{*}{$\angle I: \pm 9 I$} & ZL6+00L96S \\
\hline ES I & $\nabla / N$ & $\mathcal{E S I}$ & $f I^{24}$ Iədd $\Omega$ & & IL6t00L $96 \mathrm{~S}$ \\
\hline $8 \mathrm{~S}^{\circ} \mathrm{I}$ & $\forall / N$ & $85^{\circ} \mathrm{I}$ & I[Rप $12 \mathrm{MOT}$ & \multirow[b]{2}{*}{$91: \$ 9 I$} & $696+00 \mathrm{~L} 96 \mathrm{~S}$ \\
\hline $99^{\circ} 1$ & $\forall / N$ & $9 S^{\circ} I$ & $f I^{e y}$ Iadd $_{\Omega}$ & & $0<6+00 \mathrm{~L} 96 \mathrm{~S}$ \\
\hline$\angle S^{*} \mathrm{I}$ & $\forall / N$ & $\angle S^{\circ} \mathrm{I}$ & Jleप Iәмот) & \multirow[b]{2}{*}{$S I: \nabla 9 I$} & $\angle 96 t 00 L 96 \mathrm{~S}$ \\
\hline SSII & $\mathrm{V} / \mathrm{N}$ & SS.I & $f[$ I Iәdd $\Omega$ & & $896700 \mathrm{~L} 96 \mathrm{~S}$ \\
\hline $2 S^{\circ} 1$ & $\forall / N$ & $25^{\circ} \mathrm{I}$ & J[Еप IวMOT] & \multirow[b]{2}{*}{$\nabla I: \nabla 9[$} & $996+00196 \mathrm{~S}$ \\
\hline $9 S^{\circ} \mathrm{I}$ & $\forall / N$ & 95.1 & f[R् Iədd $\cap$ & & \$96700L96S \\
\hline$E S^{\circ} I$ & $\nabla / N$ & $\varepsilon S^{\circ} \mathrm{I}$ & „lеप Іәмо & $\varepsilon r \div \neq 9 I$ & ILLt00L96S \\
\hline $79^{\circ} \mathrm{I}$ & $\forall / N$ & $+9 \cdot 1$ & I[एप गәмот & \multirow[b]{2}{*}{$0 z: \varepsilon 9 I$} & ESSS00L96S \\
\hline$\varepsilon 9^{\circ} \mathrm{I}$ & $\forall / N$ & 89.1 & \lrcorner$[\varepsilon \varphi$ เәdd $\Omega$ & & 2S\$\$00L96S \\
\hline$I L^{\circ} I$ & $\forall / N$ & {$[L \cdot I$} & I[Rप IәMOT & \multirow[b]{2}{*}{$8 \mathrm{I}: \varepsilon 9 \mathrm{I}$} & 0LLS00L96S \\
\hline $6 S^{\circ} \mathrm{I}$ & $\forall / N$ & $6 S^{\circ} 1$ & $\pm\left[e_{4}{ }_{2} \mathrm{dd}_{\Omega}\right.$ & & 69LS00L96S \\
\hline$\angle S I$ & $\forall / N$ & $\angle S^{\circ} I$ & IlEY IOMOT & \multirow[b]{2}{*}{$9 I: \mathcal{E I}$} & LZŻS00L96S \\
\hline $9 S^{\circ} \mathrm{I}$ & $\forall / N$ & $9 S^{\circ} I$ & 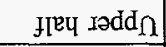 & & 922500L96S \\
\hline $19^{\circ} \mathrm{I}$ & $V / N$ & {$[9.1$} & Jןeप Iәмот & \multirow[b]{2}{*}{$\nabla I: \varepsilon 9 I$} & $6 Z \angle S 00 \mathrm{~L} 96 \mathrm{~S}$ \\
\hline$\$ 9^{\circ} \mathrm{L}$ & $\forall / N$ & S9. I & $\pm\left[{ }^{\mathbb{4}} \operatorname{Iədd}_{\Omega}\right.$ & & $8 Z L S 00 I 96 \mathrm{~S}$ \\
\hline $9 L^{\circ} \mathrm{I}$ & $V / N$ & $9 L^{\circ} \mathrm{I}$ & I IеY Iәмот & $Z I: \mathcal{E} 9 \mathrm{I}$ & 89LS00L96S \\
\hline 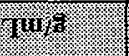 & I1968 & 19.6. & & (7) & (1) \\
\hline \%18s/gu & (1) & mus & Why & 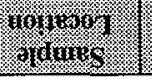 & 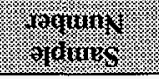 \\
\hline
\end{tabular}

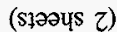

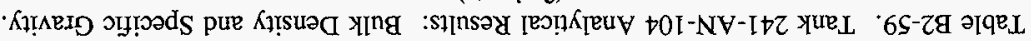




\begin{tabular}{|c|c|c|c|c|c|}
\hline $900^{\circ} \mathrm{I}$ & $10 t^{\circ} \mathrm{I}$ & IIt. I & ą!soduo & t9I 2 วroD & I86S00L96S \\
\hline $06 \varepsilon^{\circ} \mathrm{I}$ & $66 \varepsilon^{\circ} \mathrm{I}$ & $8 \varepsilon^{\prime} \mathrm{I}$ & p!nb!̣ & $\varepsilon r: \neq 9 \mathrm{I}$ & $+8 L t 00 \mathrm{~L} 96 \mathrm{~S}$ \\
\hline$I Z \nabla^{\circ} \mathrm{I}$ & $\tau \tau \nabla^{\circ} \mathrm{I}$ & $6 \mathrm{It}: \mathrm{I}$ & punb!r & $2 I:+91$ & $\varepsilon 8 \angle t 00 \mathrm{~L} 96 \mathrm{~S}$ \\
\hline tIt.I & $I Z+\cdot T$ & $90+1$ & p!nb!n & II:t9I & $28 \mathrm{~L} 000 \mathrm{~L} 96 \mathrm{~S}$ \\
\hline $8 \varepsilon^{\circ} \cdot$ & $\angle L E^{\circ} I$ & $\varepsilon 8 \varepsilon^{\circ} \mathrm{L}$ & pinb!l & $0 \mathrm{I}: t 9 \mathrm{I}$ & $6 L 6$ t00L $96 \mathrm{~S}$ \\
\hline ZIt*I & $60 t^{\circ} I$ & tIt十 I & pinb!̣ & $6:+91$ & $8 L 6+00 L 96 \mathrm{~S}$ \\
\hline $80 t^{\circ} \mathrm{I}$ & $800^{\circ} \mathrm{L}$ & $80 t^{\circ} \mathrm{L}$ & p!nb!̣ & $8: t 91$ & LL6+00L96S \\
\hline 210.1 & $2 I+1$ & $Z I+1$ & p!nb!̣] & $L: t 91$ & I8Lt00L $\mathrm{L} 96 \mathrm{~S}$ \\
\hline IIt I & $\varepsilon 0 t^{\circ} \mathrm{I}$ & $8 I t \cdot I$ & p!nb!̣ & $s: t 91$ & $9 L 6+00 \mathrm{~L}, 96 \mathrm{~S}$ \\
\hline ZIt.I & $9 \mathrm{It} \cdot \mathrm{I}$ & sitil & punb!̣ & $t: t 9 I$ & $08 L t 00 \mathrm{~L} 96 \mathrm{~S}$ \\
\hline $200^{\circ} \mathrm{I}$ & $96 \varepsilon^{\circ} \mathrm{I}$ & $\angle O D^{\circ} I$ & p!nbị & $\varepsilon: \neq 9 I$ & 6LLt00L96S \\
\hline $26 \varepsilon^{\circ} \mathrm{I}$ & $66 \varepsilon^{\circ} \mathrm{I}$ & $\nabla 8 \varepsilon^{\circ} \cdot$ & p!nb! I & $z: t 9 I$ & $8 L L+00 \mathrm{~L} 96 \mathrm{~S}$ \\
\hline $82 t \cdot I$ & $01 t^{\circ} I$ & $2 t \nabla^{\circ} 1$ & p!̣b!t & $I:+91$ & $\nabla L L+00 \mathrm{~L} 96 \mathrm{~S}$ \\
\hline $06 t^{\circ} \mathrm{I}$ & $6+1$ & $680^{\circ} \mathrm{I}$ & pụnbị & $\nabla I: \varepsilon 9 I$ & $\mathrm{I} \nabla \angle S 00 \mathrm{I} 96 \mathrm{~S}$ \\
\hline $9 S \nabla^{\circ} I$ & $\varepsilon 90^{\circ} \mathrm{I}$ & $6+5 \cdot 1$ & p!nb? & $Z \Upsilon: \varepsilon 9 \mathrm{I}$ & $99 \angle S 00 \mathrm{~L} 96 \mathrm{~S}$ \\
\hline $0+\varepsilon^{\circ} I$ & $6 E E^{\circ} \mathrm{I}$ & $\nabla \varepsilon \cdot 1$ & p!̣nb!̣ & $I I: \varepsilon 9 I$ & 6ELSO0L $96 S$ \\
\hline $\pm 6 \varepsilon^{\circ} \mathrm{I}$ & $9 z+1$ & $29 \varepsilon \cdot \mathrm{I}$ & p!nb!n & OI:£9l & 8ZSS00L96S \\
\hline $2 S E \cdot I$ & $\varsigma \mathcal{S} I$ & $t S E \cdot I$ & p̣nbṛ] & $6: \varepsilon 9 \mathrm{I}$ & $8 S 2500 \mathrm{~L} 96 \mathrm{~S}$ \\
\hline$\angle O D^{\circ} I$ & ZIt'I & $200 \cdot 1$ & p!nbit & $8: \varepsilon 91$ & LSzS00LI96S \\
\hline $\mathrm{II}+\mathrm{I}$ & $900^{\circ} \mathrm{I}$ & $9 \mathrm{It} \mathrm{I}$ & punbị & $L: \varepsilon 9 \mathrm{I}$ & SSZS00L96S \\
\hline$\nabla t \varepsilon \cdot I$ & $\varepsilon+\varepsilon^{\prime} I$ & $\nabla \nabla E I$ & p!nb!c & $9: \varepsilon 9 \mathrm{I}$ & tSSSS00L96S \\
\hline $99 \varepsilon^{\circ} \mathrm{I}$ & $\varepsilon L \varepsilon^{\prime} \mathrm{I}$ & $8 S E^{\prime} I$ & p!nb!r & $\varsigma: \varepsilon 9 \tau$ & LZSS00L96S \\
\hline$L L E^{\circ} I$ & $78 \mathcal{E}^{\circ} \mathrm{I}$ & $69 \varepsilon^{\circ} \mathrm{I}$ & p!nb!t & $t: \varepsilon 91$ & $092500 \mathrm{~L} 96 \mathrm{~S}$ \\
\hline$\angle 8 E^{\circ} I$ & $68 \varepsilon^{\circ} \mathrm{I}$ & $78 \mathcal{E}^{\circ} \mathrm{I}$ & p!̣nb!ฺ & $\mathrm{z} \varepsilon 9 \mathrm{I}$ & $9 S z \$ 00 \mathrm{~L} 96 \mathrm{~S}$ \\
\hline S52) 1818 & 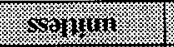 & 25, II) & 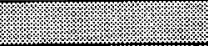 & 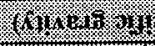 & (9arts spinting \\
\hline (1970\% & 404619 & 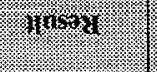 & 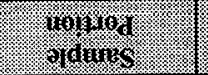 & 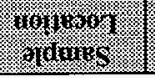 & 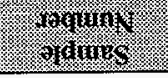 \\
\hline
\end{tabular}

(\$วəəus Z)

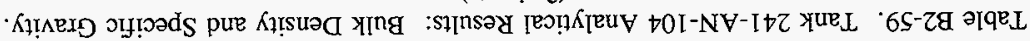


HNF-SD-WM-ER-690 Rev. 0

Table B2-60. Tank 241-AN-104 Analytical Results: Total Alpha (Alpha Radiation). (2 sheets)

\begin{tabular}{|c|c|c|c|c|c|}
\hline 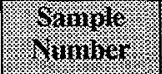 & 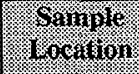 & (6) & foskin & 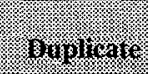 & (2) \\
\hline Monins & & & K & 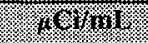 & (10 Ting \\
\hline S96T005256 & $163: 2$ & Liquid & $<0.0994$ & $<0.0822$ & $<0.0908$ \\
\hline$\$ 96 \mathrm{~T} 005260$ & $163: 4$ & Liquid & $<0.0483$ & $<0.0994$ & $<0.07385^{\mathrm{QC}: \mathrm{c}}$ \\
\hline \$967005527 & $163: 5$ & Liquid & $<0.00212$ & $<0.00226$ & $<0.00219$ \\
\hline S96T005554 & $163: 6$ & Liquid & $<0.00243$ & $<0.00212$ & $<0.002275$ \\
\hline S96T005255 & $163: 7$ & Liquid & $<0.0822$ & $<0.0649$ & $<0.07355^{\mathrm{QC}: \mathrm{e}}$ \\
\hline$\$ 96 \mathrm{~T} 005257$ & $163: 8$ & Liquid & $<0.0483$ & $<0.0483$ & $<0.0483$ \\
\hline S96T005258 & $163: 9$ & Liquid & $<0.0483$ & $<0.0483$ & $<0.0483$ \\
\hline S96T005528 & $163: 10$ & Liquid & 0.0025 & $<0.00243$ & $<0.002465$ \\
\hline S96T005739 & $163: 11$ & Liquid & $<0.00212$ & $<0.00243$ & $<0.002275$ \\
\hline S96T005766 & $163: 12$ & Liquid & 0.00362 & 0.00201 & $0.002815^{\mathrm{QC}}$ \\
\hline S96T005741 & $163: 14$ & Liquid & $<0.00243$ & $<0.00181$ & $<0.00212^{Q \mathrm{QC}: \mathrm{c}}$ \\
\hline S96T004774 & $164: 1$ & Liquid & $<0.0142$ & $<0.0142$ & $<0.0142$ \\
\hline S96T004778 & $164: 2$ & Liquid & $<0.0272$ & $<0.0142$ & $<0.0207^{\mathrm{QC}: e}$ \\
\hline S96T004779 & $164: 3$ & Liquid & $<0.0142$ & $<0.0142$ & $<0.0142$ \\
\hline S96T004780 & $164: 4$ & Liquid & $<0.0142$ & $<0.0142$ & $<0.0142$ \\
\hline S96T004976 & $164: 5$ & Liquid & $<0.0539$ & $<0.0348$ & $<0.04435^{\mathrm{QC}: \mathrm{e}}$ \\
\hline S96T004781 & $164: 7$ & $\longdiv { \text { Liquid } }$ & $<0.0645$ & $<0.0565$ & $<0.0605$ \\
\hline S96T004977 & $164: 8$ & Liquid & $<0.0189$ & $<0.0316$ & $<0.02525^{\mathrm{QC}: \mathrm{e}}$ \\
\hline S96T004978 & $164: 9$ & Liquid & $<0.0189$ & $<0.0189$ & $<0.0189$ \\
\hline S96T004979 & $164: 10$ & Liquid & $<0.0253$ & $<0.0943$ & $<0.0598^{\mathrm{QC}: \mathrm{c}}$ \\
\hline S96T004782 & $164: 11$ & Liquid & $<0.0147$ & $<0.0179$ & $<0.0163$ \\
\hline S96T004783 & $164: 12$ & Liquid & $<0.0147$ & $<0.021$ & $<0.01785^{\overline{Q C}: \mathrm{e}}$ \\
\hline S96T004784 & $164: 13$ & Liquid & $<0.0273$ & $<0.0242$ & $<0.02575$ \\
\hline S96T005978 & \multirow[t]{2}{*}{ Core 164} & Composite & 0.0276 & 0.0219 & $0.02475^{\mathrm{Qc:c}, f}$ \\
\hline S96T005979 & & Composite & $<0.0257$ & 0.0259 & $<0.0258$ \\
\hline
\end{tabular}




\begin{tabular}{|c|c|c|c|c|c|}
\hline $200056620^{\circ} 0>$ & $9610^{\circ} 0>$ & $\mathcal{E} 6 \varepsilon 0^{\circ} 0>$ & 27!soduros & 791 әлор & SL6S00L96S \\
\hline 1.0089500 & $89 \$ 0^{\circ} 0$ & $8550^{\circ} 0$ & I[एч гәмот & $0 Z:+9 I$ & $8 L Z S 00196 S$ \\
\hline$: 0, S 0 t S 0^{\circ} 0$ & $I \angle 90^{\circ} 0$ & $I+0^{\circ} 0$ & Ғ[ЕЧ Јәмот & $6 I:+9 I$ & $\downarrow \mathcal{E} 0 \mathrm{~S} 00 \mathrm{~L} 96 \mathrm{~S}$ \\
\hline จ.00 $\$ 08 \varepsilon 0^{\circ} 0$ & $\tau t 50^{\circ} 0$ & $61200^{\circ}$ & J[ЕY دәMOT] & $\angle I: \nabla 9 I$ & IEOS00.L96S \\
\hline$: 008 \varepsilon \varepsilon 0^{\circ} 0>$ & $\angle S Z O^{\circ} 0$ & $6 ! t 0^{\circ} 0>$ & ग[Еप IәмоT & $91:+9 I$ & EEOSO0L96S \\
\hline$s \nabla t t 0^{\circ} 0$ & $\angle S t 0^{\circ} 0$ & $\tau \varepsilon+0^{\circ} 0$ & J[Еบ ऽәмоТ & $S I: \neq 9 I$ & zE0S00L96S \\
\hline $\mathcal{E E E 0 ^ { \circ }} \mathrm{O}^{\circ}$ & $I S E 0^{\circ} 0>$ & SIE $0^{\circ} 0>$ & IIEU IอMOT) & $+I:+9 I$ & OE0S00L96S \\
\hline$\angle L+0^{\circ} O>$ & $\$ s+0^{\circ} 0>$ & $66+0^{\circ} 0$ & У[ЕЧ मәMOІ & $\varepsilon I: \neq 9 \mathrm{I}$ & $96 \angle t 00 \mathrm{~L} 96 \mathrm{~S}$ \\
\hline SEZt $0^{\circ} 0>$ & $2 \gg 0^{\circ} 0>$ & $\angle 2 \neq 0^{\circ} O>$ & J[Еप Іәмо I & $I: t 9 I$ & S6Lt00L $96 \mathrm{~S}$ \\
\hline 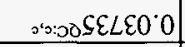 & $6+20^{\circ} 0$ & $86 t 0^{\circ} 0$ & I[EY IOMOT & $0 Z: \varepsilon 9 I$ & 6SSS00L96S \\
\hline $0: 00 \$ 8+20^{\circ} 0>$ & $\$ I 20^{\circ} 0$ & $2820^{\circ} 0>$ & J[Eप JPMOI & $8 \mathrm{I}: \varepsilon 9 \mathrm{I}$ & I8LS00L96S \\
\hline$x^{2}: 20 \$ 29700$ & 66200 & 92200 & 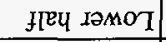 & $9 I: \mathcal{I T I}$ & 982500L96S \\
\hline$: 006 \mathrm{I} \varepsilon 0>$ & $887^{\circ} 0>$ & $\subseteq \mathcal{E} \cdot 0>$ & I[एप ऽәмот & $\nabla I: \varepsilon 9 \mathrm{I}$ & $8 \nabla L S 00 L 96 \mathrm{~S}$ \\
\hline OR:oo $S 0 Z 0>$ & $z \varepsilon z^{\prime} 0>$ & $8 \angle I^{\circ} 0>$ & I[Еप Iәмот & $Z I: \varepsilon 9 I$ & 08LS00L96S \\
\hline $0: 00<700$ & $\$ 9 \varepsilon 0^{\circ} 0$ & $\varepsilon 670^{\circ} 0$ & IIE4 IәMOT & $z: \varepsilon 9 \mathrm{I}$ & \$8ZS00.L96S \\
\hline 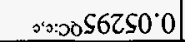 & ISZ0 0 & $8080^{\circ} 0$ & I[Еч IəмОТ] & I:\&9I & t8zs00L96S \\
\hline \% & $6 \%$ & 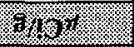 & & 前 & $3 \times 13$ \% \\
\hline then & (4) & Hoxss. & 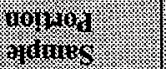 & \%oroms & ogrong \\
\hline
\end{tabular}

(słoəus z)

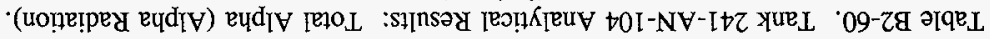


Table B2-61. Tank 241-AN-104 Analytical Results: Total Beta (Alpha).

\begin{tabular}{|c|c|c|c|c|c|}
\hline 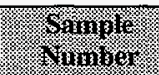 & 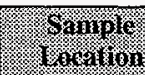 & 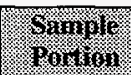 & Mosilt & SHOl a to & 40 \\
\hline \multicolumn{3}{|c|}{ 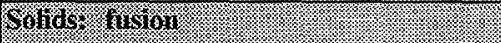 } & (c) & 16 14 & (3) \\
\hline S96T005975 & Core 164 & Composite & 415 & 385 & 400 \\
\hline YOMing & & 要 & 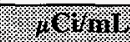 & 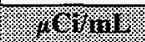 & K. W \\
\hline S96T005979 & Core 164 & Composite & 482 & 509 & 495.5 \\
\hline
\end{tabular}

Table B2-62. Tank 241-AN-104 Analytical Results: Strontium-89/90.

\begin{tabular}{|c|c|c|c|c|c|}
\hline 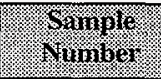 & \%ocalion & Tomirien & Resin) & Bimpingte & Merin. \\
\hline 6rofor. & & & 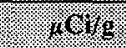 & 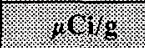 & 16ris \\
\hline \$96T005975 & Core 164 & Composite & 33.8 & 34.7 & 34.25 \\
\hline Yornas. & & & 10.111 & 1\% & \% \\
\hline S96T005980 & Core 164 & Composite & 0.334 & 0.342 & $0.338^{\mathrm{QC:F}}$ \\
\hline
\end{tabular}

Table B2-63. Tank 241-AN-104 Analytical Results: Americium-241 (Gamma Energy Analysis).

\begin{tabular}{|c|c|c|c|c|c|}
\hline STample & 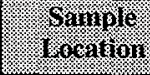 & 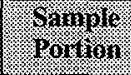 & Kesull & 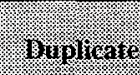 & 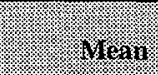 \\
\hline SH110\% - ysio & & & (x) & (x) & (x) \\
\hline S96T005975 & Core 164 & Composite & $<1.413$ & $<1.35$ & $<1.3815$ \\
\hline 164us & & & 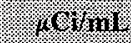 & Hothin & 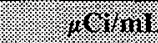 \\
\hline S96T005979 & Core 164 & Composite & $<1.364$ & $<1.35$ & $<1.357$ \\
\hline
\end{tabular}


Table B2-64. Tank 241-AN-104 Analytical Results: Cesium-137

(Gamma Energy Analysis).

\begin{tabular}{|c|c|c|c|c|c|}
\hline 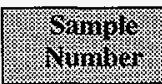 & 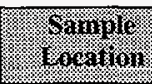 & Gamplo & 2pesuly & 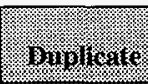 & yor \\
\hline \multicolumn{3}{|c|}{ Woings: } & reas & F) & $10 \%$ \\
\hline S96T005975 & Core 164 & Composite & 357.4 & 341 & 349.2 \\
\hline \multicolumn{2}{|l|}{ 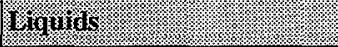 } & & \% & 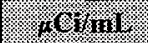 & 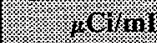 \\
\hline S96T005979 & Core 164 & Composite & 572 & 568 & 570 \\
\hline
\end{tabular}

Table B2-65. Tank 241-AN-104 Analytical Results: Cobalt-60 (Gamma Energy Analysis).

\begin{tabular}{|c|c|c|c|c|c|}
\hline $\begin{array}{l}\text { Saraple } \\
\text { Haniog }\end{array}$ & $\begin{array}{l}\text { Somplo } \\
\text { Hocatrol }\end{array}$ & Sogmin & 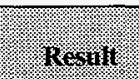 & ouplinate & Menation \\
\hline 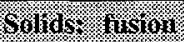 & & & 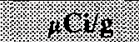 & $10 \%$ & 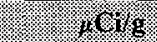 \\
\hline S96T005975 & Core 164 & Composite & $<0.02636$ & $<0.026$ & $<0.02618$ \\
\hline Houmus. & & & 16.1111 & 16\% & \% \\
\hline S96T005979 & Core 164 & Composite & $<0.01674$ & $<0.0159$ & $<0.01632$ \\
\hline
\end{tabular}

Table B2-66. Tank 241-AN-104 Analytical Results: Europium-154 (Gamma Energy Analysis).

\begin{tabular}{|c|c|c|c|c|c|}
\hline thormor & 164mole & govinger & Resulu & Binglours & Misan. \\
\hline Solids, gitsin & & 3 & (x) & 10.19 & 40 \\
\hline S96T005975 & Core 164 & Composite & $<0.09029$ & $<0.1$ & $<0.095145$ \\
\hline (xiglings & & & H Wing & 64 1111. & mo \\
\hline S96T005979 & Core 164 & Composite & $<0.1282$ & $<0.123$ & $<0.1256$ \\
\hline
\end{tabular}


Table B2-67. Tank 241-AN-104 Analytical Results: Europium-155 (Gamma Energy Analysis).

\begin{tabular}{|c|c|c|c|c|c|}
\hline Sinule & Samilo & 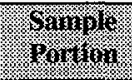 & Resin & Builere. & Ming \\
\hline Solits inston & : & r. & 1.978 & 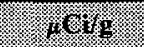 & 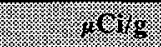 \\
\hline S96T005975 & Core 164 & Composite & $<0.6724$ & $<0.655$ & $<0.6637$ \\
\hline Marins: & & & Hocrint & fretivilu & 4. \\
\hline S96T005979 & Core 164 & Composite & $<0.6573$ & $<0.654$ & $<0.65565$ \\
\hline
\end{tabular}

Table B2-68. Tank 241-AN-104 Analytical Results: Total Uranium.

\begin{tabular}{|c|c|c|c|c|c|}
\hline $\begin{array}{l}\text { Saminle: } \\
\text { Nurnber. }\end{array}$ & $\begin{array}{l}\text { Sravile } \\
\text { I ecalion }\end{array}$ & $\begin{array}{l}\text { Sample } \\
\text { Porion }\end{array}$ & Resint & Bripluare & Wien \\
\hline Solids' Wusio & & & $1.98 \mathrm{~g}$ & 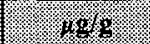 & 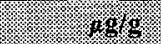 \\
\hline S96T005975 & Core 164 & Composite & 174 & 187 & 180.5 \\
\hline Mirivids & & & gromin & $180 \% 101$ & . 1.96 .111 \\
\hline S96T005981 & Core 164 & Composite & 3.3 & 4.06 & $3.68^{\mathrm{CC}: \mathrm{e}, \mathrm{f}}$ \\
\hline
\end{tabular}

Table B2-69. Tank 241-AN-104 Analytical Results: Plutonium-239/40.

\begin{tabular}{|c|c|c|c|c|c|}
\hline $\begin{array}{l}\text { Sample } \\
\text { Vimber }\end{array}$ & Somple & $\begin{array}{l}\text { Shanple } \\
\text { I orfion }\end{array}$ & Kresinll & Muptiante & Merain \\
\hline Sollos & & $1: 0$ & ncts. & $19(989$. & $1.1 \% 8$ \\
\hline S96T005975 & Core 164 & Composite & 0.00359 & 0.00371 & 0.00365 \\
\hline Migaina & & ? & ytwi & Hins: & $1101 \mathrm{nin}$ \\
\hline S96T005980 & Core 164 & Composite & $<1.740 \mathrm{E}-04$ & $<1.760 \mathrm{E}-04$ & $<1.750 \mathrm{E}-04$ \\
\hline
\end{tabular}


Table B2-70. Tank 241-AN-104 Analytical Results: Iodine-129.

\begin{tabular}{|c|c|c|c|c|c|}
\hline $\begin{array}{l}\text { Sample } \\
\text { Number }\end{array}$ & $\begin{array}{l}\text { Sample } \\
\text { Location }\end{array}$ & $\begin{array}{l}\text { Sample } \\
\text { Portion }\end{array}$ & Result & Duplicate & Mean \\
\hline \multicolumn{3}{|l|}{ Eiquids } & $\mu \mathrm{Ci} / \mathrm{mL}$ & $\mu \mathrm{Ci} / \mathrm{mL}$ & $\mu \subset \mathrm{i} / \mathrm{mL}$ \\
\hline S97T000022 & Core 164 & Composite & $1.930 \mathrm{E}-04$ & $2.220 \mathrm{E}-04$ & $2.075 \mathrm{E}-04$ \\
\hline \multicolumn{3}{|c|}{ Solids: water digest } & $\mu \mathrm{Ci} / \mathrm{g}$ & $\mu \mathrm{Cl} / \mathrm{g}$ & $\mu \mathrm{Cl} \mathrm{s}$ \\
\hline S96T005977 & Core 164 & Composite & $<0.00277$ & $<0.00276$ & $<0.002765$ \\
\hline
\end{tabular}

Table B2-71. Tank 241-AN-104 Analytical Results: Tritium (Liquid Scintillation).

\begin{tabular}{|c|c|c|c|c|c|}
\hline $\begin{array}{l}\text { Sample } \\
\text { Vumber }\end{array}$ & $\begin{array}{c}\text { Samplo } \\
\text { iocrition }\end{array}$ & $\begin{array}{l}\text { Simingle } \\
\text { Initira }\end{array}$ & Mosinge & injiticato & Geri: \\
\hline \multicolumn{2}{|c|}{ Solias? yorer digest } & & $180 \%$ & 1.109 & 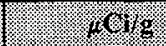 \\
\hline S96T005977 & Core 164 & Composite & 0.00971 & 0.0105 & $0.010105^{\text {QC: }}$ \\
\hline Miginas & & S: & 14010 & Hown? & 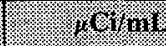 \\
\hline S97T000389 & Core 164 & Composite & 0.00159 & 0.00143 & 0.00151 \\
\hline
\end{tabular}

Table B2-72. Tank 241-AN-104 Analytical Results: Neptunium-237.

\begin{tabular}{|c|c|c|c|c|c|}
\hline $\begin{array}{l}\text { Sample } \\
\text { Numilion }\end{array}$ & $\begin{array}{l}\text { Shrove. } \\
\text { Loention }\end{array}$ & $\begin{array}{l}\text { Sample } \\
\text { Portion }\end{array}$ & nesul: & Dinilinte & Mern \\
\hline Solids: Iisio & F & & 196/2 & 1818 & 1864 \\
\hline S96T005975 & Core 164 & Composite & $<0.012$ & $<0.0112$ & $<0.0116^{\text {QC:a }}$ \\
\hline Thoundis & & & 1. & 16) WIIT & $191 \mathrm{~mL}$ \\
\hline S96T005980 & Core 164 & Composite & $<2.260 \mathrm{E}-05$ & $<3.680 \mathrm{E}-05$ & $<2.970 \mathrm{E}-05^{\mathrm{QC}}$ \\
\hline
\end{tabular}

Table B2-73. Tank 241-AN-104 Analytical Results: Technetium-99.

\begin{tabular}{|c|c|c|c|c|c|}
\hline $\begin{array}{l}\text { Sample } \\
\text { Nunber }\end{array}$ & Sample & $\begin{array}{l}\text { Sample } \\
\text { Torriteor }\end{array}$ & Resuil & Muplinate & Virean \\
\hline Sprink \% risrion & : & & 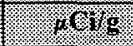 & 1.109 & 18018 \\
\hline S96T005975 & Core 164 & Composite & 0.141 & 0.128 & 0.1345 \\
\hline Uiguras: & i & & 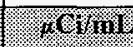 & 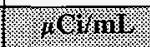 & \% (1) \\
\hline S97T000022 & Core 164 & Composite & $2.220 \mathrm{E}-04$ & $2.170 \mathrm{E}-04$ & $2.195 \mathrm{E}-04$ \\
\hline
\end{tabular}


HNF-SD-WM-ER-690 Rev. 0

Table B2-74. Tank 241-AN-104 Analytical Results: Americium-241 (Alpha Energy Analysis).

\begin{tabular}{|c|c|c|c|c|c|}
\hline 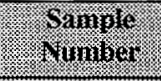 & 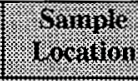 & 8 & 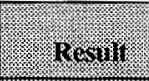 & 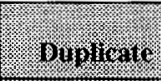 & $\%$ \\
\hline 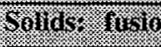 & & & \% & 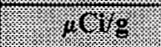 & \\
\hline $96 \mathrm{~T} 005975$ & Core 164 & Composite & 0.0188 & 0.0196 & 0.0192 \\
\hline Mrinas & & & HO & 136) & 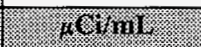 \\
\hline $6 \mathrm{~T} 005980$ & Core 164 & Composite & $<3.510 \mathrm{E}-04$ & $<3.430 \mathrm{E}-04$ & $<3.4$ \\
\hline
\end{tabular}

Table B2-75. Tank 241-AN-104 Analytical Results: Cm-243/244 (Alpha Energy Analysis).

\begin{tabular}{|c|c|c|c|c|c|}
\hline $\begin{array}{l}\text { Sinule } \\
\text { Juminer }\end{array}$ & Squpple & $\begin{array}{l}\text { Simples: } \\
\text { rodion }\end{array}$ & Resint & Buplicate & 118.11. \\
\hline Sollas , Jush & & : & (1) / & 16\% & $2.16 \mathrm{~g}$ \\
\hline S96T005975 & Core 164 & Composite & $<0.00399$ & $<0.00377$ & $<0.00388$ \\
\hline 1quinu & : & & Whour & (orno & 1. ENIII \\
\hline S96T005980 & Core 164 & Composite & $<3.510 \mathrm{E}-04$ & $<3.430 \mathrm{E}-04$ & $<3.470 \mathrm{E}-04$ \\
\hline
\end{tabular}




\section{B3.0 ASSESSMENT OF CHARACTERIZATION RESULTS}

This section discusses the overall quality and consistency of the current sampling results for tank 241-AN-104, and it provides the calculation results of an analytical-based inventory. This section also evaluates sampling and analysis factors that may impact data interpretation. These factors are used to assess overall data quality and consistency and to identify limitations in data use.

\section{B3.1 FIELD OBSERVATIONS}

The safety screening DQO (Dukelow et al. 1995) requires all vertical waste profiles be obtained from two widely spaced risers. The flammable gas DQO (McDuffie and Johnson 1995) requires at least one complete core be obtained. All expected segments were collected from core 163; segments 6 and 21 were not recovered from core 164 . The waste recovered in segments with less than full recovery was assumed to represent the whole segment. Hydrostatic head fluid was used in the sampling. The analytical results of the trace elements $\mathrm{Br}$ and $\mathrm{Li}$ indicate few segments were contaminated by $\mathrm{HHF}$.

\section{B3.1.1 Evaluation of Hydrostatic Head Fluid Contamination}

In tank 241-AN-104, the trace elements $\mathrm{Li}$ and $\mathrm{Br}$ in $\mathrm{HHF}$ were detected in solid and liquid samples, thus providing evidence of HHF extrusion in the waste samples. Tables B2-23 and B2-44 show the analytical results of $\mathrm{Li}$ and $\mathrm{Br}$. Based on the data, a corrected water content in the contaminated samples was calculated. For the core sampling event, correction of water intrusion (such as $\mathrm{HHF}$ ) based on $\mathrm{Li}$ and $\mathrm{Br}$ tracers was addressed and formulated by Winkleman (1996b). Detailed contaminated water content evaluations are given below.

According to the sample chain-of-custody data, the HHF used in sampling contains $0.3 M$ of a $\mathrm{Li}$ and $\mathrm{Br}$ solution. The analytical results of $\mathrm{Li}$ and $\mathrm{Br}$ in the sample of the HHF field blank are $1,960 \mu \mathrm{g} / \mathrm{mL}$ for $\mathrm{Li}$ and $22,000 \mu \mathrm{g} / \mathrm{mL}$ for $\mathrm{Br}$. To convert the concentration from $\mu \mathrm{g} / \mathrm{g}$ to molarity, use approximately $0.282 \mathrm{M}$ and $0.275 \mathrm{M}$ for $\mathrm{Li}$ and $\mathrm{Br}$, respectively. These are close to the $\mathrm{Li}$ and $\mathrm{Br}$ concentration $0.3 \mathrm{M}$ in the $\mathrm{HHF}$ solution prepared for field use. The data are used as a baseline for the evaluation of water contamination in the sample.

According to Bechtold (1995), lithium ions were easily formed into soluble compounds with other constituents and precipitate. Therefore, the concentrations was not a good indicator to use in calculating the amount of $\mathrm{HHF}$ in waste samples. Correction of the water content was based only on bromide data. Table B3-I lists the analytical results of Br above the detection limit. The highest value of $\mathrm{Br}$ is $1,748 \mu \mathrm{g} / \mathrm{mL}$ for the liquid sample from segment 3 , core 164 , and $1,163 \mu \mathrm{g} / \mathrm{g}$ for the solid sample from segment 19 , upper half of core 164 . Based on these results, the percent of HHF in water and corrected water content in the waste samples are calculated according to Winkelman (1996b) and listed in Table B3-1. The 
Table B3-1. Weight Percent Water Correction Based on Bromide Results for Tank 241-AN-104.

\begin{tabular}{|c|c|c|c|c|c|c|}
\hline \multirow{2}{*}{ 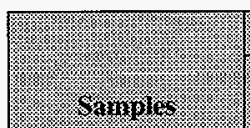 } & \multicolumn{3}{|c|}{ Br. 610 r I } & \multicolumn{3}{|c|}{ X } \\
\hline & $\begin{array}{l}\text { Sarmule } \\
\text { Revilus }\end{array}$ & 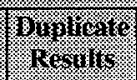 & Mran: & 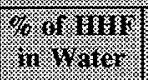 & 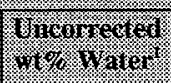 & 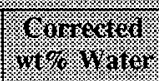 \\
\hline Solids: & \multicolumn{3}{|c|}{$\mu \mathrm{g} / \mathrm{g}$} & & & \\
\hline 163:12 Lower half & 1,038 & 1,020 & 1,029 & 9.87 & $46.99(0.16)$ & 44.6 \\
\hline 163:18 Upper half & 667.9 & 635 & 651.5 & 7.12 & $41.26(0.31)$ & 39.51 \\
\hline 164:14 Upper half & 1,158 & 1,160 & 1,159 & 11.66 & $45.84(0.79)$ & 41.85 \\
\hline 164:14 Lower half & 389.6 & 513 & 451.3 & 4.36 & $46.66(0.06)$ & 45.57 \\
\hline 164:19 Upper half & 1,165 & 1,160 & $1,162.5$ & 11.40 & $46.05(0.06)$ & 43.06 \\
\hline 164:Composite & 1,145 & $n / a$ & 1,145 & 13.0 & $39.81(1.09)$ & 36.58 \\
\hline Liquids & \multicolumn{3}{|c|}{$\mu \mathrm{g} / \mathrm{mL}$} & & & \\
\hline $163: 4$ & 916.9 & 950 & 933.45 & 5.89 & $51.95(0.18)$ & 50.57 \\
\hline $163: 5$ & 815.8 & 893 & 854.4 & 5.42 & $52.05(0.04)$ & 50.69 \\
\hline $163: 6$ & 736.3 & 748 & 742.15 & 4.76 & $52.34(0.07)$ & 51.15 \\
\hline $163: 7$ & 654.9 & 650 & 652.45 & 4.04 & $51.61(0.14)$ & 50.61 \\
\hline $163: 8$ & 731.3 & 749 & 740.15 & 4.56 & $52.03(0.10)$ & 50.89 \\
\hline $163: 9$ & 1,104 & 1,050 & 1,077 & 6.89 & $52.14(0.21)$ & 50.40 \\
\hline $163: 10$ & 1,107 & 1,080 & $1,093.5$ & 6.79 & $52.09(0.32)$ & 50.38 \\
\hline $163: 12$ & 1,128 & 1,150 & 1,139 & 6.71 & $52.58(0.14)$ & 50.89 \\
\hline $163: 14$ & 757.2 & 759 & 758.1 & 4.43 & $51.48(0.30)$ & 50.72 \\
\hline $164: 2$ & 1,167 & 1,120 & $1,143.5$ & 7.31 & $50.68(1.57)$ & 48.83 \\
\hline $164: 3$ & 1,756 & 1,740 & 1,748 & 11.02 & $51.02(0.02)$ & 48.16 \\
\hline $164: 4$ & 1,738 & 1,740 & 1,739 & 10.74 & $51.63(0.02)$ & 48.86 \\
\hline $164: 9$ & 848.7 & 844 & 846.35 & 5.25 & $51.53(0.50)$ & 50.22 \\
\hline $164: 10$ & 825.2 & 810 & 817.6 & 5.17 & $51.70(0.14)$ & 50.41 \\
\hline $164: 11$ & 1,707 & 1,750 & $1,728.5$ & 10.66 & $51.71(0.02)$ & 48.96 \\
\hline $164: 13$ & 902.5 & 887 & 894.75 & 5.63 & $51.60(0.16)$ & 50.19 \\
\hline 164:Composite & 1,520 & 1,580 & 1,550 & 9.70 & $51.25(0.12)$ & 48.76 \\
\hline
\end{tabular}

Note:

'Uncorrected $w t \%$ water is from Table B2-57. The values in parentheses are the standard deviation of wt\% water. 
results show the highest contaminations are 11.66 percent for solid samples from segment 14 , upper half of core 164 , and 11.02 percent for liquid samples from segment 3 , core 163 . The solid core composite sample has 13 percent HHF in water, and the calculation based on $\mathrm{Br}$ data was $1,145 \mathrm{ug} / \mathrm{g}$. The duplicate data is below a detection limit of $976 \mathrm{ug} / \mathrm{g}$. The corrected water content is reduced ranging from $3.9 \mathrm{wt} \%$ (solid sample from segment 14, upper half of core 164) to $0.76 \mathrm{wt} \%$ water (liquid sample from segment 14 , core 163 comparing the uncorrected value).

By incorporating the water content corrections, tank mean TGA values were recalculated as 50.4 percent for liquids and 45.31 percent for solids. The DSC and TOC values in dry weight basis were corrected accordingly. No corrected value exceeds the threshold values of the safety screening $\mathrm{DQO}$ requirements.

\section{B3.2 QUALITY CONTROL ASSESSMENT}

The usual QC assessment includes an evaluation of the appropriate standard recoveries, spike recoveries, duplicate analyses, and blanks that are performed in conjunction with the chemical analyses. All pertinent QC tests were conducted on 1996 core samples, enabling a full assessment regarding data accuracy and precision. Winkelman (1996a) established specific criteria for all analytes. Sample and duplicate pairs with one or more QC results outside the specified criteria are identified by footnotes in the data summary tables.

The standard and spike recovery results provide an estimate of analysis accuracy. If a standard or spike recovery is above or below the given criterion, the analytical results may be biased high or low, respectively. The precision is estimated by the RPD, which is defined as the absolute value of the difference between the primary and duplicate samples, divided by their mean, times 100 .

A high RPD was reported for two of 40 subsamples submitted for total alpha activity. This may have been caused by subsequent self-shielding. Reruns were deemed unnecessary because sample results were far below the action limit. Some high RPDs for IC analytes may be attributable to sample homogeneity problems. The high RPD and poor spike recovery for fluoride is because the fluoride peak is near the baseline, and it experiences interference from the slightly larger chloride peak.

Many ICP analytes also had one or more QC parameters outside specified limits. Poor spike recoveries for sodium may be caused by the high concentration of sodium in the samples. (Samples cannot be spiked to levels much greater than at present.) High concentrations of sodium required high dilutions for all ICP samples. These high dilutions can cause poor or meaningless spike recoveries and RPDs for ICP elements with very high concentrations or for those close to the detection limit. Finally, no sample exceeded the criterion for preparation blanks; therefore, contamination was not a problem. 
In summary, the vast majority of QC results were within the boundaries specified in sampling and analysis plans. The discrepancies mentioned here and footnoted in the data summary tables should not impact data validity or use.

\section{B3.3 DATA CONSISTENCY CHECKS}

Comparing different analytical methods helps in accessing data consistency and quality. Several comparisons were possible with the data set provided by the two core samples, including a comparison of phosphorous and sulfur as analyzed by ICP with phosphate and sulfate as analyzed by IC. Weight percent water data was checked using $\mathrm{Li}$ and $\mathrm{Br}$ data as described in Section B3.1.1. In addition, mass and charge balances were calculated to help assess the overall data consistency.

\section{B3.3.1 Comparison of Results from Different Analytical Methods}

The following data consistency checks compare the results from two analytical methods. A close comparison between the two methods strengthens the credibility of both results, but a poor comparison brings the reliability of the data into question.

The analytical phosphorous mean result as determined by ICP was $808 \mu \mathrm{g} / \mathrm{g}$, which converts to $2,478 \mu \mathrm{g} / \mathrm{g}$ of phosphate. This compared well with the IC phosphate mean result of $2,118 \mu \mathrm{g} / \mathrm{g}$ (see Table B3-2). The RPD between these two phosphate results was 16 percent. The ICP sulfur value of $1,955 \mu \mathrm{g} / \mathrm{g}$, which represents total sulfur, is equivalent to $5,864 \mu \mathrm{g} / \mathrm{g}$ of sulfate. The IC result for sulfate was $4,866 \mu \mathrm{g} / \mathrm{g}$ (see Table B3-2) with an RPD between the two values of 19 percent. This comparison shows that the results from ICP and IC methods agree reasonably well. Usually the calculated sulfur/phosphate values from ICP are equal or larger than the measured sulfur/phosphate from IC because the IC only measured the soluble sulfur/phosphate. 
Tabie B3-2. Comparison of Phosphate/Phosphorous and Sulfate/Sulfur Results.

\begin{tabular}{|c|c|c|c|c|}
\hline \multirow{2}{*}{ 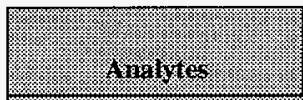 } & \multicolumn{2}{|c|}{ 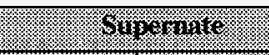 } & \multirow{2}{*}{$\sqrt{\text { Sartivinu? }}$} & \multirow{2}{*}{ Weghrod wern } \\
\hline & $48 \mathrm{~mol}$ & 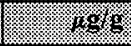 & & \\
\hline \multicolumn{5}{|c|}{ 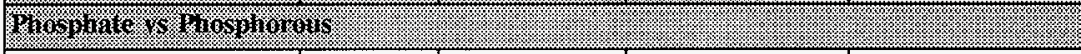 } \\
\hline P (by ICP) & 629 & 449 & 1,370 & 1,219 \\
\hline $\mathrm{PO}_{4}$ (by IC) & 1,621 & 1,158 & 3,620 & 2,118 \\
\hline $\mathrm{PO}_{4}$ (calculated from $\mathrm{P}$ ) & & 1,377 & 4,198 & 2,478 \\
\hline $\mathrm{PO}_{4}$ ratio (calculated/IC) & & 1.19 & 1.16 & 1.17 \\
\hline \multicolumn{5}{|l|}{ Sillaro - S Siltir. } \\
\hline S (by ICP) & 843 & 602 & 4,070 & 1,955 \\
\hline $\mathrm{SO}_{4}$ (by IC) & 1,143 & 816 & 11,200 & 4,866 \\
\hline $\mathrm{SO}_{4}$ (calculated from $\mathrm{S}$ ) & & 1,806 & 12,210 & 5,864 \\
\hline $\mathrm{SO}_{4}$ ratio (calculated/IC) & & 2.21 & 1.09 & 1.21 \\
\hline
\end{tabular}

Note:

${ }^{1}$ Weighted mean was calculated using the following formula: $0.61 *[$ supernatant] $+0.39 *[$ salt slurry].

\section{B3.3.2 Mass and Charge Balance}

The principal objective in performing mass and charge balances is to determine whether the measurements are consistent. In calculating the balances, only the analytes listed in Section B2.0, detected at a concentration of $1,000 \mu \mathrm{g} / \mathrm{g}$ or greater, were considered. Table B3-3 lists the analytical data and calculations for charge and mass balances. All analytical results used in calculating the supernatant mass and charge balances were first converted from $\mu \mathrm{g} / \mathrm{mL}$ to $\mu \mathrm{g} / \mathrm{g}$ (using the supernatant specific gravity mean of $1.4 \mathrm{~g} / \mathrm{mL}$ ). The weighted total concentration was generated by multiplying the concentration of analyte in supernate and slurry by 61 percent and 39 percent, respectively, based on the volumes of supernate and slurry. The anions listed in Table B3-3 were assumed present as sodium salts and were expected to balance the positive charge exhibited by the cations. Sulfur was assumed present as sulfate. Phosphate, as determined by IC, is assumed to be completely water soluble and appears only in the anion mass and charge calculations. The concentrations of cationic species, anionic species, and water were used to calculate the mass and charge balance. 


$$
\begin{aligned}
& \left\{\varepsilon^{t} \mathrm{Od}+.^{\varepsilon} \mathrm{ON}+.^{\tau} \mathrm{ON}+. \mathrm{HO}+\right.
\end{aligned}
$$

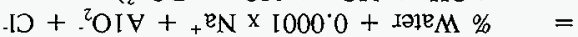

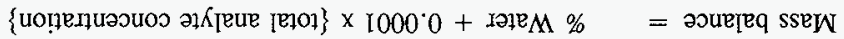

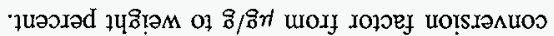

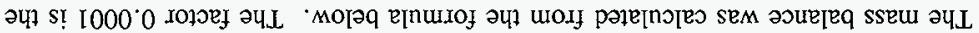

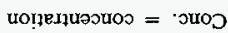

$: 210 \mathrm{~N}$

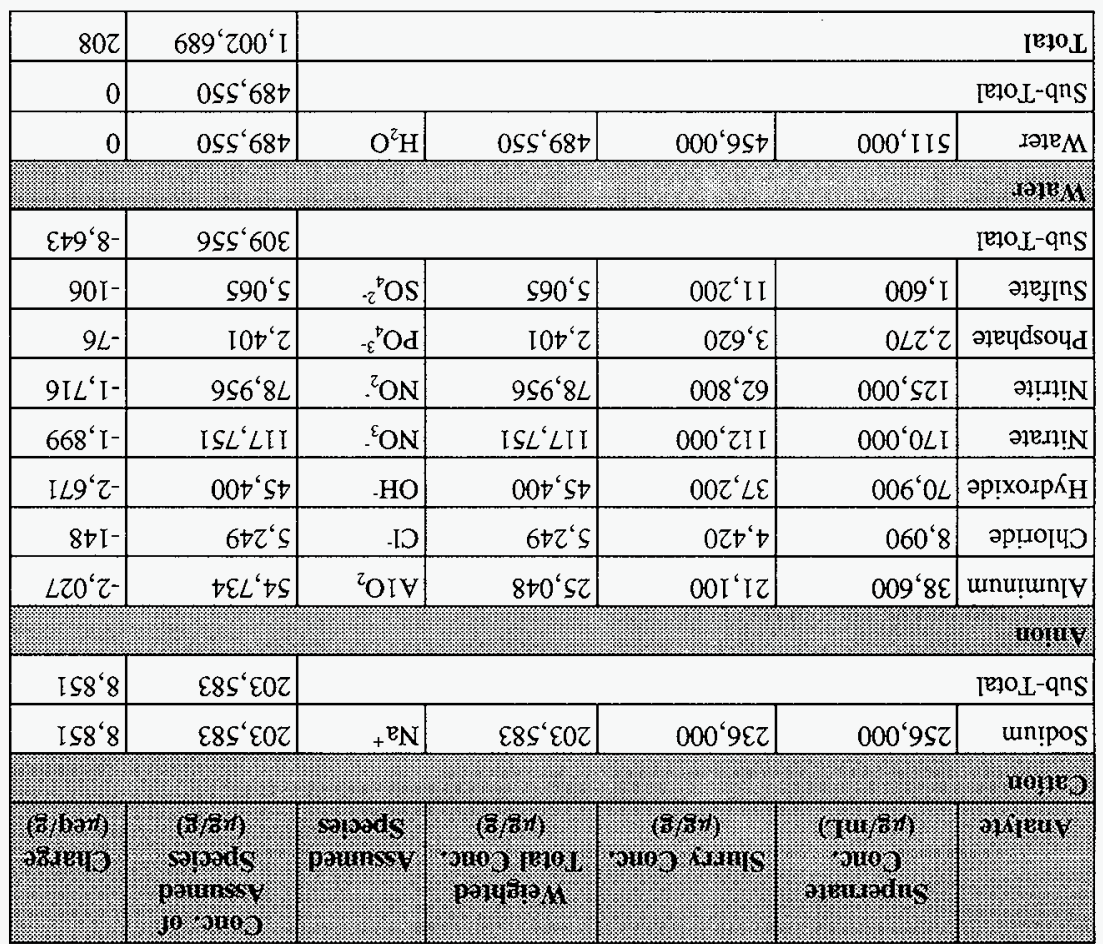

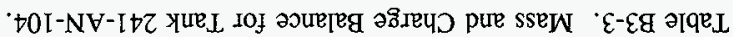


The total analyte concentration calculated from the above equation is $513,139 \mu \mathrm{g} / \mathrm{g}$. The mean weight percent water is 49 percent, or $489,550 \mu \mathrm{g} / \mathrm{g}$. The mass balance resulting from adding the percent water to the total analyte concentration is 100.2 percent (see Table B3-4).

The following equations demonstrate the derivation of total cations and total anions; the charge balance is the ratio of these two values.

Total cations $(\mu \mathrm{eq} / \mathrm{g})=\left[\mathrm{Na}^{+}\right] / 23.0=8,851 \mu \mathrm{eq} / \mathrm{g}$

Total anions $(\mu \mathrm{eq} / \mathrm{g})=\left[\mathrm{AlO}_{2}^{-} / 59\right]+\left[\mathrm{Cl}^{-}\right] / 35.5+\left[\mathrm{OH}^{-}\right] / 17.0+\left[\mathrm{NO}_{2}^{-}\right] / 46.0+$ $\left[\mathrm{NO}_{3}{ }^{-}\right] / 62.0+\left[\mathrm{PO}_{4}^{-3}\right] / 95.0 * 3+\left[\mathrm{SO}_{4}^{-2}\right] / 96.0^{*} 2=-8,643 \mu \mathrm{eq} / \mathrm{g}$

The charge balance obtained by dividing the sum of the positive charge by the sum of the negative charge was 1.02 .

In summary, the above calculations yield reasonable mass and charge balance values (close to 1.00 for charge balance and 100 percent for mass balance) indicating the analytical results are generally consistent.

\section{B3.4 MEAN CONCENTRATIONS AND CONFIDENCE INTERVALS}

The following statistical evaluation was performed using the analytical data generated from tank 241-AN-104 core samples. The core samples (163 and 164) were obtained September 1996 from two risers and included the full length of the waste in the tank (21 segments). The crust was considered to be the solids from segment 1 and a portion of segment 2 . Supernatant samples made up the remainder of segment 2 through segment 12 and a portion of segment 13. Salt slurry samples made up the remainder of segment 13 through segment 21. The three sets of data (crust, supernate, and slurry) were analyzed separately.

For each data set, a mean concentration and the associated variability were calculated for each analyte. A two-sided 95 percent $\mathrm{CI}$ for the mean concentration was also calculated for . each analyte. The CI takes into account the sampling and analytical uncertainties. The upper and lower limits (UL and LL) of a two-sided 95 percent Cl for the mean are

$$
\hat{\mu} \pm t_{(\alpha+0.025)} \times \hat{\sigma}_{\hat{\beta}} .
$$

In these equations, $\hat{\mu}$ is the estimate of the mean concentration, $\hat{\sigma}_{\hat{\mu}}$ is the estimate of the standard deviation of the mean concentration, and $\mathrm{t}_{(\mathrm{df}, 0.025)}$ is the quantile from Student's $t$ distribution with $d f$ degrees of freedom for a two-sided 95 percent $\mathrm{Cl}$. The mean, $\hat{\mu}$, and the standard deviation of the mean, $\hat{\sigma}_{\hat{\mu}}$, were estimated using restricted maximum likelihood estimation (REML) methods. 


\section{B3.4.1 Mean Concentrations}

B3.4.1.1 Supernate Data. The statistics in this section were based on analytical data from the 1996 sampling event of tank 241-AN-104. The 1996 data were statistically evaluated using two different models. The first model used a nested analysis of variance (ANOVA): the data were identified by segment within riser. The second model used a one-way ANOVA: the data were identified by one variable (the sample). Analysis of variance techniques were used to estimate the mean and its associated variability for all analytes that had at least 50 percent of the reported data as quantitative values.

For those analytes, which had a mixture of quantitative values and "less than" values, the ANOVA was computed using two methodologies.

The upper value of the "less than" (for example, 3.5 for $<3.5$ ) was used to represent all "less than" analytical values in the first computation. This produces a bias of unknown magnitude in both the mean analyte concentration and the variance associated with the mean; the mean analyte concentration is biased high. The extension ".lt" was added to the analyte name in the tables to distinguish which analyte was statistically analyzed using "less than" values.

The "less than" values were deleted in the second computation. Deleting data produces unbalanced data sets and complicates the statistical analysis. Deleting data decreases the number of degrees of freedom and produces a bias of unknown magnitude in both the mean analyte concentration and the variance associated with the mean. The extension ".nlt" was added to the analyte name in the tables to distinguish which analyte was statistically analyzed with the "less than" values deleted.

No ANOVA estimates were computed for analytes that had less than 50 percent of the reported data as quantitative values.

Tables B3-4 (nested ANOVA) and B3-5 (one-way ANOVA) provide the mean concentration estimates for the supernate layer and the two-sided 95 percent $\mathrm{CI}$ for the mean concentration for analytes with at least 50 percent of the reported data as quantitative values. For some analytes, the $\mathrm{LL}$ of the 95 percent $\mathrm{Cl}$ was a negative value caused by the magnitude of the variability. Because the actual concentration of a tank sample cannot be less than zero, the $\mathrm{LL}$ is reported as zero. The analytes in Table B3-4 where $\hat{\sigma}_{\text {riser }}$ is significantly different from zero are marked with a "*". The riser variable is an indicator of horizontal homogeneity.

For analytes where $\hat{\sigma}_{\text {riser }}$ is not significantly different from zero, the mean concentrations and the variances of the mean concentrations calculated using the two statistical models (one-way ANOVA and nested ANOVA) are not significantly different. In these cases, the result from the one-way ANOVA model is more appropriate.

Table B3-6 lists the analytes (supernate layer) which had less than 50 percent of the reported data as quantitative values, and it cites the largest value observed from analytical results. 


\begin{tabular}{|c|c|c|c|c|c|c|}
\hline $20+\exists \angle 0^{\circ} I$ & $00+3000$ & I & $00+\exists+\varepsilon\llcorner L$ & $10+\exists 1+1$ & Tu/ $8 n$ & $r^{H}+p^{*} \cdot d o r \cdot u z$ \\
\hline $00+360^{\circ} \mathrm{I}$ & $00+\exists I \varepsilon^{\circ} I$ & I & $\varepsilon 0^{-} \exists+0^{\circ} \mathrm{L}$ & $00+300^{\circ} 1$ & --- & Kఛ! \\
\hline $20+78 z^{\prime} 9$ & $00+300^{\circ} 0$ & $\mathrm{I}$ & $10+\exists \varepsilon 0^{\circ} \varepsilon$ & $\tau 0+\exists \varepsilon t^{\circ} \tau$ & Tu/ŝt & ${ }_{1} \mathrm{p}^{\cdot} \mathrm{dor} \cdot \mathrm{r}^{*}$ \\
\hline$\varepsilon 0+30 L^{\circ} t$ & $00+300^{\circ} 0$ & $\mathrm{I}$ & $z 0+\exists 9 \varepsilon^{\circ} z$ & $\mathcal{E} 0+\exists 0 L^{\circ} \mathrm{I}$ & Tui/on $n$ & I[u•or ${ }_{-z}^{+}{ }^{\dagger} \mathrm{OS}$ \\
\hline$\varepsilon 0+\exists 99^{\circ} \circ$ & $00+300^{\circ} 0$ & I & $20+30 t^{\circ} z$ & $\varepsilon 0+309^{\circ} \mathrm{I}$ & $\mathrm{Tu} / 3 n$ & $11^{\circ} 0 \Gamma^{*}-\tau^{\dagger} \mathrm{OS}$ \\
\hline$\varepsilon 0+38 t^{\circ} \mathrm{I}$ & $20+\exists Z L L^{\prime} 8$ & I & $10+\exists 30 t^{\circ} z$ & $\varepsilon 0+381 \cdot I$ & $\mathrm{~T} w / \mathfrak{s} n$ & $p^{\cdot} d o ! ̣ s$ \\
\hline $\mathrm{E} 0+\exists 20^{\circ} \mathrm{s}$ & $00+300^{\circ} 0$ & $I$ & $20+\exists L I \cdot z$ & $\varepsilon 0+\exists \angle Z \cdot z$ & $\mathrm{~T}$ w/8̂n & $\mathrm{HI} \cdot \mathrm{II}^{-} \cdot{ }_{\varepsilon}^{+} \mathrm{Od}$ \\
\hline$\varepsilon 0+\exists \subseteq t^{\circ} \mathrm{I}$ & $z 0+\exists \mathcal{E} I^{\circ} \mathcal{E}$ & $\mathrm{I}$ & $10+38 t^{\circ} t$ & $20+\exists 18^{\circ} 8$ & $\mathrm{Tu} / \mathrm{z} n$ & p.dor. d \\
\hline $90+308^{\circ} z$ & $S 0+\exists I \mathcal{E}^{\prime} Z$ & I & $\varepsilon 0+\exists Z 6^{\circ} \mathrm{I}$ & $s_{0}+79 s^{\circ} z$ & Тய/8̂n & $\mathrm{p} \cdot \mathrm{d} \jmath{ }^{\circ} \mathrm{eN}$ \\
\hline$S 0+\exists 8 L Z$ & $00+300^{\circ} 0$ & I & $\varepsilon 0+\exists \angle t \cdot 8$ & $90+30 L^{\circ} \mathrm{I}$ & $\mathrm{Tul} / \mathrm{s} n$ & $\partial !^{\cdot} \cdot{ }^{\varepsilon} \mathrm{ON}$ \\
\hline $90+\exists \angle 8^{\circ} \mathrm{Z}$ & $00+\exists 00^{\circ} 0$ & $\mathrm{I}$ & $50+\exists L z \cdot I$ & $\subseteq 0+\exists \subseteq Z^{\circ} \mathrm{I}$ & Tul/sn & $\mathrm{I}^{-2} \mathrm{ON}$ \\
\hline $10+376^{\circ} 8$ & $10+3 z I^{\circ} L$ & $\mathrm{I}$ & $\mathrm{I0}-\mathrm{B} \angle \mathrm{I}^{\circ} \angle$ & $10+\exists \mathcal{E} 0^{\circ} 8$ & ךu/ & p'dor'or \\
\hline $10+\exists \nabla 6^{\circ} t$ & $00+300^{\circ} 0$ & $\mathrm{I}$ & $00+\exists 89^{\circ} \mathrm{Z}$ & $10+\exists \varepsilon S^{\circ} I$ & Tw/sn & 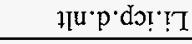 \\
\hline $10+99 I^{\circ} \mathrm{s}$ & $00+3000$ & I & $00+\exists S I^{\circ} \varepsilon$ & $10+3 \angle I^{\circ} \mathrm{I}$ & Тu/ठี & II'p·dor' \\
\hline $\mathcal{E} 0+\exists I 9^{\circ} 8$ & $\varepsilon 0+\exists 8 L t$ & I & $20+\exists 0 S^{\circ} \mathrm{I}$ & $\varepsilon 0+\exists 0 L^{\circ} 9$ & Tư/sิn & 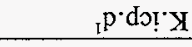 \\
\hline$\varepsilon 0+\exists L L^{\prime} \mathrm{I}$ & $20+\exists+6 . I$ & 1 & $10+\exists 12 \cdot 9$ & $20+3 \varepsilon 8^{\circ} 6$ & $\mathrm{~T} \omega / \mathrm{B} n$ & I[u'o! \\
\hline$\varepsilon 0+\exists \varepsilon 0^{\circ} z$ & $00+3000$ & I & $10+\exists 9 \nabla^{\circ} 8$ & $20+\exists 65^{\circ} 6$ & $\mathrm{Tu} / \mathrm{s} n$ & 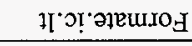 \\
\hline $10+\exists S I^{\circ} \mathrm{s}$ & $00+\exists 00^{\circ} 0$ & $I$ & $00+\exists I L \mathcal{E}$ & $00+\exists t \varepsilon \cdot t$ & 1əM $\mathrm{B} / \mathrm{C}$ & 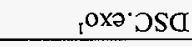 \\
\hline $20+$ GI I I & $00+300^{\circ} 0$ & I & $00+3 t 0.8$ & $00+\exists t \varepsilon \cdot 6$ & $\operatorname{sip} 8 / \Omega$ & Isp*DSC \\
\hline$\tau 0+\exists t L \cdot \varepsilon$ & $z 0+386 \cdot z$ & $\mathrm{I}$ & $00+\exists I 0^{\circ} \varepsilon$ & $20+39 \varepsilon^{\circ} \varepsilon$ & qui/sn & $p \cdot$ do! \\
\hline$t 0+\exists z \varepsilon^{\circ} \mathrm{I}$ & $00+300^{\circ} 0$ & $\mathrm{I}$ & $20+320^{\circ} \nabla$ & $\varepsilon 0+360^{\circ} 8$ & Tu/gr & ว!"-ID \\
\hline$\varepsilon 0+\exists \angle 6^{\circ} \varepsilon$ & $00+300^{\circ} 0$ & I & $20+\exists \varsigma z \cdot z$ & $\varepsilon 0+\exists I I^{*} I$ & Tu/ŝn & 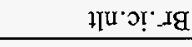 \\
\hline$\varepsilon 0+\exists \tau \varepsilon^{\circ} \varepsilon$ & $00+300^{\circ} 0$ & I & $20+39 L^{\circ} \mathrm{I}$ & $\varepsilon 0+\exists 80^{\circ} \mathrm{L}$ & Tw/8n & II'OI'_Ig \\
\hline $10+\exists 8 Z^{\circ} L$ & $\mathrm{I} 0+\exists L L \cdot \mathrm{S}$ & I & I0-38I'9 & $10+36 t^{\circ} 9$ & TU/8̂n & I[u*p'do! ${ }^{\cdot} g$ \\
\hline $10+\exists+1: L$ & 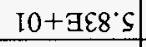 & I & I0-- $99 \mathrm{I}^{\circ} \mathrm{s}$ & $10+387^{\circ} 9$ & Tu/sin & I['p'do! $g$ \\
\hline $70+\exists 8 \tau^{\circ} t$ & $t 0+\exists \varepsilon t^{\circ} \mathcal{E}$ & $I$ & $20+\exists \not \varepsilon \mathcal{E}$ & $70+B 98^{\circ} \mathcal{E}$ & Тய/శึ & p.dor.lv \\
\hline $10+39 I^{\circ} z$ & $\mathrm{IO}+\mathrm{BSS} \mathrm{I}$ & I & $10-30 t^{\circ} z$ & $10+398^{\circ} \mathrm{I}$ & ТU/ & 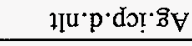 \\
\hline $10+\exists \subseteq S z$ & $00+3 t 6 \cdot 6$ & I & I0-تZI I'9 & $10+\exists L L \cdot I$ & Tw/ठิ $n$ & 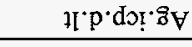 \\
\hline$\varepsilon 0+\exists I \varepsilon \cdot I$ & $00+300.0$ & I & $10+319^{\circ} \mathrm{s}$ & $20+320.9$ & TU/B⿵⺆ & 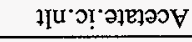 \\
\hline$\varepsilon 0+\exists I \varepsilon^{\prime} I$ & $00+300^{\circ} 0$ & I & $10+\exists \angle D \subseteq$ & $20+B z I \cdot 9$ & Tu/snt & 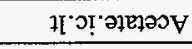 \\
\hline $10+309^{\circ} \mathrm{s}$ & $10+\exists \varepsilon 9^{\circ} \nu$ & I & $10^{-} \exists \subseteq 8^{\circ} \mathcal{E}$ & $10+3 I I S$ & $\% 1 \mathrm{M}$ & 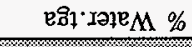 \\
\hline 刻 & 9. & 19: & 8 & the & -114. & 8) \\
\hline
\end{tabular}

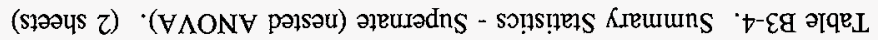


Table B3-4. Summary Statistics - Supernate (nested ANOVA). (2 sheets)

\begin{tabular}{|c|c|c|c|c|c|c|}
\hline 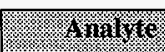 & Whis & I & 6 & of & f & (1) \\
\hline Zn.icp.d.nlt ${ }^{1}$ & $\mu \mathrm{g} / \mathrm{mL}$ & $1.53 \mathrm{E}+01$ & $7.08 \mathrm{E}+00$ & 1 & $0.00 \mathrm{E}+00$ & $1.05 \mathrm{E}+02$ \\
\hline Zr.icp.d.lt $^{1}$ & $\mu \mathrm{g} / \mathrm{mL}$ & $7.81 \mathrm{E}+00$ & $8.99 \mathrm{E}-01$ & 1 & $0.00 \mathrm{E}+00$ & $1.92 \mathrm{E}+01$ \\
\hline Zr.icp.d.nlt & $\mu \mathrm{g} / \mathrm{mL}$ & $7.86 \mathrm{E}+00$ & $3.58 \mathrm{E}-01$ & 1 & $3.31 \mathrm{E}+00$ & $1.24 \mathrm{E}+01$ \\
\hline
\end{tabular}

Note:

${ }^{1} \hat{\sigma}_{\text {tisex }}$ is significantly different from zero (evidence of horizontal heterogenetty).

Table B3-5. Summary Statistics - Supernate (one-way ANOVA). (2 sheets)

\begin{tabular}{|c|c|c|c|c|c|c|}
\hline Sinalie & 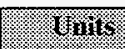 & tis. & (2) & df & III: & (11: \\
\hline$\%$ Water.tga & wt\% & $5.11 E+01$ & $3.85 \mathrm{E}-01$ & 22 & $5.03 \mathrm{E}+01$ & $5.19 \mathrm{E}+01$ \\
\hline Acetate.ic.1t & $\mu \mathrm{g} / \mathrm{mL}$ & $6.12 \mathrm{E}+02$ & $5.47 \mathrm{E}+01$ & 22 & $4.99 \mathrm{E}+02$ & $7.26 \mathrm{E}+02$ \\
\hline Acetate.ic.nlt & $\mu \mathrm{g} / \mathrm{mL}$ & $6.02 \mathrm{E}+02$ & $5.61 \mathrm{E}+01$ & 21 & $4.85 \mathrm{E}+02$ & $7.18 \mathrm{E}+02$ \\
\hline Ag.icp.d.it & $\mu \mathrm{g} / \mathrm{mL}$ & $1.77 \mathrm{E}+01$ & $4.91 \mathrm{E}-01$ & 22 & $1.67 \mathrm{E}+01$ & $1.87 \mathrm{E}+01$ \\
\hline Ag.icp.d.nlt & $\mu \mathrm{g} / \mathrm{mL}$ & $1.86 \mathrm{E}+01$ & $1.62 \mathrm{E}-01$ & 19 & $1.82 \mathrm{E}+01$ & $1.89 \mathrm{E}+01$ \\
\hline Al.icp.d & $\mu \mathrm{g} / \mathrm{mL}$ & $3.86 \mathrm{E}+04$ & $3.33 \mathrm{E}+02$ & 22 & $3.79 \mathrm{E}+04$ & $3.93 \mathrm{E}+04$ \\
\hline B.icp.d.lt & $\mu \mathrm{g} / \mathrm{mL}$ & $6.48 \mathrm{E}+01$ & $5.16 \mathrm{E}-01$ & 22 & $6.38 \mathrm{E}+01$ & $6.59 \mathrm{E}+01$ \\
\hline B.icp.d.nlt & $\mu \mathrm{g} / \mathrm{mL}$ & $6.49 \mathrm{E}+01$ & $5.16 \mathrm{E}-01$ & 22 & $6.39 \mathrm{E}+01$ & $6.60 \mathrm{E}+01$ \\
\hline Br.ic.lt & $\mu \mathrm{g} / \mathrm{mL}$ & $1.08 \mathrm{E}+03$ & $7.51 \mathrm{E}+01$ & 21 & $9.21 \mathrm{E}+02$ & $1.23 \mathrm{E}+03$ \\
\hline$\overline{\mathrm{Br}}$.ic.nlt & $\mu \mathrm{g} / \mathrm{mL}$ & $1.07 \mathrm{E}+03$ & $9.82 \mathrm{E}+01$ & 14 & $8.57 \mathrm{E}+02$ & $1.28 \mathrm{E}+03$ \\
\hline $\mathrm{Cl}$.ic & $\mu \mathrm{g} / \mathrm{mL}$ & $8.09 \mathrm{E}+03$ & $4.02 \mathrm{E}+02$ & 21 & $7.25 \mathrm{E}+03$ & $8.92 E+03$ \\
\hline Cr.icp.d & $\mu \mathrm{g} / \mathrm{mL}$ & $3.36 \mathrm{E}+02$ & $3.01 \mathrm{E}+00$ & 22 & $3.30 \mathrm{E}+02$ & $3.42 \mathrm{E}+02$ \\
\hline Formate.ic.1t & $\mu \mathrm{g} / \mathrm{mL}$ & $9.61 \mathrm{E}+02$ & $5.32 \mathrm{E}+01$ & 22 & $8.51 \mathrm{E}+02$ & $1.07 \mathrm{E}+03$ \\
\hline Formate.ic.nlt & $\mu \mathrm{g} / \mathrm{mL}$ & $9.86 \mathrm{E}+02$ & $4.95 \mathrm{E}+01$ & 21 & $8.83 \mathrm{E}+02$ & $1.09 \mathrm{E}+03$ \\
\hline Li.icp.d.lt & $\mu \mathrm{g} / \mathrm{mL}$ & $1.18 \mathrm{E}+01$ & $1.69 \mathrm{E}+00$ & 22 & $8.25 \mathrm{E}+00$ & $1.53 \mathrm{E}+01$ \\
\hline Li.icp.d.nlt & $\mu \mathrm{g} / \mathrm{mL}$ & $1.55 \mathrm{E}+01$ & $2.77 \mathrm{E}+00$ & 11 & $9.44 \mathrm{E}+00$ & $2.16 \mathrm{E}+01$ \\
\hline Mo.icp.d & $\mu \mathrm{g} / \mathrm{mL}$ & $8.03 \mathrm{E}+01$ & $7.17 \mathrm{E}-01$ & 22 & $7.88 \mathrm{E}+01$ & $8.18 \mathrm{E}+01$ \\
\hline $\mathrm{NO}_{2}^{-}$. ic & $\mu \mathrm{g} / \mathrm{mL}$ & $1.25 \mathrm{E}+05$ & $1.10 \mathrm{E}+04$ & 21 & $1.02 \mathrm{E}+05$ & $1.48 \mathrm{E}+05$ \\
\hline $\mathrm{NO}_{3}^{-}$.ic & $\mu \mathrm{g} / \mathrm{mL}$ & $1.70 \mathrm{E}+05$ & $8.47 \mathrm{E}+03$ & 21 & $1.53 \mathrm{E}+05$ & $1.88 \mathrm{E}+05$ \\
\hline Na.icp.d & $\mu \mathrm{g} / \mathrm{mL}$ & $2.56 \mathrm{E}+05$ & $1.92 \mathrm{E}+03$ & 22 & $2.52 \mathrm{E}+05$ & $2.60 \mathrm{E}+05$ \\
\hline P.icp.d & $\mu \mathrm{g} / \mathrm{mL}$ & $8.83 \mathrm{E}+02$ & $2.68 \mathrm{E}+01$ & 22 & $8.27 \mathrm{E}+02$ & $9.38 \mathrm{E}+02$ \\
\hline $\mathrm{PO}_{4}{ }^{3 *}$.ic.1t & $\mu \mathrm{g} / \mathrm{mL}$ & $2.27 \mathrm{E}+03$ & $1.44 \mathrm{E}+02$ & 21 & $1.97 \mathrm{E}+03$ & $2.57 \mathrm{E}+03$ \\
\hline
\end{tabular}


Table B3-5. Summary Statistics - Supernate (one-way ANOVA). (2 sheets)

\begin{tabular}{|c|c|c|c|c|c|c|}
\hline sharife & Wins & 1: & (1.8) & if & 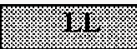 & 14 \\
\hline S.icp.d & $\mu \mathrm{g} / \mathrm{mL}$ & $1.18 \mathrm{E}+03$ & $1.92 \mathrm{E}+01$ & 22 & $1.14 \mathrm{E}+03$ & $1.22 \mathrm{E}+03$ \\
\hline $\mathrm{SO}_{4}{ }^{2-}$.ic.lt & $\mu \mathrm{g} / \mathrm{mL}$ & $1.60 \mathrm{E}+03$ & $1.08 \mathrm{E}+02$ & 21 & $1.38 \mathrm{E}+03$ & $1.83 \mathrm{E}+03$ \\
\hline $\mathrm{SO}_{4}^{2-}$.ic.nlt & $\mu \mathrm{g} / \mathrm{mL}$ & $1.69 \mathrm{E}+03$ & $1.06 \mathrm{E}+02$ & 18 & $1.47 \mathrm{E}+03$ & $1.92 \mathrm{E}+03$ \\
\hline Specific gravity & -- & $1.40 \mathrm{E}+00$ & $7.04 \mathrm{E}-03$ & 22 & $1.39 \mathrm{E}+00$ & $1.41 \mathrm{E}+00$ \\
\hline Zr.icp.d.nlt & $\mu \mathrm{g} / \mathrm{mL}$ & $7.86 \mathrm{E}+00$ & $3.58 \mathrm{E}-01$ & 12 & $7.08 \mathrm{E}+00$ & $8.64 \mathrm{E}+00$ \\
\hline
\end{tabular}

Table B3-6. Analytes (Supernate) with $>50$ Percent "Less Than" Values. (2 sheets)

\begin{tabular}{|l|l|l|}
\hline & & \\
\hline As.icp.d & $\mu \mathrm{g} / \mathrm{mL}$ & $<120$ \\
\hline Ba.icp.d & $\mu \mathrm{g} / \mathrm{mL}$ & $<60.1$ \\
\hline Be.icp.d & $\mu \mathrm{g} / \mathrm{mL}$ & $<6$ \\
\hline Bi.icp.d & $\mu \mathrm{g} / \mathrm{mL}$ & $<120$ \\
\hline Ca.icp.d & $\mu \mathrm{g} / \mathrm{mL}$ & $<120$ \\
\hline Cd.icp.d & $\mu \mathrm{g} / \mathrm{mL}$ & $<6$ \\
\hline Ce.icp.d & $\mu \mathrm{g} / \mathrm{mL}$ & $<120$ \\
\hline Co.icp.d & $\mu \mathrm{g} / \mathrm{mL}$ & 66.5 \\
\hline Cu.icp.d & $\mu \mathrm{g} / \mathrm{mL}$ & $<12$ \\
\hline Fe.icp.d & $\mu \mathrm{g} / \mathrm{mL}$ & $<60.1$ \\
\hline La.icp.d & $\mu \mathrm{g} / \mathrm{mL}$ & $<60.1$ \\
\hline Mg.icp.d & $\mu \mathrm{g} / \mathrm{mL}$ & $<120$ \\
\hline Mn.icp.d & $\mu \mathrm{g} / \mathrm{mL}$ & $<12$ \\
\hline Nd.icp.d & $\mu \mathrm{g} / \mathrm{mL}$ & $<120$ \\
\hline Ni.icp.d & $\mu \mathrm{g} / \mathrm{mL}$ & $<24$ \\
\hline Sb.icp.d & $\mu \mathrm{g} / \mathrm{mL}$ & $<72.1$ \\
\hline Se.icp.d & $\mu \mathrm{g} / \mathrm{mL}$ & $<120$ \\
\hline Sm.icp.d & $\mu \mathrm{g} / \mathrm{mL}$ & $<120$ \\
\hline Sr.icp.d & $\mu \mathrm{g} / \mathrm{mL}$ & $<12$ \\
\hline Ti.icp.d & $\mu \mathrm{g} / \mathrm{mL}$ & $<12$ \\
\hline Tl.icp.d & $\mu \mathrm{g} / \mathrm{mL}$ & $<240$ \\
\hline & & \\
\hline
\end{tabular}


Table B3-6. Analytes (Supernate) with >50 Percent "Less Than" Values. (2 sheets)

\begin{tabular}{|l|l|l|}
\hline Total Alpha & $\mu \mathrm{Ci} / \mathrm{mL}$ & $<0.0994$ \\
\hline U.icp.d & $\mu \mathrm{g} / \mathrm{mL}$ & $<600$ \\
\hline V.icp.d & $\mu \mathrm{Ci} / \mathrm{mL}$ & $<60.1$ \\
\hline
\end{tabular}

A supernate composite sample was formed from supernate subsamples from core 164 segments 1 to 13 (15 mL from segments $1,2,3,4,5,7,8,9,10,11,12$, and 13). The arithmetic mean, the associated variability, and a two-sided 95 percent $\mathrm{CI}$ for the mean concentration were calculated for each analyte with at least 50 percent of the reported data as quantitative values. The CI takes into account only the analytical uncertainty. Tables B3-7 and B3-8 list the summary statistics.

Table B3-7. Summary Statistics - Supernate Composite (core 164 , segments 1 to 13 ). (2 sheets)

\begin{tabular}{|c|c|c|c|c|c|c|}
\hline \%oly & Yritis & 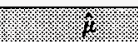 & \% & wif & \%. & X) \\
\hline$\%$ Water.tga & wt $\%$ & $5.11 \mathrm{E}+01$ & $2.55 \mathrm{E}-01$ & 1 & $4.79 \mathrm{E}+01$ & $5.44 \mathrm{E}+01$ \\
\hline Acetate.ic & $\mu \mathrm{g} / \mathrm{mL}$ & $1.88 \mathrm{E}+03$ & $1.10 \mathrm{E}+02$ & 1 & $4.82 E+02$ & $3.28 \mathrm{E}+03$ \\
\hline Al.icp.d & $\mu \mathrm{g} / \mathrm{mL}$ & $4.17 \mathrm{E}+04$ & $8.00 \mathrm{E}+02$ & 1 & $3.15 \mathrm{E}+04$ & $5.19 \mathrm{E}+04$ \\
\hline $\mathrm{Br}$.ic & $\mu \mathrm{g} / \mathrm{mL}$ & $1.55 \mathrm{E}+03$ & $3.00 \mathrm{E}+01$ & 1 & $1.17 \mathrm{E}+03$ & $1.93 \mathrm{E}+03$ \\
\hline $\mathrm{Cl}^{-}$.ic & $\mu \mathrm{g} / \mathrm{mL}$ & $9.46 \mathrm{E}+03$ & $1.60 \mathrm{E}+02$ & 1 & $7.43 \mathrm{E}+03$ & $1.15 \mathrm{E}+04$ \\
\hline Co.icp.d & $\mu \mathrm{g} / \mathrm{mL}$ & $7.13 \mathrm{E}+01$ & $3.95 \mathrm{E}+00$ & 1 & $2.11 \mathrm{E}+01$ & $1.21 \mathrm{E}+02$ \\
\hline $\mathrm{Cr}(\mathrm{VI})$ & $\mu \mathrm{g} / \mathrm{mL}$ & $2.13 \mathrm{E}+02$ & $1.50 \mathrm{E}+00$ & 1 & $1.93 \mathrm{E}+02$ & $2.32 \mathrm{E}+02$ \\
\hline Cr.icp.d & $\mu \mathrm{g} / \mathrm{mL}$ & $3.61 E+02$ & $4.00 \mathrm{E}+00$ & 1 & $3.10 \mathrm{E}+02$ & $4.12 \mathrm{E}+02$ \\
\hline${ }^{137} \mathrm{Cs}$.gea & $\mu \mathrm{Ci} / \mathrm{mL}$ & $5.70 \mathrm{E}+02$ & $2.00 \mathrm{E}+00$ & 1 & $5.45 E+02$ & $5.95 \mathrm{E}+02$ \\
\hline DSC.dry & Joules/g dry & $3.88 \mathrm{E}+01$ & $9.00 \mathrm{E}-01$ & 1 & $2.74 \mathrm{E}+01$ & $5.02 \mathrm{E}+01$ \\
\hline DSC.exo & Joules/g wet & $1.90 \mathrm{E}+01$ & $4.50 \mathrm{E}-01$ & 1 & $1.32 \mathrm{E}+01$ & $2.47 \mathrm{E}+01$ \\
\hline Formate.ic & $\mu \mathrm{g} / \mathrm{mL}$ & $3.44 \mathrm{E}+03$ & $1.00 \mathrm{E}+01$ & 1 & $3.31 \mathrm{E}+03$ & $3.57 \mathrm{E}+03$ \\
\hline${ }^{3} \mathrm{H}$ & $\mu \mathrm{Ci} / \mathrm{mL}$ & $1.51 \mathrm{E}-03$ & $8.00 \mathrm{E}-05$ & 1 & $4.94 \mathrm{E}-04$ & $2.53 \mathrm{E}-03$ \\
\hline${ }^{129} \mathrm{I}$ & $\mu \mathrm{Ci} / \mathrm{mL}$ & $2.08 \mathrm{E}-04$ & $1.45 \mathrm{E}-05$ & 1 & $2.33 \mathrm{E}-05$ & $3.92 \mathrm{E}-04$ \\
\hline K.icp.d & $\mu \mathrm{g} / \mathrm{mL}$ & $6.90 \mathrm{E}+03$ & $1.25 \mathrm{E}+02$ & 1 & $5.31 \mathrm{E}+03$ & $8.48 \mathrm{E}+03$ \\
\hline Mo.icp.d & $\mu \mathrm{g} / \mathrm{mL}$ & $8.70 \mathrm{E}+01$ & $1.95 \mathrm{E}+00$ & 1 & $6.22 \mathrm{E}+01$ & $1.12 \mathrm{E}+02$ \\
\hline $\mathrm{NO}_{2}^{-} \cdot \mathrm{ic}$ & $\mu \mathrm{g} / \mathrm{mL}$ & $1.31 \mathrm{E}+05$ & $1.50 \mathrm{E}+03$ & 1 & $1.11 \mathrm{E}+05$ & $1.50 \mathrm{E}+05$ \\
\hline $\mathrm{NO}_{3}^{-}$, ic & $\mu \mathrm{g} / \mathrm{mL}$ & $2.07 \mathrm{E}+05$ & $5.00 \mathrm{E}+02$ & 1 & $2.00 \mathrm{E}+05$ & $2.13 \mathrm{E}+05$ \\
\hline Na.icp.d & $\mu \mathrm{Ci} / \mathrm{mL}$ & $2.61 E+05$ & $6.00 \mathrm{E}+03$ & 1 & $1.85 \mathrm{E}+05$ & $3.37 \mathrm{E}+05$ \\
\hline
\end{tabular}


Table B3-7. Summary Statistics - Supernate Composite (core 164, segments 1 to 13 ). (2 sheets)

\begin{tabular}{|c|c|c|c|c|c|c|}
\hline (V) & W & W & 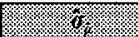 & (1) & 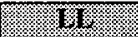 & (3) \\
\hline $\mathrm{OH}^{-}$ & $\mu \mathrm{g} / \mathrm{mL}$ & $7.09 \mathrm{E}+04$ & $5.00 \mathrm{E}+02$ & 1 & $6.45 \mathrm{E}+04$ & $7.73 \mathrm{E}+04$ \\
\hline P.icp.d & $\mu \mathrm{g} / \mathrm{mL}$ & $1.03 E+03$ & $3.25 \mathrm{E}+01$ & 1 & $6.15 \mathrm{E}+02$ & $1.44 \mathrm{E}+03$ \\
\hline $\mathrm{PO}_{4}^{3-} \cdot \mathrm{ic}$ & $\mu \mathrm{g} / \mathrm{mL}$ & $3.80 \mathrm{E}+03$ & $1.85 \mathrm{E}+02$ & 1 & $1.44 E+03$ & $6.15 \mathrm{E}+03$ \\
\hline S.icp.d & $\mu \mathrm{g} / \mathrm{mL}$ & $1.20 \mathrm{E}+03$ & $2.00 \mathrm{E}+01$ & 1 & $9.46 \mathrm{E}+02$ & $1.45 \mathrm{E}+03$ \\
\hline $\mathrm{SO}_{4}^{2-}$.ic & $\mu \mathrm{g} / \mathrm{mL}$ & $2.31 \mathrm{E}+03$ & $1.90 \mathrm{E}+02$ & 1 & $0.00 \mathrm{E}+00$ & $4.72 \mathrm{E}+03$ \\
\hline Si.icp.d & $\mu \mathrm{g} / \mathrm{mL}$ & $3.79 \mathrm{E}+02$ & $3.20 \mathrm{E}+01$ & 1 & $0.00 \mathrm{E}+00$ & $7.86 \mathrm{E}+02$ \\
\hline Specific gravity & - & $1.41 \mathrm{E}+00$ & $5.00 \mathrm{E}-03$ & 1 & $1.34 \mathrm{E}+00$ & $1.47 \mathrm{E}+00$ \\
\hline${ }^{89 / 90} \mathrm{Sr}$ & $\mu \mathrm{Ci} / \mathrm{mL}$ & $3.38 \mathrm{E}-01$ & $4.00 \mathrm{E}-03$ & 1 & $2.87 \mathrm{E}-01$ & $3.89 \mathrm{E}-01$ \\
\hline TIC & $\mu \mathrm{g} / \mathrm{mL}$ & $2.17 \mathrm{E}+03$ & $1.15 \mathrm{E}+02$ & 1 & $7.04 \mathrm{E}+02$ & $3.63 \mathrm{E}+03$ \\
\hline TOC & $\mu \mathrm{g} / \mathrm{mL}$ & $3.11 \mathrm{E}+03$ & $5.76 \mathrm{E}+02$ & 2 & $6.30 \mathrm{E}+02$ & $5.59 \mathrm{E}+03$ \\
\hline${ }^{99} \mathrm{Tc}$ & $\mu \mathrm{Ci} / \mathrm{mL}$ & $2.20 \mathrm{E}-04$ & $2.50 \mathrm{E}-06$ & 1 & $1.88 \mathrm{E}-04$ & $2.51 \mathrm{E}-04$ \\
\hline Total Alpha.1t & $\mu \mathrm{Ci} / \mathrm{mL}$ & $2.53 \mathrm{E}-02$ & $1.20 \mathrm{E}-03$ & 1 & $2.14 \mathrm{E}-02$ & $2.91 \mathrm{E}-02$ \\
\hline Total Alpha.nlt & $\mu \mathrm{Ci} / \mathrm{mL}$ & $2.51 \mathrm{E}-02$ & $1.69 \mathrm{E}-03$ & 1 & $1.79 \mathrm{E}-02$ & $3.24 \mathrm{E}-02$ \\
\hline Total Beta & $\mu \mathrm{Ci} / \mathrm{mL}$ & $4.96 \mathrm{E}+02$ & $1.35 \mathrm{E}+01$ & 1 & $3.24 \mathrm{E}+02$ & $6.67 \mathrm{E}+02$ \\
\hline U.phos & $\mu \mathrm{g} / \mathrm{mL}$ & $3.68 \mathrm{E}+00$ & $3.80 \mathrm{E}-01$ & 1 & $0.00 \mathrm{E}+00$ & $8.51 E+00$ \\
\hline
\end{tabular}

Table B3-8. Supernate Composite (core 164 , segments 1 to 13 )

Analytes with $>50$ Percent "Less Than" Values. (2 sheets)

\begin{tabular}{|c|c|c|}
\hline raqu he & W. & Magminimogomil \\
\hline Ag.icp.d & $\mu \mathrm{g} / \mathrm{mL}$ & $<1.20 \mathrm{E}+01$ \\
\hline${ }^{241}$ Am.aea & $\mu \mathrm{Ci} / \mathrm{mL}$ & $<3.51 \mathrm{E}-04$ \\
\hline${ }^{241}$ Am.gea & $\mu \mathrm{Ci} / \mathrm{mL}$ & $<1.36 \mathrm{E}+00$ \\
\hline As.icp.d & $\mu \mathrm{g} / \mathrm{mL}$ & $<1.20 \mathrm{E}+02$ \\
\hline B.icp.d & $\mu \mathrm{g} / \mathrm{mL}$ & $<6.01 \mathrm{E}+01$ \\
\hline Ba.icp.d & $\mu \mathrm{g} / \mathrm{mL}$ & $<6.01 \mathrm{E}+01$ \\
\hline Be.icp.d & $\mu \mathrm{g} / \mathrm{mL}$ & $<6.00 \mathrm{E}+00$ \\
\hline Bi.icp.d & $\mu \mathrm{g} / \mathrm{mL}$ & $<1.20 \mathrm{E}+02$ \\
\hline Ca.icp.d & $\mu \mathrm{g} / \mathrm{mL}$ & $<1.20 \mathrm{E}+02$ \\
\hline Cd.icp.d & $\mu \mathrm{g} / \mathrm{mL}$ & $<6.00 \mathrm{E}+00$ \\
\hline Ce.icp.d & $\mu \mathrm{g} / \mathrm{mL}$ & $<1.20 \mathrm{E}+02$ \\
\hline${ }^{243 / 244} \mathrm{Cm}$ & $\mu \mathrm{Ci} / \mathrm{mL}$ & $<3.51 \mathrm{E}-04$ \\
\hline
\end{tabular}


Table B3-8. Supernate Composite (core 164, segments 1 to 13)

Analytes with $>50$ Percent "Less Than" Values. (2 sheets)

\begin{tabular}{|c|c|c|}
\hline 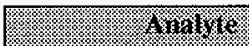 & 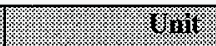 & Whorinun Rerail \\
\hline${ }^{60} \mathrm{Co}$.gea & $\mu \mathrm{Ci} / \mathrm{mL}$ & $<1.67 \mathrm{E}-02$ \\
\hline Cu.icp.d & $\mu \mathrm{g} / \mathrm{mL}$ & $<1.20 \mathrm{E}+01$ \\
\hline${ }^{154}$ Eu.gea & $\mu \mathrm{Ci} / \mathrm{mL}$ & $<1.28 \mathrm{E}-01$ \\
\hline${ }^{155}$ Eu.gea & $\mu \mathrm{Ci} / \mathrm{mL}$ & $<6.57 \mathrm{E}-01$ \\
\hline F-ic & $\mu \mathrm{g} / \mathrm{mL}$ & $<1.22 \mathrm{E}+02$ \\
\hline Fe.icp.d & $\mu \mathrm{g} / \mathrm{mL}$ & $<6.01 \mathrm{E}+01$ \\
\hline La.icp.d & $\mu \mathrm{g} / \mathrm{mL}$ & $<6.01 \mathrm{E}+01$ \\
\hline Li.icp.d & $\mu \mathrm{g} / \mathrm{mL}$ & $<1.20 \mathrm{E}+01$ \\
\hline Mg.icp.d & $\mu \mathrm{g} / \mathrm{mL}$ & $<1.20 \mathrm{E}+02$ \\
\hline Mn.icp.d & $\mu \mathrm{g} / \mathrm{mL}$ & $<1.20 \mathrm{E}+01$ \\
\hline Nd.icp.d & $\mu \mathrm{g} / \mathrm{mL}$ & $<1.20 \mathrm{E}+02$ \\
\hline Ni.icp.d & $\mu \mathrm{g} / \mathrm{mL}$ & $<2.40 \mathrm{E}+01$ \\
\hline${ }^{237} \mathrm{~Np}$ & $\mu \mathrm{Ci} / \mathrm{mL}$ & $<3.68 \mathrm{E}-05$ \\
\hline Oxalate.ic & $\mu \mathrm{g} / \mathrm{mL}$ & $<1.07 \mathrm{E}+03$ \\
\hline Pb.icp.d & $\mu \mathrm{g} / \mathrm{mL}$ & $<1.20 \mathrm{E}+02$ \\
\hline${ }^{239 / 240} \mathrm{Pu}$ & $\mu \mathrm{Ci} / \mathrm{mL}$ & $<1.76 \mathrm{E}-04$ \\
\hline Sb.icp.d & $\mu \mathrm{g} / \mathrm{mL}$ & $<7.21 \mathrm{E}+01$ \\
\hline Se.icp.d & $\mu \mathrm{g} / \mathrm{mL}$ & $<1.20 \mathrm{E}+02$ \\
\hline Sm.icp.d & $\mu \mathrm{g} / \mathrm{mL}$ & $<1.20 \mathrm{E}+02$ \\
\hline Sr.icp.d & $\mu \mathrm{g} / \mathrm{mL}$ & $<1.20 \mathrm{E}+01$ \\
\hline Ti.icp.d & $\mu \mathrm{g} / \mathrm{mL}$ & $<1.20 \mathrm{E}+01$ \\
\hline Tl.icp.d & $\mu \mathrm{g} / \mathrm{mL}$ & $<2.40 \mathrm{E}+02$ \\
\hline U.icp.d & $\mu \mathrm{g} / \mathrm{mL}$ & $<6.00 \mathrm{E}+02$ \\
\hline V.icp.d & $\mu \mathrm{g} / \mathrm{mL}$ & $<6.01 E+01$ \\
\hline Zn.icp.d & $\mu \mathrm{g} / \mathrm{mL}$ & $<1.20 \mathrm{E}+01$ \\
\hline Zr.icp.d & $\mu \mathrm{g} / \mathrm{mL}$ & $<1.20 \mathrm{E}+01$ \\
\hline
\end{tabular}




\section{B.3.4.1.2 Salt Slurry}

Tables B3-9 (nested ANOVA with the riser term) and B3-10 (nested ANOVA without the riser term) list the mean concentration estimates for the slurry layer and the two-sided 95 percent $\mathrm{CI}$ for the mean concentration for analytes with at least 50 percent of the reported data as quantitative values. For some analytes, the LL of the 95 percent $\mathrm{CI}$ was a negative value caused by the magnitude of the variability. Because the actual concentration of a tank sample cannot be less than zero, the LL is reported as zero. The analytes in Table B3-9, where $\hat{\sigma}_{\text {riser }}$ is significantly different from zero, are marked with a "*". The riser variable is an indicator of horizontal homogeneity.

For analytes where $\hat{\sigma}_{\text {riser }}$ is not significantly different from zero, the mean concentrations and the variances of the mean concentrations calculated using the two statistical models (nested ANOVA with the riser term and nested ANOVA without the riser term) are not significantly different. In these cases, the results from the nested ANOVA model without the riser term is more appropriate. Table B3-11 lists the analytes (slurry layer) which had less than 50 percent of the reported data as quantitative values. Table B3-11 cites the largest value observed from the analytical results. 
Table B3-9. Summary Statistics (per g basis) - Slurry (nested ANOVA with riser term). (2 sheets)

\begin{tabular}{|c|c|c|c|c|c|c|}
\hline (6) & Hit & t) & \% & (6) & W & 箖 \\
\hline$\%$ Water.tga ${ }^{1}$ & wt\% & $4.56 \mathrm{E}+01$ & $1.66 \mathrm{E}+00$ & 1 & $2.45 \mathrm{E}+01$ & $6.67 \mathrm{E}+01$ \\
\hline Acetate.ic.lt & $\mu \mathrm{g} / \mathrm{g}$ & $5.49 \mathrm{E}+02$ & $3.37 \mathrm{E}+01$ & 1 & $1.20 \mathrm{E}+02$ & $9.77 \mathrm{E}+02$ \\
\hline Acetate.ic.nlt & $\mu \mathrm{g} / \mathrm{g}$ & $5.83 E+02$ & $4.49 E+01$ & 1 & $1.22 \mathbf{E}+01$ & $1.15 \mathrm{E}+03$ \\
\hline Ag.icp.a.lt & $\mu \mathrm{g} / \mathrm{g}$ & $1.58 \mathrm{E}+01$ & $9.35 \mathrm{E}-01$ & 1 & $3.91 \mathrm{E}+00$ & $2.77 \mathrm{E}+01$ \\
\hline Ag.icp.a.nlt & $\mu \mathrm{g} / \mathrm{g}$ & $1.62 \mathrm{E}+01$ & $1.28 \mathrm{E}+00$ & 1 & $0.00 \mathrm{E}+00$ & $3.25 \mathrm{E}+01$ \\
\hline Al.icp.a ${ }^{1}$ & $\mu \mathrm{g} / \mathrm{g}$ & $2.06 \mathrm{E}+04$ & $1.38 \mathrm{E}+03$ & 1 & $3.04 \mathrm{E}+03$ & $3.82 E+04$ \\
\hline Al.icp.f & $\mu \mathrm{g} / \mathrm{g}$ & $2.11 \mathrm{E}+04$ & $3.99 E+02$ & 1 & $1.60 E+04$ & $2.62 E+04$ \\
\hline B.icp.a & $\mu \mathrm{g} / \mathrm{g}$ & $1.34 \mathrm{E}+02$ & $7.70 \mathrm{E}+00$ & 1 & $3.59 \mathrm{E}+01$ & $2.31 E+02$ \\
\hline Bulk Density ${ }^{1}$ & $\mathrm{~g} / \mathrm{mL}$ & $1.60 \mathrm{E}+00$ & $4.15 \mathrm{E}-02$ & 1 & $1.07 \mathrm{E}+00$ & $2.13 E+00$ \\
\hline Ca.icp.a.lt & $\mu \mathrm{g} / \mathrm{g}$ & $2.25 \mathrm{E}+02$ & $2.13 E+01$ & 1 & $0.00 \mathrm{E}+00$ & $4.96 \mathrm{E}+02$ \\
\hline Ca.icp.a.nlt & $\mu \mathrm{g} / \mathrm{g}$ & $2.25 E+02$ & $2.13 \mathrm{E}+01$ & 1 & $0.00 \mathrm{E}+00$ & $4.96 \mathrm{E}+02$ \\
\hline Cd.icp.a.lt & $\mu \mathrm{g} / \mathrm{g}$ & $1.07 \mathrm{E}+01$ & $1.58 \mathrm{E}+00$ & 1 & $0.00 \mathrm{E}+00$ & $3.09 E+01$ \\
\hline Cd.icp.a.nlt & $\mu \mathrm{g} / \mathrm{g}$ & $1.08 \mathrm{E}+01$ & $1.65 \mathrm{E}+00$ & 1 & $0.00 \mathrm{E}+00$ & $3.17 \mathrm{E}+01$ \\
\hline $\mathrm{Cl}^{-}$.ic & $\mu \mathrm{g} / \mathrm{g}$ & $4.42 \mathrm{E}+03$ & $1.48 \mathrm{E}+02$ & 1 & $2.55 \mathrm{E}+03$ & $6.30 \mathrm{E}+03$ \\
\hline Cr.icp.a & $\mu \mathrm{g} / \mathrm{g}$ & $1.65 E+03$ & $2.99 \mathrm{E}+02$ & 1 & $0.00 \mathrm{E}+00$ & $5.45 E+03$ \\
\hline Cr.icp.f & $\mu \mathrm{g} / \mathrm{g}$ & $1.71 \mathrm{E}+03$ & $1.88 \mathrm{E}+02$ & 1 & $0.00 \mathrm{E}+00$ & $4.10 \mathrm{E}+03$ \\
\hline Cu.icp.a.lt ${ }^{1}$ & $\mu \mathrm{g} / \mathrm{g}$ & $7.46 \mathrm{E}+00$ & $2.89 \mathrm{E}+00$ & 1 & $0.00 \mathrm{E}+00$ & $4.42 \mathrm{E}+01$ \\
\hline Cu.icp.a.nlt & $\mu \mathrm{g} / \mathrm{g}$ & $5.72 \mathrm{E}+00$ & $1.21 \mathrm{E}+00$ & 1 & $0.00 \mathrm{E}+00$ & $2.11 \mathrm{E}+01$ \\
\hline DSC.dry & Joules/g dry & $2.38 \mathrm{E}+01$ & $1.10 \mathrm{E}+01$ & 1 & $0.00 E+00$ & $1.64 \mathrm{E}+02$ \\
\hline DSC.exo & Joules/g wet & $1.29 \mathrm{E}+01$ & $6.07 \mathrm{E}+00$ & 1 & $0.00 \mathrm{E}+00$ & $9.01 \mathrm{E}+01$ \\
\hline $\mathrm{F}^{*}$.ic & $\mu \mathrm{g} / \mathrm{g}$ & $8.36 \mathrm{E}+02$ & $1.45 \mathrm{E}+02$ & $1^{\circ}$ & $0.00 \mathrm{E}+00$ & $2.68 \mathrm{E}+03$ \\
\hline Fe.icp.a.lt & $\mu \mathrm{g} / \mathrm{g}$ & $9.75 E+01$ & $9.31 \mathrm{E}+00$ & 1 & $0.00 \mathrm{E}+00$ & $2.16 \mathrm{E}+02$ \\
\hline Fe.icp.a.nlt & $\mu \mathrm{g} / \mathrm{g}$ & $1.04 \mathrm{E}+02$ & $1.06 \mathrm{E}+01$ & 1 & $0.00 E+00$ & $2.38 \mathrm{E}+02$ \\
\hline Formate.ic & $\mu \mathrm{g} / \mathrm{g}$ & $7.77 \mathrm{E}+02$ & $4.71 \mathrm{E}+01$ & 1 & $1.79 E+02$ & $1.37 \mathrm{E}+03$ \\
\hline K.icp. $\mathrm{a}^{1}$ & $\mu \mathrm{g} / \mathrm{g}$ & $3.57 \mathrm{E}+03$ & $2.05 \mathrm{E}+02$ & 1 & $9.69 \mathrm{E}+02$ & $6.17 \mathrm{E}+03$ \\
\hline Li.icp.a.lt & $\mu \mathrm{g} / \mathrm{g}$ & $1.68 \mathrm{E}+01$ & $5.21 \mathrm{E}+00$ & 1 & $0.00 \mathrm{E}+00$ & $8.30 \mathrm{E}+01$ \\
\hline Li.icp.a.nlt & $\mu \mathrm{g} / \mathrm{g}$ & $2.05 \mathrm{E}+01$ & $8.65 \mathrm{E}+00$ & 1 & $0.00 \mathrm{E}+00$ & $1.30 \mathrm{E}+02$ \\
\hline Mn.icp.a.lt & $\mu \mathrm{g} / \mathrm{g}$ & $1.80 E+01$ & $3.08 \mathrm{E}+00$ & 1 & $0.00 \mathrm{E}+00$ & $5.71 E+01$ \\
\hline Mn.icp.a.nlt & $\mu \mathrm{g} / \mathrm{g}$ & $1.80 \mathrm{E}+01$ & $3.14 \mathrm{E}+00$ & 1 & $0.00 \mathrm{E}+00$ & $5.79 E+01$ \\
\hline Mo.icp.a. $1 \mathrm{t}^{\mathrm{i}}$ & $\mu \mathrm{g} / \mathrm{g}$ & $4.96 \mathrm{E}+01$ & $8.17 \mathrm{E}+00$ & 1 & $0.00 \mathrm{E}+00$ & $1.53 E+02$ \\
\hline
\end{tabular}


Table B3-9. Summary Statistics (per g basis) - Slurry (nested ANOVA with riser term). (2 sheets)

\begin{tabular}{|c|c|c|c|c|c|c|}
\hline (1) & URo & 11 & 1 & if: & 141 & Y1. \\
\hline Mo.icp.a.nlt & $\mu \mathrm{g} / \mathrm{g}$ & $4.30 \mathrm{E}+01$ & $1.95 \mathrm{E}+00$ & 1 & $1.82 \mathrm{E}+01$ & $6.77 \mathrm{E}+01$ \\
\hline $\mathrm{NO}_{2}^{-}$.ic & $\mu \mathrm{g} / \mathrm{g}$ & $6.28 \mathrm{E}+04$ & $9.93 \mathrm{E}+02$ & 1 & $5.02 \mathrm{E}+04$ & $7.54 \mathrm{E}+04$ \\
\hline $\mathrm{NO}_{3}^{-}$.ic & $\mu \mathrm{g} / \mathrm{g}$ & $1.12 \mathrm{E}+05$ & $5.60 \mathrm{E}+03$ & 1 & $4.13 \mathrm{E}+04$ & $1.84 \mathrm{E}+05$ \\
\hline Na.icp.a & $\mu \mathrm{g} / \mathrm{g}$ & $1.92 \mathrm{E}+05$ & $2 . \overline{44 \mathrm{E}+03}$ & 1 & $1.61 \mathrm{E}+05$ & $2.23 \mathrm{E}+05$ \\
\hline Na.icp.f & $\mu \mathrm{g} / \mathrm{g}$ & $2.36 \mathrm{E}+05$ & $8.30 \mathrm{E}+03$ & 1 & $1.31 \mathrm{E}+05$ & $3.41 \mathrm{E}+05$ \\
\hline Ni.icp.a & $\mu \mathrm{g} / \mathrm{g}$ & $6.49 \mathrm{E}+01$ & $1.27 \mathrm{E}+01$ & 1 & $0.00 \mathrm{E}+00$ & $2.26 \mathrm{E}+02$ \\
\hline Ni.icp.f.1t & $\mu \mathrm{g} / \mathrm{g}$ & $6.37 \mathrm{E}+03$ & $4.30 \mathrm{E}+03$ & 1 & $0.00 \mathrm{E}+00$ & $6.10 \mathrm{E}+04$ \\
\hline Ni.icp.f.nlt & $\mu \mathrm{g} / \mathrm{g}$ & $6.41 E+03$ & $4.27 \mathrm{E}+03$ & 1 & $0.00 \mathrm{E}+00$ & $6.07 \mathrm{E}+04$ \\
\hline Oxalate.ic & $\mu \mathrm{g} / \mathrm{g}$ & $8.33 \mathrm{E}+03$ & $6.66 \mathrm{E}+02$ & 1 & $0.00 \mathrm{E}+00$ & $1.68 \mathrm{E}+04$ \\
\hline P.icp.a & $\mu \mathrm{g} / \mathrm{g}$ & $1.39 \mathrm{E}+03$ & $2.85 \mathrm{E}+02$ & 1 & $0.00 \mathrm{E}+00$ & $5.01 E+03$ \\
\hline $\mathrm{PO}_{4}{ }^{3-}$.ic.1t & $\mu \mathrm{g} / \mathrm{g}$ & $3.70 \mathrm{E}+03$ & $8.77 \mathrm{E}+02$ & 1 & $0.00 \mathrm{E}+00$ & $1.48 \mathrm{E}+04$ \\
\hline $\mathrm{PO}_{4}{ }^{3-}$.ic.nlt & $\mu \mathrm{g} / \mathrm{g}$ & $3.72 \mathrm{E}+03$ & $8.65 \mathrm{E}+02$ & 1 & $0.00 \mathrm{E}+00$ & $1.47 \mathrm{E}+04$ \\
\hline S.icp.a & $\mu \mathrm{g} / \mathrm{g}$ & $3.80 \mathrm{E}+03$ & $2.35 \mathrm{E}+02$ & 1 & $8.21 \mathrm{E}+02$ & $6.79 \mathrm{E}+03$ \\
\hline S.icp.f & $\mu \mathrm{g} / \mathrm{g}$ & $4.12 \mathrm{E}+03$ & $4.99 \mathrm{E}+02$ & 1 & $0.00 \mathrm{E}+00$ & $1.05 \mathrm{E}+04$ \\
\hline $\mathrm{SO}_{4}{ }^{2-}$.ic & $\mu \mathrm{g} / \mathrm{g}$ & $1.12 \mathrm{E}+04$ & $8.95 \mathrm{E}+02$ & 1 & $0.00 \mathrm{E}+00$ & $2.26 \mathrm{E}+04$ \\
\hline Si.icp.a & $\mu \mathrm{g} / \mathrm{g}$ & $6.09 \mathrm{E}+02$ & $4.25 \mathrm{E}+01$ & 1 & $6.93 \mathrm{E}+01$ & $1.15 E+03$ \\
\hline Total Alpha.1t & $\mu \mathrm{Ci} / \mathrm{g}$ & $8.06 \mathrm{E}-02$ & $3.92 \mathrm{E}-02$ & 1 & $0.00 \mathrm{E}+00$ & 5.79E-01 \\
\hline Total Alpha.nlt & $\mu \mathrm{Ci} / \mathrm{g}$ & $3.84 \mathrm{E}-02$ & $8.13 \mathrm{E}-03$ & 1 & $0.00 \mathrm{E}+00$ & $1.42 \mathrm{E}-01$ \\
\hline Zn.icp.a.lt & $\mu \mathrm{g} / \mathrm{g}$ & $2.02 \mathrm{E}+01$ & $6.10 \mathrm{E}+00$ & 1 & $0.00 \mathrm{E}+00$ & $9.78 \mathrm{E}+01$ \\
\hline Zn.icp.a.nlt ${ }^{1}$ & $\mu \mathrm{g} / \mathrm{g}$ & $2.13 \mathrm{E}+01$ & $7.20 \mathrm{E}+00$ & 1 & $0.00 \mathrm{E}+00$ & $1.13 \mathrm{E}+02$ \\
\hline Zr.icp.a.lt & $\mu \mathrm{g} / \mathrm{g}$ & $1.79 \mathrm{E}+01$ & $8.32 \mathrm{E}-01$ & 1 & $7.31 \mathrm{E}+00$ & $2.84 \mathrm{E}+01$ \\
\hline Zr.icp.a.nlt & $\mu \mathrm{Ci} / \mathrm{g}$ & $1.82 \mathrm{E}+01$ & $8.93 \mathrm{E}-01$ & 1 & $6.89 \mathrm{E}+00$ & $2.96 \mathrm{E}+01$ \\
\hline
\end{tabular}

Note:

\footnotetext{
${ }^{\prime} \hat{\sigma}_{\text {riger }}$ is significantly different from zero (evidence of horizontal heterogeneity).
} 
HNF-SD-WM-ER-690 Rev. 0

Table B3-10. Summary Statistics (per g basis) - Slurry (nested ANOVA without riser), (2 sheets)

\begin{tabular}{|c|c|c|c|c|c|c|}
\hline (6) & Wivily & 14: & 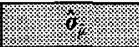 & of & \% & (T) \\
\hline Acetate.ic.lt & $\mu \mathrm{g} / \mathrm{g}$ & $5.49 \mathrm{E}+02$ & $3.37 \mathrm{E}+01$ & 11 & $4.74 \mathrm{E}+02$ & $6.23 E+02$ \\
\hline Acetate.ic.nlt & $\mu \mathrm{g} / \mathrm{g}$ & $5.83 E+02$ & $4.49 E+01$ & 11 & $4.84 \mathrm{E}+02$ & $6.82 \mathrm{E}+02$ \\
\hline Ag.icp.a.lt & $\mu \mathrm{g} / \mathrm{g}$ & $1.58 \mathrm{E}+01$ & $9.35 \mathrm{E}-01$ & 11 & $1.37 \mathrm{E}+01$ & $1.78 \mathrm{E}+01$ \\
\hline Ag.icp.a.nlt & $\mu \mathrm{g} / \mathrm{g}$ & $1.62 \mathrm{E}+01$ & $1.28 \mathrm{E}+00$ & 8 & $1.32 \mathrm{E}+01$ & $1.91 \mathrm{E}+01$ \\
\hline Al.icp.f & $\mu \mathrm{g} / \mathrm{g}$ & $2.11 \mathrm{E}+04$ & $3.99 \mathrm{E}+02$ & 11 & $2.02 \mathrm{E}+04$ & $2.20 \mathrm{E}+04$ \\
\hline B.icp.a & $\mu \mathrm{g} / \mathrm{g}$ & $1.34 \mathrm{E}+02$ & $7.70 \mathrm{E}+00$ & 11 & $1.17 \mathrm{E}+02$ & $1.51 \mathrm{E}+02$ \\
\hline Ca.icp.a.1t & $\mu \mathrm{g} / \mathrm{g}$ & $2.25 E+02$ & $2.13 E+01$ & 11 & $1.78 \mathrm{E}+02$ & $2.72 \mathrm{E}+02$ \\
\hline Ca.icp.a.nlt & $\mu \mathrm{g} / \mathrm{g}$ & $2.26 \mathrm{E}+02$ & $2.11 E+01$ & 11 & $1.79 \mathrm{E}+02$ & $2.72 \mathrm{E}+02$ \\
\hline Cd.icp.a.lt & $\mu \mathrm{g} / \mathrm{g}$ & $1.09 E+01$ & $1.26 \mathrm{E}+00$ & 11 & $8.09 \mathrm{E}+00$ & $1.36 \mathrm{E}+01$ \\
\hline Cd.icp.a.nIt & $\mu \mathrm{g} / \mathrm{g}$ & $1.09 E+01$ & $1.24 \mathrm{E}+00$ & 11 & $8.18 \mathrm{E}+00$ & $1.36 \mathrm{E}+01$ \\
\hline $\mathrm{Cl}$.ic & $\mu \mathrm{g} / \mathrm{g}$ & $4.42 \mathrm{E}+03$ & $1.48 \mathrm{E}+02$ & 11 & $4.10 \mathrm{E}+03$ & $4.75 E+03$ \\
\hline Cr.icp.a & $\mu \mathrm{g} / \mathrm{g}$ & $1.69 E+03$ & $1.81 E+02$ & 11 & $1.29 \mathrm{E}+03$ & $2.09 \mathrm{E}+03$ \\
\hline Cr.icp.f & $\mu \mathrm{g} / \mathrm{g}$ & $1.71 E+03$ & $1.88 \mathrm{E}+02$ & 11 & $1.30 \mathrm{E}+03$ & $2.13 \mathrm{E}+03$ \\
\hline Cu.icp.a.nlt & $\mu \mathrm{g} / \mathrm{g}$ & $5.99 \mathrm{E}+00$ & 7.57E-01 & 6 & $4.14 \mathrm{E}+00$ & $7.84 \mathrm{E}+00$ \\
\hline DSC.dry & $\mathrm{J} / \mathrm{g}$ dry & $2.31 E+01$ & $8.96 E+00$ & 11 & $3.40 \mathrm{E}+00$ & $4.28 \mathrm{E}+01$ \\
\hline DSC.exo & $\mathrm{g} / \mathrm{g}$ wet & $1.25 \mathrm{E}+01$ & $4.80 \mathrm{E}+00$ & 11 & $1.93 E+00$ & $2.30 \mathrm{E}+01$ \\
\hline $\mathrm{F}^{-}$.ic & $\mu \mathrm{g} / \mathrm{g}$ & $8.36 \mathrm{E}+02$ & $1.45 E+02$ & 11 & $5.16 E+02$ & $1.16 \mathrm{E}+03$ \\
\hline Fe.icp.a.lt & $\mu \mathrm{g} / \mathrm{g}$ & $9.75 E+01$ & $9.31 E+00$ & 11 & $7.70 \mathrm{E}+01$ & $1.18 \mathrm{E}+02$ \\
\hline Fe.icp.a.nit & $\mu \mathrm{g} / \mathrm{g}$ & $1.04 \mathrm{E}+02$ & $1.06 \mathrm{E}+01$ & 9 & $8.00 \mathrm{E}+01$ & $1.28 \mathrm{E}+02$ \\
\hline Formate.ic & $\mu \mathrm{g} / \mathrm{g}$ & $7.77 \mathrm{E}+02$ & $4.71 \mathrm{E}+01$ & 11 & $6.73 \mathrm{E}+02$ & $8.80 \mathrm{E}+02$ \\
\hline Li.icp.a.1t & $\mu \mathrm{g} / \mathrm{g}$ & $1.73 \mathrm{E}+01$ & $3.97 E+00$ & 11 & $8.53 E+00$ & $2.60 \mathrm{E}+01$ \\
\hline Li.icp.a.nlt & $\mu \mathrm{g} / \mathrm{g}$ & $2.05 \mathrm{E}+01$ & $6.05 E+00$ & 9 & $6.86 \mathrm{E}+00$ & $3.42 \mathrm{E}+01$ \\
\hline Mn.icp.a.lt & $\mu \mathrm{g} / \mathrm{g}$ & $1.83 \mathrm{E}+01$ & $2.00 \mathrm{E}+00$ & 11 & $1.39 \mathrm{E}+01$ & $2.27 \mathrm{E}+01$ \\
\hline Mn.icp.a.nlt & $\mu \mathrm{g} / \mathrm{g}$ & $1.84 \mathrm{E}+01$ & $1.99 \mathrm{E}+00$ & 11 & $1.40 \mathrm{E}+01$ & $2.28 \mathrm{E}+01$ \\
\hline Mo.icp.a.nit & $\mu \mathrm{g} / \mathrm{g}$ & $4.28 \mathrm{E}+01$ & $1.11 \mathrm{E}+00$ & 8 & $4.03 \mathrm{E}+01$ & $4.54 \mathrm{E}+01$ \\
\hline $\mathrm{NO}_{2}^{-} \cdot \mathrm{ic}$ & $\mu \mathrm{g} / \mathrm{g}$ & $6.28 E+04$ & $9.93 E+02$ & 11 & $6.06 \mathrm{E}+04$ & $6.50 \mathrm{E}+04$ \\
\hline $\mathrm{NO}_{3}^{-} \cdot \mathrm{ic}$ & $\mu \mathrm{g} / \mathrm{g}$ & $1.12 \mathrm{E}+05$ & $5.60 \mathrm{E}+03$ & 11 & $1.00 \mathrm{E}+05$ & $1.25 \mathrm{E}+05$ \\
\hline Na.icp.a & $\mu \mathrm{g} / \mathrm{g}$ & $1.92 \mathrm{E}+05$ & $2.27 \mathrm{E}+03$ & 11 & $1.87 \mathrm{E}+05$ & $1.97 \mathrm{E}+05$ \\
\hline Na.icp.f & $\mu \mathrm{g} / \mathrm{g}$ & $2.36 \mathrm{E}+05$ & $7.11 \mathrm{E}+03$ & 11 & $2.20 \mathrm{E}+05$ & $2.51 \mathrm{E}+05$ \\
\hline Ni.icp.a & $\mu \mathrm{g} / \mathrm{g}$ & $6.65 \mathrm{E}+01$ & $8.03 E+00$ & 11 & $4.88 \mathrm{E}+01$ & $8.41 \mathrm{E}+01$ \\
\hline
\end{tabular}


Table B3-10. Summary Statistics (per g basis) - Slurry (nested ANOVA without riser). (2 sheets)

\begin{tabular}{|c|c|c|c|c|c|c|}
\hline Amariste & (6ins & (19. & (1) & 81 & YI: & (91) \\
\hline Ni.icp.f.it & $\mu \mathrm{g} / \mathrm{g}$ & $6.01 \mathrm{E}+03$ & $3.27 \mathrm{E}+03$ & 11 & $0.00 \mathrm{E}+00$ & $1.32 \mathrm{E}+04$ \\
\hline Ni.icp.f.nlt & $\mu \mathrm{g} / \mathrm{g}$ & $6.09 E+03$ & $3.30 \mathrm{E}+03$ & 11 & $0.00 \mathrm{E}+00$ & $1.33 \mathrm{E}+04$ \\
\hline Oxalate.ic & $\mu \mathrm{g} / \mathrm{g}$ & $8.33 \mathrm{E}+03$ & $6.66 \mathrm{E}+02$ & 11 & $6.86 \mathrm{E}+03$ & $9.79 \mathrm{E}+03$ \\
\hline P.icp.a & $\mu \mathrm{g} / \mathrm{g}$ & $1.37 \mathrm{E}+03$ & $2.41 \mathrm{E}+02$ & 11 & $8.44 \mathrm{E}+02$ & $1.90 \mathrm{E}+03$ \\
\hline$\overline{\mathrm{PO}_{4}{ }^{3-} \text {.ic.lt }}$ & $\mu \mathrm{g} / \mathrm{g}$ & $3.62 \mathrm{E}+03$ & $6.46 \mathrm{E}+02$ & 11 & $2.20 \mathrm{E}+03$ & $5.05 \mathrm{E}+03$ \\
\hline $\mathrm{PO}_{4}{ }^{3-}$.ic.nlt & $\mu \mathrm{g} / \mathrm{g}$ & $3.64 \mathrm{E}+03$ & $6.43 \mathrm{E}+02$ & 11 & $2.22 \mathrm{E}+03$ & $5.06 \mathrm{E}+03$ \\
\hline S.icp.a & $\mu \mathrm{g} / \mathrm{g}$ & $3.80 \mathrm{E}+03$ & $2.35 \mathrm{E}+02$ & 11 & $3.29 \mathrm{E}+03$ & $4.32 \mathrm{E}+03$ \\
\hline S.icp.f & $\mu \mathrm{g} / \mathrm{g}$ & $4.07 \mathrm{E}+03$ & $3.58 \mathrm{E}+02$ & 11 & $3.28 \mathrm{E}+03$ & $4.86 \mathrm{E}+03$ \\
\hline $\mathrm{SO}_{4}^{2-} \cdot \mathrm{ic}$ & $\mu \mathrm{g} / \mathrm{g}$ & $1.12 \mathrm{E}+04$ & $8.95 \mathrm{E}+02$ & 11 & $9.24 \mathrm{E}+03$ & $1.32 \mathrm{E}+04$ \\
\hline Si.icp.a & $\mu \mathrm{g} / \mathrm{g}$ & $6.09 \mathrm{E}+02$ & $4.25 \mathrm{E}+01$ & 11 & $5.16 \mathrm{E}+02$ & $7.03 E+02$ \\
\hline Total Alpha.1t & $\mu \mathrm{Ci} / \mathrm{g}$ & $7.66 \mathrm{E}-02$ & $2.61 E-02$ & 11 & $1.92 \mathrm{E}-02$ & $1.34 \mathrm{E}-01$ \\
\hline Total Alpha.nlt & $\mu \mathrm{Ci} / \mathrm{g}$ & $4.04 \mathrm{E}-02$ & $4.07 \mathrm{E}-03$ & 8 & $3.10 \mathrm{E}-02$ & $4.98 \mathrm{E}-02$ \\
\hline Zn.icp.a.lt & $\mu \mathrm{g} / \mathrm{g}$ & $2.11 \mathrm{E}+01$ & $3.34 \mathrm{E}+00$ & 11 & $1.37 \mathrm{E}+01$ & $2.84 \mathrm{E}+01$ \\
\hline Zr.icp.a.lt & $\mu \mathrm{g} / \mathrm{g}$ & $1.79 \mathrm{E}+01$ & $8.32 \mathrm{E}-01$ & 11 & $1.60 \mathrm{E}+01$ & $1.97 \mathrm{E}+01$ \\
\hline Zr.icp.a.nlt & $\mu \mathrm{g} / \mathrm{g}$ & $1.83 \mathrm{E}+01$ & $9.13 \mathrm{E}-01$ & 11 & $1.63 \mathrm{E}+01$ & $2.03 \mathrm{E}+01$ \\
\hline
\end{tabular}


Table B3-11. Analytes (Slurry - per g basis) with $>50$ Percent "Less Than" Values. (2 sheets)

\begin{tabular}{|l|l|l|}
\hline & & \\
\hline Ag.icp.f & $\mu \mathrm{g} / \mathrm{g}$ & $<225$ \\
\hline As.icp.a & $\mu \mathrm{g} / \mathrm{g}$ & $<153$ \\
\hline As.icp.f & $\mu \mathrm{g} / \mathrm{g}$ & $<2250$ \\
\hline B.icp.f & $\mu \mathrm{g} / \mathrm{g}$ & $<1130$ \\
\hline Ba.icp.a & $\mu \mathrm{g} / \mathrm{g}$ & $<76.7$ \\
\hline Ba.icp.f & $\mu \mathrm{g} / \mathrm{g}$ & $<1130$ \\
\hline Be.icp.a & $\mu \mathrm{g} / \mathrm{g}$ & $<7.67$ \\
\hline Be.icp.f & $\mu \mathrm{g} / \mathrm{g}$ & $<113$ \\
\hline Bi.icp.a & $\mu \mathrm{g} / \mathrm{g}$ & $<153$ \\
\hline Bi.icp.f & $\mu \mathrm{g} / \mathrm{g}$ & $<2250$ \\
\hline Ca.icp.f & $\mu \mathrm{g} / \mathrm{g}$ & $<2250$ \\
\hline Cd.icp.f & $\mu \mathrm{g} / \mathrm{g}$ & $<113$ \\
\hline Ce.icp.a & $\mu \mathrm{g} / \mathrm{g}$ & $<153$ \\
\hline Ce.icp.f & $\mu \mathrm{g} / \mathrm{g}$ & $<2250$ \\
\hline Co.icp.a & $\mu \mathrm{g} / \mathrm{g}$ & 52.2 \\
\hline Co.icp.f & $\mu \mathrm{g} / \mathrm{g}$ & $<451$ \\
\hline Cu.icp.f & $\mu \mathrm{g} / \mathrm{g}$ & 339 \\
\hline Fe.icp.f & $\mu \mathrm{g} / \mathrm{g}$ & 3620 \\
\hline La.icp.a & $\mu \mathrm{g} / \mathrm{g}$ & $<76.7$ \\
\hline La.icp.f & $\mu \mathrm{g} / \mathrm{g}$ & $<1130$ \\
\hline Li.icp.f & $\mu \mathrm{g} / \mathrm{g}$ & $<225$ \\
\hline Mg.icp.a & $\mu \mathrm{g} / \mathrm{g}$ & $<153$ \\
\hline Mg.icp.f & $\mu \mathrm{g} / \mathrm{g}$ & $<2250$ \\
\hline Mn.icp.f & $\mu \mathrm{g} / \mathrm{g}$ & 325 \\
\hline Mo.icp.f & $\mu \mathrm{g} / \mathrm{g}$ & $<1130$ \\
\hline Nd.icp.a & $\mu \mathrm{g} / \mathrm{g}$ & $<153$ \\
\hline Nd.icp.f & $\mu \mathrm{g} / \mathrm{g}$ & $<2250$ \\
\hline P.icp.f & $\mu \mathrm{g} / \mathrm{g}$ & 5400 \\
\hline Pb.icp.a & $\mu \mathrm{g} / \mathrm{g}$ & 706 \\
\hline Pb.icp.f & $<2250$ \\
\hline & & \\
\hline
\end{tabular}


Table B3-11. Analytes (Slurry - per g basis) with $>50$ Percent "Less Than" Values. ( 2 sheets)

\begin{tabular}{|c|c|c|}
\hline (1) & 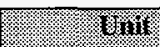 & Whanur Resin \\
\hline Sb.icp.a & $\mu \mathrm{g} / \mathrm{g}$ & $<92.1$ \\
\hline Sb.icp.f & $\mu \mathrm{g} / \mathrm{g}$ & 1590 \\
\hline Se.icp.a & $\mu \mathrm{g} / \mathrm{g}$ & 279 \\
\hline Se.icp.f & $\mu \mathrm{g} / \mathrm{g}$ & $<2250$ \\
\hline Si.icp.f & $\mu \mathrm{g} / \mathrm{g}$ & 2060 \\
\hline Sm.icp.a & $\mu \mathrm{g} / \mathrm{g}$ & $<153$ \\
\hline Sm.icp.f & $\mu \mathrm{g} / \mathrm{g}$ & $<2250$ \\
\hline Sr.icp.a & $\mu \mathrm{g} / \mathrm{g}$ & $<15.3$ \\
\hline Sr.icp.f & $\mu \mathrm{g} / \mathrm{g}$ & $<225$ \\
\hline Ti.icp.a & $\mu \mathrm{g} / \mathrm{g}$ & $<15.3$ \\
\hline Ti.icp.f & $\mu \mathrm{g} / \mathrm{g}$ & $<225$ \\
\hline Tl.icp.a & $\mu \mathrm{g} / \mathrm{g}$ & $<307$ \\
\hline Tl.icp.f & $\mu \mathrm{g} / \mathrm{g}$ & $<4510$ \\
\hline U.icp.a & $\mu \mathrm{g} / \mathrm{g}$ & $<767$ \\
\hline U.icp.f & $\mu \mathrm{g} / \mathrm{g}$ & $<11300$ \\
\hline V.icp,a & $\mu \mathrm{g} / \mathrm{g}$ & $<76.7$ \\
\hline V.icp.f & $\mu \mathrm{g} / \mathrm{g}$ & $<1130$ \\
\hline Zn.icp.f & $\mu \mathrm{g} / \mathrm{g}$ & 635 \\
\hline Zr.icp.f & $\mu \mathrm{g} / \mathrm{g}$ & $<225$ \\
\hline
\end{tabular}

A slurry composite sample was formed from slurry subsamples from core 164 , segments 13 to 20 (20 grams from segments 13 lower half (LH), 14 upper half (UH), $14 \mathrm{LH}, 15 \mathrm{UH}$, $15 \mathrm{LH}, 16 \mathrm{UH}, 16 \mathrm{LH}, 17 \mathrm{UH}, 17 \mathrm{LH}, 19 \mathrm{UH}, 19 \mathrm{LH}, 20 \mathrm{UH}$, and $20 \mathrm{LH})$. The arithmetic mean, the associated variability, and a two-sided 95 percent $\mathrm{Cr}$ for the mean concentration were calculated for each analyte with at least 50 percent of the reported data as quantitative values. The $\mathrm{CI}$ takes into account only the analytical uncertainty. Tables B3-12 and B3-13 list the summary statistics. 
Table B3-12. Summary Statistics - Slurry Composite (core 164, segments 13 to 20). (2 sheets)

\begin{tabular}{|c|c|c|c|c|c|c|}
\hline mater & yas & is & \% & (1) & \%) & 119. \\
\hline$\%$ Water.tga & wt\% & $4.09 \mathrm{E}+01$ & $4.36 \mathrm{E}+00$ & 1 & $0.00 \mathrm{E}+00$ & $9.62 \mathrm{E}+01$ \\
\hline Acetate. ic & $\mu \mathrm{g} / \mathrm{g}$ & $6.37 \mathrm{E}+02$ & $2.35 \mathrm{E}+01$ & 1 & $3.38 \mathrm{E}+02$ & $9.35 \mathrm{E}+02$ \\
\hline Ag.icp.a & $\mu \mathrm{g} / \mathrm{g}$ & $1.39 \mathrm{E}+01$ & $3.50 \mathrm{E}-01$ & 1 & $9.40 \mathrm{E}+00$ & $1.83 \mathrm{E}+01$ \\
\hline Al.icp.a & $\mu \mathrm{g} / \mathrm{g}$ & $2.08 \mathrm{E}+04$ & $1.50 \mathrm{E}+02$ & 1 & $1.88 \mathrm{E}+04$ & $2.27 \mathrm{E}+04$ \\
\hline${ }^{241} \mathrm{Am}$.aea & $\mu \mathrm{Ci} / \mathrm{g}$ & $1.92 \mathrm{E}-02$ & $4.00 \mathrm{E}-04$ & 1 & $1.41 \mathrm{E}-02$ & $2.43 \mathrm{E}-02$ \\
\hline B.icp.a & $\mu \mathrm{g} / \mathrm{g}$ & $1.46 \mathrm{E}+02$ & $1.25 \mathrm{E}+01$ & 1 & $0.00 \mathrm{E}+00$ & $3.04 \mathrm{E}+02$ \\
\hline Br.ic.lt & $\mu \mathrm{g} / \mathrm{g}$ & $1.06 E+03$ & $8.20 \mathrm{E}+01$ & 1 & $1.61 E+01$ & $2.10 E+03$ \\
\hline Bulk Density & $\mathrm{g} / \mathrm{mL}$ & $1.57 \mathrm{E}+00$ & NA & 1 & $\mathrm{NA}$ & NA \\
\hline Ca.icp.a & $\mu \mathrm{g} / \mathrm{g}$ & $2.44 \mathrm{E}+02$ & $2.55 \mathrm{E}+01$ & 1 & $0.00 \mathrm{E}+00$ & $5.68 E+02$ \\
\hline Cd.icp.a & $\mu \mathrm{g} / \mathrm{g}$ & $1.29 E+01$ & $3.00 \mathrm{E}-01$ & 1 & $9.09 E+00$ & $1.67 \mathrm{E}+01$ \\
\hline $\mathrm{Cl}$ ic & $\mu \mathrm{g} / \mathrm{g}$ & $4.09 E+03$ & $6.50 \mathrm{E}+01$ & 1 & $3.26 \mathrm{E}+03$ & $4.91 \mathrm{E}+03$ \\
\hline $\mathrm{Cr}(\mathrm{VI})$ & $\mu \mathrm{g} / \mathrm{g}$ & 2.00E+02 & $3.00 \mathrm{E}+00$ & 1 & $1.62 \mathrm{E}+02$ & $2.38 \mathrm{E}+02$ \\
\hline Cr.icp.a & $\mu \mathrm{g} / \mathrm{g}$ & $2.00 \mathrm{E}+03$ & $1.05 \mathrm{E}+02$ & 1 & $6.61 \mathrm{E}+02$ & $3.33 \mathrm{E}+03$ \\
\hline${ }^{137} \mathrm{Cs}$.gea & $\mu \mathrm{Ci} / \mathrm{g}$ & $3.49 \mathrm{E}+02$ & $8.00 \mathrm{E}+00$ & 1 & $2.47 \mathrm{E}+02$ & $4.51 E+02$ \\
\hline Cu.icp.a & $\mu \mathrm{g} / \mathrm{g}$ & $5.17 \mathrm{E}+00$ & 1.50E-01 & 1 & $3.26 \mathrm{E}+00$ & $7.08 \mathrm{E}+00$ \\
\hline DSC.dry & $\mathrm{J} / \mathrm{g}$ dry & $1.75 \mathrm{E}+02$ & $3.50 \mathrm{E}+00$ & 1 & $1.30 \mathrm{E}+02$ & $2.19 \mathrm{E}+02$ \\
\hline DSC.exo & $\mathrm{J} / \mathrm{g}$ wet & $1.03 E+02$ & $2.00 \mathrm{E}+00$ & 1 & $7.76 \mathrm{E}+01$ & $1.28 \mathrm{E}+02$ \\
\hline $\mathrm{F}$.ic & $\mu \mathrm{g} / \mathrm{g}$ & $6.58 \mathrm{E}+02$ & $1.26 \mathrm{E}+02$ & 1 & $0.00 \mathrm{E}+00$ & $2.26 \mathrm{E}+03$ \\
\hline Fe.icp.a & $\mu \mathrm{g} / \mathrm{g}$ & $9.95 \mathrm{E}+01$ & $4.55 \mathrm{E}+00$ & 1 & $4.16 \mathrm{E}+01$ & $1.57 \mathrm{E}+02$ \\
\hline Formate.ic & $\mu \mathrm{g} / \mathrm{g}$ & $7.33 \mathrm{E}+02$ & $3.05 \mathrm{E}+01$ & 1 & $3.45 E+02$ & $1.12 \mathrm{E}+03$ \\
\hline${ }^{3} \mathrm{H}$ & $\mu \mathrm{Ci} / \mathrm{g}$ & $1.01 \mathrm{E}-02$ & $3.95 \mathrm{E}-04$ & 1 & $5.09 \mathrm{E}-03$ & $1.51 \mathrm{E}-02$ \\
\hline K.icp.a & $\mu \mathrm{g} / \mathrm{g}$ & $3.72 \mathrm{E}+03$ & $9.00 \mathrm{E}+01$ & 1 & $2.58 \mathrm{E}+03$ & $4.86 \mathrm{E}+03$ \\
\hline Li.icp.a & $\mu \mathrm{g} / \mathrm{g}$ & $1.50 \mathrm{E}+01$ & $1.40 \mathrm{E}+00$ & 1 & $0.00 \mathrm{E}+00$ & $3.28 \mathrm{E}+01$ \\
\hline Mn.icp.a & $\mu \mathrm{g} / \mathrm{g}$ & $2.09 \bar{E}+01$ & $1.25 \mathrm{E}+00$ & 1 & $4.97 \mathrm{E}+00$ & $3.67 \mathrm{E}+01$ \\
\hline Mo.icp.a & $\mu \mathrm{g} / \mathrm{g}$ & $4.34 \mathrm{E}+01$ & $1.00 \mathrm{E}-01$ & 1 & $4.21 \mathrm{E}+01$ & $4.47 \mathrm{E}+01$ \\
\hline $\mathrm{NO}_{2}^{-}$.ic & $\mu \mathrm{g} / \mathrm{g}$ & $5.81 \mathrm{E}+04$ & $1.50 \mathrm{E}+02$ & 1 & $5.61 \mathrm{E}+04$ & $6.00 \mathrm{E}+04$ \\
\hline $\mathrm{NO}_{3}^{-} \cdot \mathrm{ic}$ & $\mu g / g$ & $1.01 E+05$ & $1.00 \mathrm{E}+03$ & 1 & $8.83 E+04$ & $1.14 \mathrm{E}+05$ \\
\hline Na.icp.a & $\mu \mathrm{g} / \mathrm{g}$ & $1.92 \mathrm{E}+05$ & $3.50 \mathrm{E}+03$ & 1 & $1.47 \mathrm{E}+05$ & $2.36 E+05$ \\
\hline Ni.icp.a & $\mu \mathrm{g} / \mathrm{g}$ & $7.50 \mathrm{E}+01$ & $4.50 \mathrm{E}+00$ & 1 & $1.78 \mathrm{E}+01$ & $1.32 \mathrm{E}+02$ \\
\hline $\mathrm{OH}^{-}$ & $\mu \mathrm{g} / \mathrm{g}$ & $3.72 \mathrm{E}+04$ & $1.05 \mathrm{E}+03$ & 1 & $2.38 \mathrm{E}+04$ & $5.05 E+04$ \\
\hline
\end{tabular}


Table B3-12. Summary Statistics - Slurry Composite (core 164, segments 13 to 20). ( 2 sheets)

\begin{tabular}{|c|c|c|c|c|c|c|}
\hline sinalye & I & 1. & 8 & af: & Y.1 & 181: \\
\hline Oxalate.ic & $\mu \mathrm{g} / \mathrm{g}$ & $9.67 \mathrm{E}+03$ & $1.15 \mathrm{E}+02$ & 1 & $8.20 \mathrm{E}+03$ & $1.11 \mathrm{E}+04$ \\
\hline P.icp.a & $\mu \mathrm{g} / \mathrm{g}$ & $8.95 \mathrm{E}+02$ & $1.05 \mathrm{E}+02$ & 1 & $0.00 \mathrm{E}+00$ & $2.23 \mathrm{E}+03$ \\
\hline $\mathrm{PO}_{4}^{3-}$.ic & $\mu \mathrm{g} / \mathrm{g}$ & $3.35 \mathrm{E}+03$ & $2.15 \mathrm{E}+02$ & 1 & $6.13 \mathrm{E}+02$ & $6.08 \mathrm{E}+03$ \\
\hline Pb.icp.a & $\mu \mathrm{g} / \mathrm{g}$ & $4.20 \mathrm{E}+01$ & $1.00 \mathrm{E}-01$ & 1 & $4.07 \mathrm{E}+01$ & $4.33 \mathrm{E}+01$ \\
\hline${ }^{239 / 240} \mathrm{Pu}$ & $\mu \mathrm{Ci} / \mathrm{g}$ & $3.65 \mathrm{E}-03$ & $6.00 \mathrm{E}-05$ & 1 & $2.89 \mathrm{E}-03$ & $4.41 \mathrm{E}-03$ \\
\hline S.icp.a & $\mu \mathrm{g} / \mathrm{g}$ & $3.98 \mathrm{E}+03$ & $2.40 \mathrm{E}+02$ & 1 & $9.31 \mathrm{E}+02$ & $7.03 \mathrm{E}+03$ \\
\hline $\mathrm{SO}_{4}^{2-}$. ic & $\mu \mathrm{g} / \mathrm{g}$ & $1.16 \mathrm{E}+04$ & $1.00 \mathrm{E}+02$ & 1 & $1.03 \mathrm{E}+04$ & $1.29 \mathrm{E}+04$ \\
\hline Si.icp.a & $\mu \mathrm{g} / \mathrm{g}$ & $7.62 \mathrm{E}+02$ & $7.35 \mathrm{E}+01$ & 1 & $0.00 \mathrm{E}+00$ & $1.70 \mathrm{E}+03$ \\
\hline${ }^{89 / 90} \mathrm{Sr}$ & $\mu \mathrm{Ci} / \mathrm{g}$ & $3.43 \mathrm{E}+01$ & $4.50 \mathrm{E}-01$ & 1 & $2.85 \mathrm{E}+01$ & $4.00 \mathrm{E}+01$ \\
\hline TIC & $\mu \mathrm{g} / \mathrm{g}$ & $1.47 \mathrm{E}+04$ & $3.50 \mathrm{E}+02$ & 1 & $1.02 \mathrm{E}+04$ & $1.91 \mathrm{E}+04$ \\
\hline TOC & $\mu \mathrm{g} / \mathrm{g}$ & $4.63 \mathrm{E}+03$ & $2.50 \mathrm{E}+01$ & 1 & $4.31 \mathrm{E}+03$ & $4.94 \mathrm{E}+03$ \\
\hline${ }^{99} \mathrm{Tc}$ & $\mu \mathrm{Ci} / \mathrm{g}$ & $1.35 \mathrm{E}-01$ & $6.50 \mathrm{E}-03$ & 1 & $5.19 \mathrm{E}-02$ & $2.17 \mathrm{E}-01$ \\
\hline Total Beta & $\mu \mathrm{Ci} / \mathrm{g}$ & $4.00 \mathrm{E}+02$ & $1.50 \mathrm{E}+01$ & 1 & $2.09 \mathrm{E}+02$ & $5.91 \mathrm{E}+02$ \\
\hline U.phos & $\mu \mathrm{g} / \mathrm{g}$ & $1.81 \mathrm{E}+02$ & $6.50 \mathrm{E}+00$ & 1 & $9.79 \mathrm{E}+01$ & $2.63 \mathrm{E}+02$ \\
\hline Zn.icp.a & $\mu \mathrm{g} / \mathrm{g}$ & $1.68 \mathrm{E}+01$ & $2.40 \mathrm{E}+00$ & 1 & $0.00 \mathrm{E}+00$ & $4.73 \mathrm{E}+01$ \\
\hline Zr.icp.a & $\mu \mathrm{g} / \mathrm{g}$ & $1.69 \mathrm{E}+01$ & $7.50 \mathrm{E}-01$ & 1 & $7.32 \mathrm{E}+00$ & $2.64 \mathrm{E}+01$ \\
\hline
\end{tabular}


Table B3-13. Slurry Composite (core 164 , segments 13 to 20 ) Analytes with $>50$ percent "Less Than" Values.

\begin{tabular}{|l|l|l|}
\hline & & \\
\hline${ }^{241} \mathrm{Am} . \mathrm{gea}$ & $\mu \mathrm{Ci} / \mathrm{g}$ & $<1.41 \mathrm{E}+00$ \\
\hline As.icp.a & $\mu \mathrm{g} / \mathrm{g}$ & $<3.98 \mathrm{E}+01$ \\
\hline Ba.icp.a & $\mu \mathrm{g} / \mathrm{g}$ & $<1.99 \mathrm{E}+01$ \\
\hline Be.icp.a & $\mu \mathrm{g} / \mathrm{g}$ & $<1.99 \mathrm{E}+00$ \\
\hline Bi.icp.a & $\mu \mathrm{g} / \mathrm{g}$ & $<3.98 \mathrm{E}+01$ \\
\hline Ce.icp.a & $\mu \mathrm{g} / \mathrm{g}$ & $<3.98 \mathrm{E}+01$ \\
\hline${ }^{243 i 244} \mathrm{Cm}$ & $\mu \mathrm{Ci} / \mathrm{g}$ & $<3.99 \mathrm{E}-03$ \\
\hline Co.icp.a & $\mu \mathrm{g} / \mathrm{g}$ & $<7.96 \mathrm{E}+00$ \\
\hline${ }^{60} \mathrm{Co} . g e a$ & $\mu \mathrm{Ci} / \mathrm{g}$ & $<2.64 \mathrm{E}-02$ \\
\hline${ }^{154} \mathrm{Eu} . g e a$ & $\mu \mathrm{Ci} / \mathrm{g}$ & $<1.00 \mathrm{E}-01$ \\
\hline${ }^{155} \mathrm{Eu} . g e a$ & $\mu \mathrm{Ci} / \mathrm{g}$ & $<6.72 \mathrm{E}-01$ \\
\hline${ }^{129} \mathrm{I}$ & $\mu \mathrm{Ci} / \mathrm{g}$ & $<2.77 \mathrm{E}-03$ \\
\hline La.icp.a & $\mu \mathrm{Ci} / \mathrm{g}$ & $<1.99 \mathrm{E}+01$ \\
\hline Mg.icp.a & $\mu \mathrm{g} / \mathrm{g}$ & $<3.98 \mathrm{E}+01$ \\
\hline Nd.icp.a & $\mu \mathrm{g} / \mathrm{g}$ & $<3.98 \mathrm{E}+01$ \\
\hline${ }^{237} \mathrm{~Np}$ & $\mu \mathrm{Ci} / \mathrm{g}$ & $<1.20 \mathrm{E}-02$ \\
\hline Sb.icp.a & $\mu \mathrm{g} / \mathrm{g}$ & $<2.39 \mathrm{E}+01$ \\
\hline Se.icp.a & $\mu \mathrm{Ci} / \mathrm{g}$ & $<3.98 \mathrm{E}+01$ \\
\hline Sm.icp.a & $\mu \mathrm{g} / \mathrm{g}$ & $<3.98 \mathrm{E}+01$ \\
\hline Sr.icp.a & $\mu \mathrm{g} / \mathrm{g}$ & $<3.98 \mathrm{E}+00$ \\
\hline Ti.icp.a & $\mu \mathrm{g} / \mathrm{g}$ & $<3.98 \mathrm{E}+00$ \\
\hline Tl.icp.a & $\mu \mathrm{g} / \mathrm{g}$ & $<7.96 \mathrm{E}+01$ \\
\hline Total Alpha & $\mu \mathrm{Ci} / \mathrm{g}$ & $<3.93 \mathrm{E}-02$ \\
\hline U.icp.a & $\mu \mathrm{g} / \mathrm{g}$ & $<1.99 \mathrm{E}+02$ \\
\hline V.icp.a & $\mu \mathrm{g} / \mathrm{g}$ & $<1.99 \mathrm{E}+01$ \\
\hline & & \\
\hline
\end{tabular}


B3.4.1.3 Crust. Tables B3-14 (nested ANOVA with the riser term) and Table B3-15 (nested ANOVA without the riser term) list the mean concentration estimates for the crust and the two-sided 95 percent CI for the mean concentration for analytes with at least 50 percent of the reported data as quantitative values. For some analytes, the LL of the 95 percent $\mathrm{CI}$ was a negative value caused by the magnitude of the variability. Because the actual concentration of a tank sample cannot be less than zero, the $L L$ is reported as zero. Table B3-16 lists the analytes (crust) which had less than 50 percent of the reported data as quantitative values, and it cites the largest value observed from the analytical results.

Table B3-14. Summary Statistics (per g basis) - Crust (nested ANOVA with riser term). (2 sheets)

\begin{tabular}{|c|c|c|c|c|c|c|}
\hline ( & VIIIS & 18 & 8 & 16 & W. & 11: \\
\hline$\%$ Water.tga & wt\% & $4.52 \mathrm{E}+01$ & $2.89 \mathrm{E}+00$ & 1 & $8.44 E+00$ & $8.19 \mathrm{E}+01$ \\
\hline Acetate.ic ${ }^{1}$ & $\mu \mathrm{g} / \mathrm{g}$ & $3.96 \mathrm{E}+02$ & $4.76 \mathrm{E}+01$ & 1 & $0.00 \mathrm{E}+00$ & $1.00 \mathrm{E}+03$ \\
\hline Ag.icp.a & $\mu \mathrm{g} / \mathrm{g}$ & $1.48 \mathrm{E}+01$ & $8.67 \mathrm{E}-01$ & 1 & $3.80 \mathrm{E}+00$ & $2.58 \mathrm{E}+01$ \\
\hline Al.icp.a & $\mu \mathrm{g} / \mathrm{g}$ & $1.95 \mathrm{E}+04$ & $1.10 \mathrm{E}+03$ & 1 & $5.55 \mathrm{E}+03$ & $3.34 \mathrm{E}+04$ \\
\hline Al.icp.f & $\mu \dot{\mathrm{g}} / \mathrm{g}$ & $1.95 \mathrm{E}+04$ & $2.29 \mathrm{E}+03$ & 1 & $0.00 \mathrm{E}+00$ & $4.85 \mathrm{E}+04$ \\
\hline B.icp.a & $\mu \mathrm{g} / \mathrm{g}$ & $1.57 \mathrm{E}+02$ & $2.19 \mathrm{E}+01$ & 1 & $0.00 \mathrm{E}+00$ & $4.35 \mathrm{E}+02$ \\
\hline Ca.icp.a & $\mu \mathrm{g} / \mathrm{g}$ & $1.60 \mathrm{E}+02$ & $2.14 \mathrm{E}+01$ & 1 & $0.00 \mathrm{E}+00$ & $4.32 \mathrm{E}+02$ \\
\hline Cd.icp.a & $\mu \mathrm{g} / \mathrm{g}$ & $5.25 \mathrm{E}+00$ & $1.04 \mathrm{E}+00$ & 1 & $0.00 \mathrm{E}+00$ & $1.84 \mathrm{E}+01$ \\
\hline $\mathrm{Cl}^{-}$.ic & $\mu \mathrm{g} / \mathrm{g}$ & $4.54 \mathrm{E}+03$ & $2.73 \mathrm{E}+02$ & 1 & $1.07 \mathrm{E}+03$ & $8.01 \mathrm{E}+03$ \\
\hline Cr.icp.a & $\mu \mathrm{g} / \mathrm{g}$ & $8.93 \mathrm{E}+02$ & $1.42 \mathrm{E}+02$ & 1 & $0.00 \mathrm{E}+00$ & $2.70 E+03$ \\
\hline Cr.icp.f & $\mu \mathrm{g} / \mathrm{g}$ & $1.34 \mathrm{E}+03$ & $4.05 \mathrm{E}+02$ & 1 & $0.00 \mathrm{E}+00$ & $6.49 \mathrm{E}+03$ \\
\hline Cu.icp.a.1t & $\mu \mathrm{g} / \mathrm{g}$ & $4.89 \mathrm{E}+00$ & $6.57 \mathrm{E}-01$ & 1 & $0.00 \mathrm{E}+00$ & $1.32 \mathrm{E}+01$ \\
\hline Cu.icp.a.nlt & $\mu \mathrm{g} / \mathrm{g}$ & $4.99 \mathrm{E}+00$ & $5.77 \mathrm{E}-01$ & 1 & $0.00 \mathrm{E}+00$ & $1.23 \mathrm{E}+01$ \\
\hline F-ic & $\mu \mathrm{g} / \mathrm{g}$ & $6.04 \mathrm{E}+02$ & $2.24 \mathrm{E}+02$ & 1 & $0.00 \mathrm{E}+00$ & $3.45 \mathrm{E}+03$ \\
\hline Fe.icp.a & $\mu \mathrm{g} / \mathrm{g}$ & $2.54 \mathrm{E}+02$ & $1.45 \mathrm{E}+02$ & 1 & $0.00 \mathrm{E}+00$ & $2.10 \mathrm{E}+03$ \\
\hline Formate.ic & $\mu \mathrm{g} / \mathrm{g}$ & $6.28 \mathrm{E}+02$ & $4.10 \mathrm{E}+01$ & 1 & $0.00 \mathrm{E}+00$ & $1.15 \mathrm{E}+03$ \\
\hline K.icp.a & $\mu \mathrm{g} / \mathrm{g}$ & $3.46 \mathrm{E}+03$ & $1.73 \mathrm{E}+02$ & 1 & $1.26 \mathrm{E}+03$ & $5.66 \mathrm{E}+03$ \\
\hline Mn.icp.a & $\mu \mathrm{g} / \mathrm{g}$ & $1.20 \mathrm{E}+01$ & $3.09 \mathrm{E}+00$ & 1 & $0.00 \mathrm{E}+00$ & $5.12 \mathrm{E}+01$ \\
\hline Mo.icp.a & $\mu \mathrm{g} / \mathrm{g}$ & $4.13 \mathrm{E}+01$ & $2.09 \mathrm{E}+00$ & 1 & $1.47 \mathrm{E}+01$ & $6.78 \mathrm{E}+01$ \\
\hline $\mathrm{NO}_{2} \cdot \mathrm{ic}^{1}$ & $\mu \mathrm{g} / \mathrm{g}$ & $6.57 \mathrm{E}+04$ & $2.19 \mathrm{E}+03$ & 1 & $3.79 \mathrm{E}+04$ & $9.35 \mathrm{E}+04$ \\
\hline $\mathrm{NO}_{3}{ }^{\circ}$.ic & $\mu \mathrm{g} / \mathrm{g}$ & $1.78 \mathrm{E}+05$ & $3.55 \mathrm{E}+04$ & 1 & $0.00 \mathrm{E}+00$ & $6.29 \mathrm{E}+05$ \\
\hline Na.icp.a & $\mu \mathrm{g} / \mathrm{g}$ & $1.94 \mathrm{E}+05$ & $1.45 \mathrm{E}+03$ & 1 & $1.75 \mathrm{E}+05$ & $2.12 \mathrm{E}+05$ \\
\hline Na.icp.f & $\mu \mathrm{g} / \mathrm{g}$ & $2.36 \mathrm{E}+05$ & $6.94 \mathrm{E}+03$ & 1 & $1.48 \mathrm{E}+05$ & $3.24 \mathrm{E}+05$ \\
\hline
\end{tabular}


Table B3-14. Summary Statistics (per g basis) - Crust (nested ANOVA with riser term). (2 sheets)

\begin{tabular}{|c|c|c|c|c|c|c|}
\hline anitue & 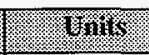 & i. & 8 & if & 14 & (61) \\
\hline Ni.icp.a & $\mu \mathrm{g} / \mathrm{g}$ & $3.16 \mathrm{E}+01$ & $5.34 \mathrm{E}+00$ & 1 & $0.00 \mathrm{E}+00$ & $9.94 \mathrm{E}+01$ \\
\hline Ni.icp.f & $\mu \mathrm{g} / \mathrm{g}$ & $3.81 \mathrm{E}+03$ & $1.17 \mathrm{E}+03$ & 1 & $0.00 \mathrm{E}+00$ & $1.87 \mathrm{E}+04$ \\
\hline Oxalate.ic & $\mu \mathrm{g} / \mathrm{g}$ & $7.59 \mathrm{E}+03$ & $1.08 \mathrm{E}+03$ & 1 & $0.00 \mathrm{E}+00$ & $2.14 \mathrm{E}+04$ \\
\hline P.icp.a & $\mu \mathrm{g} / \mathrm{g}$ & $1.53 \mathrm{E}+03$ & $3.62 \mathrm{E}+02$ & 1 & $0.00 \mathrm{E}+00$ & $6.13 \mathrm{E}+03$ \\
\hline $\mathrm{PO}_{4}^{3-}$.ic & $\mu \mathrm{g} / \mathrm{g}$ & $4.51 \mathrm{E}+03$ & $1.27 \mathrm{E}+03$ & 1 & $0.00 \mathrm{E}+00$ & $2.06 \mathrm{E}+04$ \\
\hline S.icp.a & $\mu \mathrm{g} / \mathrm{g}$ & $2.63 \mathrm{E}+03$ & $4.54 \mathrm{E}+02$ & 1 & $0.00 \mathrm{E}+00$ & $8.40 \mathrm{E}+03$ \\
\hline S.icp.f.it & $\mu \mathrm{g} / \mathrm{g}$ & $3.14 \mathrm{E}+03$ & $6.98 \mathrm{E}+02$ & 1 & $0.00 \mathrm{E}+00$ & $1.20 \mathrm{E}+04$ \\
\hline S.icp.f.nlt & $\mu \mathrm{g} / \mathrm{g}$ & $3.78 \mathrm{E}+03$ & $4.70 \mathrm{E}+02$ & 1 & $0.00 \mathrm{E}+00$ & $9.75 \mathrm{E}+03$ \\
\hline $\mathrm{SO}_{4}{ }^{2 \cdot}$. ic & $\mu \mathrm{g} / \mathrm{g}$ & $7.84 \mathrm{E}+03$ & $2.18 \mathrm{E}+03$ & 1 & $0.00 \mathrm{E}+00$ & $3.56 \mathrm{E}+04$ \\
\hline Si.icp.a & $\mu \mathrm{g} / \mathrm{g}$ & $8.82 \mathrm{E}+02$ & $2.08 \mathrm{E}+01$ & 1 & $6.17 \mathrm{E}+02$ & $1.15 \mathrm{E}+03$ \\
\hline Total Alpha.lt & $\mu \mathrm{Ci} / \mathrm{g}$ & $4.61 \mathrm{E}-02$ & $7.69 \mathrm{E}-03$ & 1 & $0.00 \mathrm{E}+00$ & $1.44 \mathrm{E}-01$ \\
\hline Total Alpha.nlt & $\mu \mathrm{Ci} / \mathrm{g}$ & $4.79 \mathrm{E}-02$ & $1.20 \mathrm{E}-02$ & 1 & $0.00 \mathrm{E}+00$ & $2.01 \mathrm{E}-01$ \\
\hline Zn.icp.a & $\mu \mathrm{g} / \mathrm{g}$ & $2.61 \mathrm{E}+01$ & $2.98 \mathrm{E}+00$ & 1 & $0.00 \mathrm{E}+00$ & $6.40 \mathrm{E}+01$ \\
\hline Zr.icp.a & $\mu \mathrm{g} / \mathrm{g}$ & $1.08 \mathrm{E}+01$ & $1.37 \mathrm{E}+00$ & 1 & $0.00 \mathrm{E}+00$ & $2.82 \mathrm{E}+01$ \\
\hline
\end{tabular}

Note:

${ }^{1} \hat{\sigma}_{\text {riest }}$ is significantly different from zero (evidence of horizontal heterogeneity). 
Table B3-15. Summary Statistics (per g basis) - Crust (nested ANOVA without riser term). (2 sheets)

\begin{tabular}{|c|c|c|c|c|c|c|}
\hline 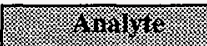 & (1) & (3) & 16 & if & (1) & (1) \\
\hline \% Water.tga & wt\% & $4.52 \mathrm{E}+01$ & $2.89 \mathrm{E}+00$ & 2 & $3.27 \mathrm{E}+01$ & $5.76 \mathrm{E}+01$ \\
\hline Ag.icp.a & $\mu \mathrm{g} / \mathrm{g}$ & $1.48 \mathrm{E}+01$ & $8.67 \mathrm{E}-01$ & 2 & $1.11 \mathrm{E}+01$ & $1.85 \mathrm{E}+01$ \\
\hline Al.icp.a & $\mu \mathrm{g} / \mathrm{g}$ & $1.95 \mathrm{E}+04$ & $1.10 \mathrm{E}+03$ & 2 & $1.48 \mathrm{E}+04$ & $2.42 \mathrm{E}+04$ \\
\hline Al.icp.f & $\mu \mathrm{g} / \mathrm{g}$ & $1.95 \mathrm{E}+04$ & $2.29 \mathrm{E}+03$ & 2 & $9.61 \mathrm{E}+03$ & $2.93 E+04$ \\
\hline B.icp.a & $\mu \mathrm{g} / \mathrm{g}$ & $1.62 \mathrm{E}+02$ & $1.67 \mathrm{E}+01$ & 2 & $9.03 E+01$ & $2.34 \mathrm{E}+02$ \\
\hline Ca.icp.a & $\mu \mathrm{g} / \mathrm{g}$ & $1.60 \mathrm{E}+02$ & $2.14 \mathrm{E}+01$ & 2 & $6.85 E+01$ & $2.52 \mathrm{E}+02$ \\
\hline Cd.icp.a & $\mu \mathrm{g} / \mathrm{g}$ & $5.25 \mathrm{E}+00$ & $1.04 \mathrm{E}+00$ & 2 & $7.88 \mathrm{E}-01$ & $9.72 \mathrm{E}+00$ \\
\hline $\mathrm{Cl}$.ic & $\mu \mathrm{g} / \mathrm{g}$ & $4.50 \mathrm{E}+03$ & $2.28 \mathrm{E}+02$ & 2 & $3.52 \mathrm{E}+03$ & $5.47 \mathrm{E}+03$ \\
\hline Cr.icp.a & $\mu \mathrm{g} / \mathrm{g}$ & $8.93 \mathrm{E}+02$ & $1.42 \mathrm{E}+02$ & 2 & $2.80 \mathrm{E}+02$ & $1.50 \mathrm{E}+03$ \\
\hline Cr.icp.f & $\mu \mathrm{g} / \mathrm{g}$ & $1.27 \mathrm{E}+03$ & $3.43 \mathrm{E}+02$ & 2 & $0.00 \mathrm{E}+00$ & $2.75 \mathrm{E}+03$ \\
\hline Cu.icp.a.It & $\mu \mathrm{g} / \mathrm{g}$ & $4.69 E+00$ & $4.48 \mathrm{E}-01$ & 2 & $2.76 \mathrm{E}+00$ & $6.62 \mathrm{E}+00$ \\
\hline Cu.icp.a.nlt & $\mu \mathrm{g} / \mathrm{g}$ & $4.99 E+00$ & $5.77 \mathrm{E}-01$ & 1 & $0.00 \mathrm{E}+00$ & $1.23 \mathrm{E}+01$ \\
\hline F-ic & $\mu \mathrm{g} / \mathrm{g}$ & $5.39 \mathrm{E}+02$ & $1.59 \mathrm{E}+02$ & 2 & $0.00 \mathrm{E}+00$ & $1.22 \mathrm{E}+03$ \\
\hline Fe.icp.a & $\mu \mathrm{g} / \mathrm{g}$ & $2.58 \mathrm{E}+02$ & $1.41 \mathrm{E}+02$ & 2 & $0.00 \mathrm{E}+00$ & $8.66 \mathrm{E}+02$ \\
\hline Formate.ic & $\mu \mathrm{g} / \mathrm{g}$ & $6.28 \mathrm{E}+02$ & $4.10 \mathrm{E}+01$ & 2 & $4.51 \mathrm{E}+02$ & $8.04 \mathrm{E}+02$ \\
\hline K.icp.a & $\mu \mathrm{g} / \mathrm{g}$ & $3.46 \mathrm{E}+03$ & $1.73 \mathrm{E}+02$ & 2 & $2.71 \mathrm{E}+03$ & $4.21 E+03$ \\
\hline Li.icp.a.lt & $\mu \mathrm{g} / \mathrm{g}$ & $1.82 \mathrm{E}+01$ & $1.39 \mathrm{E}+01$ & 2 & $0.00 \mathrm{E}+00$ & $7.81 \mathrm{E}+01$ \\
\hline Li.icp.a.nlt & $\mu \mathrm{g} / \mathrm{g}$ & $4.61 \mathrm{E}+01$ & $9.50 \mathrm{E}-01$ & 1 & $3.40 \mathrm{E}+01$ & $5.81 \mathrm{E}+01$ \\
\hline Mn.icp.a & $\mu \mathrm{g} / \mathrm{g}$ & $1.20 \mathrm{E}+01$ & $3.09 \mathrm{E}+00$ & 2 & $0.00 \mathrm{E}+00$ & $2.53 \mathrm{E}+01$ \\
\hline Mo.icp.a & $\mu \mathrm{g} / \mathrm{g}$ & $4.13 \mathrm{E}+01$ & $2.09 \mathrm{E}+00$ & 2 & $3.23 \mathrm{E}+01$ & $5.03 \mathrm{E}+01$ \\
\hline $\mathrm{NO}_{3}^{-}$.'ic & $\mu \mathrm{g} / \mathrm{g}$ & $1.78 \mathrm{E}+05$ & $3.55 \mathrm{E}+04$ & 2 & $2.55 \mathrm{E}+04$ & $3.31 \mathrm{E}+05$ \\
\hline Na.icp.a & $\mu \mathrm{g} / \mathrm{g}$ & $1.94 \mathrm{E}+05$ & $1.45 \mathrm{E}+03$ & 2 & $1.88 \mathrm{E}+05$ & $2.00 \mathrm{E}+05$ \\
\hline Na.icp.f & $\mu \mathrm{g} / \mathrm{g}$ & $2.35 \mathrm{E}+05$ & $5.49 \mathrm{E}+03$ & 2 & $2.11 \mathrm{E}+05$ & $2.58 \mathrm{E}+05$ \\
\hline Ni.icp.a & $\mu \mathrm{g} / \mathrm{g}$ & $3.16 \mathrm{E}+01$ & $5.34 \mathrm{E}+00$ & 2 & $8.59 \mathrm{E}+00$ & $5.45 \mathrm{E}+01$ \\
\hline Ni.icp.f & $\mu \mathrm{g} / \mathrm{g}$ & $3.50 \mathrm{E}+03$ & $7.86 \mathrm{E}+02$ & 2 & $1.13 \mathrm{E}+02$ & $6.88 \mathrm{E}+03$ \\
\hline Oxalate.ic & $\mu \mathrm{g} / \mathrm{g}$ & $7.59 \mathrm{E}+03$ & $1.08 \mathrm{E}+03$ & 2 & $2.93 E+03$ & $1.23 \mathrm{E}+04$ \\
\hline P.icp.a & $\mu \mathrm{g} / \mathrm{g}$ & $1.44 \mathrm{E}+03$ & $2.68 \mathrm{E}+02$ & 2 & $2.81 \mathrm{E}+02$ & $2.59 E+03$ \\
\hline $\mathrm{PO}_{4}^{3-}$.ic & $\mu \mathrm{g} / \mathrm{g}$ & $4.10 \mathrm{E}+03$ & $8.58 \mathrm{E}+02$ & 2 & $4.03 \mathrm{E}+02$ & $7.79 \mathrm{E}+03$ \\
\hline S.icp.a & $\mu \mathrm{g} / \mathrm{g}$ & $2.63 \mathrm{E}+03$ & $4.54 \mathrm{E}+02$ & 2 & $6.75 \mathrm{E}+02$ & $4.58 \mathrm{E}+03$ \\
\hline S.icp.f.lt & $\mu \mathrm{g} / \mathrm{g}$ & $3.14 \mathrm{E}+03$ & $6.98 \mathrm{E}+02$ & 2 & $1.32 \mathrm{E}+02$ & $6.14 \mathrm{E}+03$ \\
\hline
\end{tabular}


Table B3-15. Summary Statistics (per g basis) - Crust (nested ANOVA without riser term). (2 sheets)

\begin{tabular}{|c|c|c|c|c|c|c|}
\hline Nitiryt) & (6.mits & 12 & (4) & (19 & Y1. & (6) \\
\hline S.icp.f.nlt & $\mu \mathrm{g} / \mathrm{g}$ & $3.78 \mathrm{E}+03$ & $4.70 \mathrm{E}+02$ & 1 & $0.00 \mathrm{E}+00$ & $9.75 \mathrm{E}+03$ \\
\hline $\mathrm{SO}_{4}^{2-}$.ic & $\mu \mathrm{g} / \mathrm{g}$ & $7.84 \mathrm{E}+03$ & $2.18 \mathrm{E}+03$ & 2 & $0.00 \mathrm{E}+00$ & $1.72 \mathrm{E}+04$ \\
\hline Si.icp.a & $\mu \mathrm{g} / \mathrm{g}$ & $8.82 \mathrm{E}+02$ & $2.08 \mathrm{E}+01$ & 2 & $7.92 \mathrm{E}+02$ & $9.72 \mathrm{E}+02$ \\
\hline Total Alpha.It & $\mu \mathrm{Ci} / \mathrm{g}$ & $4.61 \mathrm{E}-02$ & $7.69 \mathrm{E}-03$ & 2 & $1.30 \mathrm{E}-02$ & $7.92 \mathrm{E}-02$ \\
\hline Total Alpha.nlt & $\mu \mathrm{Ci} / \mathrm{g}$ & $4.79 \mathrm{E}-02$ & $1.20 \mathrm{E}-02$ & 1 & $0.00 \mathrm{E}+00$ & $2.01 \mathrm{E}-01$ \\
\hline Zn.icp.a & $\mu \mathrm{g} / \mathrm{g}$ & $2.55 \mathrm{E}+01$ & $2.38 \mathrm{E}+00$ & 2 & $1.52 \mathrm{E}+01$ & $3.57 \mathrm{E}+01$ \\
\hline Zr.icp.a & $\mu \mathrm{g} / \mathrm{g}$ & $1.08 \mathrm{E}+01$ & $1.37 \mathrm{E}+00$ & 2 & $4.88 \mathrm{E}+00$ & $1.67 \mathrm{E}+01$ \\
\hline
\end{tabular}

Table B3-16. Analytes (Crust - per g basis) with $>50$ Percent "Less Than" Values. (2 sheets)

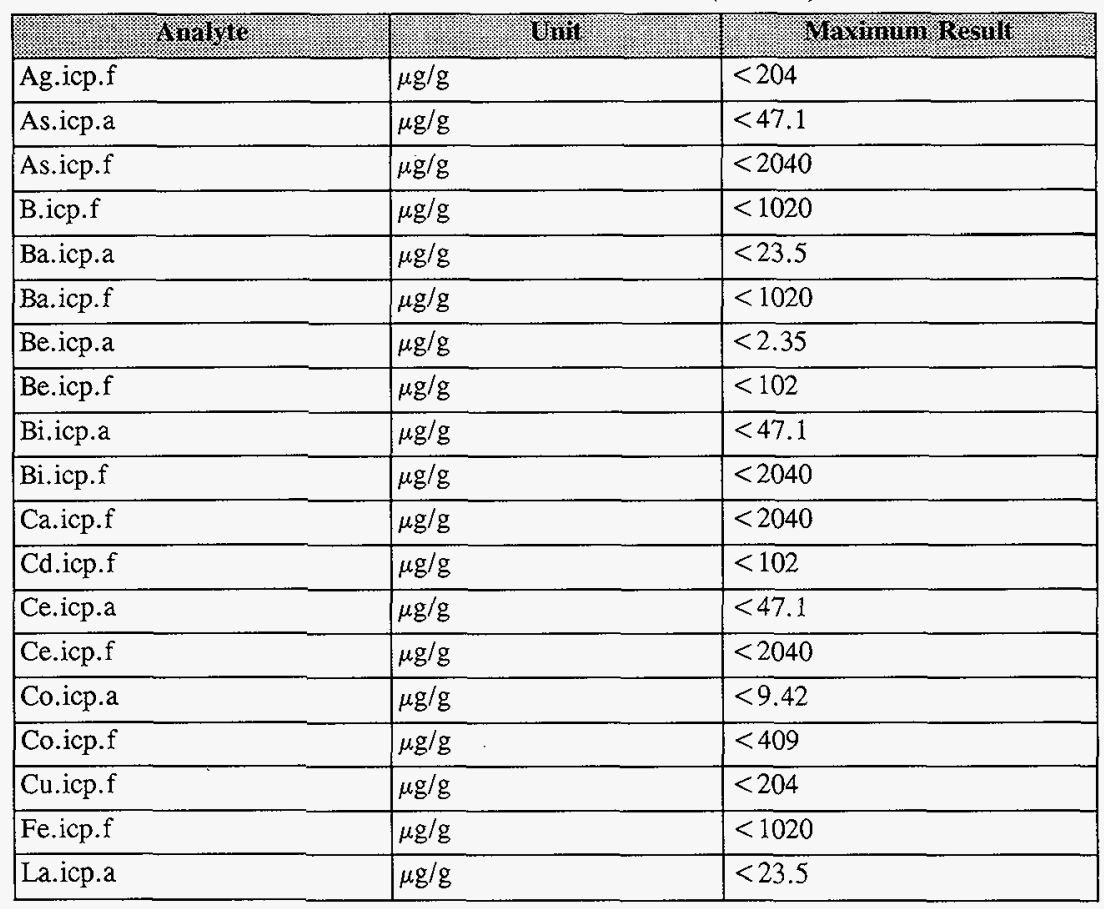


Table B3-16. Analytes (Crust - per g basis) with

$>50$ Percent "Less Than" Values. (2 sheets)

\begin{tabular}{|l|l|l|}
\hline & & \\
\hline La.icp.f & $\mu \mathrm{g} / \mathrm{g}$ & $<1020$ \\
\hline Li.icp.f & $\mu \mathrm{g} / \mathrm{g}$ & $<204$ \\
\hline Mg.icp.a & $\mu \mathrm{g} / \mathrm{g}$ & $<47.1$ \\
\hline Mg.icp.f & $\mu \mathrm{g} / \mathrm{g}$ & $<2040$ \\
\hline Mn.icp.f & $\mu \mathrm{g} / \mathrm{g}$ & $<204$ \\
\hline Mo.icp.f & $\mu \mathrm{g} / \mathrm{g}$ & $<1020$ \\
\hline Nd.icp.a & $\mu \mathrm{g} / \mathrm{g}$ & $<47.1$ \\
\hline Nd.icp.f & $\mu \mathrm{g} / \mathrm{g}$ & $<2040$ \\
\hline P.icp.f & $\mu \mathrm{g} / \mathrm{g}$ & $<4090$ \\
\hline Pb.icp.a & $\mu \mathrm{g} / \mathrm{g}$ & $<47.1$ \\
\hline Pb.icp.f & $\mu \mathrm{g} / \mathrm{g}$ & $<2040$ \\
\hline Sb.icp.a & $\mu \mathrm{g} / \mathrm{g}$ & $<28.3$ \\
\hline Sb.icp.f & $\mu \mathrm{g} / \mathrm{g}$ & $<1230$ \\
\hline Se.icp.a & $\mu \mathrm{g} / \mathrm{g}$ & $<47.1$ \\
\hline Se.icp.f & $\mu \mathrm{g} / \mathrm{g}$ & $<2040$ \\
\hline Si.icp.f & $\mu \mathrm{g} / \mathrm{g}$ & $<1180$ \\
\hline Sm.icp.a & $\mu \mathrm{g} / \mathrm{g}$ & $<47.1$ \\
\hline Sm.icp.f & $\mu \mathrm{g} / \mathrm{g}$ & $<2040$ \\
\hline Sr.icp.a & $\mu \mathrm{g} / \mathrm{g}$ & $<4.71$ \\
\hline Sr.icp.f & $\mu \mathrm{g} / \mathrm{g}$ & $<204$ \\
\hline Ti.icp.a & $\mu \mathrm{g} / \mathrm{g}$ & $<4.71$ \\
\hline Ti.icp.f & $\mu \mathrm{g} / \mathrm{g}$ & $<204$ \\
\hline Tl.icp.a & $\mu \mathrm{g} / \mathrm{g}$ & $<94.2$ \\
\hline Tl.icp.f & $\mu \mathrm{g} / \mathrm{g}$ & $<4090$ \\
\hline U.icp.a & $\mu \mathrm{g} / \mathrm{g}$ & $<235$ \\
\hline U.icp.f & $\mu \mathrm{g} / \mathrm{g}$ & $<10200$ \\
\hline V.icp.a & $\mu \mathrm{g} / \mathrm{g}$ & $<23.5$ \\
\hline V.icp.f & $\mu \mathrm{g} / \mathrm{g}$ & $<1020$ \\
\hline Zn.icp.f & $\mu \mathrm{g} / \mathrm{g}$ & $<466$ \\
\hline Zr.icp.f & $\mu \mathrm{g} / \mathrm{g}$ & $<204$ \\
\hline & & \\
\hline
\end{tabular}




\section{B3.4.2 Analysis of Variance Model}

A statistical model is needed to account for the spatial and measurement variability in $\hat{\sigma}_{\hat{\mu}}$. This cannot be done using an ordinary standard deviation of the data (Snedecor and Cochran 1980).

B3.4.2.1 Supernate. The data were statistically evaluated using two models. The first model used a nested analysis of variance. The nested analysis of variance statistical model used to describe the structure of the data is

where

$$
\begin{gathered}
Y_{i j k}=\mu+R_{i}+S_{i j}+A_{i j k}, \\
i=1,2, \ldots, a ; j=1,2, \ldots, b_{i} ; k=1,2, \ldots, n_{i j} ;
\end{gathered}
$$

$$
\begin{aligned}
& \mathrm{Y}_{\mathrm{ijk}} \quad=\quad \text { concentration from the } \mathrm{k}^{\mathrm{th}} \text { analytical result from the } \mathrm{j}^{\text {th }} \text { segment } \\
& \text { from the } i^{\text {th }} \text { riser } \\
& \mu \quad=\quad \text { the grand mean } \\
& \mathbf{R}_{\mathrm{i}} \quad=\quad \text { the effect of the } \mathrm{i}^{\mathrm{th}} \text { riser } \\
& \mathrm{S}_{\mathrm{ij}} \quad=\quad \text { the effect of the } \mathrm{j}^{\mathrm{th}} \text { segment from the } \mathrm{i}^{\mathrm{th}} \text { riser } \\
& \mathrm{A}_{\mathrm{ijk}} \quad=\quad \text { the effect of the } \mathrm{k}^{\text {th }} \text { analytical result from the } \mathrm{j}^{\text {th }} \text { segment from } \\
& \text { the } i^{\text {th }} \text { riser } \\
& \text { a }=\text { the number of risers } \\
& b_{i} \quad=\quad \text { the number of segments from the } i^{\text {th }} \text { riser } \\
& n_{\mathrm{ij}} \quad=\quad \text { the number of analytical results from the } \mathrm{j}^{\text {th }} \text { segment from the } \\
& i^{\text {th }} \text { riser. }
\end{aligned}
$$

The variables $R_{i}$ and $S_{i j}$ are assumed to be a random effects. These variables and $A_{i j k}$ are assumed to be uncorrelated and normally distributed with means zero and variances $\sigma^{2}(R)$, $\sigma^{2}(\mathrm{~S})$, and $\sigma^{2}(\mathrm{~A})$, respectively. Estimates of $\sigma^{2}(\mathrm{R}), \sigma^{2}(\mathrm{~S})$, and $\sigma^{2}(\mathrm{~A})$ were obtained using REML methods. This method applied to variance component estimation is described in Harville (1977). The results using the REML methods were obtained using the statistical analysis package S-PLUS ${ }^{1}$ (Statistical Sciences 1993). The $d f$ associated with the standard deviation of the mean (a function of $\sigma^{2}(R), \sigma^{2}(S)$, and $\sigma^{2}(A)$ ) is the number of risers minus one.

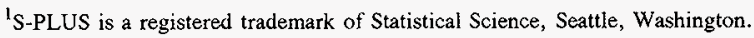


The second model used one-way analysis of variance. The one-way analysis of variance statistical model used to describe the structure of the data is

where

$$
\begin{gathered}
\mathrm{Y}_{\mathrm{ij}}=\mu+\mathrm{S}_{\mathrm{i}}+\mathrm{A}_{\mathrm{ij}}, \\
\mathrm{i}=1,2, \ldots, \mathrm{a}, \mathrm{j}=1,2, \ldots, \mathrm{n}_{\mathrm{i}},
\end{gathered}
$$

$\begin{array}{lll}\mathrm{Y}_{\mathrm{ij}} & = & \text { concentration from the } j^{\text {th }} \text { analytical result from the } \mathrm{i}^{\text {th }} \text { sample } \\ \mu & = & \text { the grand mean } \\ \mathrm{S}_{\mathrm{i}} & = & \text { the effect of the } \mathrm{i}^{\text {th }} \text { sample } \\ \mathrm{A}_{\mathrm{ij}} & = & \text { the effect of the } \mathrm{j}^{\text {th }} \text { analytical result from the } \mathrm{i}^{\text {th }} \text { sample } \\ \mathrm{a} & = & \text { the number of samples } \\ \mathrm{n}_{\mathrm{i}} & = & \text { the number of analytical results from the } \mathrm{i}^{\text {th }} \text { sample. }\end{array}$

The variable $S_{i}$ is assumed to be a random effect. This variable and $A_{i j}$ are assumed to be uncorrelated and normally distributed with means zero and variances $\sigma^{2}(S)$ and $\sigma^{2}(A)$, respectively. Estimates of $\sigma^{2}(\mathrm{~S})$ and $\sigma^{2}(\mathrm{~A})$ were obtained using REML methods. The results were obtained using the statistical analysis package S-PLUS ${ }^{\circledR}$ (Statistical Sciences 1993). The $d f$ associated with the standard deviation of the mean (a function of $\sigma^{2}(\mathrm{~S})$ and $\sigma^{2}(\mathrm{~A})$ ) is the number of samples minus one.

B3.4.2.2 Salt Slurry and Crust. The data were statistically evaluated using two models. The first model used a nested analysis of variance with a riser term. The nested analysis of variance statistical model used to describe the structure of the data is

where

$$
\begin{gathered}
\mathrm{Y}_{\mathrm{ijkl}}=\mu+\mathrm{R}_{\mathrm{i}}+\mathrm{S}_{\mathrm{ij}}+\mathrm{L}_{\mathrm{ijk}}+\mathrm{A}_{\mathrm{ijk} \mathbf{l},} \\
\mathrm{i}=1,2, \ldots, \mathrm{a} ; \mathrm{j}=1,2, \ldots, \mathrm{b}_{\mathrm{i}} ; \mathrm{k}=1,2, \ldots, \mathrm{c}_{\mathrm{ij} ;} ; \mathrm{l}=1,2, \ldots, \mathrm{n}_{\mathrm{ijk}} ;
\end{gathered}
$$

$$
\begin{aligned}
& Y_{\mathrm{ijk} 1}=\quad \text { concentration from the } \mathrm{l}^{\mathrm{th}} \text { analytical result from the } \mathrm{k}^{\text {th }} \text { location } \\
& \text { from the } \mathrm{j}^{\text {th }} \text { segment from the } \mathrm{i}^{\text {th }} \text { riser } \\
& \mu \quad=\quad \text { the grand mean } \\
& \mathrm{R}_{\mathrm{i}} \quad=\quad \text { the effect of the } \mathrm{i}^{\text {th }} \text { riser } \\
& \mathrm{S}_{\mathrm{ij}}=\text { the effect of the } \mathrm{j}^{\text {th }} \text { segment from the } \mathrm{i}^{\text {th }} \text { riser } \\
& \mathrm{L}_{\mathrm{ijk}}=\quad \text { the effect of the } \mathrm{k}^{\mathrm{th}} \text { location from the } \mathrm{j}^{\text {th }} \text { segment from the } \\
& i^{\text {th }} \text { riser }
\end{aligned}
$$




\begin{tabular}{|c|c|c|}
\hline $\mathrm{A}_{\mathrm{ijk \textrm {i }}}$ & $=$ & $\begin{array}{l}\text { the effect of the } \mathrm{l}^{\text {th }} \text { analytical result from the } \mathrm{k}^{\text {th }} \text { location from } \\
\text { the } \mathrm{j}^{\text {th }} \text { segment from the } \mathrm{i}^{\text {th }} \text { riser }\end{array}$ \\
\hline a & $=$ & the number of risers \\
\hline$b_{i}$ & $=$ & the number of segments from the $i^{\text {th }}$ riser \\
\hline$c_{i j}$ & $=$ & the number of locations from the $j^{\text {th }}$ segment from the $i^{\text {th }}$ riser \\
\hline $\mathrm{n}_{\mathrm{ijk}}$ & $=$ & $\begin{array}{l}\text { the number of analytical results from the } \mathrm{k}^{\mathrm{th}} \text { location from the } \mathrm{j}^{\mathrm{th}} \\
\text { segment from the } \mathrm{i}^{\mathrm{th}} \text { riser. }\end{array}$ \\
\hline
\end{tabular}

The variables $R_{i}, S_{i j}$, and $L_{i j k}$ are assumed to be a random effects. These variables and $A_{i j k l}$ are assumed to be uncorrelated and normally distributed with means zero and variances $\sigma^{2}(\mathrm{R}), \sigma^{2}(\mathrm{~S}), \sigma^{2}(\mathrm{~L})$, and $\sigma^{2}(\mathrm{~A})$, respectively. Estimates of $\sigma^{2}(\mathrm{R}), \sigma^{2}(\mathrm{~S}), \sigma^{2}(\mathrm{~L})$, and $\sigma^{2}(\mathrm{~A})$ were obtained using REML methods. This method applied to variance component estimation is described in Harville (1977). The results using the REML methods were obtained using the statistical analysis package S-PLUS ${ }^{\circledR}$ (Statistical Sciences 1993). The $d f$ associated with the standard deviation of the mean (a function of $\sigma^{2}(\mathrm{R}), \sigma^{2}(\mathrm{~S}), \sigma^{2}(\mathrm{~L})$, and $\sigma^{2}(\mathrm{~A})$ ) is the number of risers minus one.

The second model used nested analysis of variance without the riser term. This nested ANOVA model is

where

$$
\begin{gathered}
Y_{i j k}=\mu+S_{i}+L_{i j}+A_{i j k}, \\
i=1,2, \ldots, a ; j=1,2, \ldots, b_{i} ; k=1,2, \ldots, n_{i j} ;
\end{gathered}
$$

$\begin{array}{ll}Y_{i j k} & =\quad \begin{array}{l}\text { concentration from the } k^{\text {th }} \text { analytical result from the } j^{\text {th }} \text { location } \\ \text { from the } i^{\text {th }} \text { segment }\end{array} \\ \mu & =\text { the grand mean } \\ S_{i} & =\text { the effect of the } i^{\text {th }} \text { segment } \\ L_{i j} & =\quad \text { the effect of the } j^{\text {th }} \text { location from the } i^{\text {th }} \text { segment } \\ A_{i j k} & \text { the } i^{\text {th }} \text { segment }\end{array}$




$$
\begin{array}{ll}
\mathrm{n}_{\mathrm{ij}} & =\quad \begin{array}{l}
\text { the number of analytical results from the } \mathrm{j}^{\mathrm{th}} \text { location from the } \\
\mathrm{i}^{\text {th }} \text { segment. }
\end{array}
\end{array}
$$

The variables $S_{i}$ and $L_{i j}$ are assumed to be a random effects. These variables and $A_{i j k}$ are assumed to be uncorrelated and normally distributed with means zero and variances $\sigma^{2}(S)$, $\sigma^{2}(\mathrm{~L})$, and $\sigma^{2}(\mathrm{~A})$, respectively. Estimates of $\sigma^{2}(\mathrm{~S}), \sigma^{2}(\mathrm{~L})$, and $\sigma^{2}(\mathrm{~A})$ were obtained using REML methods. This method applied to variance component estimation is described in Harville (1977). The results using the REML methods were obtained using the statistical analysis package S-PLUS ${ }^{\circledR}$ (Statistical Sciences 1993). The $d f$ associated with the standard deviation of the mean (a function of $\sigma^{2}(\mathrm{~S}), \sigma^{2}(\mathrm{~L})$, and $\sigma^{2}(\mathrm{~A})$ ) is the number of segments minus one.

\section{B4.0 APPENDIX B REFERENCES}

Bechtold, D. B., 1995, Use of LiBr as Tracer for HHF and Correction of Results, "internal memorandum 75980-PCS95-045 to J. Jo, May 13), Westinghouse Hanford Company, Richland, Washington.

Dukelow, G. T., J. W. Hunt, H. Babad, and J. E. Meacham, 1995, Tank Safety Screening Data Quality Objective, WHC-SD-WM-SP-004, Rev. 2, Westinghouse Hanford Company, Richland, Washington.

Harville, D. A., 1977, "Maximum Likelihood Approaches to Variance Component Estimation and to Related Problems," Journal of the American Statistical Association, pp. 320-340.

Jansky, M. T., 1984a, Laboratory for Tank Farm Samples from Tanks 103 and 104 AN, (internal letter 65453-84-164 to P.J. Certa, July 2), Rockwell Hanford Operation, Richland, Washington.

Jansky, M. T., 1984b, Laboratory Support for Upcoming 242-A Evaporator Campaign, (internal memorandum 65453-84-134 to E. G. Gratny, May 10), Rockwell Hanford Operation, Richland, Washington.

Kristofzski, J. G., 1996, Directions for "Opportunistic Analyses," (memorandum 75310-96 to J. H. Baldwin et al., September 11), Westinghouse Hanford Company, Richland, Washington.

Mauss, B. M., 1984a, Chemical Compositions of 102-AY, 101-AW, 105-AN and 104-AW, (internal memorandum 65453-84-348 to E. G. Gratny, November 9), Rockwell Hanford Operation, Richland, Washington. 
Mauss, B. M., and M. T. Jansky, 1985, 102-AW Laboratory Boildowns in Support of Evaporator Run 84-5, (internal memorandum 65453-85-013 to E. G. Gratny and N. L. Pontious, January 18), Rockwell Hanford Operation, Richland, Washington.

McDuffie, N. G., and G. D Johnson, 1995, Flammable Gas Tank Safety Program: Data Requirements for Core Sample Analysis Developed Through the Data Quality Objectives (DQO) Process, WHC-SD-WM DQO-004, Rev. 2, Westinghouse Hanford Company, Richland, Washington.

Shekarriz, A., D. R. Rector, N. S. Cannon, L. A. Mahoney, B. E. Hey, M. A. Chieda, C. G. Linshooten, J. M. Bates, F. J. Reitz, R. E. Bauer, and E. R. Siciliano, 1997, Composition and Quantities of Retained Gas Measured in Hanford Waste Tanks 241-AW-101, $A-101, A N-105, A N-104$, and $A N-103$, PNNL-11450, Rev. 1, Pacific Northwest National Laboratory, Richland, Washington.

Stewart, C. W., J. M. Alzheimer, M. E. Brewster, G. Chen, R. E. Mendoza, H. C. Reid, C. L. Shepard, and G. Terrones, 1996, In Situ Rheology and Gas Volume in Hanford Double-shell Waste Tanks, PNNL-11296, Pacific Northwest National Laboratory, Richland, Washington.

Snedecor, G. W., and W. G. Cochran, 1980, Statistical Methods, 7th Edition, Iowa State University Press, Ames, lowa.

Statistical Sciences, Inc., 1993, S-PLUS Reference Manual, Version 3.2, StatSci, (a division of MathSoft, Inc.), Statistical Sciences, Inc., Seattle, Washington.

Steen, F. H., 1997, Final Report for Tank 24l-AN-104, Cores 163 and 164, HNF-SD-WM-DP-226, Rev. 1A, Rust Federal Services of Hanford, Inc. for Fluor Daniel Hanford Inc, Richland, Washington.

Winkelman, W. D., 1996a, Tank 241-AN-104 Push Mode Core Sampling and Analysis Plan, WHC-SD-WM-TSAP-086, Rev. 0, Westinghouse Hanford Company, Richland, Washington.

Winkelman, W. D., 1996b, Technical Basis and Spreadsheet Documentation for Correcting Waste Tank Core Samples for Water Intrusion Based on a LiBr Tracer, WHC-SD-WM-CSWD-081, Rev. 0, Westinghouse Hanford Company, Richland, Washington. 
HNF-SD-WM-ER-690 Rev. 0

APPENDIX C

STATISTICAL ANALYSIS FOR ISSUE RESOLUTION

C-1 
HNF-SD-WM-ER-690 Rev. 0

This page intentionally left blank. 


\section{APPENDIX C}

\section{STATISTICAL ANALYSIS FOR ISSUE RESOLUTION}

Appendix C reports the analysis results of the safety screening DQO (Dukelow et al. 1995) as it relates to tank 241-AN-104. Specifically, confidence intervals were needed to support the DSC and plutonium (criticality) threshold limits.

\section{C1.0 STATISTICS FOR SAFETY SCREENING DATA QUALITY OBJECTIVES}

The safety screening DQO (Dukelow et al, 1995) defines acceptable decision confidence limits in terms of one-sided 95 percent confidence intervals. The confidence limits are calculated from the 1996 sampling event for tank 241-AN-104.

Confidence intervals were computed for each sample number from tank 241-AN-104 analytical data (Steen 1997). The UL of a one-sided 95 percent Clfor the mean is

$$
\hat{\mu}+t_{(\mathrm{df}, 0.05)} * \hat{\sigma}_{\hat{\mu}} .
$$

In this equation, $\hat{\mu}$ is the arithmetic mean of the data, $\hat{\sigma}_{\hat{\mu}}$ is the estimate of the standard deviation of the mean, and $\mathrm{t}_{(\mathrm{dr}, 0.05)}$ is the quantile from Student's $t$ distribution with $d f$ degrees of freedom for a one-sided 95 percent CI. For tank 241-AN-104 data (per sample number), $d f$ equals the number of observations minus one.

\section{C1.1 DIFFERENTIAL SCANNING CALORIMETRY IN DRY}

The UL of the 95 percent $\mathrm{CI}$ was calculated for each subsample that had DSC measurements. The UL for each sample number is listed in Table Cl-1. Each $\mathrm{CI}$ can be used to make the following statement. If the UL is less than $480 \mathrm{~J} / \mathrm{g}$ dry, reject the null hypothesis that DSC is greater than or equal to $480 \mathrm{~J} / \mathrm{g}$ dry at the 0.05 level of significance. All but one DSC dry upper limit was less than $480 \mathrm{~J} / \mathrm{g}$ dry. Thus, the hypothesis that the DSC results are greater than $480 \mathrm{~J} / \mathrm{g}$ dry is rejected for all subsamples except core 163 , segment 16 , upper half.

\section{C1.2 TOTAL ALPHA}

The UL of the 95 percent CI was calculated for each subsample that had at least 50 percent of the reported total alpha data as quantitative values. The sample numbers and the UL of the 95 percent $\mathrm{CI}$ are listed in Table $\mathrm{C} 1-1$. Each $\mathrm{Cl}$ can be used to make the following statement. If the UL is less than $61.5 \mu \mathrm{Ci} / \mathrm{mL}$ or $38.4 \mu \mathrm{Ci} / \mathrm{g}$, reject the null hypothesis that 
total alpha is greater than or equal to $61.5 \mu \mathrm{Ci} / \mathrm{mL}$ (or $38.4 \mu \mathrm{Ci} / \mathrm{g}$ ) at the 0.05 level of significance. For all subsamples with at least 50 percent of the reported total alpha data as quantitative values, the UL were less than $61.5 \mu \mathrm{Ci} / \mathrm{mL}$ or $38.4 \mu \mathrm{Ci} / \mathrm{g}$. In subsamples where both analytical results were "less than" values, the maximum "less than" value is orders of magnitude smaller than either $61.5 \mu \mathrm{Ci} / \mathrm{mL}$ or $38.4 \mu \mathrm{Ci} / \mathrm{g}$. Thus, the hypothesis that Total alpha results are greater than $61.5 \mu \mathrm{Ci} / \mathrm{mL}$ or $38.4 \mu \mathrm{Ci} / \mathrm{g}$ is rejected for all subsamples.

Table C1-1. Tank 241-AN-104 Differential Scanning Calorimetry Results (J/g dry). (2 sheets)

\begin{tabular}{|c|c|c|c|c|c|c|}
\hline Wannple Minnop & 6. & segnent & 1691601 & 10. & . & 16is \\
\hline S96T005256 & 163 & 2 & $\mathrm{DL}$ & $0.00 E+00$ & $0.00 \mathrm{E}+00$ & $0.00 \mathrm{E}+00$ \\
\hline$\$ 96 \mathrm{~T} 005260$ & 163 & 4 & DL & $1.53 \mathrm{E}+01$ & $1.53 \mathrm{E}+01$ & $1.12 \mathrm{E}+02$ \\
\hline$\$ 967005527$ & 163 & 5 & $\overline{\mathrm{DL}}$ & $6.90 E+01$ & $1.12 \mathrm{E}+01$ & $1.39 \mathrm{E}+02$ \\
\hline S96'T005554 & 163 & 6 & $\overline{\mathrm{DL}}$ & $0.00 \mathrm{E}+00$ & $0.00 \mathrm{E}+00$ & $0.00 \mathrm{E}+00$ \\
\hline S96T005255 & 163 & 7 & $\overline{\mathrm{DL}}$ & $0.00 \mathrm{E}+00$ & $0.00 \mathrm{E}+00$ & $0.00 \mathrm{E}+00$ \\
\hline S96T005257 & 163 & 8 & $\overline{\mathrm{DL}}$ & $8.75 E+00$ & $8.75 E+00$ & $6.40 \mathrm{E}+01$ \\
\hline S96T005258 & 163 & 9 & $\mathrm{DL}$ & $1.53 \mathrm{E}+01$ & $1.53 \mathrm{E}+01$ & $1.12 \mathrm{E}+02$ \\
\hline S96T005528 & 163 & 10 & $\mathrm{DL}$ & $4.10 \mathrm{E}+01$ & $4.10 \mathrm{E}+01$ & $3.00 E+02$ \\
\hline \$96T005739 & 163 & 11 & $\overline{\mathrm{DL}}$ & $2.75 \mathrm{E}+01$ & $2.75 \mathrm{E}+01$ & $2.01 \mathrm{E}+02$ \\
\hline S96T005766 & 163 & $\overline{12}$ & $\overline{\mathrm{DL}}$ & $0.00 \mathrm{E}+00$ & $0.00 \mathrm{E}+00$ & $0.00 \mathrm{E}+00$ \\
\hline S96T005741 & 163 & 14 & $\overline{\mathrm{DL}}$ & $1.51 \mathrm{E}+01$ & $1.51 \mathrm{E}+01$ & $1.10 \mathrm{E}+02$ \\
\hline S96T004774 & 164 & 1 & $\mathrm{DL}$ & $0.00 \mathrm{E}+00$ & $0.00 \mathrm{E}+00$ & $0.00 \mathrm{E}+00$ \\
\hline S96T004778 & 164 & 2 & $\mathrm{DL}$ & $0.00 \mathrm{E}+00$ & $0.00 \mathrm{E}+00$ & $0.00 \mathrm{E}+00$ \\
\hline \$96T004779 & 164 & 3 & DL & $0.00 \mathrm{E}+00$ & $0.00 \mathrm{E}+00$ & $0.00 \mathrm{E}+00$ \\
\hline S96T004780 & 164 & 4 & $\mathrm{DL}$ & $1.63 \mathrm{E}+01$ & $1.63 \mathrm{E}+01$ & $1.19 \mathrm{E}+02$ \\
\hline S96T004976 & 164 & 5 & $\mathrm{DL}$ & $0.00 E+00$ & $0.00 \mathrm{E}+00$ & $0.00 \mathrm{E}+00$ \\
\hline S96T004781 & 164 & 7 & DL & $0.00 \mathrm{E}+00$ & $0,00 \mathrm{E}+00$ & $0.00 \mathrm{E}+00$ \\
\hline S96T004977 & 164 & 8 & $\mathrm{DL}$ & $0.00 \mathrm{E}+00$ & $0.00 \mathrm{E}+00$ & $0.00 \mathrm{E}+00$ \\
\hline S96T004978 & 164 & 9 & $\mathrm{DL}$ & $0.00 \mathrm{E}+00$ & $0.00 \mathrm{E}+00$ & $0.00 E+00$ \\
\hline S96T004979 & 164 & 10 & $\mathrm{DL}$ & $0.00 \mathrm{E}+00$ & $0.00 \mathrm{E}+00$ & $0.00 \mathrm{E}+00$ \\
\hline S96T004782 & 164 & 11 & $\overline{D L}$ & $0.00 \mathrm{E}+00$ & $0.00 \mathrm{E}+00$ & $0.00 \mathrm{E}+00$ \\
\hline S96T004783 & 164 & 12 & $\mathrm{DL}$ & $0.00 \mathrm{E}+00$ & $0.00 \mathrm{E}+00$ & $0.00 \mathrm{E}+00$ \\
\hline S96T004784 & 164 & 13 & $\overline{\mathrm{DL}}$ & $0.00 \mathrm{E}+00$ & $0.00 \mathrm{E}+00$ & $0.00 \mathrm{E}+00$ \\
\hline S96T005978 & 164 & COMP & $\overline{--}$ & $3.88 \mathrm{E}+01$ & $9.00 \mathrm{E}-01$ & $4.45 \mathrm{E}+01$ \\
\hline
\end{tabular}


HNF-SD-WM-ER-690 Rev. 0

Table C1-1. Tank 241-AN-104 Differential Scanning Calorimetry Results (J/g dry). (2 sheets)

\begin{tabular}{|c|c|c|c|c|c|c|}
\hline 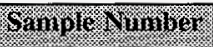 & 8698 & oerimould & $18 \%$ 16\% & \% & 8 & 14 \\
\hline S96T005269 & 163 & 1 & $\overline{\mathrm{LH}}$ & $0.00 \mathrm{E}+00$ & $0.00 \mathrm{E}+00$ & $0.00 \mathrm{E}+00$ \\
\hline \$96T005270 & 163 & 2 & LH & $0.00 \mathrm{E}+00$ & $0.00 \mathrm{E}+00$ & $0.00 \mathrm{E}+00$ \\
\hline S96T005771 & 163 & 12 & LH & $5.43 \mathrm{E}+01$ & $5.35 E+00$ & $8.80 E+01$ \\
\hline \$96T005744 & 163 & 14 & LH & $0.00 \mathrm{E}+00$ & $0.00 \mathrm{E}+00$ & $0.00 \mathrm{E}+00$ \\
\hline S96T005743 & 163 & 14 & $\overline{\mathrm{UH}}$ & $0.00 \mathrm{E}+00$ & $0.00 \mathrm{E}+00$ & $0.00 \mathrm{E}+00$ \\
\hline S96T005272 & 163 & 16 & $\mathrm{LH}$ & $5.06 \mathrm{E}+01$ & $6.56 E+00$ & $9.20 \overline{\mathrm{E}}+01$ \\
\hline S96T0052712 & 163 & 16 & UH & $1.33 \mathrm{E}+02$ & $7.06 \bar{E}+01$ & $5.79 \mathrm{E}+02$ \\
\hline S96T005773 & 163 & 18 & $\mathrm{LH}$ & $3.84 \mathrm{E}+01$ & $1.25 \mathrm{E}+00$ & $4.62 \mathrm{E}+01$ \\
\hline \$96T005772 & 163 & 18 & UH & $3.67 \mathrm{E}+01$ & $3.50 \mathrm{E}+00$ & $5.88 \mathrm{E}+01$ \\
\hline S96T005549 & 163 & 20 & $\mathrm{LH}$ & $0.00 \mathrm{E}+00$ & $0.00 \mathrm{E}+00$ & $0.00 \mathrm{E}+00$ \\
\hline S96T005548 & 163 & 20 & $\overline{\mathrm{UH}}$ & $0.00 \mathrm{E}+00$ & $0.00 \mathrm{E}+00$ & $0.00 E+00$ \\
\hline S96T004772 & 164 & 1 & $\overline{\mathrm{LH}}$ & $0.00 \mathrm{E}+00$ & $0.00 \mathrm{E}+00$ & $0.00 E+00$ \\
\hline \$96T004773 & 164 & 13 & LH & $0.00 \mathrm{E}+00$ & $0.00 \mathrm{E}+00$ & $0.00 \mathrm{E}+00$ \\
\hline S96T004985 & 164 & 14 & $\mathrm{LH}$ & $0.00 \mathrm{E}+00$ & $0.00 \mathrm{E}+00$ & $0.00 \mathrm{E}+00$ \\
\hline \$96T004984 & 164 & 14 & $\mathrm{UH}$ & $0.00 \mathrm{E}+00$ & $0.00 \mathrm{E}+00$ & $0.00 \mathrm{E}+00$ \\
\hline S96T004987 & $\overline{164}$ & 15 & $\overline{\mathrm{LH}}$ & $5.46 \mathrm{E}+01$ & $7.05 \mathrm{E}+00$ & $9.91 \mathrm{E}+01$ \\
\hline \$96T004986 & 164 & 15 & $\mathrm{UH}$ & $5.25 \mathrm{E}+01$ & $1.52 E+01$ & $1.48 \mathrm{E}+02$ \\
\hline S96T004989 & 164 & 16 & $\overline{\mathrm{LH}}$ & $1.60 \mathrm{E}+01$ & $1.60 \bar{E}+01$ & $1.17 \mathrm{E}+02$ \\
\hline S96T004988 & 164 & 16 & $\mathrm{UH}$ & $5.35 \mathrm{E}+01$ & $7.15 E+00$ & $9.86 \mathrm{E}+01$ \\
\hline S96T004991 & 164 & 17 & $\mathrm{LH}$ & $6.66 \mathrm{E}+00$ & $2.41 \mathrm{E}+00$ & $2.19 \mathrm{E}+01$ \\
\hline \$96T004990 & 164 & 17 & $\overline{U H}$ & $7.00 \mathrm{E}+00$ & $7.00 \mathrm{E}+00$ & $5.12 E+01$ \\
\hline \$96T004993 & 164 & 19 & $\mathrm{LH}$ & $0.00 \mathrm{E}+00$ & $0.00 \mathrm{E}+00$ & $0.00 \mathrm{E}+00$ \\
\hline S96T004992 & 164 & 19 & $\mathrm{UH}$ & $0.00 \mathrm{E}+00$ & $0.00 \mathrm{E}+00$ & $0.00 \mathrm{E}+00$ \\
\hline S96T005250 & 164 & 20 & $\mathrm{LH}$ & $0.00 \mathrm{E}+00$ & $0.00 \mathrm{E}+00$ & $0.00 \mathrm{E}+00$ \\
\hline S96T005249 & 164 & 20 & UH & $0.00 \mathrm{E}+00$ & $0.00 \mathrm{E}+00$ & $0.00 \mathrm{E}+00$ \\
\hline S96T005972 & 164 & COMP & $-\cdots$ & $1.75 \mathrm{E}+02$ & $3.50 E+00$ & $1.97 E+02$ \\
\hline
\end{tabular}

Notes:
$\mathrm{DL}=$ drainable liquid
LH $=$ lower half
$\mathrm{UH} \quad=$ upper half
COMP $=$ composite

${ }^{1} \mathrm{UL}$ is greater than the action limit of $480 \mathrm{~J} / \mathrm{g}$ dry. 
Table C1-2. Tank 241-AN-104 Total Alpha Results. (2 sheets)

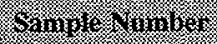

Liquid Samples

\begin{tabular}{|l|c|c|c|c|c|c|}
\hline S96T005256 & 163 & 2 & $\mathrm{DL}$ & $<9.94 \mathrm{E}-02$ & $\mathrm{n} / \mathrm{a}$ & $\mathrm{n} / \mathrm{a}$ \\
\hline S96T005260 & 163 & 4 & $\mathrm{DL}$ & $<9.94 \mathrm{E}-02$ & $\mathrm{n} / \mathrm{a}$ & $\mathrm{n} / \mathrm{a}$ \\
\hline S96T005527 & 163 & 5 & $\mathrm{DL}$ & $<2.26 \mathrm{E}-03$ & $\mathrm{n} / \mathrm{a}$ & $\mathrm{n} / \mathrm{a}$ \\
\hline S96T005554 & 163 & 6 & $\mathrm{DL}$ & $<2.43 \mathrm{E}-03$ & $\mathrm{n} / \mathrm{a}$ & $\mathrm{n} / \mathrm{a}$ \\
\hline S96T005255 & 163 & 7 & $\mathrm{DL}$ & $<8.22 \mathrm{E}-02$ & $\mathrm{n} / \mathrm{a}$ & $\mathrm{n} / \mathrm{a}$ \\
\hline S96T005257 & 163 & 8 & $\mathrm{DL}$ & $<4.83 \mathrm{E}-02$ & $\mathrm{n} / \mathrm{a}$ & $\mathrm{n} / \mathrm{a}$ \\
\hline 96T005258 & 163 & 9 & $\mathrm{DL}$ & $<4.83 \mathrm{E}-02$ & $\mathrm{n} / \mathrm{a}$ & $\mathrm{n} / \mathrm{a}$ \\
\hline S96T0055281 & 163 & 10 & $\mathrm{DL}$ & $2.47 \mathrm{E}-03$ & $3.50 \mathrm{E}-05$ & $2.69 \mathrm{E}-03$ \\
\hline S96T005739 & 163 & 11 & $\mathrm{DL}$ & $<2.43 \mathrm{E}-03$ & $\mathrm{n} / \mathrm{a}$ & $\mathrm{n} / \mathrm{a}$ \\
\hline S96T005766 & 163 & 12 & $\mathrm{DL}$ & $2.82 \mathrm{E}-03$ & $8.05 \mathrm{E}-04$ & $7.90 \mathrm{E}-03$ \\
\hline S96T005741 & 163 & 14 & $\mathrm{DL}$ & $<2.43 \mathrm{E}-03$ & $\mathrm{n} / \mathrm{a}$ & $\mathrm{n} / \mathrm{a}$ \\
\hline S96T004774 & 164 & 1 & $\mathrm{DL}$ & $<1.42 \mathrm{E}-02$ & $\mathrm{n} / \mathrm{a}$ & $\mathrm{n} / \mathrm{a}$ \\
\hline S96T004778 & 164 & 2 & $\mathrm{DL}$ & $<2.72 \mathrm{E}-02$ & $\mathrm{n} / \mathrm{a}$ & $\mathrm{n} / \mathrm{a}$ \\
\hline S96T004779 & 164 & 3 & $\mathrm{DL}$ & $<1.42 \mathrm{E}-02$ & $\mathrm{n} / \mathrm{a}$ & $\mathrm{n} / \mathrm{a}$ \\
\hline S96T004780 & 164 & 4 & $\mathrm{DL}$ & $<1.42 \mathrm{E}-02$ & $\mathrm{n} / \mathrm{a}$ & $\mathrm{n} / \mathrm{a}$ \\
\hline S96T004976 & 164 & 5 & $\mathrm{DL}$ & $<5.39 \mathrm{E}-02$ & $\mathrm{n} / \mathrm{a}$ & $\mathrm{n} / \mathrm{a}$ \\
\hline S96T004781 & 164 & 7 & $\mathrm{DL}$ & $<6.45 \mathrm{E}-02$ & $\mathrm{n} / \mathrm{a}$ & $\mathrm{n} / \mathrm{a}$ \\
\hline S96T004977 & 164 & 8 & $\mathrm{DL}$ & $<3.16 \mathrm{E}-02$ & $\mathrm{n} / \mathrm{a}$ & $\mathrm{n} / \mathrm{a}$ \\
\hline S96T004978 & 164 & 9 & $\mathrm{DL}$ & $<1.89 \mathrm{E}-02$ & $\mathrm{n} / \mathrm{a}$ & $\mathrm{n} / \mathrm{a}$ \\
\hline S96T004979 & 164 & 10 & $\mathrm{DL}$ & $<9.43 \mathrm{E}-02$ & $\mathrm{n} / \mathrm{a}$ & $\mathrm{n} / \mathrm{a}$ \\
\hline S96T004782 & 164 & 11 & $\mathrm{DL}$ & $<1.79 \mathrm{E}-02$ & $\mathrm{n} / \mathrm{a}$ & $\mathrm{n} / \mathrm{a}$ \\
\hline S96T004783 & 164 & 12 & $\mathrm{DL}$ & $<2.10 \mathrm{E}-02$ & $\mathrm{n} / \mathrm{a}$ & $\mathrm{n} / \mathrm{a}$ \\
\hline S96T004784 & 164 & 13 & $\mathrm{DL}$ & $<2.73 \mathrm{E}-02$ & $\mathrm{n} / \mathrm{a}$ & $\mathrm{n} / \mathrm{a}$ \\
\hline $\begin{array}{l}\text { S96T005978,9 } \\
\text { all 4 results }\end{array}$ & 164 & $\mathrm{COMP}$ & --- & $2.53 \mathrm{E}-02$ & $2.41 \mathrm{E}-03$ & $3.09 \mathrm{E}-02$ \\
\hline $\begin{array}{l}\text { S96T005978,9 } \\
\text { 3 results }(<\text { deleted) }\end{array}$ & 164 & $\mathrm{COMP}$ & --- & $2.51 \mathrm{E}-02$ & $2.93 \mathrm{E}-03$ & $3.37 \mathrm{E}-02$ \\
\hline
\end{tabular}


HNF-SD-WM-ER-690 Rev. 0

Table C1-2. Tank 241-AN-104 Total Alpha Results. (2 sheets)

\begin{tabular}{|c|c|c|c|c|c|c|}
\hline 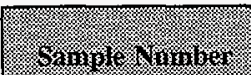 & 4010 & 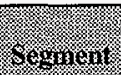 & 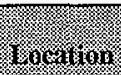 & 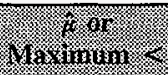 & $\sqrt{1}$ & 1) \\
\hline \multicolumn{7}{|c|}{ Slurry samples $(\mu \mathrm{Ci} / \mathrm{g})$} \\
\hline S96T005284 & 163 & 1 & $\overrightarrow{\mathrm{LH}}$ & $5.30 \mathrm{E}-02$ & $2.79 \mathrm{E}-02$ & $2.29 \mathrm{E}-01$ \\
\hline S96T005285 & 163 & 2 & $\overline{\mathrm{LH}}$ & $4.29 \mathrm{E}-02$ & $6.40 \mathrm{E}-03$ & $8.33 \mathrm{E}-02$ \\
\hline \$96T005780 & 163 & 12 & LH & $<2.32 \mathrm{E}-01$ & $\mathrm{n} / \mathrm{a}$ & $n / a$ \\
\hline S96T005748 & 163 & 14 & LH & $<3.50 \mathrm{E}-01$ & $\mathrm{n} / \mathrm{a}$ & $\mathrm{n} / \mathrm{a}$ \\
\hline \$96T005286 & 163 & 16 & LH & $2.63 \mathrm{E}-02$ & $3.65 \mathrm{E}-03$ & $4.93 \mathrm{E}-02$ \\
\hline S96T005781 & 163 & 18 & LH & $2.49 \mathrm{E}-02$ & $3.35 \mathrm{E}-03$ & $4.60 \mathrm{E}-02$ \\
\hline S96T005559 & 163 & 20 & $\mathrm{LH}$ & $3.74 \mathrm{E}-02$ & $1.25 \mathrm{E}-02$ & $1.16 \mathrm{E}-01$ \\
\hline S96T004795 & 164 & 1 & $\overline{\mathrm{LH}}$ & $<4.27 \mathrm{E}-02$ & $\mathrm{n} / \mathrm{a}$ & $n / a$ \\
\hline S96T004796 & 164 & 13 & $\overline{\mathrm{LH}}$ & $4.77 \mathrm{E}-02$ & $2.20 \mathrm{E}-03$ & $6.16 \mathrm{E}-02$ \\
\hline \$96T005030 & 164 & 14 & LH & $<3.51 \mathrm{E}-02$ & $\mathrm{n} / \mathrm{a}$ & $\mathrm{n} / \mathrm{a}$ \\
\hline \$96T005032 & 164 & 15 & $\overline{\mathrm{LH}}$ & $4.45 \mathrm{E}-02$ & $1.25 \mathrm{E}-03$ & $5.23 \mathrm{E}-02$ \\
\hline S96T005033' & 164 & 16 & $\overline{\mathrm{LH}}$ & $3.38 \mathrm{E}-02$ & $8.10 \mathrm{E}-03$ & $8.49 \mathrm{E}-02$ \\
\hline S96T005031 & 164 & 17 & LH & $3.81 E-02$ & $1.62 \mathrm{E}-02$ & $1.40 \mathrm{E}-01$ \\
\hline S96T005034 & 164 & 19 & $\widehat{\mathrm{LH}}$ & $5.41 \mathrm{E}-02$ & $1.31 \mathrm{E}-02$ & $1.36 \mathrm{E}-01$ \\
\hline S96T005278 & 164 & 20 & LH & $5.58 \mathrm{E}-02$ & $\begin{array}{c}0.00 \mathrm{E}+0 \\
0\end{array}$ & $5.58 \mathrm{E}-02$ \\
\hline S96T005975 & 164 & COMP & -- & $<3.93 \mathrm{E}-02$ & $n / a$ & $n / a$ \\
\hline
\end{tabular}

Notes:

${ }^{1}$ One of two results was a less than value; it was treated as a real number. 


\section{C2.0 APPENDIX C REFERENCES}

Dukelow, G. T., J. W. Hunt, H. Babad, and J. E. Meacham, 1995, Tank Safety Screening Data Quality Objective, WHC-SD-WM-SP-004, Rev. 2, Westinghouse Hanford Company, Richland, Washington.

Steen, F. H., 1997, Final Report for Tank 241-AN-104, Cores 163 and 164, HNF-SD-WM-DP-226, Rev. 1A, Rush Federal Services of Hanford, Inc. for Fluor Daniel Hanford, Inc., Richland, Washington. 


\section{APPENDIX D}

\section{EVALUATION TO ESTABLISH BEST-BASIS} INVENTORY FOR TANK 241-AN-104 
HNF-SD-WM-ER-690 Rev. 0

This page intentionally left blank. 


\section{APPENDIX D}

\section{EVALUATION TO ESTABLISH BEST-BASIS INVENTORY FOR TANK 241-AN-104}

An effort is underway to provide waste inventory estimates that will serve as standard characterization source terms for waste management activities (Hodgson and LeClair 1996). As part of this effort, an evaluation of available information for tank 241-AN-104 was performed, and a best-basis inventory was established. This work follows the methodology that was established by the standard inventory task.

\section{D1.O CHEMICAL INFORMATION SOURCES}

Available composition information for the waste in tank 241-AN-104 is as follows:

- Appendix B provides characterization results from the June 1996 core sampling event.

- A feed projection document that supported grout treatment facility studies provides estimates of the waste in tank 241-AN-104 based on 242-A Evaporator slurry product data (Hendrickson 1994).

- The HDW model document (Agnew et al. 1997) provides tank content estimates derived from the Los Alamos National Laboratory model in terms of component concentrations and inventories.

\section{D2.0 COMPARISON OF COMPONENT INVENTORY VALUES}

There have been no transfers into or out of tank 241-AN-104 since the third quarter of 1985. The HDW model provides composition estimates for the waste in tank 241-AN-104 as of January 1, 1994. Sample-based inventories, derived from the September 1996 core samples and inventories generated by the HDW model (Agnew et al. 1997), are compared in Tables D2-1 and D2-2. A tank volume of 3,994 kL (1,055 kgal) was used to generate the sample-based and HDW inventories (Hanlon 1997). The chemical species are reported without charge designation per the best-basis inventory convention. 
Table D2-1. Sampling And Hanford Defined Waste Model Inventory Estimates for Nonradioactive Components in Double-Shell Tank 241-AN-104.

\begin{tabular}{|c|c|c|c|c|}
\hline 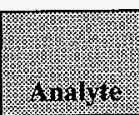 & 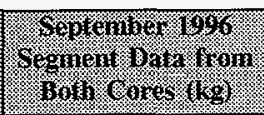 & 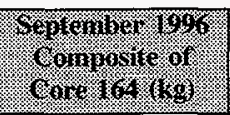 & 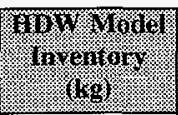 & 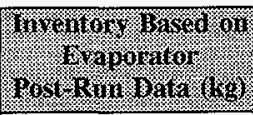 \\
\hline $\mathrm{Al}$ & 143,000 & 151,000 & 136,0000 & 150,000 \\
\hline$\overline{\mathrm{Bi}}$ & $<667$ & 108 & 793 & NR \\
\hline $\mathrm{Ca}$ & 885 & 928 & 5,110 & NR \\
\hline $\mathrm{Cl}$ & 29,900 & 32,600 & 26,900 & 30,000 \\
\hline $\mathrm{CO}_{3}$ & NR & 220,000 & 121,0000 & 113,000 \\
\hline $\mathrm{Cr}$ & 5,190 & 6,740 & 10,500 & 2,700 \\
\hline $\mathrm{F}$ & 2,160 & 2,050 & 7,590 & NR \\
\hline $\mathrm{Fe}$ & $<395$ & 410 & 2,020 & 44.6 \\
\hline $\mathrm{Hg}$ & NR & $\overline{\mathrm{NR}}$ & 7.34 & NR \\
\hline $\mathrm{K}$ & NR & 25,700 & 15,600 & 24,500 \\
\hline $\mathrm{La}$ & $<334$ & 191 & 7.04 & NR \\
\hline $\mathrm{Mn}$ & 74.6 & 83.2 & 1,470 & NR \\
\hline $\mathrm{Na}$ & $1.19 \mathrm{E}+06$ & $1.11 \mathrm{E}+06$ & $1.02 \mathrm{E}+06$ & $1.10 \mathrm{E}+06$ \\
\hline $\mathrm{Ni}$ & $<226$ & 256 & 1,340 & NR \\
\hline $\mathrm{NO}_{2}$ & 448,000 & 455,000 & 316,000 & 353,000 \\
\hline $\mathrm{NO}_{3}$ & 680,000 & 747,000 & 156,000 & 768,000 \\
\hline$\widehat{\mathrm{OH}}$ & NR & 261,000 & 502,000 & 277,000 \\
\hline $\mathrm{Pb}$ & $<382$ & 387 & 785 & NR \\
\hline $\mathrm{PO}_{4}$ & 17,100 & $17 \overline{700}$ & 43,700 & $11, \overline{200}$ \\
\hline $\mathrm{Si}$ & 1,590 & 2,920 & 6,850 & NR \\
\hline $\mathrm{SO}_{4}$ & 37,100 & 39,400 & 80,800 & 26,200 \\
\hline$\overline{\mathrm{Sr}}$ & $<66.7$ & 38.1 & NR & NR \\
\hline TOC & NR & 20,100 & 61,845 & 18,400 \\
\hline $\bar{U}$ & $<3,430$ & 498 & 8,650 & NR \\
\hline $\mathrm{Zr}$ & 64.1 & 73.0 & 273 & NR \\
\hline$\%$ water & 48 & 48 & 42.8 & NR \\
\hline $\begin{array}{l}\text { Density } \\
(\mathrm{g} / \mathrm{mL})\end{array}$ & 1.45 & 1.46 & 1.47 & 1.50 \\
\hline
\end{tabular}

Note:

$\mathrm{NR}=$ not reported 
Table D2-2. Sampling and Hanford Defined Waste Model Inventory Estimates for Radioactive Components in Double-Shell Tank 241-AN-104 (Decayed to January 1, 1994).

\begin{tabular}{|c|c|c|c|c|}
\hline 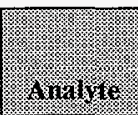 & 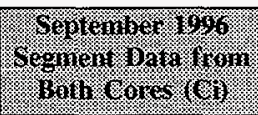 & 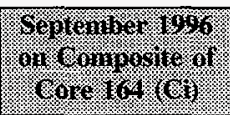 & 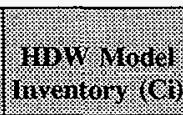 & 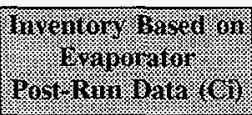 \\
\hline${ }^{3} \mathbf{H}$ & NR & 42.8 & 744 & NR \\
\hline${ }^{60} \mathrm{Co}$ & NR & $<147$ & 135 & $\mathrm{NR}$ \\
\hline${ }^{79} \mathrm{Se}$ & NR & NR & 11.3 & NR \\
\hline${ }^{90} \mathrm{Sr}$ & NR & 92,700 & 354,000 & 39,300 \\
\hline${ }^{90} \mathrm{Y}$ & $\mathrm{NR}$ & 92,700 & NR & $\overline{N R}$ \\
\hline${ }^{99} \mathrm{Tc}$ & NR & 340 & 782 & NR \\
\hline${ }^{129} \mathrm{~T}$ & NR & 7.45 & 1.51 & $\mathrm{NR}$ \\
\hline${ }^{137} \mathrm{Cs}$ & NR & $2.31 \mathrm{E}+06$ & 783,000 & $3.02 \mathrm{E}+06$ \\
\hline${ }^{137} \mathrm{mBa}$ & NR & $2.19 \mathrm{E}+06$ & NR & $2.87 \mathrm{E}+06$ \\
\hline${ }^{154} \mathrm{Eu}$ & NR & $<671$ & 2,130 & NR \\
\hline${ }^{155} \mathrm{Eu}$ & NR & $<4,700$ & 899 & NR \\
\hline${ }^{237} \mathrm{~Np}$ & NR & $<30.3$ & 2.77 & NR \\
\hline${ }^{238} \mathrm{Pu}$ & NR & NR & 7.97 & $\mathrm{NR}$ \\
\hline${ }^{239} \mathrm{Pu}$ & NR & NR & NR & NR \\
\hline${ }^{239 / 240} \mathrm{Pu}$ & NR & $<9.59$ & 205 & 41.6 \\
\hline${ }^{241} \mathrm{Am}$ & NR & $<49.4$ & 223 & NR \\
\hline${ }^{244} \mathrm{Cm}$ & NR & $<11.9$ & 0.520 & $\mathrm{NR}$ \\
\hline
\end{tabular}

The sample-based densities are $1.40 \mathrm{~g} / \mathrm{mL}$ for the liquid and $1.58 \mathrm{~g} / \mathrm{mL}$ for the slurry. The slurry density is based on measurements of centrifuged solids; it may not be the same as the slurry density of the slurry. Gases generated in the tank are trapped in the slurry layer and form gas pockets; therefore, a lower density is expected in tank waste than in laboratory waste. In tank 241-AN-104, the retained gas is 5.7 vol\% of the slurry volume (Shekarriz et al. 1996). The mean slurry density was corrected by multiplying by 0.943 ( 1 - vol\% retained gas). The corrected slurry density is $1.51 \mathrm{~g} / \mathrm{mL}$. The HDW model bulk density is $1.47 \mathrm{~g} / \mathrm{mL}$.

The sample-based concentrations were taken from the inventory tables in Section B3.4.1. The means which included the less than values (those having a "lt" extension in Appendix B) were used to calculate the sample-based inventories. 
The slurry volume, determined from temperature profiles, rheometer and core sample data is $1,700 \mathrm{~kL}$ (449 kgal) (Stauffer 1997). Based on the extrusion reports, a $5.1 \mathrm{~cm}$ (2 in.) crust layer exists in tank 241-AN-104. This constitutes a volume of $21 \mathrm{~kL}(5.5 \mathrm{kgal})$ as saltcake. The supernatant and slurry volume, equal to the total volume minus the slurry and crust volumes, is $2,273 \mathrm{~kL}$ (600.5 kgal). The supernatant volume in Hanlon (1997) are out of date estimates and should be updated to the newer basis presented here barring changes to the total measured volume.

The HDW model estimates for tank 241-AN-104 were derived by combining supernatant streams that were believed to have been concentrated in the 242-A Evaporator, then sent to tank 241-AN-104. (This part of the HDW model is called the SMM.) The supernatant compositions were derived largely from estimates of bulk compositions of the process waste streams that left processing facilities. To obtain the supernatant fractions, the bulk compositions were multiplied by solubility factors determined from the highest concentrations found among supernatant sample analyses. Normally, these solubility assumptions result in higher inventories in the HDW model estimates for double-shell tank supernatants and slurries when compared to their respective laboratory sample results.

In the case of tank 241-AN-104, the higher inventory estimates made by the HDW model are noted in Table D2-1 for most minor $(<100,000 \mathrm{~kg}$ ) waste constituents. In a departure from other double-shell tank comparisons, the HDW model estimates for major components such as $\mathrm{Al}, \mathrm{Na}, \mathrm{NO}_{2}$, and $\mathrm{NO}_{3}$ are in reasonable agreement with the sample-based inventories. This may be caused by the higher percent of salt-slurry waste types used to derive the tank inventories in the HDW model. The salt-slurry waste types, as defined in the HDW model, are based more on sample data. In this case, the overstated solubility assumptions would not impact the composition as much as other double-shell tanks.

The periods in which tank 241-AN-104 received double-shell slurry feed from the 242-A Evaporator and other waste tanks are known (see Appendix A). Slurry product concentration data from 242-A Evaporator post-run documents can be obtained for each batch of feed processed. This data combined with the fill history of tank 241-AN-104 can be used to estimate the composition of tank 241-AN-104. This can serve as a check against the samplebased and HDW model inventories.

In 1994, Hendrickson performed such an analysis of the double-shell slurry feed in tank 241-AN-104 (Hendrickson 1994). Column 5 of Table D2-1 shows the results for tank 241-AN-104, the average of the segment data from 1996 for core samples 163 and 164, the core composite for core 163 , and the HDW model inventory estimates. This table demonstrates close agreement between 242-A Evaporator slurry data and the September 1996 core samples for many major components including $\mathrm{Al}, \mathrm{K}, \mathrm{Na}, \mathrm{OH}, \mathrm{NO}_{2}$, and $\mathrm{NO}_{3}, \mathrm{PO}_{4}$, and TOC. This agreement suggests that the sample-based inventories are a better basis than the HDW model. 


\section{D3.0 COMPONENT INVENTORY EVALUATION}

Table D3-1 shows concentrations for the supernatant, slurry, and crust portions based on the means of the segment data for both cores and the core 163 composite. Core 163 was taken from riser $10 \mathrm{~A}$, and core 164 was taken from riser $12 \mathrm{~A}$. Mean concentrations from segment data are based on segments from both cores. Mean concentrations from composite data are based on a composite of core 164 only.

Acid and fusion digests and were performed on individual segments to obtain cation concentrations, but only acid digests were performed on the core composite. Fusion digests with $\mathrm{KOH}$ or $\mathrm{NaOH}$ are usually a more complete method of dissolving solids than acid dissolution.

The segment-based data are a better source of information than the core composite data even though the core composite and segment-based concentrations agree. However, the composite core data contain information on several components not found in the segment-based data including $\mathrm{CO}_{3}, \mathrm{OH}$, and TOC. The composite data are also the only source of individual radionuclide concentrations from this sampling event.

The best-basis inventory is a combination of segment and core composite analysis values. Core composite concentrations are used for components not included in the segment analyses. Note that liquid concentrations from the core composite are approximately 5 percent to 30 percent higher than the segment-based data.

Table D3-1 shows concentrations for the $5.1 \mathrm{~cm} \mathrm{(2} \mathrm{in.)} \mathrm{crust.} \mathrm{The} \mathrm{crustis} \mathrm{similar} \mathrm{to} \mathrm{the}$ slurry below it. Although the density of the crust was not reported, it is assumed that it is equal to the supernatant density because it floats.

Table D3-1. Comparison of September 1996 Core Sample Concentrations. (2 sheets)

\begin{tabular}{|c|c|c|c|c|c|}
\hline 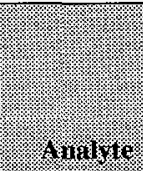 & 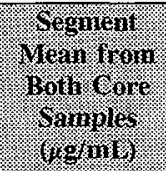 & 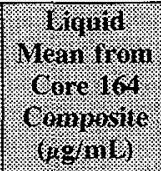 & 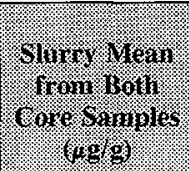 & 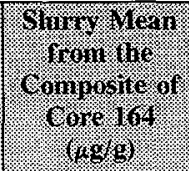 & 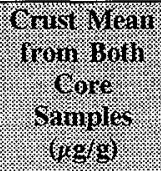 \\
\hline $\mathrm{Al}$ & 38,600 & 41,700 & 21,100 & 20,800 & 19,500 \\
\hline$B i$ & $<120$ & NR & $<153$ & $<39.8$ & $<47.1$ \\
\hline $\mathrm{Ca}$ & $<120$ & $<120$ & 225 & 244 & 160 \\
\hline $\mathrm{Cl}$ & 8,090 & 9,500 & 4,420 & 4,090 & 4,500 \\
\hline $\mathrm{CO}_{3}$ & NR & 10,900 & NR & 73,500 & $\mathrm{NR}$ \\
\hline $\mathrm{Cr}$ & 336 & 574 & 1,710 & 2,200 & 1,270 \\
\hline
\end{tabular}


Table D3-1. Comparison of September 1996 Core Sample Concentrations. (2 sheets)

\begin{tabular}{|c|c|c|c|c|c|}
\hline : & 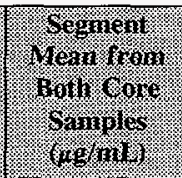 & 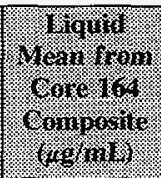 & 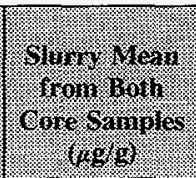 & 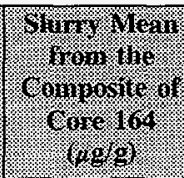 & 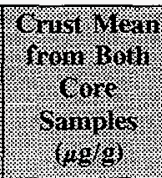 \\
\hline $\mathrm{F}$ & NR & $<122$ & 836 & 658 & 539 \\
\hline $\mathrm{Fe}$ & $<60.1$ & $<60.1$ & 97.5 & 99.5 & 258 \\
\hline $\mathrm{Hg}$ & NR & NR & NR & NR & NR \\
\hline $\mathrm{K}$ & NR & 6,900 & 3,720 & 3,720 & 3,460 \\
\hline $\mathrm{La}$ & $<60.1$ & $<60.1$ & $<76.7$ & $<19.9$ & $<23.5$ \\
\hline $\mathrm{Mn}$ & $<12.0$ & $<12.0$ & 18.3 & 20.9 & 12.0 \\
\hline $\mathrm{Na}$ & 256,000 & 261,000 & 236,000 & 192,000 & 235,000 \\
\hline $\mathrm{Ni}$ & $<24.0$ & $<24.0$ & 66.5 & $75 . \overline{0}$ & 31.5 \\
\hline $\mathrm{NO}_{2}$ & 125,000 & 131,000 & 62,800 & 58,100 & 66,400 \\
\hline $\mathrm{NO}_{3}$ & 170,000 & 207,000 & 112,000 & 101,000 & 178,000 \\
\hline $\mathrm{OH}$ & NR & 70,900 & NR & 37,200 & NR \\
\hline $\mathrm{Pb}$ & NR & $<120$ & NR & 42.0 & $<47.1$ \\
\hline$\widehat{\mathrm{PO}_{4}}$ & 2,710 & 3,800 & 4,200 & 3,350 & $4, \overline{420}$ \\
\hline $\mathrm{Si}$ & NR & 379 & 609 & 761.5 & 882 \\
\hline $\mathrm{SO}_{4}$ & 3,540 & 3,600 & 11,200 & 11,600 & 9,410 \\
\hline $\mathrm{Sr}$ & $<12.0$ & $<12.0$ & $<15.3$ & $<3.98$ & $<4.71$ \\
\hline TOC & NR & 3,110 & NR & 4,630 & NR \\
\hline$\overline{\mathrm{U}}$ & $<600$ & 3.68 & $<767$ & $<181$ & $<235$ \\
\hline $\mathrm{Zr}$ & 7.86 & $<12.0$ & 17.9 & 16.9 & 10.8 \\
\hline Volume (L) & $2.275 \mathrm{E}+06$ & $2.275 \mathrm{E}+06$ & $1.700 \mathrm{E}+06$ & $1.7000 \mathrm{E}+06$ & 20,820 \\
\hline $\begin{array}{l}\text { Density } \\
(\mathrm{g} / \mathrm{mL})\end{array}$ & 1.40 & 1.41 & 1.51 & 1.48 & $1.40^{\mathrm{a}}$ \\
\hline
\end{tabular}




\section{D4.0 DEFINE THE BEST-BASIS AND ESTABLISH COMPONENT INVENTORIES}

Inventories based on the September 1996 sampling event should serve as the basis for the best estimate inventory to tank $241-\mathrm{AN}-104$ for the following reasons:

1. The September 1996 sampling event provides the most recent data for the waste.

2. Estimates based on 242-A Evaporator product sample data agree with the September 1996 analytical data.

3. Although the HDW model estimates are in reasonable agreement with sampling data, they do not agree well with the evaporator product data.

Once the best-basis inventories were determined, the hydroxide inventory was calculated by performing a charge balance with the valence of other analytes. In some cases, this approach requires the other analyte (for example, sodium or nitrate) inventories be adjusted to achieve the charge balance. During such adjustments, significant figures are retained. This charge balance approach is consistent with that used by Agnew et al. (1997).

Best-basis tank inventory values are derived for 46 key radionuclides (Kupfer et al. 1997) decayed to a common report date, January 1, 1994. Often, waste sample anaiyses have only reported ${ }^{90} \mathrm{Sr},{ }^{13} 7 \mathrm{Cs},{ }^{239 / 240} \mathrm{Pu}$, and total uranium, or total beta and total alpha, while other key radionuclides such as ${ }^{60} \mathrm{Co},{ }^{99} \mathrm{Tc},{ }^{129} \mathrm{I},{ }^{154} \mathrm{Eu},{ }^{155} \mathrm{Eu}$, and ${ }^{241} \mathrm{Am}$ etc. have been infrequently reported. For this reason, it was necessary to derive most 46 key radionuclides by computer models. These models estimate radionuclide activity in batches of reactor fuel, account for the split of radionuclides to various separations plant waste streams, and track their movement with tank waste transactions. The computer models are described in Kupfer et al. (1997) and Watrous and Wootan (1997).

Model-generated values for radionuclides in the 177 tanks are reported in Agnew et al. (1997). The best-basis value for any one analyte may be a model result or a sample of engineering assessment-based result, if available. No attempt was made to ratio or normalize model results for the 46 radionuclides when values for measured radionuclides disagree with the model. For a discussion of typical error between model derived values and sample derived values, refer to Kupfer et al. (1997).

Tables D4-1 through D4-6 show best-basis inventory estimates of the crust, supernatant, and sludge layers for tank 241-AN-104. Table D4-7 and D4-8 list best basis inventory estimates for the whole tank. Radionuclide values are decayed to January 1, 1994. 
Table D4-1. Best-Basis Inventory Estimates for Nonradioactive Components in Tank 241-AN-104 Supernatant as of May 31, $1997 .{ }^{1}$

\begin{tabular}{|c|c|c|c|}
\hline : vilute & 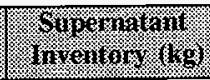 & 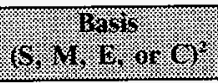 & Cominant \\
\hline $\mathrm{Al}$ & 87,800 & $\mathrm{~S}$ & \\
\hline$\overline{\mathrm{Bi}}$ & $<273$ & $\mathrm{~S}$ & \\
\hline $\mathrm{Ca}$ & $<273$ & $\mathrm{~S}$ & \\
\hline $\mathrm{Cl}$ & 18,400 & $S$ & \\
\hline TIC as $\mathrm{CO}_{3}$ & 24,700 & $\mathrm{~S}$ & Not reported in segment-based data \\
\hline $\mathrm{Cr}$ & 765 & $S$ & \\
\hline F & $<278$ & $S$ & Not reported in segment-based data \\
\hline $\mathrm{Fe}$ & $<137$ & $S$ & \\
\hline $\mathrm{K}$ & 15,200 & $S$ & \\
\hline $\mathrm{La}$ & $<137$ & $\mathrm{~S}$ & \\
\hline $\mathrm{Mn}$ & $<27.3$ & $\mathrm{~S}$ & \\
\hline $\mathrm{Na}$ & 582,000 & $\mathrm{~S}$ & \\
\hline $\mathrm{Ni}$ & $<54.6$ & $S$ & \\
\hline $\mathrm{NO}_{2}$ & 284,000 & $S$ & \\
\hline $\mathrm{NO}_{3}$ & 387,000 & $\mathrm{~S}$ & \\
\hline $\mathrm{OH}$ & 362,000 & $\mathrm{C}$ & Composite-based inventory $=161,000$ \\
\hline $\mathrm{Pb}$ & 273 & $S$ & Composite $<154$ \\
\hline $\mathrm{P}$ as $\mathrm{PO}_{4}$ & 6,160 & $S$ & \\
\hline $\mathrm{Si}$ & 862 & $\mathrm{~S}$ & Not reported in segment-based data \\
\hline $\mathrm{SO}_{4}$ & 8,022 & $\mathrm{~s}$ & \\
\hline $\mathrm{Sr}$ & $<27.3$ & $S$ & \\
\hline TOC & 7,760 & $S$ & Not reported in segment-based data \\
\hline$\overline{\mathrm{U}_{\text {TOTAL }}}$ & $<1,370$ & $S$ & \\
\hline$\overline{\mathrm{Zr}}$ & 17.8 & $S$ & \\
\hline$\%$ water & 51.1 & $S$ & \\
\hline Density $\mathrm{g} / \mathrm{mL}$ & 1.40 & $S$ & \\
\hline
\end{tabular}

Notes:

'The tank is active. Waste transfers will change tank contents.

${ }^{2} \mathrm{~S}=$ sample-based, $\mathrm{M}=\mathrm{HDW}$ model-based, $\mathrm{E}=$ engineering assessment-based,

$\mathrm{C}=$ calculated by charge balance; includes oxides as hydroxides, not including $\mathrm{CO}_{3}, \mathrm{NO}_{2}, \mathrm{NO}_{3}$,

$\mathrm{PO}_{4}, \mathrm{SO}_{4}$, and $\mathrm{SiO}_{3}$ 
Table D4-2. Best-Basis Inventory Estimates for Radioactive Components in Tank 241-AN-104 Supernatant as of May 31, 1997 (Decayed to January 1, 1994). ${ }^{1}$

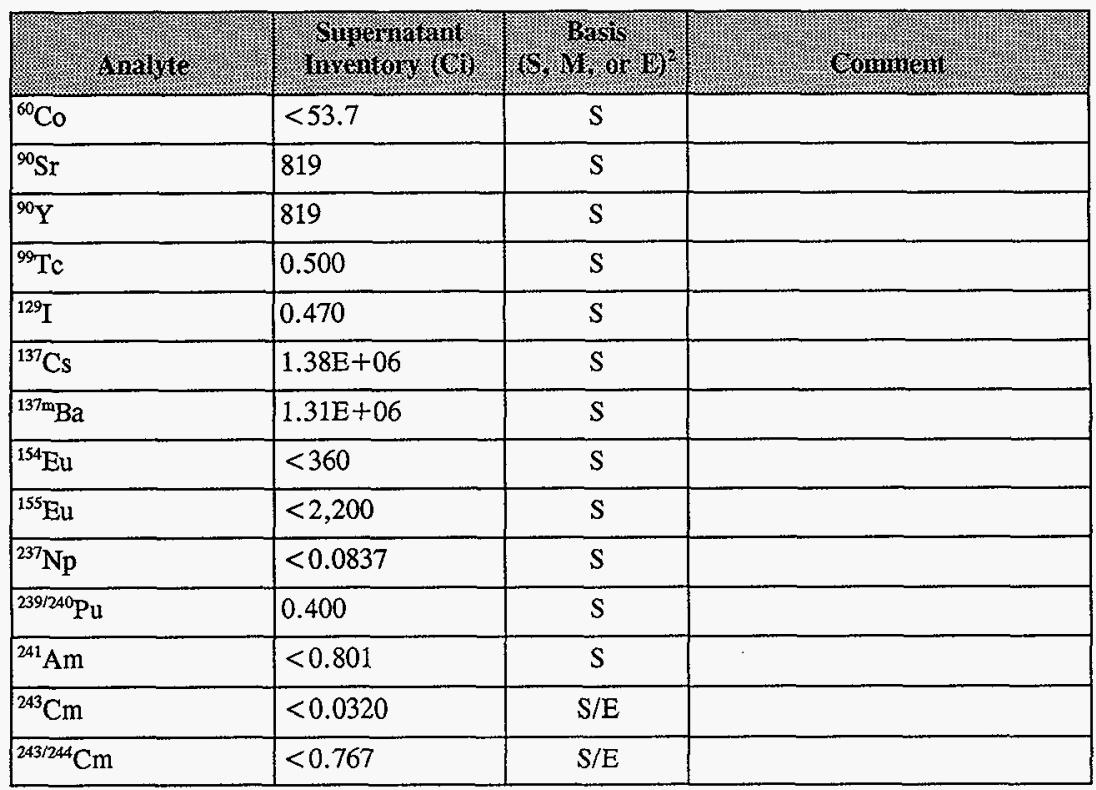

Notes:

'The tank is active. Waste transfers will change tank contents.

${ }^{1} \mathrm{~S}=$ sample-based, $\mathrm{M}=\mathrm{HDW}$ model-based, $\mathrm{E}=$ engineering assessment-based,

$\mathrm{C}=$ calculated by charge balance; includes oxides as hydroxides, not including $\mathrm{CO}_{3}, \mathrm{NO}_{2}, \mathrm{NO}_{3}$,

$\mathrm{PO}_{4}, \mathrm{SO}_{4}$, and $\mathrm{SiO}_{3}$ 
HNF-SD-WM-ER-690 Rev. 0

Table D4-3. Best-Basis Inventory Estimates for Nonradioactive Components in Tank 241-AN-104 Salt Slurry as of May 31, 1997. ${ }^{1}$ (2 sheets)

\begin{tabular}{|c|c|c|c|}
\hline (vinates & 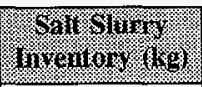 & 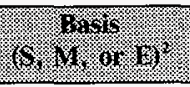 & (ovment \\
\hline $\mathrm{Al}$ & 54,100 & $S$ & \\
\hline $\mathrm{Bi}$ & $<393$ & $S$ & \\
\hline $\mathrm{Ca}$ & 577 & $S$ & \\
\hline $\mathrm{Cl}$ & 11,300 & $S$ & \\
\hline $\mathrm{TIC}$ as $\mathrm{CO}_{3}$ & 189,000 & $\bar{S}$ & Not reported in segment-based data. \\
\hline $\mathrm{Cr}$ & 4,390 & $\mathrm{~S}$ & \\
\hline $\mathrm{F}$ & 2,150 & $S$ & \\
\hline $\mathrm{Fe}$ & 250 & $S$ & \\
\hline$\widehat{K}$ & 9,550 & $S$ & \\
\hline $\mathrm{La}$ & $<197$ & $\bar{S}$ & \\
\hline $\mathrm{Mn}$ & 47.0 & $\mathrm{~S}$ & \\
\hline $\mathrm{Na}$ & 606,000 & $\bar{S}$ & \\
\hline $\mathrm{Ni}$ & 171 & $S$ & \\
\hline $\mathrm{NO}_{2}$ & 161,000 & $S$ & \\
\hline $\mathrm{NO}_{3}$ & 287,000 & $S$ & \\
\hline $\mathrm{OH}$ & 285,000 & C & Composite-based inventory $=95,300$ \\
\hline $\mathrm{Pb}$ & 108 & $S$ & \\
\hline $\mathrm{PO}_{4}$ & 10,800 & $\mathrm{~S}$ & \\
\hline $\mathrm{Si}$ & 1,560 & $S$ & \\
\hline $\mathrm{SO}_{4}$ & 28,700 & $\mathrm{~S}$ & \\
\hline $\mathrm{Sr}$ & $<39.3$ & $\mathrm{~S}$ & \\
\hline TOC & 11,900 & $\mathrm{~S}$ & Not reported in segment-based data. \\
\hline $\mathrm{U}_{\text {TOTAL }}$ & $<1,970$ & $S$ & Not reported in segment-based data. \\
\hline
\end{tabular}


Table D4-3. Best-Basis Inventory Estimates for Nonradioactive Components in Tank 241-AN-104 Salt Slurry as of May 31, 1997. ${ }^{1}$ (2 sheets)

\begin{tabular}{|c|c|c|c|}
\hline 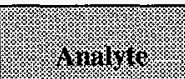 & 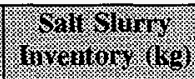 & 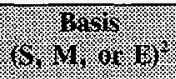 & (1) \\
\hline $\mathrm{Zr}$ & 45.9 & $S$ & \\
\hline$\%$ water & 45.6 & $\bar{S}$ & \\
\hline Density $(\mathrm{g} / \mathrm{mL})$ & 1.51 & $S$ & \\
\hline
\end{tabular}

Notes:

${ }^{1}$ The tank is active. Waste transfers will change tank contents.

${ }^{2} S=$ sample-based, $M=H D W$ model-based, $E$ = engineering assessment-based,

$\mathrm{C}=$ calculated by charge balance; includes oxides as hydroxides, not including $\mathrm{CO}_{3}, \mathrm{NO}_{2}, \mathrm{NO}_{3}$,

$\mathrm{PO}_{4}, \mathrm{SO}_{4}$, and $\mathrm{SiO}_{3}$

Table D4-4. Best-Basis Inventory Estimates for Radioactive Components in Tank 241-AN-104 Salt Slurry as of May 31, 1997

(Decayed to January 1, 1994).' (2 sheets)

\begin{tabular}{|c|c|c|c|}
\hline sinjo & Srif Slowy & (S. & (rininan \\
\hline${ }^{3} \mathrm{H}$ & 42.8 & $S$ & \\
\hline${ }^{60} \mathrm{Co}$ & $<93.9$ & $S$ & \\
\hline${ }^{90} \mathrm{Sr}$ & 91,900 & $S$ & \\
\hline${ }^{90} \mathrm{Y}$ & 91,900 & $S$ & \\
\hline${ }^{99} \mathrm{TC}$ & 340 & $\bar{S}$ & \\
\hline${ }^{129} \mathrm{I}$ & $<6.97$ & $\bar{S}$ & \\
\hline${ }^{137} \mathrm{Cs}$ & 934,000 & $S$ & \\
\hline${ }^{137 \mathrm{~m}} \mathrm{Ba}$ & 887,000 & $S$ & \\
\hline${ }^{154} \mathrm{Eu}$ & $<312$ & $S$ & \\
\hline${ }^{155} \mathrm{Eu}$ & $<2,490$ & $\bar{S}$ & \\
\hline${ }^{237} \mathrm{~Np}$ & $<30.2$ & $S$ & \\
\hline${ }^{239 / 240} \mathrm{Pu}$ & 9.19 & $S$ & \\
\hline
\end{tabular}


Table D4-4. Best-Basis Inventory Estimates for Radioactive Components in Tank 241-AN-104 Salt Slurry as of May 31, 1997

(Decayed to January 1, 1994). ${ }^{1}$ (2 sheets)

\begin{tabular}{|c|c|c|c|}
\hline Arnalgt & 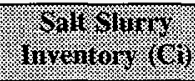 & $(5.17 .0 \%$ G & Connent \\
\hline${ }^{241} \mathrm{Am}$ & 48.6 & $\mathrm{~S} / \mathrm{E}$ & \\
\hline${ }^{243} \mathrm{Cm}$ & $<0.472$ & $S$ & \\
\hline $243 / 244 \mathrm{Cm}$ & $<11.4$ & $S / E$ & \\
\hline
\end{tabular}

Notes:

'The tank is active. Waste transfers will change tank contents.

${ }^{2} \mathrm{~S}=$ sample-based, $\mathrm{M}=\mathrm{HDW}$ model-based, $\mathrm{E}$ = engineering assessment-based,

$\mathrm{C}=$ calculated by charge balance; includes oxides as hydroxides, not including $\mathrm{CO}_{3}, \mathrm{NO}_{2}, \mathrm{NO}_{3}$,

$\mathrm{PO}_{4}, \mathrm{SO}_{4}$, and $\mathrm{SiO}_{3}$

Table D4-5. Best-Basis Inventory Estimates for Nonradioactive Components in Tank 241-AN-104 Crust as of May 31, 1997.' (2 sheets)

\begin{tabular}{|c|c|c|c|}
\hline : & 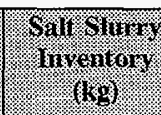 & (6. & T: \\
\hline $\mathrm{Al}$ & 611 & $\mathrm{~S}$ & \\
\hline $\mathrm{Bi}$ & $<1.48$ & $S$ & \\
\hline $\mathrm{Ca}$ & 5.04 & $S$ & \\
\hline $\mathrm{Cl}$ & 141 & $S$ & \\
\hline TIC as $\mathrm{CO}_{3}$ & 2,310 & $S$ & $\begin{array}{l}\text { Assumed to be equal to slurry-based } \\
\text { concentration. }\end{array}$ \\
\hline$\overline{\mathrm{Cr}}$ & 40.0 & $\bar{S}$ & \\
\hline $\bar{F}$ & 16.9 & $\bar{S}$ & \\
\hline $\mathrm{Fe}$ & 8.10 & $S$ & \\
\hline $\mathrm{K}$ & 109 & $S$ & \\
\hline $\mathrm{La}$ & $<0.738$ & $S$ & \\
\hline $\mathrm{Mn}$ & 0.376 & $S$ & \\
\hline $\mathrm{Na}$ & 7,370 & $S$ & \\
\hline $\mathrm{Ni}$ & 0.991 & $S$ & \\
\hline $\mathrm{NO}_{2}$ & 2,090 & $S$ & \\
\hline
\end{tabular}


Table D4-5. Best-Basis Inventory Estimates for Nonradioactive Components in Tank 241-AN-104 Crust as of May 31, 1997.' (2 sheets)

\begin{tabular}{|c|c|c|c|}
\hline : & 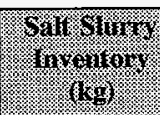 & (6) & Commen! \\
\hline $\mathrm{NO}_{3}$ & 5,600 & $S$ & \\
\hline$\overline{\mathrm{OH}}$ & 2,730 & $\mathrm{C}$ & Composite slurry-based inventory $=1,170$ \\
\hline$\overline{\mathrm{Pb}}$ & $<1.48$ & $S$ & \\
\hline $\mathrm{PO}_{4}$ & 139 & $S$ & \\
\hline$\overline{\mathrm{Si}}$ & 27.7 & $\bar{S}$ & \\
\hline $\mathrm{SO}_{4}$ & 296 & S & \\
\hline $\mathrm{Sr}$ & $<0.148$ & $\mathrm{~S}$ & \\
\hline TOC & 145 & $S$ & $\begin{array}{l}\text { Assumed to be equal to slurry-based } \\
\text { concentration. }\end{array}$ \\
\hline $\mathrm{U}_{\text {TOTAL }}$ & $<7.38$ & $S$ & \\
\hline$\overline{\mathrm{Zr}}$ & 0.339 & $S$ & \\
\hline$\%$ water & NR & $S$ & \\
\hline $\begin{array}{l}\text { Density } \\
(\mathrm{g} / \mathrm{mL})\end{array}$ & 1.40 & $S$ & Assumed to be equal to supernatant density. \\
\hline
\end{tabular}

Notes:

'The tank is active. Waste transfers will change tank contents.

${ }^{2} S=$ sample-based, $M=$ HDW model-based, $E=$ engineering assessment-based,

$\mathrm{C}$ = calculated by charge balance; includes oxides as hydroxides, not including $\mathrm{CO}_{3}, \mathrm{NO}_{2}, \mathrm{NO}_{3}$, $\mathrm{PO}_{4}, \mathrm{SO}_{4}$, and $\mathrm{SiO}_{3}$ 
Table D4-6. Best-Basis Inventory Estimates for Radioactive Components in

Tank 241-AN-104 Crust as of May 31, $1997^{1}$ (Decayed to January 1, 1994).

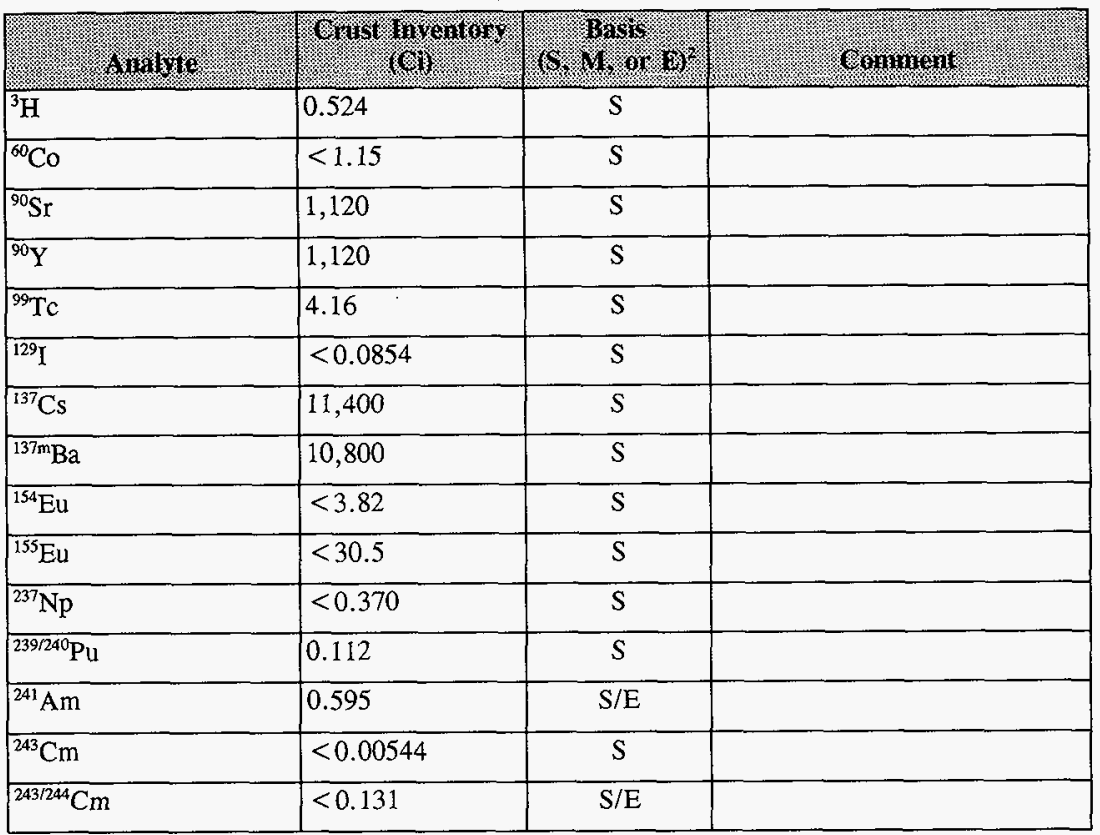

Notes:

${ }^{1}$ The tank is active. Waste transfers will change tank contents.

${ }^{2} \mathrm{~S}=$ sample-based, $\mathrm{M}=\mathrm{HDW}$ model-based, $\mathrm{E}=$ engineering assessment-based,

$\mathrm{C}=$ calculated by charge balance; includes oxides as hydroxides, not including $\mathrm{CO}_{3}, \mathrm{NO}_{2}, \mathrm{NO}_{3}$,

$\mathrm{PO}_{4}, \mathrm{SO}_{4}$, and $\mathrm{SiO}_{3}$ 
HNF-SD-WM-ER-690 Rev. 0

Table D4-7. Best-Basis Total Inventory Estimates for Nonradioactive Components in Tank 241-AN-104 as of May 31, 1997.' (2 sheets)

\begin{tabular}{|c|c|c|c|}
\hline 7 (ninglo & 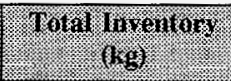 & $(6,11 ., 00.672$ & 㐨 \\
\hline $\mathrm{Al}$ & 143,000 & $\mathrm{~S}$ & \\
\hline $\mathrm{Bi}$ & $<667$ & $S$ & \\
\hline $\mathrm{Ca}$ & $<855$ & $S$ & \\
\hline$\overline{\mathrm{Cl}}$ & 29,900 & $S$ & \\
\hline TIC as $\mathrm{CO}_{3}$ & $\longdiv { 2 1 0 , 0 0 0 }$ & $S$ & \\
\hline $\mathrm{Cr}$ & 5,190 & $S$ & \\
\hline $\bar{F}$ & 2,160 & $S$ & \\
\hline $\mathrm{Fe}$ & $<395$ & $S$ & \\
\hline $\mathrm{K}$ & 24,900 & $S$ & \\
\hline $\mathrm{La}$ & $<334$ & $s$ & \\
\hline $\mathrm{Mn}$ & $<74.6$ & $\mathrm{~S}$ & \\
\hline$\overline{\mathrm{Na}}$ & $1.19 \mathrm{E}+06$ & $S$ & \\
\hline$\widehat{\mathrm{Ni}}$ & $<226$ & $S$ & \\
\hline $\mathrm{NO}_{2}$ & 448,000 & $\mathrm{~S}$ & \\
\hline$\overline{\mathrm{NO}_{3}}$ & 680,000 & $S$ & \\
\hline $\mathrm{OH}$ & 650,000 & $\mathrm{C}$ & Sample-based $=255,000$ \\
\hline $\mathrm{Pb}$ & $<382$ & $S$ & \\
\hline $\mathrm{PO}_{4}$ & 17,100 & $S$ & \\
\hline Si & 1,590 & $S$ & \\
\hline $\mathrm{SO}_{4}$ & 37,100 & $\mathrm{~s}$ & \\
\hline $\mathrm{Sr}$ & $<66.7$ & $\bar{S}$ & \\
\hline TOC & 18,700 & $\bar{s}$ & \\
\hline $\mathrm{U}_{\text {TOTAL }}$ & $<3,340$ & $S$ & \\
\hline
\end{tabular}


Table D4-7. ' Best-Basis Total Inventory Estimates for Nonradioactive Components in Tank 241-AN-104 as of May 31, 1997. ${ }^{1}$ (2 sheets)

\begin{tabular}{|c|c|c|c|}
\hline Anafinte & 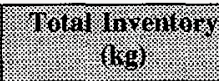 & (5. $14 ., 0,6)^{2}$ & Conninuril \\
\hline $\mathrm{Zr}$ & 64.2 & $\mathrm{~S}$ & \\
\hline$\%$ water & 48.4 & $S$ & \\
\hline Density $(\mathrm{g} / \mathrm{mL})$ & 1.45 & $\mathrm{~s}$ & \\
\hline
\end{tabular}

Notes:

${ }^{1}$ The tank is active. Waste transfers will change tank contents.

${ }^{2} \mathrm{~S}=$ sample-based, $\mathrm{M}=\mathrm{HDW}$ model-based, $\mathrm{E}=$ engineering assessment-based,

$\mathrm{C}=$ calculated by charge balance; includes oxides as hydroxides, not including $\mathrm{CO}_{3}, \mathrm{NO}_{2}, \mathrm{NO}_{3}$, $\mathrm{PO}_{4}, \mathrm{SO}_{4}$, and $\mathrm{SiO}_{3}$ 
Table D4-8. Best-Basis Inventory Estimates for Radioactive Components in

Tank 241-AN-104 as of May 31, 1997' (Decayed to January 1, 1994). (2 sheets)

\begin{tabular}{|c|c|c|c|}
\hline 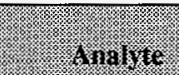 & 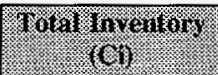 & 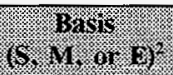 & (6) \\
\hline${ }^{3} \mathrm{H}$ & 43.3 & $\mathrm{~S}$ & Slurry/crust only \\
\hline${ }^{14} \mathrm{C}$ & 104 & $M$ & \\
\hline${ }^{59} \mathrm{Ni}$ & 5.61 & $\bar{M}$ & \\
\hline${ }^{60} \mathrm{Co}$ & $<149$ & $S$ & \\
\hline${ }^{63} \mathrm{Ni}$ & 552 & $\overrightarrow{\mathrm{M}}$ & \\
\hline${ }^{79} \mathrm{Se}$ & 11.3 & $\mathrm{M}$ & \\
\hline${ }^{90} \mathrm{Sr}$ & 93,800 & $S$ & \\
\hline${ }^{90} \mathrm{Y}$ & 93,800 & $\bar{S}$ & Referenced to ${ }^{90} \mathrm{Sr}$. \\
\hline${ }^{93} \mathrm{Zr}$ & 55.3 & $\mathrm{M}$ & \\
\hline${ }^{93 \mathrm{~m}} \mathrm{Nb}$ & $\longdiv { 4 0 . 2 }$ & $\mathrm{M}$ & \\
\hline${ }^{99} \mathrm{Tc}$ & 345 & $\mathrm{~S}$ & \\
\hline${ }^{106} \mathrm{Ru}$ & 0.0323 & $\mathrm{M}$ & \\
\hline${ }^{113 m} \mathrm{Cd}$ & 290 & $\mathrm{M}$ & \\
\hline${ }^{125} \mathrm{Sb}$ & 745 & $\bar{M}$ & \\
\hline${ }^{126} \mathrm{Sn}$ & 17.2 & $\overline{\mathrm{M}}$ & \\
\hline${ }^{129} \mathrm{I}$ & $<7.53$ & $S$ & \\
\hline${ }^{134} \mathrm{Cs}$ & 123 & $\mathrm{M}$ & \\
\hline${ }^{137} \mathrm{Cs}$ & $2.32 \mathrm{E}+06$ & $\bar{S}$ & \\
\hline${ }^{137 \mathrm{~m}} \mathrm{Ba}$ & $2.20 \mathrm{E}+06$ & $S$ & Referenced to ${ }^{137} \mathrm{Cs}$ \\
\hline${ }^{151} \mathrm{Sm}$ & 40,000 & $\bar{M}$ & \\
\hline${ }^{152} \mathrm{Eu}$ & 14.5 & $M$ & \\
\hline${ }^{154} \mathrm{Eu}$ & $<676$ & $S$ & \\
\hline${ }^{155} \mathrm{Eu}$ & $<4,730$ & $S$ & \\
\hline${ }^{226} \mathrm{Ra}$ & $4.63 \mathrm{E}-04$ & $\bar{M}$ & \\
\hline${ }^{227} \mathrm{Ac}$ & 0.00286 & $\overrightarrow{\mathrm{M}}$ & \\
\hline${ }^{228} \mathrm{Ra}$ & 1.03 & $\mathrm{M}$ & \\
\hline
\end{tabular}


Table D4-8. Best-Basis Inventory Estimates for Radioactive Components in Tank 241-AN-104 as of May 31, 1997' (Decayed to January 1, 1994). (2 sheets)

\begin{tabular}{|c|c|c|c|}
\hline (1) & 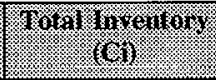 & 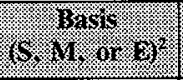 & (1) Gomint \\
\hline${ }^{229} \mathrm{Th}$ & 0.0239 & $\mathrm{M}$ & \\
\hline${ }^{231} \mathrm{~Pa}$ & 0.0128 & $M$ & \\
\hline${ }^{232} \mathrm{Th}$ & 0.109 & $\mathbf{M}$ & \\
\hline${ }^{232} \mathrm{U}$ & 3.17 & $M$ & \\
\hline${ }^{233} \mathrm{U}$ & 12.1 & $\mathbf{M}$ & \\
\hline${ }^{234} \mathrm{U}$ & 3.59 & $\mathbf{M}$ & \\
\hline${ }^{235} \mathrm{U}$ & 0.140 & $\mathrm{M}$ & \\
\hline${ }^{236} \mathrm{U}$ & 0.193 & M & \\
\hline${ }^{237} \mathrm{~Np}$ & $<30.7$ & $S$ & \\
\hline${ }^{238} \mathrm{Pu}$ & 7.97 & $\mathrm{M}$ & \\
\hline${ }^{238} \mathrm{U}$ & 3.86 & M & \\
\hline $2399240 \mathrm{Pu}$ & 9.70 & $S$ & \\
\hline${ }^{241} \mathrm{Am}$ & $<50.0$ & $S$ & Slurry $=9.04 \mathrm{Ci}$ \\
\hline${ }^{241} \mathrm{Pu}$ & 664 & $\bar{M}$ & \\
\hline${ }^{242} \mathrm{Cm}$ & 0.524 & $\mathrm{M}$ & \\
\hline${ }^{242} \mathrm{Pu}$ & 0.00306 & $\mathrm{M}$ & \\
\hline${ }^{243} \mathrm{Am}$ & 0.00124 & M & \\
\hline${ }^{243} \mathrm{Cm}$ & $<0.48$ & $S$ & \\
\hline${ }^{244} \mathrm{Cm}$ & $<Y 2.0$ & $S$ & \\
\hline
\end{tabular}

Notes:

'The tank was active. Waste transfers will change tank contents.

${ }^{2} \mathrm{~S}$ = sample-based, $\mathrm{M}=\mathrm{HDW}$ model-based, $\mathrm{E}=$ engineering assessment-based,

$\mathrm{C}=$ calculated by charge balance; includes oxides as hydroxides, not including $\mathrm{CO}_{3}, \mathrm{NO}_{2}, \mathrm{NO}_{3}$, $\mathrm{PO}_{4}, \mathrm{SO}_{4}$, and $\mathrm{SiO}_{3}$ 


\section{D5.0 APPENDIX D REFERENCES}

Agnew, S. F., 1997, Hanford Tank Chemical and Radionuclide Inventories: HDW Model Rev. 4, Los Alamos National Laboratory, Los Alamos, New Mexico.

Hanlon, B. M., 1997, Waste Tank Summary Report for Month Ending May 31, 1997, HNF-EP-0182-110, Lockheed Martin Hanford Corp. for Fluor Daniel Hanford Inc., Richland, Washington.

Hendrickson, D. W., 1994, Grout Treatment Facility Waste Feed Projections, WHC-SD-WM-TI-528, Rev. 0, Westinghouse Hanford Company, Richland, Washington.

Hodgson, K. M., and M. D. LeClair, 1996, Work Plan for Defining a Standard Inventory Estimate for Wastes Stored in Hanford Site Underground Tanks, WHC-SD-WM-WP-311, Rev, 1, Lockheed Martin Hanford Corp. for Fluor Daniel Hanford, Inc., Richland, Washington.

Kupfer, M. J., A. L. Boldt, B. A. Higley, K. M. Hodgson, L. W. Shelton, R. A. Watrous, S. L. Lambert, D. E. Place, R. M. Orme, G. L. Borsheim, N. G. Colton, M. D. LeClair, R. T. Winward, and W. W. Schulz, 1997, Standard Inventories of Chemicals and Radionuclides in Hanford Site Tank Wastes, HNF-SD-WM-TI-740, Rev. 0, Lockheed Martin Hanford Corp. for Fluor Daniel Hanford, Richland, Washington.

Shekarriz, A., J. M. Bates, R. E. Bauer, N. S. Cannon, M. A. Chieda, B. E. Hey, C. G. Linschooten, L. A. Mahoney, D. R. Rector, and E. R. Siciliano, 1997, Composition and Quantities of Retained Gas Measured in Hanford Waste Tanks 241-AW-101,A-101, $A N-105, A N-104$, and $A N-103$, PNNL-11450, Rev. 1, Pacific Northwest National Laboratory, Richland, Washington.

Stauffer, L. A., 1997, Solids Level Changes for Tanks 241-AN-103, 241-AN-104, 241-AN-105, 241-AW-101, and 241-SY-103, (internal memorandum 74330-97-105 to B. M. Hanlon, April 3), Lockheed Martin Hanford Corp. for Fluor Daniel Hanford, Inc., Richland, Washington.

Watrous, R. A., and D. W. Wootan, 1997, Activity of Fuel Batches Processed Through Hanford Separations Plants, 1944 Through 1989, Lockheed Martin Hanford Corp. for Fluor Daniel Hanford Inc., Richland, Washington. 
HNF-SD-WM-ER-690 Rev. 0

This page intentionally left blank. 
APPENDIX E

BIBLIOGRAPHY FOR TANK 241-AN-104 
HNF-SD-WM-ER-690 Rev. 0

This page intentionally left blank. 


\section{APPENDIX E}

\section{BIBLIOGRAPHY FOR TANK 241-AN-104}

Appendix $\mathrm{E}$ is a bibliography that supports the characterization of tank 241-AN-104. The bibliography represents an in-depth literature search of all known information sources that provide sampling, analysis, surveillance, and modeling information, as well as processing occurrences associated with tank 241-AN-104 and its respective waste types.

The references in this bibliography are separated into three broad categories containing references broken down into subgroups. These categories and their subgroups are listed below.

\section{NON-ANALYTICAL DATA}

Ia. Models/Waste Type Inventories/Campaign Information

Ib. Fill History/Waste Transfer Records

Ic. Surveillance/Tank Configuration

Id. Sample Planning/Tank Prioritization

Ie. Data Quality Objectives/Customers of Characterization Data

\section{ANALYTICAL DATA - SAMPLING OF TANK WASTE AND WASTE TYPES}

IIa. Sampling of tank 241-AN-104

IIb. Sampling of 242-A Evaporator Streams

\section{COMBINED ANALYTICAL/NON-ANALYTICAL DATA}

IIIa. Inventories using both Campaign and Analytical Information (Best Estimate)

IIIb. Compendium of Existing Physical and Chemical Documented Data Sources

This bibliography is broken down into the appropriate sections with an annotation at the end of each reference. Where possible, a reference is provided for information sources. A majority of the information listed below is available in the Tank Characterization safety and Resource Center. 


\section{NON-ANALYTICAL DATA}

Ia. Models/Waste Type Inventories/Campaign Information

Agnew, S. F., J. Boyer, R. A. Corbin, T. B. Duran, J. R. Fitzpatrick, K. A. Jurgensen, T. P. Ortiz, and B. L. Young, 1997, Hanford Tank Chemical and Radionuclide Inventories: HDW Model Rev. 4, LA-UR-96-3680, Rev. 0, Los Alamos National Laboratory, Los Alamos, New Mexico.

- Contains tank layer and supernatant modeis, the historical tank content estimate for Hanford Site underground waste storage tanks, and a list of Hanford Site waste types.

Ib. Fill History/Waste Transfer Records

Agnew, S. F., R. A. Corbin, T. B. Duran, K. A. Jurgensen, T. P. Ortiz, and B. L. Young, 1994, Waste Status and Transaction Record Summary, WSTRS, Rev. 4, LA-UR-97-311, Rev. 0, Los Alamos National Laboratory, Los Alamos, New Mexico.

- Contains spreadsheets showing all available data on tank additions and transfers.

Koreski, G. M., and J. N. Strode, 1996, Operational Waste Volume Projections, WHC-SD-WM-ER-029, Rev. 22, Westinghouse Hanford Company, Richland, Washington.

- Contains an account of waste transfers for double-shell tanks, including waste type and volume, source, and destination.

Ic. Surveillance/Tank Configuration

Lipnizki, J., 1997, Waste Tank Risers Available for Sampling, WHC-SD-WM-TI-710, Rev. 4, Westinghouse Hanford Company, Richland, Washington.

- Describes riser information.

Salazar, B. E. 1994, Double-Shell Underground Waste Storage Tank Riser Survey, WHC-SD-RE-TI-093, Rev. 4, Westinghouse Hanford Company, Richland, Washington.

- Describes the double-shell underground waste tank riser survey. 
Tran, T. T., 1993, Thermocouple Status Single-Shell and Double-Shell Waste Tanks, WHC-SD-WM-TI-553, Rev. 0, Westinghouse Hanford Company, Richland, Washington.

- Contains information about the thermocouple trees installed in the Hanford Site underground waste tanks, for example, installation date, material condition, riser number, height of individual thermocouples above the tank bottom, high temperature reading, frequency of surveillance, and type of thermocouple.

Id. Sample Planning/Tank Prioritization

Baldwin, J. H., 1997, Push Mode Core Sampling and Analysis Plan for Tank 241-AN-104 in Support of the Privatization Request Tank for Proposal, WHC-SD-WM-TSAP-111, Rev. 0A, Westinghouse Hanford Company, Richland, Washington.

- Contains detailed sampling and analysis procedure information for tank 241-AN-104 based on TWRS Privatization's Request for Proposal.

Bell, K. E., 1993, Tank Waste Remediation System Tank Waste Characterization Plan, WHC-SD-WM-PLN-047, Rev. 1, Westinghouse Hanford Company, Richland, Washington.

- Coordinates the activities of the tank farms and the laboratories by establishing standard sample procurement and analysis procedures, standard quality control procedures and criteria, and prioritizing tank samples.

Brown, T. M., J. W. Hunt, and L. J. Fergestrom, 1997, Tank Waste Characterization Basis, HNF-SD-WM-TA-164, Rev. 3, lockheed Martin Hanford Corp. for Fluor Daniel Hanford, Inc., Richland, Washington.

- Summarizes the technical basis for characterizing tank waste and assigns a priority number to each tank.

Winkelman, W. D., 1996, Tank 241-AN-104 Push Mode Core Sampling and Analysis Plan, WHC-SD-WM-TSAP-086, Rev. 0, Westinghouse Hanford Company, Richland, Washington.

- Contains detailed sampling and analysis procedure information for tank 241-AN-104 based on applicable DQOs. 
Grimes, G. W., 1977, Hanford Long-Term Defense High-Level Waste Management Program Waste Sampling and Characterization Plan, RHO-CD-137, Rockwell Hanford Operations, Richland, Washington.

- Early characterization planning document.

Phillips, J. R., A. Shekarriz, and J. M. Bates, 1996, Sampling Plan for Tank 241-AN-104 Retained Gas Samples Deployment, PNNLMIT:030796, Pacific Northwest National Laboratory, Richland, Washington.

- Letter report and May 30, 1996 addendum provide RGS requirements for the 241-AN-104 TSAP.

Winkelman, W. D., 1996, Tank 241-AN-104 Tank Characterization Plan, WHC-SD-WM-SD-TP-384, Rev. 3, Westinghouse Hanford Company, Richland, Washington.

- Discusses all relevant DQOs and how they will be met for tank 241-AN-104.

Winkelman, W. D., J. W. Hunt, and L. J. Fergstrom, 1996, FY 1997 Tank Waste Analysis Plan, WHC-SD-WM-PLN-120, Rev. 1, Lockheed Martin Hanford Corp. for Fluor Daniel Hanford, Inc., Richland, Washington.

- Contains Tri-Party Agreement (see Ecology et al. 1996 listing in Section 5.0) requirement-driven TWRS Characterization Program information and a list of tanks addressed in fiscal year 1997.

Winters, W. I., 1996, Privatization Samples for Envelopes " $A$, " $B$, " and " $C$," WHC-SD-WM-TP-495, Rev. 2, Westinghouse Hanford Company, Richland, Washington.

- Contains test plan to describe the method for sampling and analysis for privatization.

Ie. Data Quality Objectives (DQO) and Customers of Characterization Data

Carothers, K. G., 1994, Data Quality Objectives for the Waste Compatibility Program, WHC-SD-WM-DQO-001, Rev. 0, Westinghouse Hanford Company, Richland, Washington.

- Provides requirements for safe and efficient combination of waste from different tanks and tank systems. 
Dukelow, G. T., J. W. Hunt, H. Babad, and J. E. Meacham, 1995, Tank Safety Screening Data Quality Objective, WHC-SD-WM-SP-004, Rev, 2, Westinghouse Hanford Company, Richland, Washington.

- Determines whether tanks are under safe operating conditions.

Jones, T. E., and K. D. Wiemers, 1996, Data Requirements for TWRS Privatization Characterization of Potential Low Activity Waste Feed, WHC-SD-WM-DQO-023, Rev. 0, Westinghouse Hanford Company, Richland, Washington.

- Documents the need for the privatization function within TWRS.

McDuffie, N. G., and G. D. Johnson, 1995, Flammable Gas Tank Safety Program: Data Requirements for Core Sample Analysis Developed Through the Data Quality Objectives (DQO) Process, WHC-SD-WM-DQO-004, Rev. 2, Westinghouse Hanford Company, Richland Washington.

- Provides data needs for evaluating flammability issues in the tank.

Minteer, D. J., 1996, Waste Tank 214-AN-105 Gas Release Response Recommendations, WHC-SD-WM-ER-524, Rev. 0, Westinghouse Hanford Company, Richland, Washington.

- Describes gas release response recommendations.

Slankas, T. J., M. J. Kupfer, and W. W. Schultz, 1995, Data Needs and Attendant Data Quality Objectives for Tank Waste Pretreatment and Disposal, WHC-SD-WM-DQO-022, Rev. 0, Westinghouse Hanford Company, Richland, Washington.

- Documents the needs for the pretreatment function within TWRS.

\section{ANALYTICAL DATA - SAMPLING OF TANK WASTE AND WASTE TYPES}

IIa. Sampling of tank 241-AN-104

Harmon, H. D., 1991, Watch List for Tanks Which May Have Hydrogen Buildup, (letter 9001478B.R1 to R. E. Gerton, February 8), Westinghouse Hanford Company, Richland, Washington.

- Reports the results of the slurry growth study of actual waste. 
Jansky, M. T., and K. T. Patterson, 1984, Laboratory Batch Boil Down on Actual Double-Shell Slurry Feed: $A N-104 \& A N-105$ Blend, (letter 65453-84-090 to L. M. Sasaki, April 2), Rockwell Hanford Operation, Richland, Washington.

- Reports the results of the slurry growth study of actual waste.

Jansky, M. T., 1984, Boil Down \& Slurry Growth Experiment with Tank $A N-104 \& A N-105$ Double-Shell Slurry Feed (DSSF), (letter 65611-84-008 to L. M. Sasaki, January 19), Rockwell Hanford Operation, Richland, Washington.

- Provide information in support of double-shell flowsheet and for determining scale-up effects on slurry growth.

King, A. G., 1996, TWRS Privatization Private Contractor Samples for LowActivity Waste Envelope B Tank 241-AN-104 Preliminary Analytical Report, (letter 9655753 to C. G. Mattsson, November 20), Rust Federal Services of Hanford, Inc. for Fluor Daniel Hanford, Inc., Richland, Washington.

- Provides analyses of TWRS privatization contract samples, low-activity waste feed envelope B Tank.

Mauss, B. M., 1984, Chemical Compositions of 102-AY, 101-AW, 105-AN, and $104-A N$, (internal memorandum $65453-84-348$ to E. G. Gratny, November 9), Rockwell Hanford Operation, Richland, Washington.

- Provides the chemical compositions of 105-AN tank.

Payne, M. A., 1992, Addition of Water to Watch List Tanks, (letter 9251519 to R. E. Gerton, March 12), Westinghouse Hanford Company, Richland, Washington.

- Reports the results of the slurry growth study of actual waste.

Sasaki L. M., 1984, Results of Organic Analysis of Double Shell Slurry, (letter 65611-84-053 to D. J. Flesher, April 5), Rockwell Hanford Operation, Richland, Washington.

- Reports the results of the slurry growth study of actual waste. Also supports complexant removal studies. 
Stewart, C. W., J. M. Alzheimer, C. L. Shepard, G. Terrones, G. Chen, and N. E. Wilkins, 1996, In Situ Determination of Rheological Properties and Void Fraction: Hanford Waste Tank 241-AN-104, PNNLMIT:021696, Pacific Northwest National Laboratory, Richland, Washington.

- Provides results from operating the ball rheometer and void fraction instrument in Hanford Site waste tank 241-AN-104.

Steen, F. H., 1997, Final Report for Tank 241-AN-104, Cores 163 and 164, HNF-SD-WM-DP-226, Rev. 1A, Rust Federal Services of Hanford, Inc. for Fluor Daniel Hanford, Inc., Richland, Washington.

- Contains sample analyses from 1996 tank 241-AN-104 core sampling event.

Shekarriz, A., R. E. Bauer, D. R. Rector, N. S. Cannon, L. A. Mahoney, B. E. Hey, M. A. Chieda, C. G. Linshooten, J. M. Bates, F. J. Reitz, and E. R. Siciliano., 1997, Retained Gas Sampler Measurement Results for Hanford Waste Tanks 241-AW-101, 241-A-101, 241-AN-104, 241-AN-104 241-AN-103, PNNL-11450, Rev. 1, Pacific Northwest National Laboratory, Richland, Washington.

- Contains the measurements resulting from retained gas sampling tanks.

Wilkins, N. E., 1995, Results of Gas Monitoring of Double-Shell Flammable Gas Watch List Tanks, WHC-SD-WM-TI-682, Rev. OA, Westinghouse Hanford Company, Richland, Washington.

- Describes gas monitoring of double-shell tanks.

Wilkins, N. E., R. E. Bauer, and D. M. Ogden, 1996, Results of Vapor Space Monitoring of Flammable Gas Watch List Tanks, HNF-SD-WM-TI-797, Rev. 1, Lockheed Martin Hanford Corp. for Fluor Daniel Hanford, Inc., Richland, Washington.

- Contains results of the standard hydrogen monitoring system, vapor grab sampling, and gas character system monitoring of tank 241-AN-104 and other watch list tanks. 
IIb. Sampling of 242-A Evaporator Streams

Cash, R. J., 1996, Flammable Gas Safety Program Report on May 30, 1996 Gas Release Event in Tank AN-105, 1996, (letter 9652960 to J. K. McClusky, July 3), Westinghouse Hanford Company, Richland, Washington.

- Reports assessment and actions in response to gas release event of May 30, 1996.

Jansky, M. T., 1984, Laboratory Support for Upcoming 242-Evaporator Campaign, Run 84-5, (letter 65453-84-134 to E. F. Gratny, May 10), Rockwell Hanford Operation, Richland, Washington.

- Reports analytical data to support Evaporator Campaign, Run 84-5.

Johnson, G. D., and M. A. Payne, 1994, Tank AN-105 Gas Release Event of May 1994, (letter 9455249 to distribution, July 29), Westinghouse Hanford Company, Richland, Washington.

- Reviews data and evaluates the safety implications of gas release event of May 17, 1994.

Pontious, N. L., 1986, 242-A Evaporator/Crystal; FY-1985 Campaign 85-3 Post-Run Document, WHC-SD-WM-PE-023, Rev. 0, Westinghouse Hanford Company, Richland, Washington.

- Describes Evaporator Campaign Run 85-3.

Von Bergen, B. H., 1987, 242-A Evaporator Run Schedule for Room 88-1, (internal memorandum 13331-87-975 to G. L. Dunford, December 18), Westinghouse Hanford Company, Richland, Washington.

- Contains 1988 evaporator run planning information. 


\section{COMBINED ANALYTICAL/NON-ANALYTICAL DATA}

IIIa. Inventories from Campaign and Analytical Information

Agnew, S. F., J. Boyer, R. A. Corbin, T. B. Duran, J. R. Fitzpatrick, K. A. Jurgensen, T. P. Ortiz, and B. L. Young, 1997, Hanford Tank Chemical and Radionuclide Inventories: HDW Rev. 4, LA-UR-96-3680, Rev. 0, Los Alamos National Laboratory, Los Alamos, New Mexico.

- Contains waste type summaries and primary chemical compound/analyte and radionuclide estimates for sludge, supernatant, and solids.

IIIb. Compendium of data from other sources physical and chemical

Agnew, S. F., and J. G. Watkin, 1994, Estimation of Limiting Solubilities for lonic Species in Hanford Waste Tank Supernates, LAUR-94-3590, Los Alamos National Laboratory, Los Alamos, New Mexico.

- Provides solubility ranges used for key chemical and radionuclide components based on supernatant sample analyses.

Brevick, C. H., L. A. Gaddis, and E. D. Johnson, 1995, Tank Waste Source Term Inventory Validation, Vol I \& II., WHC-SD-WM-ER-400, Rev. 0, Westinghouse Hanford Company, Richland, Washington.

- Contains a quick reference to sampling information in spreadsheet or graphical form for 23 chemicals and 11 radionuclides for all tanks.

Brevick, C. H., L. A. Gaddis, and S. D. Consort, 1995, Supporting Document for the Southeast Quadrant Historical Tank Content Estimate for AN Tank Farm (Vol I and II), WHC-SD-WM-ER-314, Rev. 0, Westinghouse Hanford Company, Richland, Washington.

- Describes the AN Tank Farm.

Hanlon, B. M., 1997, Waste Tank Summary Repeat for Month Ending May 31 , 1997, HNF-EP-0182-110, Lockheed Martin Hanford Corp., for Fluor Daniel Hanford Inc., Richland, Washington.

- Describes the waste tank summary report. 
Husa, E. I., 1993, Hanford Site Waste Storage Tank Information Notebook, WHC-EP-0625, Westinghouse Hanford Company, Richland, Washington.

- Contains in-tank photos and summaries on the tank description, leak detection system, and tank status.

Husa, E. I., 1995, Hanford Waste Tank Preliminary Dryness Evaluation, WHC-SD-WM-TI-703, Rev. 0, Westinghouse Hanford Company, Richland, Washington.

- Assesses relative dryness between tanks.

Olson, N. J., 1989, Electrochemical Testing A537 Carbon Steel Purex Scrub Solutions For Corrosion Behavior-Preliminary Report, (letter LET-011689 to D. A. Reynolds, January 16), Pacific Northwest Laboratory, Richland, Washington.

- Presents the results from corrosion testing with scrub solutions for corrosion behavior.

Shelton, L. W., 1995, Chemical and Radionuclide Inventory for Single- and Double-Shell Tanks, (internal memorandum 74A20-96-30 to D. J. Washenfelder, February 28), Westinghouse Hanford Company, Richland, Washington.

- Contains a tank inventory estimate based on analytical information.

Shelton, L. W., 1995, Chemical and Radionuclide Inventory for Single- and Double-Shell Tanks, (internal memorandum 75520-95-007 to R. M. Orme, August 8), Westinghouse Hanford Company, Richland, Washington.

- Contains a tank inventory estimate based on analytical information.

Shelton, L. W., 1995, Chemical and Radionuclide Inventory for Single- and Double-Shell Tanks, (internal memorandum 71320-95-002 to F. M. Cooney, February 14), Westinghouse Hanford Company, Richland, Washington.

- Contains a tank estimate based on analytical information. 
Van Vleet, R. J., 1993, Radionuclide and Chemical Inventories for DoubleShell Tanks, WHC-SD-WM-TI-543, Rev. 1, Westinghouse Hanford Company, Richland, Washington.

- Contains radionuclide chemical inventories for double-shell tanks. 
HNF-SD-WM-ER-690 Rev. 0

This page intentionally left blank. 


\section{DISTRIBUTION SHEET}

\begin{tabular}{|c|c|c|c|c|c|}
\hline \multirow{2}{*}{ To } & \multirow{2}{*}{\multicolumn{3}{|c|}{ From }} & \multicolumn{2}{|l|}{ Page 1 of 2} \\
\hline & & & & \multicolumn{2}{|c|}{$05 / 08 / 97$} \\
\hline \multicolumn{4}{|l|}{ Project Title/Work Order } & \multicolumn{2}{|c|}{ EDT No. EDT-617664 } \\
\hline \multicolumn{4}{|c|}{$\begin{array}{l}\text { Tank Characterization Report for Double-She } 11 \text { Tank 241-AN-104, } \\
\text { HNF-SD-WM-ER-690. Rev. } 0\end{array}$} & \multicolumn{2}{|c|}{ ECN No. $\quad N / A$} \\
\hline Name & MSIN & $\begin{array}{l}\text { Text } \\
\text { With All } \\
\text { Attach. }\end{array}$ & Text Only & $\begin{array}{l}\text { Attach./ } \\
\text { Appendix } \\
\text { Only }\end{array}$ & $\begin{array}{c}\text { EDT/ECN } \\
\text { Only }\end{array}$ \\
\hline
\end{tabular}

\section{OFFSITE}

Sandia Nationa] Laboratory

P.0. Box 5800

MS-0744, Dept. 6404

Albuquerque, NM 87815

D. Powers

Nuclear Consulting Services Inc.

P. O. Box 29151

Columbus, OH 43229-01051

J. L. Kovach

$x$

Chemical Reaction Sub-TAP

P.0. Box 271

Lindsborg, KS 67456

B. C. Hudson

SAIC

20300 Century Boulevard. Suite 200-B

Germantown, MD 20874

H. Sutter

Los Alamos Laboratory

CST-14 MS-J586

P. 0. Box 1663

Los Alamos, NM 87545

S. F. Agnew

Los Alamos Technical Associates

T. T. Tran

B1-44 X

Tank Advisory Panel

102 Windham Road

Oak Ridge, TN 37830

D. 0. Campbe 17 


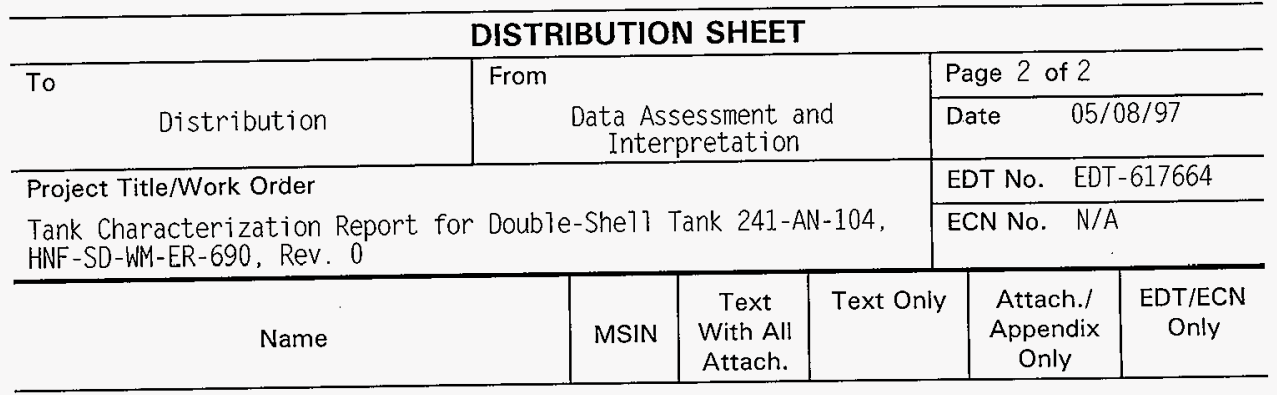

\section{ONSITE}

Department of Energy - Richland Operations

J. F. Thompson

W. S. Liou

J. A. Poppiti

N. W. Withis

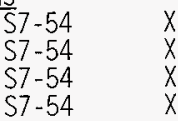

DE\&S Hanford. Inc.

R. J. Cash

W. L. Cowley

G. L. Dunford

G. D. Johnson

J. E. Meacham

S7-14 $\quad X$

R2-54 $\quad X$

A2-34 $X$

S7-14 $\quad X$

S7-14 $\quad X$

Fluor Daniel Northwest

J. L. Stroup

S3-09 $\quad x$

Lockheed Martin Hanford, Corp.

K. M. Hodgson

T. A. Hu

T. J. Kelley

L. M. Sasaki

B. C. Simpson

L. R. Webb

ERC (Environmental Resource Center)

T.C.S.R.C.

$\mathrm{HO}-34 \quad \mathrm{X}$

R2-12

S7-21

R2 -12

R2 -12

R2 -12

R1-51

R1-10

$x$
$x$
$x$

Lockheed Martin Services, Inc.

B. G. Lauzon

Central Files

EDMC

R1 -08

A3-88

H6-08

$x$
$X$
$x$
$x$
$X$
$X$
$x$
5

Numatec Hanford Corporation

J. S. Garfield

J. S. Hertze]

R. A. Kirkbride

D. L. Lamberd

H5- 49

H5-61

H5-27

H5- 61

Pacific Northwest National Laboratory

A. F. Noonan

K9-91 X

Rust Federal Services of Hanford, Inc. C. T. Narquis 